OAK RIDGE

NATIONAL LABORATORY

MANAGED BY UT-BATTELLE

FOR THE DEPARTMENT OF ENERGY

\title{
Validation and Comparison of KENO V.a and KENO-VI
}

\author{
P. B. Fox \\ L. M. Petrie
}



Nuclear Science and Technology Division (94)

\title{
Validation and Comparison of KENO V.a and KENO-VI
}

\author{
P. B. Fox and L. M. Petrie \\ Oak Ridge National Laboratory, \\ P.O. Box 2008, \\ Oak Ridge, TN 37831-6370
}

Date Published: May 2002

Prepared by the

OAK RIDGE NATIONAL LABORATORY

Oak Ridge, Tennessee 37831

managed and operated by

UT-Battelle, LLC

for the

U.S. DEPARTMENT OF ENERGY

under contract DE-AC05-00OR22725 



\section{CONTENTS}

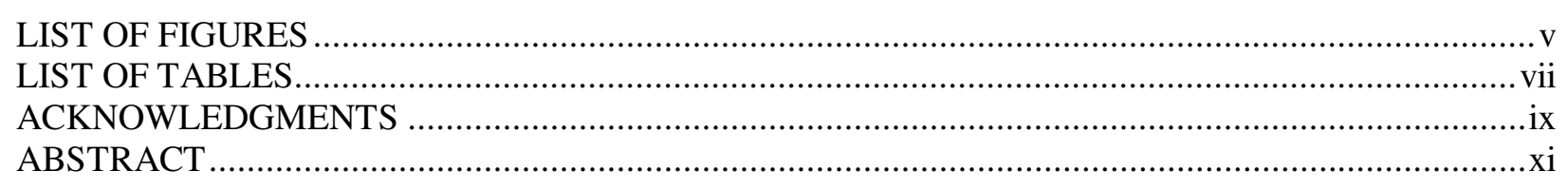

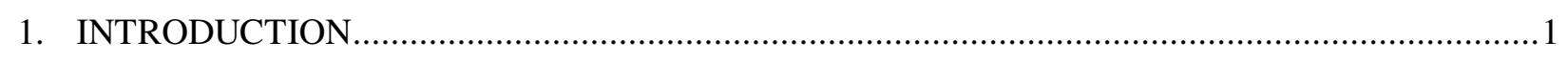

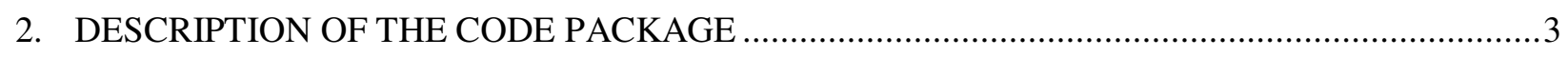

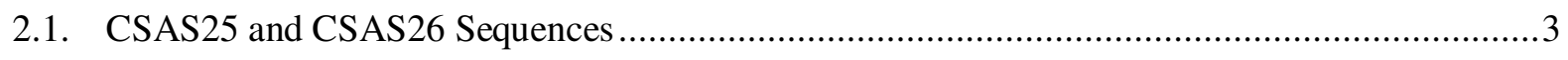

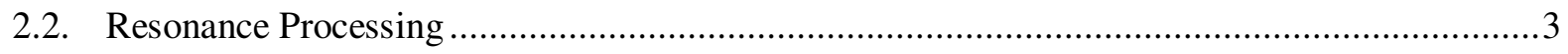

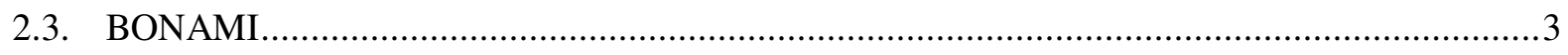

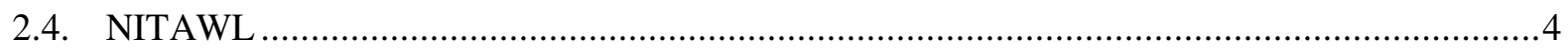

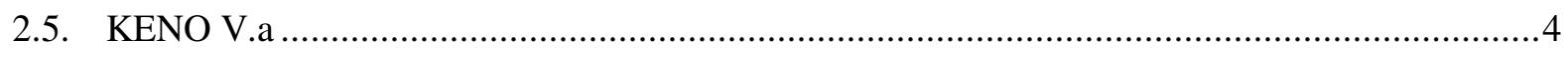

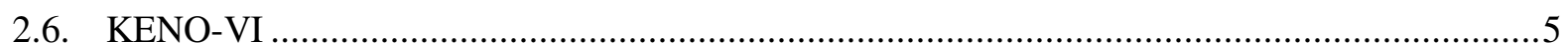

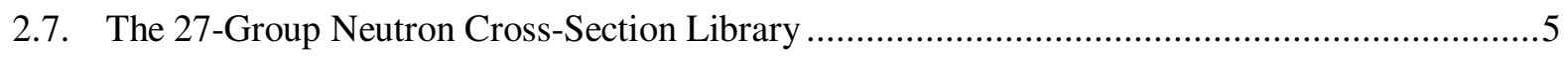

2.8. The 238-Group Neutron Cross-Section Library .......................................................

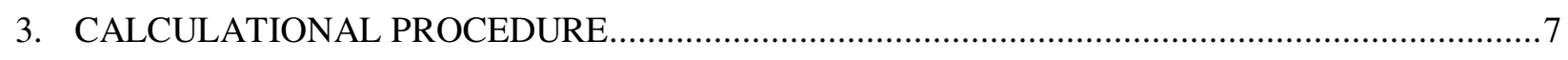

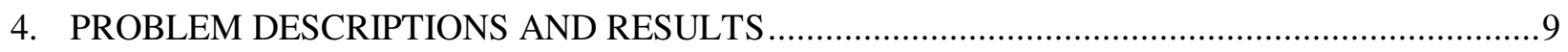

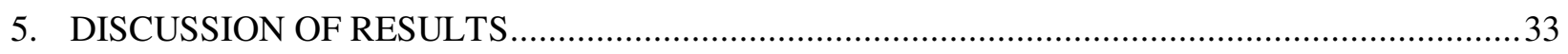

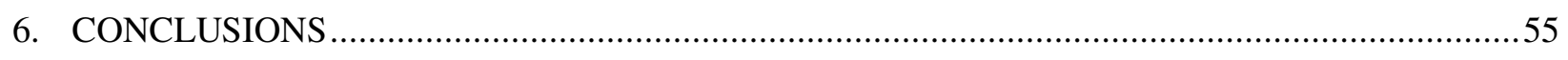

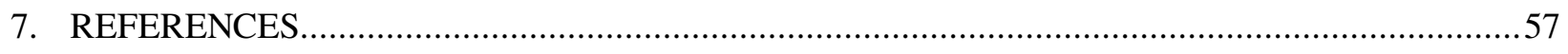

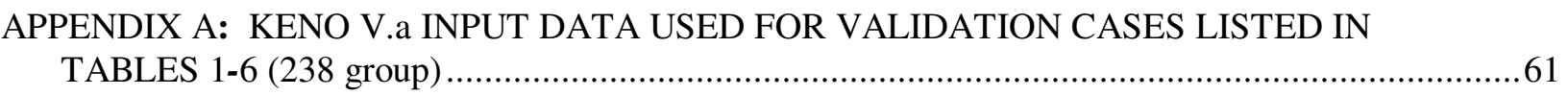

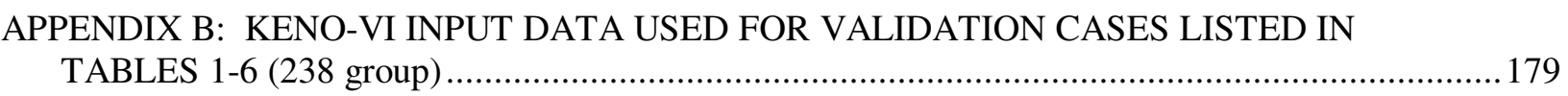




\section{LIST OF FIGURES}

Figure $\quad \underline{\text { Page }}$

1. Calculated K-effective values from Table 1.......................................................................36

2. Calculated K-effective values from Table 2 .........................................................................

3. Calculated K-effective values from Table 3 .....................................................................38

4. Calculated K-effective values from Table 4 .......................................................................39

5. Calculated K-effective values from Table 5 ........................................................................4

6. Calculated K-effective values from Table 6 ..........................................................................4

7. $\quad$ KENO V.a k-effective vs. enrichment level low enrichment experiments.....................................42

8. KENO-VI k-effective vs. enrichment level low enrichment experiments.......................................43

9. $\quad$ KENO V.a k-effective vs. enrichment level high enrichment experiments.....................................44

10. KENO-VI k-effective vs. enrichment level high enrichment experiments.....................................45

11. KENO V.a and KENO-VI (238 group) k-effective vs. enrichment level low enrichment experiments ...................................................................................................................

12. KENO V.a and KENO-VI (238 group) k-effective vs. enrichment level high enrichment

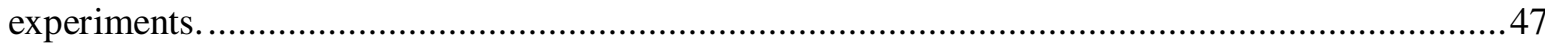

13. KENO-VI 238-group results for low enriched homogeneous and heterogeneous systems (Table 1)

14. KENO-VI 238-group results for large diameter low enriched uranium cylinders in various lattice arrangements (Table 2).

15. KENO-VI 238-group results for low enriched homogeneous uranium systems (Table 3),...............50

16. KENO-VI 238-group results for highly enriched uranium solutions (Table 4)..............................51

17. KENO-VI 238-group results for highly enriched uranium metal systems (Table 5) .......................52

18. KENO-VI 238 group results for highly enriched uranium solution systems (Table 5).....................53

19. KENO-VI 238-group results for highly enriched uranium solution systems (Table 6)....................54 


\section{LIST OF TABLES}

$\underline{\text { Table }}$

Page

1. KENO V.a and KENO-VI comparison, Calculated $k_{\text {eff }}$ for low enriched homogeneous and

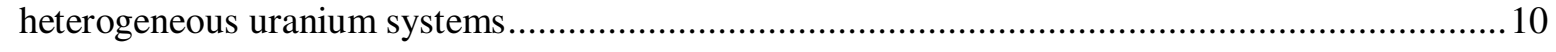

2. KENO V.a and KENO-VI comparison, Calculated $k_{\text {eff }}$ for large diameter low enriched uranium cylinders in various lattice arrangements ....

3. KENO V.a and KENO-VI comparison, Calculated $k_{\text {eff }}$ for low enriched homogeneous uranium systems.

4. KENO V.a and KENO-VI comparison, Calculated $k_{\text {eff }}$ for highly enriched uranium solution

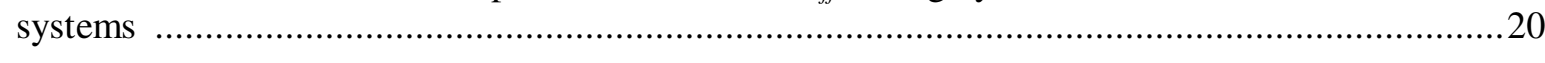

5. KENO V.a and KENO-VI comparison, Calculated $k_{e f f}$ highly enriched uranium metal and solution systems

6. KENO V.a and KENO-VI comparison, Calculated $k_{\text {eff }}$ for highly enriched uranium

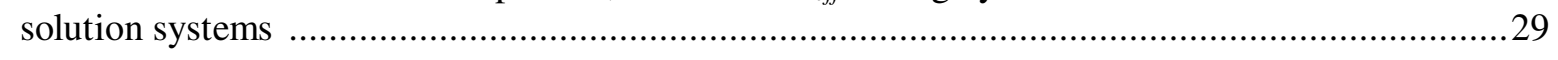

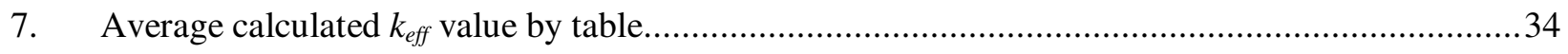

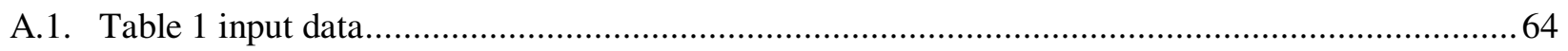

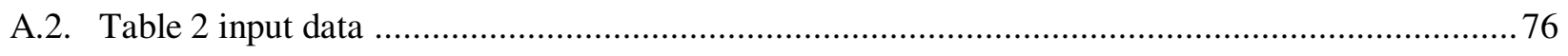

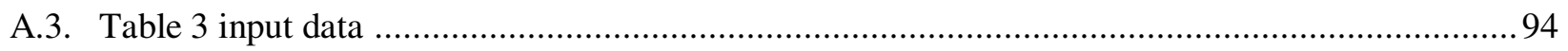

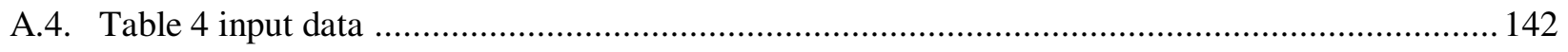

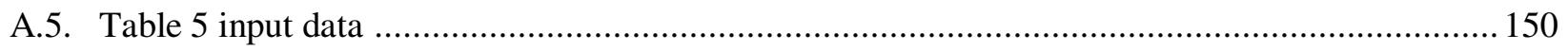

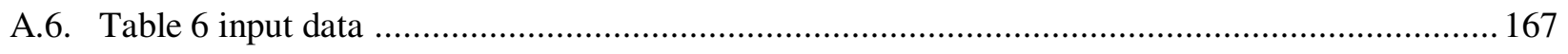

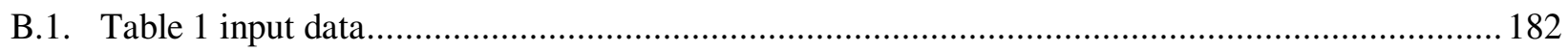

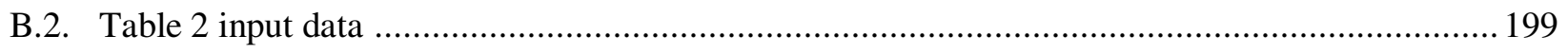

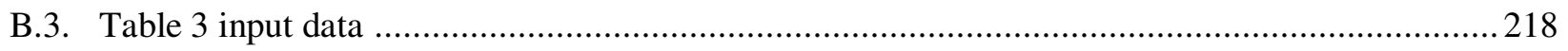

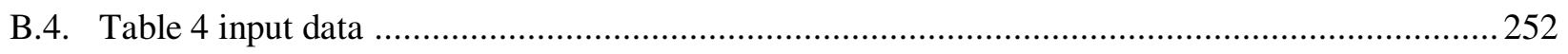

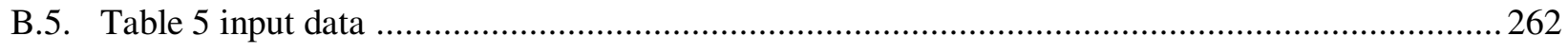

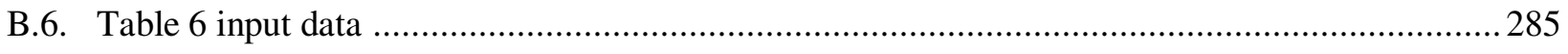




\section{ACKNOWLEDGMENTS}

This validation study of KENO-VI, as well as much of the earlier code development and the continuing software maintenance, has been sponsored jointly by the U.S. Department of Energy (DOE) Office of Nuclear Safety Policy and Standards (DOE/EH-53) and the U.S. DOE Office of Nuclear Material and Spent Fuel (DOE/EM-21). Currently, this work area is also supported by the DOE/NNSA Office of Facilities Management and ES\&H Support (DOE/NA-117). The work is being performed as part of the DOE Nuclear Criticality Safety Program. The authors are grateful for the technical reviews and comments provided by R. M. Westfall, D. F. Hollenbach, and S. M. Bowman and the guidance of C. V. Parks. A special acknowledgement is extended to Willena C. Carter for her patient assistance in preparing this report. 


\begin{abstract}
This report documents the validation and comparison of KENO V.a and KENO-VI using the SCALE 27-group ENDF/B-IV and 238-group ENDF/B-V cross-section libraries against critical experiments. Experiments considered were primarily high- or low-enriched uranium systems. The results indicate that KENO V.a and KENO-VI Monte Carlo Criticality Programs accurately calculate a broad range of critical experiments. A number of the calculations showed a positive or negative bias in excess of $1 \frac{1}{2} \%$ in k-effective $\left(k_{\text {eff }}\right)$. Classes of criticals that show a bias include $3 \%$ enriched green blocks, highly-enriched uranyl fluoride slab arrays, and highly-enriched uranyl nitrate arrays. If these biases are properly taken into account, KENO V.a and KENO-VI can be used with confidence for the design and criticality safety analysis of uranium-containing systems. KENO V.a and KENO-VI calculate the same $k_{\text {eff }}$ for a system within statistics and thus may be used interchangeably as long as the geometry of the problem can be modeled.
\end{abstract}




\section{INTRODUCTION}

The intent of this study is to validate the KENO V.a 1 and ${\mathrm{KENO}-\mathrm{VI}^{2}}^{2}$ Monte Carlo criticality programs against critical experiments. KENO V.a and KENO-VI are part of the SCALE computer code system. The sequences being validated are for KENO V.a the CSAS25 sequence of CSAS and for KENO-VI the CSAS26 sequence of CSAS6. Both are documented in the SCALE manual. ${ }^{3}$ The 27-group ENDF/B-IV and 238-group ENDF/B-V libraries were used for both the KENO V.a and KENO-VI calculations. Comparisons of KENO V.a and KENO-VI results, both 27- and 238-group, are reported in Section 4.

ANSI Standard ANS-8.1 ${ }^{4}$ requires that calculational methods used for criticality safety be validated and any bias be determined by correlating the results of critical experiments with calculations. It is essential that the computational methods used for nuclear criticality safety purposes be sufficiently accurate so that one can be confident of subcriticality when adequate safety margins are applied. It is also important that the applied safety margins not be unduly conservative.

The purpose of this report is to validate the use of KENO-VI for several types of applications. This validation is based primarily on uranium experiments ${ }^{5-9}$ Low-enriched $\left(<5 \%{ }^{235} \mathrm{U}\right)$ and highlyenriched $\left(\sim 90 \%{ }^{235} \mathrm{U}\right)$ experiments from ORNL/TM-238 ${ }^{10}$ were selected to validate and compare KENO V.a and KENO-VI. These experiments include various solution systems, varying fuel density systems, and uranium oxide and metal systems. 


\section{DESCRIPTION OF THE CODE PACKAGE}

\subsection{CSAS25 and CSAS26 Sequences}

The CSAS25 and CSAS26 control sequences in SCALE allow simplified data input to the functional modules (i.e., BONAMI, NITAWL and KENO V.a / KENO-VI, respectively). These sequences will calculate atomic densities for both mixtures and standard solutions. They also generate input data for BONAMI and NITAWL, allowing various options for treatment of the cross sections in the resonance region for both homogeneous and heterogeneous systems.

\subsection{Resonance Processing}

BONAMI and NITAWL perform resonance processing in the SCALE sequences. Both of these codes are able to process resolved and unresolved resonance data using different methods discussed in the following sections. A common characteristic of both modules is that neither BONAMI nor NITAWL treat resonance overlap or resonance interference. Several system characteristics may cause problems with the resonance treatments. One such problem, resonance overlap, which occurs when two nuclides in a mixture have resonances at the same or nearly the same energies, as discussed in Sect. M7.A of the SCALE manual. When resonance overlap is ignored, the flux used to shield the cross section is incorrect and thus the group cross section can be in error. Another form of improper resonance processing can occur when the same resonance nuclide appears in different regions (mixtures) of a geometry specification, because SCALE currently only processes one region at a time. Again, because an incorrect flux is used to shield the cross sections, the group cross sections can be in error. An example of this is in a dissolver where a fuel lump is surrounded by fissile solution containing the same resonance absorbers.

Resonance interference is similar to resonance overlap. When two resonances are close together, the higher-energy resonance affects the flux shape in the lower-energy resonance, because the flux does not recover to the asymptotic slowing-down flux form over the lower-energy resonance. Resonance interference can occur between resonances of different nuclides or two closely spaced resonances of the same nuclide. The limitations and approximations used in BONAMI and NITAWL will be discussed below.

NITAWL performs temperature broadening during resonance processing. If temperature data are included in the library, BONAMI performs temperature broadening at the user-specified problem temperature during resonance processing. Starting with SCALE 4.3, NITAWL also performs a temperature interpolation of thermal-scattering data on the master library.

\subsection{BONAMI}

The BONAMI module self-shields cross sections with Bondarenko data using the shielding-factor methodology. Nuclides with Bondarenko data carry an infinite dilute cross section on the master library and tables of dilution-dependent shielding factors. BONAMI performs an iteration for each nuclide and each energy group that has shielding factors. Convergence is achieved when the shielded total cross section changes by less than some $\varepsilon$ for all nuclides, groups, and zones. In this manner, the problem-dependent self-shielded cross sections for each nuclide and group are determined while accounting for interactions. When CSAS calls BONAMI, heterogenous geometry effects are accounted for in the escape cross section that is passed to BONAMI. 
The escape cross section is determined based on the system geometry specified in the crosssection processing portion of the SCALE input. The geometry type, materials, characteristic dimensions, and the Dancoff factor are all used to determine an escape cross section that has units equivalent to a macroscopic cross-section. The escape cross section is added to other cross sections to account for geometry effects. This method allows all nuclides to be processed by BONAMI as infinite homogeneous media in the CSAS sequences.

The performance of data shielded by the Bondarenko method depends on the adequacy of the approximations used to generate the Bondarenko data. The typical approach is to use the narrow resonance approximation to generate these data, which is adequate for a broad range of applications. When a resonance is not narrow relative to the slowing down in the system, the narrow resonance approximation breaks down and the resonance corrections for the cross sections can be in error. This breakdown has been observed for libraries that use the Bondarenko method to shield the low-energy resolved resonances for ${ }^{238} \mathrm{U}$ for systems with low hydrogen moderation ${ }^{11}$ and for many nuclides when the principal moderator is an intermediate-mass nuclide. The solution to this type of breakdown is to either carry sufficient cross-section energy groups to march across the resonance and/or to use more appropriate flux-generating methods to compute the Bondarenko factors. This problem does not occur in the SCALE END/B-IV and ENDF/B-V libraries because Bondarenko data are only used in the unresolved resonance range.

\subsection{NITAWL}

The NITAWL-II module shields cross sections with resonance data utilizing the Nordheim integral transport method. In the SCALE implementation, the infinite-dilute multi-group cross sections are adjusted by a correction value determined by NITAWL. The correction is calculated by first determining the infinite dilute contribution of each resonance to the group cross section and then by calculating what the contribution would be if the resonance was shielded for the specific problem. The geometry type, materials, characteristic dimensions, and Dancoff factor are all passed to NITAWL for determining the details of the approximations used to self-shield the cross sections. NITAWL uses two internal moderators when reconstructing the shielded flux. The slowing-down mass and scatter cross section for the principal material (first moderator) mixed with the fuel are used explicitly. The remaining materials (second moderator) are treated using an averaged slowing-down mass and scatter cross section.

A fundamental assumption of the Nordheim method is that resonances are widely spaced, both within a particular nuclide and between nuclides. If this assumption is not correct, the flux used to construct the resonance contribution to the group cross section is incorrect. Breakdowns have been observed when NITAWL was used to self-shield cross sections of fissile nuclides with overlapping resonances in dissolver-type systems $\sqrt{12}$ and in systems with intermediate-mass moderators and intermediate-mass resonance materials.

\subsection{KENO V.a}

KENO V.a is a Monte Carlo criticality program that includes an enhanced geometry package that allows modeling of a wide variety of complex three-dimensional geometries. The geometry package allows nested arrays and "holes" to be placed in the geometry model. The code allows the use of reflector options, including mirror reflection, periodic reflection, and differential albedo reflection. Automated biasing provides the capability to use reflector region weighting functions that are based on onedimensional adjoint calculations for common reflector materials. The reflector options simplify geometry data input and/or reduce the running time of a specific problem. The CSAS control module handles 
cross-section input and atom density input. Most of the major KENO parameters have defaults that work for a wide variety of problems, but which can be overridden by the analyst.

\subsection{KENO-VI}

KENO-VI is an extension of the KENO V.a Monte Carlo criticality program. KENO-VI contains features currently in KENO V.a plus a more complex geometry package. The geometry package in KENO-VI is capable of modeling any volume that can be constructed using quadratic equations. In addition, such features as geometry intersections, body rotation, hexagonal arrays, and array boundaries have been included to make the code more flexible. These features allow the user to readily solve large geometrically complex problems whose computer storage requirements and geometric complexity preclude solution by the previous versions of KENO.

A set of 13 predefined geometry shapes has been incorporated into KENO-VI. Additional volumes can be constructed using the QUADRATIC geometry card that allows the user to specify any volume that can be modeled using quadratic equations. The ability to intersect the volumes makes it possible to exactly model such things as pipe intersections, which was previously impossible in KENO V.a. The ability to rotate bodies means volumes no longer must be positioned parallel to a major axis. Hexagonal arrays were added to simplify the construction of triangular pitch arrays. The use of array boundaries makes it possible to fill a noncuboidal volume with an array, specifying the boundary where a particle leaves and enters the array.

KENO-VI is primarily an eigenvalue $k$-effective code. Other calculated quantities include lifetime, generation time, energy-dependent leakages, energy- and region-dependent absorptions, fissions, fluxes, and fission densities.

KENO-VI retains the KENO V.a features such as flexible data input, the capability of supergrouping energy-dependent data, a $P_{n}$ scattering model in the cross sections, a procedure for matching lethargy boundaries between albedos and cross sections to extend the usefulness of the albedo feature, and restart capabilities. However, due to the increased flexibility in the geometric modeling capabilities, KENO-VI usually requires more CPU time than KENO V.a to solve the same problem.

\subsection{The 27-Group Neutron Cross-Section Library}

The 27-group ENDF/B-IV library is the broad-group companion library to the 218-group ENDF/B-IV library. The 218-group library was flux collapsed using MALOCS and the MT 1099 flux file carried with the fine-group cross sections. (This flux file is the energy group representation of the original weighting spectrum used to generate the 218-group cross sections from ENDF/B-IV data.) Because of the $1 /\left(E \sigma_{t}\right)$ weighting in the resolved resonance range, the broad-group library calculates many systems nearly as well as the fine-group library does. Trends and biases in the 218-group library are preserved in the 27-group library. The library has 14 fast groups and 13 thermal groups (below $3 \mathrm{eV}$ ). The group structure was chosen to match the 16-group Hansen-Roach structure with two additional fast groups and nine additional thermal groups. The additional groups were chosen such that, for the systems considered, the broad-group calculations meet an acceptance criterion of $\Delta k / k<0.3 \%$ when compared with the reference 218-group calculation using the XSDRNPM code. This criterion was relaxed to $1 \%$ for ${ }^{238} \mathrm{U}$ in systems where the median fission energy was $>1 \mathrm{eV}$ and $<100 \mathrm{eV}$. The resonance data and the thermal-scattering data carried with the 27-group library and the 218-group library are the same and are processed by NITAWL-II. This library was conceived as a general purpose criticality analysis library, with a special interest in applicability to shipping cask analysis and thermal neutron systems. 
The 27-group library has been extensively validated against critical experiments, $1,10,13-21$ Areas of validation include highly-enriched uranium metal, compound and solution systems, moderated low-enriched uranium, heterogeneous and homogeneous systems, and plutonium metal and solution systems.

\subsection{The 238-Group Neutron Cross-Section Library}

The 238-group ENDF/B-V library ${ }^{22}$ is a general purpose criticality analysis library, and the most complete library available in SCALE. This library is also known as the LAW (Library to Analyze Radioactive $\underline{\mathbf{W}}$ aste) Library. It was initially released in version 4.3 of SCALE. The library contains data for all nuclides (more than 300) available in ENDF/B-V processed by the AMPX-77 system. ${ }^{23}$ It also contains data for ENDF/B-VI evaluations of ${ }^{14} \mathrm{~N},{ }^{15} \mathrm{~N},{ }^{16} \mathrm{O},{ }^{154} \mathrm{Eu}$, and ${ }^{155} \mathrm{Eu}$. The library has 148 fast groups and 90 thermal groups (below $3 \mathrm{eV}$ ).

Most resonance nuclides in the 238-group ENDF/B-V library have resonance data (to be processed by NITAWL-II) in the resolved resonance range and Bondarenko factors (to be processed by BONAMI) for the unresolved range. This library contains resolved resonance data for $s$-wave, $p$-wave, and $d$-wave resonances $(\mathrm{R}=0, \mathrm{R}=1$, and $\mathrm{R}=2$, respectively). These data can have a significant effect on results for undermoderated, intermediate-energy problems. Resonance structures in several light-tointermediate mass nonresonance ENDF nuclides (i.e., ${ }^{7} \mathrm{Li},{ }^{19} \mathrm{~F},{ }^{27} \mathrm{Al},{ }^{28} \mathrm{Si}$ ) are accounted for using Bondarenko shielding factors. These structures can also be important in intermediate-energy problems. The ${ }^{235} \mathrm{U}$ ENDF/B-V data result in slightly too much fission, while the ${ }^{238} \mathrm{U}$ data result in slightly too much capture. Although better than the ENDF/B-IV data, the thermal-plutonium data still appear to have problems.

All nuclides in the 238-group LAW Library use the same weighting spectrum, consisting of

1. Maxwellian spectrum (peak at $300 \mathrm{~K}$ ) from $10^{-5}$ to $0.125 \mathrm{eV}$,

2. a $1 / \mathrm{E}$ spectrum from $0.125 \mathrm{eV}$ to $67.4 \mathrm{keV}$,

3. a fission spectrum (effective temperature at $1.273 \mathrm{MeV}$ ) from $67.4 \mathrm{keV}$ to $10 \mathrm{MeV}$, and

4. a $1 / \mathrm{E}$ spectrum from 10 to $20 \mathrm{MeV}$.

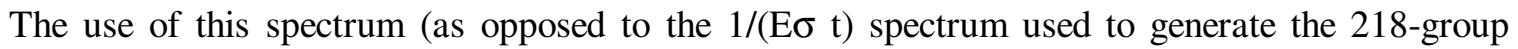
library) makes it difficult to collapse a general-purpose broad-group library that is valid over a wide range of problems.

All nuclides use a $\mathrm{P}_{5}$ Legendre expansion to fit the elastic and discrete-level inelastic scattering processes in the fast range, thereby making the library suitable for both reactor and shielding applications. $\mathrm{A}_{3}$ fit was used for thermal scattering. Thermal scattering kernels are provided at temperatures $(\mathrm{K})$ as presented in Table M4.2.7 (Sect. M4) of the SCALE manual. All other scattering processes use $\mathrm{P}_{0}$ fits. 


\section{CALCULATIONAL PROCEDURE}

In the validation and comparison process, many of the available options in CSAS25, CSAS26, KENO V.a and KENO-VI were exercised. These options include the automatic atomic density generation features, the various treatments in NITAWL, and the geometry options in KENO V.a and KENO-VI. The majority of the calculations are repeat calculations, which were modeled by different people at different times. Usually there are multiple ways that a problem may be specified, all of which are equally correct. The original input data were left substantially unchanged in the KENO V.a models. In the original input data, most problems were run for 203 generations of 600 neutrons per generation; therefore, the KENO-VI problems were run in a similar manner. KENO V.a and KENO-VI defaults have now been changed to 1000 neutrons per generation. Complete listings of the input data for KENO V.a and KENO-VI using the 238-group library are given in Appendices A and B, respectively.

With KENO V.a and KENO-VI, there is no single $k_{\text {eff }}$ that is "the answer" for the problem. The code starts with an initial neutron distribution and calculates the effective multiplication for a generation of neutrons. The next generation uses a starting distribution based on the fission points of the previous generation and calculates the effective multiplication for the current generation. Since 203 generations were run, there are 203 different answers. In the ideal situation, once the source has converged and the original (and usually arbitrary) starting distribution has decayed, the difference in the batch-to-batch $k_{\text {eff }}$ may be attributed to the statistical nature of the Monte Carlo calculation, and the "best" answer should be the average of the remaining calculations. The common practice in KENO V.a and KENO-VI is to tabulate the average $k_{\text {eff }}$ as a function of the number of generations skipped. Judgment is then required in determining the answer. Many calculations do not approach the ideal situation. For example, undersampling may exist where the number of neutrons per generation is not sufficient to sustain a proper source distribution, or the number of generations is insufficient to converge the source distribution. In order to remove the element of judgment from the interpretation of the results and to mechanize the process of validating and comparing results to previous validation, the average $k_{\text {eff }}$ by generation skipped with the lowest calculated standard deviation is reported here. This number appears as the "final value" in the plot of the average $k_{\text {eff }}$ by generation skipped in the KENO V.a and KENO-VI output. 


\section{PROBLEM DESCRIPTIONS AND RESULTS}

Brief problem descriptions and results are presented in Tables 1-6. Low-enrichment results are presented in Tables 1-3 while high-enrichment results are presented in Tables 4-6.

The experimental models used to generate Table 1 are from low-enriched uranium experiments obtained from ref. 9. These include experiments for homogeneous single-unit criticals, both reflected and unreflected, as well as heterogeneous uranium metal cylinders in water. The fissile compounds considered were $\mathrm{UO}_{2} \mathrm{~F}_{2}, \mathrm{U}$ metal, and $\mathrm{U}_{3} \mathrm{O}_{8}$, all at $4.89 \%{ }^{235} \mathrm{U}$ enrichment. The moderating materials were water, stereotex, and $\mathrm{UO}_{2} \mathrm{~F}_{2}$ solution.

The experimental models used to generate Table 2 are from low-enriched uranium experiments. These $3.85 \%$ enriched uranium metal cylinders and annuli in water were obtained from ref. 24. The critical experiments were modeled in detail from descriptions in the experiment logbook.

Table 3 includes models from ref. 8. These experiments are single-unit criticals both reflected and unreflected, at several enrichments ranging from 1.4 to $4.98 \%$. The first part of the table includes $\mathrm{UF}_{4} /$ paraffin-moderated systems and $\mathrm{UO}_{2} \mathrm{~F}_{2}$ solution systems at various moderation levels. The remainder of the table is $4.46 \%$ enriched damp oxide experiments performed at Rocky Flats.

Highly-enriched uranium experiments from ref. 9 were used to generate Table 4. These are $\mathrm{UO}_{2} \mathrm{~F}_{2}$ and $\mathrm{UO}_{2}\left(\mathrm{NO}_{3}\right)_{2}$ solution systems. Both single-unit and array systems were analyzed under a variety of reflection conditions including unreflected, fully water reflected, concrete reflected, and Plexiglas reflected.

Table 5 includes experiments from ref. 7. Single-unit reflected and unreflected systems for uranium metal, uranium alloy, $\mathrm{UO}_{2} \mathrm{~F}_{2}$ solution and $\mathrm{UO}_{2}\left(\mathrm{NO}_{3}\right)_{2}$ solutions were considered. Calculations were performed on arrays, including (1) arrays of metal units with and without interstitial moderation, (2) arrays of $\mathrm{UO}_{2} \mathrm{~F}_{2}$ slab systems, and (3) arrays of 5-liter containers of $\mathrm{UO}_{2}\left(\mathrm{NO}_{3}\right)_{2}$ having varying thickness of paraffin and/or Plexiglas.

Table 6 contains the experimental models and results from the eta experiments performed at Y-12. 5 -26 These experiments are $\mathrm{UO}_{2}\left(\mathrm{NO}_{3}\right)_{2}$ solution experiments in simple unreflected geometry. The systems are typically very dilute. The experiments included boron poisoned solutions and ${ }^{233} \mathrm{U}$ systems. 
Table 1. KENO V.a and KENO-VI comparison

Calculated $k_{\text {eff }}$ for low enriched homogeneous and heterogeneous uranium systems

\begin{tabular}{|c|c|c|c|c|c|c|}
\hline \multirow[b]{3}{*}{ Case } & \multirow[b]{3}{*}{ Experimental description } & \multirow[b]{3}{*}{ Ref. } & \multicolumn{4}{|c|}{$k_{\text {eff }} \pm \sigma$} \\
\hline & & & \multicolumn{2}{|c|}{27 group } & \multicolumn{2}{|c|}{238 group } \\
\hline & & & KENO V.a & KENO-VI & KENO V.a & KENO-VI \\
\hline CAA01 & $\begin{array}{l}\text { Experiment 1A. } 401- \\
4.89 \% \text { U rods, } 0.762-\mathrm{cm} \\
\text { diam, 30-cm long, } 1.3-\mathrm{cm} \\
\text { pitch, } \mathrm{H}_{2} \mathrm{O} \text { moderated and } \\
\text { reflected }\end{array}$ & 27 & $0.992 \pm 0.003$ & $0.989 \pm 0.002$ & $0.988 \pm .0 .003$ & $0.991 \pm 0.002$ \\
\hline CAA04 & $\begin{array}{l}\text { Experiment 6A. } 203- \\
4.89 \% \text { U rods, } 0.762-\mathrm{cm} \\
\text { diam, 30-cm long, } 2.05-\mathrm{cm} \\
\text { pitch, } \mathrm{H}_{2} \mathrm{O} \text { moderated and } \\
\text { reflected }\end{array}$ & 27 & $0.991 \pm 0.003$ & $0.991 \pm 0.002$ & $0.992 \pm 0.003$ & $0.992 \pm 0.002$ \\
\hline CAA06 & $\begin{array}{l}\text { Experiment 14A. } 398- \\
4.89 \% \text { U rods, } 0.762-\mathrm{cm} \\
\text { diam, } 30-\mathrm{cm} \text { long, } 1.3-\mathrm{cm} \\
\text { pitch, } \mathrm{H}_{2} \mathrm{O} \text { top reflector, } \\
\text { Plexiglas bottom reflector, } \\
\text { Pb reflected } 1 \text { face }\end{array}$ & 27 & $0.996 \pm 0.003$ & $0.997 \pm 0.002$ & $0.998 \pm 0.003$ & $0.996 \pm 0.002$ \\
\hline CAA08 & $\begin{array}{l}\text { Experiment 28A. } 255- \\
4.89 \% \text { U rods, } 0.762-\mathrm{cm} \\
\text { diam, } 30-\mathrm{cm} \text { long, } 1.3-\mathrm{cm} \\
\text { pitch, } \mathrm{H}_{2} \mathrm{O} \text { moderated, } \\
\mathrm{H}_{2} \mathrm{O} \text { top reflector, } \\
\text { Plexiglas bottom reflector, } \\
\mathrm{Pb} \text { reflected } 4 \text { faces }\end{array}$ & 27 & $0.995 \pm 0.002$ & $0.997 \pm 0.002$ & $0.993 \pm 0.002$ & $0.992 \pm 0.003$ \\
\hline CAA10 & $\begin{array}{l}\text { Experiment } 30 \mathrm{C} .494- \\
4.89 \% \text { U rods, } 0.762-\mathrm{cm} \\
\text { diam, } 30-\mathrm{cm} \text { long, } 1.3-\mathrm{cm} \\
\text { pitch, } \mathrm{H}_{2} \mathrm{O} \text { moderated, } \\
\mathrm{H}_{2} \mathrm{O} \text { top reflector, } \\
\text { Plexiglas bottom reflector, } \\
\mathrm{Pb} \text { reflected } 4 \text { faces, } \\
\text { boral in row } 6\end{array}$ & 27 & $1.001 \pm 0.003$ & $1.001 \pm 0.002$ & $1.002 \pm 0.002$ & $1.001 \pm 0.002$ \\
\hline CAA11 & $\begin{array}{l}\text { Experiment } 31 \mathrm{C} .494- \\
4.89 \% \mathrm{U} \text { rods, } 0.762-\mathrm{cm} \\
\text { diam, } 30-\mathrm{cm} \text { long, } 1.3-\mathrm{cm} \\
\text { pitch, } \mathrm{H}_{2} \mathrm{O} \text { moderated, } \\
\mathrm{H}_{2} \mathrm{O} \text { top reflector, } \\
\text { Plexiglas bottom reflector, } \\
\mathrm{Pb} \text { reflected } 4 \text { faces, } \\
\mathrm{Cd} \text { in row } 6\end{array}$ & 27 & $0.998 \pm 0.003$ & $0.997 \pm 0.002$ & $0.997 \pm 0.003$ & $0.997 \pm 0.002$ \\
\hline
\end{tabular}


Table 1 (continued)

Calculated $k_{\text {eff }}$ for low enriched homogeneous and heterogeneous uranium systems

\begin{tabular}{|c|c|c|c|c|c|c|}
\hline \multirow[b]{3}{*}{ Case } & \multirow[b]{3}{*}{ Experimental description } & \multirow[b]{3}{*}{ Ref. } & \multicolumn{4}{|c|}{$k_{e f f} \pm \sigma$} \\
\hline & & & \multicolumn{2}{|c|}{27 group } & \multicolumn{2}{|c|}{238 group } \\
\hline & & & KENO V.a & KENO-VI & KENO V.a & KENO-VI \\
\hline CAA14 & $\begin{array}{l}\text { Experiment } 36 \mathrm{~S}, \\
9 \times 10 \text { array of } 4.89 \% \mathrm{U} \\
\text { rods in } \sim 300 \mathrm{~g} \mathrm{U} / 1 \mathrm{UO}_{2} \mathrm{~F}_{2} \\
\text { solution, } 0.762-\mathrm{cm} \mathrm{diam,} \\
2.453-\mathrm{cm} \text { pitch in } \\
95.88-\mathrm{cm} \text {-diam tank }\end{array}$ & 27 & $0.988 \pm 0.002$ & $0.989 \pm 0.002$ & $0.990 \pm 0.002$ & $0.990 \pm 0.002$ \\
\hline CAA16 & $\begin{array}{l}\text { Experiment } 42 \mathrm{~S} \text {. } \\
6 \times 7 \text { array of } 4.89 \% \mathrm{U} \\
\text { rods in } \sim 300 \mathrm{~g} \mathrm{U} / 1 \mathrm{UO}_{2} \mathrm{~F}_{2} \\
\text { solution, } 1.31-\mathrm{cm} \mathrm{diam,} \\
3.40-\mathrm{cm} \text { pitch, in } 95.88-\mathrm{cm} \\
\text { diam tank }\end{array}$ & 27 & $0.987 \pm 0.002$ & $0.989 \pm 0.003$ & $0.991 \pm 0.002$ & $0.987 \pm 0.003$ \\
\hline CAA17 & $\begin{array}{l}\text { Experiment } 44 \mathrm{~S} \text {. } \\
6 \times 7 \text { array of } 4.89 \% \mathrm{U} \\
\text { rods in } \sim 300 \mathrm{~g} \mathrm{U} / 1 \mathrm{UO}_{2} \mathrm{~F}_{2} \\
\text { solution, } 1.31-\mathrm{cm} \mathrm{diam,} \\
3.94-\mathrm{cm} \text { pitch, in } 95.88-\mathrm{cm} \\
\text { diam tank }\end{array}$ & 27 & $0.990 \pm 0.002$ & $0.990 \pm 0.002$ & $0.989 \pm 0.002$ & $0.992 \pm 0.002$ \\
\hline CAA20 & $\begin{array}{l}4.89 \% \mathrm{U}_{3} \mathrm{O}_{8}-\text { Stereotex } \\
\text { blocks } 40.6 \mathrm{~g} \mathrm{U}-235 / 1 \\
(\mathrm{H} / \mathrm{X}=395.0) \text {, unreflected }\end{array}$ & 28 & $1.000 \pm 0.003$ & $1.000 \pm 0.004$ & $1.002 \pm 0.002$ & $1.003 \pm 0.003$ \\
\hline CAA22 & $\begin{array}{l}4.89 \% \mathrm{U}_{3} \mathrm{O}_{8}-\text { Stereotex } \\
\text { blocks } 33.3 \mathrm{~g} \mathrm{U}-235 / 1 \\
(\mathrm{H} / \mathrm{X}=503.6), \text { unreflected }\end{array}$ & 28 & $0.997 \pm 0.002$ & $0.997 \pm 0.002$ & $0.994 \pm 0.002$ & $1.000 \pm 0.002$ \\
\hline CAA25 & $\begin{array}{l}4.89 \% \mathrm{U}_{3} \mathrm{O}_{8}-\text { Stereotex } \\
\text { blocks } 40.6 \mathrm{~g} \mathrm{U}-235 / 1 \\
(\mathrm{H} / \mathrm{X}=396.7) \text {, paraffin top } \\
\text { reflector, } \mathrm{H}_{2} \mathrm{O} \text { reflected } \\
\text { bottom and sides }\end{array}$ & 28 & $0.990 \pm 0.003$ & $0.993 \pm 0.002$ & $0.997 \pm 0.003$ & $0.995 \pm 0.002$ \\
\hline CAA27 & $\begin{array}{l}4.89 \% \mathrm{U}_{3} \mathrm{O}_{8}-\text { Stereotex } \\
\text { blocks } 81.1 \mathrm{~g} \mathrm{U}-235 / 1 \\
(\mathrm{H} / \mathrm{X}=146.8) \text {, paraffin top } \\
\text { reflector, } \mathrm{H}_{2} \mathrm{O} \text { reflected } \\
\text { bottom and sides }\end{array}$ & 28 & $0.991 \pm 0.003$ & $0.990 \pm 0.002$ & $0.990 \pm 0.003$ & $0.989 \pm 0.003$ \\
\hline CAA29 & $\begin{array}{l}4.89 \% \mathrm{U}_{3} \mathrm{O}_{8}-\text { Stereotex } \\
\text { blocks } 33.3 \mathrm{~g} \mathrm{U}-235 / 1 \\
(\mathrm{H} / \mathrm{X}=504.1) \text {, paraffin top } \\
\text { reflector, } \mathrm{H}_{2} \mathrm{O} \text { reflected } \\
\text { bottom and sides }\end{array}$ & 28 & $0.995 \pm 0.002$ & $0.998 \pm 0.002$ & $0.998 \pm 0.002$ & $0.998 \pm 0.002$ \\
\hline
\end{tabular}


Table 1 (continued)

Calculated $k_{\text {eff }}$ for low enriched homogeneous and heterogeneous uranium systems

\begin{tabular}{|c|c|c|c|c|c|c|}
\hline \multirow[b]{3}{*}{ Case } & \multirow[b]{3}{*}{ Experimental description } & \multirow[b]{3}{*}{ Ref. } & \multicolumn{4}{|c|}{$k_{e f f} \pm \sigma$} \\
\hline & & & \multicolumn{2}{|c|}{27 group } & \multicolumn{2}{|c|}{238 group } \\
\hline & & & KENO V.a & KENO-VI & KENO V.a & KENO-VI \\
\hline CAA30 & $\begin{array}{l}4.89 \% \mathrm{UO}_{2} \mathrm{~F}_{2}-\text { solution } \\
42.54 \mathrm{~g} \mathrm{U}-235 / 1 \\
(\mathrm{H} / \mathrm{X}=524) \text {, in a } 20 \text {-in.- } \\
\text { diam SS cylinder, } \\
\text { unreflected }\end{array}$ & 28 & $0.989 \pm 0.003$ & $0.990 \pm 0.003$ & $0.995 \pm 0.002$ & $0.994 \pm 0.003$ \\
\hline CAA33 & $\begin{array}{l}4.89 \% \mathrm{UO}_{2} \mathrm{~F}_{2}-\text { solution } \\
24.04 \mathrm{~g} \mathrm{U}-235 / 1 \\
(\mathrm{H} / \mathrm{X}=1002) \text {, in a } 27.3-\mathrm{in} . \\
\text { diam aluminum sphere, } \\
\text { unreflected }\end{array}$ & 28 & $0.988 \pm 0.002$ & $0.991 \pm 0.002$ & $0.992 \pm 0.002$ & $0.996 \pm 0.002$ \\
\hline CAA35 & $\begin{array}{l}4.89 \% \mathrm{UO}_{2} \mathrm{~F}_{2}-\text { solution } \\
42.54 \mathrm{~g} \mathrm{U}-235 / 1 \\
(\mathrm{H} / \mathrm{X}=524) \text {, in a } 15 \text {-in.- } \\
\text { diam SS cylinder, } \\
\mathrm{H}_{2} \mathrm{O} \text { reflected }\end{array}$ & 28 & $1.002 \pm 0.003$ & $0.999 \pm 0.003$ & $0.998 \pm 0.003$ & $1.002 \pm 0.003$ \\
\hline CAA37 & $\begin{array}{l}4.89 \% \mathrm{UO}_{2} \mathrm{~F}_{2}-\text { solution } \\
31.79 \mathrm{~g} \mathrm{U}-235 / 1 \\
(\mathrm{H} / \mathrm{X}=735) \text {, in a } 15 \text {-in.- } \\
\text { diam } \mathrm{SS} \text { cylinder, } \\
\mathrm{H}_{2} \mathrm{O} \text { reflected }\end{array}$ & 28 & $0.997 \pm 0.002$ & $0.997 \pm 0.002$ & $1.001 \pm 0.002$ & $1.000 \pm 0.002$ \\
\hline CAA39 & $\begin{array}{l}4.89 \% \mathrm{UO}_{2} \mathrm{~F}_{2}-\text { solution } \\
24.22 \mathrm{~g} \mathrm{U}-235 / 1 \\
(\mathrm{H} / \mathrm{X}=994) \text {, in a } 20 \text {-in.- } \\
\text { diam SS cylinder, } \mathrm{H}_{2} \mathrm{O} \\
\text { reflected }\end{array}$ & 28 & $0.996 \pm 0.002$ & $0.996 \pm 0.002$ & $1.001 \pm 0.001$ & $0.998 \pm 0.002$ \\
\hline
\end{tabular}


Table 2. KENO V.a and KENO-VI comparison

Calculated $k_{\text {eff }}$ for large diameter low enriched uranium cylinders in various lattice arrangements

\begin{tabular}{|c|c|c|c|c|c|c|}
\hline \multirow[b]{3}{*}{ Case } & \multirow[b]{3}{*}{ Experimental description } & \multirow[b]{3}{*}{ Ref. } & \multicolumn{4}{|c|}{$k_{e f f} \pm \sigma$} \\
\hline & & & \multicolumn{2}{|c|}{27 group } & \multicolumn{2}{|c|}{238 group } \\
\hline & & & KENO V.a & KENO-VI & KENO V.a & KENO-VI \\
\hline CAB01 & $\begin{array}{l}\text { (EBJ.1) lattice } 2.15-3.85 \% \\
\text { U rods, } 7.2 \text { in. diam } \times 30 \mathrm{in} \text {. } \\
\text { long in square lattice, } 7.2 \mathrm{in} \text {. } \\
\text { center-to-center, } 77.8 \mathrm{~cm} \\
\text { water height }\end{array}$ & 29 & $1.003 \pm 0.002$ & $0.999 \pm 0.002$ & $1.000 \pm 0.002$ & $0.996 \pm 0.002$ \\
\hline CAB07 & $\begin{array}{l}\text { (EBJ.4) lattice } 3.16-3.85 \% \\
\text { U rods, } 7.2 \text { in. diam } \times \\
30 \text { in. long in triangular } \\
\text { lattice, } 7.45 \text { in. center-to- } \\
\text { center, } 53.1 \mathrm{~cm} \text { water } \\
\text { height }\end{array}$ & 29 & $0.992 \pm 0.002$ & $0.991 \pm 0.002$ & $0.984 \pm 0.002$ & $0.985 \pm 0.002$ \\
\hline CAB10 & $\begin{array}{l}(\mathrm{EBJ} .8) \text { lattice } 5.6-3.85 \% \\
\mathrm{U} \text { rods, } 7.2 \text { in. } \mathrm{OD} \times 2.6 \mathrm{in} \text {. } \\
\text { ID } \times 30 \text { in. long in square } \\
\text { lattice, } 7.95 \text { in. center-to- } \\
\text { center, } 91.4 \mathrm{~cm} \text { water height } \\
(2 \text { subcritical })\end{array}$ & 29 & $1.008 \pm 0.002$ & $1.013 \pm 0.002$ & $0.995 \pm 0.002$ & $0.997 \pm 0.003$ \\
\hline CAB12 & $\begin{array}{l}\text { (EBJ.10) lattice } 14.20- \\
3.85 \% \text { U rods, } 7.2 \text { in. OD } \times \\
2.6 \text { in. ID } \times 30 \text { in. long in } \\
\text { square lattice, } 9.07 \mathrm{in} . \\
\text { center-to-center, } 79 \mathrm{~cm} \\
\text { water height }\end{array}$ & 29 & $1.007 \pm 0.002$ & $1.006 \pm 0.002$ & $0.997 \pm 0.002$ & $0.998 \pm 0.002$ \\
\hline CAB13 & $\begin{array}{l}\text { (EBJ.13) lattice } 9.15-3.85 \% \\
\text { U rods, } 2.5 \text { in. diam } \times \\
30 \text { in. long in square lattice, } \\
3.25 \text { in. center-to-center, } \\
64.8 \mathrm{~cm} \text { water height }\end{array}$ & 29 & $1.006 \pm 0.002$ & $1.008 \pm 0.002$ & $1.003 \pm 0.002$ & $0.996 \pm 0.002$ \\
\hline CAB15 & $\begin{array}{l}\text { (EBJ.13) lattice } 9.15-3.85 \% \\
\text { U rods, } 2.5 \text { in. diam } \times 30 \text { in. } \\
\text { long in square lattice, } \\
3.25 \text { in. center-to-center, } \\
64.8 \mathrm{~cm} \text { water height }\end{array}$ & 29 & $1.007 \pm 0.002$ & $1.009 \pm 0.002$ & $0.991 \pm 0.002$ & $0.990 \pm 0.002$ \\
\hline
\end{tabular}


Table 2 (continued)

Calculated $k_{\text {eff }}$ for large diameter low enriched uranium cylinders in various lattice arrangements

\begin{tabular}{|c|c|c|c|c|c|c|}
\hline \multirow[b]{3}{*}{ Case } & \multirow[b]{3}{*}{ Experimental description } & \multirow[b]{3}{*}{ Ref. } & \multicolumn{4}{|c|}{$k_{e f f} \pm \sigma$} \\
\hline & & & \multicolumn{2}{|c|}{27 group } & \multicolumn{2}{|c|}{238 group } \\
\hline & & & KENO V.a & KENO-VI & KENO V.a & KENO-VI \\
\hline CAB16 & $\begin{array}{l}\text { (EBJ.14) lattice } 2.23- \\
3.85 \% \text { U rods, } \\
2.5 \text { in. diam } \times 30 \mathrm{in.} \mathrm{long} \\
\text { in square lattice } 4.00 \mathrm{in} . \\
\text { center-to-center, } \\
68.9 \mathrm{~cm} \text { water height }\end{array}$ & 29 & $1.000 \pm 0.002$ & $0.999 \pm 0.002$ & $0.992 \pm 0.002$ & $0.990 \pm 0.002$ \\
\hline
\end{tabular}


Table 3. KENO V.a and KENO-VI comparison

Calculated $k_{\text {eff }}$ for low enriched homogeneous uranium systems

\begin{tabular}{|c|c|c|c|c|c|c|}
\hline \multirow[b]{3}{*}{ Case } & \multirow[b]{3}{*}{ Experimental description } & \multirow[b]{3}{*}{ Ref. } & \multicolumn{4}{|c|}{$k_{e f f} \pm \sigma$} \\
\hline & & & \multicolumn{2}{|c|}{27 group } & \multicolumn{2}{|c|}{238 group } \\
\hline & & & KENO V.a & KENO-VI & KENO V.a & KENO-VI \\
\hline CAS04 & $\begin{array}{l}\text { An unreflected rectangular } \\
\text { parallelepiped of } \\
\text { homogeneous } \mathrm{U}(1.4) \mathrm{F}_{4} \\
\text { and paraffin with an } \mathrm{H} / \mathrm{U}- \\
235 \text { atomic ratio of } 421.8 \text {; } \\
93.1 \mathrm{~cm} \times 93.0 \mathrm{~cm} \times \\
123.8 \mathrm{~cm}\end{array}$ & 30 & $0.987 \pm .0 .002$ & 0.988 土. 0.002 & $0.993 \pm 0.002$ & $0.993 \pm 0.002$ \\
\hline CAS05 & $\begin{array}{l}\text { An unreflected rectangular } \\
\text { parallelepiped of } \\
\text { homogeneous } \mathrm{U}(1.4) \mathrm{F}_{4} \\
\text { and paraffin with an } \mathrm{H} / \mathrm{U}- \\
235 \text { atomic ratio of } 421.8 \text {; } \\
100.0 \mathrm{~cm} \times 99.9 \mathrm{~cm} \times \\
103.1 \mathrm{~cm}\end{array}$ & 30 & $0.989 \pm 0.002$ & $0.986 \pm 0.002$ & $0.992 \pm 0.002$ & $0.991 \pm 0.002$ \\
\hline CAS06 & $\begin{array}{l}\text { An unreflected rectangular } \\
\text { parallelepiped of } \\
\text { homogeneous } \mathrm{U}(1.4) \mathrm{F}_{4} \text { and } \\
\text { paraffin with an } \mathrm{H} / \mathrm{U}-235 \\
\text { atomic ratio of } 421.8 ; \\
130.7 \mathrm{~cm} \times 130.6 \mathrm{~cm} \times \\
74.2 \mathrm{~cm}\end{array}$ & 30 & $0.985 \pm 0.002$ & $0.985 \pm 0.002$ & $0.994 \pm 0.002$ & $0.992 \pm 0.002$ \\
\hline CAS11 & $\begin{array}{l}\text { A reflected rectangular } \\
\text { parallelepiped of } \\
\text { homogeneous } \mathrm{U}(2) \mathrm{F}_{4} \text { and } \\
\text { paraffin with an } \mathrm{H} / \mathrm{U}-235 \\
\text { atomic ratio of } 421.8\end{array}$ & 31 & $0.994 \pm 0.003$ & $0.995 \pm 0.003$ & $1.001 \pm 0.002$ & $1.000 \pm 0.003$ \\
\hline CAS12 & $\begin{array}{l}\text { An unreflected rectangular } \\
\text { parallelepiped of } \\
\text { homogeneous } \mathrm{U}(2) \mathrm{F}_{4} \text { and } \\
\text { paraffin with an } \mathrm{H} / \mathrm{U}-235 \\
\text { atomic ratio of } 195.2 ; \\
71.47 \mathrm{~cm} \times 71.47 \mathrm{~cm} \times \\
94.14 \mathrm{~cm}\end{array}$ & 31 & $0.992 \pm 0.002$ & $0.996 \pm 0.002$ & $0.995 \pm 0.002$ & $0.995 \pm 0.002$ \\
\hline
\end{tabular}


Table 3 (continued)

Calculated $k_{\text {eff }}$ for low enriched homogeneous uranium systems

\begin{tabular}{|c|c|c|c|c|c|c|}
\hline \multirow[b]{3}{*}{ Case } & \multirow[b]{3}{*}{ Experimental description } & \multirow[b]{3}{*}{ Ref. } & \multicolumn{4}{|c|}{$k_{e f f} \pm \sigma$} \\
\hline & & & \multicolumn{2}{|c|}{27 group } & \multicolumn{2}{|c|}{238 group } \\
\hline & & & KENO V.a & KENO-VI & KENO V.a & KENO-VI \\
\hline CAS14 & $\begin{array}{l}\text { An unreflected rectangular } \\
\text { parallelepiped of } \\
\text { homogeneous } U(2) \mathrm{F}_{4} \\
\text { and paraffin with an } \\
\mathrm{H} / \mathrm{U}-235 \text { atomic ratio of } \\
293.9 ; 56.22 \mathrm{~cm} \times \\
56.22 \mathrm{~cm} \times 122.47 \mathrm{~cm}\end{array}$ & 31 & $0.997 \pm 0.002$ & $0.997 \pm 0.002$ & $1.000 \pm 0.002$ & $1.002 \pm 0.002$ \\
\hline CAS15 & $\begin{array}{l}\text { An unreflected rectangular } \\
\text { parallelepiped of } \\
\text { homogeneous } \mathrm{U}(2) \mathrm{F}_{4} \text { and } \\
\text { paraffin with an } \\
\mathrm{H} / \mathrm{U}-235 \text { atomic ratio of } \\
406.3 ; 53.67 \mathrm{~cm} \times \\
53.67 \mathrm{~cm} \times 54.29 \mathrm{~cm} \text {, } \\
\text { reflected with } 15.2 \mathrm{~cm} \text { of } \\
\text { paraffin on top and sides } \\
\text { and } 15.2 \mathrm{~cm} \text { of Plexiglas } \\
\text { on the bottom }\end{array}$ & 31 & $0.995 \pm 0.002$ & $0.998 \pm 0.002$ & $0.999 \pm 0.002$ & $1.003 \pm 0.002$ \\
\hline CAS16 & $\begin{array}{l}\text { A reflected rectangular } \\
\text { parallelepiped of } \\
\text { homogeneous } \mathrm{U}(2) \mathrm{F}_{4} \text { and } \\
\text { paraffin with an } \\
\mathrm{H} / \mathrm{U}-235 \text { atomic ratio of } \\
495.9 ; 46.00 \mathrm{~cm} \times \\
46.00 \mathrm{~cm} \times 96.57 \mathrm{~cm} \text {, } \\
\text { reflected with } 15.2 \mathrm{~cm} \text { of } \\
\text { paraffin on top and sides } \\
\text { and } 15.2 \mathrm{~cm} \text { of Plexiglas } \\
\text { on the bottom }\end{array}$ & 31 & $0.997 \pm 0.002$ & $0.999 \pm 0.002$ & $0.994 \pm 0.002$ & $0.999 \pm 0.002$ \\
\hline CAS19 & $\begin{array}{l}\text { A reflected rectangular } \\
\text { parallelepiped of } \\
\text { homogeneous } \mathrm{U}(2) \mathrm{F}_{4} \text { and } \\
\text { paraffin with an } \\
\mathrm{H} / \mathrm{U}-235 \text { atomic ratio of } \\
971.7 ; 76.51 \mathrm{~cm} \times \\
76.44 \mathrm{~cm} \times 82.42 \mathrm{~cm} \text {, } \\
\text { reflected with } 5.2 \mathrm{~cm} \text { of } \\
\text { polyethylene on top and } \\
\text { sides and } 15.2 \mathrm{~cm} \text { of } \\
\text { Plexiglas on the bottom }\end{array}$ & 31 & $0.988 \pm 0.002$ & $0.986 \pm 0.002$ & $0.997 \pm 0.002$ & $0.997 \pm 0.002$ \\
\hline
\end{tabular}


Table 3 (continued)

Calculated $k_{\text {eff }}$ for low enriched homogeneous uranium systems

\begin{tabular}{|c|c|c|c|c|c|c|}
\hline \multirow[b]{3}{*}{ Case } & \multirow[b]{3}{*}{ Experimental description } & \multirow[b]{3}{*}{ Ref. } & \multicolumn{4}{|c|}{$k_{e f f} \pm \sigma$} \\
\hline & & & \multicolumn{2}{|c|}{27 group } & \multicolumn{2}{|c|}{238 group } \\
\hline & & & KENO V.a & KENO-VI & KENO V.a & KENO-VI \\
\hline CAS20 & $\begin{array}{l}\text { An unreflected rectangular } \\
\text { parallelepiped of } \\
\text { homogeneous } \mathrm{U}(2) \mathrm{F}_{4} \text { and } \\
\text { paraffin with an } \\
\mathrm{H} / \mathrm{U}-235 \text { atomic ratio of } \\
971.7 ; 81.45 \mathrm{~cm} \times \\
86.70 \mathrm{~cm} \times 88.22 \mathrm{~cm}\end{array}$ & 31 & $0.987 \pm 0.002$ & $0.981 \pm 0.002$ & $0.991 \pm 0.002$ & $0.991 \pm 0.002$ \\
\hline CAS22 & $\begin{array}{l}\text { A reflected rectangular } \\
\text { parallelepiped of } \\
\text { homogeneous } \mathrm{U}(3) \mathrm{F}_{4} \text { and } \\
\text { paraffin with an } \\
\mathrm{H} / \mathrm{U}-235 \text { atomic ratio of } \\
133.4,43.47 \mathrm{~cm} \times \\
43.47 \mathrm{~cm} \times 86.39 \mathrm{~cm} \text {, } \\
\text { reflected with } 15.2 \mathrm{~cm} \text { of } \\
\text { paraffin on top and sides } \\
\text { and } 15.2 \mathrm{~cm} \text { of Plexiglas } \\
\text { on the bottom }\end{array}$ & 31 & $1.007 \pm 0.002$ & $1.009 \pm 0.002$ & $1.001 \pm 0.002$ & $1.015 \pm 0.002$ \\
\hline CAS24 & $\begin{array}{l}\text { A reflected rectangular } \\
\text { parallelepiped of } \\
\text { homogeneous } \mathrm{U}(3) \mathrm{F}_{4} \text { and } \\
\text { paraffin with an } \\
\mathrm{H} / \mathrm{U}-235 \text { atomic ratio of } \\
133.4 ; 56.25 \mathrm{~cm} \times \\
56.25 \mathrm{~cm} \times 43.41 \mathrm{~cm} \text {, } \\
\text { reflected with } 15.2 \mathrm{~cm} \text { of } \\
\text { paraffin on top and sides } \\
\text { and } 15.2 \mathrm{~cm} \text { of Plexiglas } \\
\text { on the bottom }\end{array}$ & 31 & $1.008 \pm 0.003$ & $1.011 \pm 0.003$ & $1.011 \pm 0.002$ & $1.012 \pm 0.002$ \\
\hline CAS26 & $\begin{array}{l}\text { An unreflected rectangular } \\
\text { parallelepiped of } \\
\text { homogeneous } \mathrm{U}(3) \mathrm{F}_{4} \text { and } \\
\text { paraffin with an } \\
\mathrm{H} / \mathrm{U}-235 \text { atomic ratio of } \\
133.4 ; 56.47 \mathrm{~cm} \times \\
56.47 \mathrm{~cm} \times 86.64 \mathrm{~cm}\end{array}$ & 31 & $1.014 \pm 0.003$ & $1.012 \pm 0.003$ & $1.013 \pm 0.002$ & $1.013 \pm 0.002$ \\
\hline
\end{tabular}


Table 3 (continued)

Calculated $k_{\text {eff }}$ for low enriched homogeneous uranium systems

\begin{tabular}{|c|c|c|c|c|c|c|}
\hline \multirow[b]{3}{*}{ Case } & \multirow[b]{3}{*}{ Experimental description } & \multirow[b]{3}{*}{ Ref. } & \multicolumn{4}{|c|}{$k_{e f f} \pm \sigma$} \\
\hline & & & \multicolumn{2}{|c|}{27 group } & \multicolumn{2}{|c|}{238 group } \\
\hline & & & KENO V.a & KENO-VI & KENO V.a & KENO-VI \\
\hline CAS28 & $\begin{array}{l}\text { An unreflected } \\
\text { rectangular parallelepiped } \\
\text { of homogeneous } \mathrm{U}(3) \mathrm{F}_{4} \\
\text { and paraffin with an } \\
\mathrm{H} / \mathrm{U}-235 \text { atomic ratio of } \\
133.4 ; 61.4 \mathrm{~cm} \times 61.4 \mathrm{~cm} \\
\times 66.0 \mathrm{~cm}\end{array}$ & 31 & $1.006 \pm 0.002$ & $1.009 \pm 0.002$ & $1.014 \pm 0.002$ & $1.014 \pm 0.002$ \\
\hline CAS30 & $\begin{array}{l}\text { An unreflected rectangular } \\
\text { parallelepiped of } \\
\text { homogeneous } \mathrm{U}(3) \mathrm{F}_{4} \text { and } \\
\text { paraffin with an } \\
\mathrm{H} / \mathrm{U}-235 \text { atomic ratio of } \\
276.9 ; 40.90 \mathrm{~cm} \times \\
40.93 \mathrm{~cm} \times 116.80 \mathrm{~cm}\end{array}$ & 31 & $1.010 \pm 0.003$ & $1.010 \pm 0.002$ & $1.016 \pm 0.003$ & $1.016 \pm 0.003$ \\
\hline CAS33 & $\begin{array}{l}\text { A composite } \\
\text { cadmium/steel/water side } \\
\text { reflected stainless steel } \\
\text { cylinder of } 0.079 \mathrm{~cm} \text { wall } \\
\text { thickness and } 19.545 \mathrm{~cm} \\
\text { IR filled to a height of } \\
54.45 \mathrm{~cm} \text { with }(4.98) \mathrm{O}_{2} \mathrm{~F}_{2} \\
\text { solution at an } \mathrm{H} / \mathrm{U}-235 \\
\text { atomic ratio of } 488\end{array}$ & 31 & $1.000 \pm 0.003$ & $1.000 \pm 0.003$ & $1.001 \pm 0.002$ & $1.007 \pm 0.002$ \\
\hline CAS36 & $\begin{array}{l}\text { An unreflected stainless } \\
\text { steel cylinder of } \\
0.07874 \mathrm{~cm} \text { wall } \\
\text { thickness and a } 19.55 \mathrm{~cm} \\
\text { IR filled to a height of } \\
101.7 \mathrm{~cm} \text { with } \\
\mathrm{U}(4.98) \mathrm{O}_{2} \mathrm{~F}_{2} \text { solution at } \\
\text { an } \mathrm{H} / \mathrm{U}-235 \text { atomic ratio } \\
\text { of } 496\end{array}$ & 33 & $0.993 \pm 0.003$ & $1.004 \pm 0.003$ & $0.998 \pm 0.003$ & $0.994 \pm 0.003$ \\
\hline CAR01 & $\begin{array}{l}\text { Experiment } 1 . \\
4.46 \% \text { enriched } \mathrm{U}_{3} \mathrm{O}_{8} \\
\mathrm{H} / \mathrm{U}=0.77,42 \text { fuel cans } \\
\text { with } 2.44 \mathrm{~cm} \text { interstitial } \\
\text { moderation, plastic } \\
\text { reflected }\end{array}$ & 34 & $1.010 \pm 0.002$ & $1.016 \pm 0.004$ & $1.016 \pm 0.002$ & $1.015 \pm 0.002$ \\
\hline
\end{tabular}


Table 3 (continued)

Calculated $k_{\text {eff }}$ for low enriched homogeneous uranium systems

\begin{tabular}{|c|c|c|c|c|c|c|}
\hline \multirow[b]{3}{*}{ Case } & \multirow[b]{3}{*}{ Experimental description } & \multirow[b]{3}{*}{ Ref. } & \multicolumn{4}{|c|}{$k_{e f f} \pm \sigma$} \\
\hline & & & \multicolumn{2}{|c|}{27 group } & \multicolumn{2}{|c|}{238 group } \\
\hline & & & KENO V.a & KENO-VI & KENO V.a & KENO-VI \\
\hline CAR05 & $\begin{array}{l}\text { Experiment } 2 . \\
4.46 \% \text { enriched } \mathrm{U}_{3} \mathrm{O}_{8} \\
\mathrm{H} / \mathrm{U}=0.77,98 \text { fuel cans } \\
\text { with } 0.929 \mathrm{~cm} \text { interstitial } \\
\text { moderation, concrete } \\
\text { reflected }\end{array}$ & 34 & $1.003 \pm 0.002$ & $0.999 \pm 0.003$ & $0.999 \pm 0.002$ & $1.003 \pm 0.003$ \\
\hline CAR11 & $\begin{array}{l}\text { Experiment } \mathrm{A} \text {. } \\
4.46 \% \text { enriched } \mathrm{U}_{3} \mathrm{O}_{8} \\
\mathrm{H} / \mathrm{U}=1.25,38 \text { fuel cans } \\
\text { with } 2.44 \mathrm{~cm} \text { interstitial } \\
\text { moderation, plastic } \\
\text { reflected }\end{array}$ & 36 & $1.011 \pm 0.003$ & $1.010 \pm 0.004$ & $1.009 \pm 0.002$ & $1.015 \pm 0.002$ \\
\hline CAR13 & $\begin{array}{l}\text { Experiment } \mathrm{C} \text {. } \\
4.46 \% \text { enriched } \mathrm{U}_{3} \mathrm{O}_{8} \\
\mathrm{H} / \mathrm{U}=1.25,80 \text { fuel cans } \\
\text { with } 0.929 \mathrm{~cm} \text { interstitial } \\
\text { moderation, plastic } \\
\text { reflected }\end{array}$ & 36 & $1.014 \pm 0.003$ & $1.014 \pm 0.004$ & $1.017 \pm 0.003$ & $1.019 \pm 0.003$ \\
\hline CAR15 & $\begin{array}{l}4.46 \% \text { enriched } \mathrm{U}_{3} \mathrm{O}_{8} \\
\mathrm{H} / \mathrm{U}=1.255, \text { driven by } \\
\text { high concentration } \\
(12,400 \mathrm{~kg} 351.65 \mathrm{~g} \mathrm{U} / \mathrm{l}), \\
93.17 \% \text { enriched } \\
\mathrm{UO}_{2}\left(\mathrm{NO}_{3}\right)_{2}, 119+2 \mathrm{~S} \text { fuel } \\
\text { cans, plastic reflected }\end{array}$ & 36 & $0.994 \pm 0.003$ & $1.003 \pm 0.003$ & $0.994 \pm 0.003$ & $1.001 \pm 0.003$ \\
\hline CAR20 & $\begin{array}{l}\text { Experiment E. } 4.46 \% \\
\text { enriched } \mathrm{U}_{3} \mathrm{O}_{8} \mathrm{H} / \mathrm{U}= \\
2.03 \text {, driven by } 93.12 \% \\
\text { enriched hollow uranium } \\
\text { metal sphere }(12.786 \mathrm{~kg}) \text {, } \\
120+4 \mathrm{~S} \text { fuel cans, } \\
\text { plastic reflected }\end{array}$ & 37 & $0.991 \pm 0.003$ & $1.000 \pm 0.002$ & $0.997 \pm 0.002$ & $0.999 \pm 0.002$ \\
\hline
\end{tabular}


Table 4. KENO V.a and KENO-VI comparison

Calculated $k_{\text {eff }}$ for highly enriched uranium solution systems

\begin{tabular}{|c|c|c|c|c|c|c|}
\hline \multirow[b]{3}{*}{ Case } & \multirow[b]{3}{*}{ Experimental description } & \multirow[b]{3}{*}{ Ref. } & \multicolumn{4}{|c|}{$k_{e f f} \pm \sigma$} \\
\hline & & & \multicolumn{2}{|c|}{27 group } & \multicolumn{2}{|c|}{238 group } \\
\hline & & & KENO V.a & KENO-VI & KENO V.a & KENO-VI \\
\hline CAA01 & $\begin{array}{l}\mathrm{U}(93.2) \mathrm{O}_{2} \mathrm{~F}_{2} \text { solution } \\
\text { sphere, } \mathrm{H} / \mathrm{X}=1112, \\
\text { unreflected }\end{array}$ & 38 & $1.004 \pm 0.002$ & $1.002 \pm 0.002$ & $1.006 \pm 0.002$ & $1.007 \pm 0.002$ \\
\hline CAA04 & $\begin{array}{l}\mathrm{U}(93.2) \mathrm{O}_{2} \mathrm{~F}_{2} \text { solution } \\
\text { sphere, } \mathrm{H} / \mathrm{X}=76.1, \\
\mathrm{H}_{2} \mathrm{O} \text { reflected }\end{array}$ & 38 & $1.004 \pm 0.003$ & $1.007 \pm 0.003$ & $1.004 \pm 0.003$ & $1.007 \pm 0.003$ \\
\hline CAA07 & $\begin{array}{l}\mathrm{U}(93.2) \mathrm{O}_{2} \mathrm{~F}_{2} \text { solution } \\
\text { sphere, } \mathrm{H} / \mathrm{X}=268.8, \\
\mathrm{H}_{2} \mathrm{O} \text { reflected }\end{array}$ & 39 & $1.008 \pm 0.003$ & $1.008 \pm 0.003$ & $1.001 \pm 0.004$ & $1.001 \pm 0.003$ \\
\hline CAA10 & $\begin{array}{l}\mathrm{U}(93.2) \mathrm{O}_{2} \mathrm{~F}_{2} \text { solution } \\
\text { sphere, } \mathrm{H} / \mathrm{X}=239.3, \\
\mathrm{H}_{2} \mathrm{O} \text { reflected }\end{array}$ & 39 & $1.028 \pm 0.003$ & $1.028 \pm 0.003$ & $1.017 \pm 0.003$ & $1.021 \pm 0.003$ \\
\hline CAA13 & $\begin{array}{l}\mathrm{U}(93.2) \mathrm{O}_{2}\left(\mathrm{NO}_{3}\right)_{2} \text { solution } \\
357.71 \mathrm{~g} \mathrm{U} / 1,28.01 \mathrm{~cm} \\
\text { diam cylinder, unreflected }\end{array}$ & 40 & $1.016 \pm 0.003$ & $1.010 \pm 0.003$ & $1.003 \pm 0.001$ & $1.007 \pm 0.003$ \\
\hline CAA16 & $\begin{array}{l}\mathrm{U}(93.2) \mathrm{O}_{2}\left(\mathrm{NO}_{3}\right)_{2} \text { solution } \\
357.71 \mathrm{~g} \mathrm{U} / 1,28.01 \mathrm{~cm} \\
\text { diam cylinder, unreflected }\end{array}$ & 40 & $1.004 \pm 0.003$ & $0.998 \pm 0.003$ & $1.000 \pm 0.003$ & $0.995 \pm 0.003$ \\
\hline CAA19 & $\begin{array}{l}\mathrm{U}(93.2) \mathrm{O}_{2}\left(\mathrm{NO}_{3}\right)_{2} \text { solution } \\
144.38 \mathrm{~g} \mathrm{U} / 1,33.01 \mathrm{~cm} \\
\text { diam cylinder, concrete } \\
\text { reflected }\end{array}$ & 40 & $1.013 \pm 0.002$ & $1.010 \pm 0.004$ & $1.006 \pm 0.003$ & $1.000 \pm 0.003$ \\
\hline CAA22 & $\begin{array}{l}\mathrm{U}(93.2) \mathrm{O}_{2}\left(\mathrm{NO}_{3}\right)_{2} \text { solution } \\
334.77 \mathrm{~g} \mathrm{U} / 1,33.01 \mathrm{~cm} \\
\text { diam cylinder, concrete } \\
\text { reflected }\end{array}$ & 40 & $1.006 \pm 0.003$ & $1.006 \pm 0.004$ & $0.998 \pm 0.003$ & $1.002 \pm 0.003$ \\
\hline CAA25 & $\begin{array}{l}\mathrm{U}(93.2) \mathrm{O}_{2}\left(\mathrm{NO}_{3}\right)_{2} \text { solution } \\
147.66 \mathrm{~g} \mathrm{U} / 1,33.01 \mathrm{~cm} \\
\text { diam cylinder, Plexiglas } \\
\text { reflected }\end{array}$ & 40 & $1.004 \pm 0.003$ & $1.002 \pm 0.003$ & $1.000 \pm 0.003$ & $1.000 \pm 0.003$ \\
\hline CAA28 & $\begin{array}{l}\mathrm{U}(93.2) \mathrm{O}_{2}\left(\mathrm{NO}_{3}\right)_{2} \text { solution } \\
345.33 \mathrm{~g} \mathrm{U} / 1,33.01 \mathrm{~cm} \\
\text { diam cylinder, concrete } \\
\text { reflected }\end{array}$ & 40 & $1.008 \pm 0.003$ & $1.011 \pm 0.003$ & $1.005 \pm 0.003$ & $1.000 \pm 0.003$ \\
\hline
\end{tabular}


Table 4 (continued)

Calculated $k_{\text {eff }}$ for highly enriched uranium solution systems

\begin{tabular}{|c|c|c|c|c|c|c|}
\hline \multirow[b]{3}{*}{ Case } & \multirow[b]{3}{*}{ Experimental description } & \multirow[b]{3}{*}{ Ref. } & \multicolumn{4}{|c|}{$k_{e f f} \pm \sigma$} \\
\hline & & & \multicolumn{2}{|c|}{27 group } & \multicolumn{2}{|c|}{238 group } \\
\hline & & & KENO V.a & KENO-VI & KENO V.a & KENO-VI \\
\hline CAA30 & $\begin{array}{l}\mathrm{U}(93.2) \mathrm{O}_{2}\left(\mathrm{NO}_{3}\right)_{2} \text { solution } \\
364.11 \mathrm{~g} \mathrm{U} / 1,21.12 \mathrm{~cm} \\
\text { diam cylinder, in a } 4 \times 4 \\
\text { array, concrete reflected }\end{array}$ & 40 & $1.009 \pm 0.003$ & $1.001 \pm 0.003$ & $1.000 \pm 0.003$ & $0.998 \pm 0.003$ \\
\hline CAA32 & $\begin{array}{l}\mathrm{U}(93.2) \mathrm{O}_{2}\left(\mathrm{NO}_{3}\right)_{2} \text { solution } \\
76.09 \mathrm{~g} \mathrm{U} / 1,21.12 \mathrm{~cm} \\
\text { diam cylinder, in a } 2 \times 2 \\
\text { array, concrete reflected }\end{array}$ & 40 & $1.011 \pm 0.003$ & $1.012 \pm 0.003$ & $1.007 \pm 0.003$ & $1.008 \pm 0.003$ \\
\hline CAA34 & $\begin{array}{l}\mathrm{U}(93.2) \mathrm{O}_{2}\left(\mathrm{NO}_{3}\right)_{2} \text { solution } \\
364.11 \mathrm{~g} \mathrm{U} / 1,21.12 \mathrm{~cm} \\
\text { diam cylinder, in a } 2 \times 2 \\
\text { array, concrete reflected }\end{array}$ & 40 & $1.003 \pm 0.003$ & $0.996 \pm 0.003$ & $0.991 \pm 0.003$ & $0.993 \pm 0.003$ \\
\hline CAA36 & $\begin{array}{l}\mathrm{U}(93.2) \mathrm{O}_{2}\left(\mathrm{NO}_{3}\right)_{2} \text { solution } \\
359.55 \mathrm{~g} \mathrm{U} / 1,16.12 \mathrm{~cm} \\
\text { diam cylinder, in a } 2 \times 4 \\
\text { array, concrete reflected }\end{array}$ & 40 & $1.011 \pm 0.002$ & $1.009 \pm 0.003$ & $1.010 \pm 0.003$ & $1.011 \pm 0.003$ \\
\hline CAA38 & $\begin{array}{l}\mathrm{U}(93.2) \mathrm{O}_{2}\left(\mathrm{NO}_{3}\right)_{2} \text { solution } \\
76.09 \mathrm{~g} \mathrm{U} / 1,21.12 \mathrm{~cm} \\
\text { diam cylinder, in a } 2 \times 2 \\
\text { array, concrete reflected }\end{array}$ & 40 & $0.998 \pm 0.003$ & $1.000 \pm 0.003$ & $0.999 \pm 0.002$ & $0.994 \pm 0.003$ \\
\hline CAA40 & $\begin{array}{l}\mathrm{U}(93.2) \mathrm{O}_{2}\left(\mathrm{NO}_{3}\right)_{2} \text { solution } \\
355.94 \mathrm{~g} \mathrm{U} / 1,16.12 \mathrm{~cm} \\
\text { diam cylinder, in a } 4 \times 4 \\
\text { array, Plexiglas reflected }\end{array}$ & 40 & $1.001 \pm 0.003$ & $1.001 \pm 0.003$ & $0.999 \pm 0.003$ & $0.995 \pm 0.003$ \\
\hline CAA42 & $\begin{array}{l}\mathrm{U}(93.2) \mathrm{O}_{2}\left(\mathrm{NO}_{3}\right)_{2} \text { solution } \\
355.94 \mathrm{~g} \mathrm{U} / 1,21.12 \mathrm{~cm} \\
\text { diam cylinder, in a } 2 \times 2 \\
\text { array, Plexiglas reflected }\end{array}$ & 40 & $1.009 \pm 0.003$ & $1.001 \pm 0.004$ & $0.999 \pm 0.002$ & $1.007 \pm 0.003$ \\
\hline
\end{tabular}


Table 5. KENO V.a and KENO-VI comparison

Calculated $k_{\text {eff }}$ highly enriched uranium metal and solution systems

\begin{tabular}{|c|c|c|c|c|c|c|}
\hline \multirow[b]{3}{*}{ Case } & \multirow[b]{3}{*}{ Experimental description } & \multirow[b]{3}{*}{ Ref. } & \multicolumn{4}{|c|}{$k_{e f f} \pm \sigma$} \\
\hline & & & \multicolumn{2}{|c|}{27 group } & \multicolumn{2}{|c|}{238 group } \\
\hline & & & KENO V.a & KENO-VI & KENO V.a & KENO-VI \\
\hline CAS01 & $\begin{array}{l}\text { 93.8\% U metal sphere, } \\
\text { unreflected (GODIVA) }\end{array}$ & 41 & $1.003 \pm 0.003$ & $0.999 \pm 0.003$ & $0.991 \pm 0.002$ & $0.990 \pm 0.002$ \\
\hline CAS05 & $\begin{array}{l}93.2 \% \mathrm{UO}_{2} \mathrm{~F}_{2} \text { solution, } \\
19.992 \mathrm{~g} \mathrm{U} / 1, \text { in } \mathrm{Al} \\
\text { sphere, unreflected }\end{array}$ & 38 & $1.001 \pm 0.002$ & $1.001 \pm 0.002$ & $1.006 \pm 0.002$ & $1.002 \pm 0.002$ \\
\hline CAS07 & $\begin{array}{l}93.5 \% \text { U metal } \\
\text { hemispherical shell, } \\
\mathrm{H}_{2} \mathrm{O} \text { reflected }\end{array}$ & 42 & $1.009 \pm 0.002$ & $1.012 \pm 0.003$ & $1.006 \pm 0.002$ & $1.005 \pm 0.002$ \\
\hline CAS09 & $\begin{array}{l}94 \% \text { U metal cuboid, } \\
\text { natural U reflected }\end{array}$ & 42 & $1.008 \pm 0.002$ & $1.011 \pm 0.002$ & $1.000 \pm 0.002$ & $0.999 \pm 0.002$ \\
\hline CAS11 & $\begin{array}{l}93.1 \% \text { U metal } \\
\text { hemispherical shell, steel } \\
\text { center and oil reflected }\end{array}$ & 43 & $1.007 \pm 0.002$ & $1.004 \pm 0.002$ & $0.994 \pm 0.002$ & $0.995 \pm 0.003$ \\
\hline CAS03 & $\begin{array}{l}93.172 \% \mathrm{UO}_{2}\left(\mathrm{NO}_{3}\right)_{2} \\
\text { solution, } 346.7 \mathrm{~g} \mathrm{U} / 1 \text {, in } \\
\text { SS cylinder, unreflected }\end{array}$ & 48 & $1.016 \pm 0.003$ & $1.011 \pm 0.003$ & $1.006 \pm 0.004$ & $1.013 \pm 0.003$ \\
\hline CAS25 & $\begin{array}{l}93.2 \% \mathrm{U} \text { metal cylinders, } \\
2 \times 2 \times 2 \text { array, each unit } \\
\text { in the array is a smaller } \\
\text { cylinder capped on each } \\
\text { end by a larger cylinder, } \\
\text { unreflected }\end{array}$ & 44 & $1.002 \pm 0.002$ & $1.003 \pm 0.002$ & $0.996 \pm 0.002$ & $0.992 \pm 0.003$ \\
\hline CAS29 & $\begin{array}{l}92.6 \% \mathrm{UO}_{2}\left(\mathrm{NO}_{3}\right)_{2} \\
\text { solution, } 415 \mathrm{~g} \mathrm{U} / 1 \text {, in } \\
\text { Plexiglas cylinders, } \\
3 \times 3 \times 3 \text { array, } \\
\text { unreflected }\end{array}$ & 45 & $0.994 \pm 0.003$ & $0.992 \pm 0.003$ & $0.991 \pm 0.003$ & $0.989 \pm 0.003$ \\
\hline
\end{tabular}


Table 5 (continued)

Calculated $k_{\text {eff }}$ for highly enriched uranium metal and solution systems

\begin{tabular}{|c|c|c|c|c|c|c|}
\hline \multirow[b]{3}{*}{ Case } & \multirow[b]{3}{*}{ Experimental description } & \multirow[b]{3}{*}{ Ref. } & \multicolumn{4}{|c|}{$k_{e f f} \pm \sigma$} \\
\hline & & & \multicolumn{2}{|c|}{27 group } & \multicolumn{2}{|c|}{238 group } \\
\hline & & & KENO V.a & KENO-VI & KENO V.a & KENO-VI \\
\hline CAS13 & $\begin{array}{l}92.6 \% \mathrm{UO}_{2}\left(\mathrm{NO}_{3}\right)_{2} \\
\text { solution, } 63.3 \mathrm{~g} \mathrm{U} / 1 \text {, in } \\
\text { Plexiglas cylinders, } \\
3 \times 3 \times 3 \text { array, } \\
\text { unreflected }\end{array}$ & 45 & $0.988 \pm 0.003$ & $0.991 \pm 0.003$ & $0.992 \pm 0.003$ & $0.991 \pm 0.003$ \\
\hline CAS15 & $\begin{array}{l}93.2 \% \text { U metal cylinders, } \\
2 \times 2 \times 2 \text { array, graphite } \\
\text { moderated and } \\
\text { polyethylene reflected }\end{array}$ & 46 & $1.002 \pm 0.002$ & $1.002 \pm 0.002$ & $0.993 \pm 0.002$ & $0.994 \pm 0.002$ \\
\hline CAS17 & $\begin{array}{l}92.6 \% \mathrm{UO}_{2}\left(\mathrm{NO}_{3}\right)_{2} \\
\text { solution, } 415 \mathrm{~g} \mathrm{U} / 1 \text {, in } \\
\text { Plexiglas cylinders, } \\
3 \times 3 \times 3 \text { array, paraffin } \\
\text { reflected }\end{array}$ & 45 & $1.019 \pm 0.003$ & $1.020 \pm 0.003$ & $1.016 \pm 0.003$ & $1.019 \pm 0.003$ \\
\hline CAS19 & $\begin{array}{l}93.1 \% \mathrm{UO}_{2}\left(\mathrm{NO}_{3}\right)_{2} \\
\text { solution, } 450.8 \mathrm{~g} \mathrm{U} / 1 \text {, in } \\
\text { SS containers, square } \\
\text { central column with } 8 \\
\text { perpendicular cylindrical } \\
\text { arms unreflected }\end{array}$ & 48,49 & $1.036 \pm 0.003$ & $1.035 \pm 0.003$ & $1.023 \pm 0.003$ & $1.028 \pm 0.003$ \\
\hline CAS21 & $\begin{array}{l}93.17 \% \mathrm{UO}_{2}\left(\mathrm{NO}_{3}\right)_{2} \\
\text { solution, } 364.1 \mathrm{~g} \mathrm{U} / 1 \text {, in } \\
\text { Al cylinders, } 4 \times 4 \text { array, } \\
\text { concrete reflected }\end{array}$ & 40 & $1.024 \pm 0.003$ & $1.024 \pm 0.003$ & $1.020 \pm 0.003$ & $1.020 \pm 0.003$ \\
\hline CAS33 & $\begin{array}{l}\text { Problem S333SP1R } \\
93.2 \% \mathrm{UO}_{2} \mathrm{~F}_{2} \\
\text { solution } 81.8 \mathrm{~g} \mathrm{U} / 1 \text {, in } \mathrm{Al} \\
\text { slabs, } 37.62 \mathrm{~cm} \text { slabs in } \\
3 \times 1 \text { array, } 2.54 \mathrm{~cm} \\
\text { separation, } \mathrm{H}_{2} \mathrm{O} \text { reflected }\end{array}$ & 49 & $1.003 \pm 0.002$ & $0.998 \pm 0.003$ & $0.993 \pm 0.003$ & $1.000 \pm 0.003$ \\
\hline
\end{tabular}


Table 5 (continued)

Calculated $k_{\text {eff }}$ highly enriched uranium metal and solution systems

\begin{tabular}{|c|c|c|c|c|c|c|}
\hline \multirow[b]{3}{*}{ Case } & \multirow[b]{3}{*}{ Experimental description } & \multirow[b]{3}{*}{ Ref. } & \multicolumn{4}{|c|}{$k_{e f f} \pm \sigma$} \\
\hline & & & \multicolumn{2}{|c|}{27 group } & \multicolumn{2}{|c|}{238 group } \\
\hline & & & KENO V.a & KENO-VI & KENO V.a & KENO-VI \\
\hline CAS39 & $\begin{array}{l}\text { Problem S333SP5R } \\
93.2 \% \mathrm{UO}_{2} \mathrm{~F}_{2} \text { solution } \\
81.8 \mathrm{~g} \mathrm{U} / 1 \text {, in } \mathrm{Al} \text { slabs, } \\
37.62 \mathrm{~cm} \text { slabs in } \\
3 \times 1 \text { array, } 13.97 \mathrm{~cm} \\
\text { separation, } \mathrm{H}_{2} \mathrm{O} \text { reflected }\end{array}$ & 49 & $0.999 \pm 0.003$ & $1.006 \pm 0.003$ & $1.005 \pm 0.003$ & $0.998 \pm 0.002$ \\
\hline CAS41 & $\begin{array}{l}\text { Problem S36SP15 93.2\% } \\
\mathrm{UO}_{2} \mathrm{~F}_{2} \text { solution } 81.8 \mathrm{~g} \mathrm{U} / 1 \text {, } \\
\text { in } \mathrm{Al} \text { slabs, } 7.62 \mathrm{~cm} \text { and } \\
14.834 \mathrm{~cm} \text { slabs in } \\
2 \times 1 \text { array, } 38.1 \mathrm{~cm} \\
\text { separation, unreflected }\end{array}$ & 49 & $0.976 \pm 0.003$ & $0.980 \pm 0.003$ & $0.973 \pm 0.003$ & $0.979 \pm 0.003$ \\
\hline CAS45 & $\begin{array}{l}\text { Problem S363SPO 93.2\% } \\
\mathrm{UO}_{2} \mathrm{~F}_{2} \text { solution } 81.8 \mathrm{~g} \mathrm{U} / \mathrm{l} \text {, } \\
\text { in Al slabs, } 7.62 \mathrm{~cm} \text { and } \\
14.834 \mathrm{~cm} \text { slabs in } \\
3 \times 1 \text { array, } 0 \mathrm{~cm} \\
\text { separation, unreflected }\end{array}$ & 49 & $0.988 \pm 0.003$ & $0.983 \pm 0.003$ & $0.979 \pm 0.003$ & $0.984 \pm 0.003$ \\
\hline CAS47 & $\begin{array}{l}\text { Problem } \mathrm{S} 363 \mathrm{SP} 20 \\
93.2 \% \mathrm{UO}_{2} \mathrm{~F}_{2} \\
\text { solution } 81.8 \mathrm{~g} \mathrm{U} / 1, \\
\text { in } \mathrm{Al} \mathrm{slabs,} 7.62 \mathrm{~cm} \text { and } \\
14.834 \mathrm{~cm} \text { slabs in } \\
2 \times 1 \text { array, } 38.1 \mathrm{~cm} \\
\text { separation, unreflected }\end{array}$ & 49 & $0.977 \pm 0.003$ & $0.976 \pm 0.003$ & $0.978 \pm 0.003$ & $0.974 \pm 0.003$ \\
\hline CAS49 & $\begin{array}{l}\text { Problem S63SP12 } 93.2 \% \\
\mathrm{UO}_{2} \mathrm{~F}_{2} \text { solution } 81.8 \mathrm{~g} \mathrm{U} / \mathrm{l} \text {, } \\
\text { in Al slabs, one slab is } \\
\text { made up from two } \\
7.62 \mathrm{~cm} \text { slabs snugly fit } \\
\text { together, the other is } \\
7.62 \mathrm{~cm}, 2 \times 1 \text { array, } \\
30.48 \mathrm{~cm} \text { separation, } \\
\text { unreflected }\end{array}$ & 49 & $0.986 \pm 0.003$ & $0.983 \pm 0.003$ & $0.987 \pm 0.003$ & $0.982 \pm 0.003$ \\
\hline
\end{tabular}


Table 5 (continued)

Calculated $k_{\text {eff }}$ highly enriched uranium metal and solution systems

\begin{tabular}{|c|c|c|c|c|c|c|}
\hline \multirow[b]{3}{*}{ Case } & \multirow[b]{3}{*}{ Experimental description } & \multirow[b]{3}{*}{ Ref. } & \multicolumn{4}{|c|}{$k_{e f f} \pm \sigma$} \\
\hline & & & \multicolumn{2}{|c|}{27 group } & \multicolumn{2}{|c|}{238 group } \\
\hline & & & KENO V.a & KENO-VI & KENO V.a & KENO-VI \\
\hline CAS51 & $\begin{array}{l}\text { Problem S63SP30 93.2\% } \\
\mathrm{UO}_{2} \mathrm{~F}_{2} \text { solution } 81.8 \mathrm{~g} \mathrm{U} / 1 \text {, } \\
\text { in Al slabs, one slab is } \\
\text { made up from two } \\
7.62 \mathrm{~cm} \text { slabs snugly fit } \\
\text { together, the other is } \\
7.62 \mathrm{~cm}, 2 \times 1 \text { array, } \\
76.2 \mathrm{~cm} \text { separation, } \\
\text { unreflected }\end{array}$ & 49 & $0.981 \pm 0.003$ & $0.985 \pm 0.003$ & $0.977 \pm 0.003$ & $0.982 \pm 0.003$ \\
\hline CAS58 & $\begin{array}{l}\text { Problem S63SP6 } 93.2 \% \\
\mathrm{UO}_{2} \mathrm{~F}_{2} \text { solution } 81.8 \mathrm{~g} \mathrm{U} / 1 \text {, } \\
\text { in } \mathrm{Al} \text { slabs, one slab is } \\
14.834 \text { and the other is } \\
\text { made of from two } \\
7.62 \mathrm{~cm} \text { slabs snugly fit } \\
\text { together } 2 \times 1 \text { array, } \\
15.24 \mathrm{~cm} \text { separation, } \\
\text { unreflected }\end{array}$ & 49 & $0.983 \pm 0.003$ & $0.982 \pm 0.003$ & $0.983 \pm 0.003$ & $0.978 \pm 0.003$ \\
\hline CAS57 & $\begin{array}{l}\text { Problem S66SP48 } 93.2 \% \\
\mathrm{UO}_{2} \mathrm{~F}_{2} \text { solution } 81.8 \mathrm{~g} \mathrm{U} / 1 \text {, } \\
\text { in } \mathrm{Al} \text { slabs, one slab is } \\
14.834 \text { and the other is } \\
\text { made of from two } \\
7.62 \mathrm{~cm} \text { slabs snugly fit } \\
\text { together } 2 \times 1 \text { array, } \\
121.92 \mathrm{~cm} \text { separation, } \\
\text { unreflected }\end{array}$ & 49 & $0.984 \pm 0.003$ & $0.987 \pm 0.003$ & $0.986 \pm 0.003$ & $0.980 \pm 0.003$ \\
\hline CAS91 & $\begin{array}{l}\text { Problem } \mathrm{U} 6 \mathrm{~B} 271 \mathrm{~F} \text {. } \\
92.6 \% \mathrm{UO}_{2}\left(\mathrm{NO}_{3}\right)_{2} \\
\text { solution, } 63.3 \mathrm{~g} \mathrm{U} / 1 \text {, in } \\
\text { Plexiglas cylinders, } \\
3 \times 3 \times 3 \text { array, } \\
\text { unreflected, walls, floor, } \\
\text { and tank in experiment } \\
\text { room included }\end{array}$ & 45 & $1.008 \pm 0.003$ & $1.007 \pm 0.003$ & $1.003 \pm 0.004$ & $1.000 \pm 0.003$ \\
\hline CAS61 & $\begin{array}{l}\text { Problem U2B81F. } 92.6 \% \\
\mathrm{UO}_{2}\left(\mathrm{NO}_{3}\right)_{2} \text { solution, } \\
279 \mathrm{~g} \mathrm{U} / 1 \text {, in Plexiglas } \\
\text { cylinders, } 2 \times 2 \times 2 \text { array, } \\
\text { unreflected, walls, floor, } \\
\text { and tank in experiment } \\
\text { room included }\end{array}$ & 45 & $1.007 \pm 0.003$ & $1.006 \pm 0.003$ & $1.001 \pm 0.003$ & $0.997 \pm 0.003$ \\
\hline
\end{tabular}


Table 5 (continued)

Calculated $k_{\text {eff }}$ highly enriched uranium metal and solution systems

\begin{tabular}{|c|c|c|c|c|c|c|}
\hline \multirow[b]{3}{*}{ Case } & \multirow[b]{3}{*}{ Experimental description } & \multirow[b]{3}{*}{ Ref. } & \multicolumn{4}{|c|}{$k_{e f f} \pm \sigma$} \\
\hline & & & \multicolumn{2}{|c|}{27 group } & \multicolumn{2}{|c|}{238 group } \\
\hline & & & KENO V.a & KENO-VI & KENO V.a & KENO-VI \\
\hline CAS63 & $\begin{array}{l}\text { Problem } \mathrm{U} 4 \mathrm{~B} 271 \mathrm{~F} \text {. } \\
92.6 \% \mathrm{UO}_{2}\left(\mathrm{NO}_{3}\right)_{2} \\
\text { solution, } 415 \mathrm{~g} \mathrm{U} / 1 \text {, in } \\
\text { Plexiglas cylinders, } \\
3 \times 3 \times 3 \text { array, } \\
\text { unreflected, walls, floor, } \\
\text { and tank in experiment } \\
\text { room included }\end{array}$ & 45 & $0.998 \pm 0.003$ & $1.001 \pm 0.003$ & $0.991 \pm 0.003$ & $0.990 \pm 0.004$ \\
\hline CAS90 & $\begin{array}{l}\text { Problem U4U2B27. } \\
92.6 \% \mathrm{UO}_{2}\left(\mathrm{NO}_{3}\right)_{2} \\
\text { solution, } 63.3 \mathrm{~g} \mathrm{U} / 1 \text {, in } \\
\text { Plexiglas cylinders, } \\
3 \times 3 \times 3 \text { array, } \\
\text { unreflected, walls, floor, } \\
\text { and tank in experiment } \\
\text { room included }\end{array}$ & 45 & $1.001 \pm 0.003$ & $1.000 \pm 0.003$ & $0.999 \pm 0.003$ & $0.995 \pm 0.003$ \\
\hline CAS67 & $\begin{array}{l}\text { Problem U4R27B1F. } \\
92.6 \% \mathrm{UO}_{2}\left(\mathrm{NO}_{3}\right)_{2} \\
\text { solution, } 415 \mathrm{~g} \mathrm{U} / 1, \text { in } \\
\text { Plexiglas cylinders, } \\
3 \times 3 \times 3 \text { array, reflected, } \\
15.24 \mathrm{~cm} \text { paraffin on } \\
\text { bottom, } 2.54 \mathrm{~cm} \text { Plexiglas } \\
\text { on other faces. }\end{array}$ & 45 & $1.018 \pm 0.003$ & $1.017 \pm 0.003$ & $1.004 \pm 0.003$ & $1.005 \pm 0.003$ \\
\hline CAS71 & $\begin{array}{l}\text { Problem U4R27F1F. } \\
92.6 \% \mathrm{UO}_{2}\left(\mathrm{NO}_{3}\right)_{2} \\
\text { solution, } 415 \mathrm{~g} \mathrm{U} / 1, \text { in } \\
\text { Plexiglas cylinders, } \\
3 \times 3 \times 3 \text { array, reflected, } \\
15.24 \mathrm{~cm} \text { paraffin on } \\
\text { bottom, } 7.62 \mathrm{~cm} \text { paraffin } \\
\text { on other faces. }\end{array}$ & 45 & $1.031 \pm 0.003$ & $1.034 \pm 0.003$ & $1.023 \pm 0.003$ & $1.027 \pm 0.003$ \\
\hline CAS73 & $\begin{array}{l}\text { Problem } \mathrm{U} 4 \mathrm{R} 27 \mathrm{H} 1 \mathrm{~F} \text {. } \\
92.6 \% \mathrm{UO}_{2}\left(\mathrm{NO}_{3}\right)_{2} \\
\text { solution, } 415 \mathrm{~g} \mathrm{U} / 1 \text {, in } \\
\text { Plexiglas cylinders, } \\
3 \times 3 \times 3 \text { array, reflected, } \\
1.27 \mathrm{~cm} \text { paraffin all faces }\end{array}$ & 45 & $1.009 \pm 0.003$ & $1.008 \pm 0.003$ & $1.002 \pm 0.003$ & $1.004 \pm 0.003$ \\
\hline
\end{tabular}


Table 5 (continued)

Calculated $k_{\text {eff }}$ highly enriched uranium metal and solution systems

\begin{tabular}{|c|c|c|c|c|c|c|}
\hline \multirow[b]{3}{*}{ Case } & \multirow[b]{3}{*}{ Experimental description } & \multirow[b]{3}{*}{ Ref. } & \multicolumn{4}{|c|}{$k_{e f f} \pm \sigma$} \\
\hline & & & \multicolumn{2}{|c|}{27 group } & \multicolumn{2}{|c|}{238 group } \\
\hline & & & KENO V.a & KENO-VI & KENO V.a & KENO-VI \\
\hline CAS76 & $\begin{array}{l}\text { Problem U4R27H1F. } \\
92.6 \% \mathrm{UO}_{2}\left(\mathrm{NO}_{3}\right)_{2} \\
\text { solution, } 415 \mathrm{~g} \mathrm{U} / 1 \text {, in } \\
\text { Plexiglas cylinders, } \\
2 \times 2 \times 2 \text { array, reflected, } \\
15.24 \mathrm{~cm} \text { paraffin on } \\
\text { bottom, } 1.27 \mathrm{~cm} \text { Plexiglas } \\
\text { on other faces }\end{array}$ & 45 & $1.019 \pm 0.004$ & $1.008 \pm 0.003$ & $1.005 \pm 0.003$ & $1.004 \pm 0.003$ \\
\hline CAS79 & $\begin{array}{l}\text { Problem U4R8D1F. } \\
92.6 \% \mathrm{UO}_{2}\left(\mathrm{NO}_{3}\right)_{2} \\
\text { solution, } 415 \mathrm{~g} \mathrm{U} / 1 \text {, in } \\
\text { Plexiglas cylinders, } \\
2 \times 2 \times 2 \text { array, reflected, } \\
15.24 \mathrm{~cm} \text { paraffin on } \\
\text { bottom, } 2.54 \mathrm{~cm} \text { Plexiglas } \\
\text { on other faces }\end{array}$ & 45 & $1.012 \pm 0.003$ & $1.012 \pm 0.003$ & $1.012 \pm 0.003$ & $1.007 \pm 0.003$ \\
\hline CAS81 & $\begin{array}{l}\text { Problem } \mathrm{U} 4 \mathrm{R} 28 \mathrm{~F} 1 \mathrm{~F} \text {. } \\
92.6 \% \mathrm{UO}_{2}\left(\mathrm{NO}_{3}\right)_{2} \\
\text { solution, } 415 \mathrm{~g} \mathrm{U} / 1 \text {, in } \\
\text { Plexiglas cylinders, } \\
2 \times 2 \times 2 \text { array, reflected, } \\
15.24 \mathrm{~cm} \text { paraffin on } \\
\text { bottom, } 6.35 \mathrm{~cm} \text { Plexiglas } \\
\text { on other faces }\end{array}$ & 45 & $1.022 \pm 0.003$ & $1.028 \pm 0.003$ & $1.012 \pm 0.003$ & $1.019 \pm 0.003$ \\
\hline CAS84 & $\begin{array}{l}\text { Problem U4R8I1F. } 92.6 \% \\
\mathrm{UO}_{2}\left(\mathrm{NO}_{3}\right)_{2} \text { solution, } \\
415 \mathrm{~g} \mathrm{U} / 1 \text {, in Plexiglas } \\
\text { cylinders, } 2 \times 2 \times 2 \text { array, } \\
\text { reflected, } 15.24 \mathrm{~cm} \\
\text { paraffin on bottom, } \\
7.62 \mathrm{~cm} \text { Plexiglas on } \\
\text { other faces }\end{array}$ & 45 & $1.022 \pm 0.004$ & $1.022 \pm 0.003$ & $1.013 \pm 0.003$ & $1.009 \pm 0.003$ \\
\hline CAS86 & $\begin{array}{l}\text { Problem } \mathrm{U} 4 \mathrm{R} 8 \mathrm{~K} 1 \mathrm{~F} \text {. } \\
92.6 \% \mathrm{UO}_{2}\left(\mathrm{NO}_{3}\right)_{2} \\
\text { solution, } 415 \mathrm{~g} \mathrm{U} / 1 \text {, in } \\
\text { Plexiglas cylinders, } \\
2 \times 2 \times 2 \text { array, reflected, } \\
1.27 \mathrm{~cm} \text { paraffin on all } \\
\text { faces }\end{array}$ & 45 & $1.012 \pm 0.003$ & $1.011 \pm 0.003$ & $1.004 \pm 0.003$ & $1.009 \pm 0.003$ \\
\hline
\end{tabular}


Table 5 (continued)

Calculated $k_{\text {eff }}$ highly enriched uranium metal and solution systems

\begin{tabular}{|c|c|c|c|c|c|c|}
\hline \multirow[b]{3}{*}{ Case } & \multirow[b]{3}{*}{ Experimental description } & \multirow[b]{3}{*}{ Ref. } & \multicolumn{4}{|c|}{$k_{e f f} \pm \sigma$} \\
\hline & & & \multicolumn{2}{|c|}{27 group } & \multicolumn{2}{|c|}{238 group } \\
\hline & & & KENO V.a & KENO-VI & KENO V.a & KENO-VI \\
\hline CAS 88 & $\begin{array}{l}\text { Problem U4R8N1F. } \\
92.6 \% \mathrm{UO}_{2}\left(\mathrm{NO}_{3}\right)_{2} \\
\text { solution, } 415 \mathrm{~g} \mathrm{U} / 1 \text {, in } \\
\text { Plexiglas cylinders, } \\
2 \times 2 \times 2 \text { array, reflected, } \\
7.62 \mathrm{~cm} \text { paraffin on all } \\
\text { faces }\end{array}$ & 45 & $1.016 \pm 0.003$ & $1.023 \pm 0.003$ & $1.013 \pm 0.004$ & $1.012 \pm 0.003$ \\
\hline
\end{tabular}


Table 6. KENO V.a and KENO-VI comparison

Calculated $k_{\text {eff }}$ for highly enriched uranium solution systems

\begin{tabular}{|c|c|c|c|c|c|c|}
\hline \multirow[b]{3}{*}{ Case } & \multirow[b]{3}{*}{ Experimental description } & \multirow[b]{3}{*}{ Ref. } & \multicolumn{4}{|c|}{$k_{e f f} \pm \sigma$} \\
\hline & & & \multicolumn{2}{|c|}{27 group } & \multicolumn{2}{|c|}{238 group } \\
\hline & & & KENO V.a & KENO-VI & KENO V.a & KENO-VI \\
\hline CAS01 & $\begin{array}{l}\text { Experiment } 1 \text {. } \\
\mathrm{U}(93.2) \mathrm{O}_{2}\left(\mathrm{NO}_{3}\right)_{2} \\
\mathrm{H} / \mathrm{X}=1378 \text { in } 27.24 \text {-in. } \\
\text { diam sphere }\end{array}$ & 25,26 & $0.999 \pm 0.002$ & $0.997 \pm 0.002$ & $0.996 \pm 0.002$ & $1.003 \pm 0.002$ \\
\hline CAS02 & $\begin{array}{l}\text { Experiment } 2 . \\
\mathrm{U}(93.2) \mathrm{O}_{2}\left(\mathrm{NO}_{3}\right)_{2} \\
\mathrm{H} / \mathrm{X}=1177, \mathrm{~B} \text { poisoned, } \\
\text { in } 27.24 \text {-in. diam sphere }\end{array}$ & 25,26 & $0.998 \pm 0.002$ & $1.001 \pm 0.002$ & $1.003 \pm 0.002$ & $0.998 \pm 0.002$ \\
\hline CAS03 & $\begin{array}{l}\text { Experiment } 3 \text {. } \\
\mathrm{U}(93.2) \mathrm{O}_{2}\left(\mathrm{NO}_{3}\right)_{2} \\
\mathrm{H} / \mathrm{X}=1033, \mathrm{~B} \text { poisoned, } \\
\text { in } 27.24 \text {-in. diam sphere }\end{array}$ & 25,26 & $0.996 \pm 0.002$ & $0.993 \pm 0.002$ & $0.993 \pm 0.002$ & $0.993 \pm 0.002$ \\
\hline CAS04 & $\begin{array}{l}\text { Experiment } 4 . \\
\mathrm{U}(93.2) \mathrm{O}_{2}\left(\mathrm{NO}_{3}\right)_{2} \\
\mathrm{H} / \mathrm{X}=972, \mathrm{~B} \text { poisoned, } \\
\text { in 27.24-in. diam sphere }\end{array}$ & 25,26 & $0.995 \pm 0.002$ & $0.998 \pm 0.002$ & $0.998 \pm 0.002$ & $1.000 \pm 0.002$ \\
\hline CAS05 & $\begin{array}{l}\text { Experiment } 5 \text {. } \\
\mathrm{U}-233 \mathrm{O}_{2}\left(\mathrm{NO}_{3}\right)_{2} \\
\mathrm{H} / \mathrm{X}=1533 \text {, in } 27.24 \text {-in. } \\
\text { diam sphere }\end{array}$ & 25,26 & $0.998 \pm 0.002$ & $1.003 \pm 0.002$ & $0.995 \pm 0.002$ & $0.999 \pm 0.002$ \\
\hline CAS06 & $\begin{array}{l}\text { Experiment } 6 . \\
\mathrm{U}-233 \mathrm{O}_{2}\left(\mathrm{NO}_{3}\right)_{2} \\
\mathrm{H} / \mathrm{X}=1470, \mathrm{~B} \text { poisoned, } \\
\text { in } 27.24 \text {-in. diam sphere }\end{array}$ & 25,26 & $1.002 \pm 0.002$ & $0.999 \pm 0.002$ & $0.996 \pm 0.002$ & $0.994 \pm 0.002$ \\
\hline CAS07 & $\begin{array}{l}\text { Experiment } 7 . \\
\mathrm{U}-233 \mathrm{O}_{2}\left(\mathrm{NO}_{3}\right)_{2} \\
\mathrm{H} / \mathrm{X}=1417, \mathrm{~B} \text { poisoned, } \\
\text { in } 27.24 \text {-in. diam sphere }\end{array}$ & 25,26 & $1.002 \pm 0.002$ & $0.999 \pm 0.002$ & $0.997 \pm 0.002$ & $0.995 \pm 0.002$ \\
\hline CAS08 & $\begin{array}{l}\text { Experiment } 8 . \\
\mathrm{U}-233 \mathrm{O}_{2}\left(\mathrm{NO}_{3}\right)_{2} \\
\mathrm{H} / \mathrm{X}=1368, \mathrm{~B} \text { poisoned, } \\
\text { in } 27.24 \text {-in. diam sphere }\end{array}$ & 25,26 & $1.003 \pm 0.002$ & $1.003 \pm 0.002$ & $0.993 \pm 0.002$ & $0.991 \pm 0.002$ \\
\hline CAS09 & $\begin{array}{l}\text { Experiment } 9 . \\
\mathrm{U}-233 \mathrm{O}_{2}\left(\mathrm{NO}_{3}\right)_{2} \\
\mathrm{H} / \mathrm{X}=1308, \mathrm{~B} \text { poisoned, } \\
\text { in } 27.24 \text {-in. diam sphere }\end{array}$ & 25,26 & $1.005 \pm 0.002$ & $1.006 \pm 0.002$ & $1.000 \pm 0.002$ & $0.996 \pm 0.002$ \\
\hline
\end{tabular}


Table 6 (continued)

Calculated $k_{\text {eff }}$ for highly enriched uranium solution systems

\begin{tabular}{|c|c|c|c|c|c|c|}
\hline \multirow[b]{3}{*}{ Case } & \multirow[b]{3}{*}{ Experimental description } & \multirow[b]{3}{*}{ Ref. } & \multicolumn{4}{|c|}{$k_{e f f} \pm \sigma$} \\
\hline & & & \multicolumn{2}{|c|}{27 group } & \multicolumn{2}{|c|}{238 group } \\
\hline & & & KENO V.a & KENO-VI & KENO V.a & KENO-VI \\
\hline CAS10 & $\begin{array}{l}\text { Experiment } 10 \text {. } \\
\mathrm{U}(93.2) \mathrm{O}_{2}\left(\mathrm{NO}_{3}\right)_{2} \\
\mathrm{H} / \mathrm{X}=1835 \text {, in } 48.04-\mathrm{in} . \\
\text { diam sphere }\end{array}$ & 25,26 & $0.998 \pm 0.002$ & $0.995 \pm 0.001$ & $1.000 \pm 0.002$ & $0.998 \pm 0.001$ \\
\hline CAS11 & $\begin{array}{l}\text { Experiment } 11 . \\
\mathrm{U}-233 \mathrm{O}_{2}\left(\mathrm{NO}_{3}\right)_{2} \\
\mathrm{H} / \mathrm{X}=1986 \text {, in 48.04-in. } \\
\text { diam sphere }\end{array}$ & 25,26 & $0.994 \pm 0.002$ & $1.000 \pm 0.001$ & $0.996 \pm 0.002$ & $0.994 \pm 0.001$ \\
\hline CAS12 & $\begin{array}{l}\text { Experiment } 12 \text {. } \\
\mathrm{U}(93.2) \mathrm{O}_{2}\left(\mathrm{NO}_{3}\right)_{2} \\
\mathrm{H} / \mathrm{X}=1604 \text {, in } 60.92-\mathrm{in} . \\
\text { diam cylinder }\end{array}$ & 25,26 & $0.991 \pm 0.002$ & $0.992 \pm 0.002$ & $0.996 \pm 0.001$ & $0.994 \pm 0.002$ \\
\hline CAS13 & $\begin{array}{l}\text { Experiment } 13 \text {. } \\
\mathrm{U}(93.2) \mathrm{O}_{2}\left(\mathrm{NO}_{3}\right)_{2} \\
\mathrm{H} / \mathrm{X}=1634 \text {, in } 60.92 \text {-in. } \\
\text { diam cylinder }\end{array}$ & 25,26 & $0.994 \pm 0.002$ & $0.992 \pm 0.001$ & $0.999 \pm 0.002$ & $0.995 \pm 0.002$ \\
\hline CAS14 & $\begin{array}{l}\text { Experiment } 14 \text {. } \\
\mathrm{U}(93.2) \mathrm{O}_{2}\left(\mathrm{NO}_{3}\right)_{2} \\
\mathrm{H} / \mathrm{X}=1821 \text {, in } 60.92-\text { in. } \\
\text { diam cylinder }\end{array}$ & 25,26 & $0.999 \pm 0.001$ & $0.997 \pm 0.001$ & $0.997 \pm 0.002$ & $1.000 \pm 0.001$ \\
\hline CAS15 & $\begin{array}{l}\text { Experiment } 15 \text {. } \\
\mathrm{U}(93.2) \mathrm{O}_{2}\left(\mathrm{NO}_{3}\right)_{2} \\
\mathrm{H} / \mathrm{X}=1905 \text {, in } 60.92-\mathrm{in} . \\
\text { diam cylinder }\end{array}$ & 25,26 & $0.999 \pm 0.001$ & $0.999 \pm 0.001$ & $0.999 \pm 0.002$ & $1.002 \pm 0.001$ \\
\hline CAS16 & $\begin{array}{l}\text { Experiment } 16 \text {. } \\
\mathrm{U}(93.2) \mathrm{O}_{2}\left(\mathrm{NO}_{3}\right)_{2} \\
\mathrm{H} / \mathrm{X}=1981 \text {, in } 60.92-\mathrm{in} . \\
\text { diam cylinder }\end{array}$ & 25,26 & $0.996 \pm 0.001$ & $0.995 \pm 0.001$ & $0.996 \pm 0.001$ & $1.003 \pm 0.001$ \\
\hline CAS17 & $\begin{array}{l}\text { Experiment } 17 \text {. } \\
\mathrm{U}-233 \mathrm{O}_{2}\left(\mathrm{NO}_{3}\right)_{2} \\
\mathrm{H} / \mathrm{X}=1819 \text {, in } 60.92 \text {-in. } \\
\text { diam cylinder }\end{array}$ & 25,26 & $0.993 \pm 0.001$ & $0.992 \pm 0.002$ & $0.993 \pm 0.001$ & $0.991 \pm 0.002$ \\
\hline CAS18 & $\begin{array}{l}\text { Experiment } 18 . \\
\mathrm{U}-233 \mathrm{O}_{2}\left(\mathrm{NO}_{3}\right)_{2} \\
\mathrm{H} / \mathrm{X}=1900 \text {, in } 60.92 \text {-in. } \\
\text { diam cylinder }\end{array}$ & 25,26 & $0.996 \pm 0.001$ & $0.996 \pm 0.001$ & $0.995 \pm 0.001$ & $0.997 \pm 0.001$ \\
\hline
\end{tabular}


Table 6 (continued)

Calculated $k_{\text {eff }}$ for highly enriched uranium solution systems

\begin{tabular}{|c|c|c|c|c|c|c|}
\hline \multirow[b]{3}{*}{ Case } & \multirow[b]{3}{*}{ Experimental description } & \multirow[b]{3}{*}{ Ref. } & \multicolumn{4}{|c|}{$k_{e f f} \pm \sigma$} \\
\hline & & & \multicolumn{2}{|c|}{27 group } & \multicolumn{2}{|c|}{238 group } \\
\hline & & & KENO V.a & KENO-VI & KENO V.a & KENO-VI \\
\hline CAS19 & $\begin{array}{l}\text { Experiment } 19 . \\
\mathrm{U}-233 \mathrm{O}_{2}\left(\mathrm{NO}_{3}\right)_{2} \\
\mathrm{H} / \mathrm{X}=1996 \text {, in } 60.92 \text {-in. } \\
\text { diam cylinder }\end{array}$ & 25,26 & $0.994 \pm 0.002$ & $0.994 \pm 0.001$ & $0.995 \pm 0.001$ & $0.997 \pm 0.001$ \\
\hline CAS20 & $\begin{array}{l}\text { Experiment } 20 \text {. } \\
\mathrm{U}-233 \mathrm{O}_{2}\left(\mathrm{NO}_{3}\right)_{2} \\
\mathrm{H} / \mathrm{X}=2106 \text {, in } 60.92 \text {-in. } \\
\text { diam cylinder }\end{array}$ & 25,26 & $0.992 \pm 0.002$ & $0.993 \pm 0.001$ & $0.995 \pm 0.001$ & $0.994 \pm 0.002$ \\
\hline CAS21 & $\begin{array}{l}\text { Experiment } 21 . \\
\mathrm{U}(93.2) \mathrm{O}_{2}\left(\mathrm{NO}_{3}\right)_{2} \\
\mathrm{H} / \mathrm{X}=1955 \text {, in } 107.7 \text {-in. } \\
\text { diam cylinder }\end{array}$ & 25,26 & $0.993 \pm 0.001$ & $0.996 \pm 0.001$ & $0.997 \pm 0.001$ & $1.002 \pm 0.001$ \\
\hline CAS22 & $\begin{array}{l}\text { Experiment } 22 . \\
\mathrm{U}(93.2) \mathrm{O}_{2}\left(\mathrm{NO}_{3}\right)_{2} \\
\mathrm{H} / \mathrm{X}=2004 \text {, in } 107.7-\mathrm{in} . \\
\text { diam cylinder }\end{array}$ & 25,26 & $0.995 \pm 0.001$ & $0.994 \pm 0.001$ & $0.999 \pm 0.001$ & $0.999 \pm 0.001$ \\
\hline CAS23 & $\begin{array}{l}\text { Experiment } 23 . \\
\mathrm{U}(93.2) \mathrm{O}_{2}\left(\mathrm{NO}_{3}\right)_{2} \\
\mathrm{H} / \mathrm{X}=2052 \text {, in } 107.7 \text {-in. } \\
\text { diam cylinder }\end{array}$ & 25,26 & $0.996 \pm 0.001$ & $0.996 \pm 0.001$ & $0.999 \pm 0.001$ & $1.000 \pm 0.001$ \\
\hline
\end{tabular}




\section{DISCUSSION OF RESULTS}

Table 7 gives the mean, median, minimum, and maximum $k$-effectives for each table of results. The deviations for the mean values are the root mean square of each table. The mean values of $k_{\text {eff }}$ are in excellent agreement with the experiments.

The spread in data, however, indicates that there may be problems with certain types of calculations. The low-enriched uranium systems reported in Table 3 and the highly-enriched uranium systems in Table 5 show a wide spread in the data. This could be caused by the detail of the descriptions of the experiments.

Figures 1-6 are plots of calculated $k_{\text {eff }}$ values for each case from Tables 1-6. These plots show the comparisons between KENO-VI and KENO V.a using both the 27- and 238-group cross-section libraries.

Figures 7-10 show the calculated value of $k_{\text {eff }}$ as a function of the assay for low-enriched and high-enriched experiments. Figures 7 and 8 show the comparison of 27- and 238-group cross sections for low-enrichment experiments using KENO V.a and KENO-VI, respectively. Figures 9 and 10 show the same comparison for high-enrichment experiments.

Figure 11 shows the comparison of KENO V.a and KENO-VI for low-enrichment experiments using 238-group cross sections. Figure 12 shows the same comparison for high-enrichment experiments.

Figures 13-19 are plots of $k_{\text {eff }}$ versus energy of average lethargy causing fission (EALF) for each table of results. Table 5 was split into two portions because of the large range in EALF. Figure 17 presents the EALF for the uranium metal systems of Table 5 while Figure 18 presents the EALF for the uranium solutions for Table 5. These plots do not show a trend in the results. 
Table 7. Average calculated $k_{\text {eff }}$ value by table

\begin{tabular}{|c|c|c|c|c|c|}
\hline \multicolumn{6}{|c|}{ Table $=1$} \\
\hline \multirow{2}{*}{\multicolumn{2}{|c|}{$\begin{array}{l}\text { Total } \\
\text { Cases }\end{array}$}} & \multicolumn{2}{|c|}{27 group } & \multicolumn{2}{|c|}{238 group } \\
\hline & & KENO V.a & KENO-VI & KENO V.a & KENO-VI \\
\hline \multirow[t]{5}{*}{19} & & & & & \\
\hline & Mean $k_{e f f}$ & & $0.994 \pm 0.004$ & $0.995 \pm 0.005$ & $0.996 \pm 0.005$ \\
\hline & Median $k_{\text {eff }}$ & $0.995 \pm 0.002$ & $0.993 \pm 0.002$ & $0.995 \pm 0.002$ & $0.996 \pm 0.002$ \\
\hline & Minimum $k_{e f f}$ & $0.987 \pm 0.002$ & $0.989 \pm 0.002$ & $0.988 \pm 0.003$ & $0.989 \pm 0.003$ \\
\hline & Maximum $k_{e f f}$ & $1.002 \pm 0.003$ & $1.001 \pm 0.002$ & $1.002 \pm 0.002$ & $1.003 \pm 0.003$ \\
\hline \multicolumn{6}{|c|}{ Table $=2$} \\
\hline \multirow{2}{*}{\multicolumn{2}{|c|}{$\begin{array}{l}\text { Total } \\
\text { Cases }\end{array}$}} & \multicolumn{2}{|c|}{27 group } & \multicolumn{2}{|c|}{238 group } \\
\hline & & KENO V.a & KENO-VI & KENO V.a & KENO-VI \\
\hline \multirow[t]{5}{*}{7} & & & & & \\
\hline & Mean $k_{e f f}$ & $1.003 \pm 0.006$ & $1.004 \pm 0.008$ & & \\
\hline & Median $k_{e f f}$ & $1.006 \pm 0.002$ & $1.006 \pm 0.002$ & $0.995 \pm 0.002$ & $0.996 \pm 0.002$ \\
\hline & Minimum $k_{e f f}$ & $0.992 \pm 0.002$ & $0.991 \pm 0.002$ & $0.984 \pm 0.002$ & $0.985 \pm 0.002$ \\
\hline & Maximum $k_{e f f}$ & $1.008 \pm 0.003$ & $1.013 \pm 0.002$ & $1.003 \pm 0.002$ & $0.998 \pm 0.003$ \\
\hline \multicolumn{6}{|c|}{ Table $=3$} \\
\hline \multirow{2}{*}{\multicolumn{2}{|c|}{$\begin{array}{l}\text { Total } \\
\text { Cases }\end{array}$}} & \multicolumn{2}{|c|}{27 group } & \multicolumn{2}{|c|}{238 group } \\
\hline & & KENO V.a & KENO-VI & KENO V.a & KENO-VI \\
\hline \multirow[t]{5}{*}{23} & & & & & \\
\hline & Mean $k_{e f f}$ & $0.999 \pm 0.010$ & $1.000 \pm 0.009$ & $1.002 \pm 0.002$ & $1.003 \pm 0.009$ \\
\hline & Median $k_{\text {eff }}$ & $0.997 \pm 0.002$ & $0.999 \pm 0.002$ & $0.999 \pm 0.003$ & $1.001 \pm 0.003$ \\
\hline & Minimum $k_{e f f}$ & $0.985 \pm 0.002$ & $0.981 \pm 0.002$ & $0.986 \pm 0.003$ & $0.990 \pm 0.003$ \\
\hline & Maximum $k_{e f f}$ & $1.014 \pm 0.003$ & $1.016 \pm 0.004$ & $1.017 \pm 0.003$ & $1.019 \pm 0.003$ \\
\hline
\end{tabular}


Table 7 (continued)

\begin{tabular}{|c|c|c|c|c|c|}
\hline \multicolumn{6}{|c|}{ Table $=4$} \\
\hline \multirow{2}{*}{\multicolumn{2}{|c|}{$\begin{array}{c}\text { Total } \\
\text { Cases } \\
\end{array}$}} & \multicolumn{2}{|c|}{27 group } & \multicolumn{2}{|c|}{238 group } \\
\hline & & KENO V.a & KENO-VI & KENO V.a & KENO-VI \\
\hline \multirow[t]{5}{*}{17} & & & & & \\
\hline & Mean $k_{e f f}$ & $1.009 \pm 0.007$ & $1.006 \pm 0.007$ & $1.002 \pm 0.007$ & $1.003 \pm 0.007$ \\
\hline & Median $k_{e f f}$ & $1.008 \pm 0.003$ & $1.005 \pm 0.003$ & $1.000 \pm 0.003$ & $1.001 \pm 0.003$ \\
\hline & Minimum $k_{e f f}$ & $0.998 \pm 0.003$ & $0.996 \pm 0.003$ & $0.991 \pm 0.003$ & $0.993 \pm 0.003$ \\
\hline & Maximum $k_{e f f}$ & $1.028 \pm 0.003$ & $1.028 \pm 0.004$ & $1.017 \pm 0.003$ & $1.021 \pm 0.003$ \\
\hline \multicolumn{6}{|c|}{ Table $=5$} \\
\hline \multirow{2}{*}{\multicolumn{2}{|c|}{$\begin{array}{l}\text { Total } \\
\text { Cases } \\
\end{array}$}} & \multicolumn{2}{|c|}{27 group } & \multicolumn{2}{|c|}{238 group } \\
\hline & & KENO V.a & KENO-VI & KENO V.a & KENO-VI \\
\hline \multirow[t]{5}{*}{35} & & & & & \\
\hline & Mean $k_{e f f}$ & $1.005 \pm 0.015$ & $1.005 \pm 0.015$ & $0.999 \pm 0.013$ & $1.004 \pm 0.014$ \\
\hline & Median $k_{e f f}$ & $1.007 \pm 0.002$ & $1.006 \pm 0.003$ & $1.001 \pm 0.003$ & $0.998 \pm 0.002$ \\
\hline & Minimum $k_{\text {eff }}$ & $0.976 \pm 0.003$ & & $0.973 \pm 0.003$ & \\
\hline & Maximum $k_{e f f}$ & $1.036 \pm 0.003$ & $1.035 \pm 0.004$ & $1.023 \pm 0.003$ & $1.028 \pm 0.003$ \\
\hline \multicolumn{6}{|c|}{ Table $=6$} \\
\hline \multirow{2}{*}{\multicolumn{2}{|c|}{$\begin{array}{l}\text { Total } \\
\text { Cases }\end{array}$}} & \multicolumn{2}{|c|}{27 group } & \multicolumn{2}{|c|}{238 group } \\
\hline & & KENO V.a & KENO-VI & KENO V.a & KENO-VI \\
\hline \multirow[t]{5}{*}{23} & & & & & \\
\hline & Mean $k_{\text {eff }}$ & $0.997 \pm 0.004$ & $0.997 \pm 0.004$ & $0.997 \pm 0.003$ & $0.997 \pm 0.004$ \\
\hline & Median $k_{e f f}$ & $0.996 \pm 0.002$ & $0.996 \pm 0.001$ & $0.996 \pm 0.001$ & $0.997 \pm 0.001$ \\
\hline & Minimum $k_{e f f}$ & $1.005 \pm 0.002$ & $1.006 \pm 0.002$ & $1.003 \pm 0.002$ & $1.003 \pm 0.002$ \\
\hline & Maximum $k_{e f f}$ & $1.005 \pm 0.002$ & $1.006 \pm 0.002$ & $1.003 \pm 0.002$ & $1.003 \pm 0.002$ \\
\hline
\end{tabular}




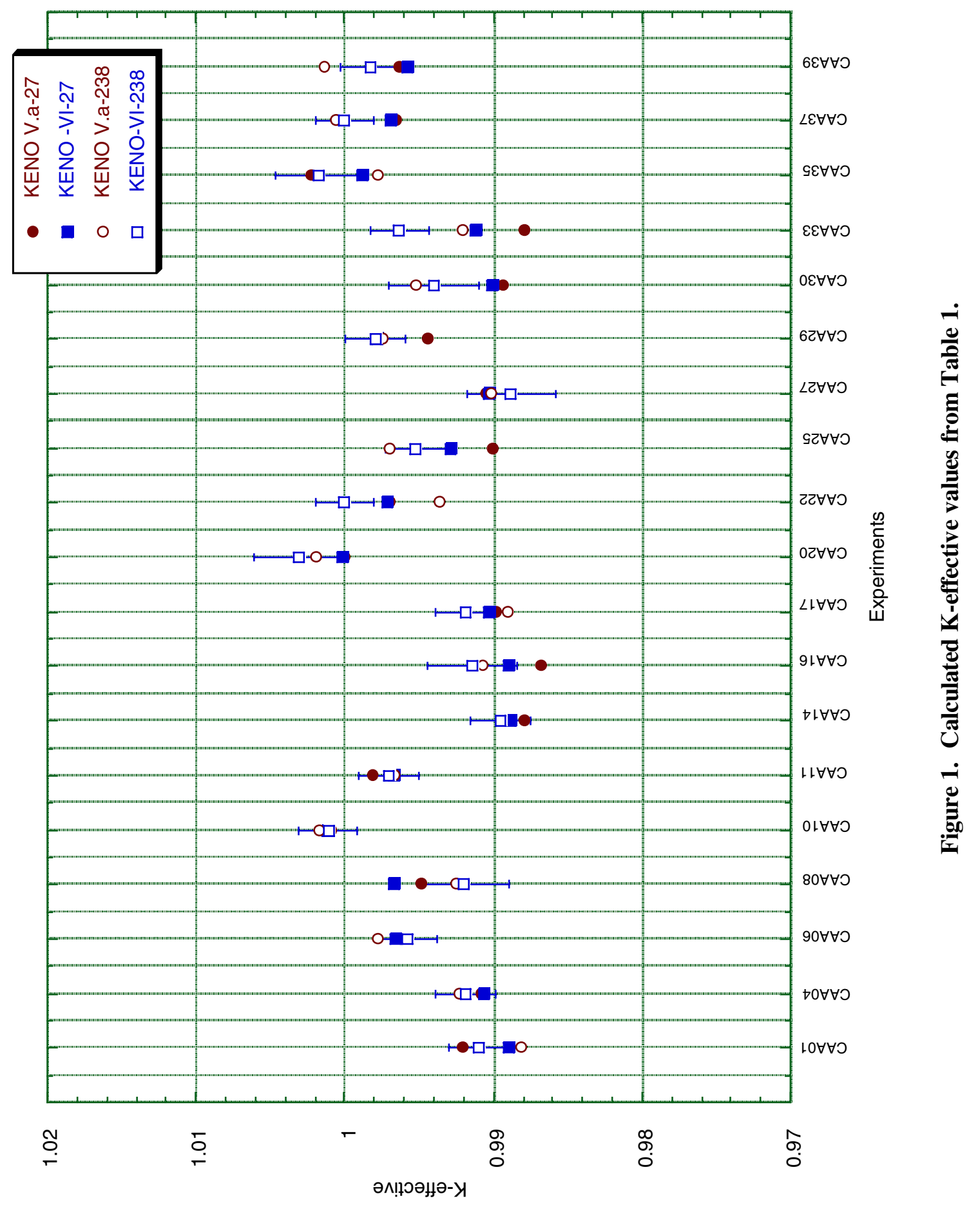




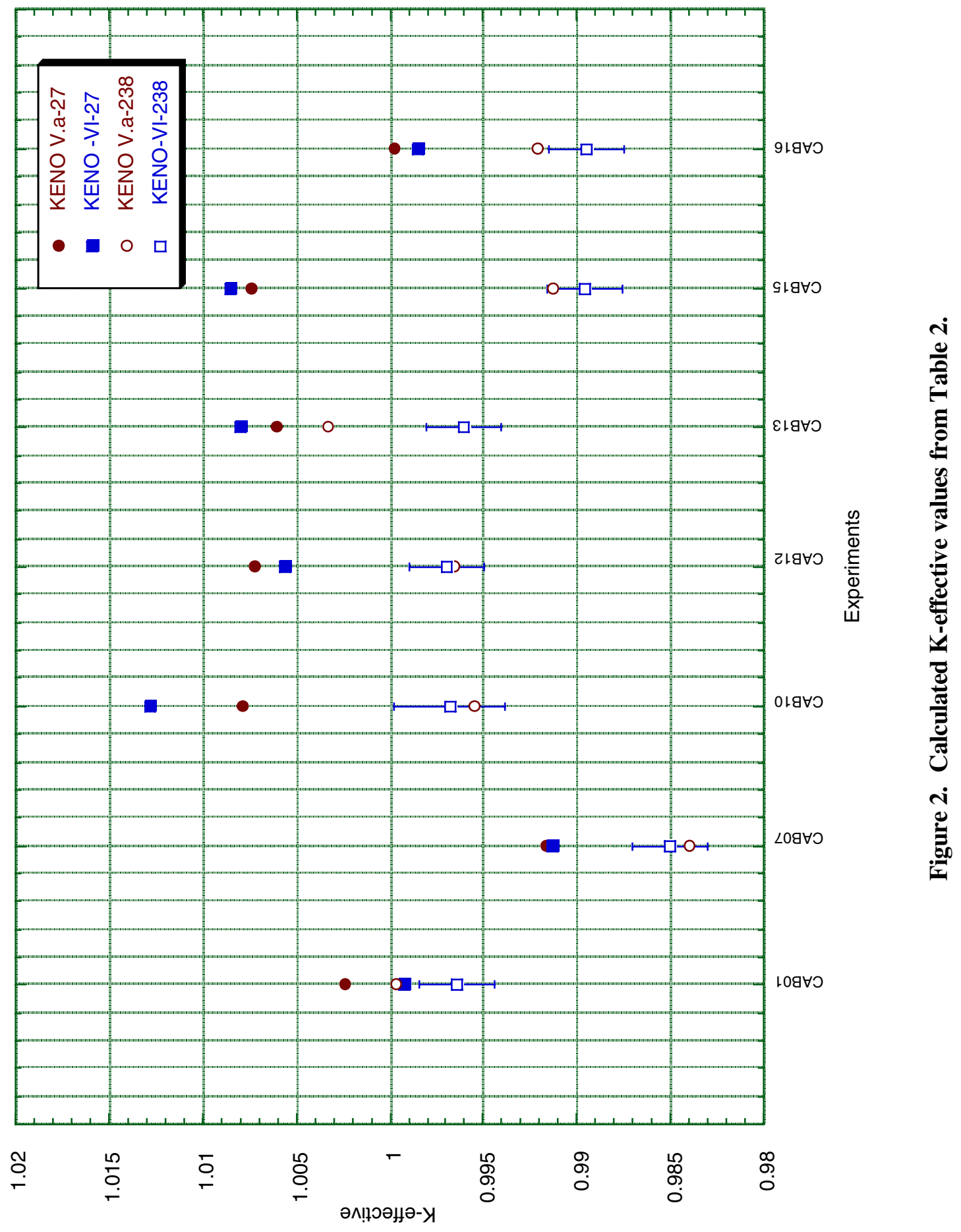




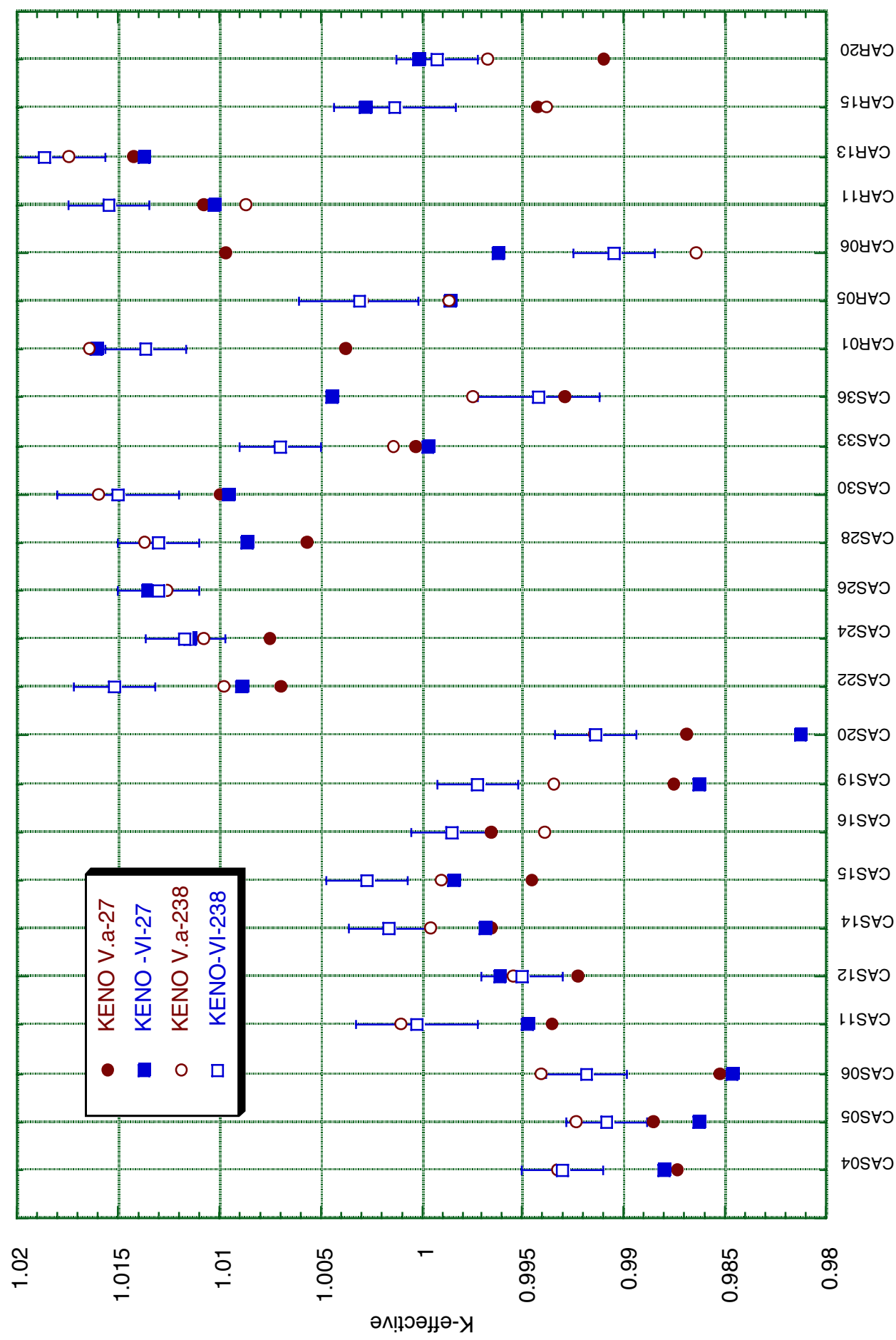

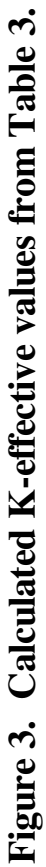




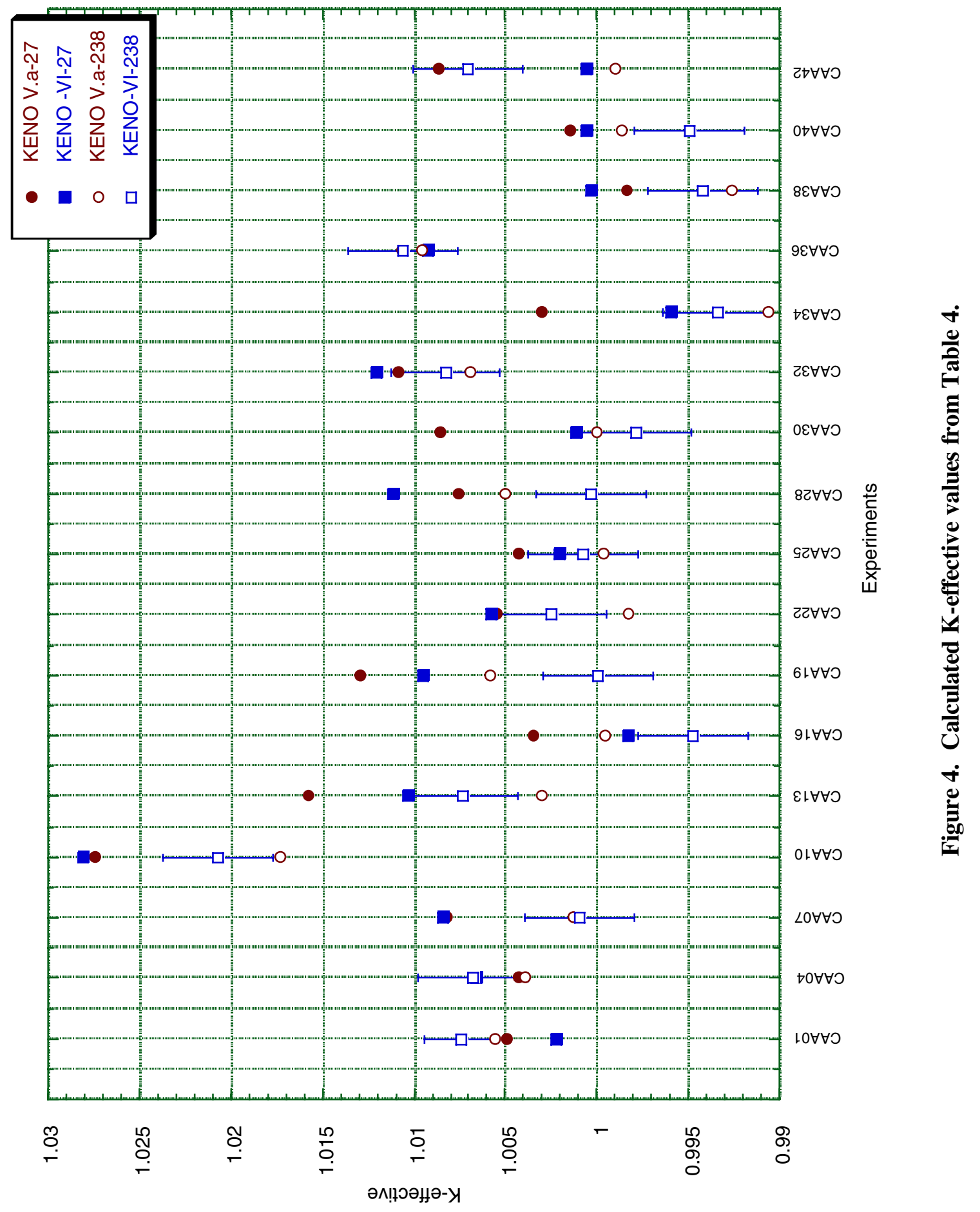




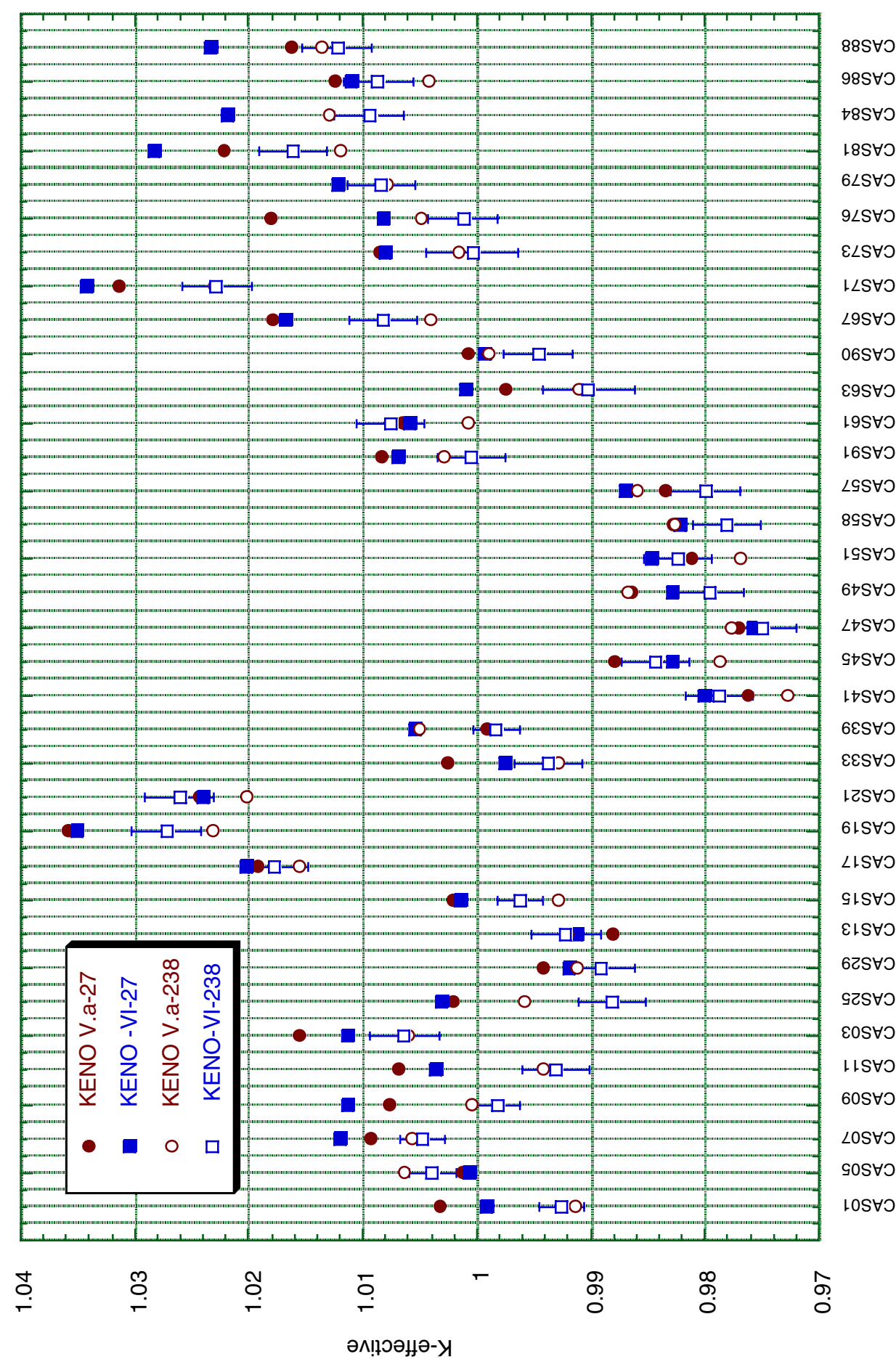

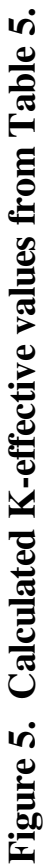




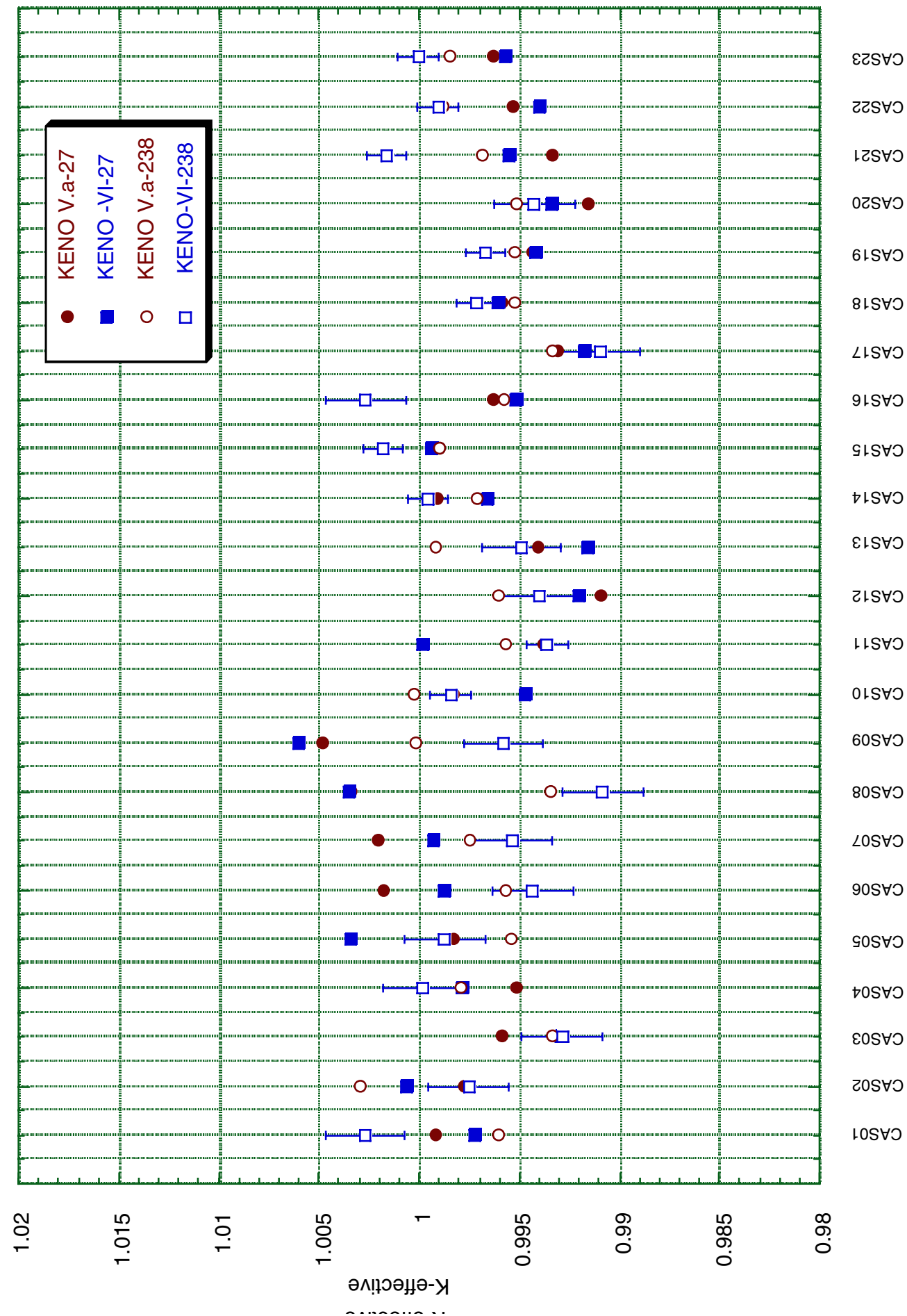




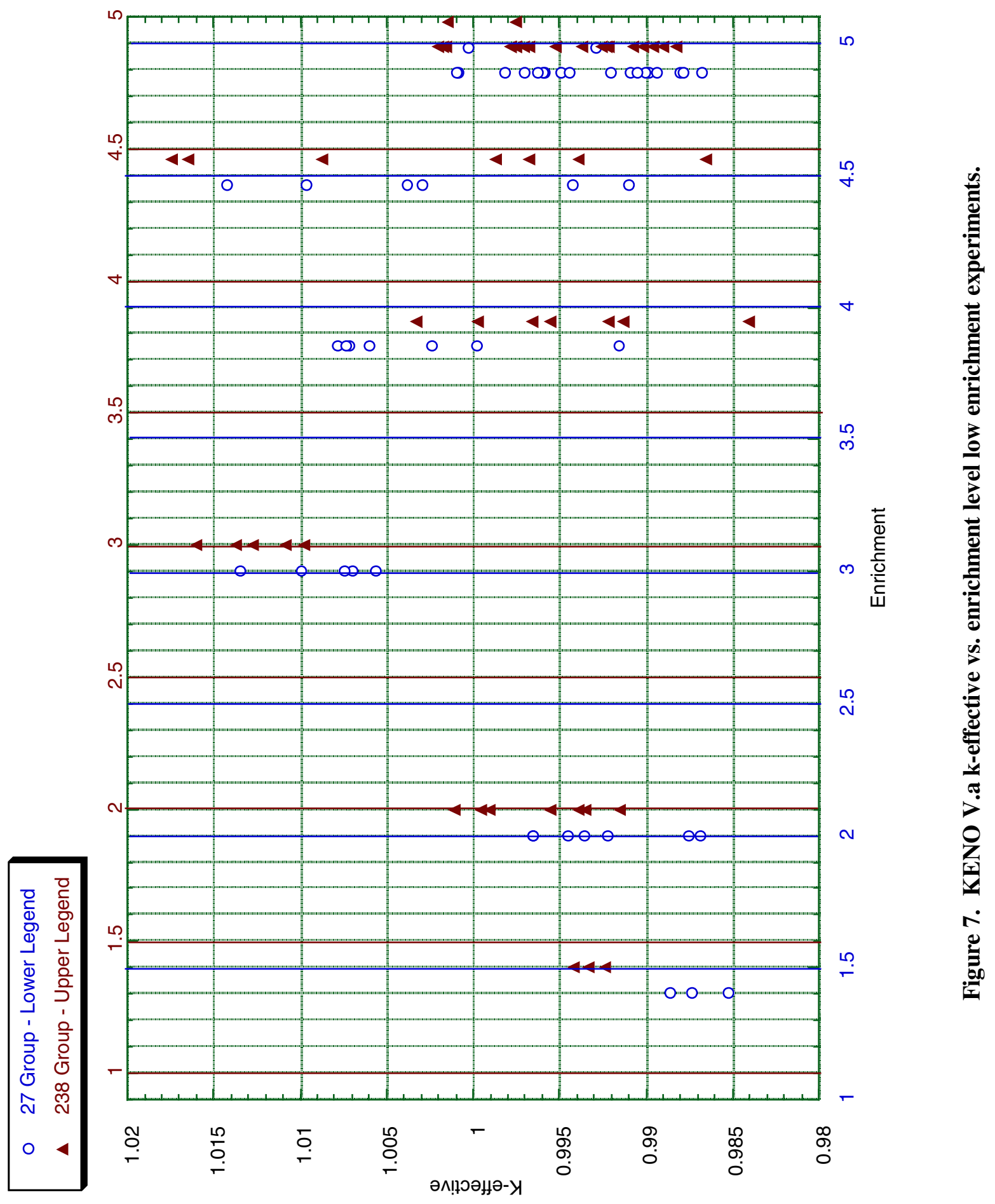




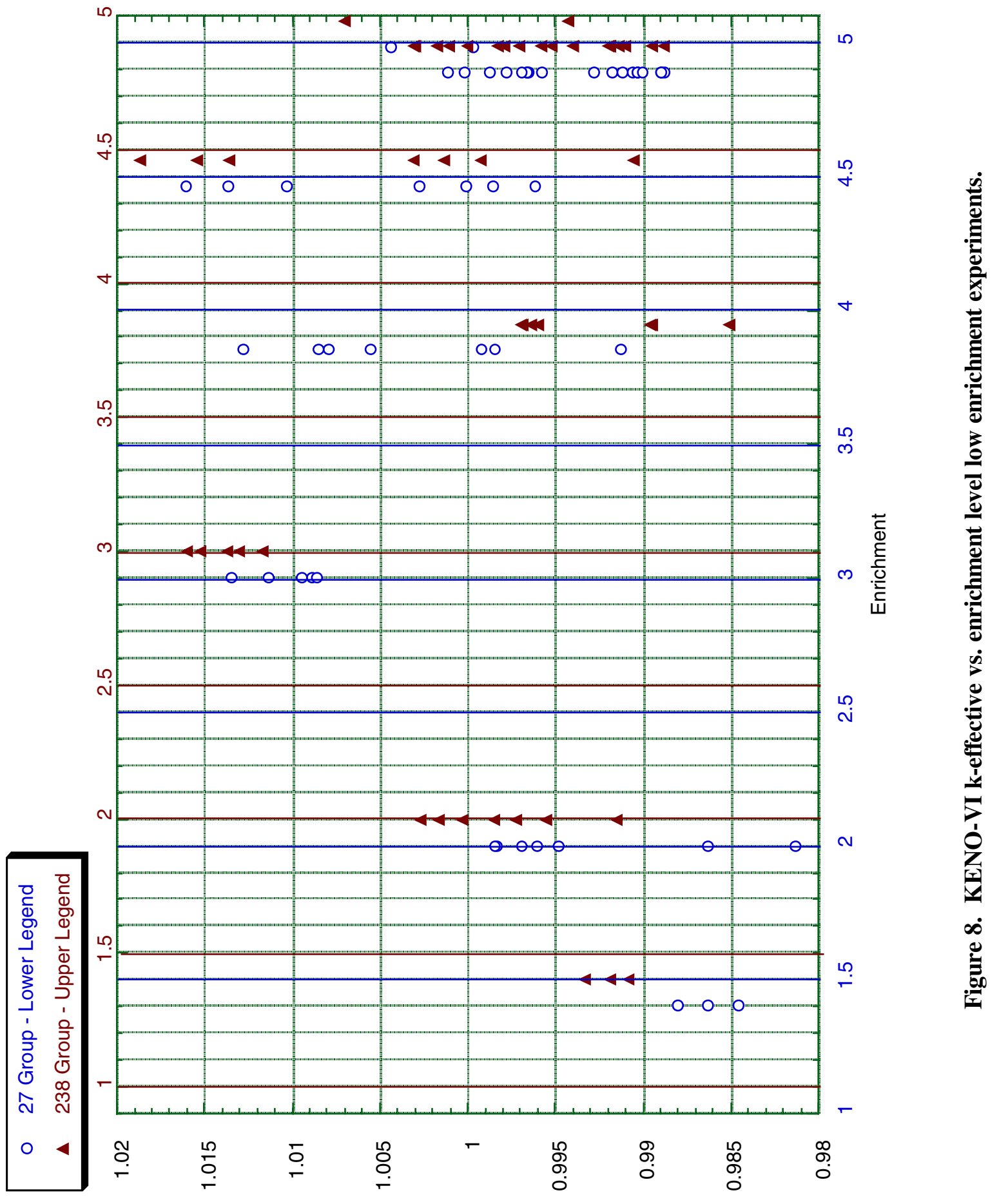




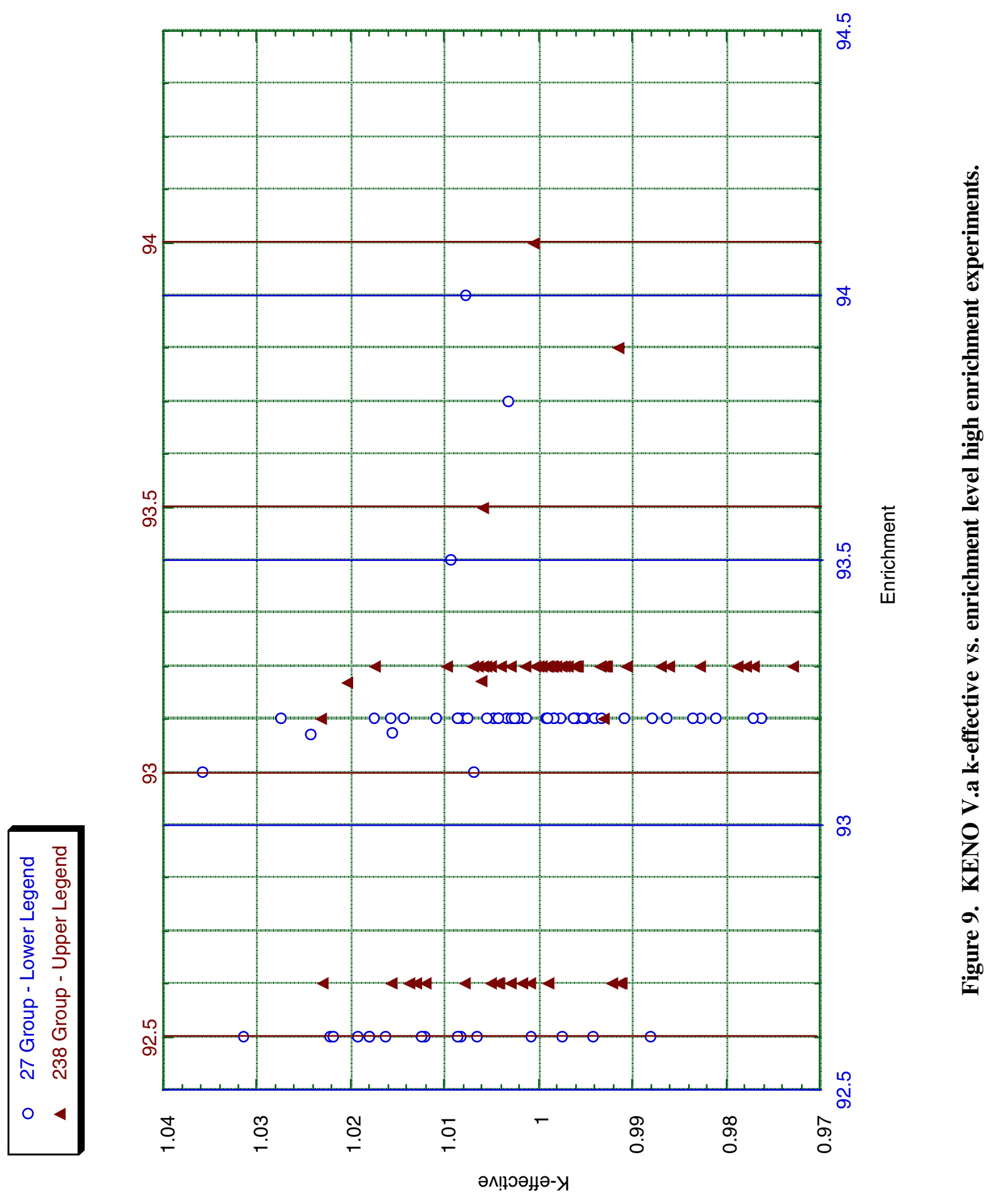




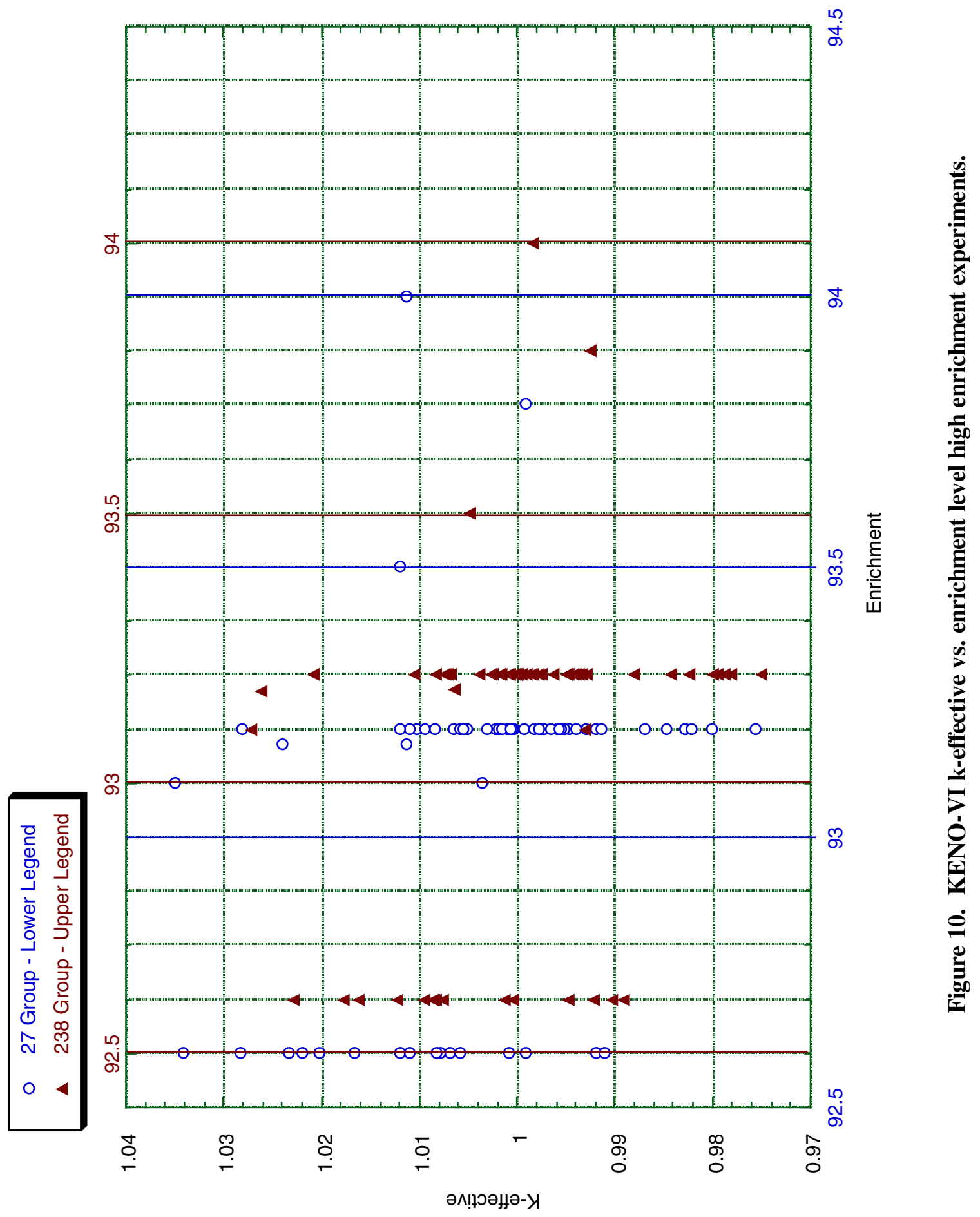




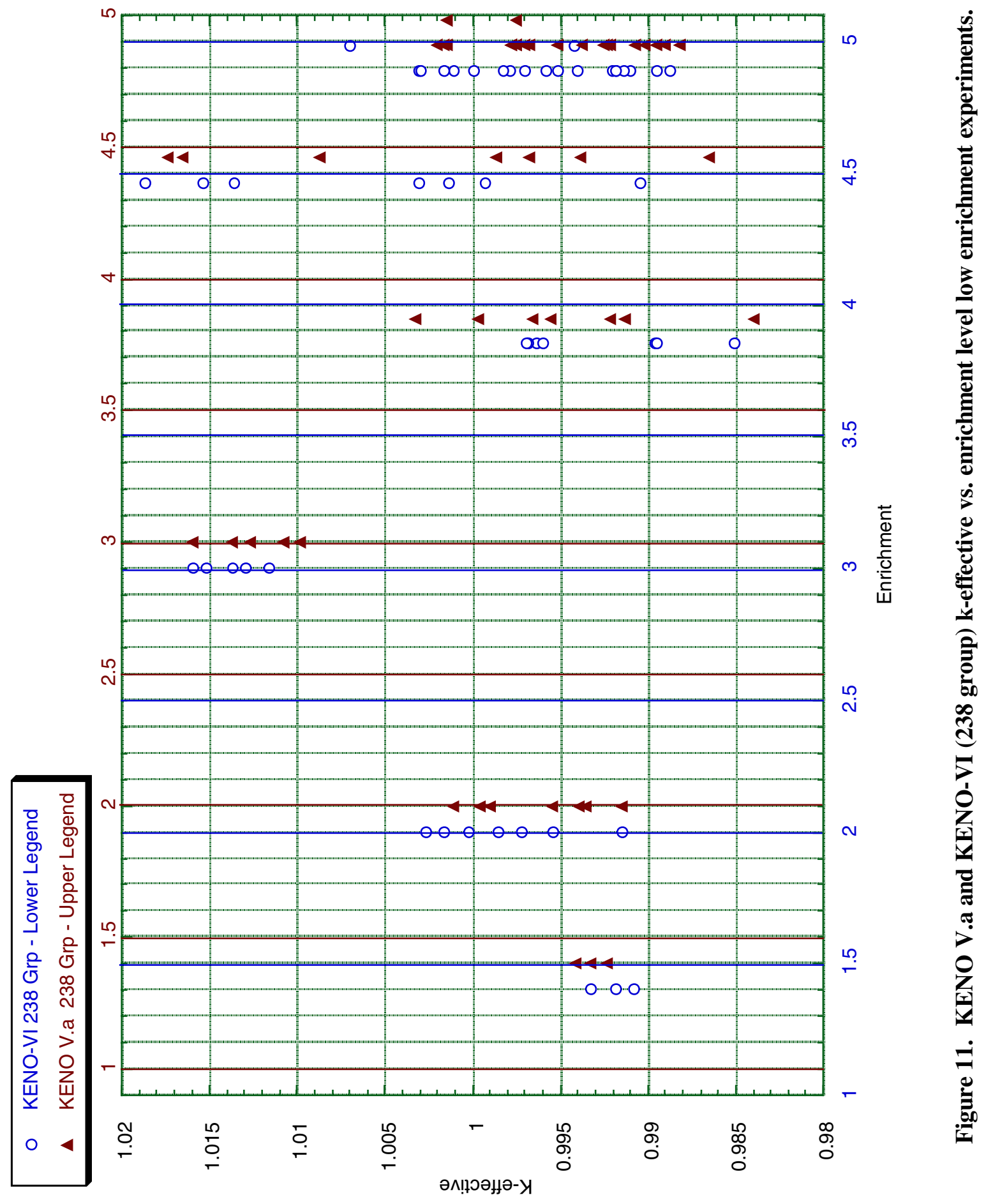




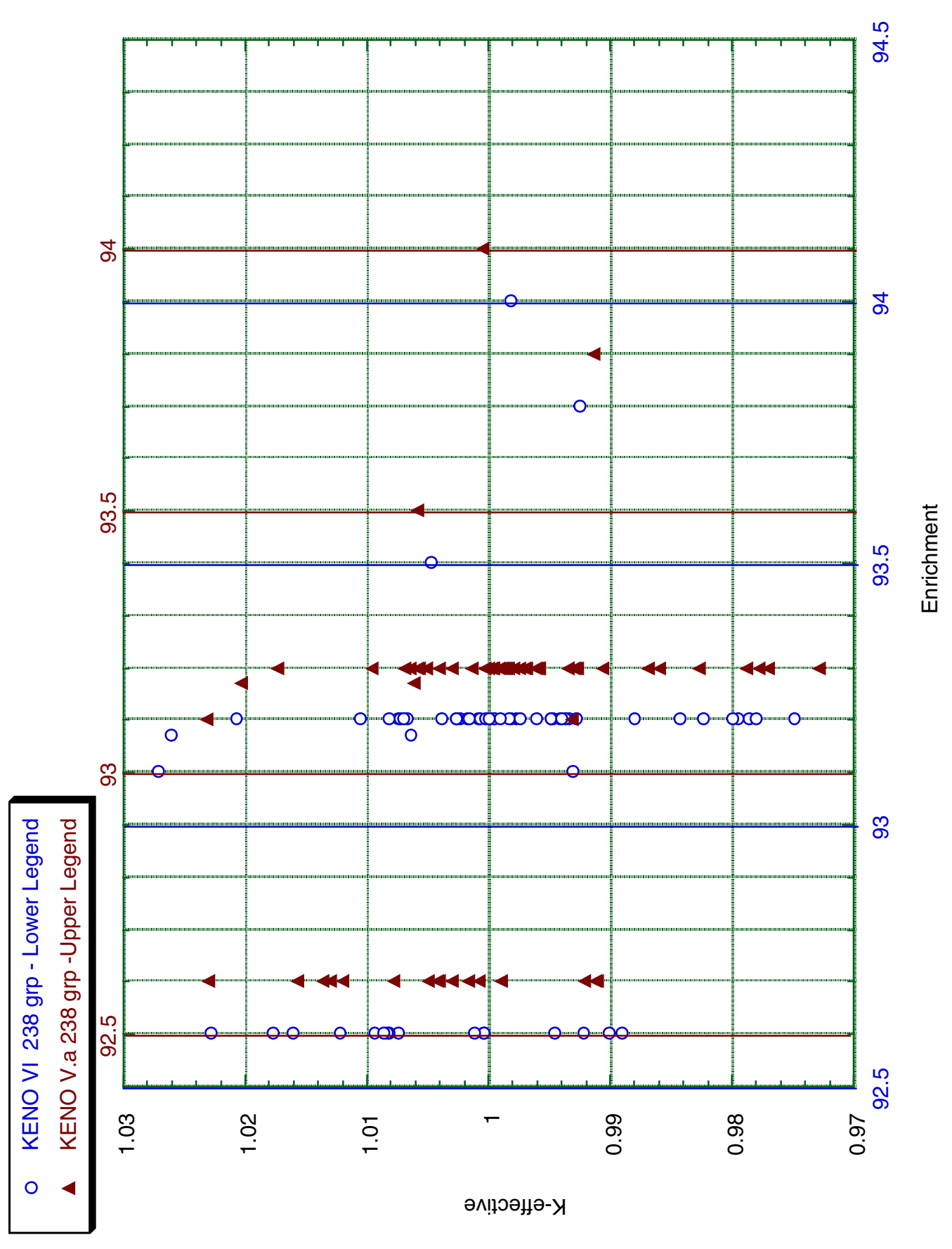

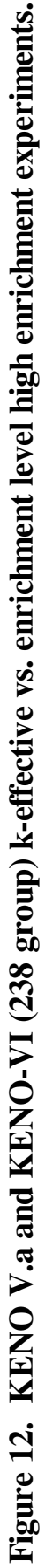




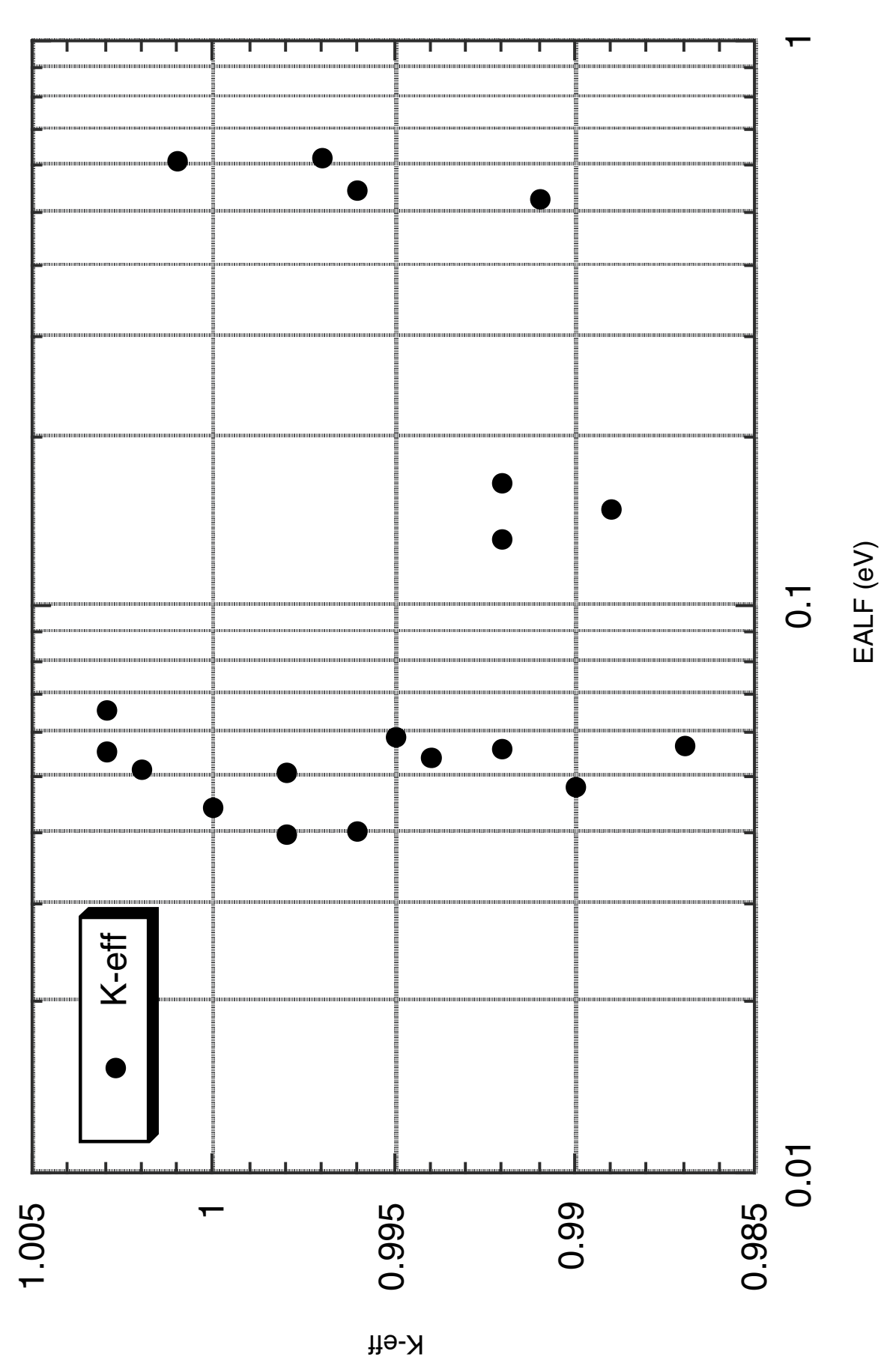

完 


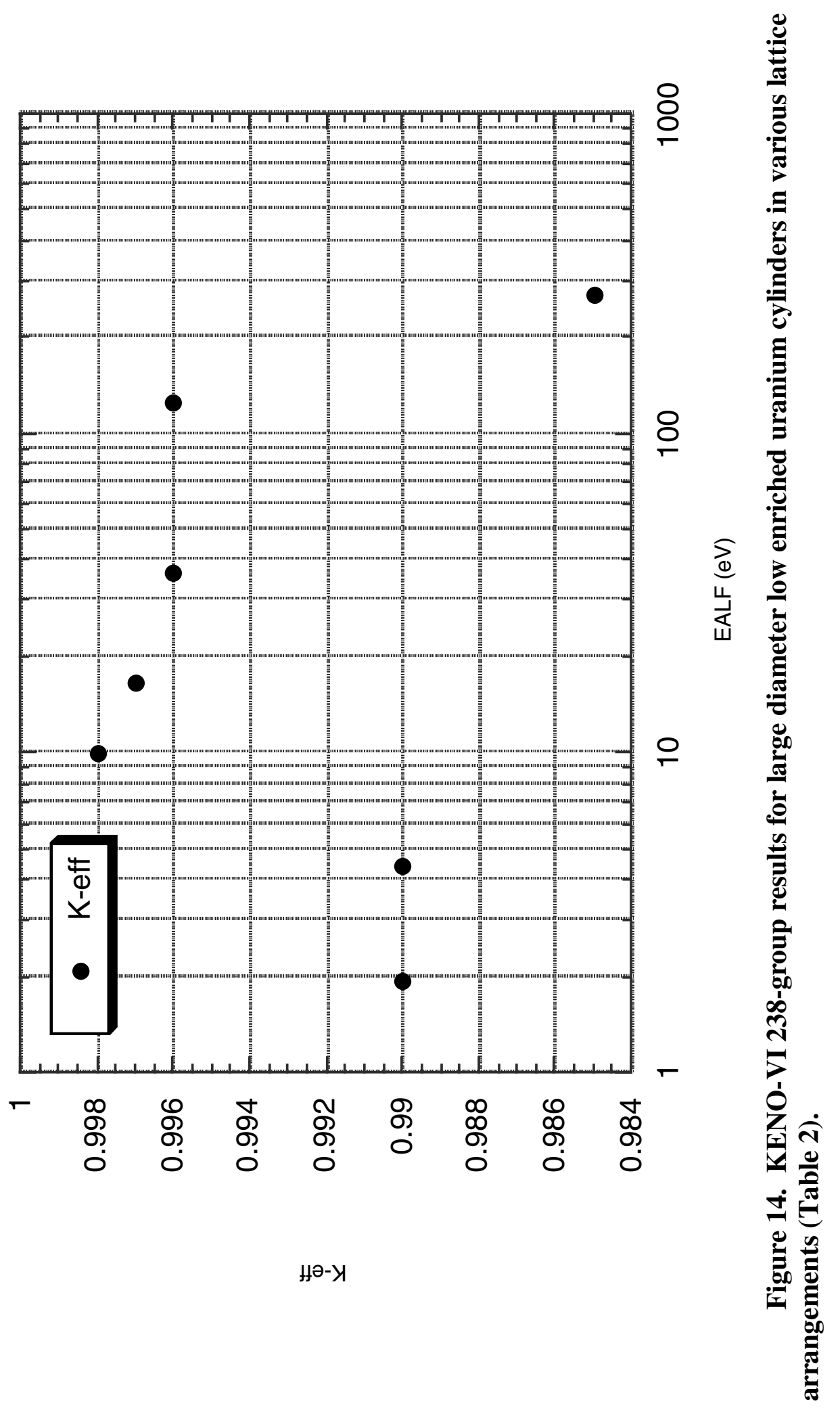




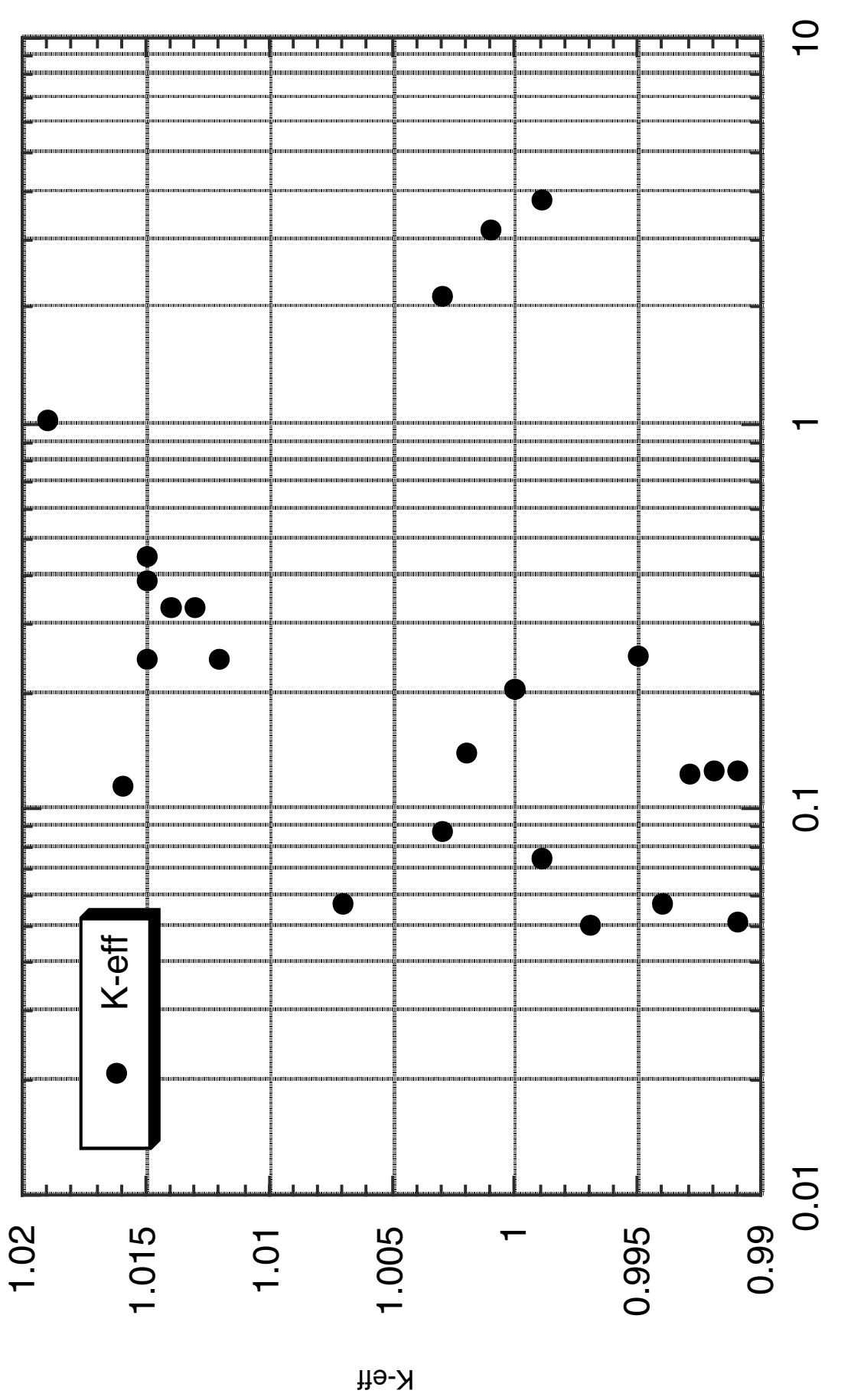

مే 


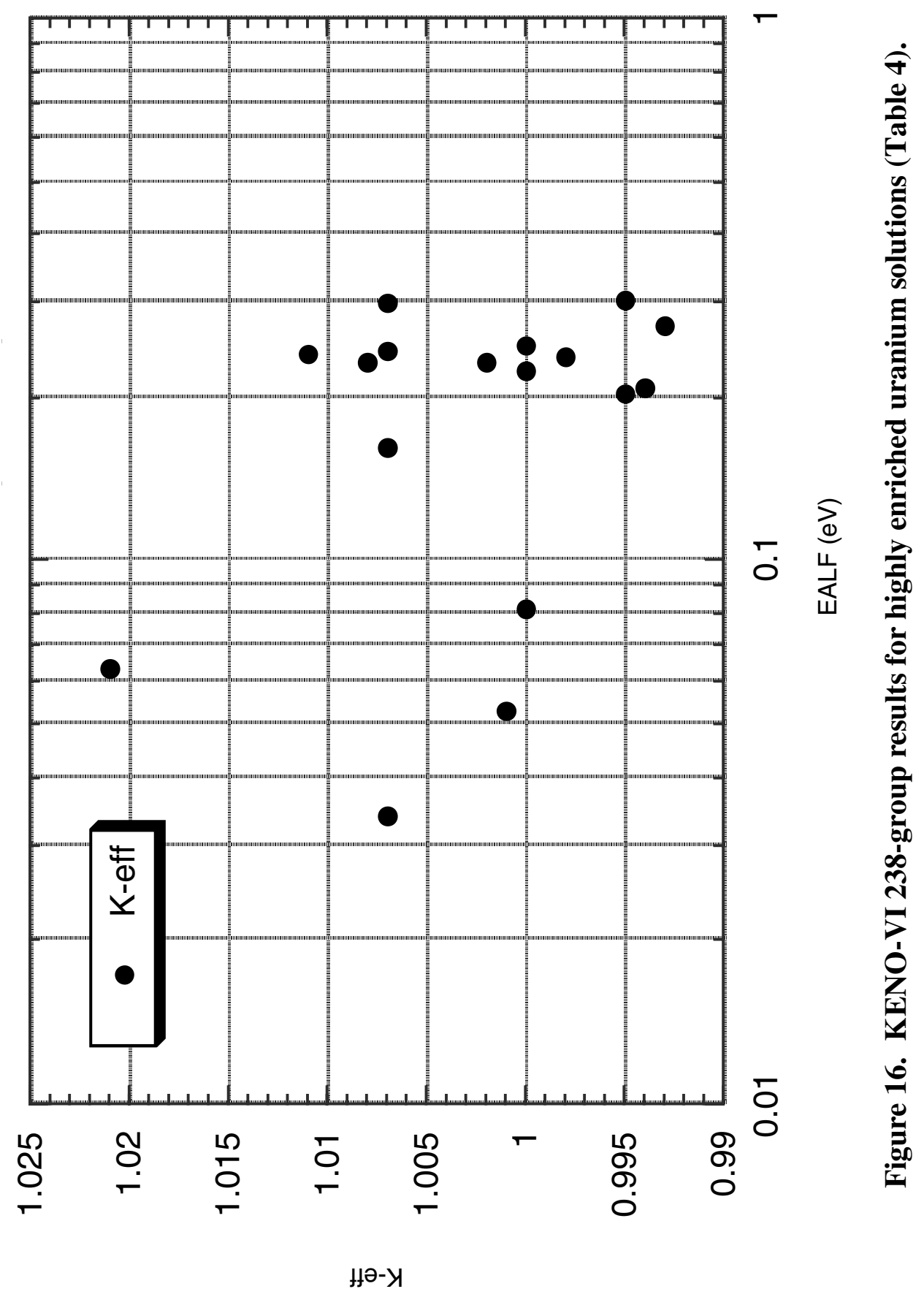




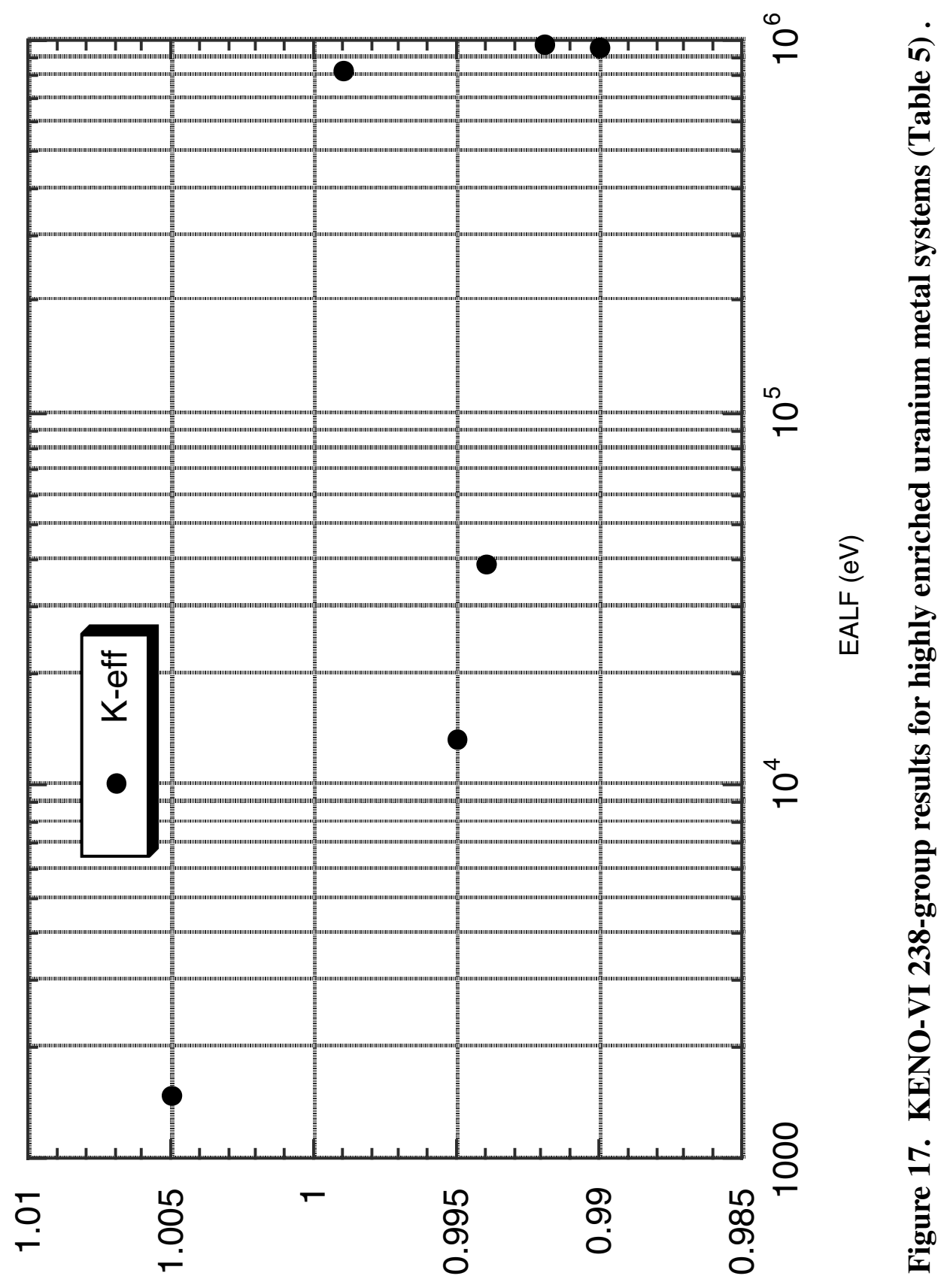

Нә-Х્ર 


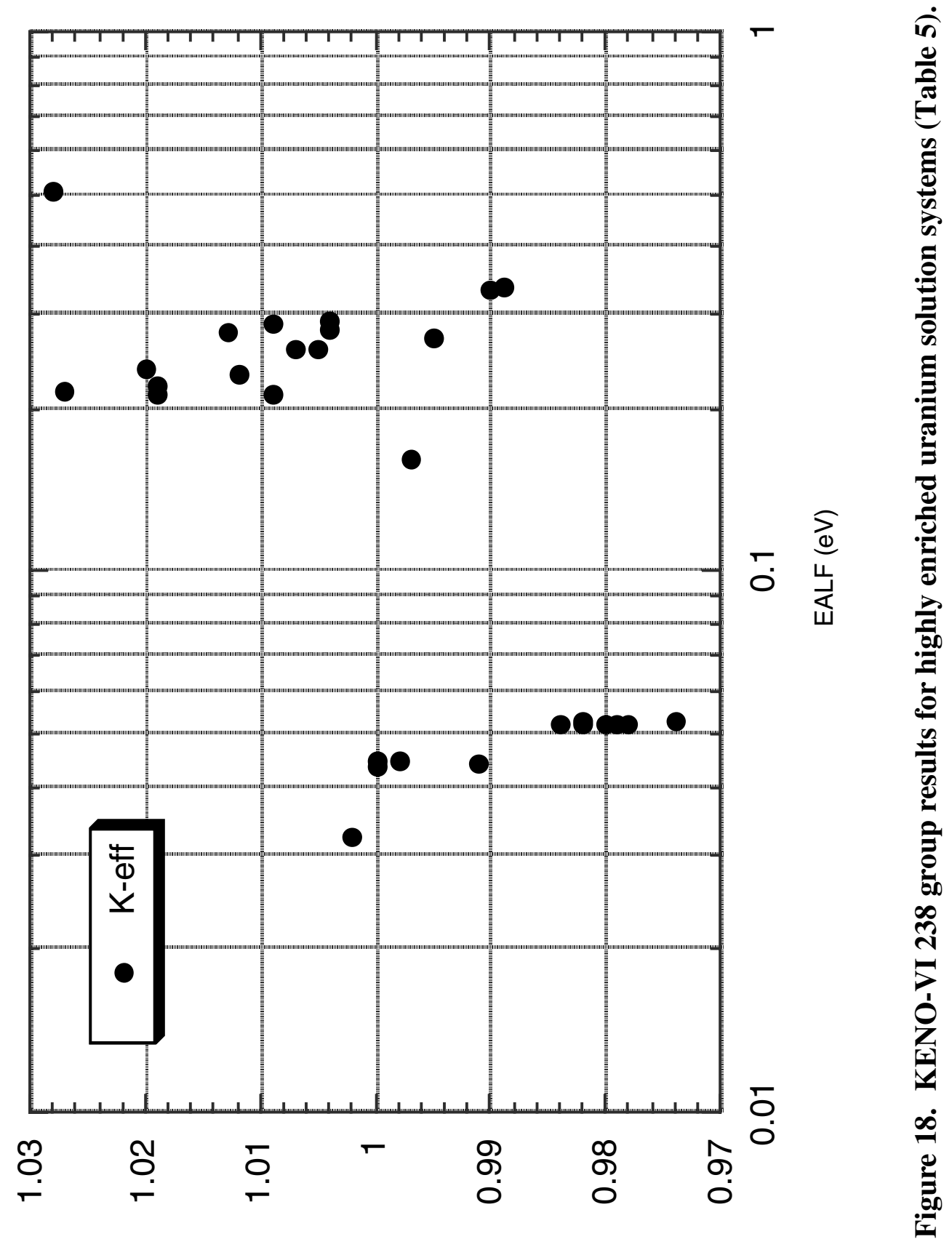

Hə-У 


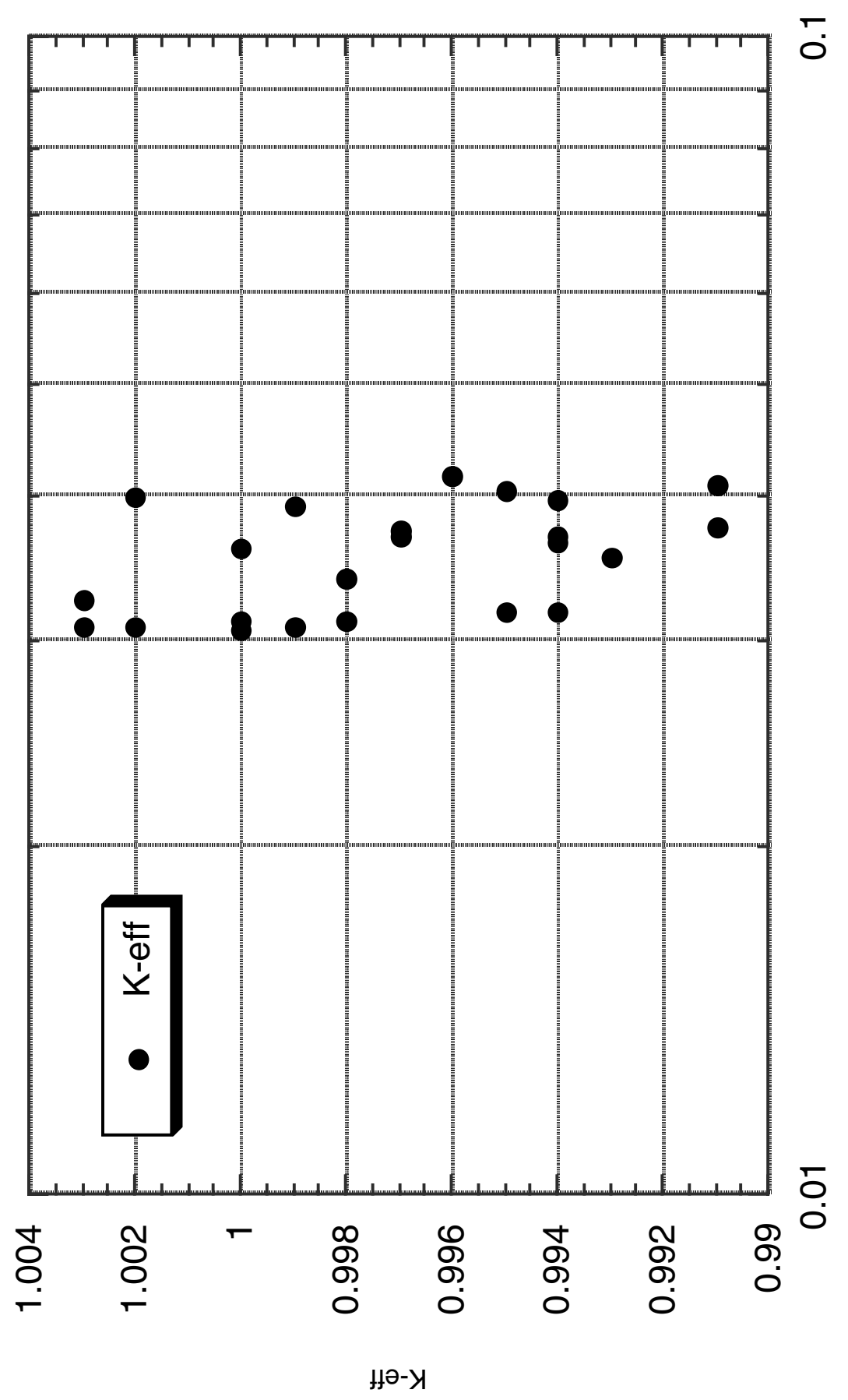

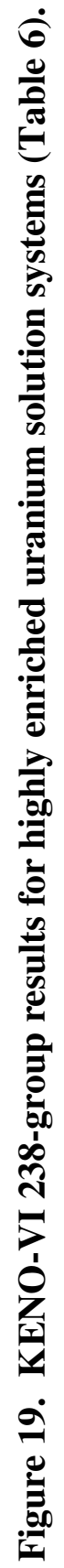
Нә-Х્ર 


\section{CONCLUSIONS}

The KENO V.a and KENO-VI criticality codes accurately calculate a broad range of critical experiments. All KENO V.a and KENO-VI results using the same cross-section library agree within statistical uncertainties. In review, a number of the calculations show a positive or negative bias in excess of $1 \frac{1}{2} \%$ in $k_{\text {eff. }}$ Classes of criticals that show a bias include $3 \%$ enriched green blocks, highly-enriched uranyl fluoride slab arrays, and highly enriched uranyl nitrate arrays. The consistency of the results for two different Monte Carlo codes using two different cross-section libraries is the basis for a high level of confidence in the analytical methods. The implication is that these biases are due to poor performance of the cross sections and/or inadequate descriptions of the experiments. If these biases are properly taken into account, the KENO V.a and KENO-VI codes can be used with great confidence for the design and criticality safety analysis of uranium-containing systems. Note that only a fraction of the available options and geometry types have been exercised. It must be emphasized that the calculated $k_{\text {eff }}$ value from KENO V.a and KENO-VI is an estimate of the eigenvalue of the system and has an associated degree of uncertainty due to the statistical nature of the code. Several suggestions are offered to the users of these codes.

1. Never base the design or safety of a system on a single calculation. Make at least two calculations in which the random sequence is somehow changed (starting random number, slight change in starting distribution, geometry or materials, etc.) to ensure that the calculated $k_{\text {eff }}$ is a reasonable estimate of the calculational mean for the system.

2. Substantial efforts may be required to ensure that the problem has converged to a reasonable estimate of the true $k_{\text {eff }}$ of the system. Undersampling (insufficient neutrons per generation) or problems with convergence of the source distribution (insufficient generations or a very poor initial starting distribution) commonly occur.

3. Only validated options, geometry types, and materials should be used for the criticality safety design of a system. Biases should be based upon validation calculations of critical experiments with similar characteristics (e.g., geometry, materials, spectrum) to the systems being analyzed. 


\section{REFERENCES}

1. L. M. Petrie and N. F. Landers, "KENO V.a An Improved Monte Carlo Criticality Program With Supergrouping," Vol. II, Sect. F11 of SCALE: A Modular Code System for Performing Standardized Computer Analyses for Licensing Evaluation, NUREG/CR-0200, Rev. 6 (ORNL/NUREG/CSD-2/R6), Vols. I, II, and III, May 2000. Available from Radiation Safety Information Computational Center at Oak Ridge National Laboratory as CCC-545.

2. D. F. Hollenbach, L. M. Petrie, and N. F. Landers, "KENO-VI: A General Quadratic Version of the KENO Program," Vol. II, Sect. F17 of SCALE: A Modular Code System for Performing Standardized Computer Analyses for Licensing Evaluation, NUREG/CR-0200, Rev. 6 (ORNL/NUREG/CSD-2/R6), Vols. I, II, and III, May 2000. Available from Radiation Safety Information Computational Center at Oak Ridge National Laboratory as CCC-545.

3. SCALE: A Modular Code System for Performing Standardized Computer Analyses for Licensing Evaluation, NUREG/CR-0200, Rev. 6 (ORNL/NUREG/CSD-2/R6), Vols. I, II, and III, May 2000. Available from Radiation Safety Information Computational Center at Oak Ridge National Laboratory as CCC-545.

4. $\quad$ Nuclear Criticality Safety in Operations with Fissionable Material Outside Reactors, ANSI/ANS8.1, American Natl. Standards Institute, New York, 1998.

5. G. R. Handley and C. M. Hopper, Validation of the KENO Code for Nuclear Criticality Safety Calculations of Moderated Low-Enriched Uranium Systems, Y-1948, Union Carbide Corporation, Nuclear Division, Oak Ridge Y-12 Plant, 1974.

6. G. R. Handley et al., Validation of the Monte Carlo Criticality Program KENO IV and the HansenRoach Sixteen-Energy-Group Cross Sections for High Assay Uranium Systems, Y-2234, Union Carbide Corporation, Nuclear Division, Oak Ridge Y-12 Plant, 1981.

7. J. R. Knight, Validation of the Monte Carlo Criticality Program KENO V.a for Highly Enriched Uranium Systems, ORNL/CSD/TM-221, Martin Marietta Energy Systems, Inc., Oak Ridge National Laboratory, 1984.

8. M. E. Easter, Validation of KENO V.a and Two Cross-Section Libraries for Criticality Calculations of Low-Enriched Uranium Systems, ORNL/CSD/T-233, K/HS-74, Martin Marietta Energy Systems, Inc., Oak Ridge National Laboratory, 1985.

9. L. M. Petrie and J. T. Thomas, Assessment of Computational Performance in Nuclear Criticality, ORNL/CSD/TM-224, Martin Marietta Energy Systems, Inc., Oak Ridge National Laboratory, 1985.

10. W. C. Jordan, N. F. Landers, and L. M. Petrie, Validation of KENO V.a: Comparison with Critical Experiments, ORNL/CSD/TM-238, Martin Marietta Energy Systems, Oak Ridge National Laboratory, December 1986.

11. S. M. Bowman, R. Q. Wright, M. D. DeHart, C. V. Parks, and L. M. Petrie, "Recent Validation Experience with Multigroup Cross-Section Libraries and SCALE," ICNC'95 Fifth International Conference on Nuclear Criticality Safety, Albuquerque, NM, September 17-12, 1995. 
12. H. J. Smith and A. Santamarina, "Analysis of the International Criticality Benchmark No. 19 of a Realistic Fuel Dissolver," Criticality Calculations Working Group, OECD/NEA Committee on Reactor Physics, NEACRP-L-325, January, 1991.

13. R. M. Westfall and J. R. Knight, "SCALE System Cross Section Validation with Shipping Cask Critical Experiments," Trans. Am. Nucl. Soc. 33, 368 (1979).

14. M. E. Easter, Validation of KENO V.a and Two Cross-Section Libraries for Criticality Calculations of Low-Enriched Uranium Systems, ORNL/CSD/T-223, K/HS-74, Martin Marietta Energy Systems, Inc., Oak Ridge National Laboratory, 1985.

15. L. M. Petrie and J. T. Thomas, Assessment of Computational Performance in Nuclear Criticality, ORNL/CSD/TM-224, Martin Marietta Energy Systems, Oak Ridge National Laboratory, 1985.

16. M. E. Easter and R. T. Primm, III, Validation of the SCALE Code System and Two Cross-Section Libraries for Plutonium Benchmark Experiments, ORNL/TM-9402, Martin Marietta Energy Systems, Oak Ridge National Laboratory, January 1985.

17. H. R. Dyer, Recalculation of a Few Bare Plutonium Critical Arrays, ORNL/CSD/TM-242, Martin Marietta Energy Systems, Oak Ridge National Laboratory, April 1987.

18. H. R. Dyer and R. M. Westfall, "KENO V.a Validation of Fuel Pin Experiments," Trans. Am. Nucl. Soc. 55, 382-385 (1987).

19. G. R. Smolen and H. Funabashi, "Validation Studies Performed with Low-Concentration Mixed $\mathrm{Pu}+\mathrm{U}$ Aqueous Critical Experiments," Trans. Am. Nucl. Soc. 55, 380-381 (1987).

20. S. M. Bowman, C. V. Parks, and S. R. Bierman, "Validation of SCALE-4 for LWR Fuel in Transportation and Storage Cask Condition," Trans. Am. Nucl. Soc. 62, 338-340 (1990).

21. M. B. Emmett and W. C. Jordan, Guide to Verification and Validation of the SCALE-4 Criticality Safety Software, NUREG/CR-6483 (ORNL/TM-12834), U.S. Nuclear Regulatory Commission, Oak Ridge National Laboratory, November 1996.

22. N. M. Greene, J. W. Arwood, R. Q. Wright, and C. V. Parks, The LAW Library - A Multigroup Cross-Section Library for Use in Radioactive Waste Analysis Calculations, ORNL/TM-12370, Martin Marietta Energy Systems, Inc., Oak Ridge National Laboratory, August 1994.

23. N. M. Greene, W. E. Ford III, L. M. Petrie, and J. W. Arwood, AMPX-77: A Modular Code System for Generating Coupled Multigroup Neutron-Gamma Cross-Section Libraries from ENDF/B-IV and/or ENDF/B-V, ORNL/CSD/TM-283, Martin Marietta Energy Systems, Inc., Oak Ridge National Laboratory, October 1992.

24. E. B. Johnson, "Criticality of U(3.85) Rods and Cylindrical Annuli in Water Cylinders and Annuli in Water," Tran. Am. Nucl. Soc. 13, 379 (1970).

25. R. Gwin and D. W. Magnuson, Critical Experiments for Reactor Physics Studies, ORNL/CF-60-412, Union Carbide Corporation, Nuclear Division, Oak Ridge National Laboratory, 1960.

26. R. Gwin and D. W. Magnuson, "The Measurement of Eta and Other Nuclear Properties of U-233 and U-235 in Critical Aqueous Solutions," Nucl. Sci. Eng. 12, 364-380 (1962). 
27. E. B. Johnson, The Criticality of Large Uranium Metal Units of Low Enrichment, to be published at Oak Ridge National Laboratory.

28. D. F. Cronin, Critical Mass Studies, Part X, ORNL-2968, Union Carbide Nuclear Corporation, Oak Ridge National Laboratory, 1960.

29. E. B. Johnson, The Criticality of Large Uranium Metal Units of Low Enrichment in U-235, to be published at Oak Ridge National Laboratory.

30. F. Abbey, comp, Handbook of Experimental Criticality Data, AHSB(s) Handbook 5, 1968.

31. S. J. Raffety and J. T. Mihalczo, Homogenized Critical Assemblies of 2 and 3\% Enriched Uranium in Paraffin, Y-DR-14, Union Carbide Corporation, Nuclear Division, Oak Ridge Y-12 Plant, 1969.

32. E. B. Johnson, "Effect of Steel-Water Reflectors on the Criticality of Low Enriched Uranyl Fluoride Solution," p. 47 in Neutron Physics Division Annual Progress Report for Period Ending May 31, 1968, ORNL-4280, Union Carbide Corporation, Nuclear Division, Oak Ridge National Laboratory, 1968.

33. J. W. Webster and E. B. Johnson, Criticality of a Single Unit of Aqueous Uranyl Fluoride Solution Enriched to 5\% in U-235, ORNL-TM-1195, Union Carbide Corporation, Nuclear Division, Oak Ridge National Laboratory, 1965.

34. E. R. Rothe et al., Critical Experiments with Interstitially Moderated Arrays of Low Enriched Uranium Oxide, NUREG/CR-1071, RFP-3008, U.S. Nuclear Regulatory Commission, 1980.

35. G. Tuck and I. Oh, Benchmark Critical Experiments on Low-Enriched Uranium Oxide Systems With $H / U=0.77$, NUREG/CR-0674, U.S. Nuclear Regulatory Commission, 1979.

36. G. R. Goebel et al., Critical Experiments on Low-Enriched Uranium Oxide Systems With H/U = 1.25, NUREG/CR-1653, RFP-3129, U.S. Nuclear Regulatory Commission, 1980.

37. R. E. Rothe and G. R. Groebel, Critical Experiments on Low-Enriched Uranium Oxide Systems With $H / U=2.03$, NUREG/CR-2500, RFP-3277, U.S. Nuclear Regulatory Commission, 1982.

38. J. K. Fox, "Critical Parameters of Uranium Solutions in Simple Geometry," p. 42 in Neutron Physics Division Annual Progress Report for Period Ending September 1, 1958, ORNL-2609, Union Carbide Corporation, Nuclear Division, Oak Ridge National Laboratory, 1958.

39. J. T. Thomas et al., "A Direct Comparison of Some Nuclear Properties of U-233 and U-235," Nucl. Sci. Eng. 1, 20 and 32 (1956).

40. R. E. Rothe, Benchmark Critical Experiments on High Enriched Uranyl Nitrate Solution Systems, NUREG/CR-0041, RFP-2710, U.S. Nuclear Regulatory Commission, 1978.

41. G. E. Hansen, "Properties of Elementary Fast Neutron Critical Assemblies," p. 84 in Proceedings of the Second United Nations International Conference on Peaceful Uses of Atomic Energy, Vol. 12, Geneva, 1958. 
42. H. C. Paxton, Los Alamos Critical Mass Data, LAMS-3067, Los Alamos National Laboratory, Los Alamos, New Mexico, 1964.

43. G. Tuck, "Critical Masses of Spherical and Hemispherical Enriched Uranium," J. Nucl. Energy 23, 663 (1969).

44. J. T. Thomas, Critical Three-Dimensional Arrays of Neutron-Interacting Units, Part II - U(93.2) Metal, ORNL-TM-868, Union Carbide Corporation, Nuclear Division, Oak Ridge National Laboratory, 1964.

45. J. T. Thomas, Critical Three-Dimensional Arrays of Neutron-Interacting Units, ORNL-TM-719, Union Carbide Corporation, Nuclear Division, Oak Ridge National Laboratory, 1963.

46. E. C. Crume and J. T. Thomas, Critical and Near Critical Graphite Moderated Arrays of U(93.2) Cylinders, Y-DD-32, Union Carbide Corporation, Nuclear Division, Oak Ridge Y-12 Plant, 1969.

47. B. B. Ernst and C. L. Schuske, Empirical Method for Calculating Pipe Intersections Containing Fissile Solution, RFP-1197, Rockwell International, Rocky Flats, Golden, CO, 1968.

48. D. Dickinson and C. L. Schuske, "An Empirical Model for Safe Pipe Interactions Containing Fissile Solution," Nucl. Technol. 10, 179-187 (1971).

49. J. K. Fox and L. W. Gilley, Preliminary Report of Critical Experiments in Slab Geometry, ORNL/CF-56-7-148, Union Carbide Corporation, Nuclear Division, Oak Ridge National Laboratory, 1956. 


\section{APPENDIX A}

\section{KENO V.a INPUT DATA USED FOR VALIDATION CASES \\ LISTED IN TABLES 1-6 (238 group)}




\section{APPENDIX A}

\section{KENO V.A INPUT DATA USED FOR VALIDATION CASES LISTED IN TABLES 1-6 (238 GROUP)}

Input data for the validation appears in the same order as listed in Tables 1-6 of Section 1 of this report.

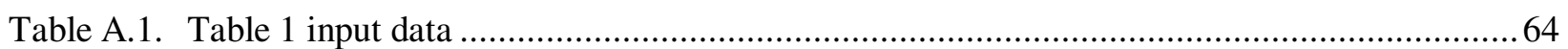

Table A.2. Table 2 input data …........................................................................................................ 76

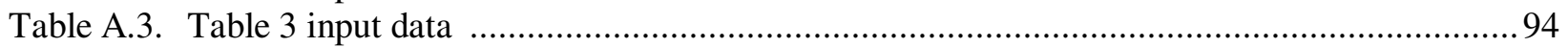

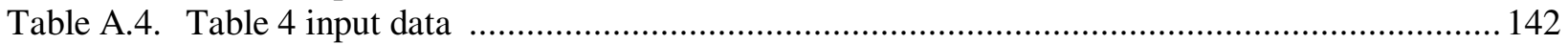

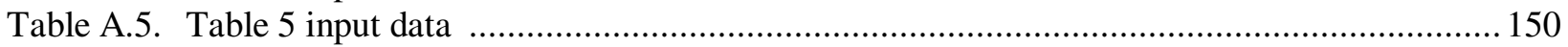

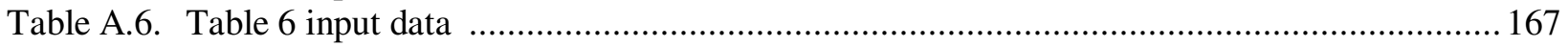


Table A.1. Table 1 input data

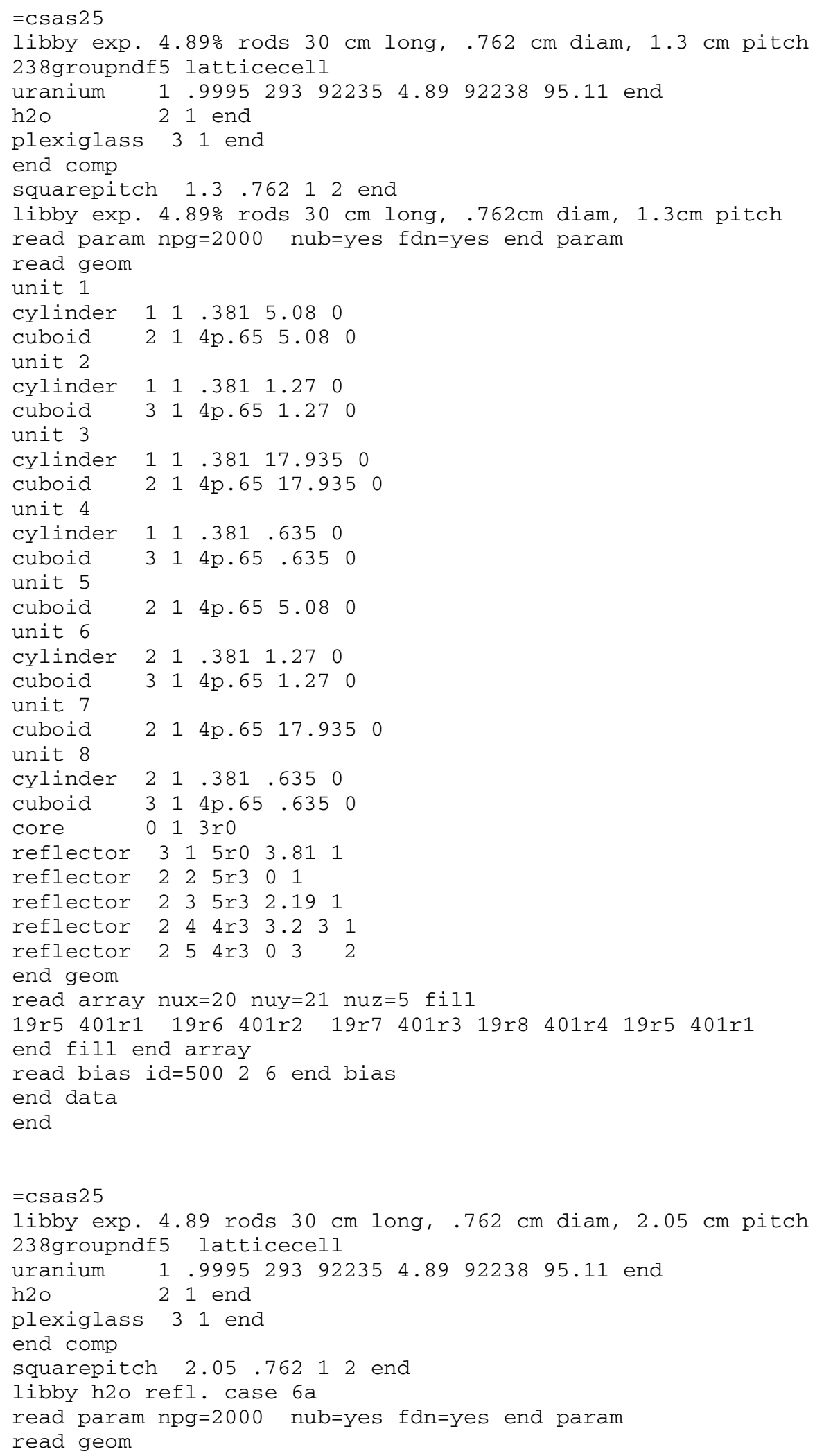




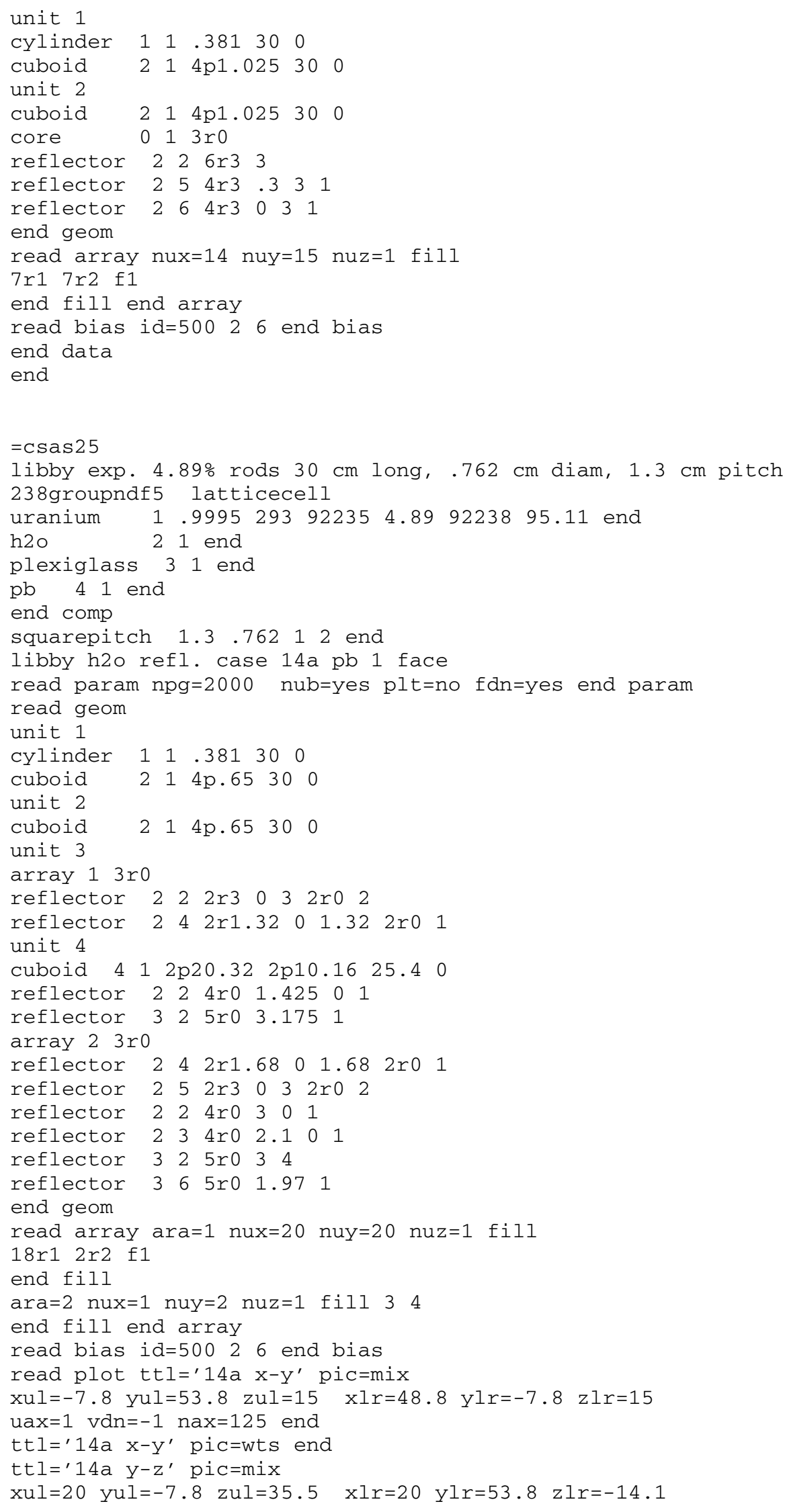




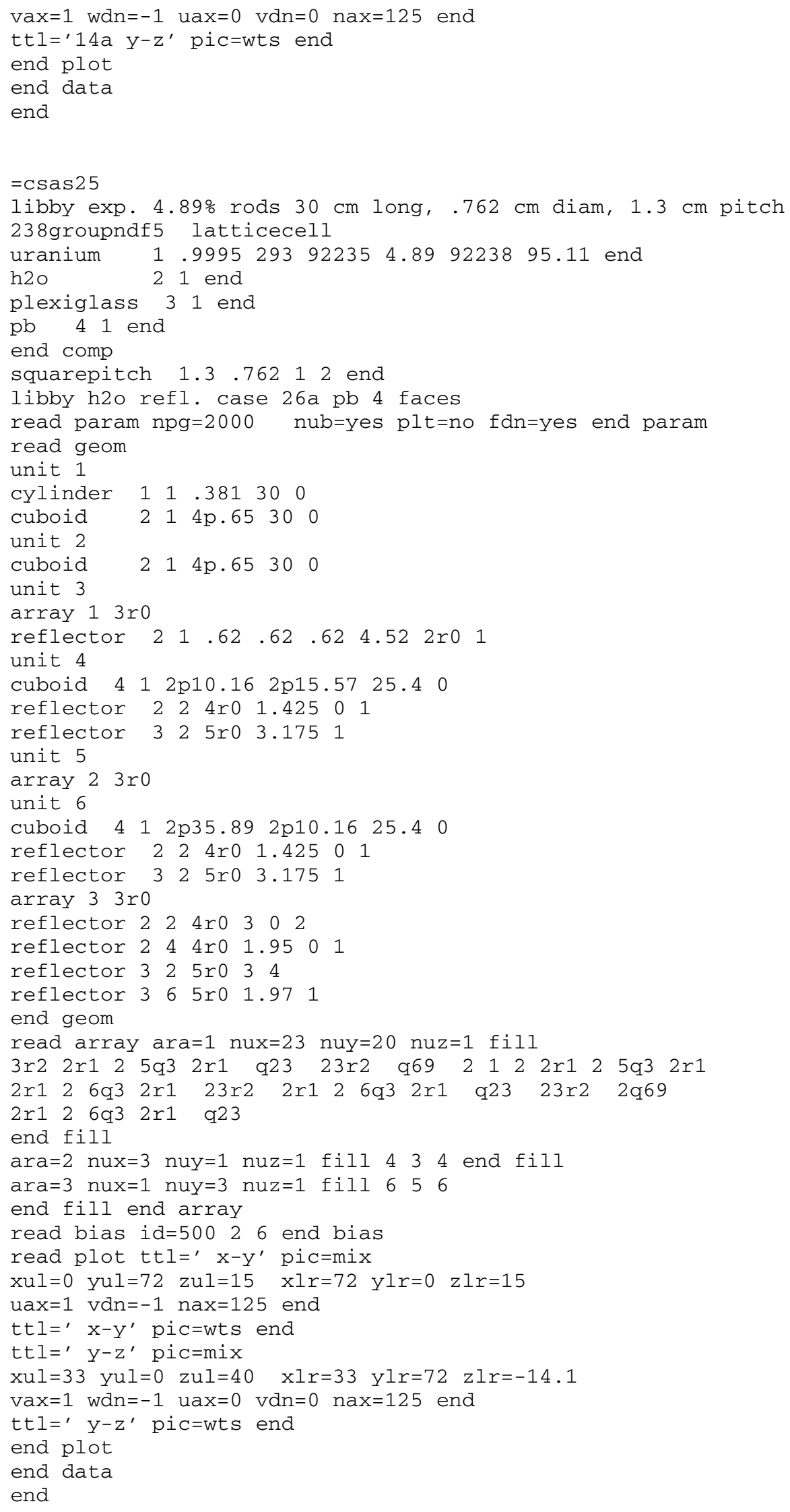




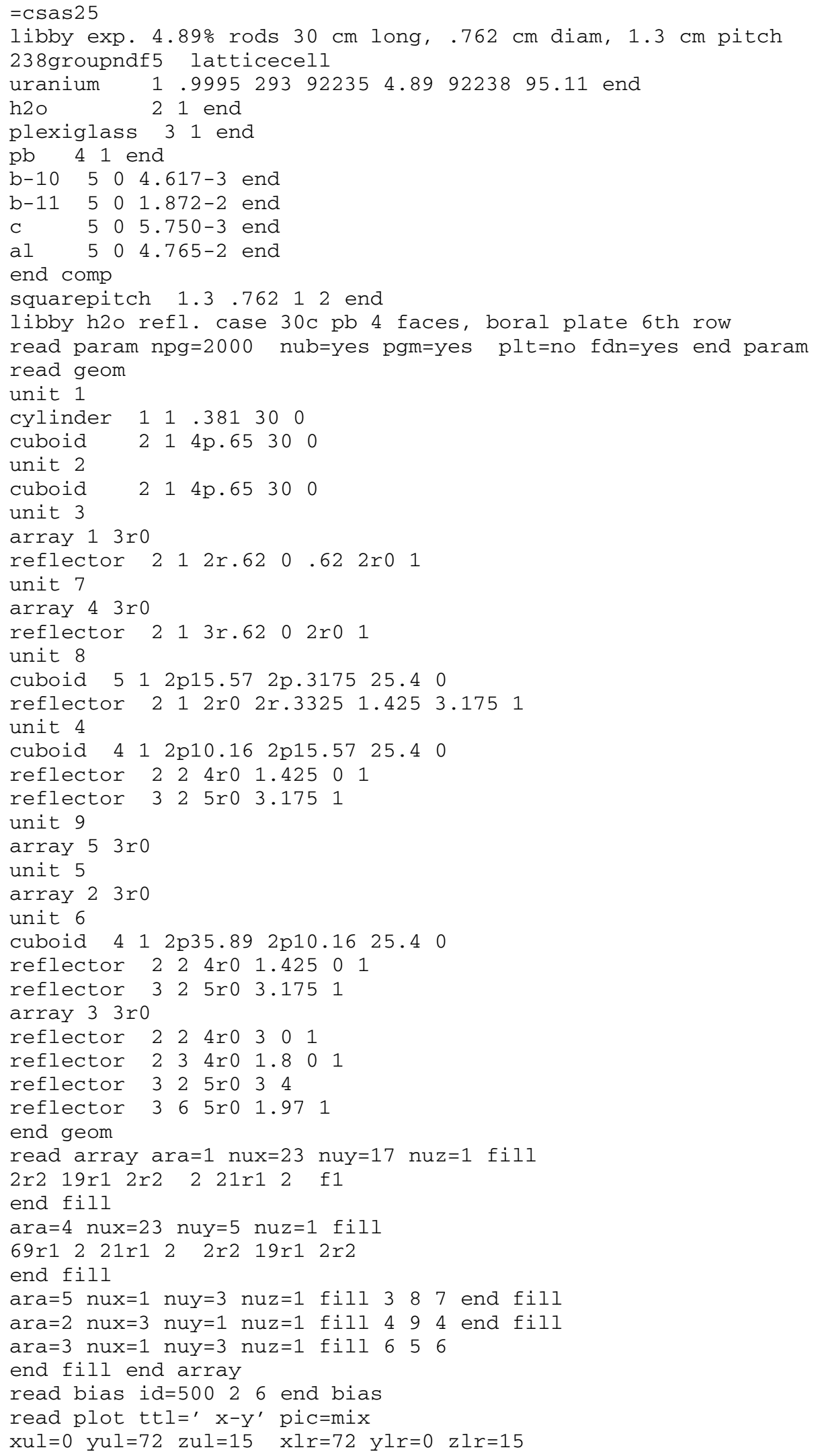




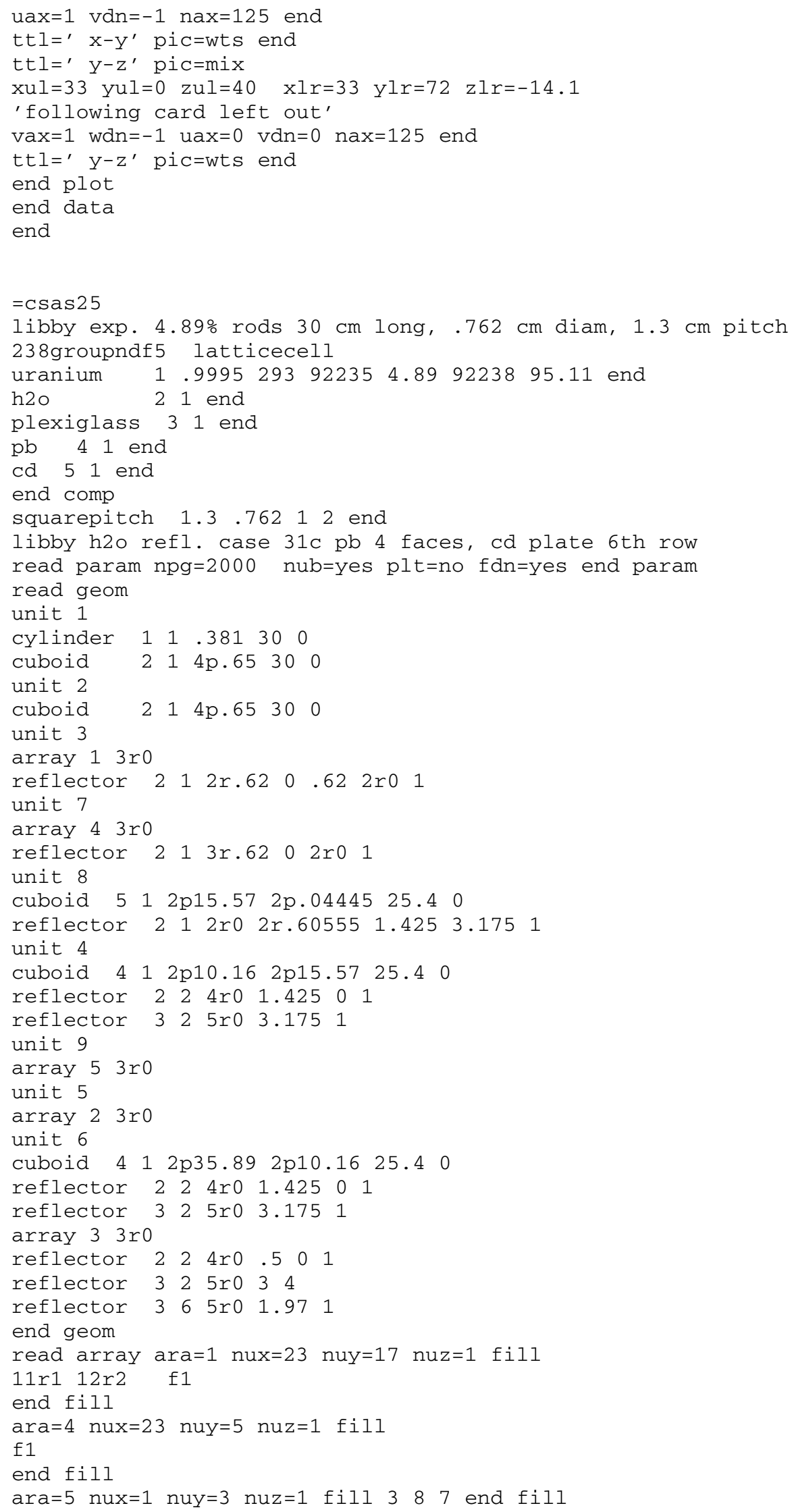




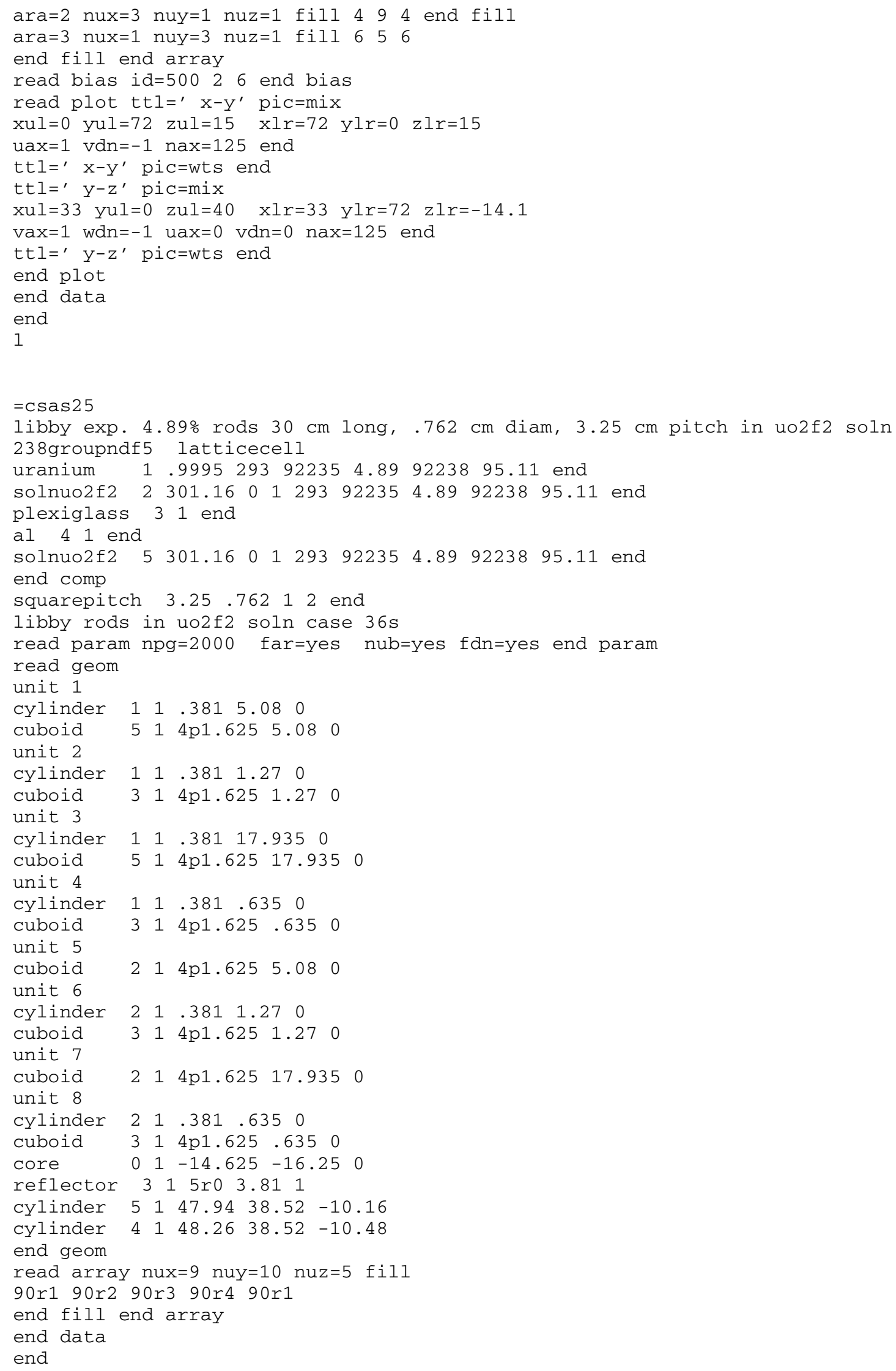




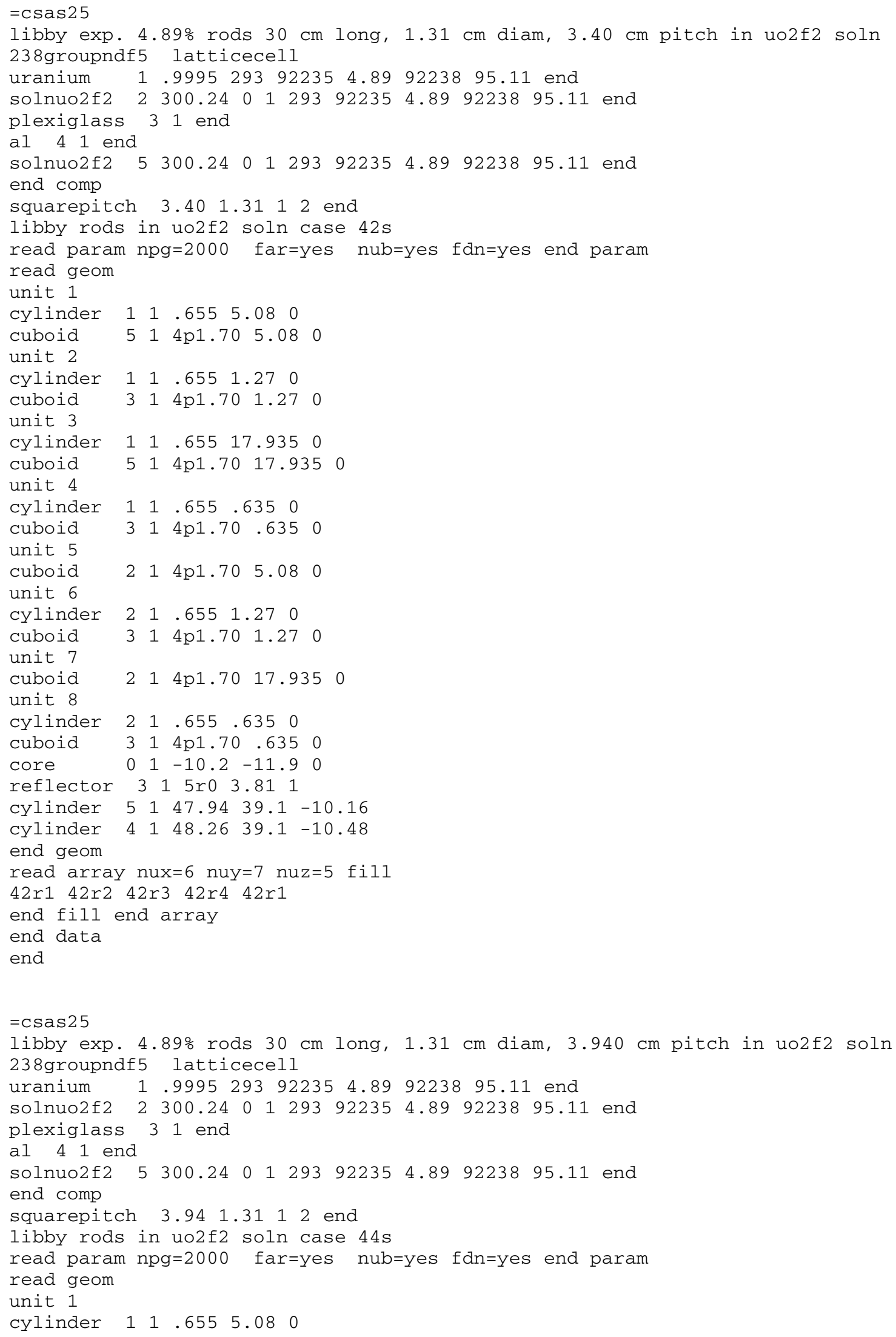




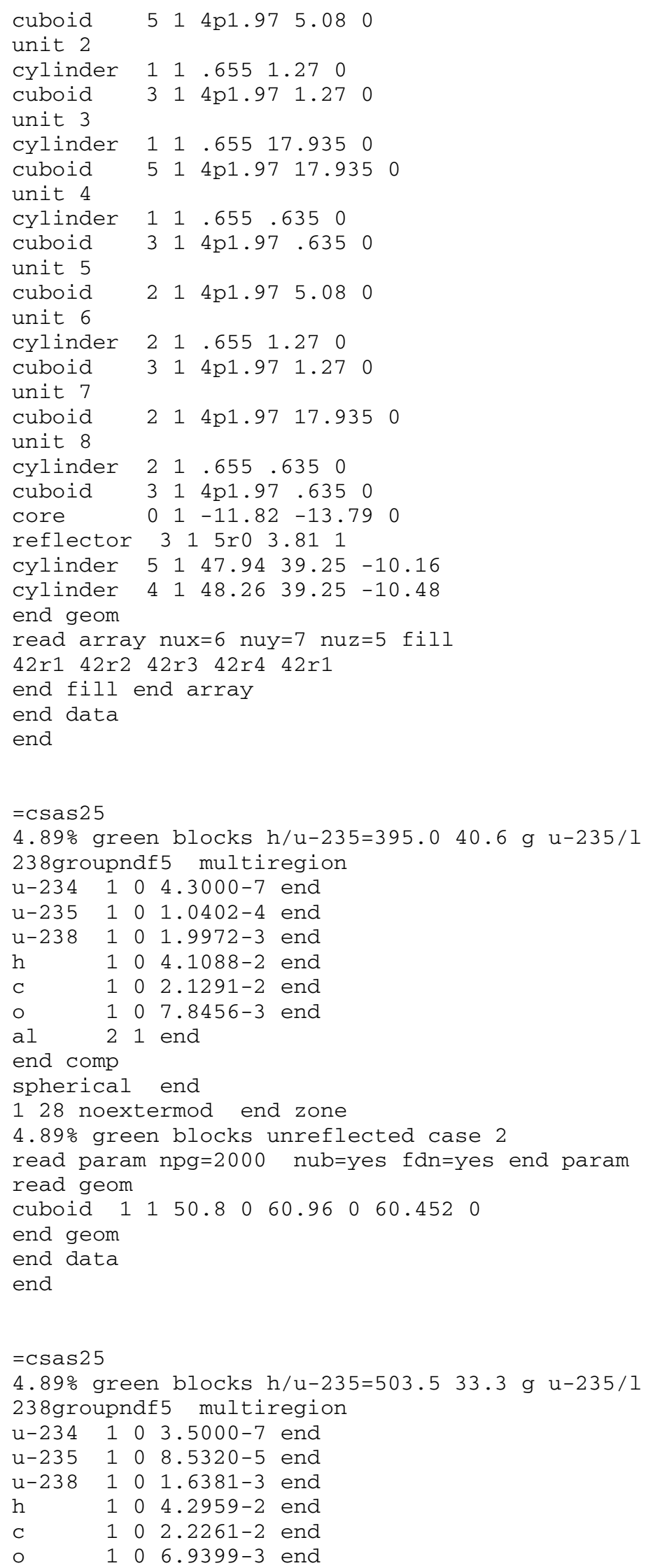




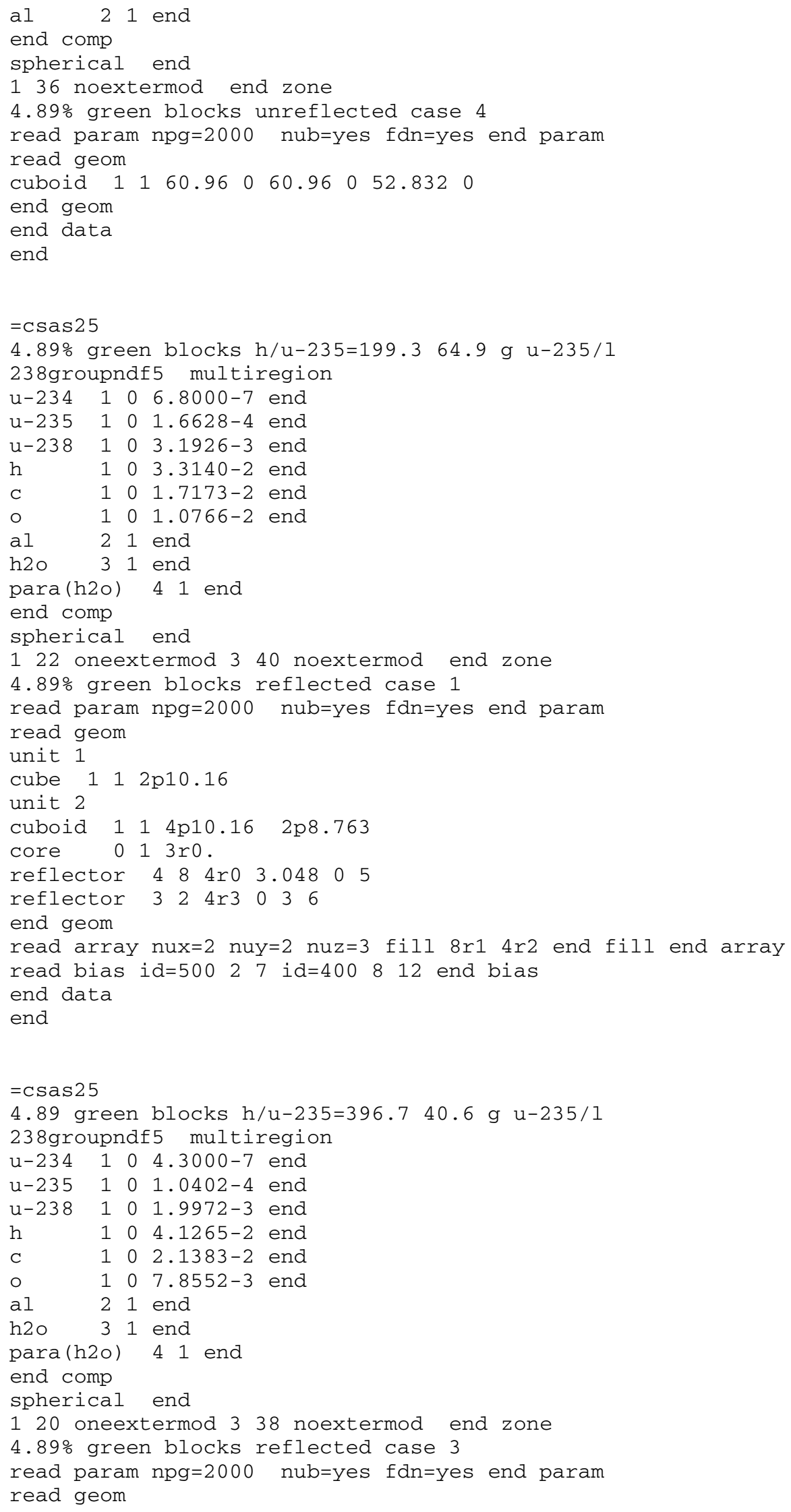




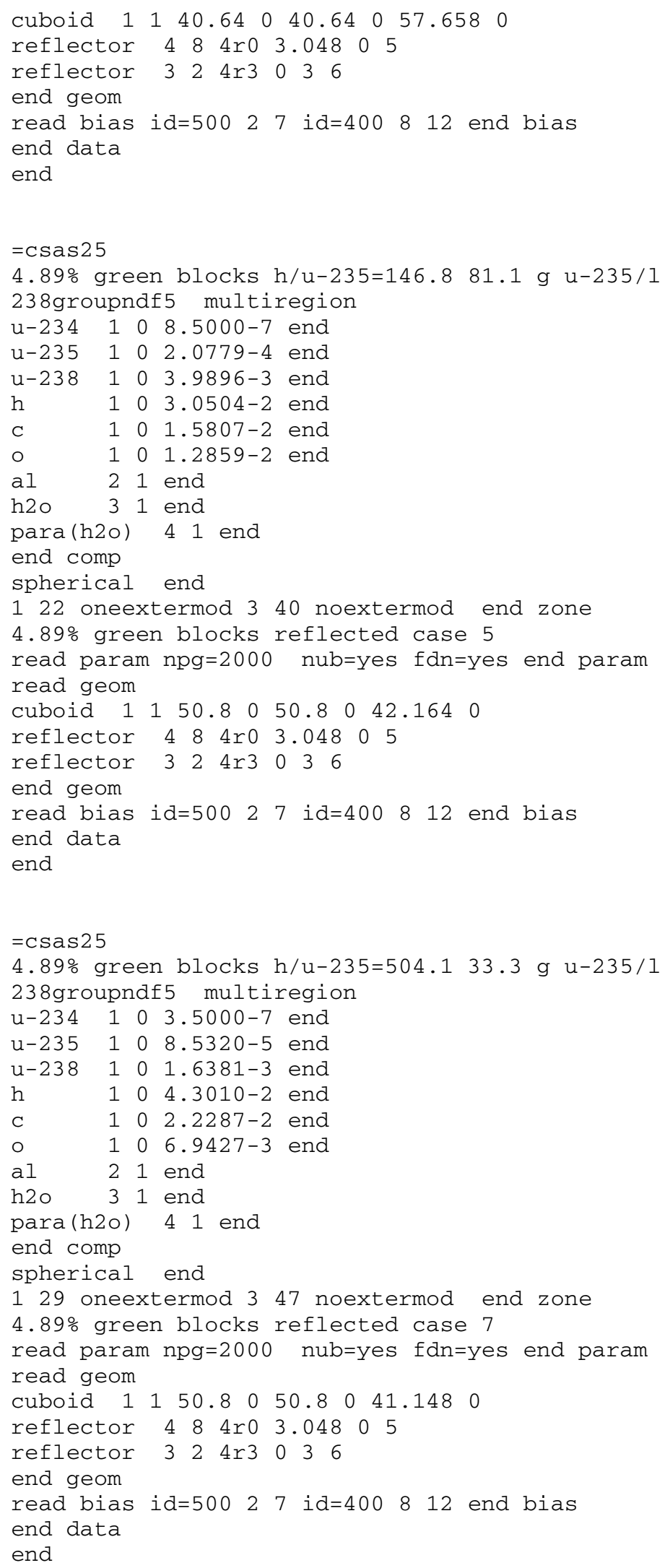




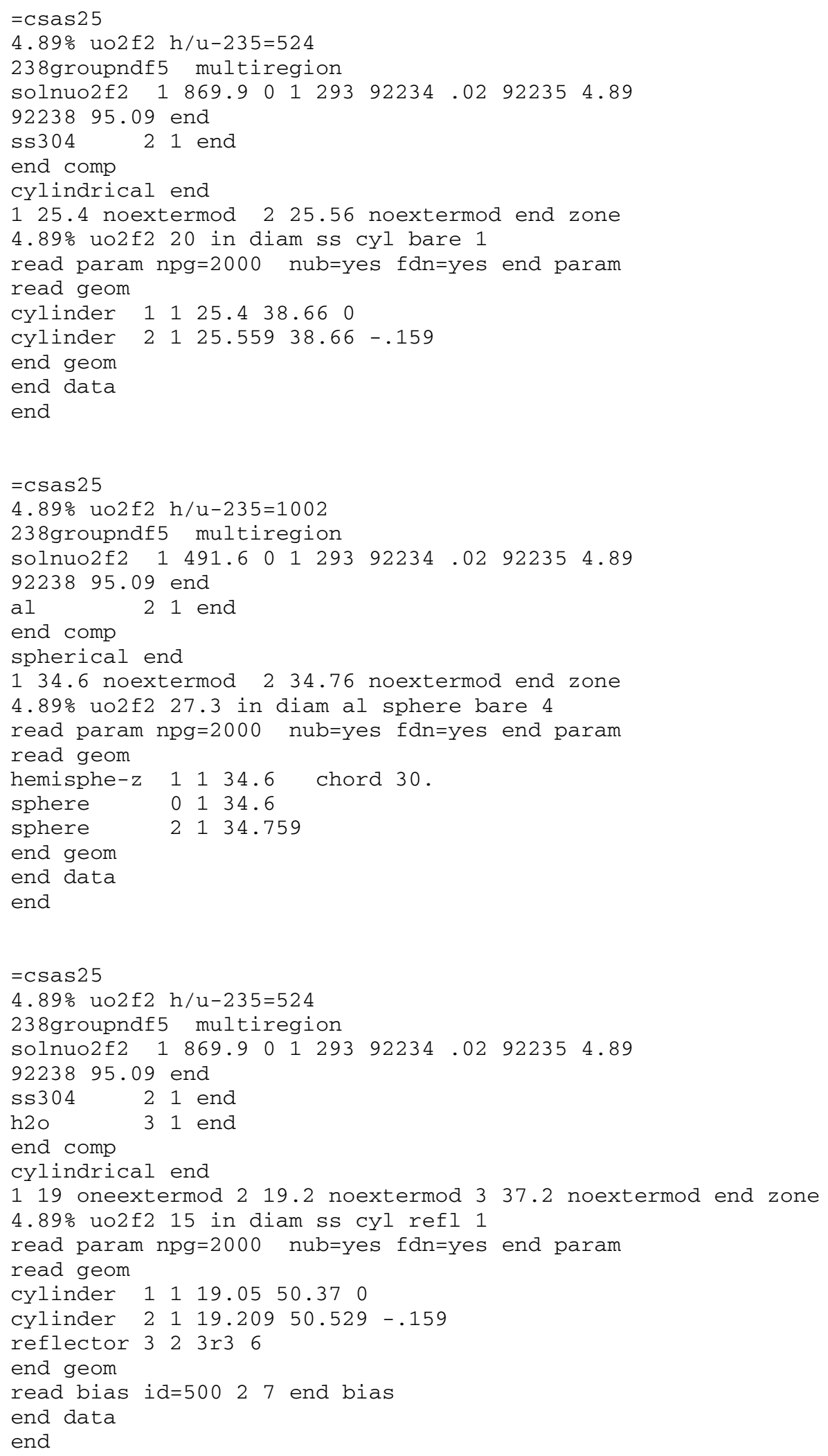




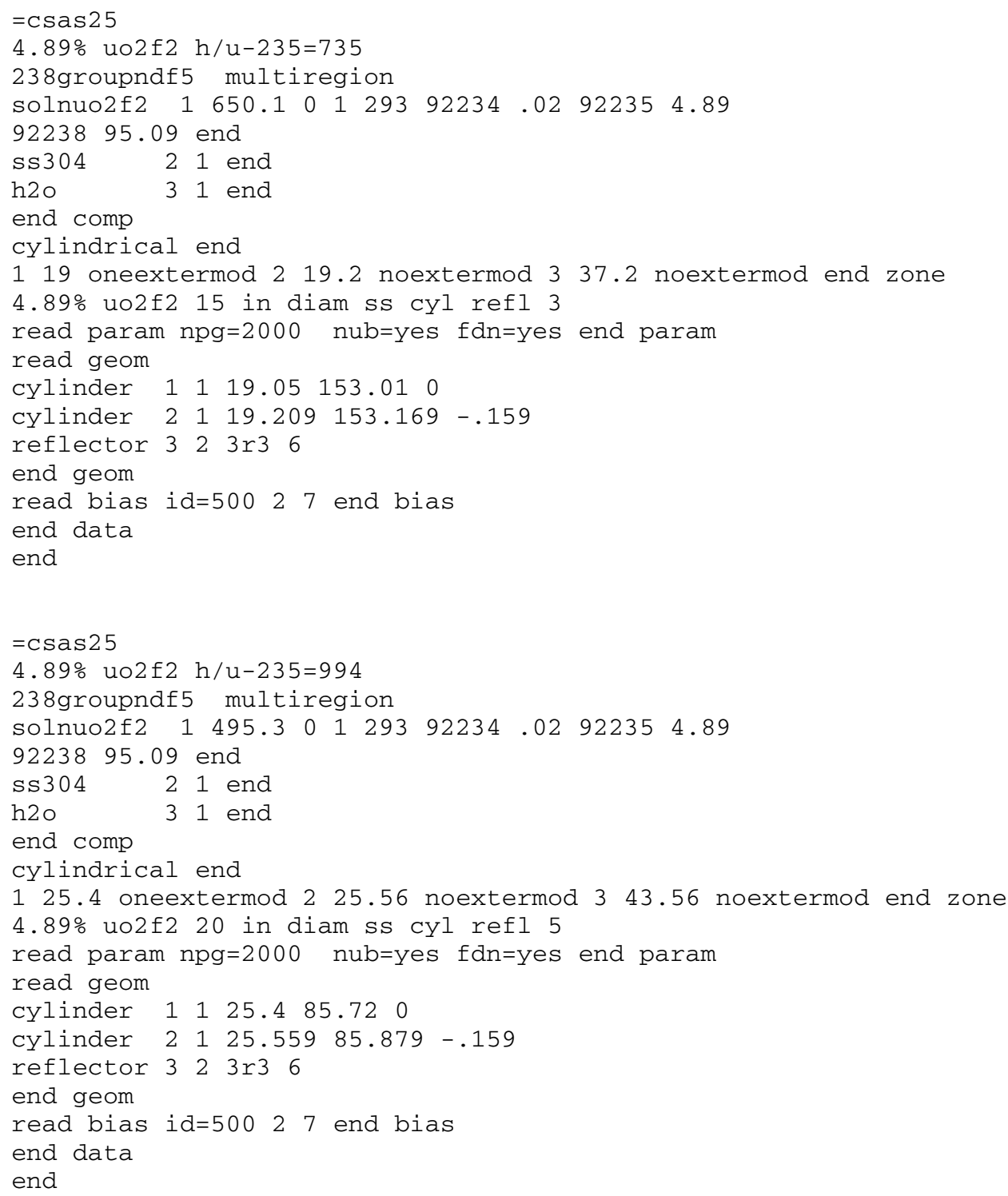


Table A.2. Table 2 input data

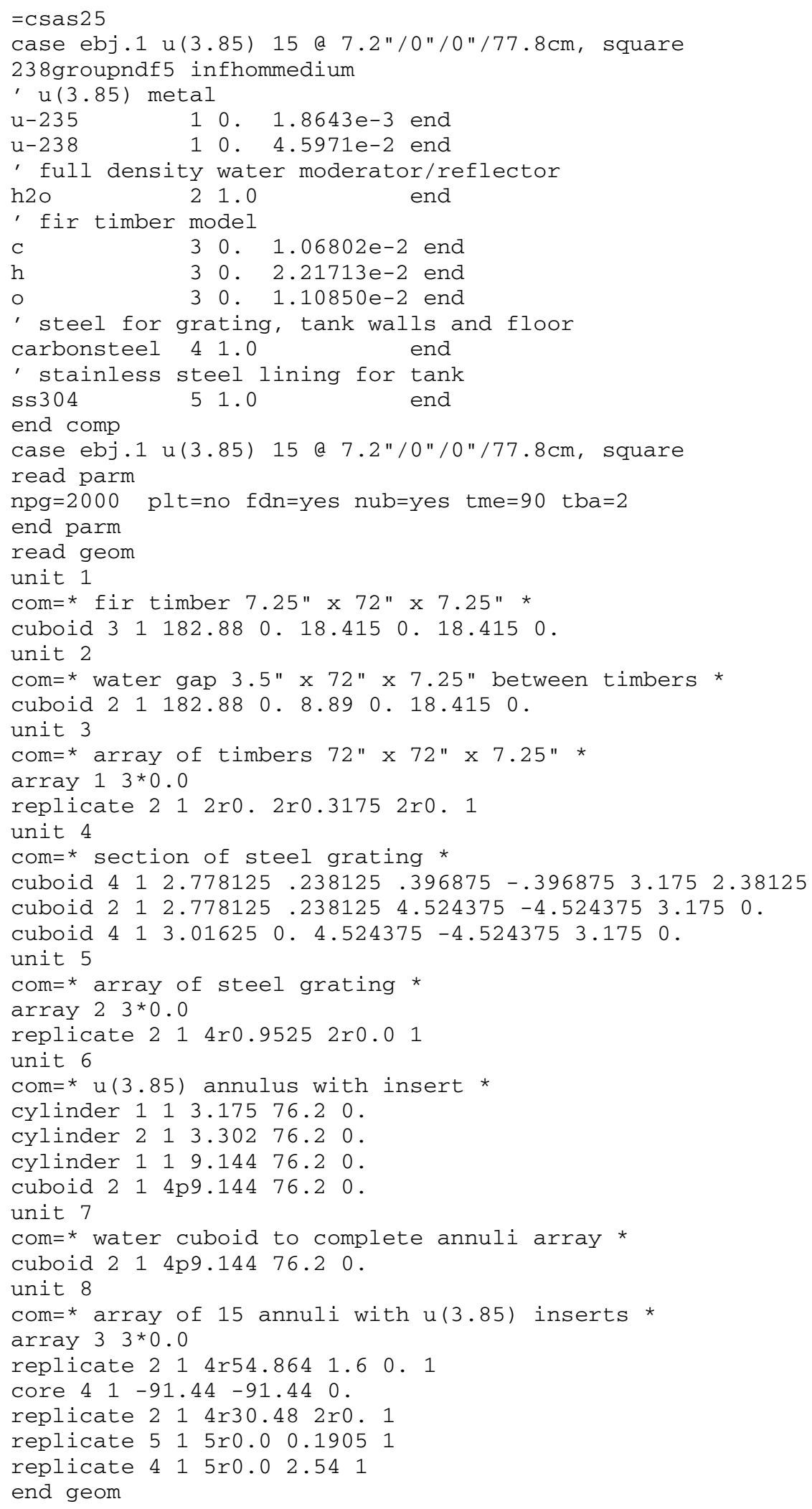




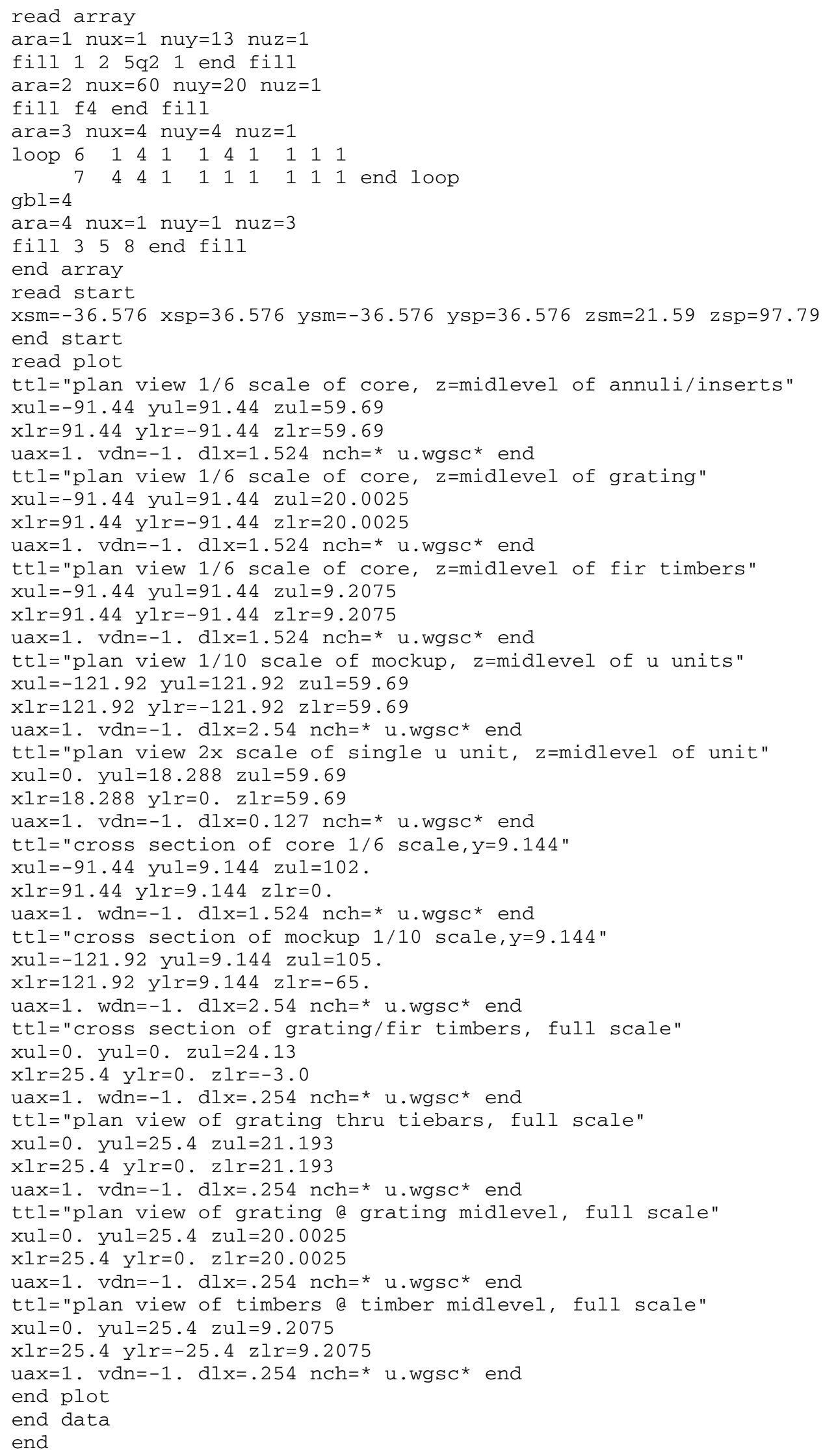




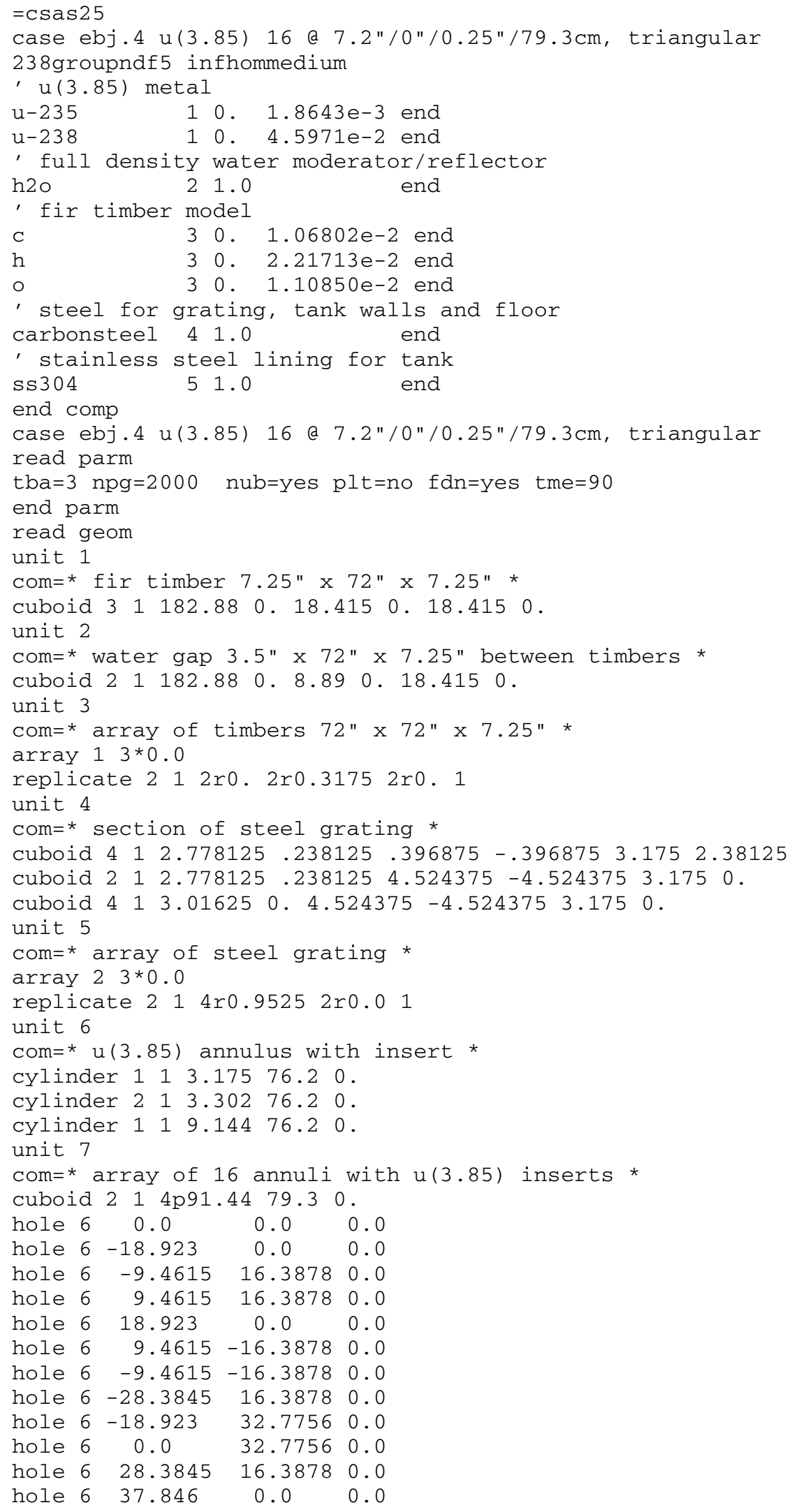




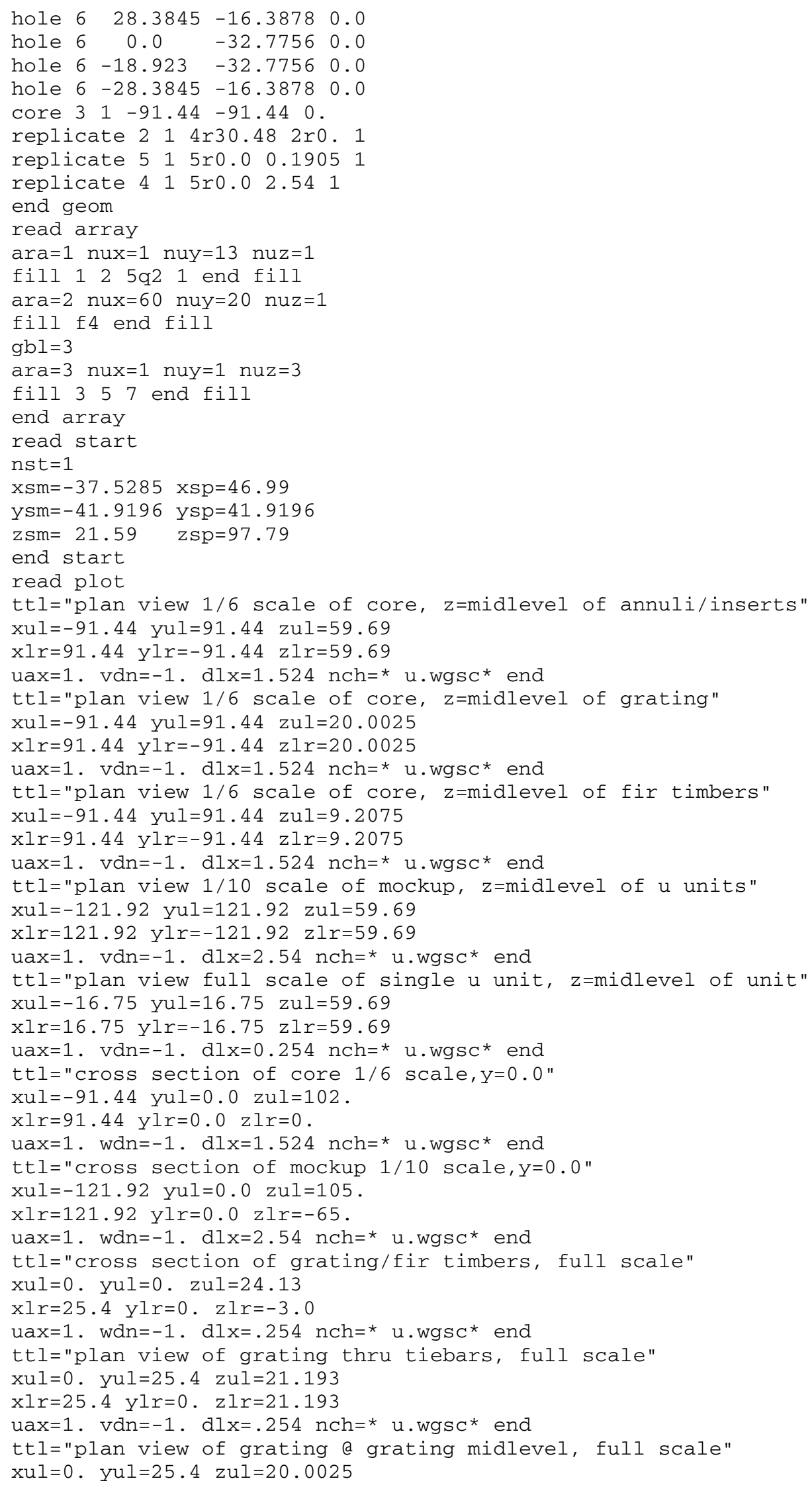




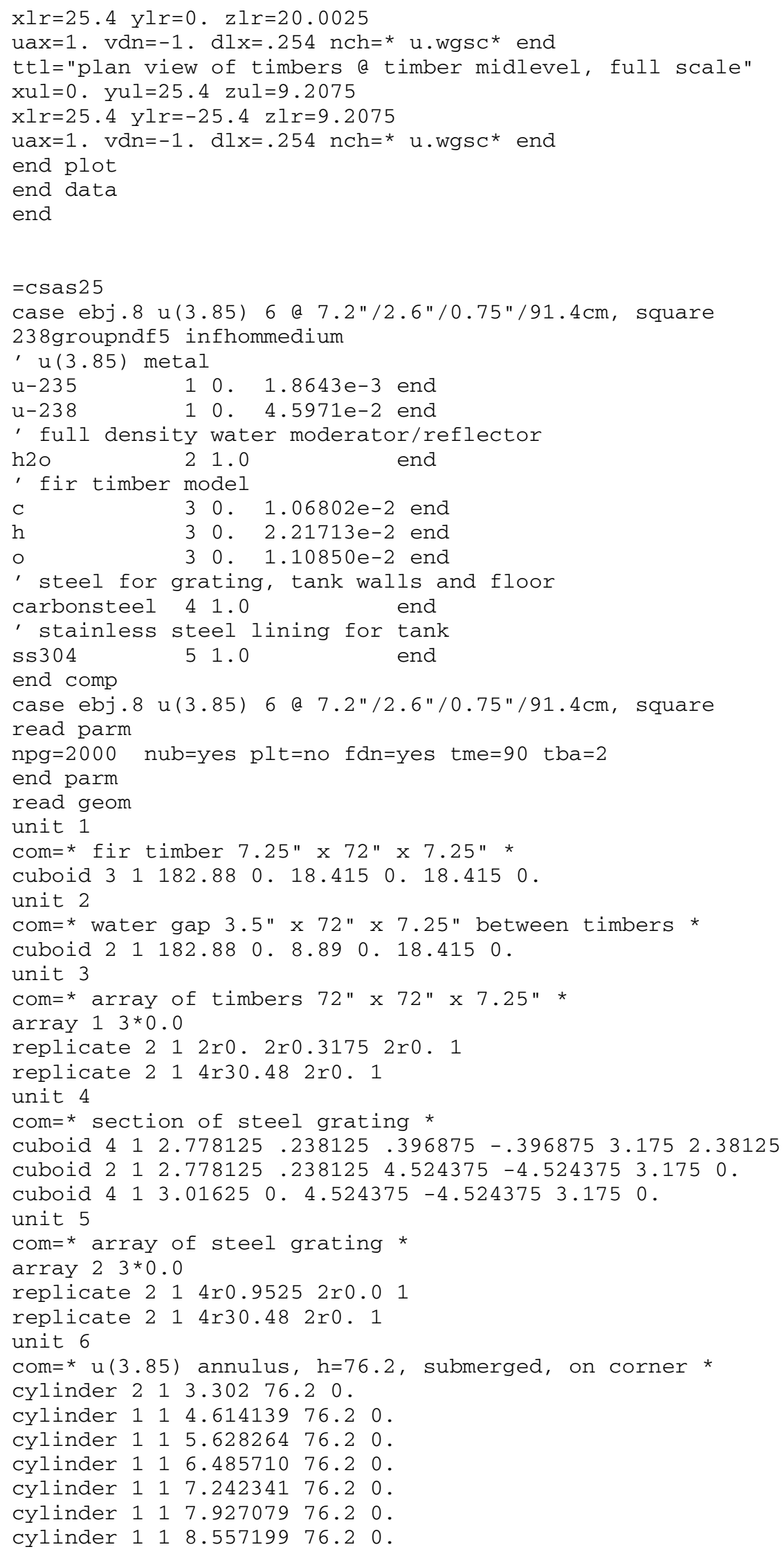


cylinder 119.14400076 .20 . cuboid 21 4p10.0965 91.40 . unit 7

com $=$ * $u(3.85)$ annulus, $h=76.2$, submerged, on side *

cylinder 213.30276 .20 .

cylinder 114.61413976 .20$.

cylinder 115.62826476 .20 .

cylinder 116.48571076 .20 .

cylinder 117.24234176 .20$.

cylinder 117.92707976 .20 .

cylinder 118.55719976 .20$.

cylinder 119.14400076 .20 .

cuboid 214 p10.0965 91.40 .

unit 8

com $=$ * array of 6 annuli, $\mathrm{h}=76.2$, submerged *

array $3 \quad 3 * 0.0$

replicate 21 2r61.1505 2r71.247 2ro. 1

replicate $214 r 30.48$ 2ro. 1

core $41-121.92-121.920$.

replicate 51 5r0.0 0.19051

replicate $415 r 0.02 .541$

end geom

read array

ara=1 nux=1 nuy=13 nuz=1

fill 12 5q2 1 end fill

ara $=2$ nux=60 nuy=20 nuz=1

fill f4 end fill

ara=3 nux=3 nuy=2 nuz=1

fill $6 \begin{array}{lllllll} & 7 & 6 & 6 & 7 & 6 & \text { end fill }\end{array}$

$\mathrm{gbl}=4$

ara=4 nux=1 nuy=1 nuz=3

fill 358 end fill

end array

read start

nst $=1$

$\mathrm{xsm}=-30.2895 \mathrm{xsp}=30.2895$

$\mathrm{ysm}=-20.193 \mathrm{ysp}=20.193$

$\mathrm{zsm}=21.59 \mathrm{zsp}=97.79$

end start

read plot

ttl="plan view $1 / 6$ scale of core, $z=$ midlevel of annuli/inserts"

$\mathrm{xul}=-91.44 \mathrm{yul}=91.44 \mathrm{zul}=59.69$

$\mathrm{xlr}=91.44 \mathrm{ylr}=-91.44$ zlr $=59.69$

uax $=1 . v d n=-1 . d l x=1.524$ nch $=$ * u.wgs* end

ttl $="$ plan view $1 / 6$ scale of core, $z=$ midlevel of grating"

$\mathrm{xul}=-91.44 \mathrm{yul}=91.44 \mathrm{zul}=20.0025$

$\mathrm{xlr}=91.44 \mathrm{ylr}=-91.44 \mathrm{zlr}=20.0025$

uax $=1 . \operatorname{vdn}=-1 . d \mathrm{dx}_{1}=1.524 \mathrm{nch}=* \mathrm{u} \cdot \mathrm{wgs}$ * end

ttl="plan view $1 / 6$ scale of core, $z=$ midlevel of fir timbers"

$\mathrm{xul}=-91.44 \mathrm{yul}=91.44 \mathrm{zul}=9.2075$

$\mathrm{xlr}=91.44 \mathrm{ylr}=-91.44 \mathrm{zlr}=9.2075$

uax $=1 . \operatorname{vdn}=-1 . \mathrm{dlx}=1.524 \mathrm{nch}=$ * u.wgs * end

tt $l="$ plan view $1 / 10$ scale of mockup, $z=$ midlevel of u units"

$\mathrm{xul}=-135 . \mathrm{yul}=135 . \mathrm{zul}=59.69$

$\mathrm{xlr}=135 . \mathrm{ylr}=-135 . \mathrm{zlr}=59.69$

uax $=1 . \operatorname{vdn}=-1 . \mathrm{d} l \mathrm{x}=2.54 \mathrm{nch}=$ * u.wgs * end

tt $l=" p l a n$ view $2 x$ scale of single u unit, $z=$ midlevel of unit"

$\mathrm{xul}=0.0 \mathrm{yul}=20.193 \mathrm{zul}=59.69$

$\mathrm{xlr}=20.193 \mathrm{ylr}=0.0 \mathrm{zlr}=59.69$

$u a x=1 . v d n=-1 . d l x=0.127$ nch=* u.wgs * end

tt $1=$ "cross section of core $1 / 6$ scale, $y=10.0965 "$

$\mathrm{xul}=-91.44 \mathrm{yul}=10.0965 \mathrm{zul}=125$.

$\mathrm{xlr}=91.44 \mathrm{ylr}=10.0965 \mathrm{zlr}=-10$. 


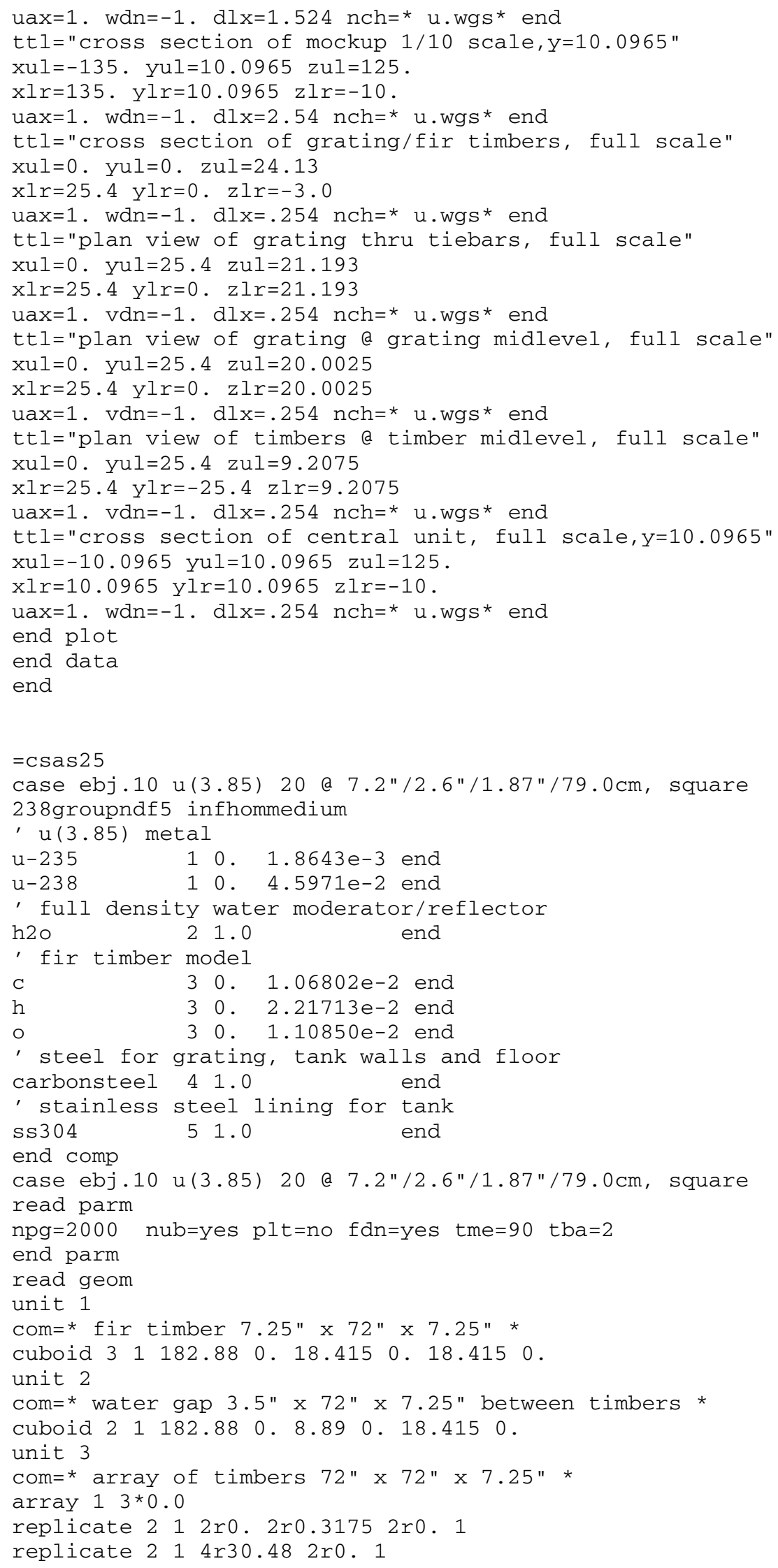




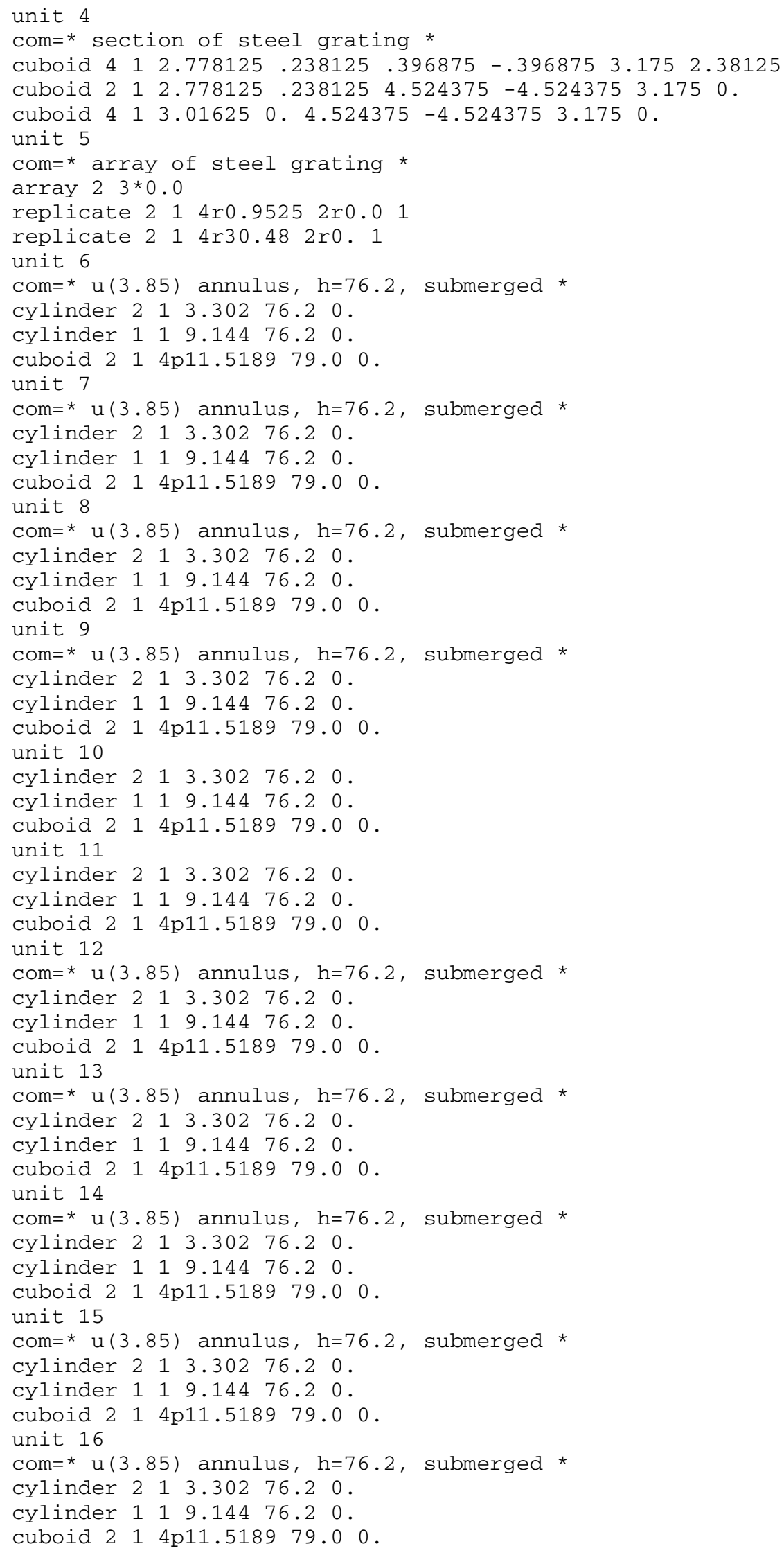


unit 17

com $=$ * $u(3.85)$ annulus, $h=76.2$, submerged * cylinder 213.30276 .20 .

cylinder 119.14476 .20 .

cuboid $214 \mathrm{p} 11.518979 .00$.

unit 18

$\mathrm{com}=* \mathrm{u}(3.85)$ annulus, $\mathrm{h}=76.2$, submerged * cylinder 213.30276 .20 .

cylinder $119.144 \quad 76.20$.

cuboid $214 \mathrm{p} 11.518979 .00$.

unit 19

$\mathrm{com}=* \mathrm{u}(3.85)$ annulus, $\mathrm{h}=76.2$, submerged * cylinder 213.30276 .20 .

cylinder 119.14476 .20$.

cuboid $214 \mathrm{p} 11.518979 .00$.

unit 20

com $=$ * $u(3.85)$ annulus, $h=76.2$, submerged * cylinder 213.30276 .20 .

cylinder 119.14476 .20$.

cuboid $214 \mathrm{p} 11.518979 .00$.

unit 21

$\mathrm{com}=$ * u(3.85) annulus, $\mathrm{h}=76.2$, submerged *

cylinder 213.30276 .20 .

cylinder $119.144 \quad 76.20$.

cuboid $214 \mathrm{p} 11.518979 .00$.

unit 22

com $=$ * $u(3.85)$ annulus, $h=76.2$, submerged *

cylinder 213.30276 .20 .

cylinder 119.14476 .20 .

cuboid 214 p11.5189 79.00 .

unit 23

com $=$ * $u(3.85)$ annulus, $h=76.2$, submerged * cylinder 213.30276 .20$.

cylinder $119.144 \quad 76.20$.

cuboid $214 \mathrm{p} 11.518979 .00$.

unit 24

com $=$ * $u(3.85)$ annulus, $h=76.2$, submerged * cylinder 213.30276 .20 .

cylinder 119.14476 .20$.

cuboid 214 p11.5189 79.00 .

unit 25

com $=$ * $u(3.85)$ annulus, $h=76.2$, submerged *

cylinder 213.30276 .20 .

cylinder $119.144 \quad 76.20$.

cuboid $214 \mathrm{p} 11.518979 .00$.

unit 26

com $=$ * water cuboid to complete array *

cuboid $214 \mathrm{p} 11.518979 .00$.

unit 27

com $=$ * array of 20 annuli, $h=76.2$, submerged * array $3 \quad 3 * 0.0$

replicate $214 r 33.84552 r 0.1$

replicate $214 r 30.482$ ro. 1

core $41-121.92-121.920$.

replicate 51 5r0.0 0.19051

replicate $415 r 0.02 .541$

end geom

read array

ara=1 nux=1 nuy=13 nuz=1

fill 12 5q2 1 end fill

ara $=2$ nux=60 nuy=20 nuz=1

fill f4 end fill

ara=3 nux=5 nuy=5 nuz=1 
$\begin{array}{lllllllllllllllllllll}\text { fill } & 26 & 26 & 6 & 7 & 26 & 8 & 9 & 10 & 11 & 12 & 13 & 14 & 15 & 16 & 17 & 18 & 19 & 20 & 21 & 22\end{array}$

$\begin{array}{lllllll}26 & 23 & 24 & 25 & 26 & \text { end fill }\end{array}$

$\mathrm{gbl}=4$

ara=4 nux=1 nuy=1 nuz=3

fill $3 \quad 5 \quad 27$ end fill

end array

read start

nst $=1$

$\mathrm{Xsm}=-57.5945 \mathrm{xsp}=57.5945$

ysm $=-57.5945$ ysp $=57.5945$

$\mathrm{zsm}=21.59 \mathrm{zsp}=97.79$

end start

read plot

ttl="plan view $1 / 6$ scale of core, $z=$ midlevel of annuli/inserts"

$\mathrm{xul}=-91.44 \mathrm{yul}=91.44 \mathrm{zul}=59.69$

$\mathrm{xlr}=91.44 \mathrm{ylr}=-91.44 \mathrm{zlr}=59.69$

$\mathrm{uax}=1 . \operatorname{vdn}=-1 . \mathrm{dlx}=1.524 \mathrm{nch}=$ * u.wgs * end

tt $1="$ plan view $1 / 6$ scale of core, $z=$ midlevel of grating"

$\mathrm{xul}=-91.44 \mathrm{yul}=91.44 \mathrm{zul}=20.0025$

$\mathrm{xlr}=91.44 \mathrm{ylr}=-91.44 \mathrm{zlr}=20.0025$

uax $=1 . \operatorname{vdn}=-1 . \mathrm{dlx}=1.524 \mathrm{nch}=$ * u.wgs* end

ttl="plan view $1 / 6$ scale of core, $z=$ midlevel of fir timbers"

$\mathrm{xul}=-91.44 \mathrm{yul}=91.44 \mathrm{zul}=9.2075$

$\mathrm{xlr}=91.44 \mathrm{ylr}=-91.44 \mathrm{zlr}=9.2075$

$\mathrm{uax}=1 . \operatorname{vdn}=-1 . \mathrm{dlx}=1.524 \mathrm{nch}=$ * u.wgs * end

ttl="plan view $1 / 10$ scale of mockup, $z=$ midlevel of u units"

$\mathrm{xul}=-135 . \mathrm{yul}=135 . \mathrm{zul}=59.69$

$\mathrm{xlr}=135 . \mathrm{ylr}=-135 . \mathrm{zlr}=59.69$

uax $=1 . \operatorname{vdn}=-1 . \mathrm{dlx}=2.54 \mathrm{nch}=$ * u.wgs * end

ttl="plan view $2 x$ scale of single u unit, $z=$ midlevel of unit"

$\mathrm{xul}=-11.5189 \mathrm{yul}=11.5189 \mathrm{zul}=59.69$

$\mathrm{xlr}=11.5189$ ylr $=-11.5189$ zlr $=59.69$

$\mathrm{uax}=1 . \mathrm{vdn}=-1 . \mathrm{dlx}=0.127 \mathrm{nch}=$ * u.wgs * end

tt $l="$ cross section of core $1 / 6$ scale, $y=0.0 "$

$\mathrm{xul}=-91.44 \mathrm{yul}=0.0 \mathrm{zul}=110$.

$\mathrm{xlr}=91.44$ ylr=0.0 zlr $=-10$.

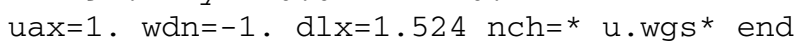

ttl $="$ cross section of mockup $1 / 10$ scale, $y=0.0 "$

$\mathrm{xul}=-135$. $\mathrm{yul}=0.0 \mathrm{zul}=125$.

$\mathrm{xlr}=135$. ylr=0.0 zlr $=-10$.

$u a x=1$. $w d n=-1 . d l x=2.54$ nch=* u.wgs * end

ttl="cross section of grating/fir timbers, full scale"

$\mathrm{xul}=0 . \mathrm{yul}=0 . \mathrm{zul}=24.13$

$\mathrm{xlr}=25.4 \mathrm{ylr}=0 . \mathrm{zlr}=-3.0$

uax $=1$. wdn $=-1 . d \mathrm{dx}=.254 \mathrm{nch}=$ * u.wgs * end

ttl="plan view of grating thru tiebars, full scale"

$\mathrm{xul}=0$. yul=25.4 zul=21.193

$\mathrm{xlr}=25.4$ ylr=0. $\mathrm{zlr}=21.193$

uax=1. $\operatorname{vdn}=-1 . \mathrm{dlx}=.254 \mathrm{nch}=$ * u.wgs * end

ttl="plan view of grating a grating midlevel, full scale"

$\mathrm{xul}=0 . \mathrm{yul}=25.4 \mathrm{zul}=20.0025$

$\mathrm{xlr}=25.4 \mathrm{ylr}=0 . \mathrm{zlr}=20.0025$

uax $=1 . \operatorname{vdn}=-1 . \mathrm{dlx}=.254 \mathrm{nch}=$ * u.wgs * end

ttl="plan view of timbers a timber midlevel, full scale"

$\mathrm{xul}=0 . \mathrm{yul}=25.4 \mathrm{zul}=9.2075$

$\mathrm{xlr}=25.4 \mathrm{ylr}=-25.4 \mathrm{zlr}=9.2075$

uax $=1 . \operatorname{vdn}=-1 . \mathrm{dlx}=.254 \mathrm{nch}=$ * u.wgs * end

ttl $="$ cross section of central unit, full scale, $y=0.0 "$

$\mathrm{xul}=-11.5189 \mathrm{yul}=0.0 \mathrm{zul}=110$.

$\mathrm{xlr}=11.5189$ ylr=0.0 zlr $=-10$.

uax $=1$. wdn $=-1 . \mathrm{dlx}=.254 \mathrm{nch}=$ * u.wgs * end

end plot

end data 


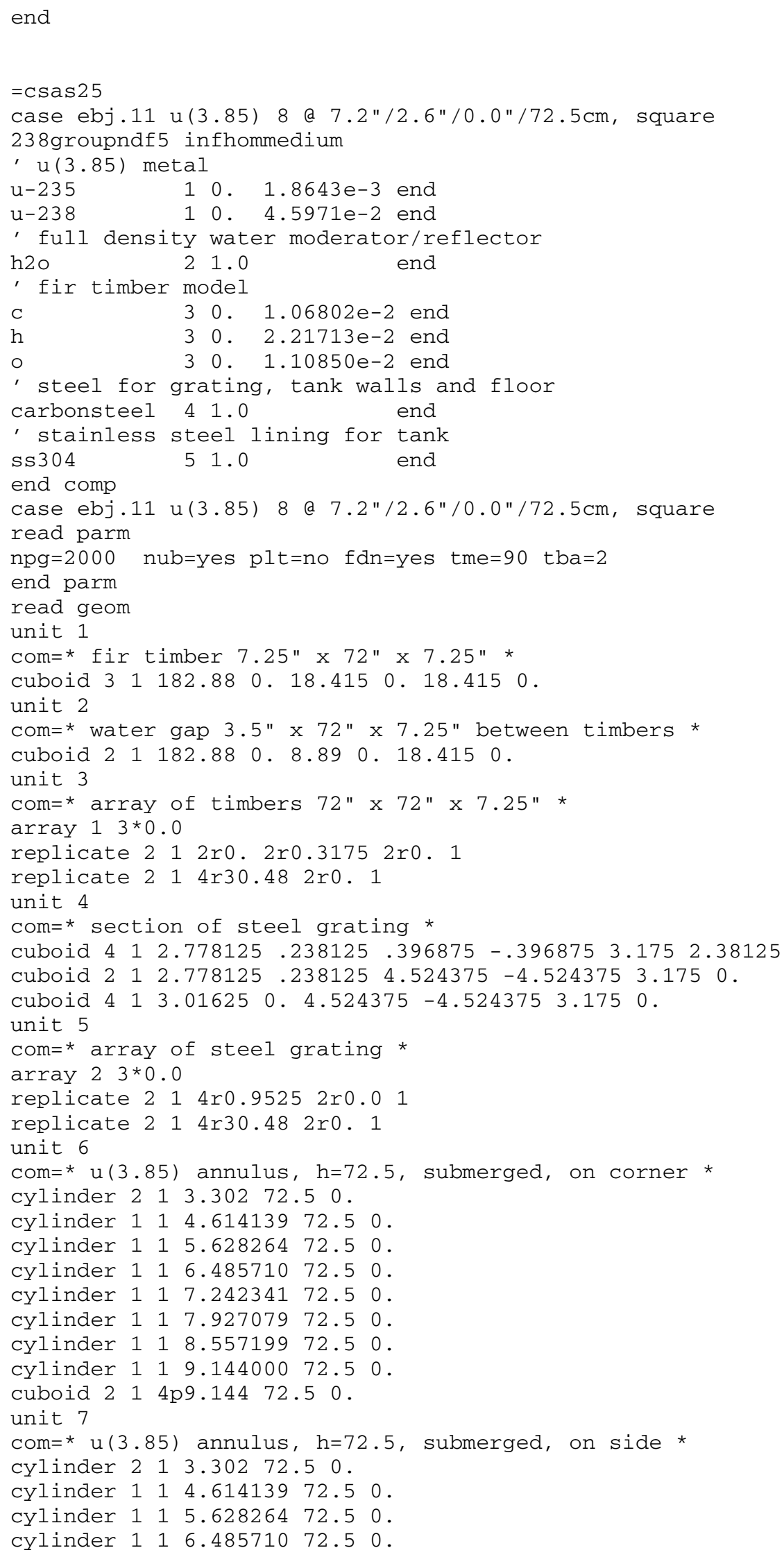


cylinder $1 \quad 1 \quad 7.242341 \quad 72.5 \quad 0$.

cylinder 117.92707972 .50 .

cylinder 118.55719972 .50 .

cylinder 119.14400072 .50 .

cuboid $214 \mathrm{p} 9.14472 .50$.

unit 8

$\mathrm{com}=$ * $\mathrm{u}(3.85)$ annulus, $\mathrm{h}=3.7$, dry, on corner *

cylinder $0 \begin{array}{llllll} & 3.302 & 3.7 & 0 .\end{array}$

cylinder 114.6141393 .70$.

cylinder 115.6282643 .70 .

cylinder $116.4857103 .7 \quad 0$.

cylinder 117.2423413 .70$.

cylinder 1107.9270793 .70$.

cylinder 118.5571993 .70 .

cylinder 119.1440003 .70 .

cuboid 014 p9.144 3.70 .

unit 9

com $=$ * u(3.85) annulus, $\mathrm{h}=3.7, \mathrm{dry}$, on side *

cylinder $0 \quad 1 \quad 3.302 \quad 3.7 \quad 0$.

cylinder 114.6141393 .70 .

cylinder 115.6282643 .70 .

cylinder $116.4857103 .7 \quad 0$.

cylinder 1117.2423413 .70 .

cylinder 1107.9270793 .70 .

cylinder 118.5571993 .70 .

cylinder 119.1440003 .70 .

cuboid 0 1 4p9.144 3.70 .

unit 10

com $=$ * array of 8 annuli, $\mathrm{h}=72.5$, submerged *

array $3 \quad 3 * 0.0$

replicate $212 r 54.8642 r 73.152 \quad 2 r 0.1$

replicate $214 r 30.48$ 2ro. 1

unit 11

com=* array of 8 annuli, $h=3.7$, dry *

array $4 \quad 3 * 0.0$

replicate 012 2r54.864 $2 r 73.152 \quad 2 r 0.1$

replicate $014 r 30.48$ 2r0. 1

core $51-121.92-121.920$.

replicate 51 5r0.0 0.19051

replicate $415 r 0.02 .541$

end geom

read array

ara=1 nux=1 nuy=13 nuz=1

fill 12 5q2 1 end fill

ara=2 nux=60 nuy=20 nuz=1

fill f4 end fill

ara $=3$ nux=4 nuy=2 nuz=1

fill $6 \begin{array}{lllllll}7 & 7 & 6 & 6 & 7 & 6 & \text { end fill }\end{array}$

ara $=4$ nux=4 nuy=2 nuz=1

fill $8 \begin{array}{llllllll} & 9 & 9 & 8 & 8 & 9 & 9 & 8\end{array}$ end fill

$\mathrm{gbl}=5$

ara=5 nux=1 nuy=1 nuz=4

fill 351011 end fill

end array

'read start

'nst $=1$

' $\mathrm{xsm}=-36.576 \mathrm{xsp}=36.576$

'ysm $=-18.288$ ysp=18.288

' $\mathrm{zsm}=21.59 \mathrm{zsp}=94.09$

' end start

read plot

ttl="plan view $1 / 6$ scale of core, $z=$ midlevel of annuli/inserts"

$\mathrm{xul}=-91.44 \mathrm{yul}=91.44 \mathrm{zul}=59.69$ 


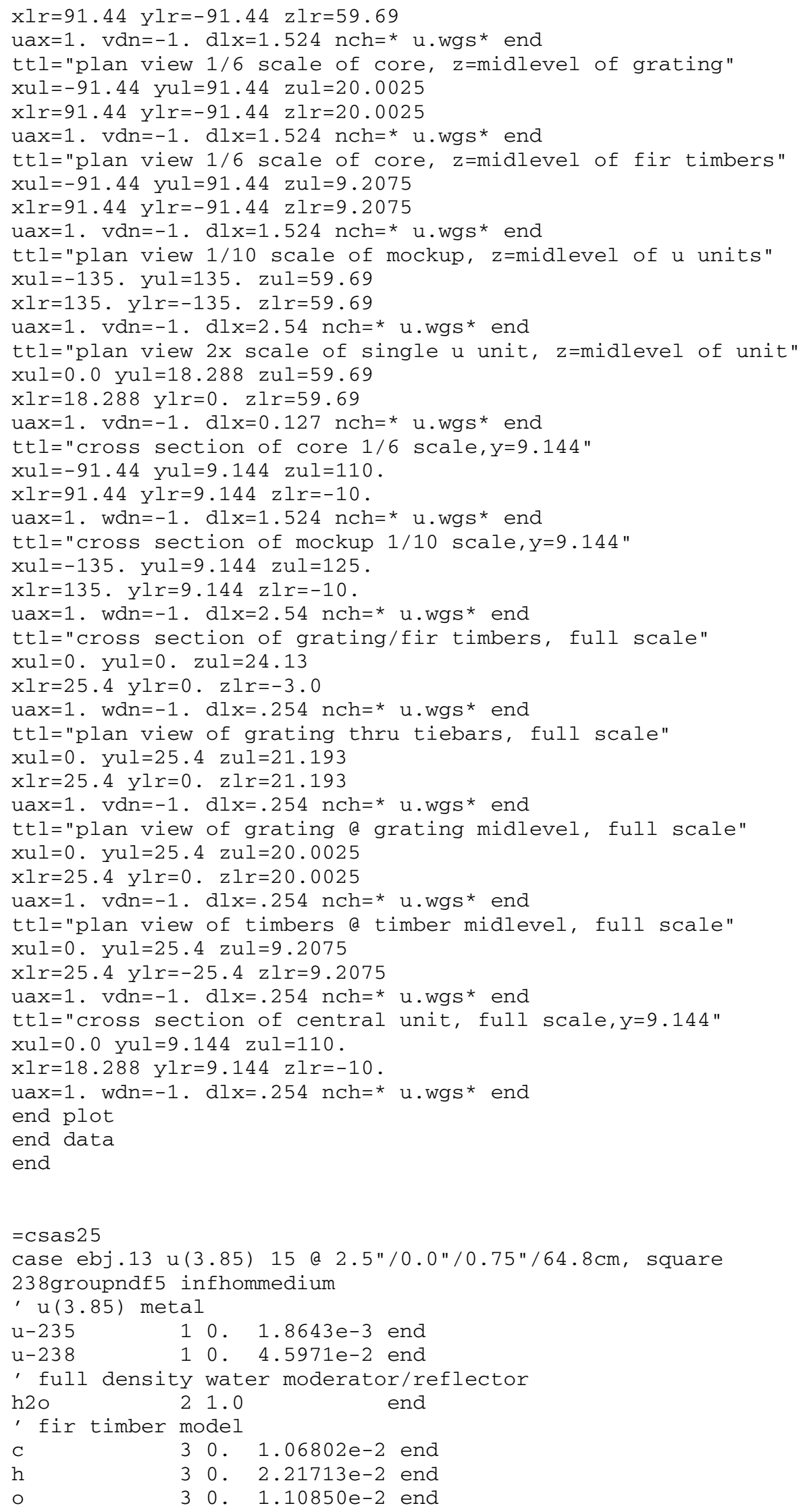




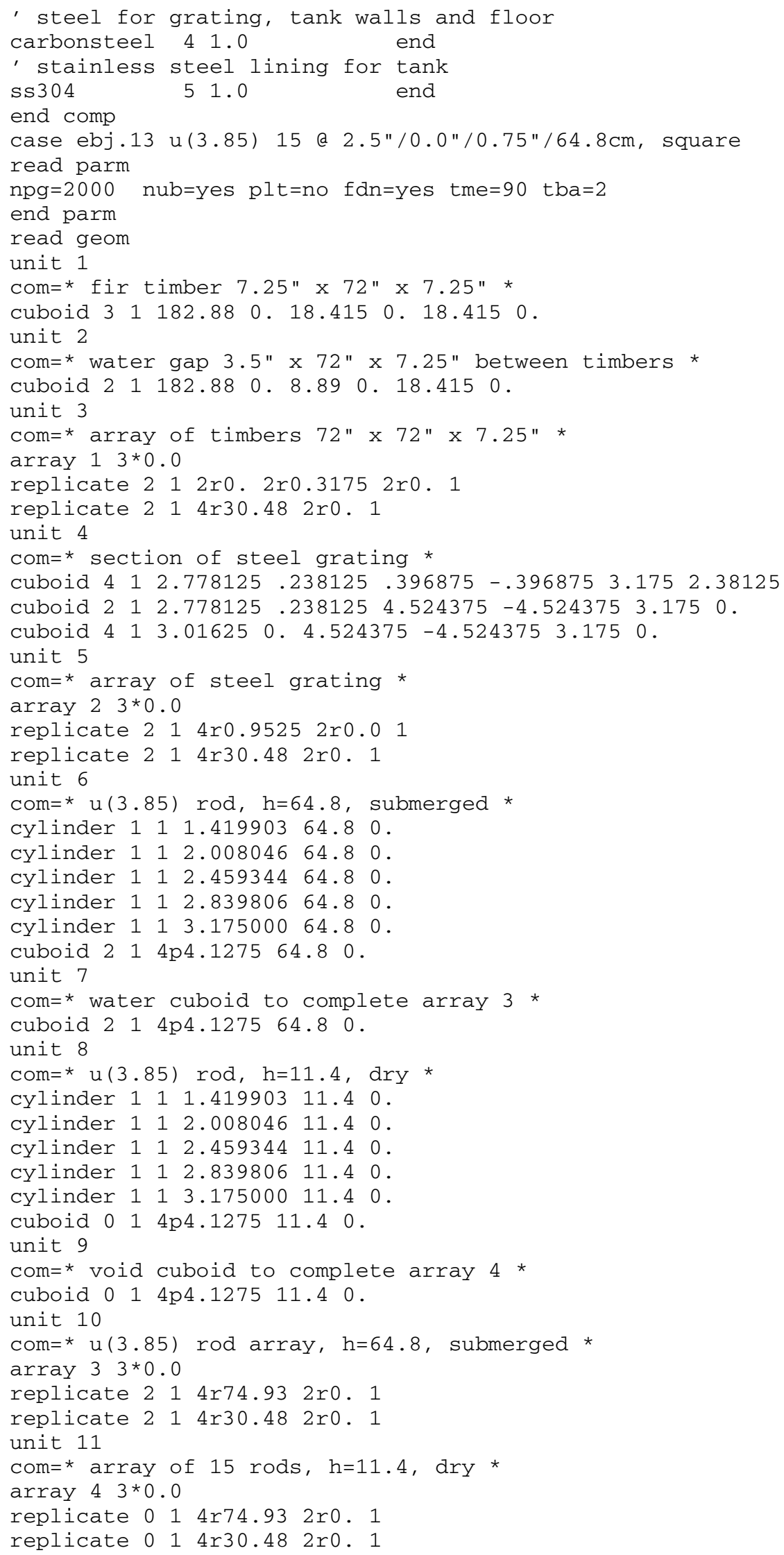




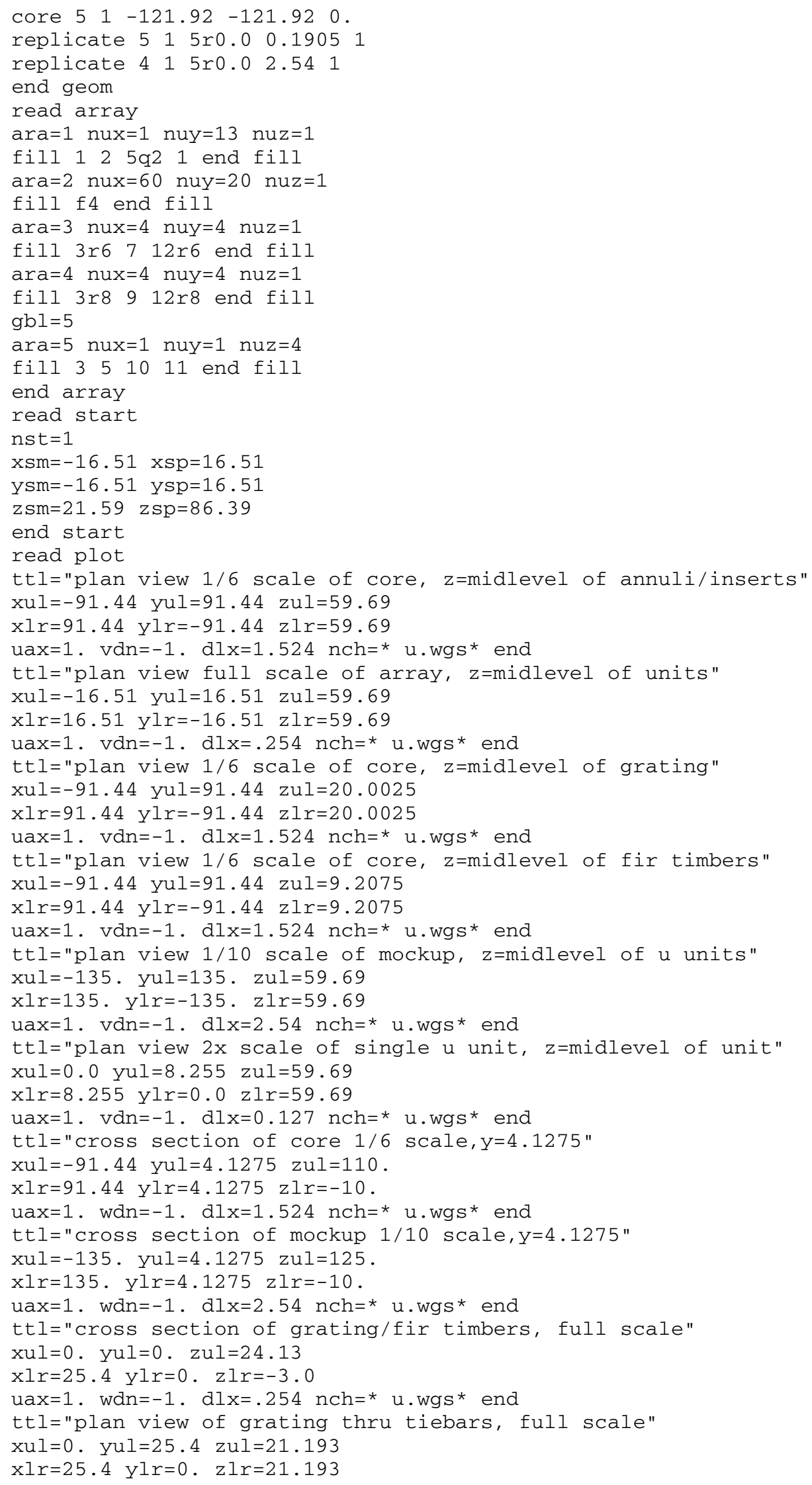




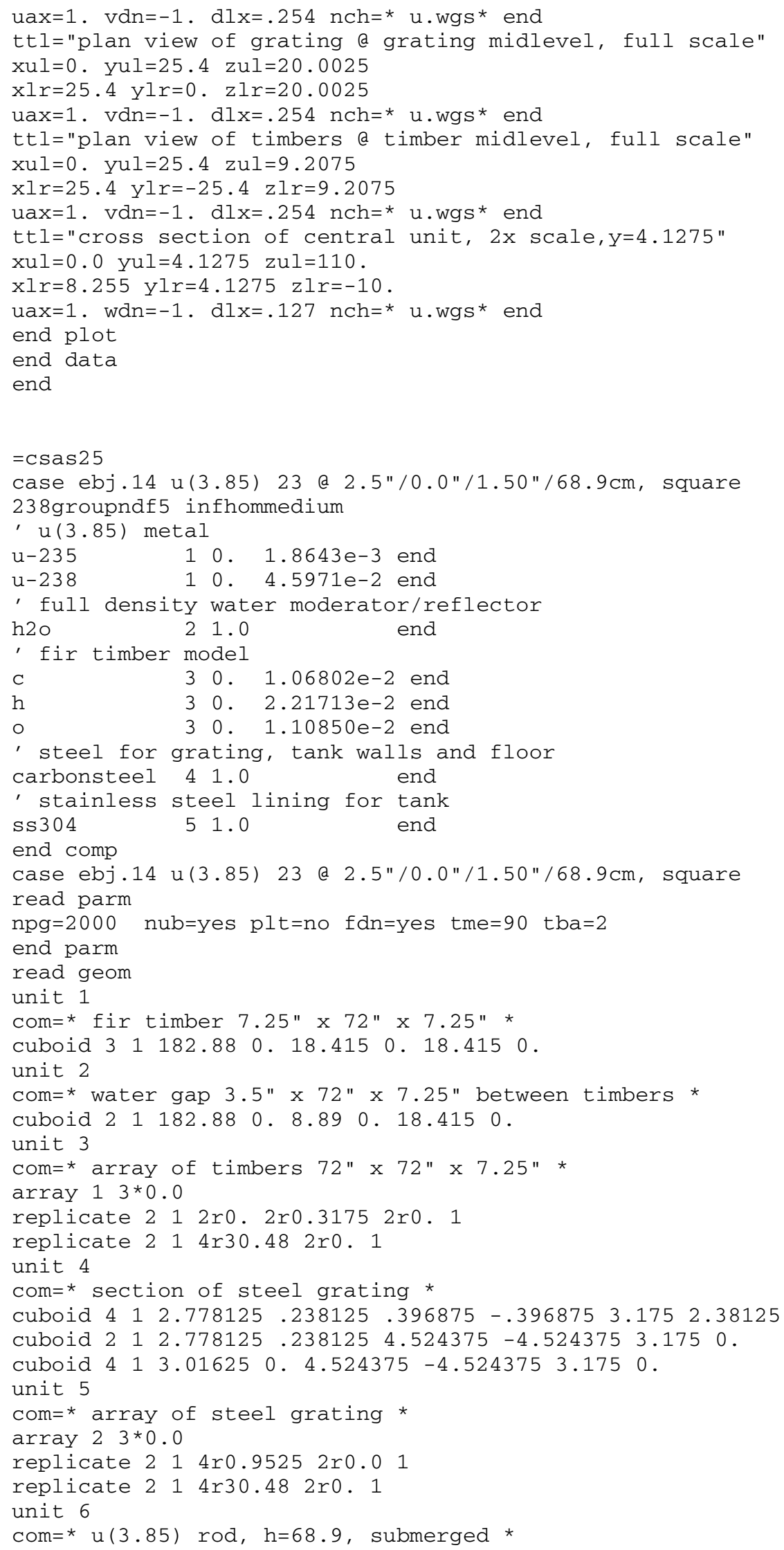




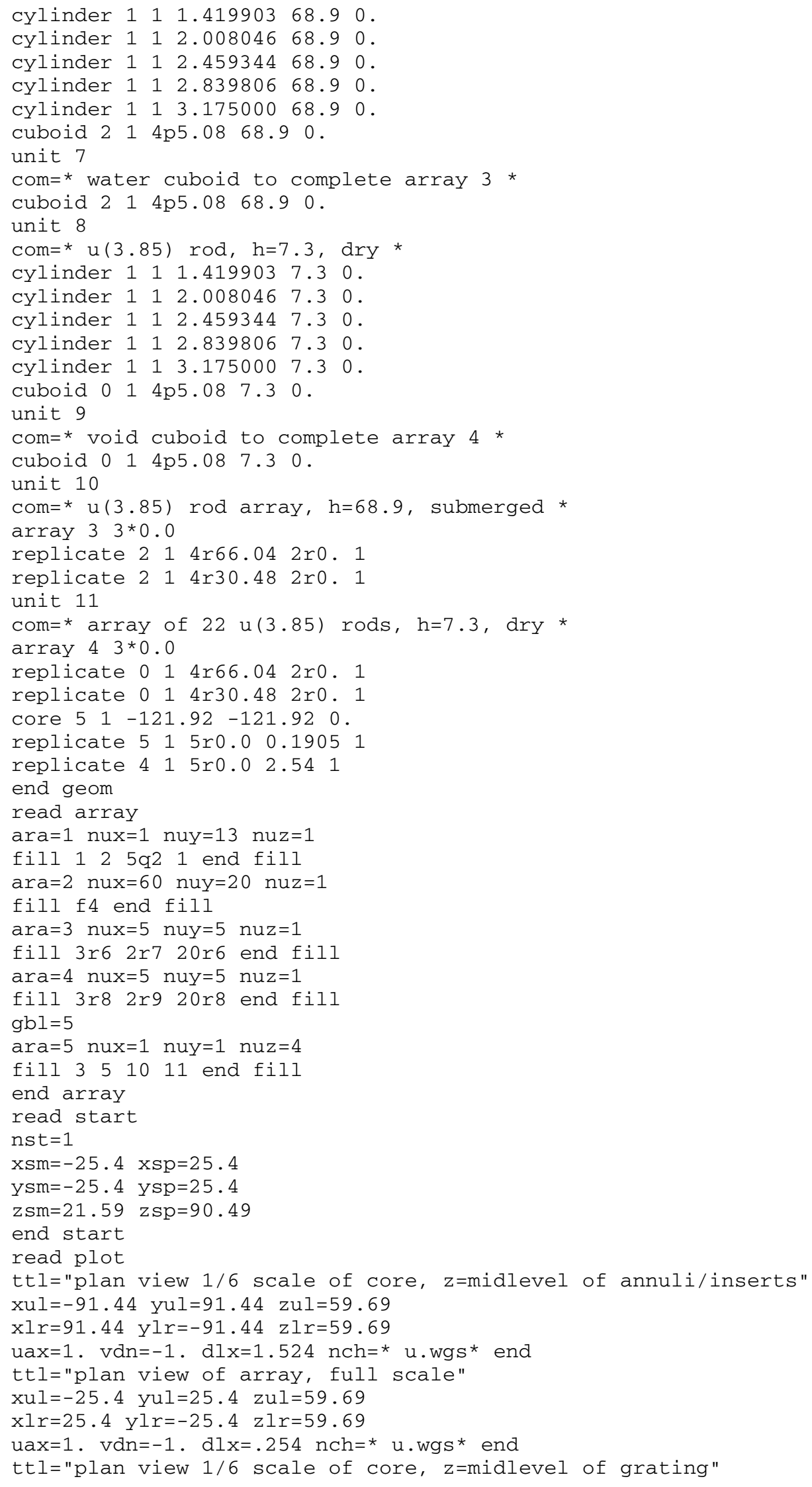




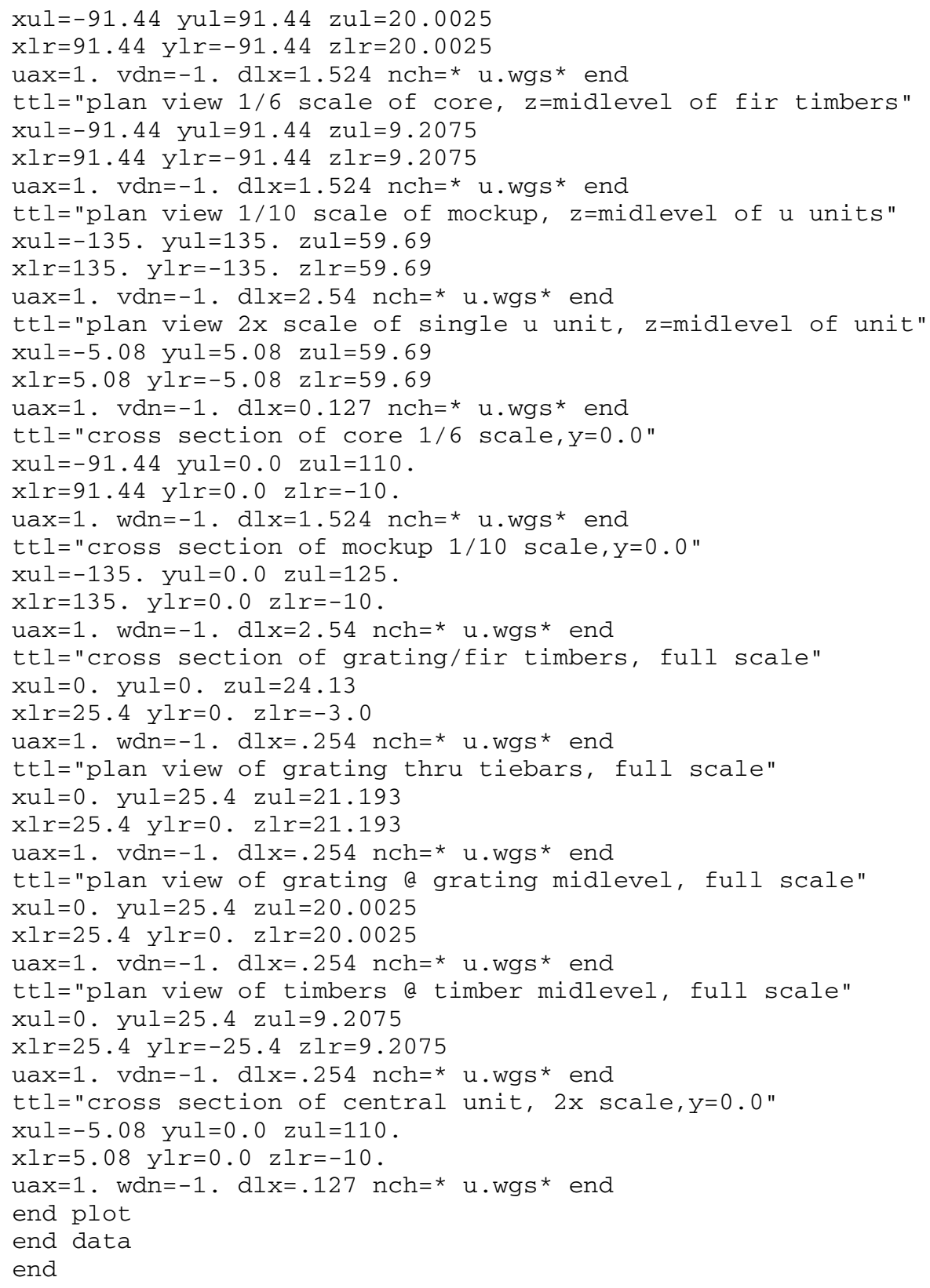


Table A.3. Table 3 input data

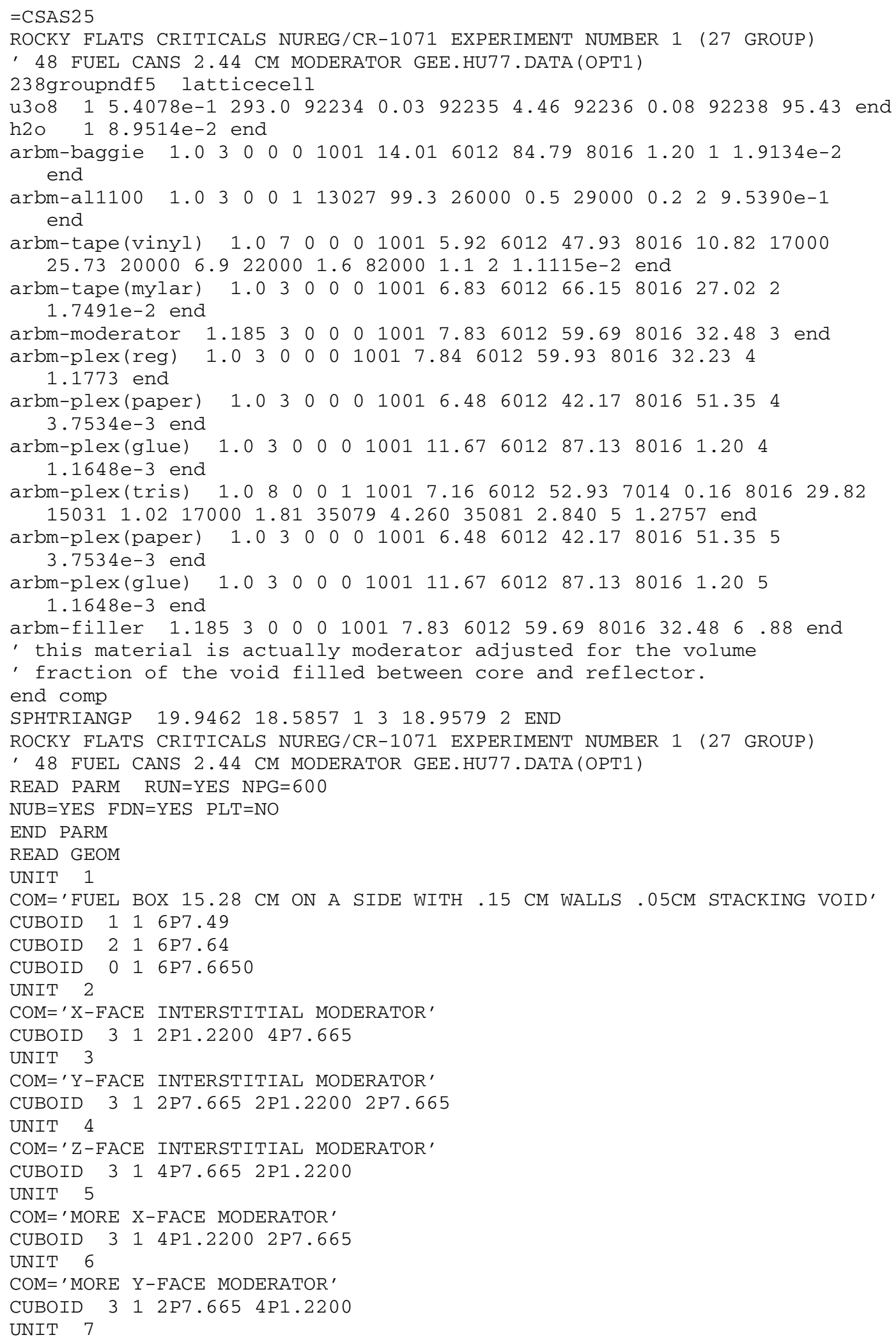




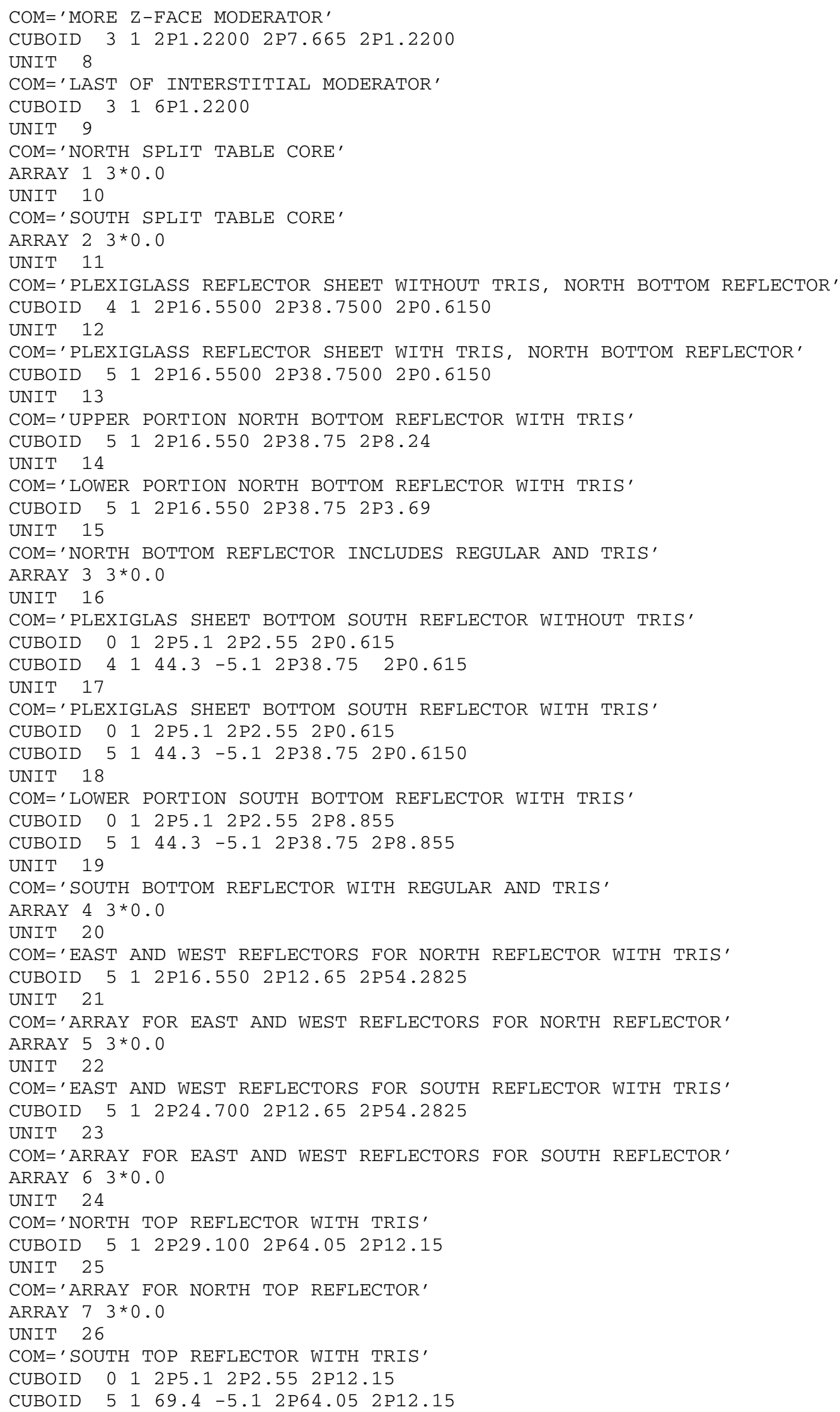




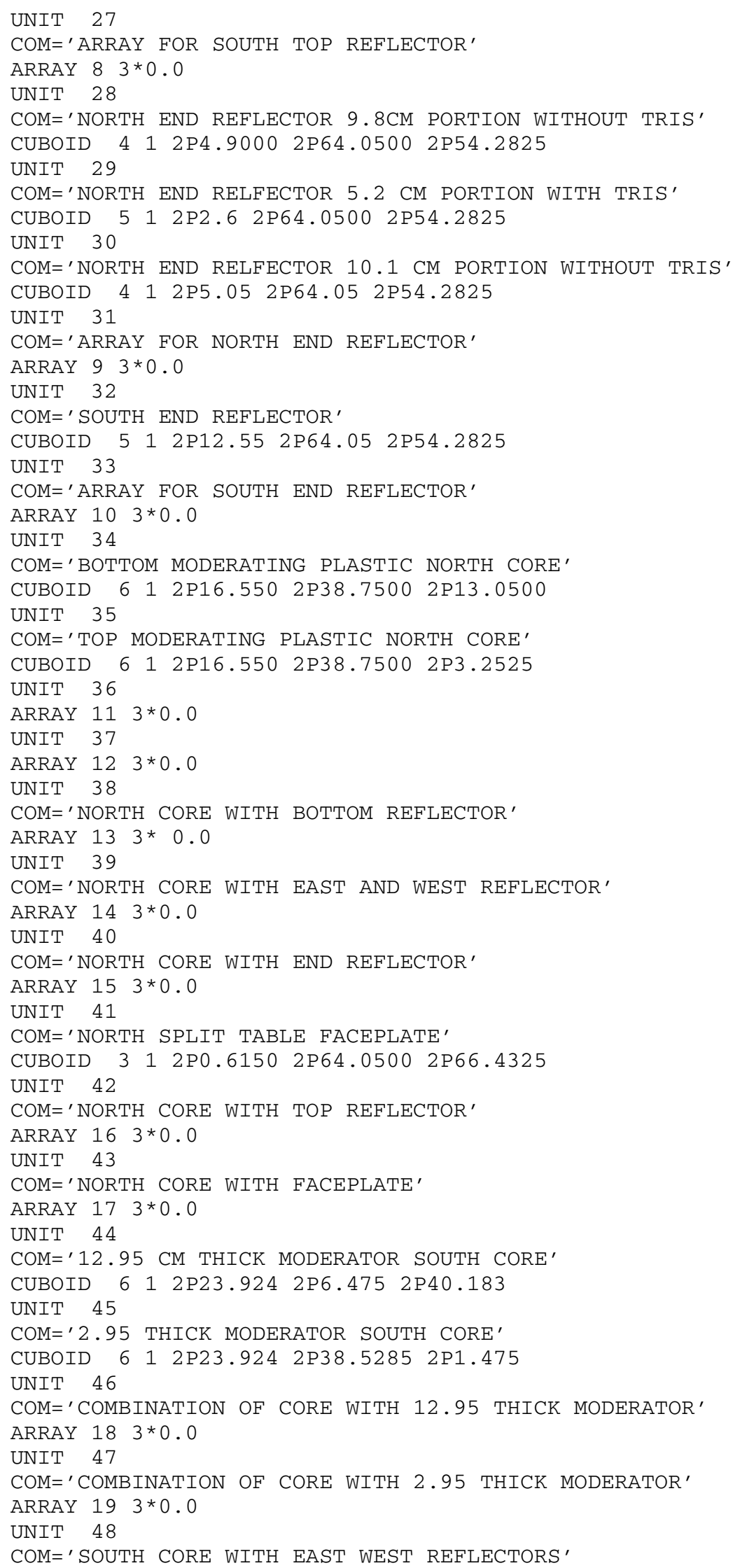




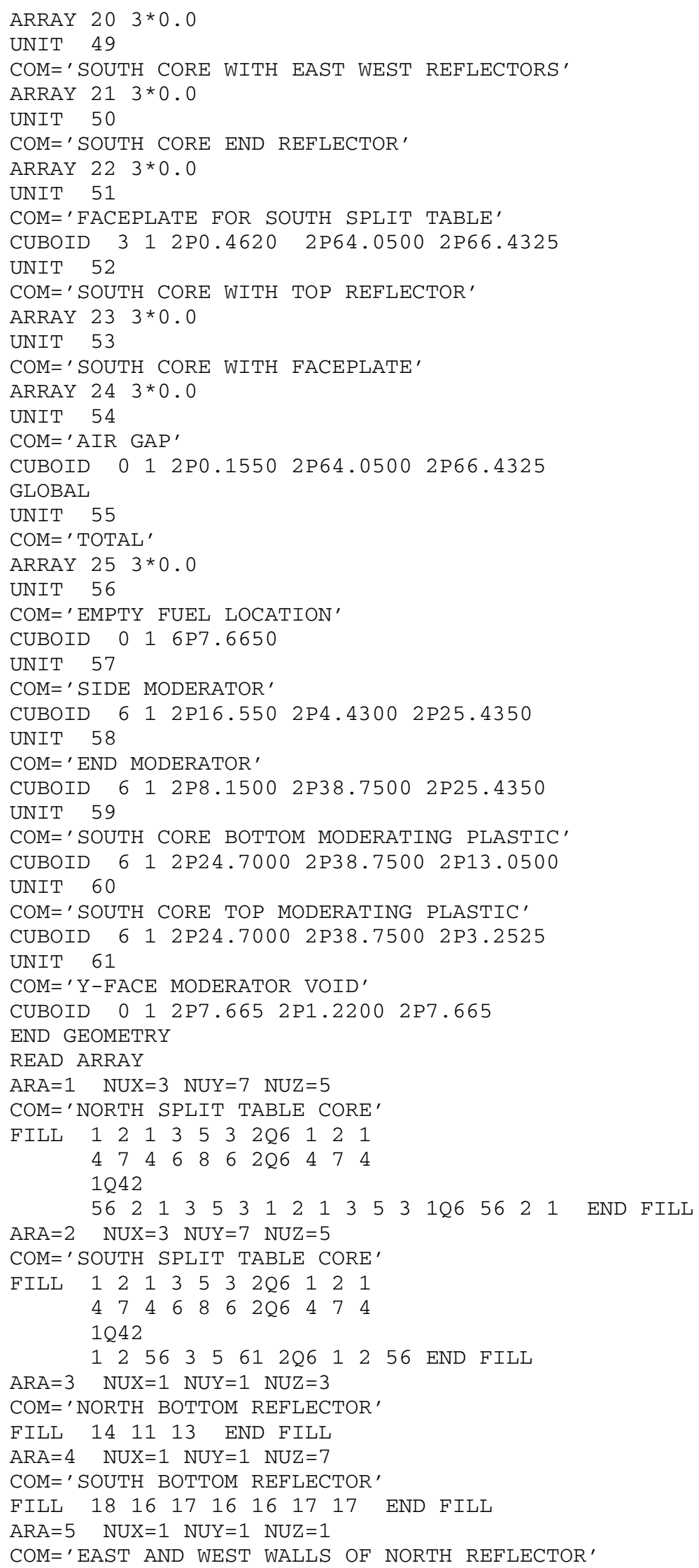


FILL 20 END FILL

$A R A=6 \quad N U X=1 \quad N U Y=1 \quad N U Z=1$

COM='EAST AND WEST WALLS OF SOUTH REFLECTOR'

FILL 22 END FILL

$\mathrm{ARA}=7 \quad \mathrm{NUX}=1 \quad \mathrm{NUY}=1 \quad \mathrm{NUZ}=1$

COM='ARRAY FOR NORTH TOP REFLECTOR'

FILL 24 END FILL

$A R A=8 \quad N U X=1 \quad N U Y=1 \quad N U Z=1$

COM='ARRAY FOR SOUTH TOP REFLECTOR'

FILL 26 END FILL

$A R A=9 \quad N U X=3 \quad N U Y=1 \quad N U Z=1$

COM='ARRAY FOR NORTH END REFLECTOR'

FILL 282930 END FILL

$A R A=10 \quad N U X=1 \quad N U Y=1 \quad N U Z=1$

COM='ARRAY FOR SOUTH END REFLECTOR'

FILL 32 END FILL

$A R A=11 \quad N U X=1 \quad N U Y=2 \quad N U Z=1$

COM='COMBINARION OF CORE WITH SIDE MODERATOR'

FILL 957 END FILL

$A R A=12$ NUX=1 NUY=1 NUZ=3

COM $=$ 'COMBINATION OF PREVIOUS ARRAY WITH TOP AND BOTTOM MODERATOR'

FILL $34 \quad 36 \quad 35$ END FILL

$A R A=13 \quad N U X=1 \quad N U Y=1 \quad N U Z=2$

COM='COMBINATION OF NORTH CORE WITH BOTTOM REFLECTOR'

FILL $15 \quad 37$ END FILL

$A R A=14 \quad N U X=1 \quad N U Y=3 \quad N U Z=1$

COM='NORTH CORE WITH SIDE REFLECTORS'

FILL $21 \quad 38 \quad 21$ END FILL

$A R A=15 \quad N U X=2 \quad N U Y=1 \quad N U Z=1$

COM='NORTH CORE WITH END REFLECTOR'

FILL 3139 END FILL

$A R A=16 \quad N U X=1 \quad N U Y=1 \quad N U Z=2$

$\mathrm{COM}=$ 'NORTH CORE WITH TOP REFLECTOR'

FILL 4025 END FILL

$A R A=17 \quad N U X=2 \quad N U Y=1 \quad N U Z=1$

COM='NORTH CORE WITH FACEPLATE'

FILL $42 \quad 41$ END FILL

$A R A=18 \quad N U X=1 \quad N U Y=2 \quad N U Z=1$

COM='COMBINATION OF $S$. CORE WITH SIDE MODERATOR'

FILL 1057 END FILL

$A R A=19 \quad N U X=2 \quad N U Y=1 \quad N U Z=1$

COM $=$ ' COMBINATION OF CORE WITH END MODERATOR'

FILL 4658 END FILL

$\mathrm{ARA}=20 \quad \mathrm{NUX}=1 \quad \mathrm{NUY}=1 \quad \mathrm{NUZ}=4$

COM='SOUTH CORE WITH BOTTOM MODERATOR AND REFLECTOR'

FILL $1959 \quad 47 \quad 60$ END FILL

$A R A=21$ NUX=1 NUY=3 NUZ=1

COM='SOUTH CORE WITH EAST WEST REFLECTORS'

FILL $23 \quad 48 \quad 23$ END FILL

$A R A=22 \quad N U X=2 \quad N U Y=1 \quad N U Z=1$

$\mathrm{COM}=$ 'SOUTH CORE WITH END REFLECTOR'

FILL 4933 END FILL

$\mathrm{ARA}=23 \mathrm{NUX}=1 \quad \mathrm{NUY}=1 \quad \mathrm{NUZ}=2$

COM $=$ 'COMBINATION OF CORE WITH TOP REFLECTOR'

FILL 5027 END FILL

$\mathrm{ARA}=24 \quad \mathrm{NUX}=2 \quad \mathrm{NUY}=1 \quad \mathrm{NUZ}=1$

COM='SOUTH CORE WITH FACEPLATE'

FILL 5152 END FILL

$A R A=25 \quad N U X=3 \quad N U Y=1 \quad N U Z=1$

$\mathrm{COM}={ }^{\prime}$ TOTAL ${ }^{\prime}$

FILL $43 \quad 54 \quad 53$ END FILL

END ARRAY

READ PLOT TTL='XZ SLICE OF RFP1 SHOWING MATERIAL REGIONS' 


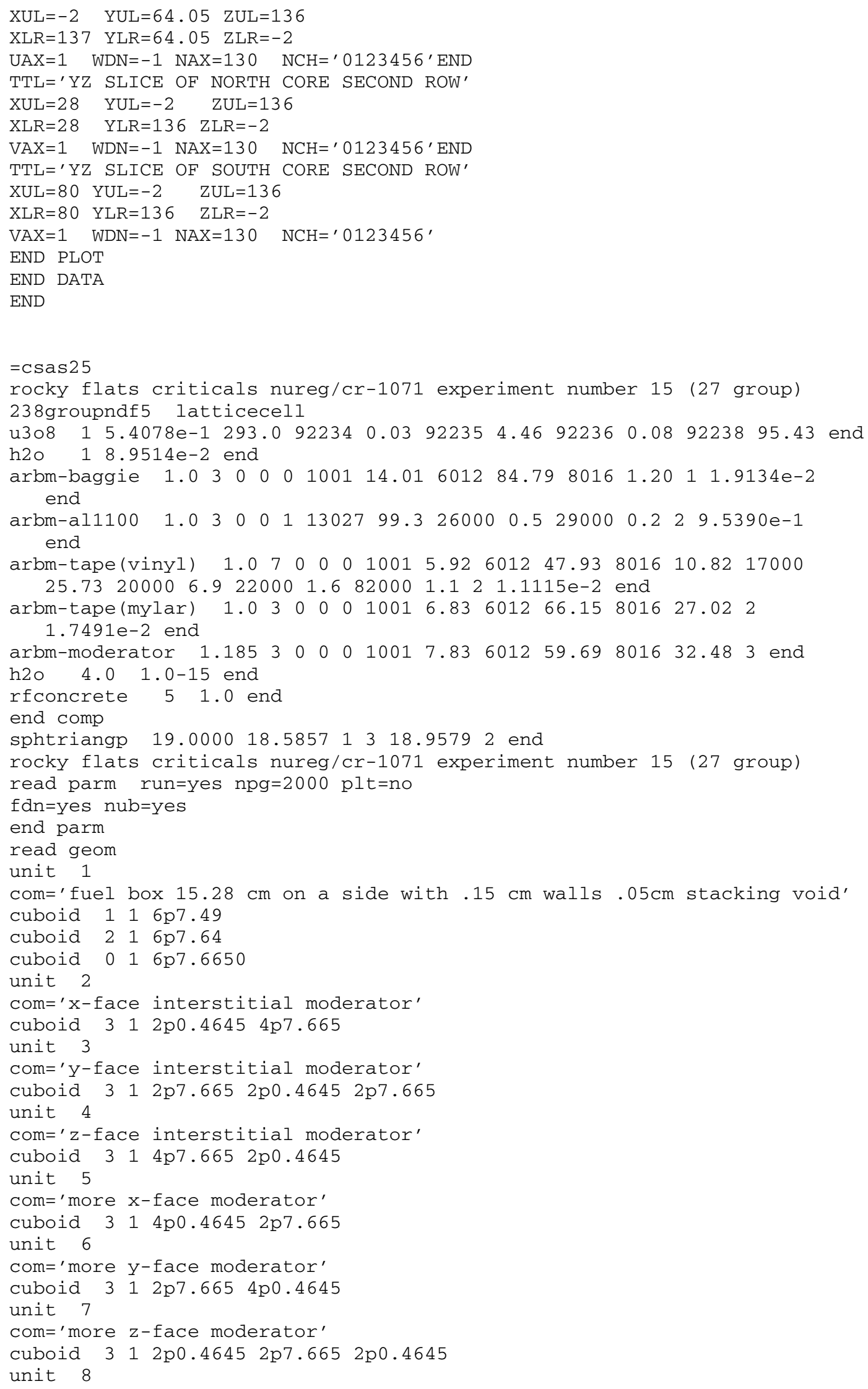




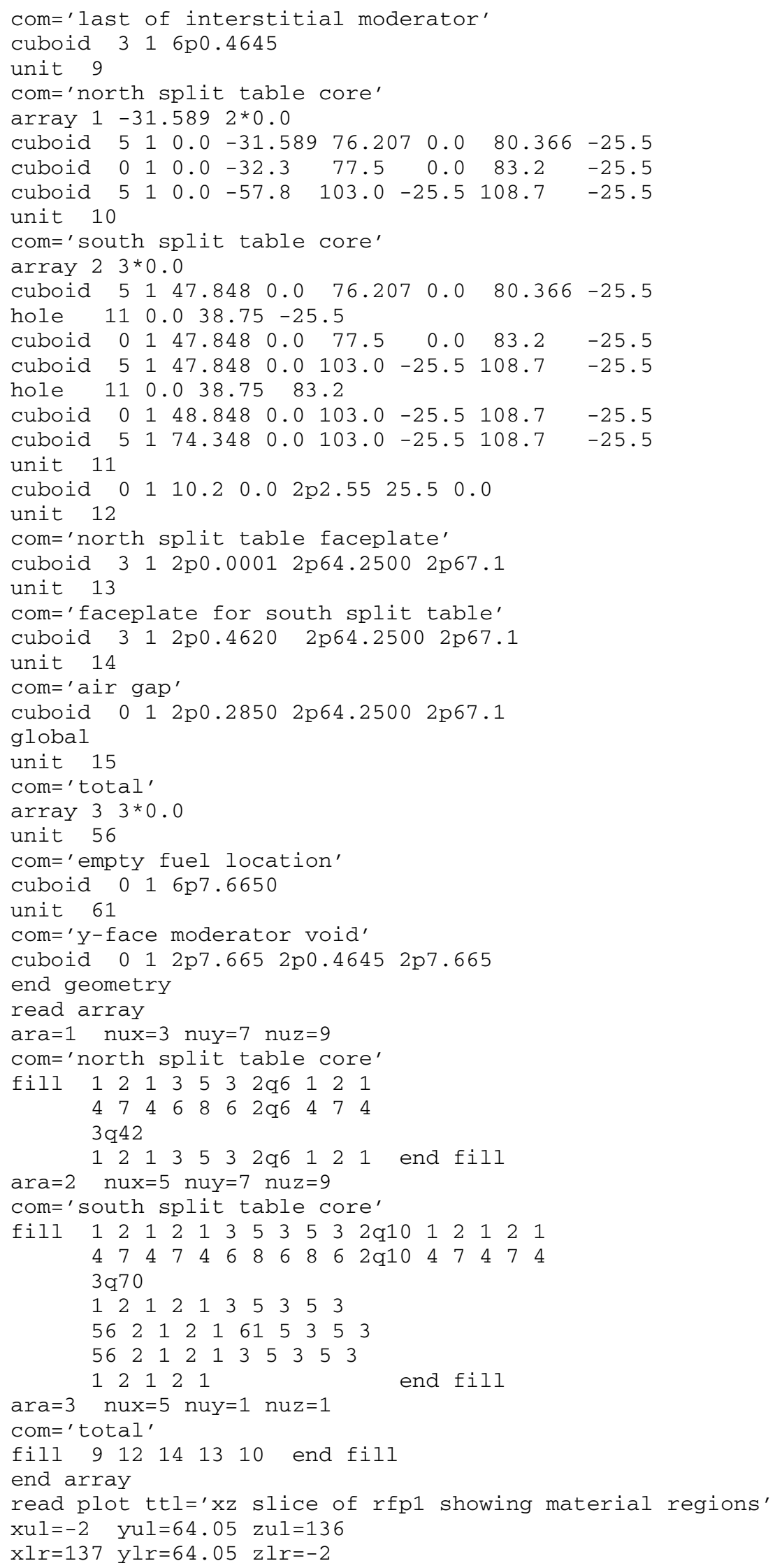




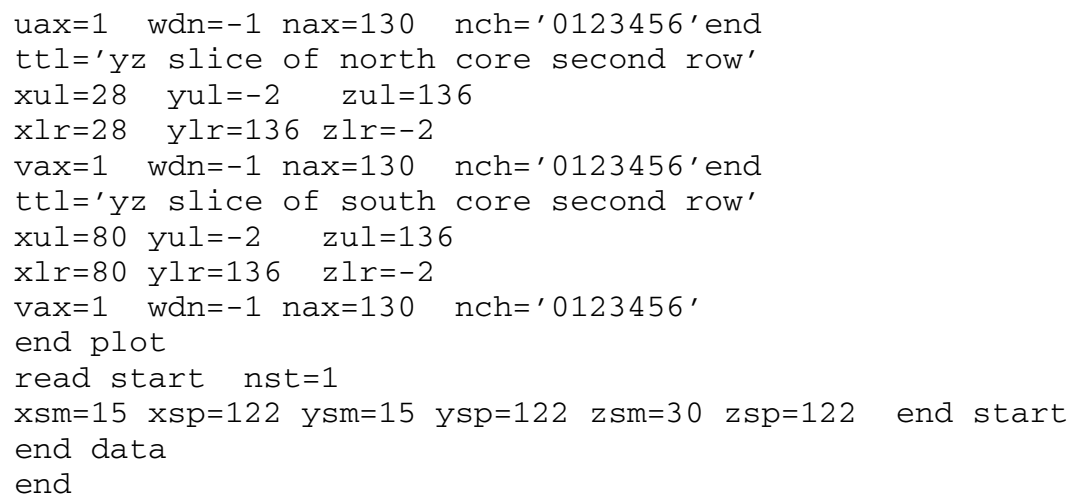




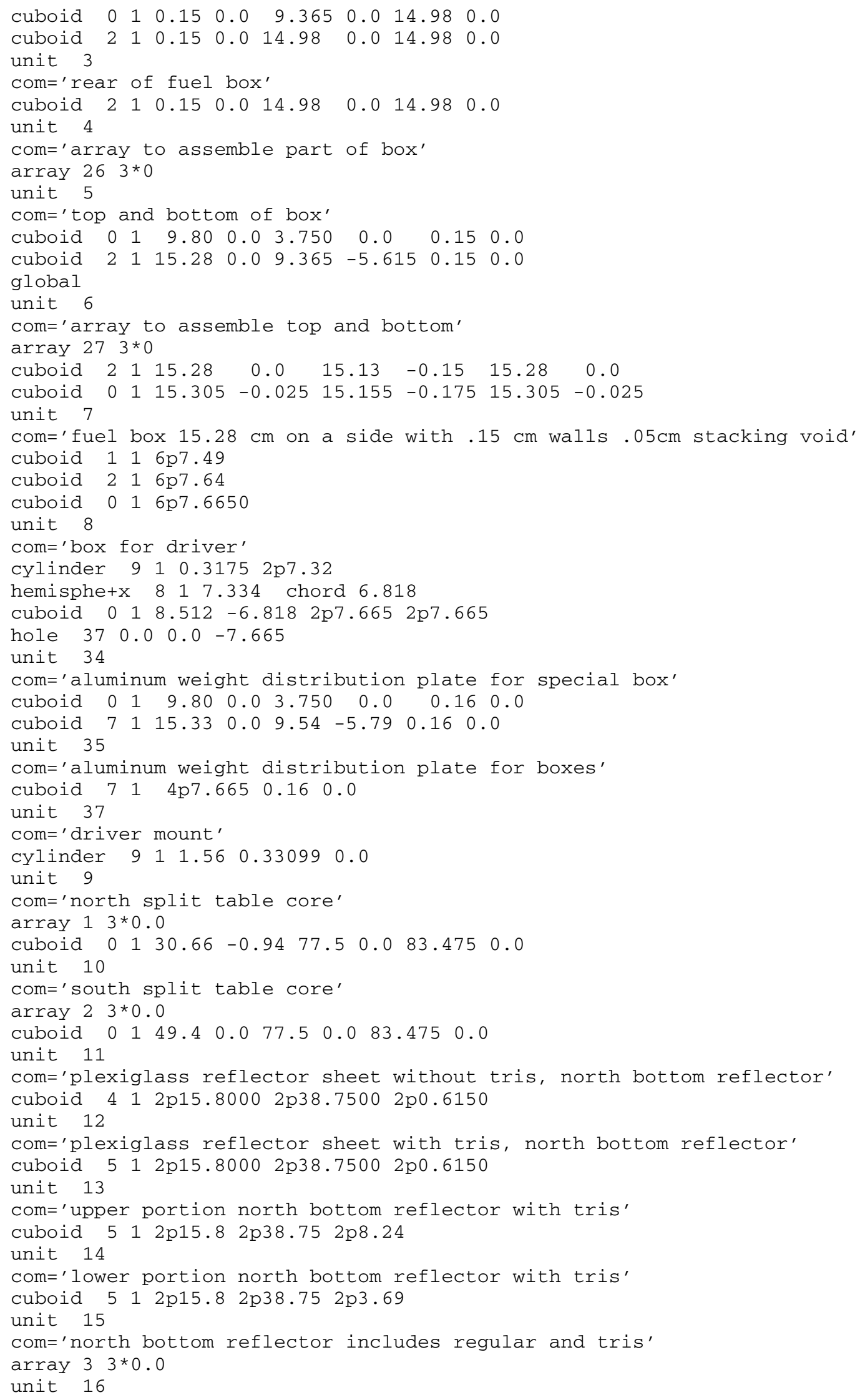


com='plexiglas sheet bottom south reflector without tris' cuboid 0 1 2p5.1 2p2.5 2p0.6150

cuboid $4 \quad 1 \quad 44.3 \quad-5.1 \quad 2 p 38.75 \quad 2 p 0.615$

unit 17

com='plexiglas sheet bottom south reflector with tris'

cuboid 0 1 $2 \mathrm{p} 5.12 \mathrm{p} 2.5 \quad 2 \mathrm{p} 0.6150$

cuboid $5144.3 \quad-5.12 \mathrm{p} 38.75 \quad 2 \mathrm{p} 0.6150$

unit 18

com='lower portion south bottom reflector with tris'

cuboid $0 \quad 1 \quad 2 \mathrm{p} 5.1 \quad 2 \mathrm{p} 2.5 \quad 2 \mathrm{p} 8.855$

cuboid $5144.3 \quad-5.1 \quad 2 p 38.75 \quad 2 p 8.855$

unit 19

com='south bottom reflector with regular and tris'

array $4 \quad 3 * 0.0$

unit 20

com='east and west reflectors for north reflector with tris'

cuboid $5 \quad 12 \mathrm{p} 15.8 \quad 2 \mathrm{p} 12.65 \quad 2 \mathrm{p} 54.2825$

unit 21

com='array for east and west reflectors for north reflector'

array $5 \quad 3 * 0.0$

unit 22

com='east and west reflectors for south reflector with tris'

cuboid $512 \mathrm{p} 24.700 \quad 2 \mathrm{p} 12.65 \quad 2 \mathrm{p} 54.2825$

unit 23

com='array for east and west reflectors for south reflector'

array $6 \quad 3 * 0.0$

unit 24

com='north top reflector with tris'

cuboid $512 \mathrm{p} 28.352 \mathrm{p} 64.052 \mathrm{p} 12.15$

unit 25

com='array for north top reflector'

array $7 \quad 3 * 0.0$

unit 26

com='south top reflector with tris'

cuboid $0 \quad 1 \quad 2 \mathrm{p} 5.1 \quad 2 \mathrm{p} 2.5 \quad 2 \mathrm{p} 12.15$

cuboid $5 \quad 1 \quad 69.4 \quad-5.1 \quad 2 p 64.05 \quad 2 p 12.15$

unit 27

com='array for south top reflector'

array $83 * 0.0$

unit 28

com='north end reflector $9.8 \mathrm{~cm}$ portion without tris'

cuboid $412 \mathrm{p} 4.90002 \mathrm{p} 64.05002 \mathrm{p} 54.2825$

unit 29

com='north end relfector $5.2 \mathrm{~cm}$ portion with tris'

cuboid $512 \mathrm{p} 2.62 \mathrm{p} 64.0500$ 2p54.2825

unit 30

com='north end relfector $10.1 \mathrm{~cm}$ portion without tris'

cuboid 412 p5.05 2p64.05 2p54.2825

unit 31

com='array for north end reflector'

array $93 * 0.0$

unit 32

com='south end reflector'

cuboid $512 \mathrm{p} 12.55$ 2p64.05 2p54.2825

unit 33

com='array for south end reflector'

array $103 * 0.0$

unit 38

com='north core with bottom reflector'

array $133 * 0.0$

unit 39

com='north core with east and west reflector'

array $143 * 0.0$ 


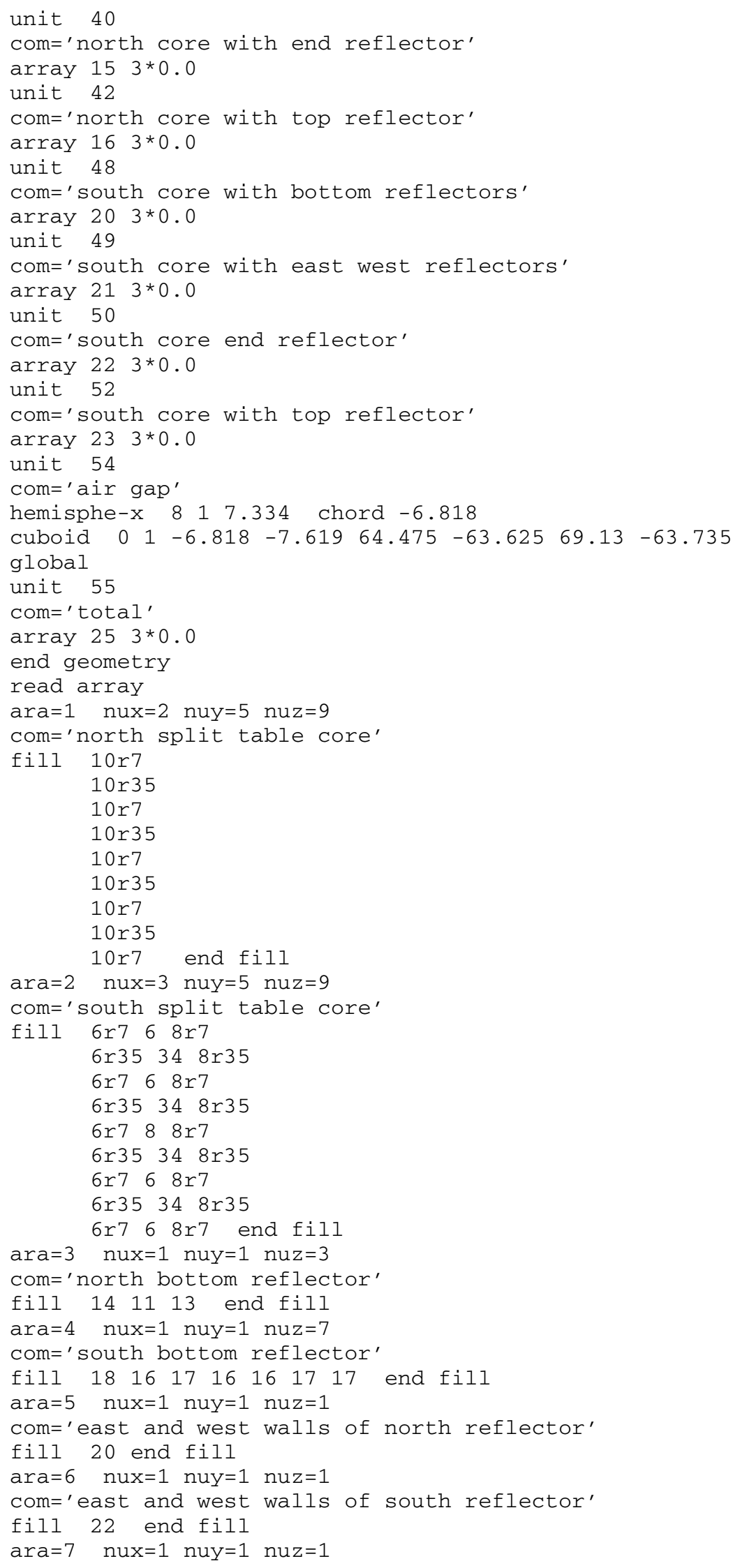




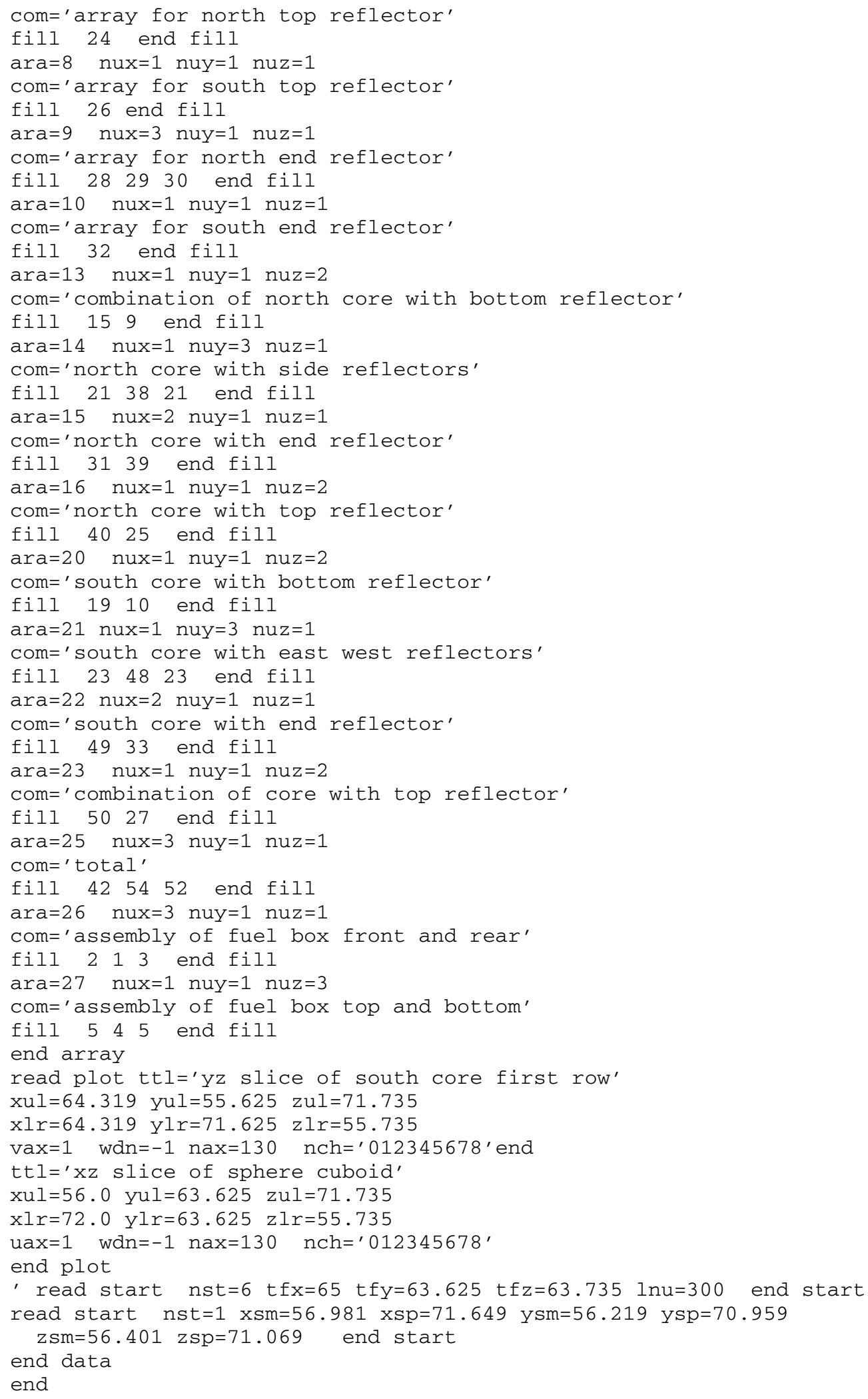




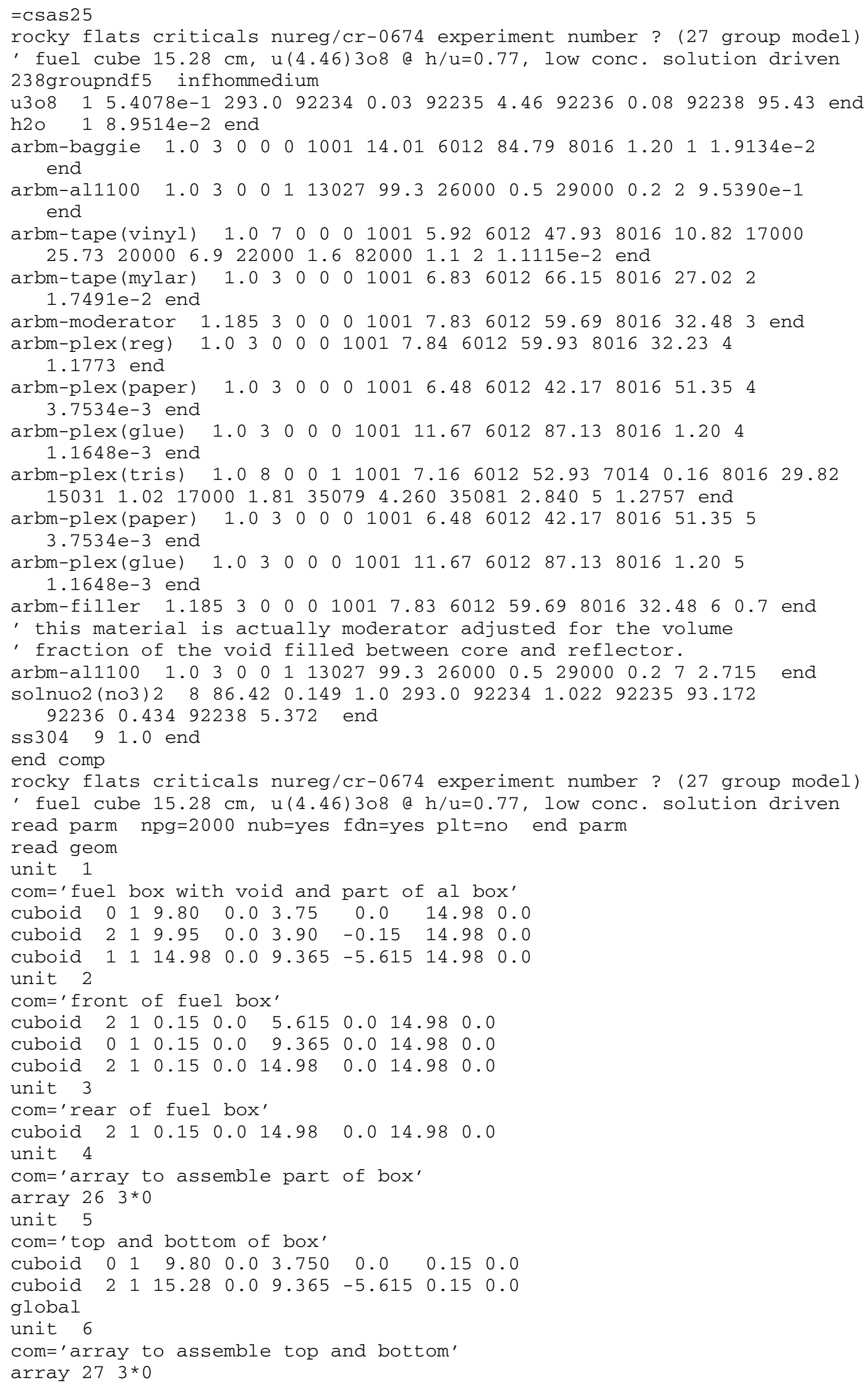


$\begin{array}{lllllllll}\text { cuboid } 2 & 1 & 15.28 & 0.0 & 15.13 & -0.15 & 15.28 & 0.0\end{array}$

cuboid $0115.305-0.02515 .155-0.175 \quad 15.305-0.025$

unit 7

com='fuel box $15.28 \mathrm{~cm}$ on a side with $.15 \mathrm{~cm}$ walls $.05 \mathrm{~cm}$ stacking void'

cuboid $116 \mathrm{p} 7.49$

cuboid $216 \mathrm{p} 7.64$

cuboid $0 \quad 16 \mathrm{p} 7.6650$

unit 34

com='aluminum weight distribution plate for special box'

cuboid $\begin{array}{llllllll} & 1 & 9.80 & 0.0 & 3.750 & 0.0 & 0.16 & 0.0\end{array}$

$\begin{array}{llllllllll}\text { cuboid } & 7 & 1 & 15.33 & 0.0 & 9.54 & -5.79 & 0.16 & 0.0\end{array}$

unit 35

com='aluminum weight distribution plate for boxes'

cuboid 714 p $7.6650 .16 \quad 0.0$

unit 9

com='north split table core'

array $13 * 0.0$

cuboid $0130.66-0.94 \quad 77.5 \quad 0.0 \quad 83.475 \quad 0.0$

unit 10

com='south split table core'

array $23 * 0.0$

cuboid $0 \begin{array}{llllllll} & 49.4 & 0.0 & 77.5 & 0.0 & 83.475 & 0.0\end{array}$

unit 11

com='plexiglass reflector sheet without tris, north bottom reflector'

cuboid $412 \mathrm{p} 15.80002 \mathrm{p} 38.7500$ 2p0.6150

unit 12

com='plexiglass reflector sheet with tris, north bottom reflector'

cuboid $512 \mathrm{p} 15.80002 \mathrm{p} 38.7500 \quad 2 \mathrm{p} 0.6150$

unit 13

com='upper portion north bottom reflector with tris'

cuboid 5 1 2 p15.8 2 p38.75 2 p8.24

unit 14

com='lower portion north bottom reflector with tris'

cuboid $512 \mathrm{p} 15.8 \quad 2 \mathrm{p} 38.75 \quad 2 \mathrm{p} 3.69$

unit 15

com='north bottom reflector includes regular and tris'

array $3 \quad 3 * 0.0$

unit 16

com='plexiglas sheet bottom south reflector without tris'

cuboid $0 \quad 1 \quad 2 \mathrm{p} 5.1 \quad 2 \mathrm{p} 2.5 \quad 2 \mathrm{p} 0.6150$

cuboid $4 \quad 1 \quad 44.3 \quad-5.1 \quad 2 p 38.75 \quad 2 p 0.615$

unit 17

com='plexiglas sheet bottom south reflector with tris'

cuboid $0 \quad 12 \mathrm{p} 5.12 \mathrm{p} 2.5 \quad 2 \mathrm{p} 0.6150$

cuboid 5 1 $44.3 \quad-5.12 \mathrm{p} 38.75 \quad 2 \mathrm{p} 0.6150$

unit 18

com='lower portion south bottom reflector with tris'

cuboid $0 \quad 12 \mathrm{p} 5.12 \mathrm{p} 2.5 \quad 2 \mathrm{p} 8.855$

cuboid 5 1 $44.3 \quad-5.1 \quad 2 \mathrm{p} 38.75 \quad 2 \mathrm{p} 8.855$

unit 19

com='south bottom reflector with regular and tris'

array $43 * 0.0$

unit 20

com='east and west reflectors for north reflector with tris'

cuboid 5 1 2p15.8 2p12.65 2p54.2825

unit 21

com='array for east and west reflectors for north reflector'

array $5 \quad 3 * 0.0$

unit 22

com='east and west reflectors for south reflector with tris'

cuboid $512 \mathrm{p} 24.700 \quad 2 \mathrm{p} 12.65 \quad 2 \mathrm{p} 54.2825$

unit 23

com='array for east and west reflectors for south reflector' 


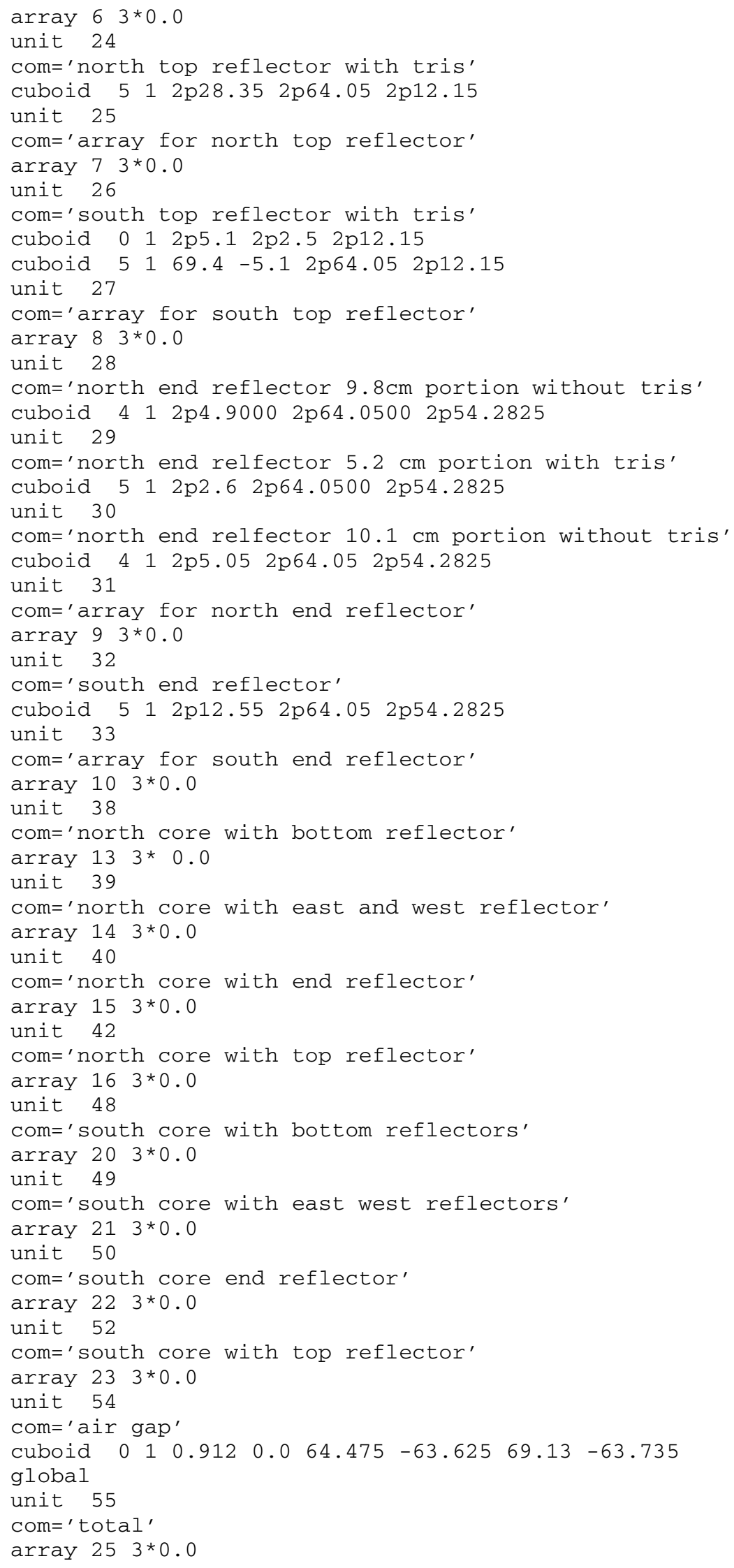




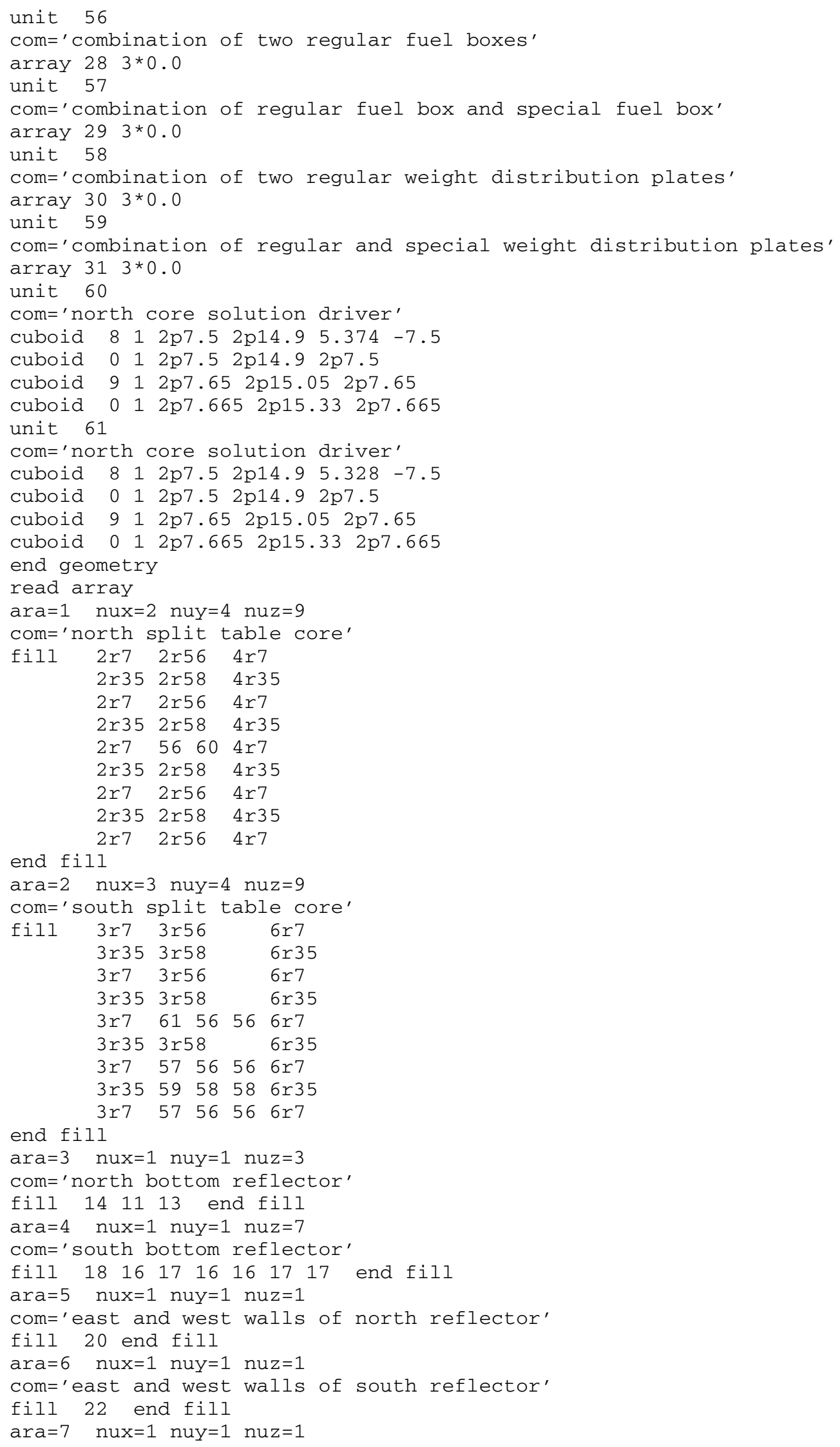




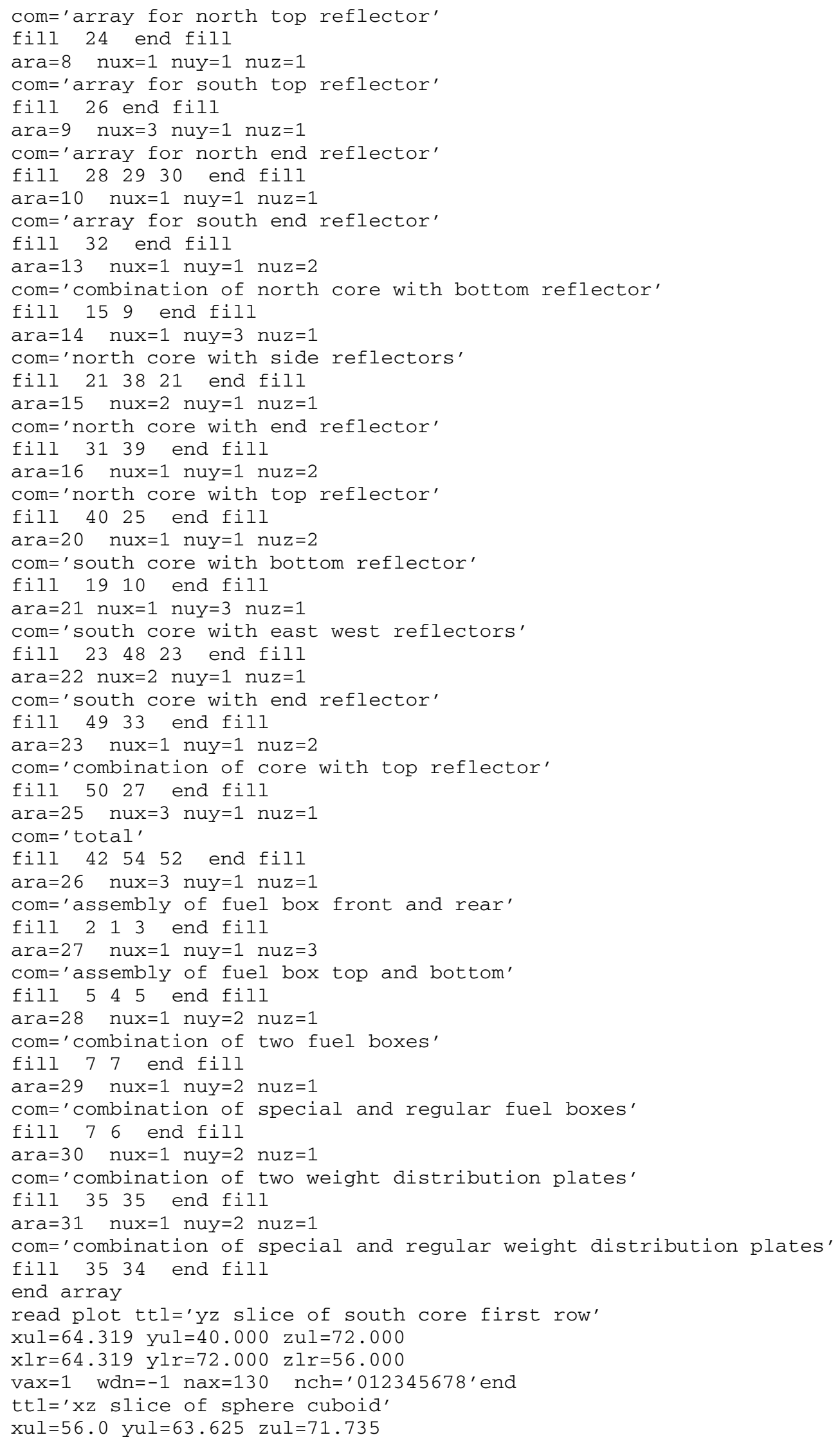




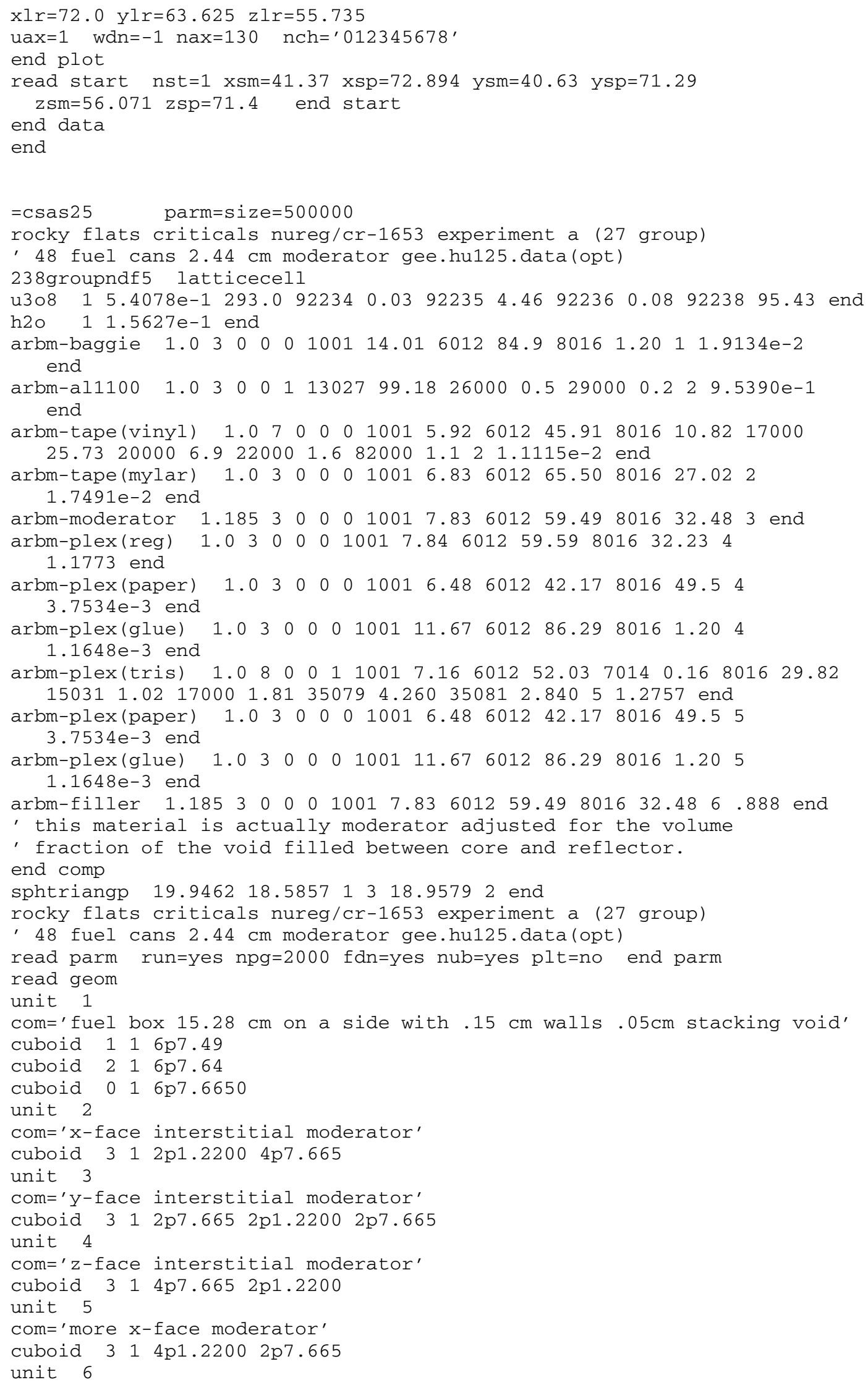




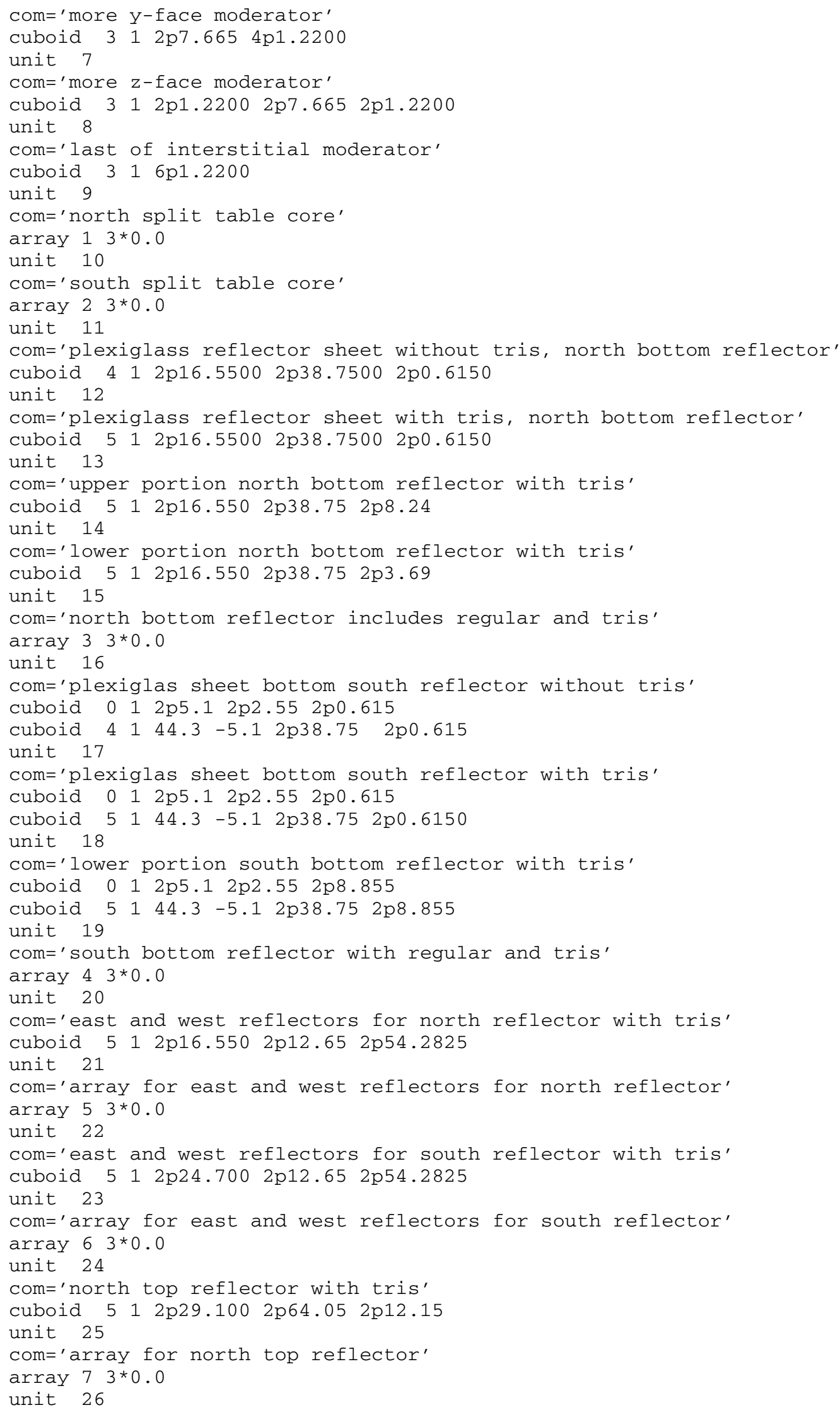




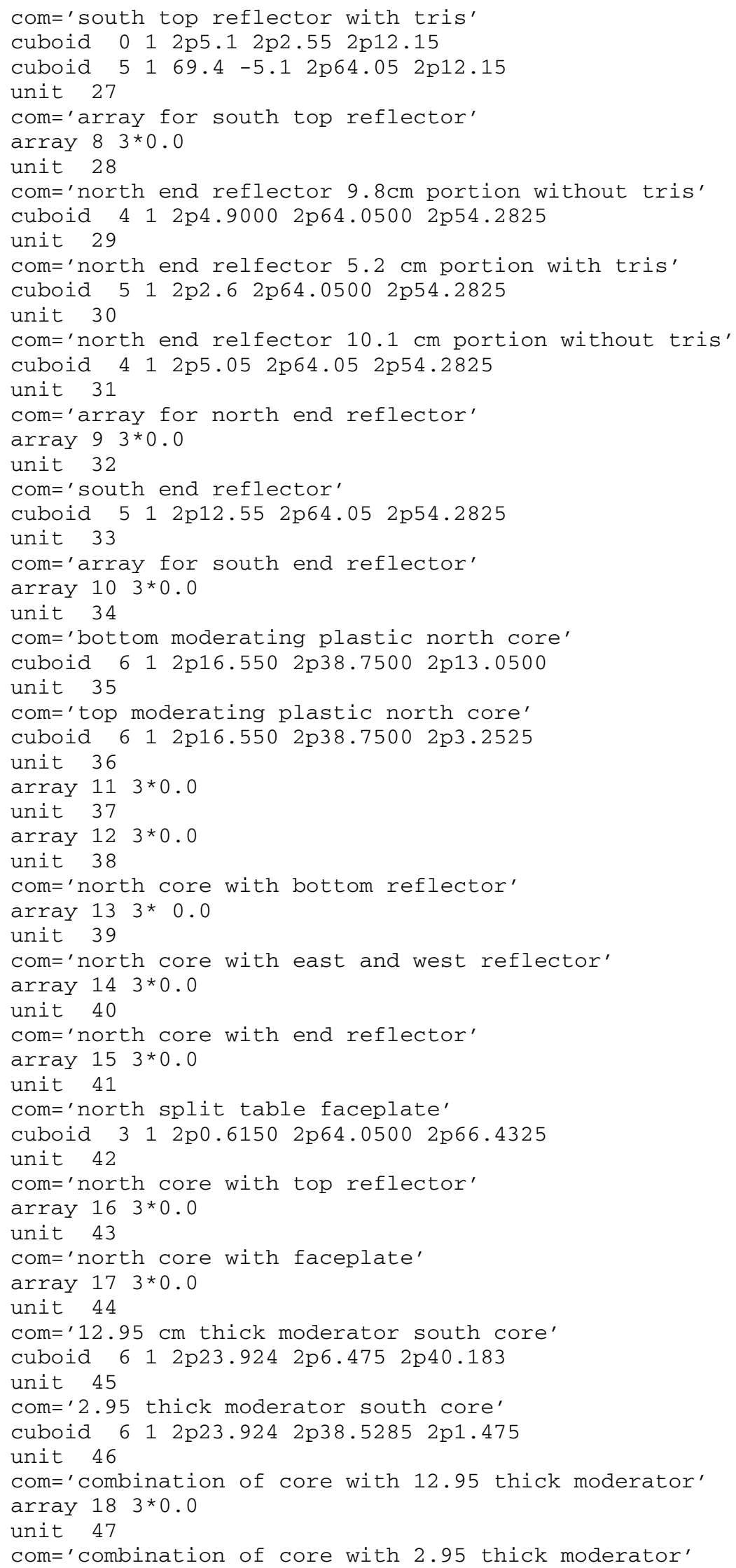




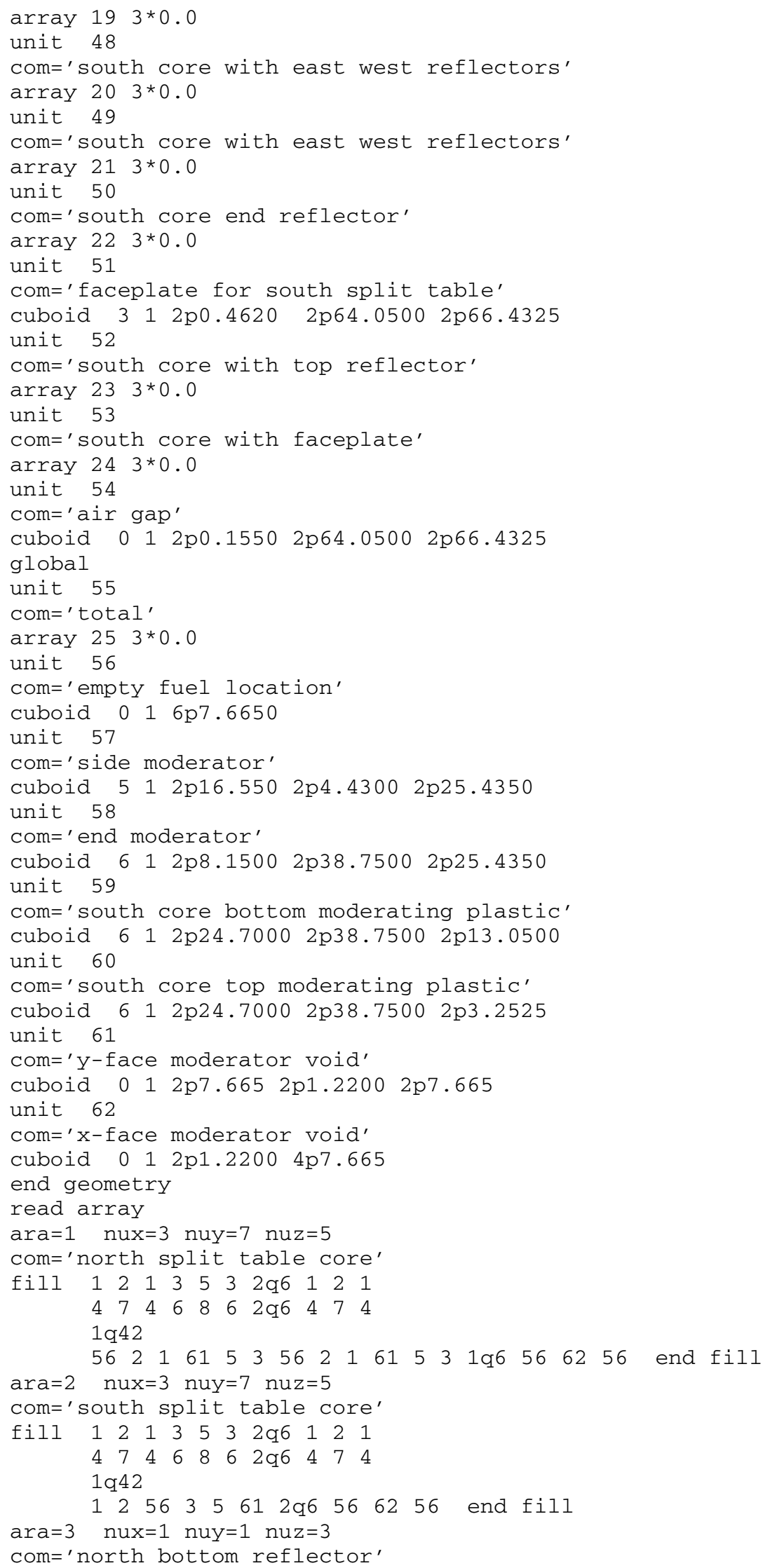


fill $14 \quad 11 \quad 13$ end fill

ara $=4$ nux=1 nuy=1 nuz=7

com='south bottom reflector'

fill $18 \quad 16 \quad 17 \quad 16 \quad 16 \quad 17 \quad 17$ end fill

ara $=5$ nux=1 nuy=1 nuz=1

com='east and west walls of north reflector'

fill 20 end fill

ara $=6$ nux=1 nuy=1 nuz=1

$\mathrm{com}=$ 'east and west walls of south reflector'

fill 22 end fill

ara=7 nux=1 nuy=1 nuz=1

com='array for north top reflector'

fill 24 end fill

ara $=8$ nux=1 nuy=1 nuz=1

$\mathrm{com}=$ 'array for south top reflector'

fill 26 end fill

ara $=9$ nux=3 nuy=1 nuz=1

com='array for north end reflector'

fill 282930 end fill

ara $=10$ nux=1 nuy=1 nuz=1

com='array for south end reflector'

fill 32 end fill

ara=11 nux=1 nuy=2 nuz=1

com='combinarion of core with side moderator'

fill 957 end fill

ara=12 nux=1 nuy=1 nuz=3

com='combination of previous array with top and bottom moderator'

fill 343635 end fill

ara=13 nux=1 nuy=1 nuz=2

com='combination of north core with bottom reflector'

fill 1537 end fill

ara $=14$ nux=1 nuy=3 nuz=1

com='north core with side reflectors'

fill 213821 end fill

ara=15 nux=2 nuy=1 nuz=1

com='north core with end reflector'

fill 3139 end fill

ara=16 nux=1 nuy=1 nuz=2

com='north core with top reflector'

fill 4025 end fill

ara=17 nux=2 nuy=1 nuz=1

com='north core with faceplate'

fill 4241 end fill

ara=18 nux=1 nuy=2 nuz=1

$\mathrm{com}=$ 'combination of $\mathrm{s}$. core with side moderator'

fill 1057 end fill

ara=19 nux=2 nuy=1 nuz=1

com $=$ 'combination of core with end moderator'

fill 4658 end fill

ara $=20$ nux=1 nuy=1 nuz=4

com='south core with bottom moderator and reflector'

fill $1959 \quad 47 \quad 60$ end fill

ara $=21$ nux=1 nuy=3 nuz=1

com='south core with east west reflectors'

fill $23 \quad 4823$ end fill

ara $=22$ nux=2 nuy=1 nuz=1

com='south core with end reflector'

fill 4933 end fill

ara=23 nux=1 nuy=1 nuz=2

com='combination of core with top reflector'

fill 5027 end fill

ara=24 nux=2 nuy=1 nuz=1

com='south core with faceplate' 
fill 5152 end fill

ara $=25$ nux=3 nuy=1 nuz=1

$\mathrm{com}=$ 'total'

fill 435453 end fill

end array

read plot ttl='xz slice of rfpl showing material regions'

$\mathrm{xul}=-2 \quad \mathrm{yul}=64.05 \mathrm{zul}=136$

$\mathrm{xlr}=137$ ylr=64.05 zlr $=-2$

uax=1 wdn=-1 nax=130 nch='0123456' end

ttl='yz slice of north core first row'

$\mathrm{xul}=48 \quad \mathrm{yul}=-2 \quad \mathrm{zul}=136$

$\mathrm{xlr}=48$ ylr=136 $\mathrm{zlr}=-2$

vax=1 wdn=-1 nax=130 nch='0123456'end

ttl $=$ 'yz slice of north core second row'

$\mathrm{xul}=28 \quad \mathrm{yul}=-2 \quad \mathrm{zul}=136$

$\mathrm{xlr}=28 \quad \mathrm{ylr}=136 \quad \mathrm{zlr}=-2$

$\operatorname{vax}=1$ wdn=-1 nax=130 nch='0123456' end

ttl $=$ 'yz slice of south core first row'

$\mathrm{xul}=62$ yul=-2 $\mathrm{zul}=136$

$\mathrm{xlr}=62$ ylr $=136 \quad \mathrm{zl} r=-2$

$\operatorname{vax}=1$ wdn=-1 nax=130 nch='0123456' end

ttl='yz slice of south core second row'

$\mathrm{xul}=80$ yul $=-2 \quad \mathrm{zul}=136$

$\mathrm{xlr}=80$ ylr $=136 \quad \mathrm{zl} r=-2$

$\operatorname{vax}=1$ wdn=-1 nax=130 nch='0123456' end

end plot

end data

end

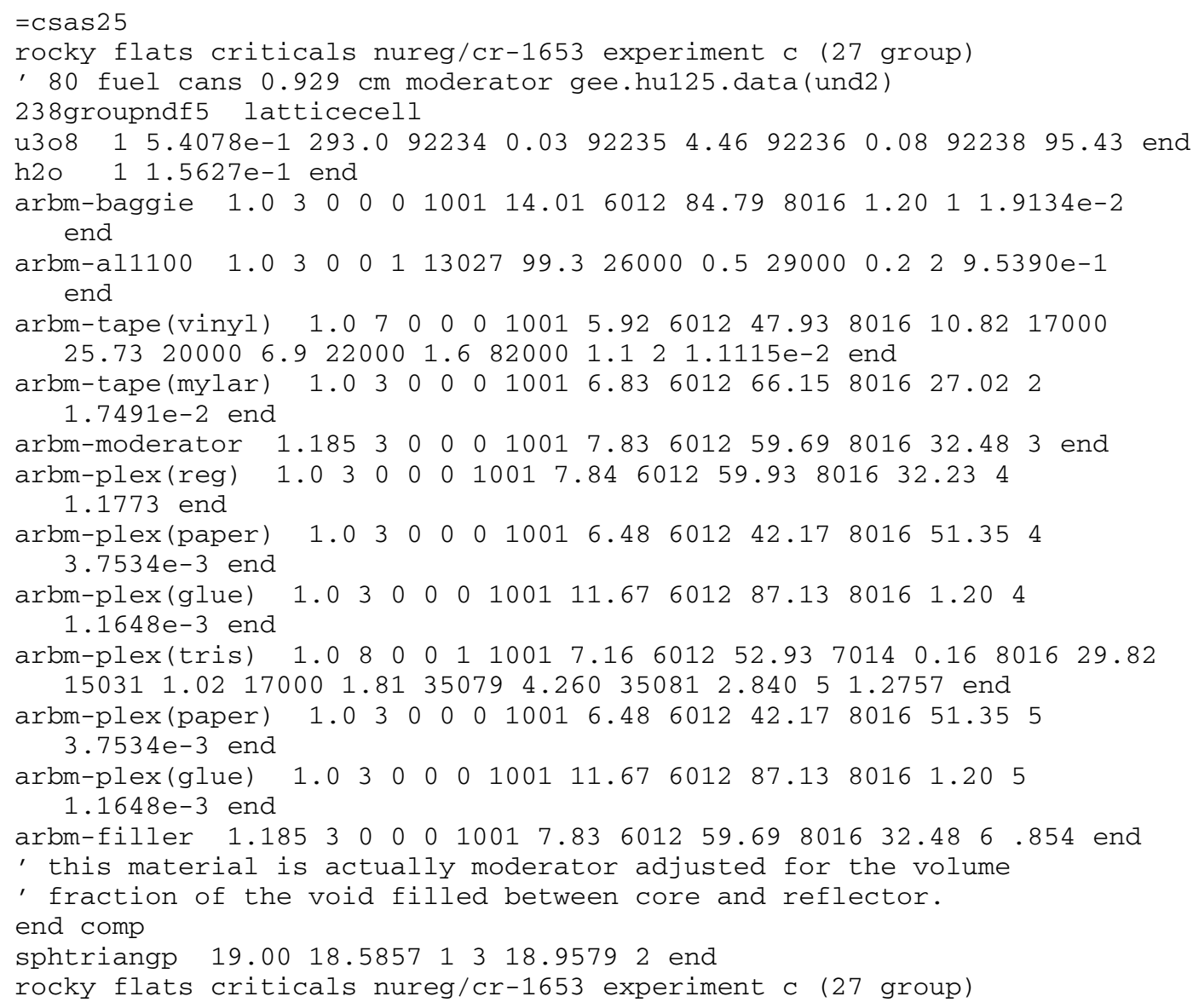




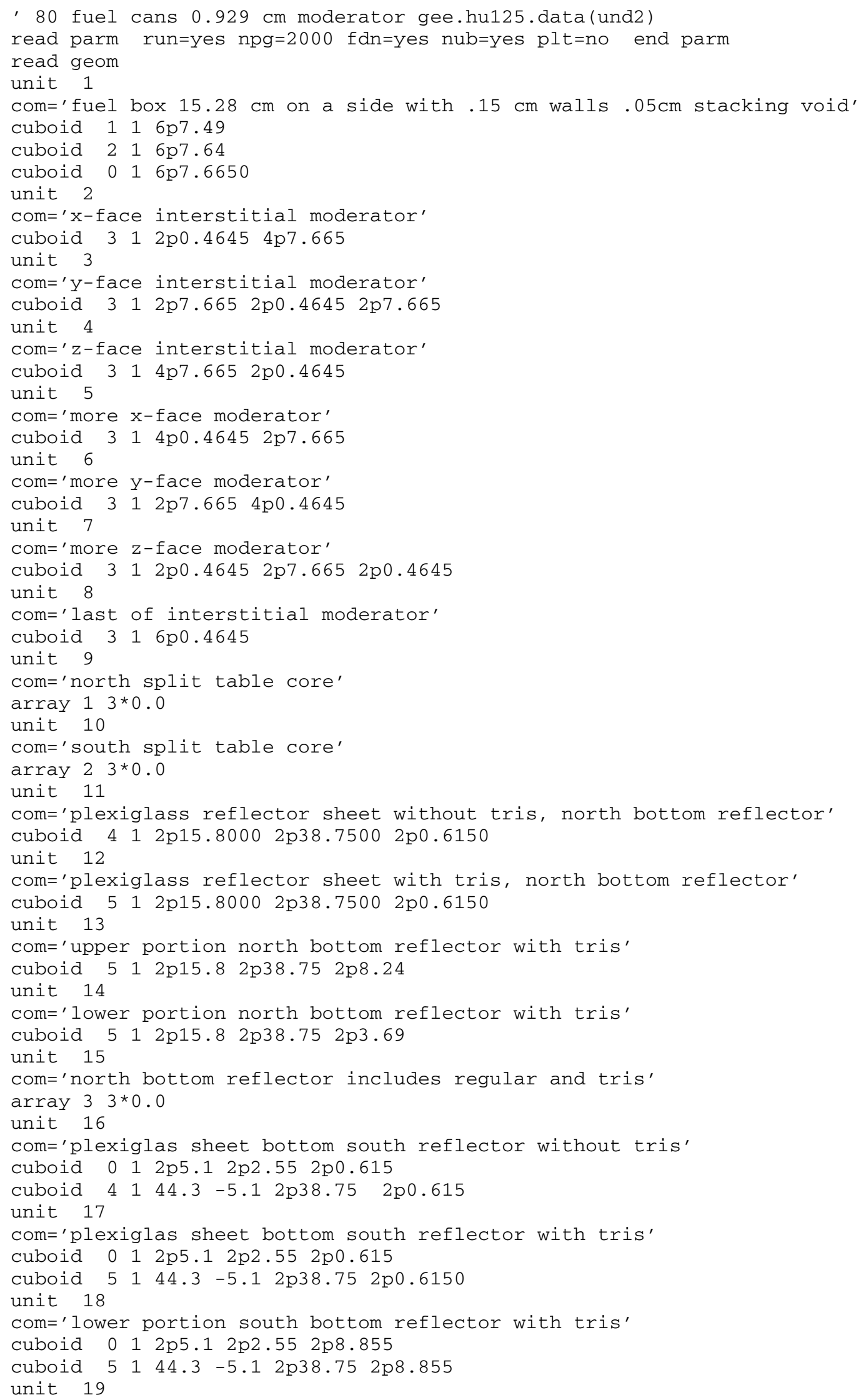


com='south bottom reflector with regular and tris'

array $43 * 0.0$

unit 20

com='east and west reflectors for north reflector with tris' cuboid $5 \quad 12 \mathrm{p} 15.8$ 2p12.65 2p54.2825

unit 21

com='array for east and west reflectors for north reflector' array $53 * 0.0$

unit 22

com='east and west reflectors for south reflector with tris' cuboid $512 \mathrm{p} 24.7002 \mathrm{p} 12.65 \quad 2 \mathrm{p} 54.2825$

unit 23

com='array for east and west reflectors for south reflector' array $63 * 0.0$

unit 24

com='north top reflector with tris'

cuboid $512 \mathrm{p} 28.352 \mathrm{p} 64.05 \quad 2 \mathrm{p} 12.15$

unit 25

com='array for north top reflector'

array $7 \quad 3 * 0.0$

unit 26

com='south top reflector with tris'

cuboid $0 \begin{array}{lllll} & 1 & 2 \mathrm{p} 5.1 & 2 \mathrm{p} 2.55 & 2 \mathrm{p} 12.15\end{array}$

cuboid $5 \quad 1 \quad 69.4 \quad-5.1 \quad 2 p 64.05 \quad 2 p 12.15$

unit 27

com='array for south top reflector'

array $8 \quad 3 * 0.0$

unit 28

com='north end reflector $9.8 \mathrm{~cm}$ portion without tris'

cuboid $412 \mathrm{p} 4.9000$ 2p64.0500 2p54.2825

unit 29

com='north end relfector $5.2 \mathrm{~cm}$ portion with tris'

cuboid $512 \mathrm{p} 2.6 \quad 2 \mathrm{p} 64.0500 \quad 2 \mathrm{p} 54.2825$

unit 30

com='north end relfector $10.1 \mathrm{~cm}$ portion without tris'

cuboid $412 \mathrm{p} 5.052 \mathrm{p} 64.052 \mathrm{p} 54.2825$

unit 31

com='array for north end reflector'

array $93 * 0.0$

unit 32

com='south end reflector'

cuboid $512 \mathrm{p} 12.552 \mathrm{p} 64.052 \mathrm{p} 54.2825$

unit 33

com='array for south end reflector'

array $103 * 0.0$

unit 34

com='12.95 thick moderating plastic north core'

cuboid $612 \mathrm{p} 15.7945 \quad 2 \mathrm{p} 6.4750 \quad 2 \mathrm{p} 32.0535$

unit 35

com='2.95 thick moderating plastic north core'

cuboid $612 \mathrm{p} 15.79452 \mathrm{p} 38.52852 \mathrm{p} 1.475$

unit 36

array $113 * 0.0$

unit 37

array $123 * 0.0$

replicate $\begin{array}{lllllllllll}0 & 1 & 0.0 & 0.011 & 0.443 & 0.0 & 0.1590 & 0.0 & 1\end{array}$

unit 38

com='north core with bottom reflector'

array $133 * 0.0$

unit 39

com='north core with east and west reflector'

array $143 * 0.0$

unit 40 


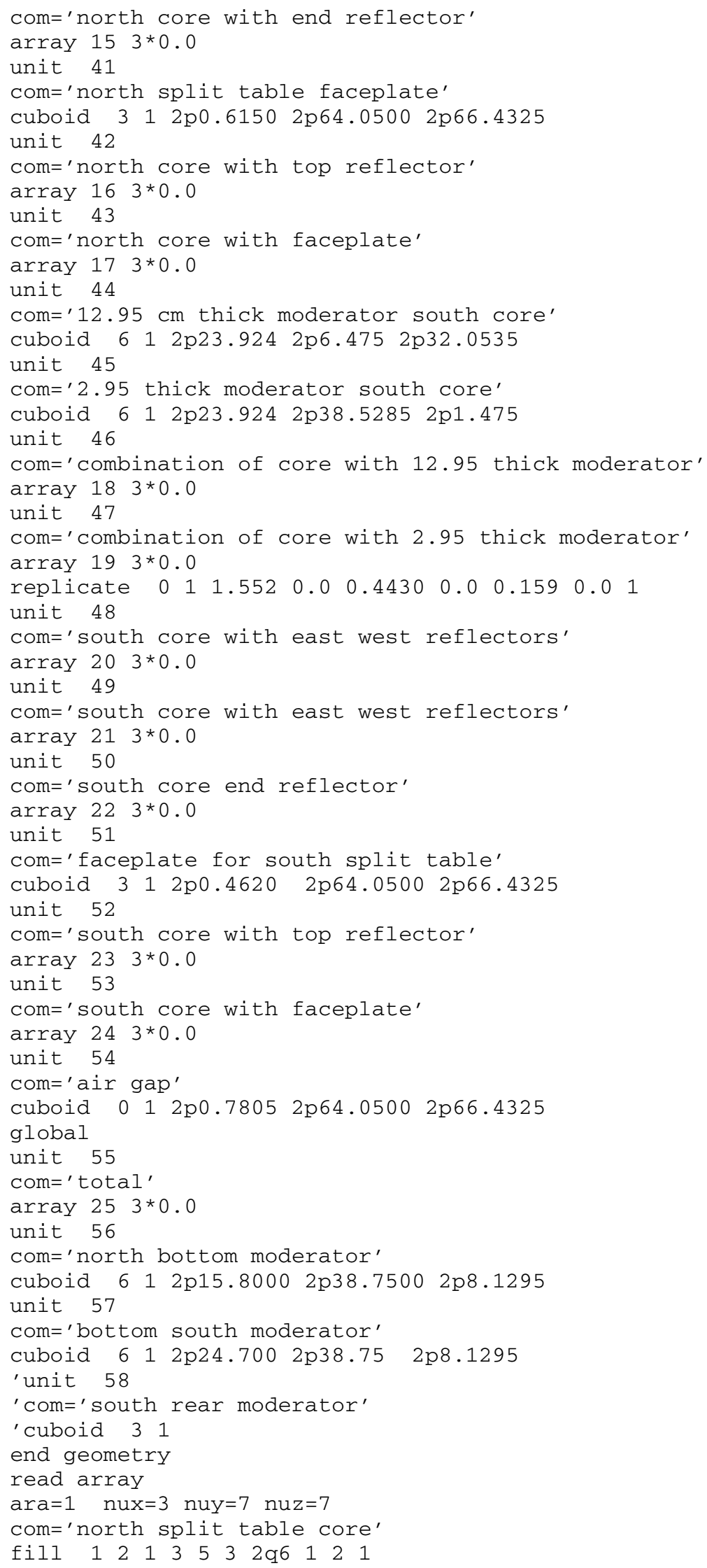




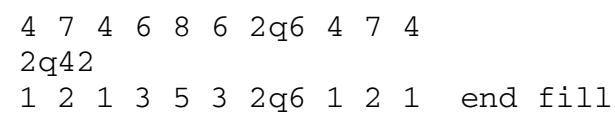


ara=21 nux=1 nuy=3 nuz=1

com='south core with east west reflectors'

fill $23 \quad 4823$ end fill

ara=22 nux=2 nuy=1 nuz=1

com='south core with end reflector'

fill 4933 end fill

ara $=23$ nux=1 nuy=1 nuz=2

com='combination of core with top reflector'

fill 5027 end fill

ara $=24$ nux=2 nuy=1 nuz=1

com='south core with faceplate'

fill 5152 end fill

$\operatorname{ara}=25$ nux=3 nuy=1 nuz=1

$\mathrm{com}={ }^{\prime}$ total'

fill 435453 end fill

end array

read plot ttl='xz slice of rfp3 showing material regions'

$\mathrm{xul}=-1 \quad \mathrm{yul}=64.05 \mathrm{zul}=135$

$\mathrm{xlr}=136 \mathrm{ylr}=64.05 \mathrm{zlr}=-2$

uax=1 wdn=-1 nax=130 nch='0123456' end

ttl $=$ 'yz slice of north core second row'

$\mathrm{xul}=28 \quad \mathrm{yul}=-2 \quad \mathrm{zul}=136$

$\mathrm{xlr}=28$ ylr=136 $\mathrm{zlr}=-2$

$\operatorname{vax}=1$ wdn=-1 nax=130 nch='0123456' end

ttl $=$ 'yz slice of south core second row'

$\mathrm{xul}=80$ yul=-2 $\mathrm{zul}=136$

$\mathrm{xlr}=80$ ylr=136 $\mathrm{zlr}=-2$

$\operatorname{vax}=1$ wan $=-1$ nax $=130$ nch='0123456'

end plot

end data

end

$=\operatorname{csas} 25$

rocky flats criticals nureg/cr-1653 experiment number ? (27 group model)

' fuel cube $15.28 \mathrm{~cm}, \mathrm{u}(4.46) 308$ @ h/u=1.25, hi conc. solution driven

238 groupndf5 infhommedium

u3o8 $15.4078 e-1 \quad 293.0 \quad 92234 \quad 0.03 \quad 92235 \quad 4.4692236 \quad 0.089223895 .43$ end

h2o $11.5627 \mathrm{e}-1$ end

arbm-baggie $1.0 \quad 300001001 \quad 14.01601284 .798016 \quad 1.2011 .9134 e-2$ end

arbm-al1100 $1.0 \quad 3 \quad 0 \quad 0 \quad 1 \quad 13027 \quad 99.326000 \quad 0.5 \quad 29000 \quad 0.2 \quad 2 \quad 9.5390 e-120$ end

arbm-tape (vinyl) $\quad 1.0 \quad 7 \quad 0 \quad 0 \quad 0 \quad 1001 \quad 5.92 \quad 6012 \quad 47.93 \quad 8016 \quad 10.82 \quad 17000$

$25.73200006 .9220001 .6820001 .121 .1115 e-2$ end

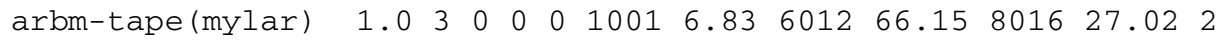

$1.7491 \mathrm{e}-2$ end

arbm-moderator $\quad 1.185 \quad 3 \quad 0 \quad 0 \quad 0 \quad 1001 \quad 7.83 \quad 6012 \quad 59.69 \quad 8016 \quad 32.48 \quad 3$ end

arbm-plex(reg) $\quad 1.0 \quad 3 \quad 0 \quad 0 \quad 0 \quad 1001 \quad 7.84 \quad 6012 \quad 59.93 \quad 8016 \quad 32.23 \quad 4$

1.1773 end

arbm-plex(paper) $\quad 1.0 \quad 3 \quad 0 \quad 0 \quad 0 \quad 1001 \quad 6.48 \quad 6012 \quad 42.17 \quad 8016 \quad 51.35 \quad 4$

$3.7534 \mathrm{e}-3$ end

$\begin{array}{llllllllllllll}\text { arbm-plex(glue) } & 1.0 & 3 & 0 & 0 & 0 & 1001 & 11.67 & 6012 & 87.13 & 8016 & 1.20 & 4\end{array}$

$1.1648 e-3$ end

arbm-plex(tris) $\quad 1.0 \quad 8 \quad 0 \quad 0 \quad 1 \quad 1001 \quad 7.16 \quad 6012 \quad 52.93 \quad 7014 \quad 0.16 \quad 8016 \quad 29.82$

$\begin{array}{lllllllllll}15031 & 1.02 & 17000 & 1.81 & 35079 & 4.260 & 35081 & 2.840 & 5 & 1.2757 & \text { end }\end{array}$

arbm-plex(paper) $\quad 1.0 \quad 3 \quad 0 \quad 0 \quad 0 \quad 1001 \quad 6.48 \quad 6012 \quad 42.17 \quad 8016 \quad 51.35 \quad 5$

$3.7534 \mathrm{e}-3$ end

arbm-plex(glue) $\quad 1.0 \quad 3 \quad 0 \quad 0 \quad 0 \quad 1001 \quad 11.67 \quad 6012 \quad 87.13 \quad 8016 \quad 1.20 \quad 5$

$1.1648 e-3$ end

arbm-filler $1.185300001001 \quad 7.83 \quad 6012 \quad 59.698016 \quad 32.48 \quad 6 \quad 0.7$ end

' this material is actually moderator adjusted for the volume

' fraction of the void filled between core and reflector. 


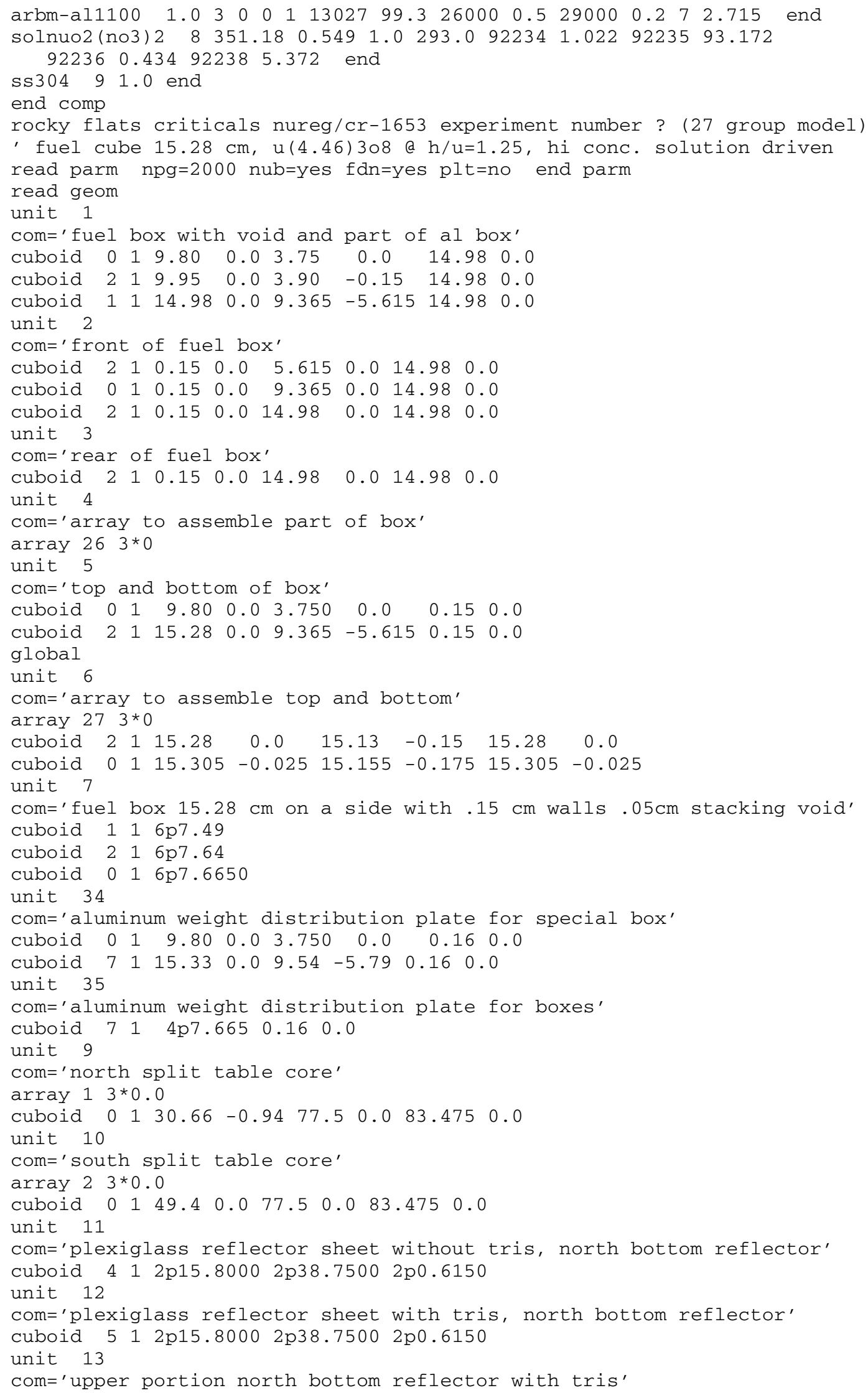


cuboid $5 \quad 1 \quad 2 p 15.8 \quad 2 p 38.75 \quad 2 p 8.24$

unit 14

com='lower portion north bottom reflector with tris'

cuboid 5 1 2 p15.8 $2 \mathrm{p} 38.75 \quad 2 \mathrm{p} 3.69$

unit 15

com='north bottom reflector includes regular and tris'

array $3 \quad 3 * 0.0$

unit 16

com='plexiglas sheet bottom south reflector without tris'

cuboid $0 \quad 12 \mathrm{p} 5.12 \mathrm{p} 2.5 \quad 2 \mathrm{p} 0.6150$

cuboid $4 \quad 1 \quad 44.3 \quad-5.12 \mathrm{p} 38.75 \quad 2 \mathrm{p} 0.615$

unit 17

com='plexiglas sheet bottom south reflector with tris'

cuboid $0 \quad 1 \quad 2 \mathrm{p} 5.12 \mathrm{p} 2.5 \quad 2 \mathrm{p} 0.6150$

cuboid 5 1 44.3 -5.1 2 p38.75 $2 \mathrm{p} 0.6150$

unit 18

com='lower portion south bottom reflector with tris'

cuboid $0 \begin{array}{lllll} & 2 \mathrm{p} 5.1 & 2 \mathrm{p} 2.5 & 2 \mathrm{p} 8.855\end{array}$

cuboid $5 \quad 1 \quad 44.3 \quad-5.1 \quad 2 \mathrm{p} 38.75 \quad 2 \mathrm{p} 8.855$

unit 19

com='south bottom reflector with regular and tris'

array $43 * 0.0$

unit 20

com='east and west reflectors for north reflector with tris'

cuboid $5 \quad 12 \mathrm{p} 15.8 \quad 2 \mathrm{p} 12.65 \quad 2 \mathrm{p} 54.2825$

unit 21

com='array for east and west reflectors for north reflector'

array $5 \quad 3 * 0.0$

unit 22

com='east and west reflectors for south reflector with tris'

cuboid $512 \mathrm{p} 24.700 \quad 2 \mathrm{p} 12.65 \quad 2 \mathrm{p} 54.2825$

unit 23

com='array for east and west reflectors for south reflector'

array $6 \quad 3 * 0.0$

unit 24

com $=$ 'north top reflector with tris'

cuboid $512 \mathrm{p} 28.352 \mathrm{p} 64.05 \quad 2 \mathrm{p} 12.15$

unit 25

com='array for north top reflector'

array $7 \quad 3 * 0.0$

unit 26

com='south top reflector with tris'

cuboid 0 1 $2 \mathrm{p} 5.12 \mathrm{p} 2.5 \quad 2 \mathrm{p} 12.15$

cuboid 5 1 $69.4 \quad-5.1 \quad 2 \mathrm{p} 64.05 \quad 2 \mathrm{p} 12.15$

unit 27

com='array for south top reflector'

array $8 \quad 3 * 0.0$

unit 28

com='north end reflector $9.8 \mathrm{~cm}$ portion without tris'

cuboid $412 \mathrm{p} 4.9000$ 2p64.0500 2p54.2825

unit 29

com='north end relfector $5.2 \mathrm{~cm}$ portion with tris'

cuboid $512 \mathrm{p} 2.6$ 2p64.0500 2p54.2825

unit 30

com='north end relfector $10.1 \mathrm{~cm}$ portion without tris'

cuboid $412 \mathrm{p} 5.052 \mathrm{p} 64.052 \mathrm{p} 54.2825$

unit 31

com='array for north end reflector'

array $93 * 0.0$

unit 32

com='south end reflector'

cuboid 512 p12.55 2p64.05 2p54.2825 


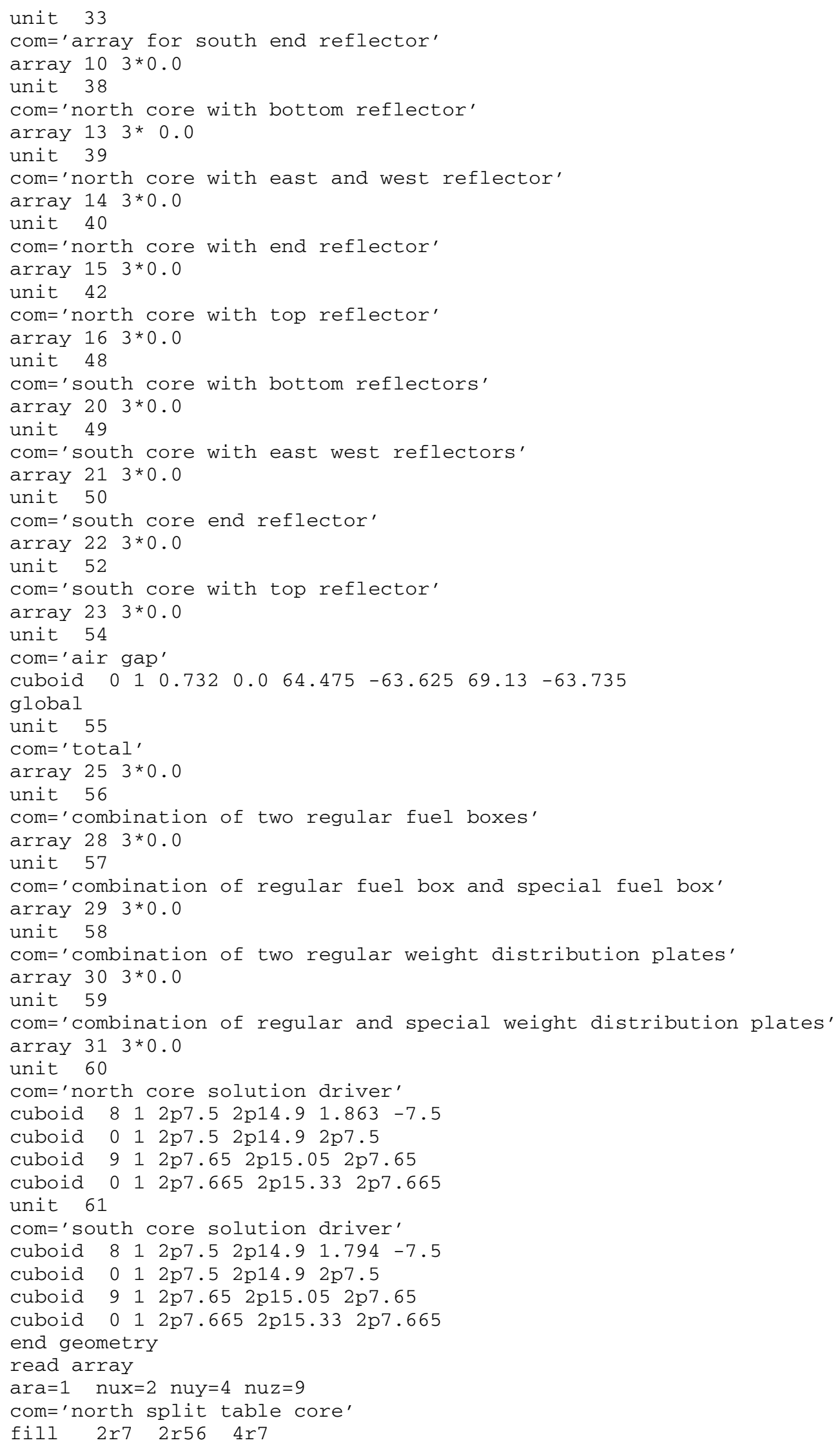




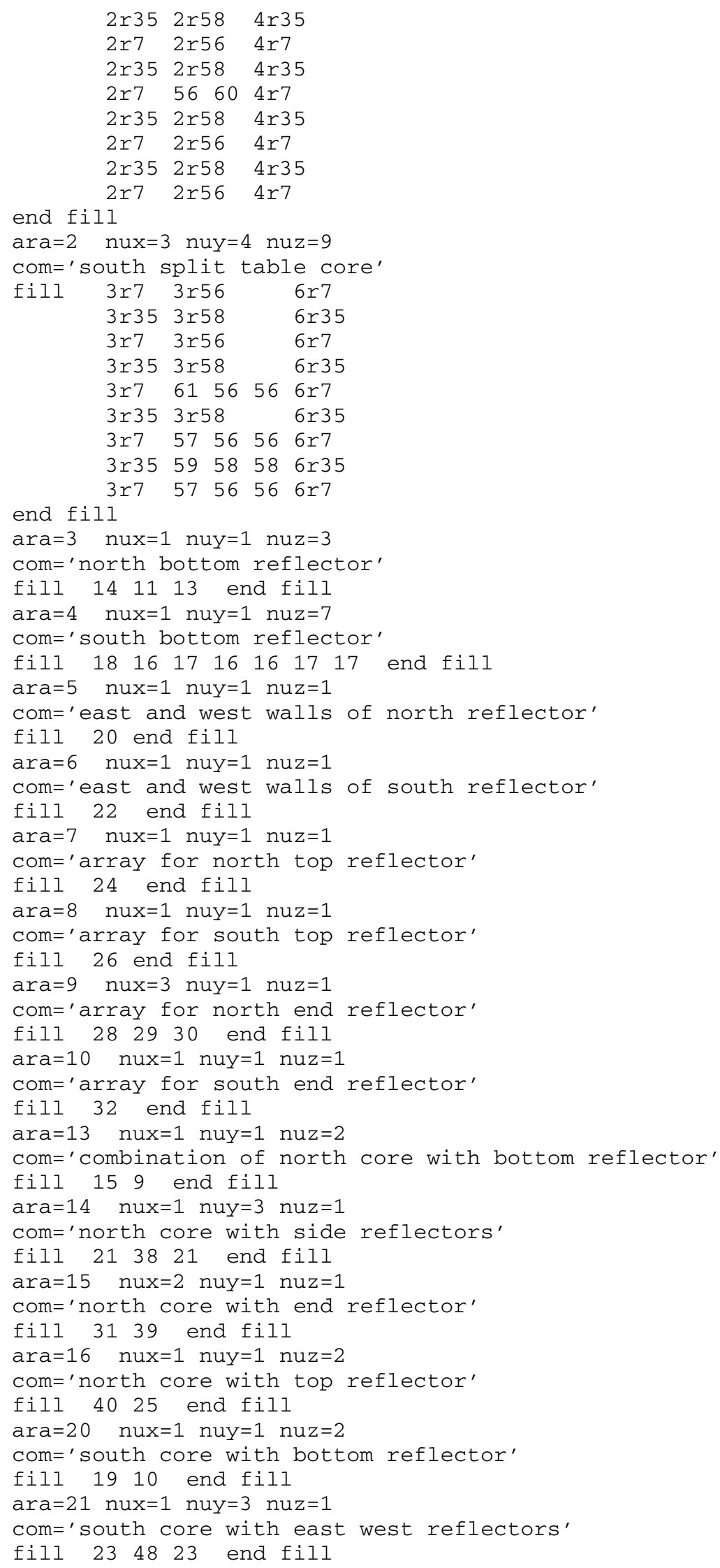




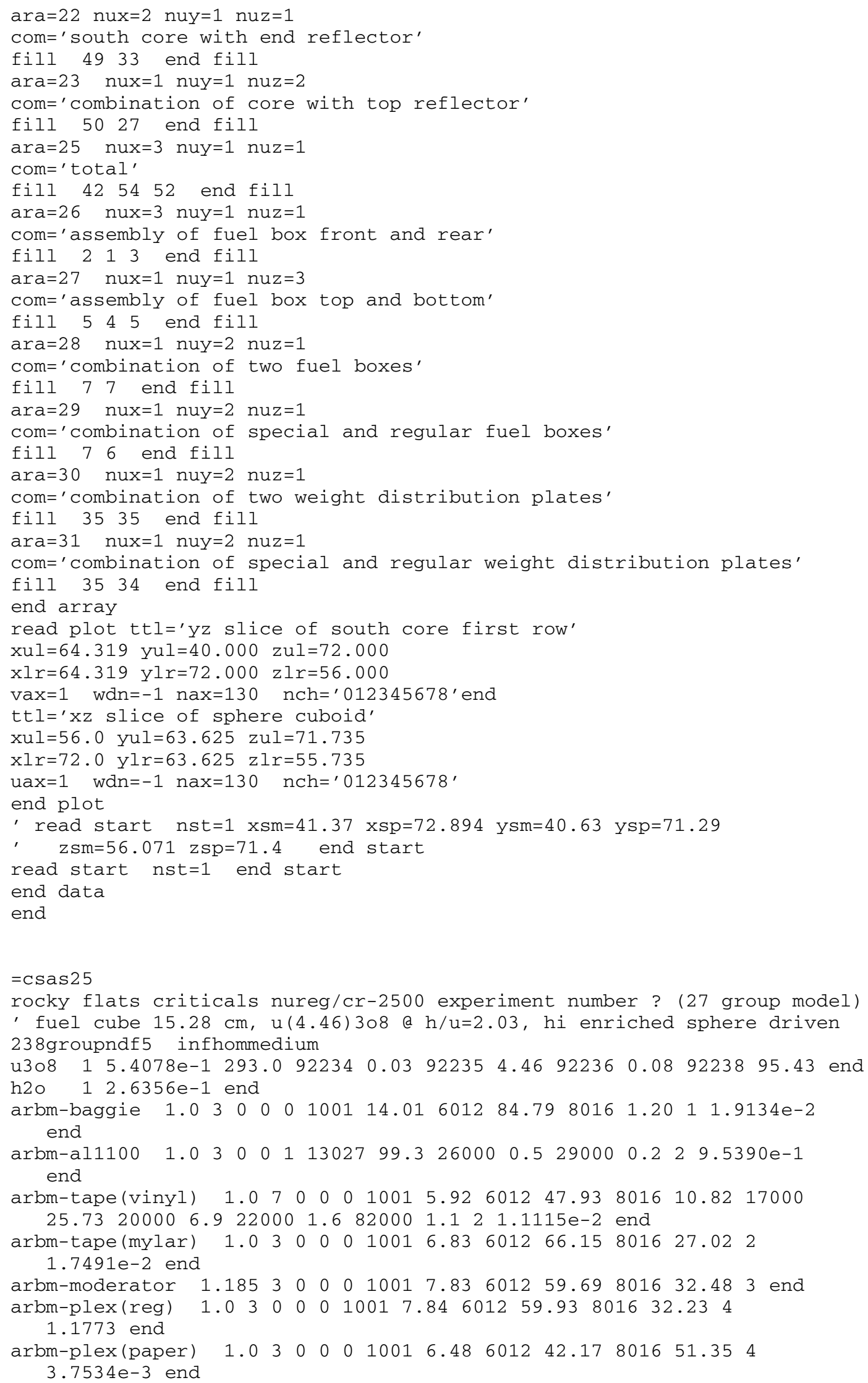




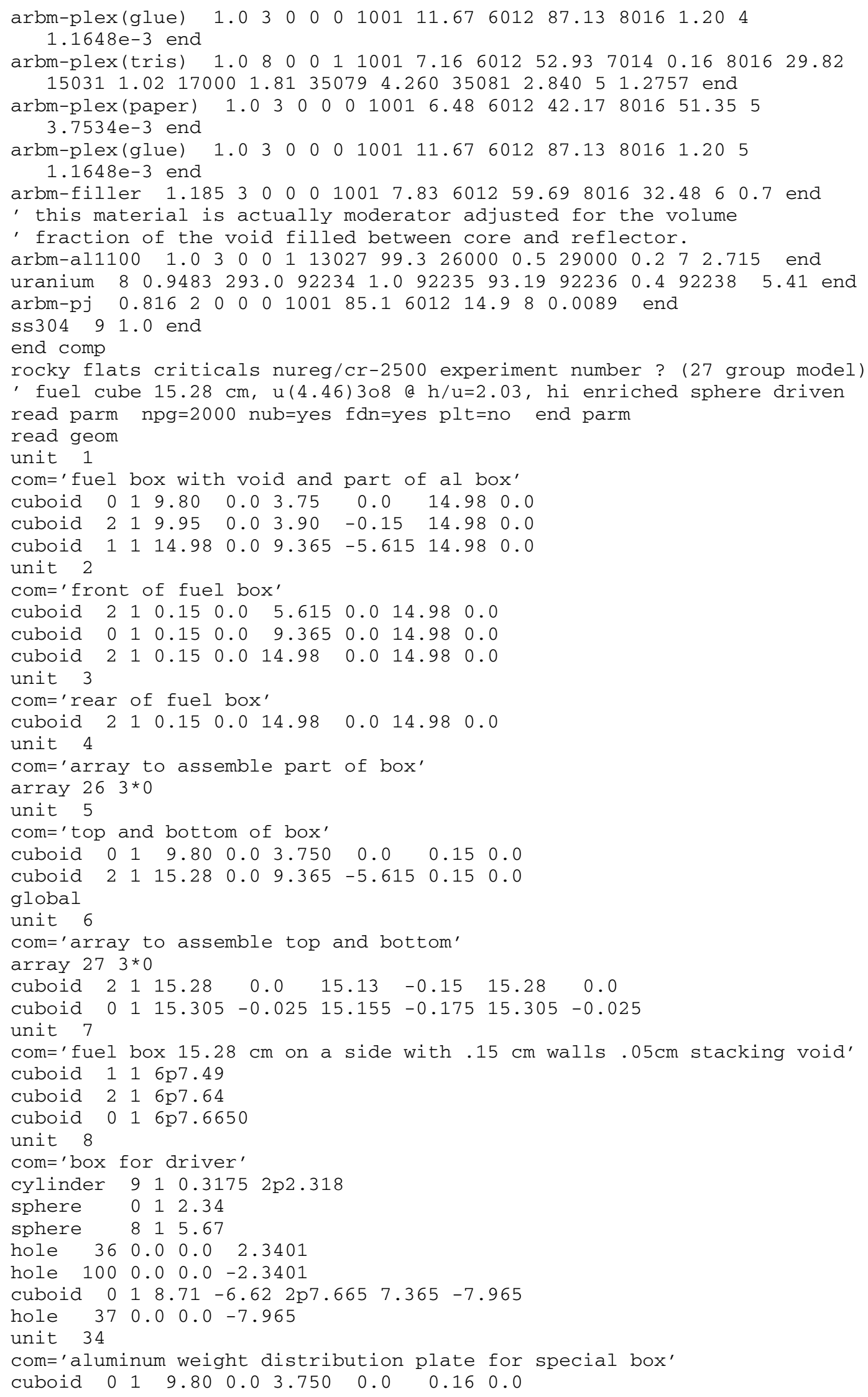




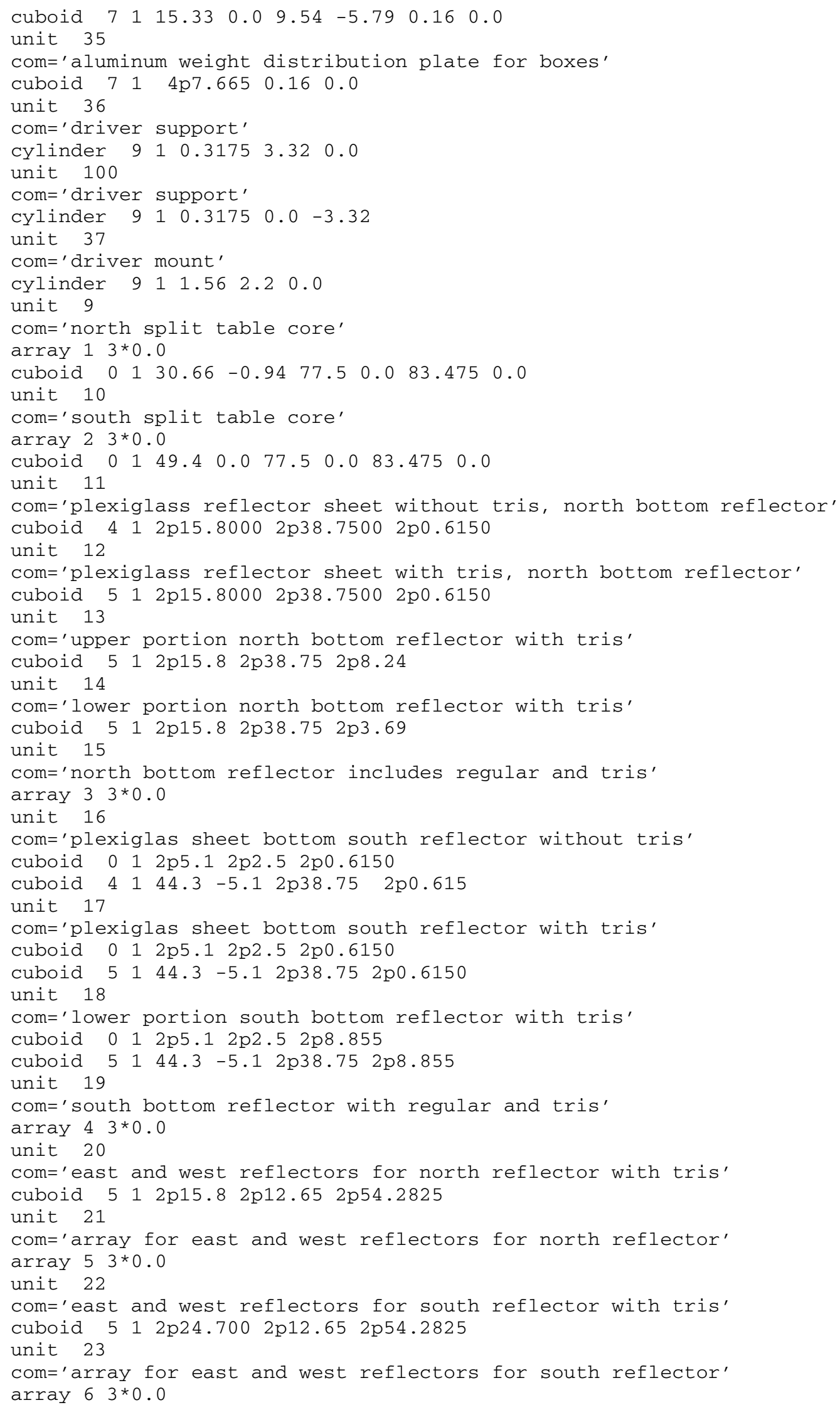




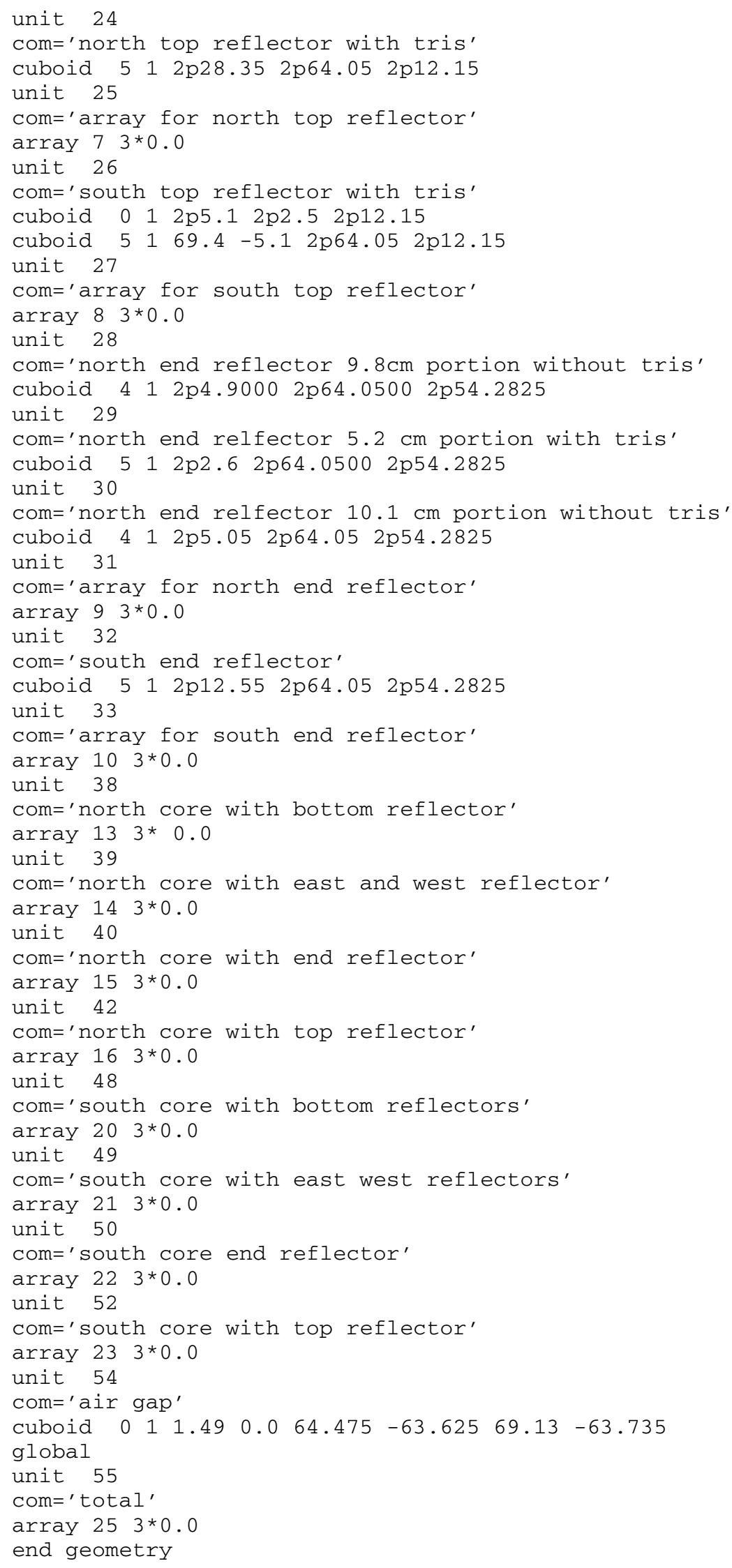




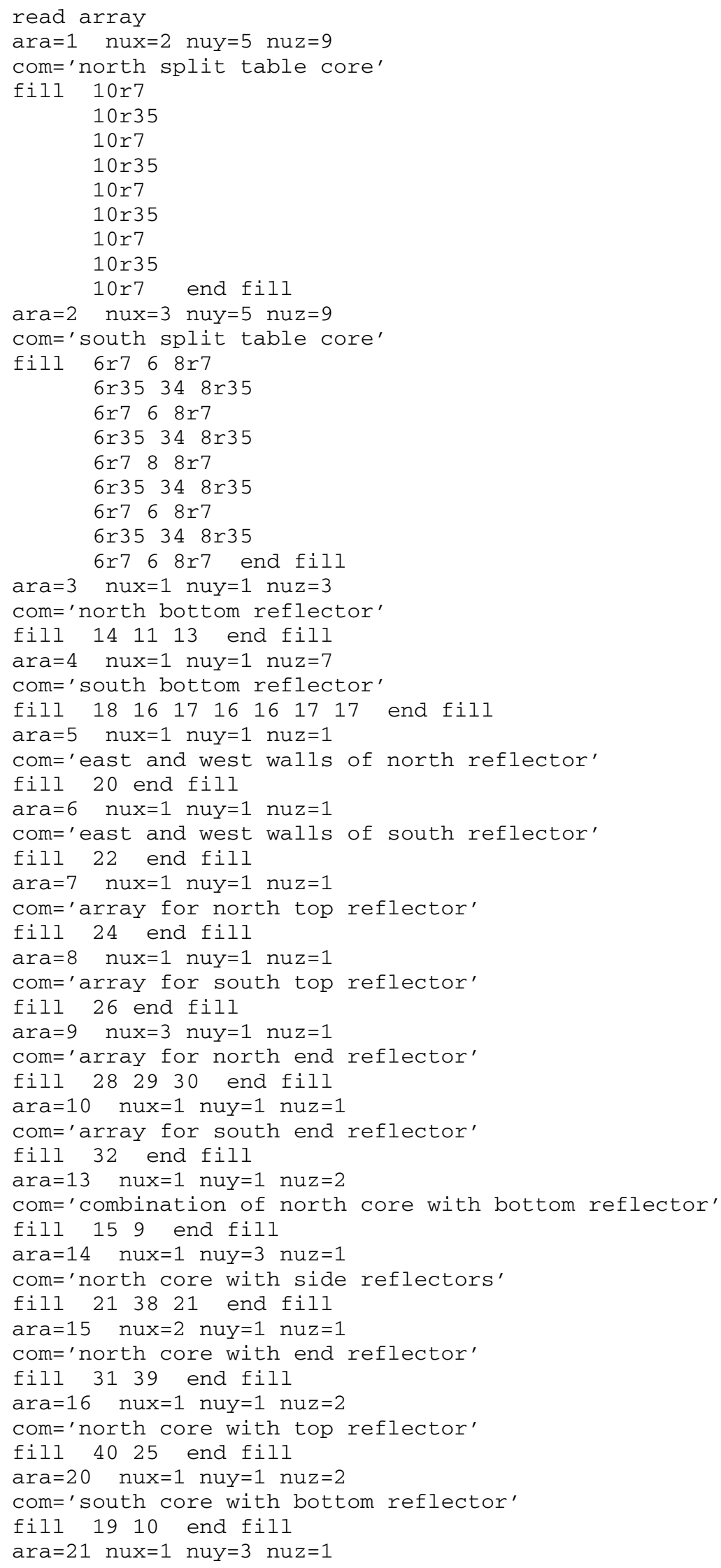




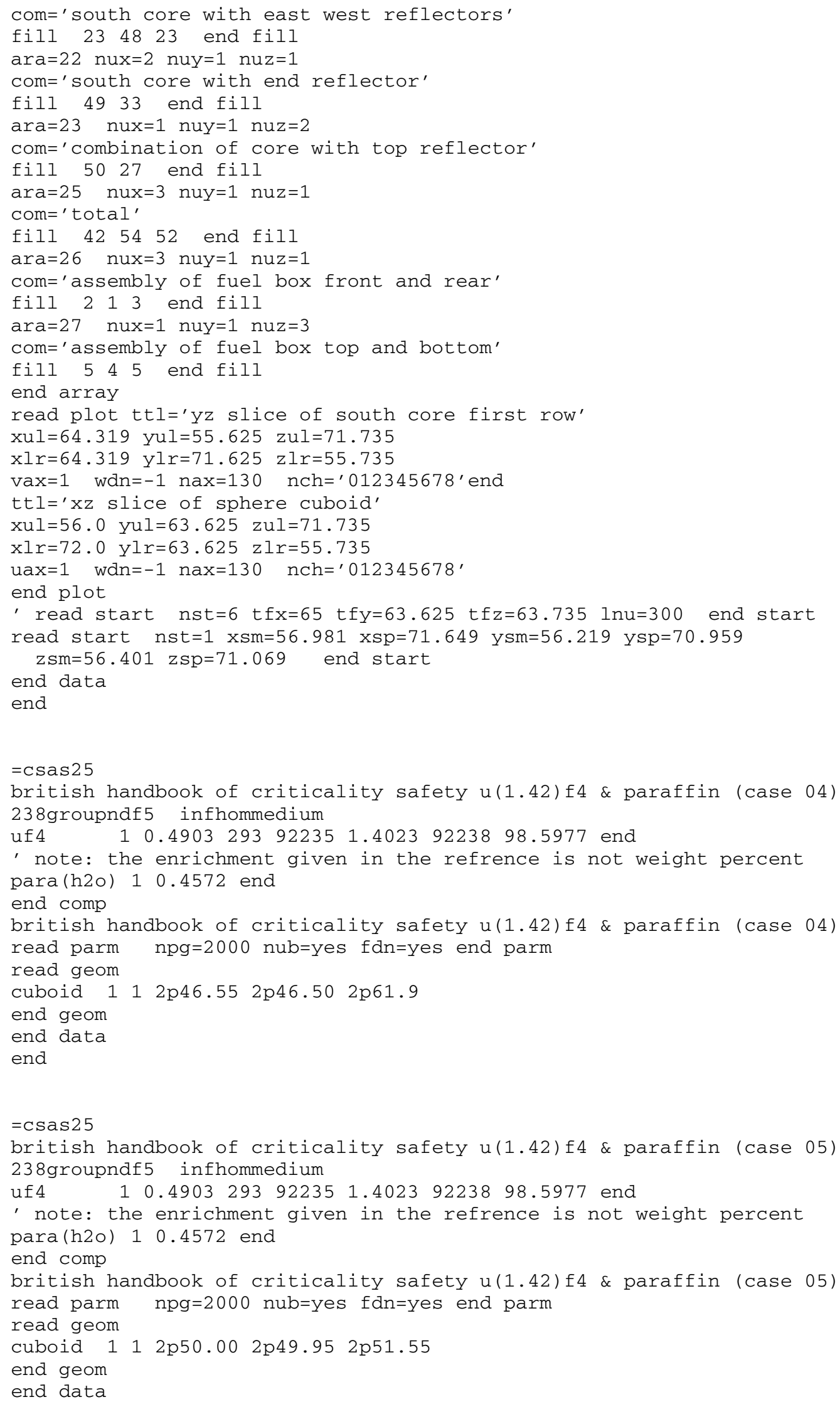




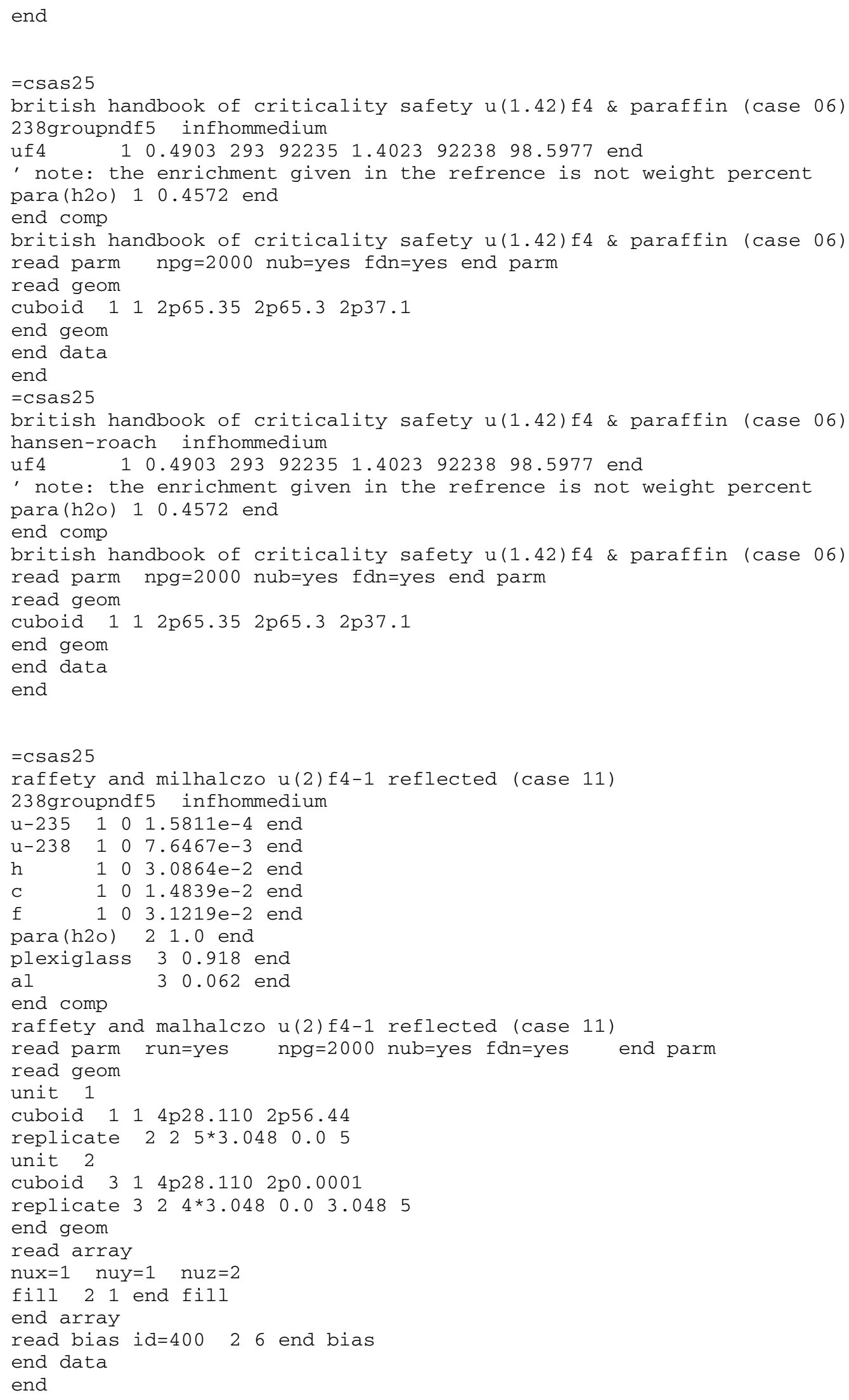




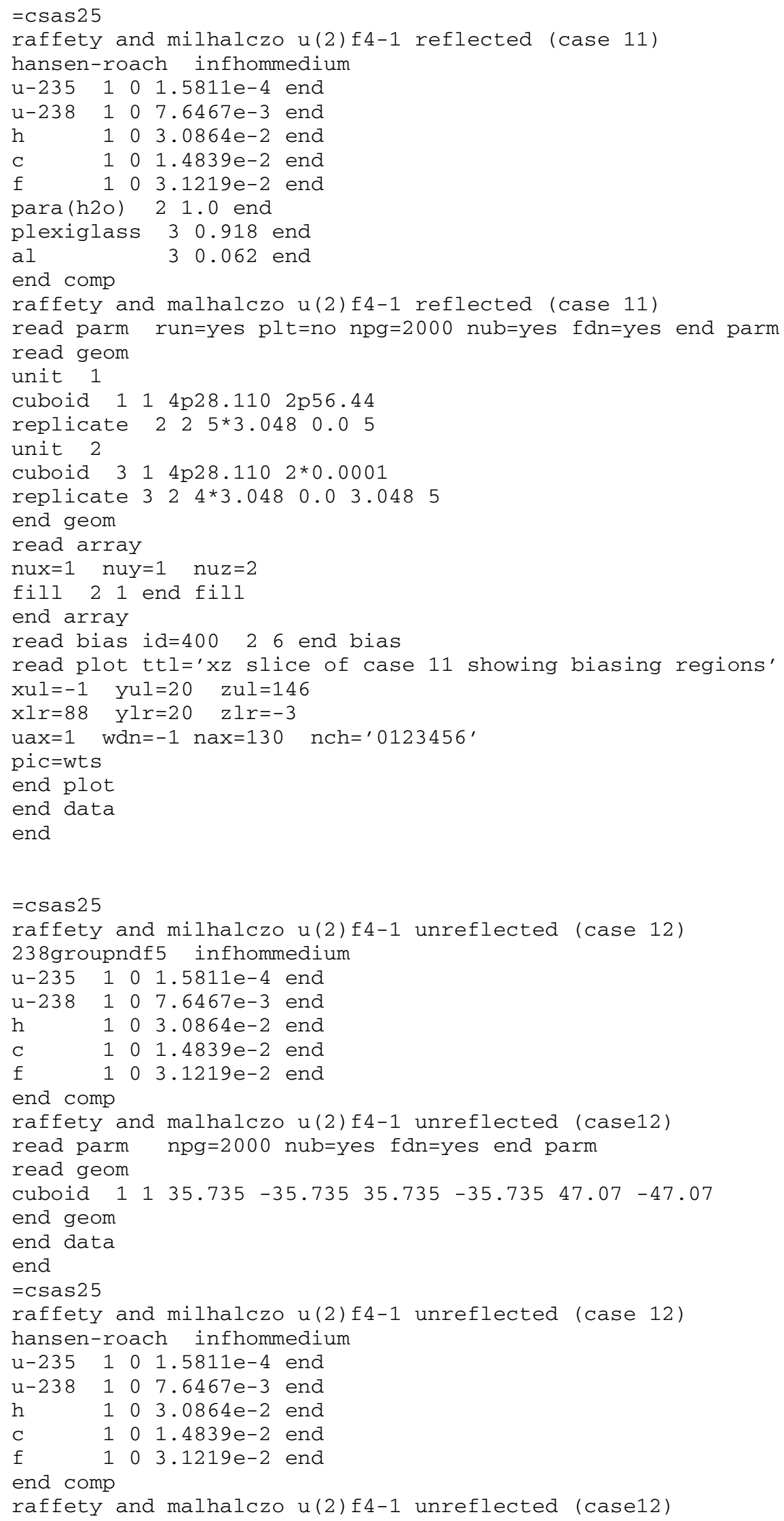




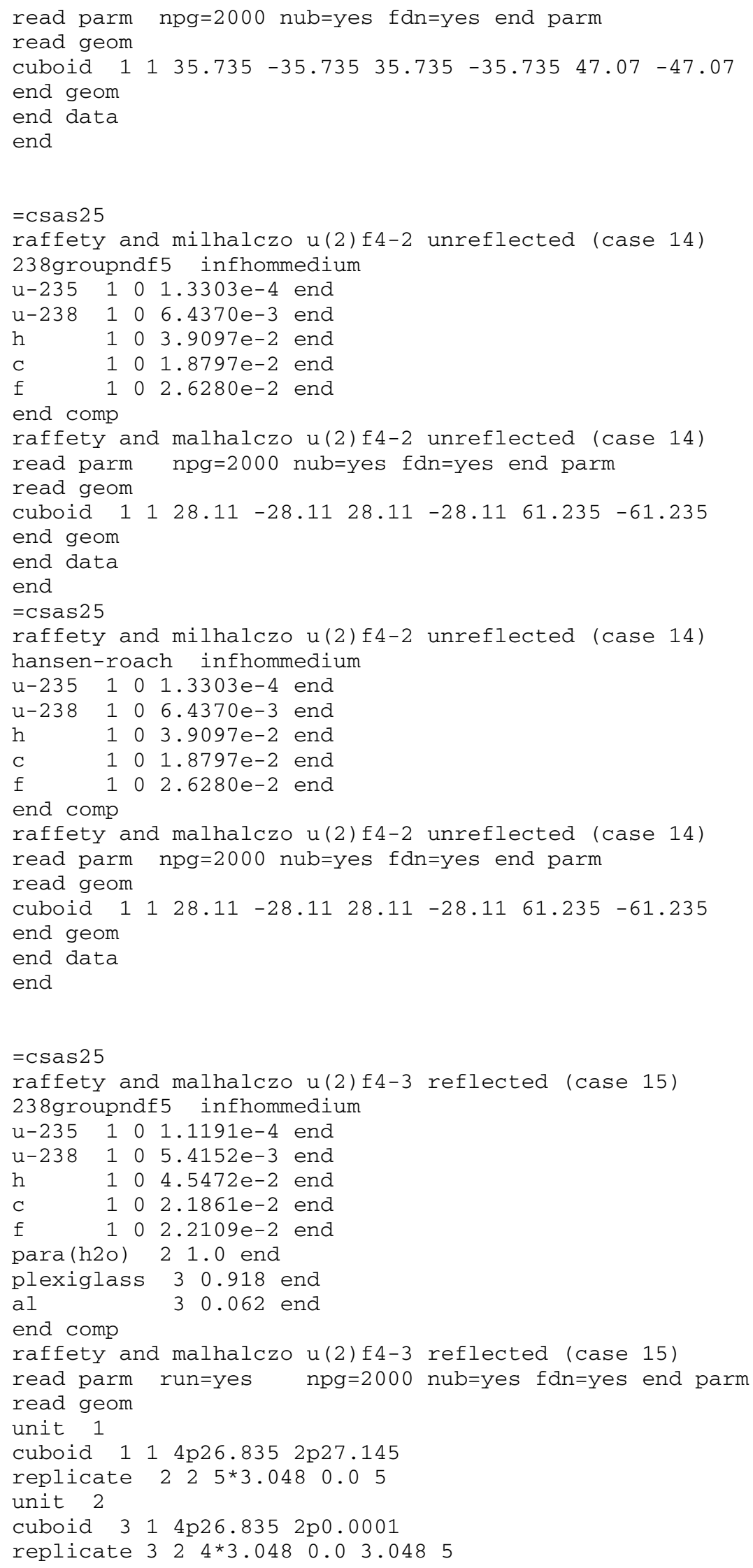




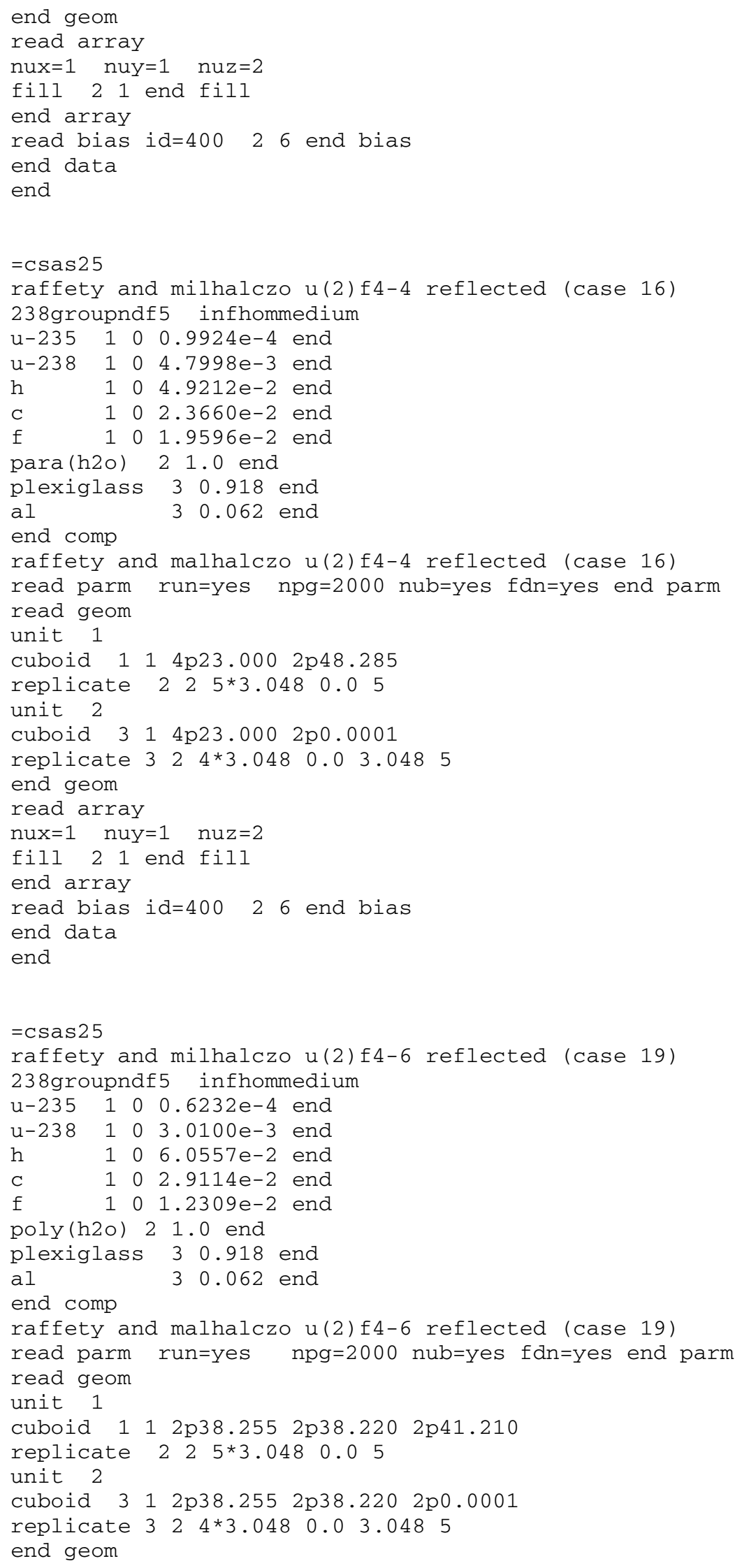




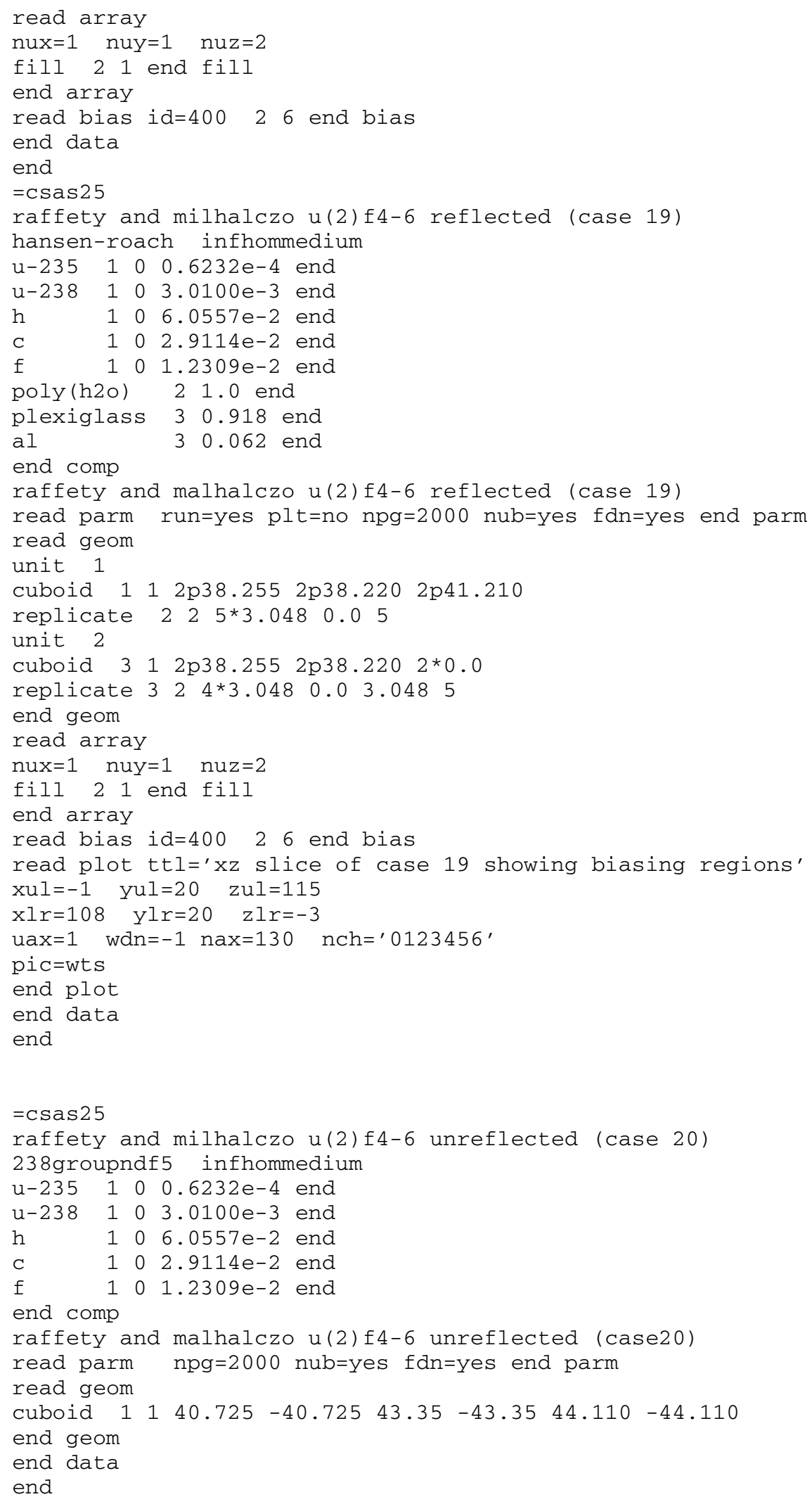




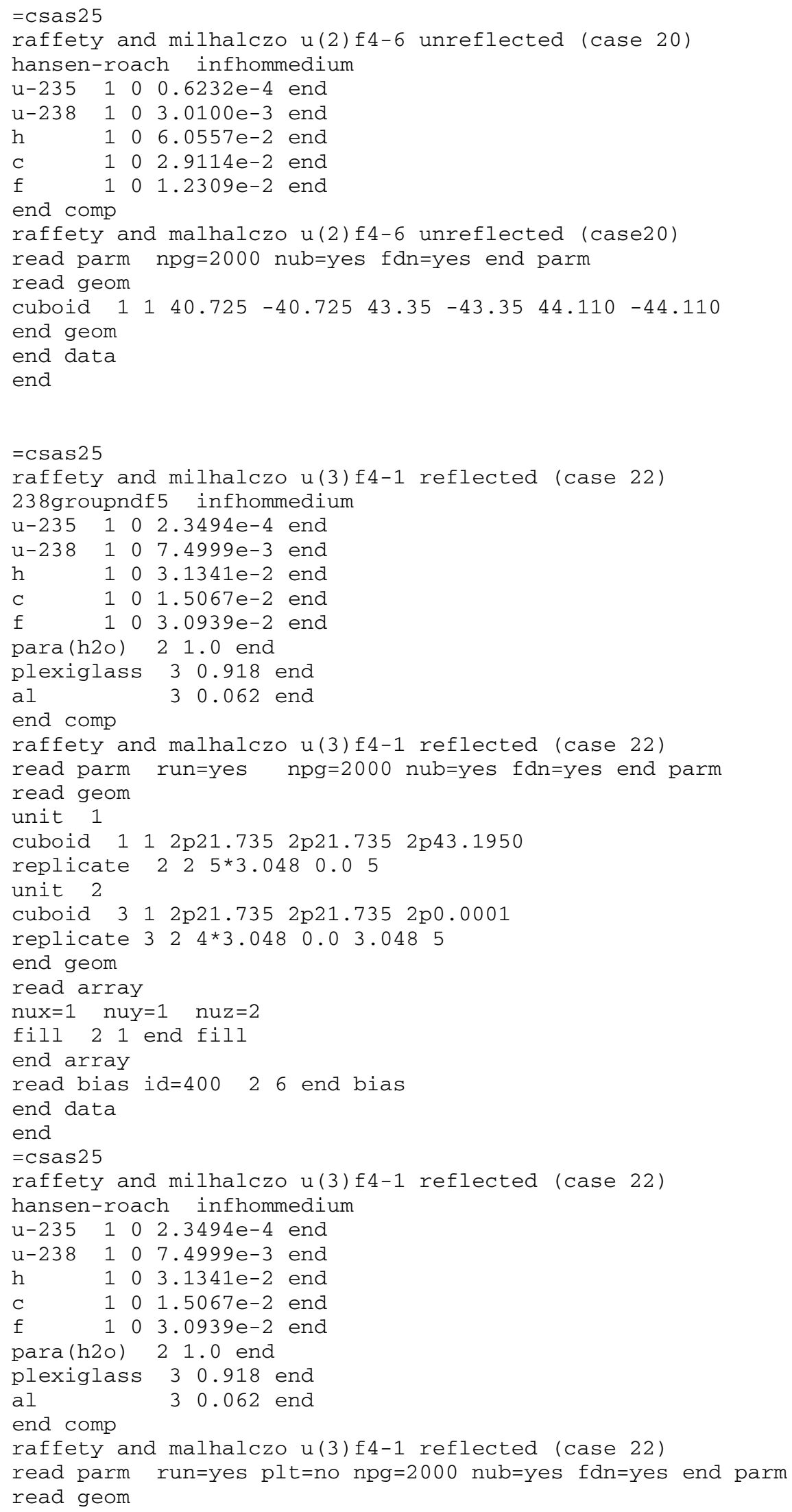




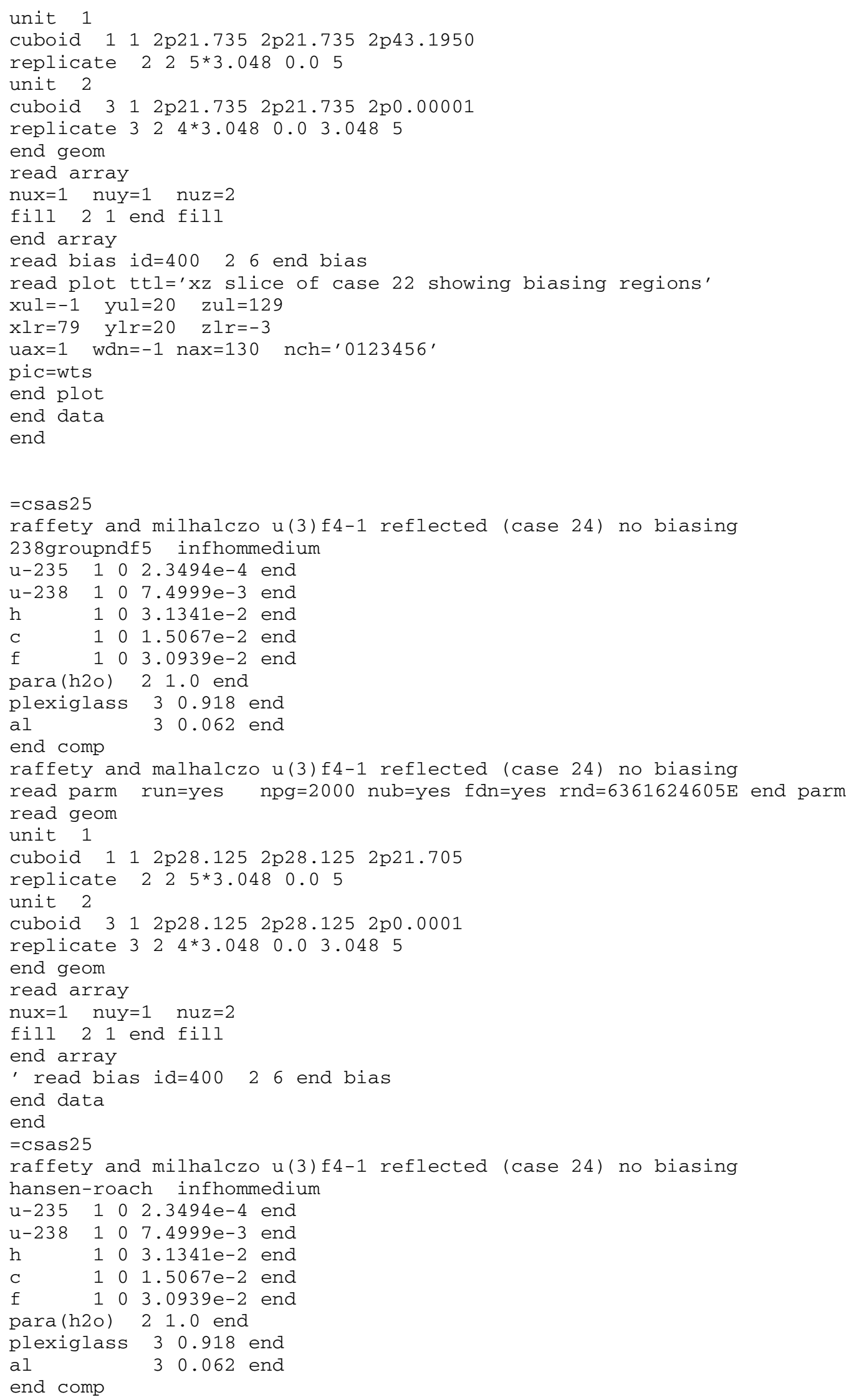




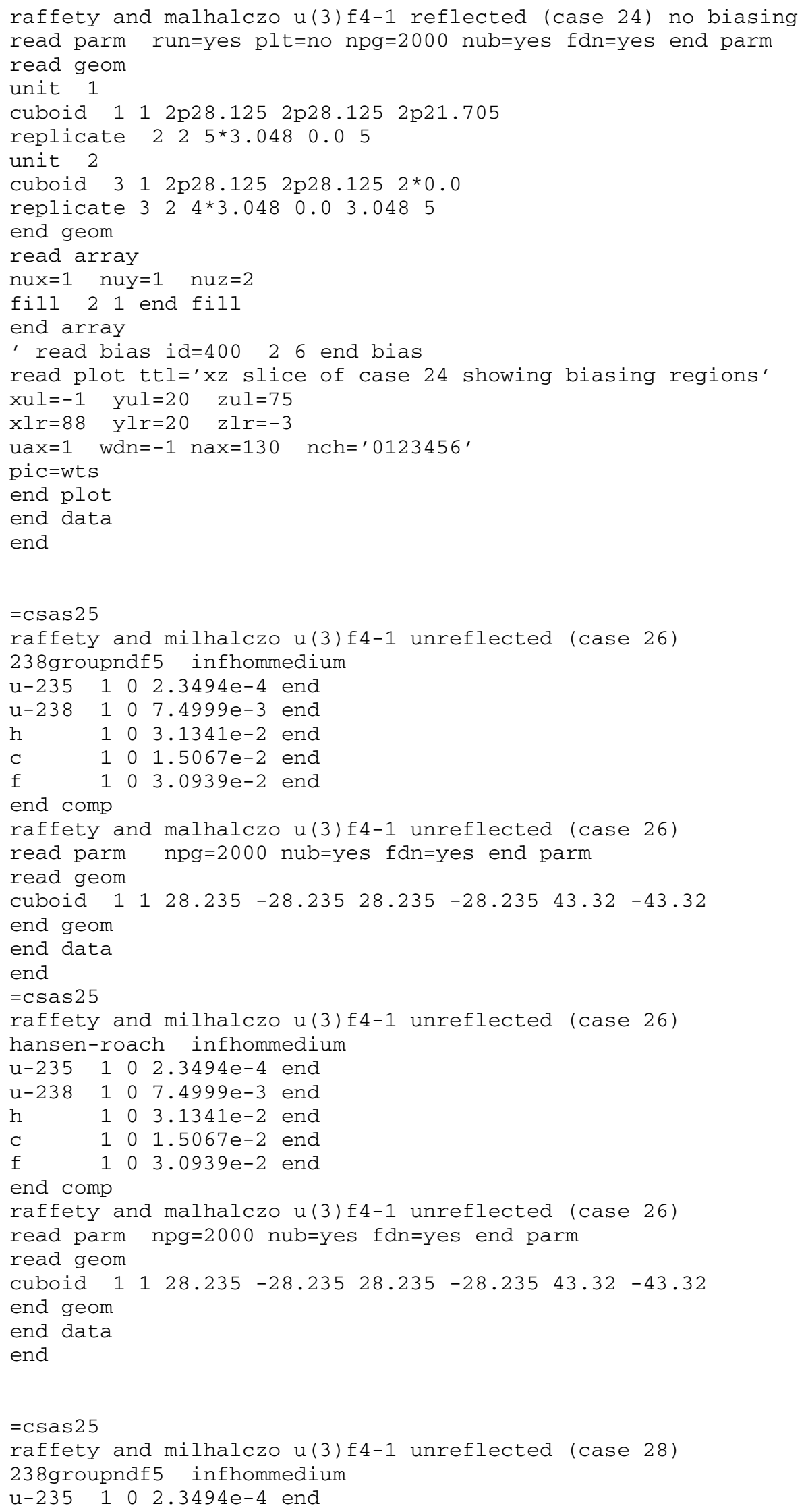




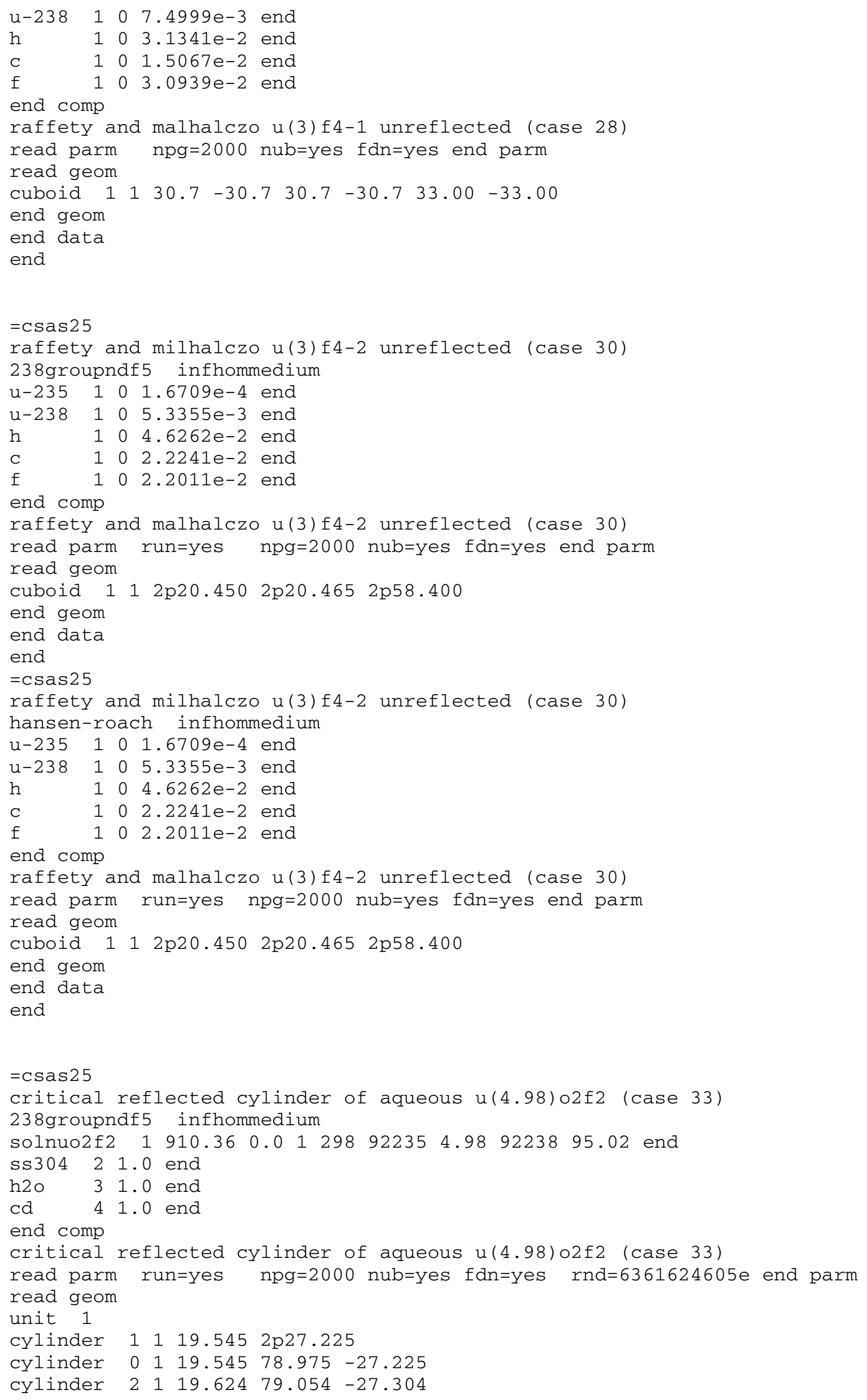




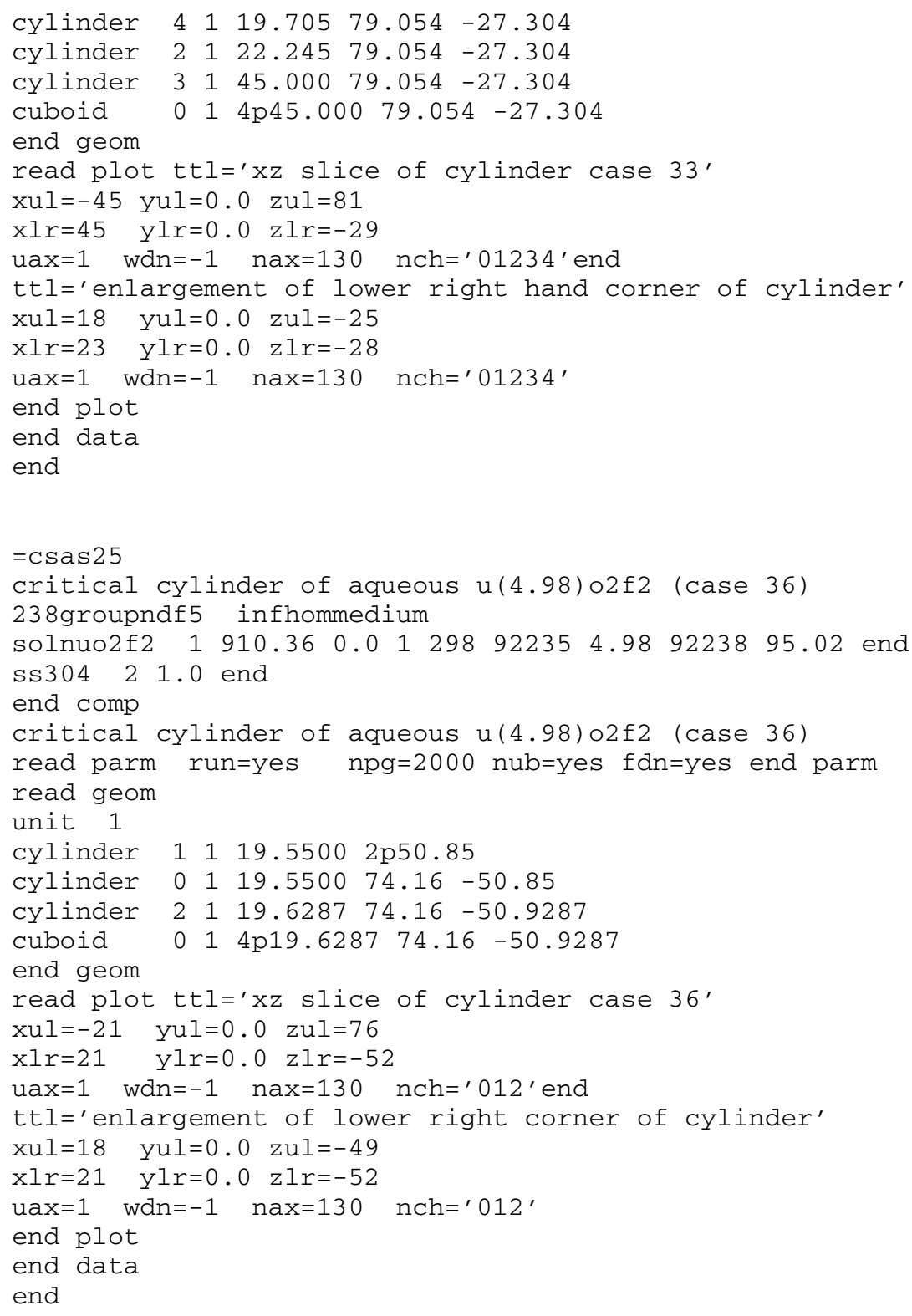


Table A.4. Table 4 input data

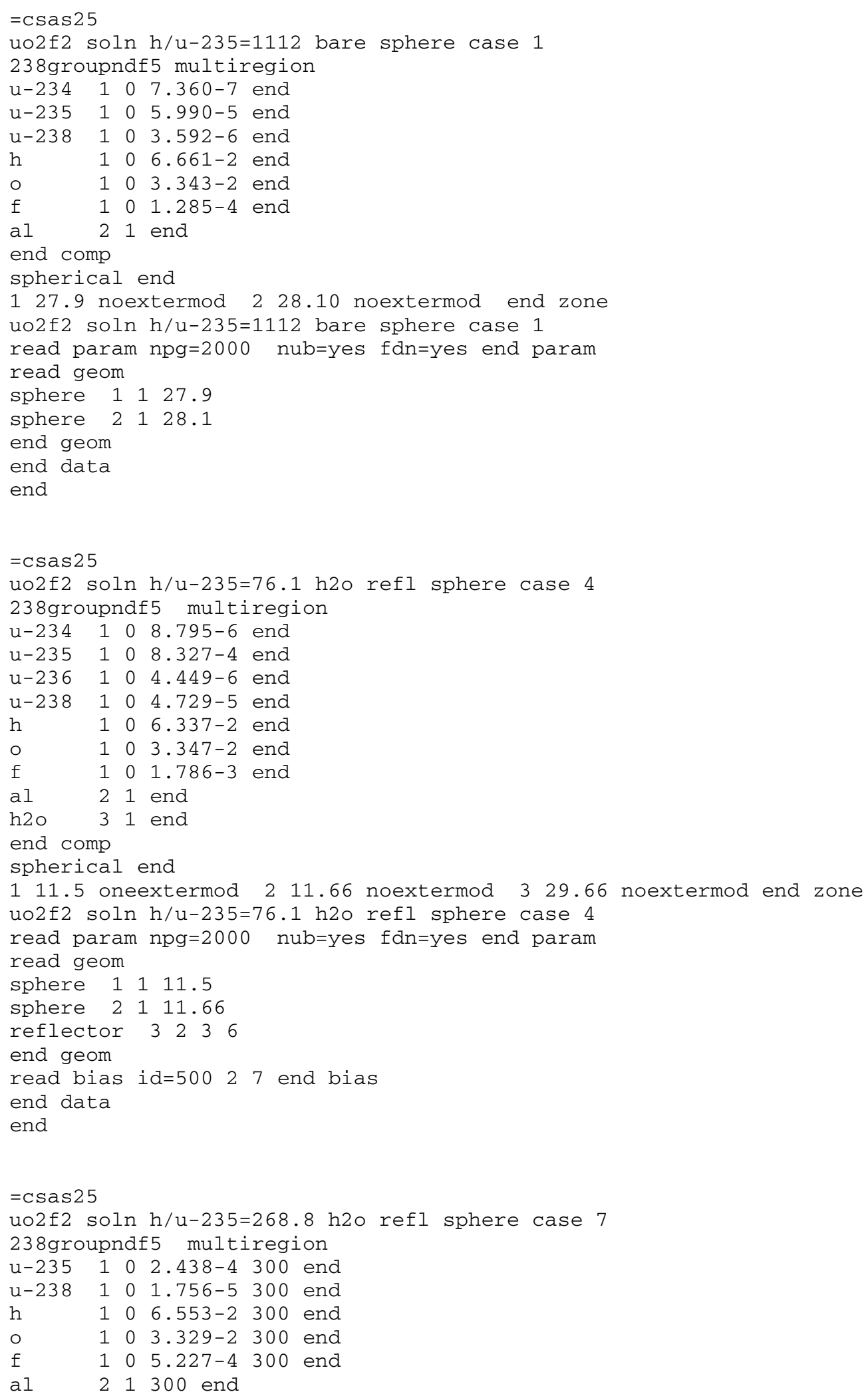




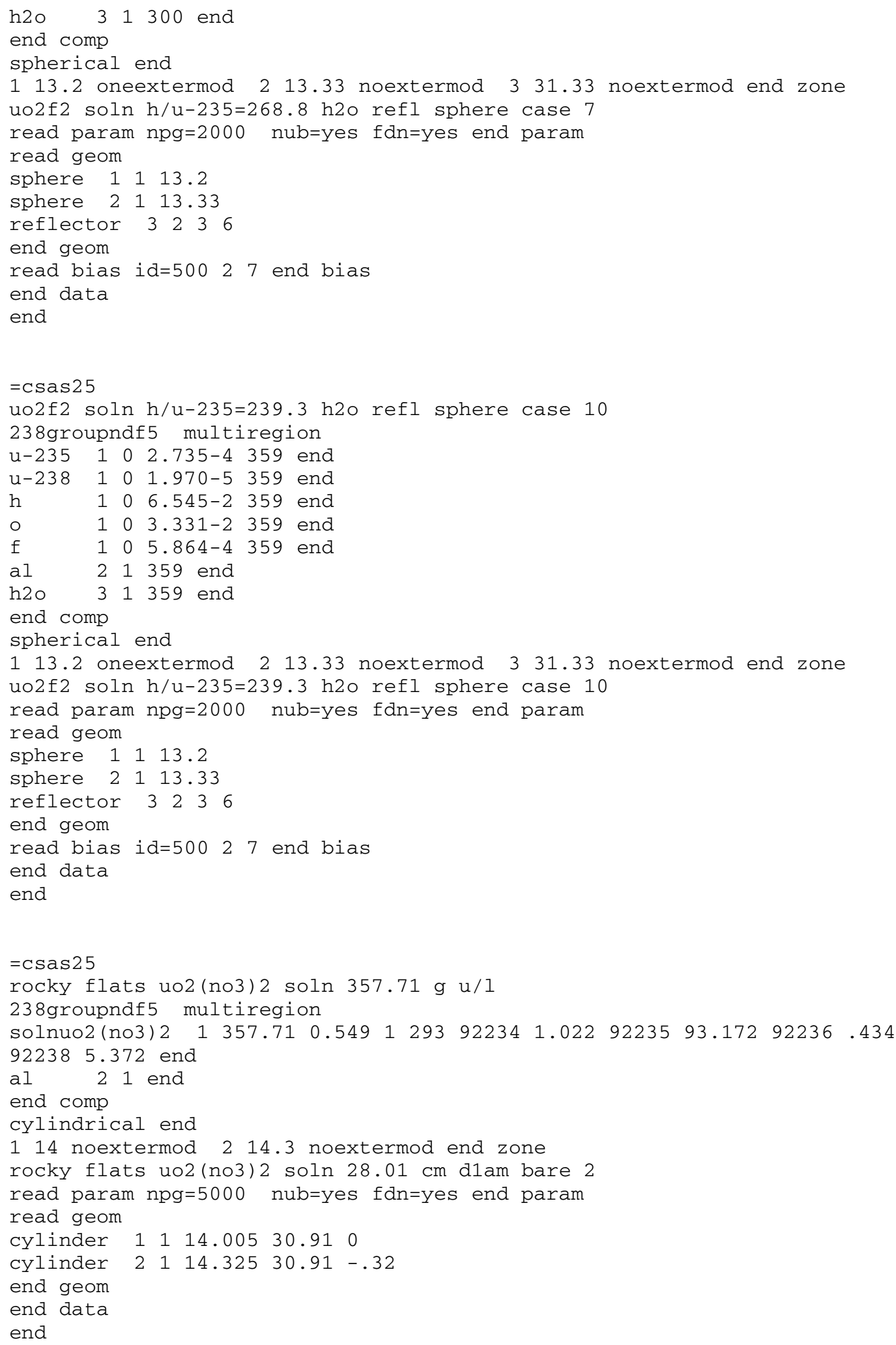




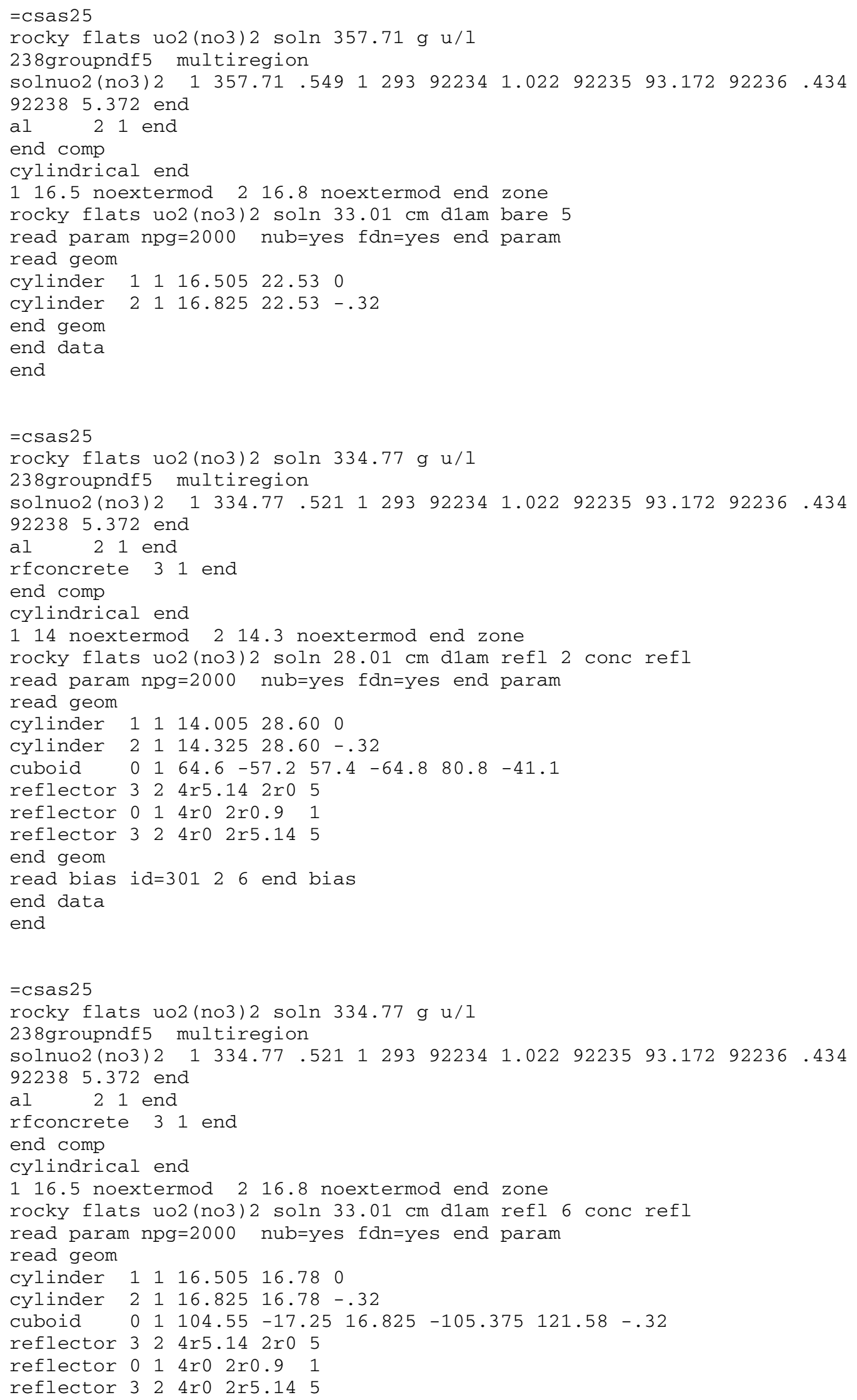




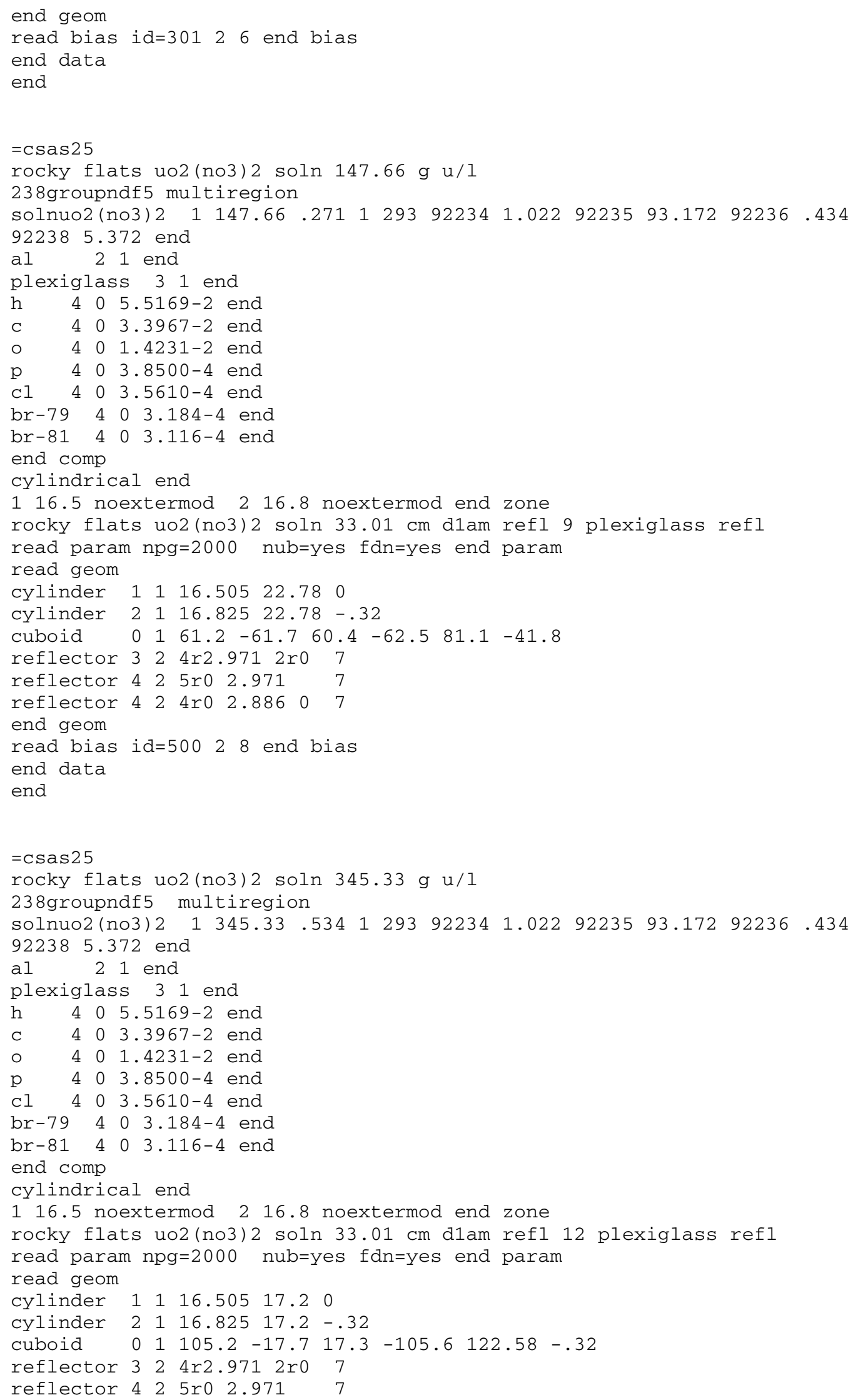




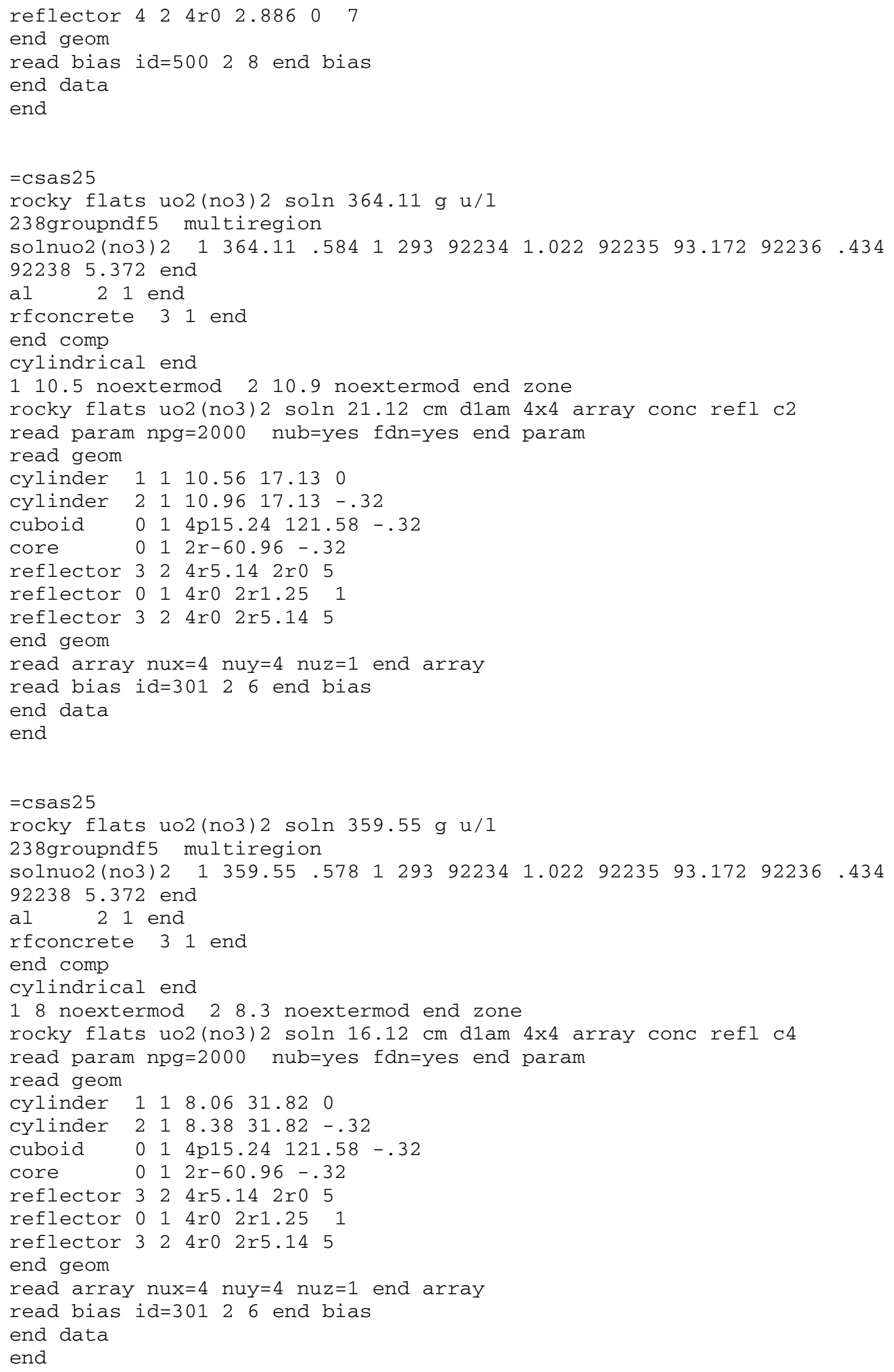




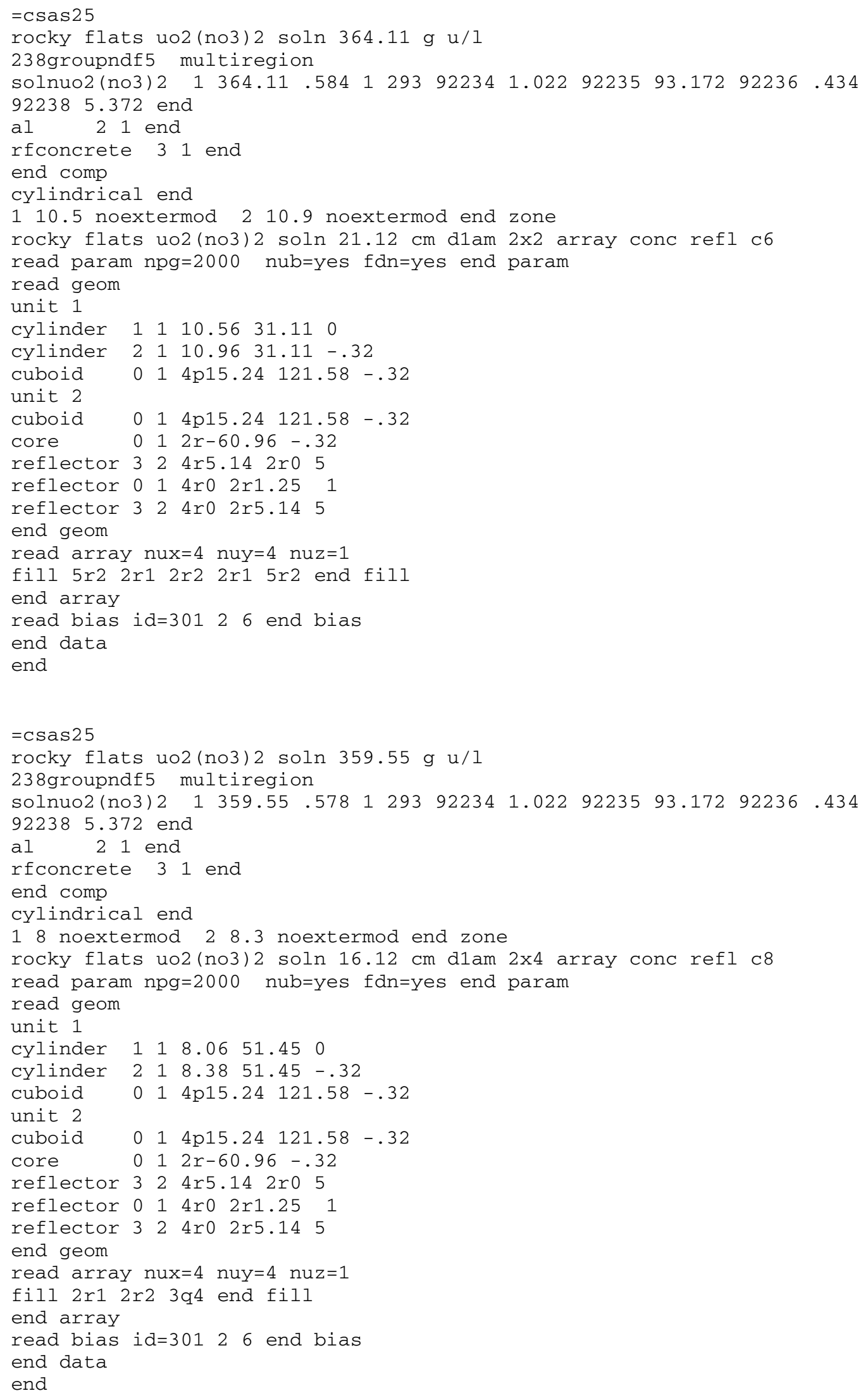




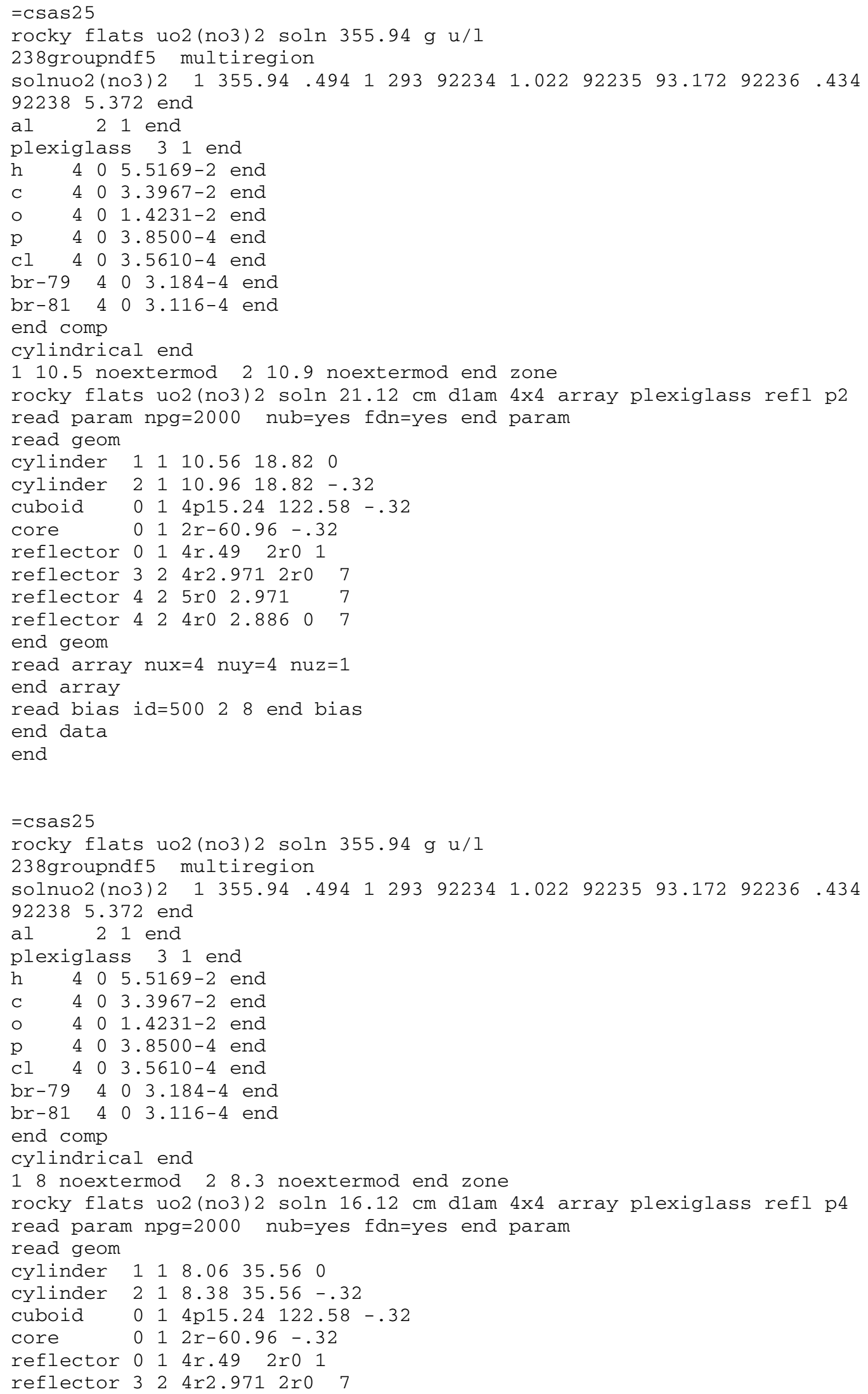




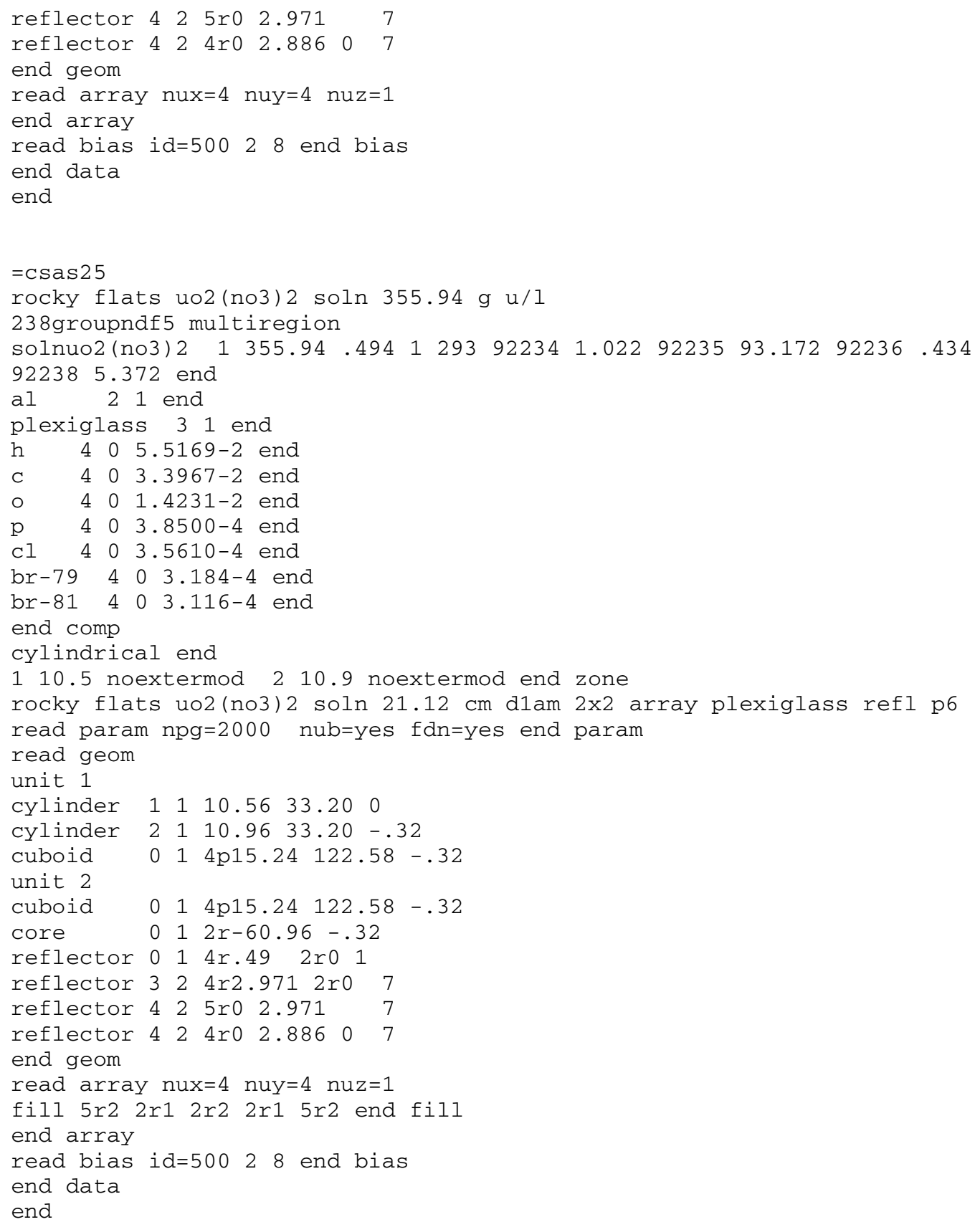


Table A.5. Table 5 input data

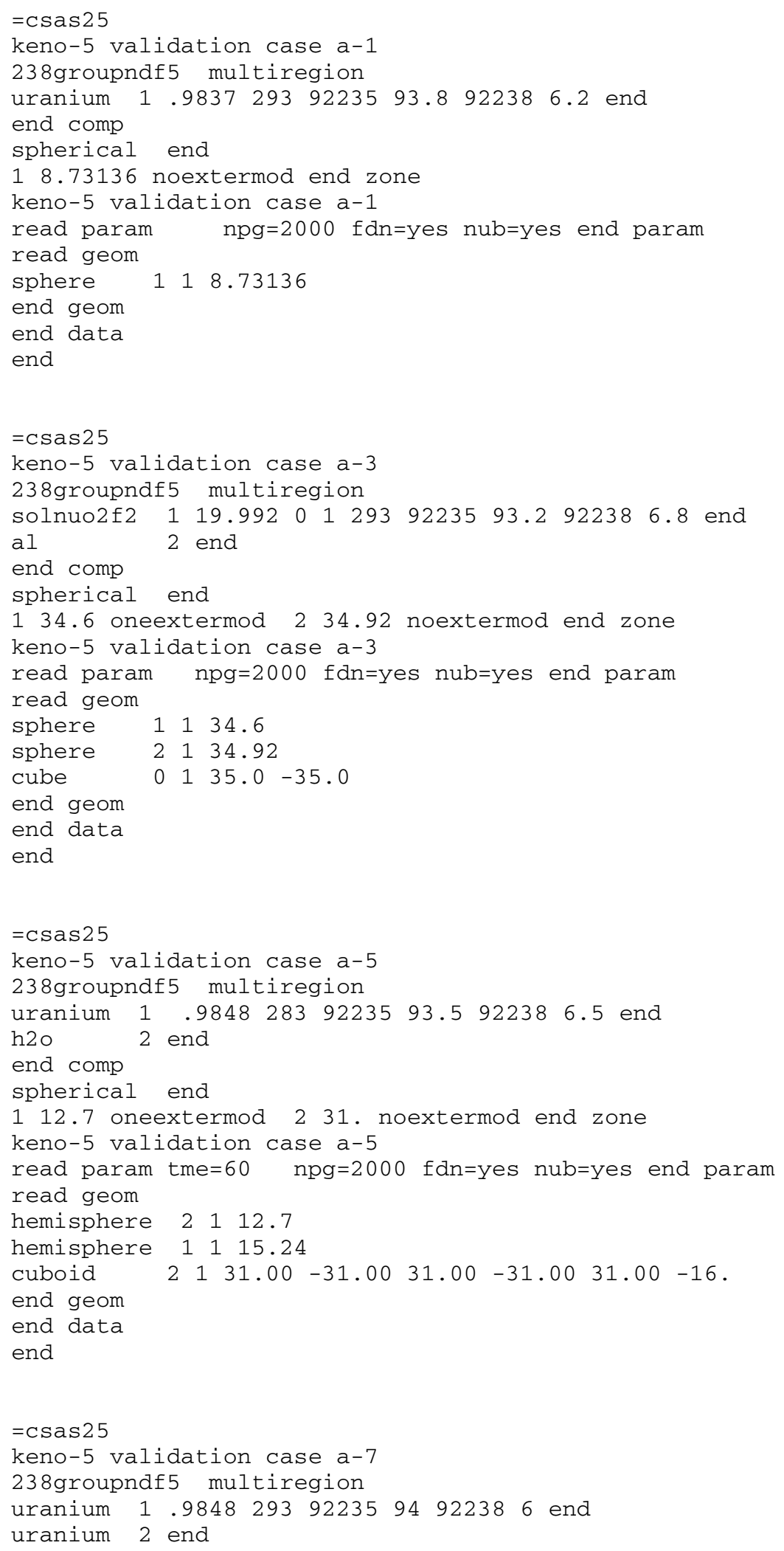




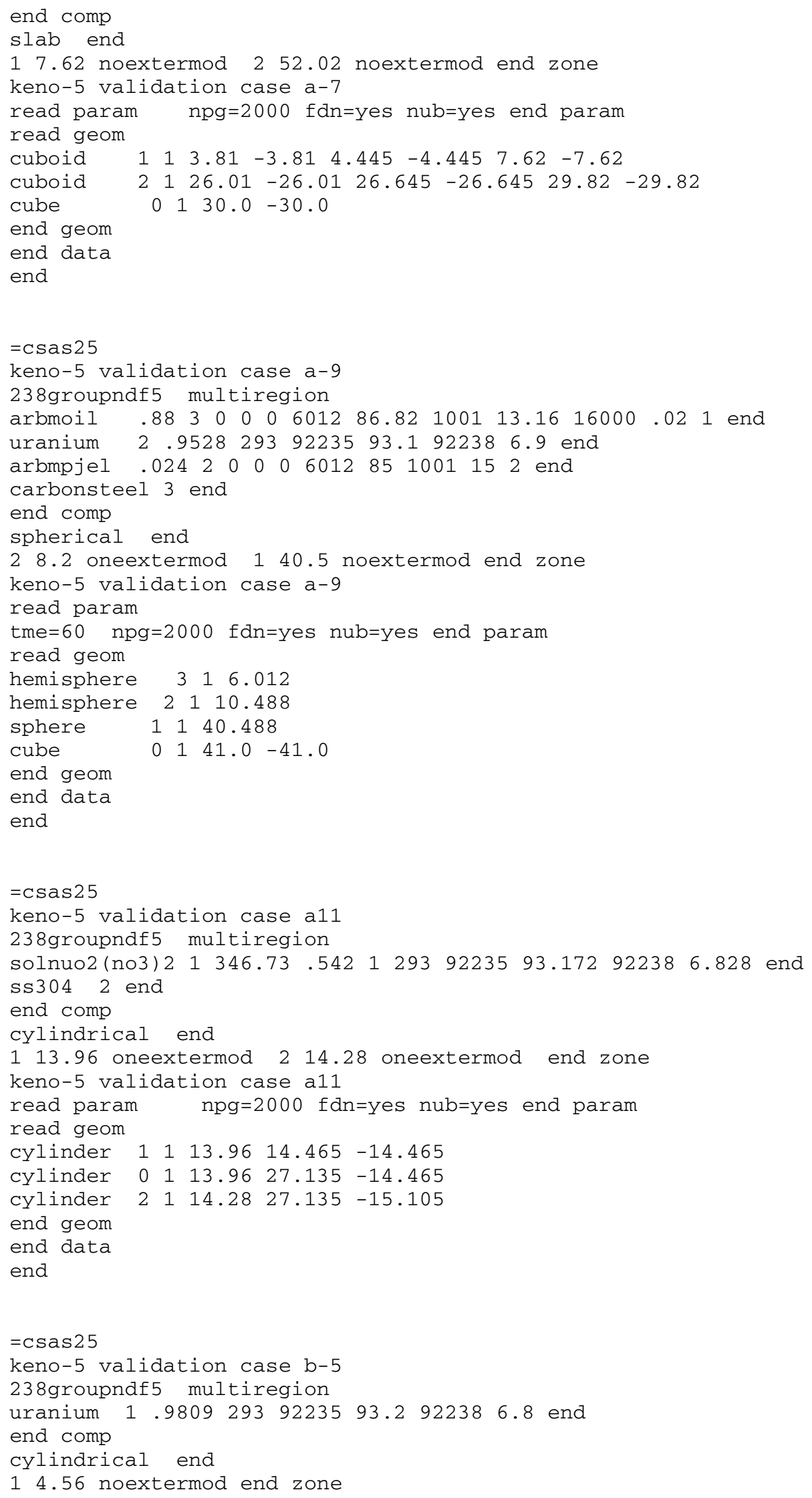




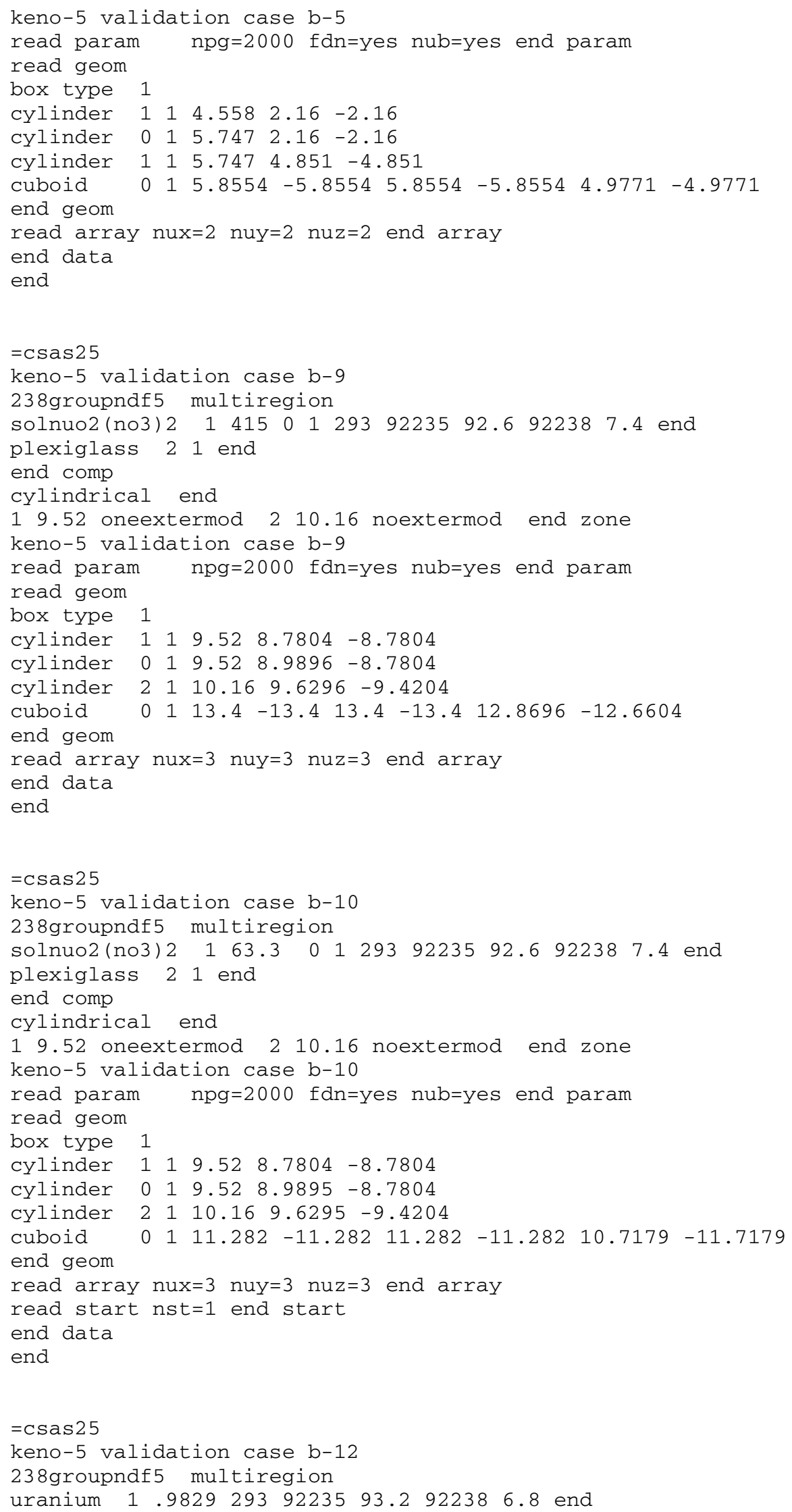




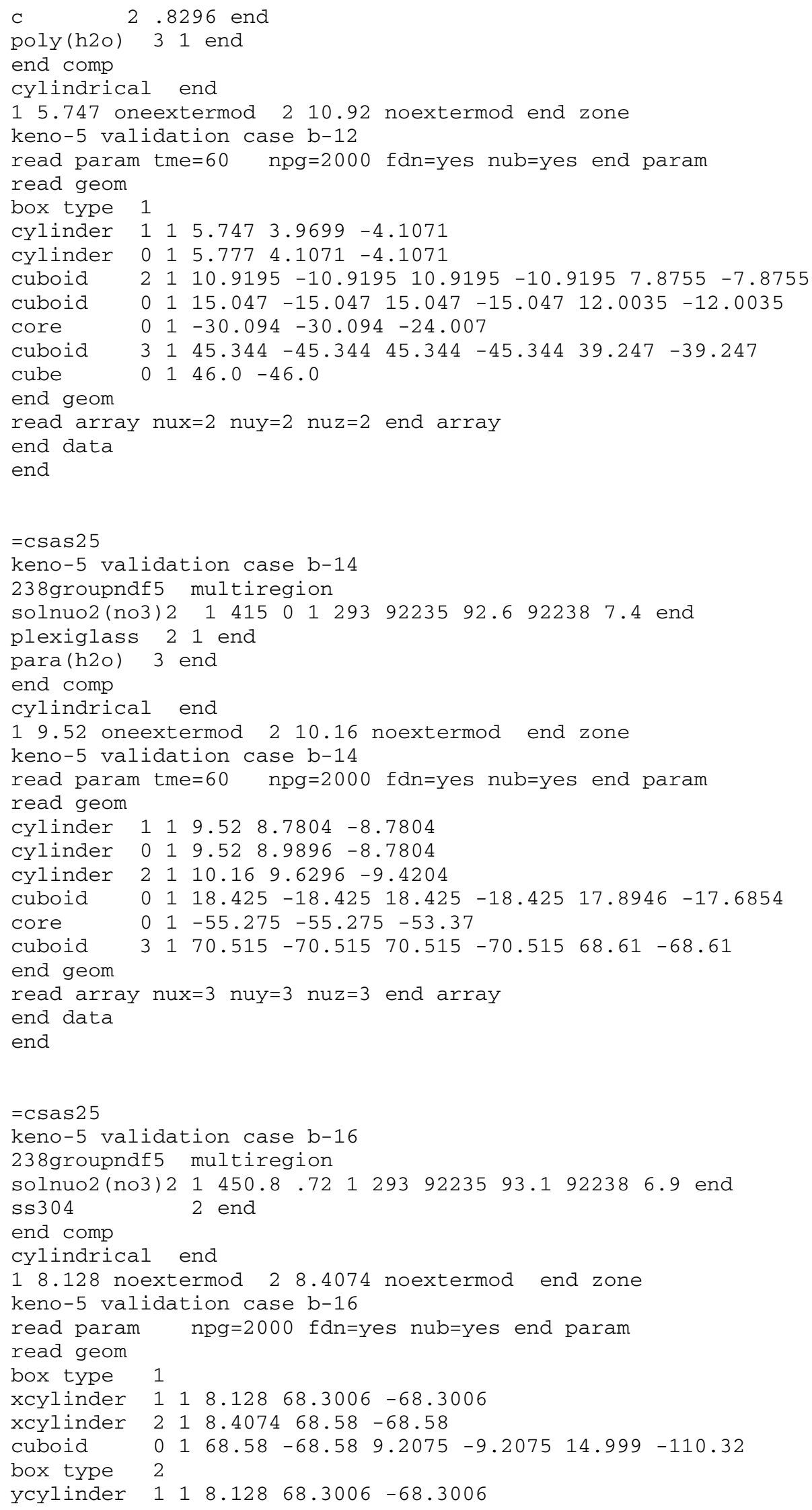




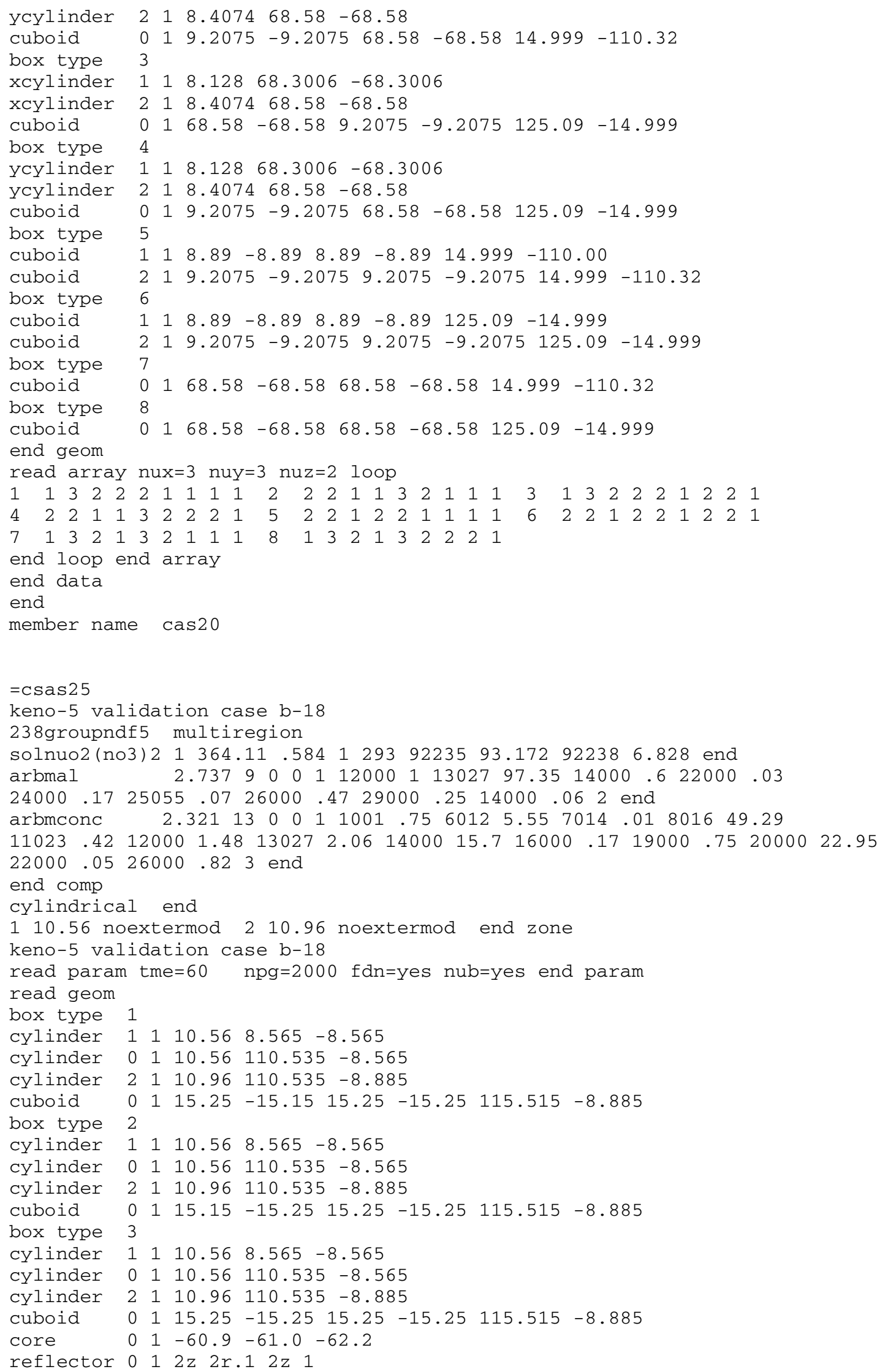




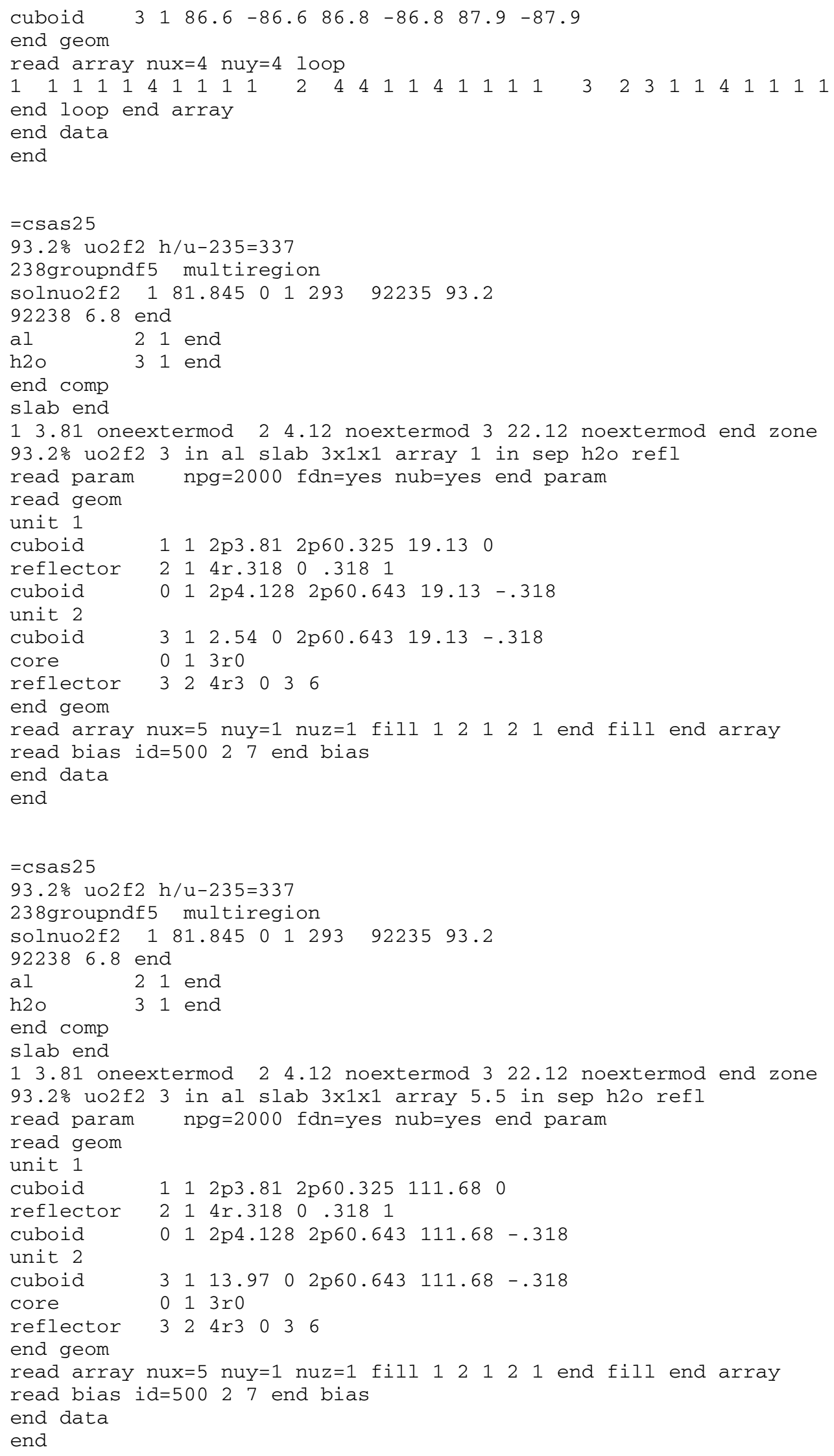




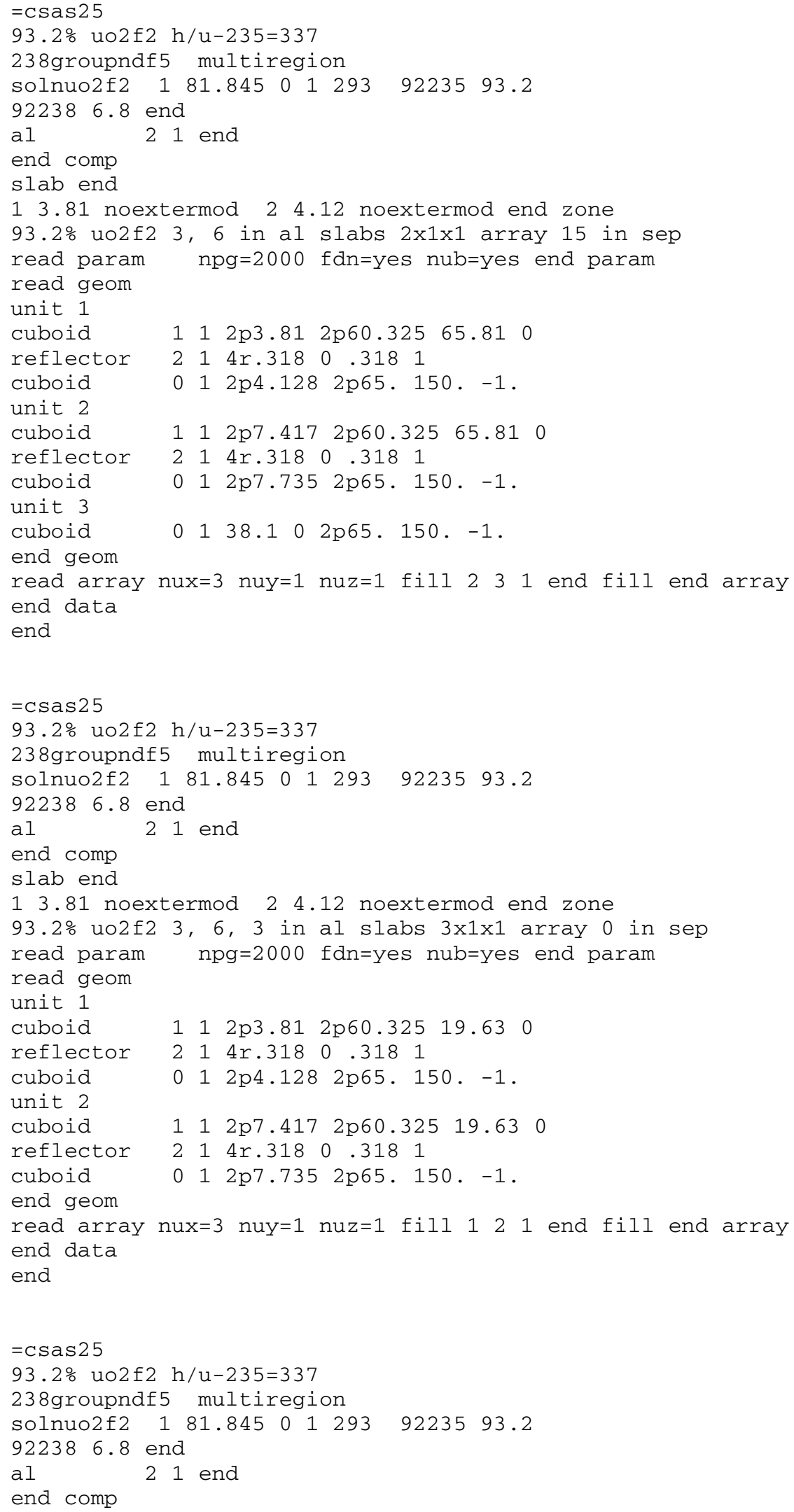




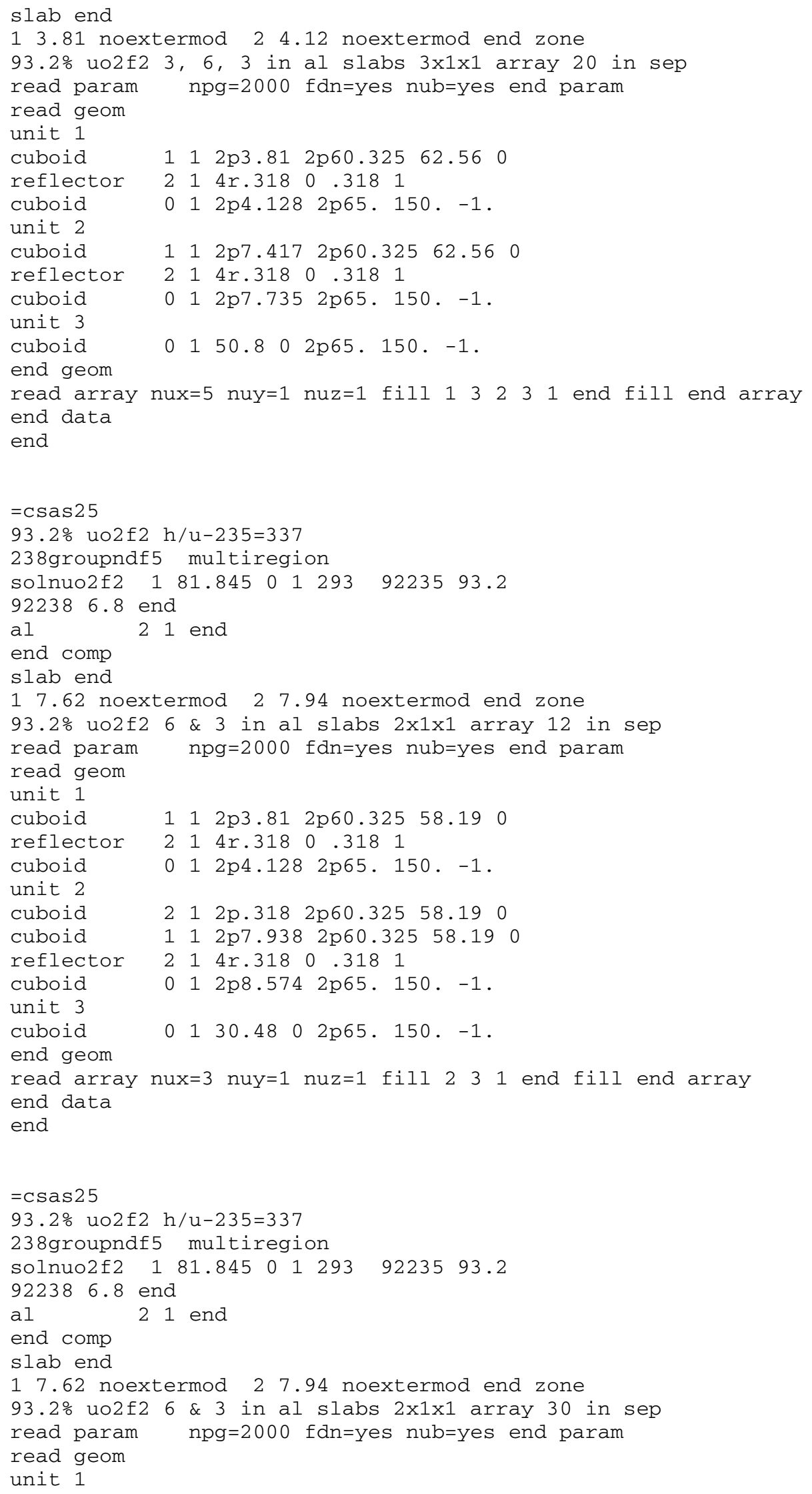




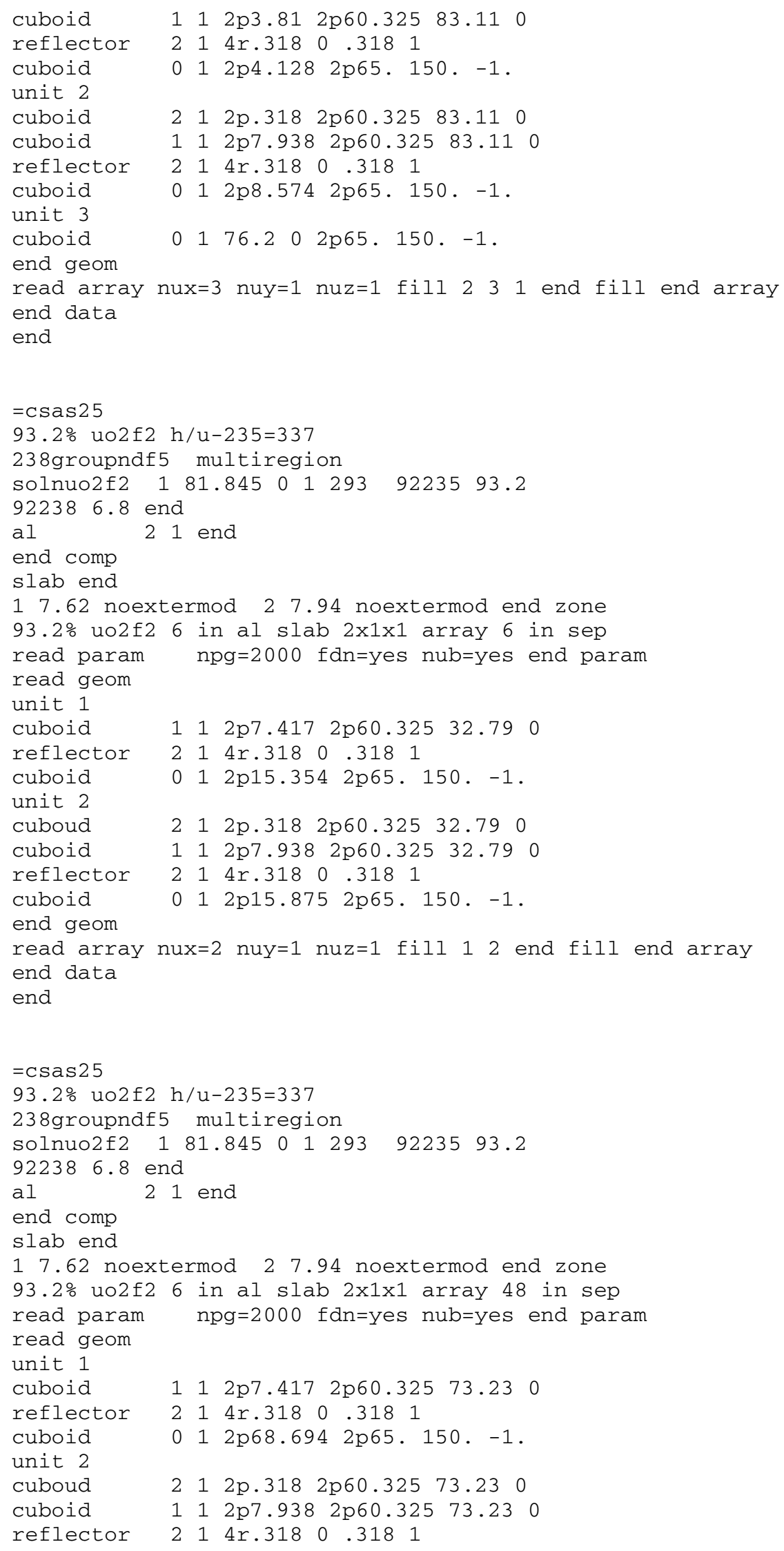




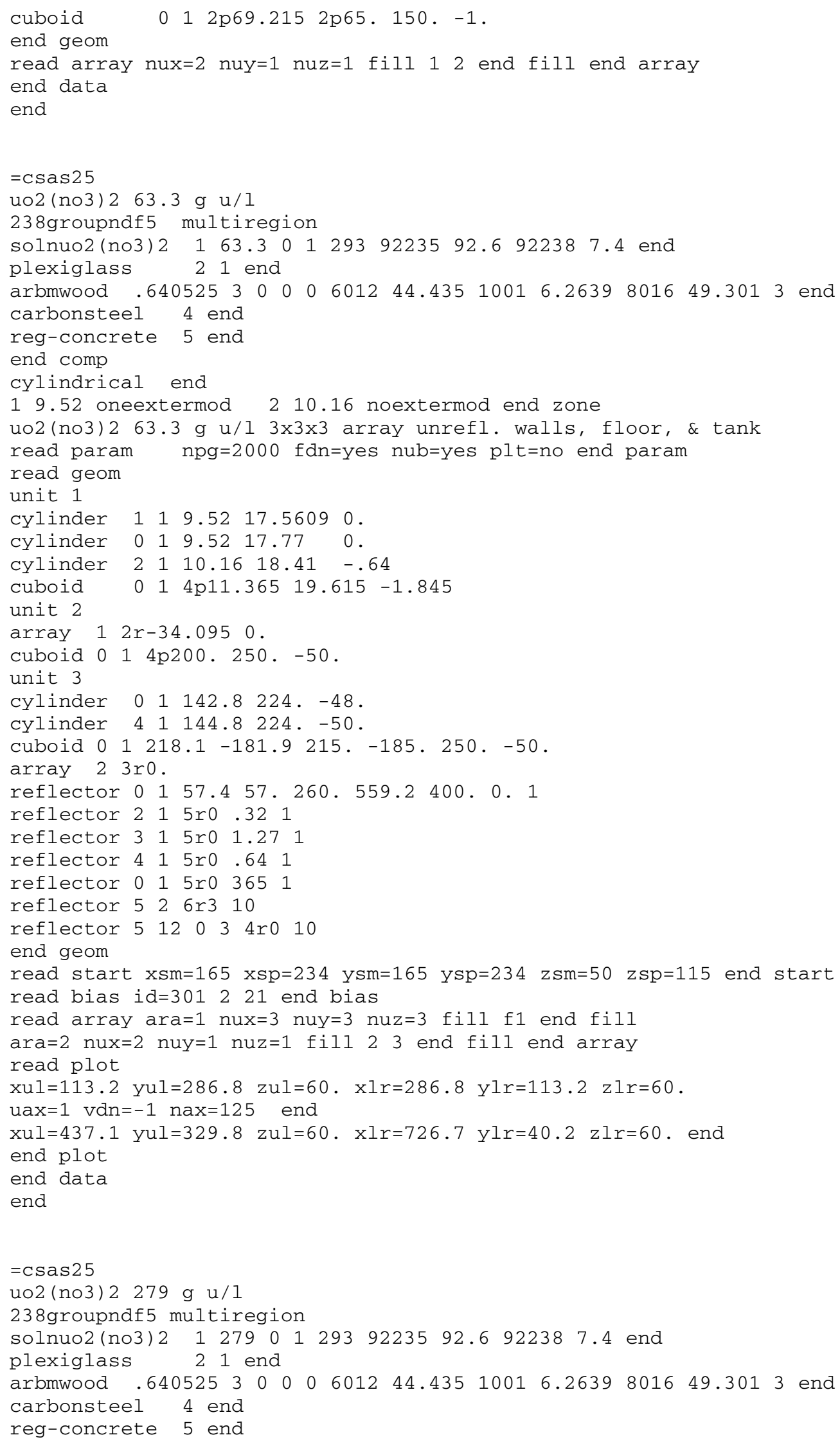




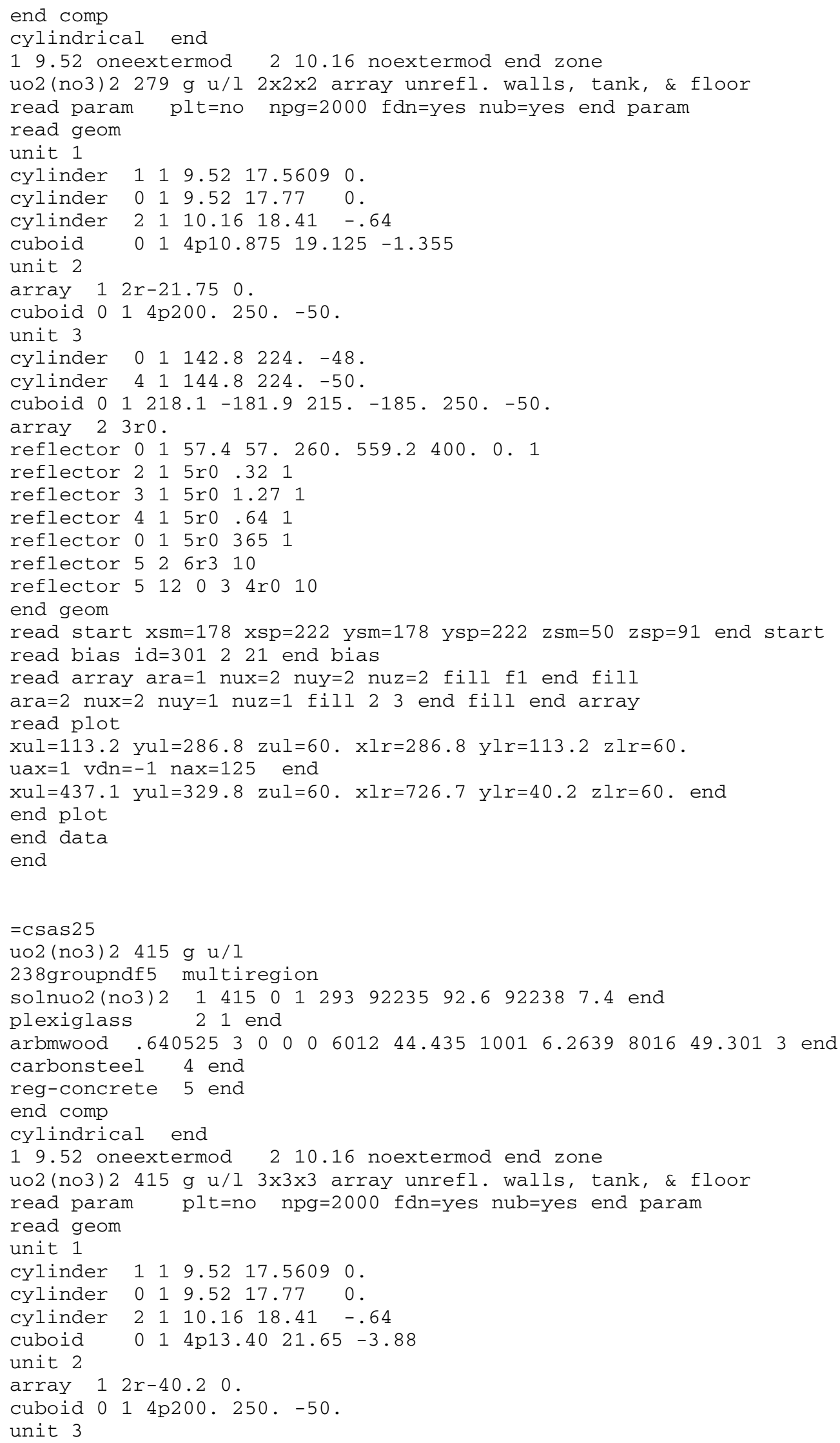




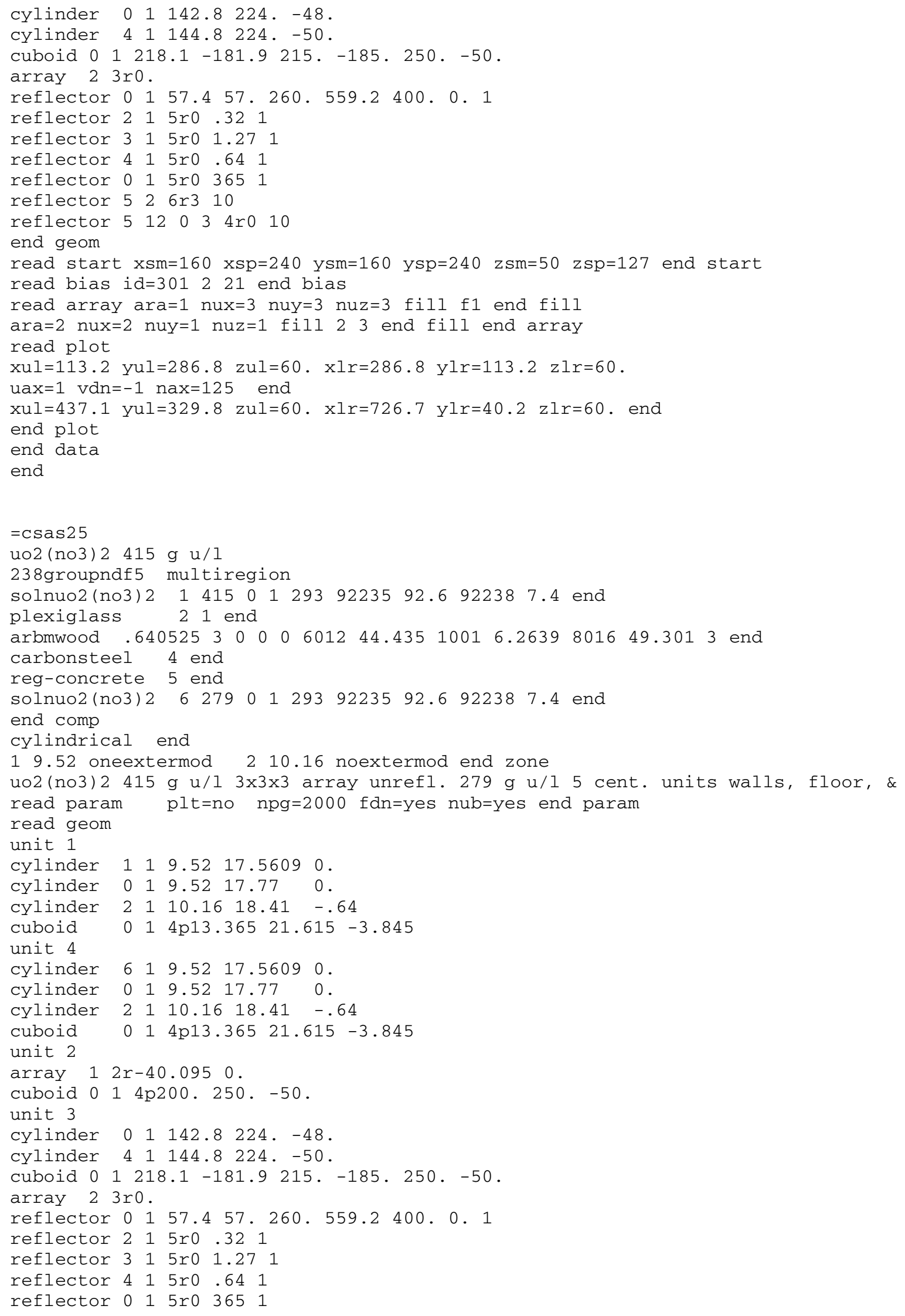




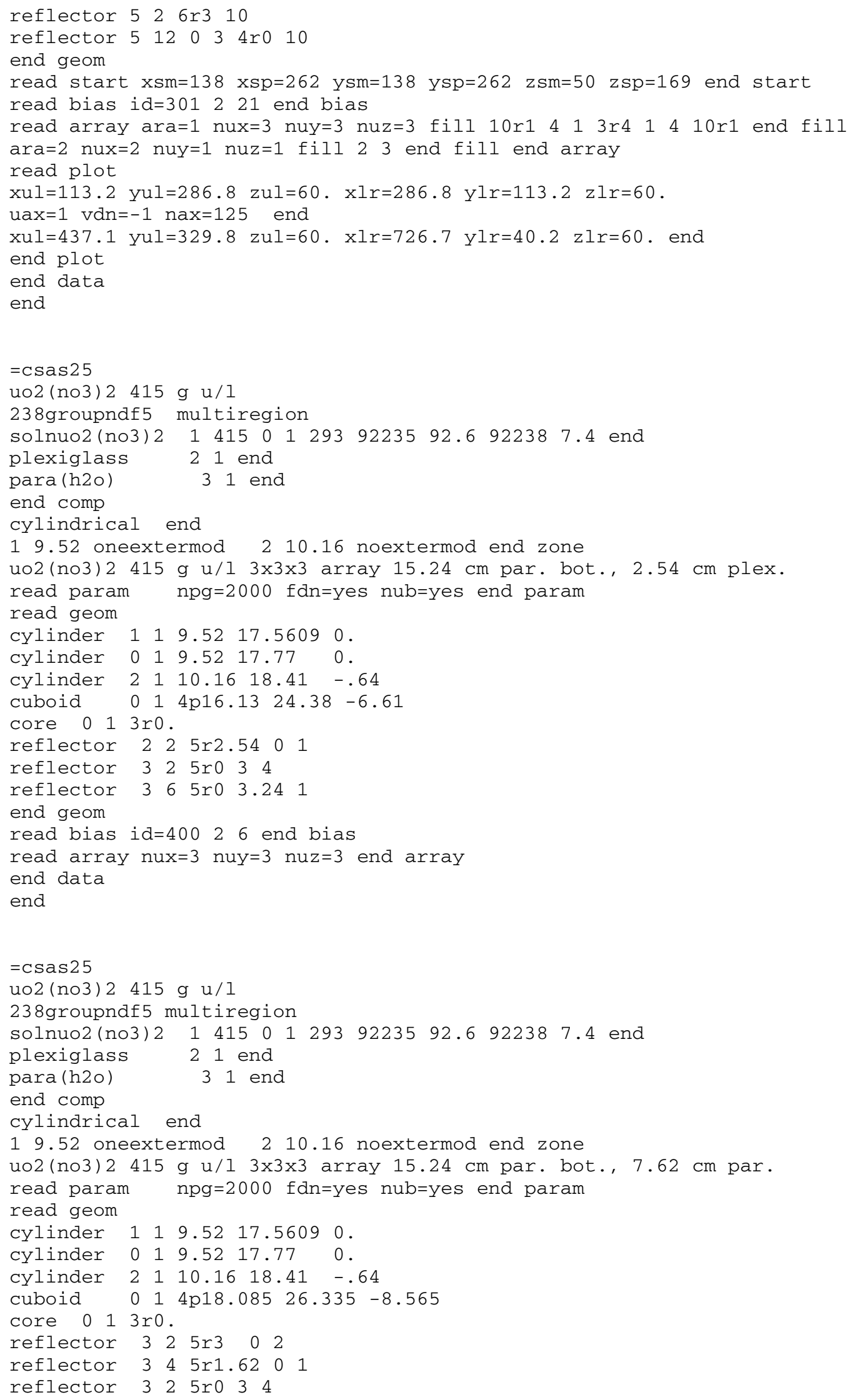




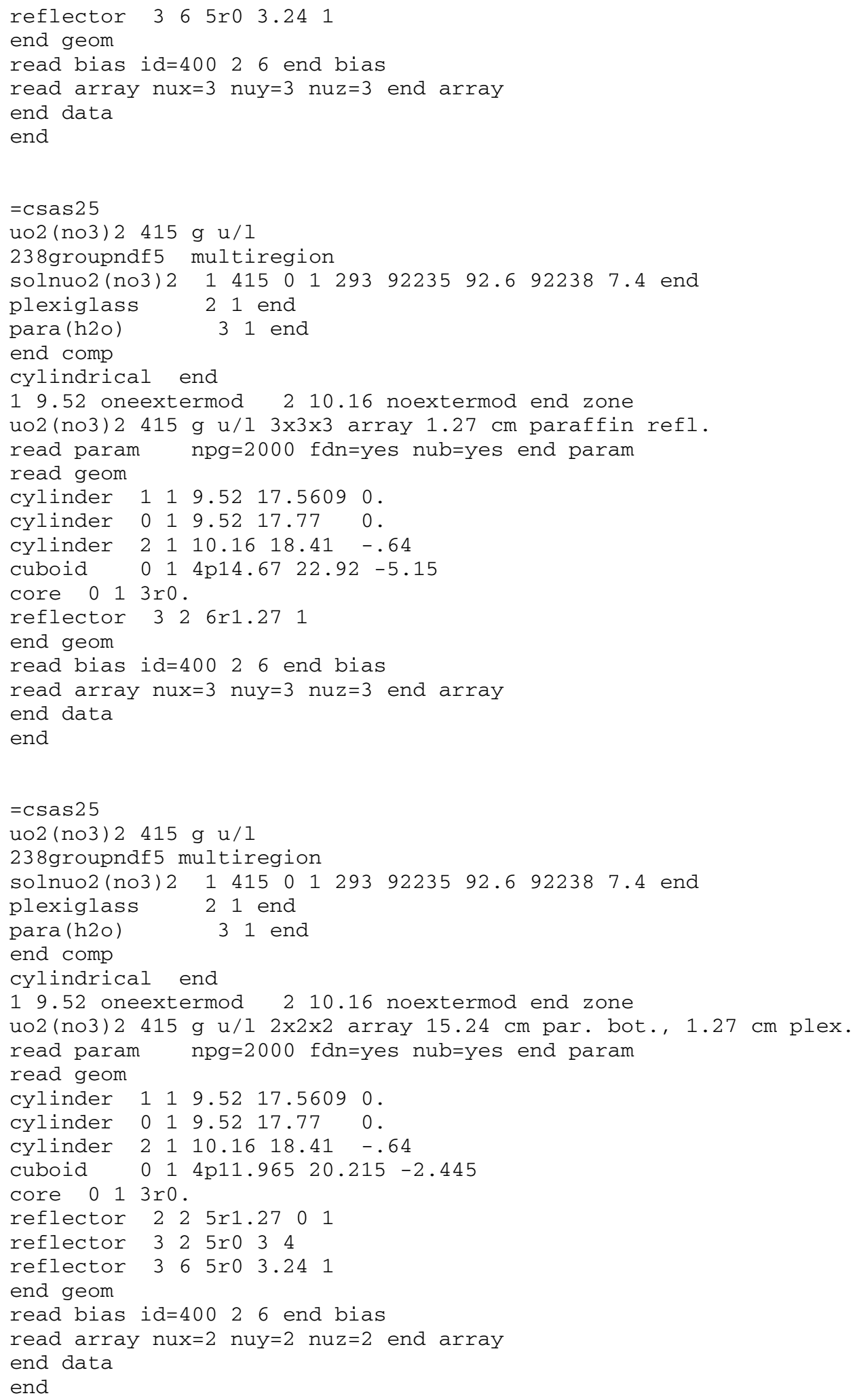




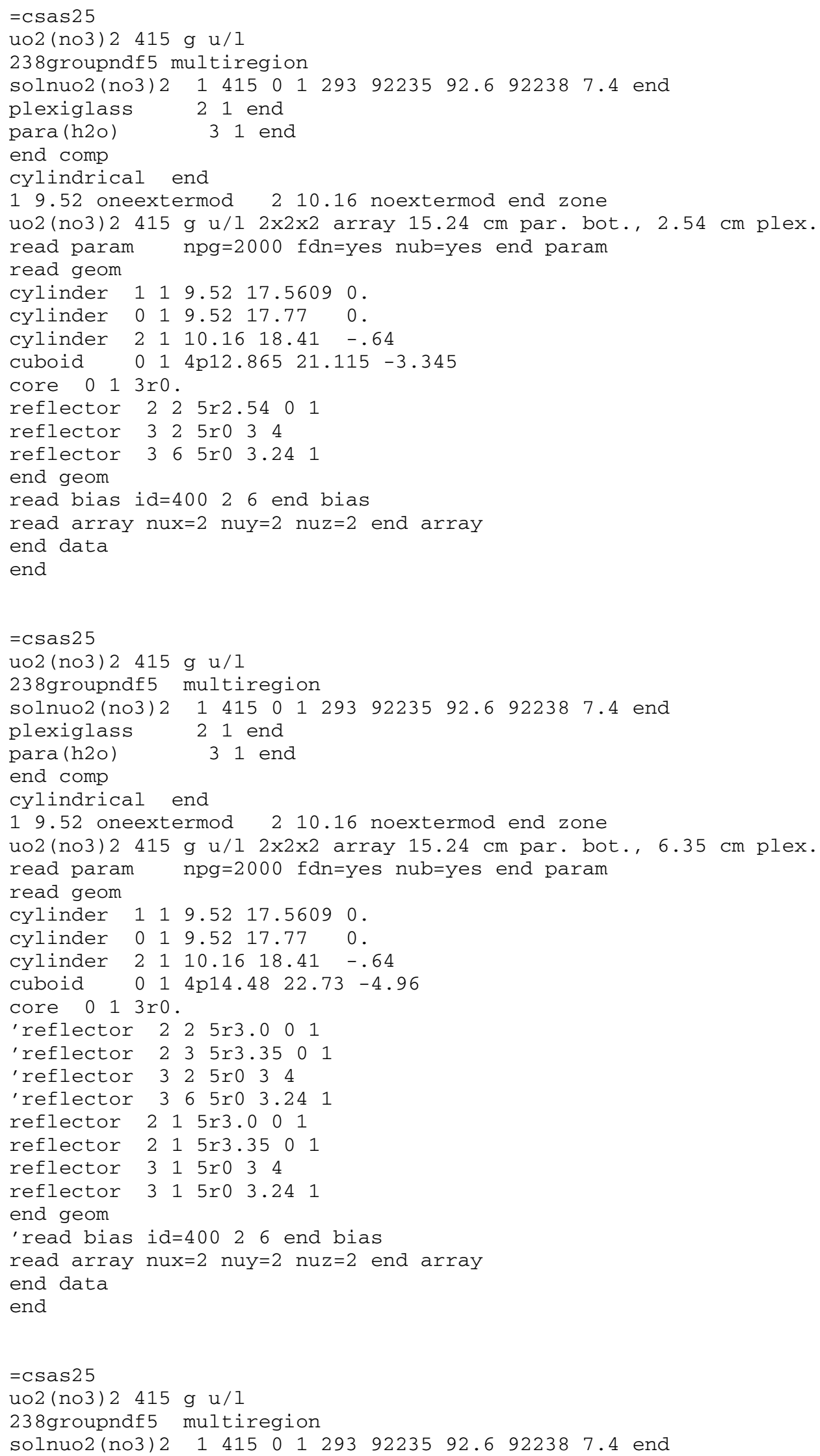




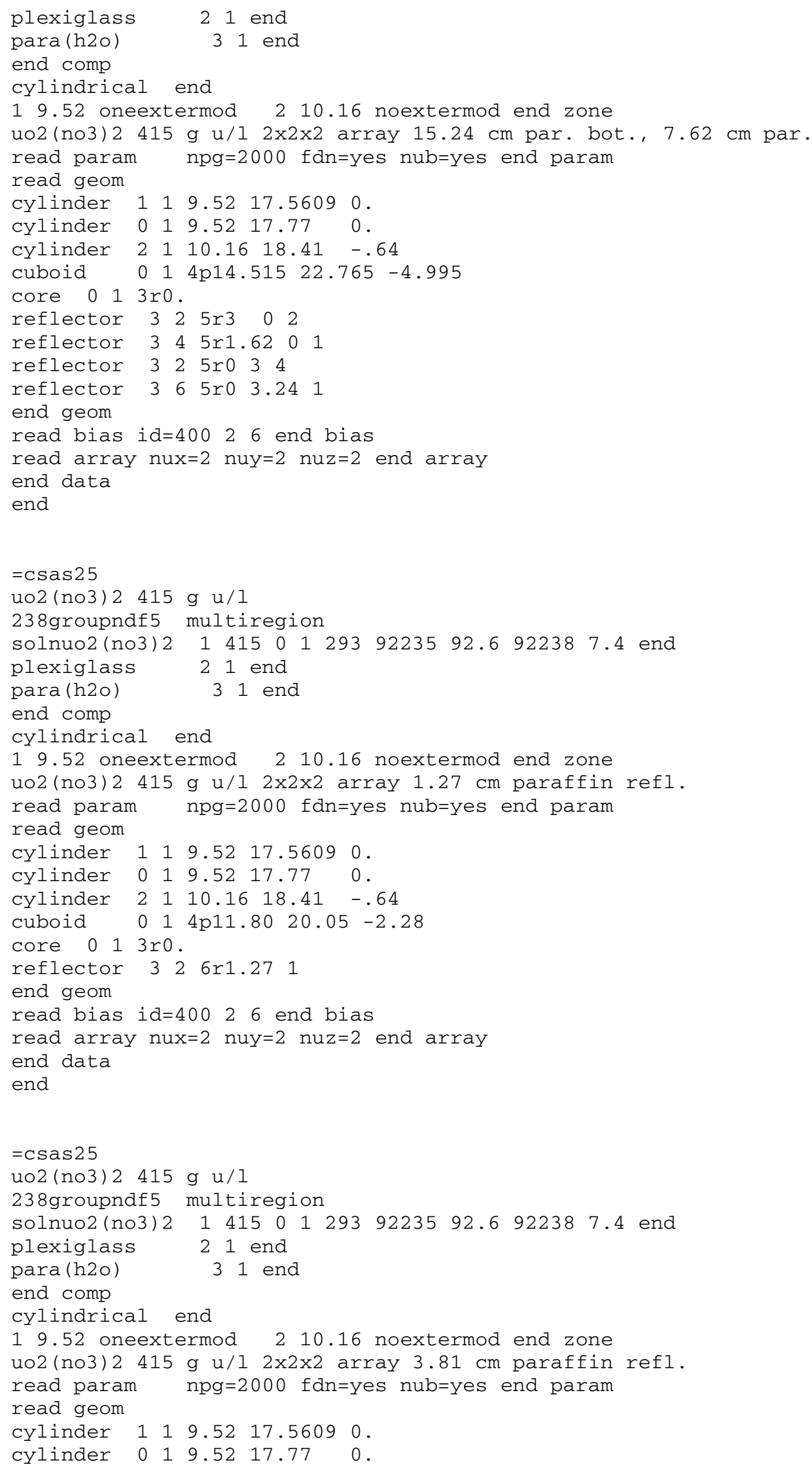


cylinder $2 \quad 1 \quad 10.16 \quad 18.41 \quad-.64$

cuboid $014 \mathrm{p} 13.615 \quad 21.865 \quad-4.095$

core $013 r 0$.

reflector $326 r 3.811$

end geom

read bias id $=40026$ end bias

read array nux $=2$ nuy $=2$ nuz $=2$ end array

end data

end 
Table A.6. Table 6 input data

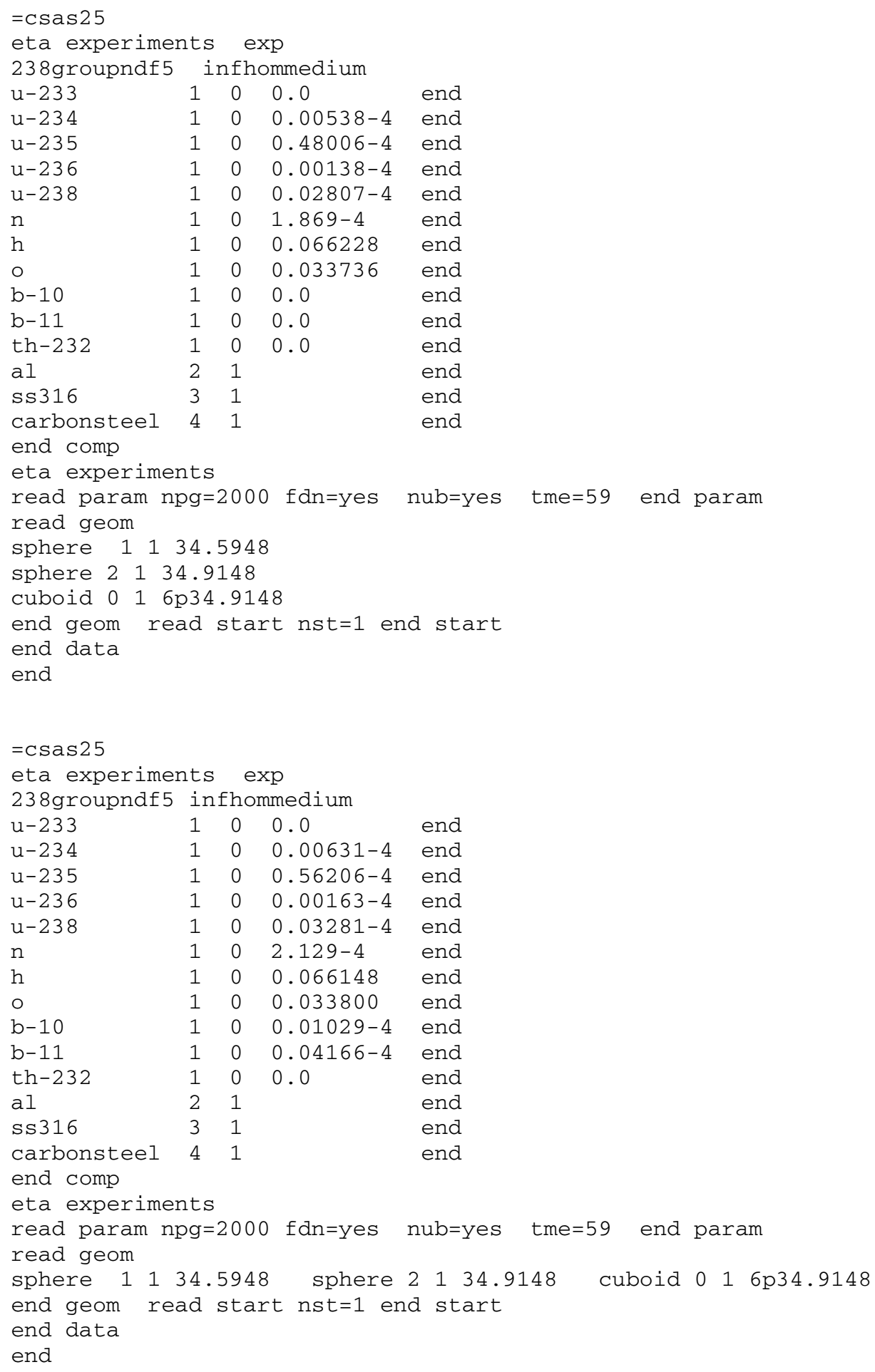




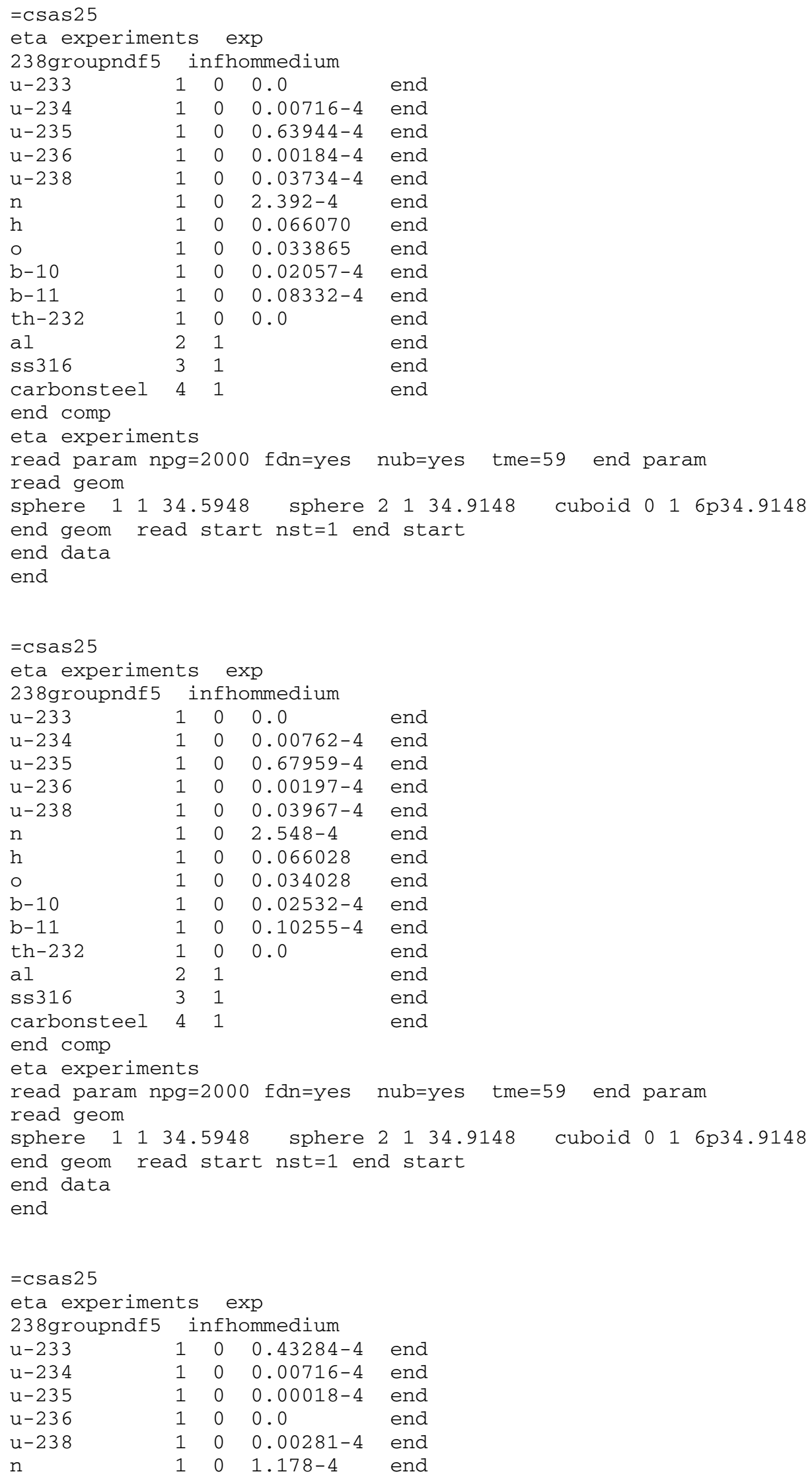




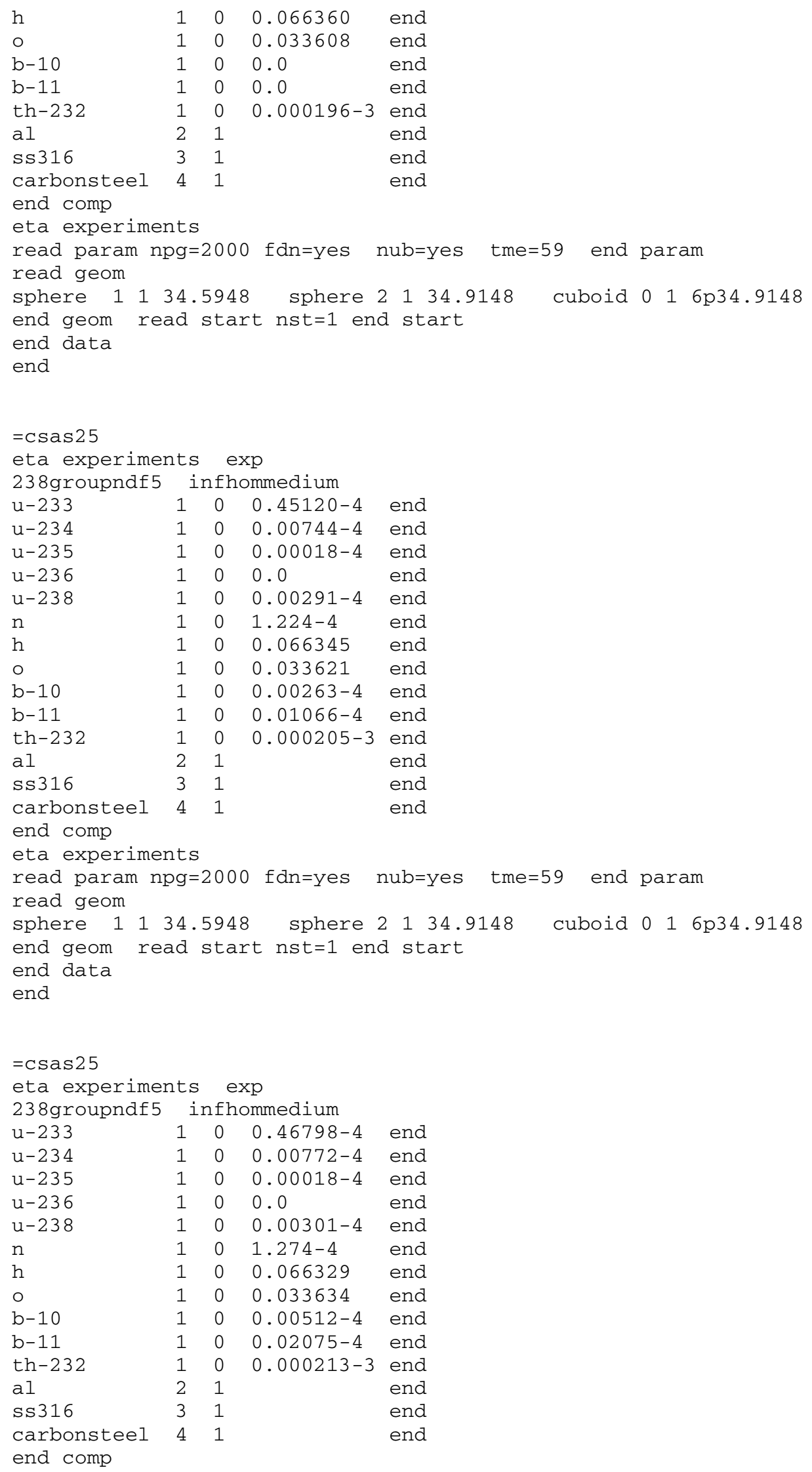




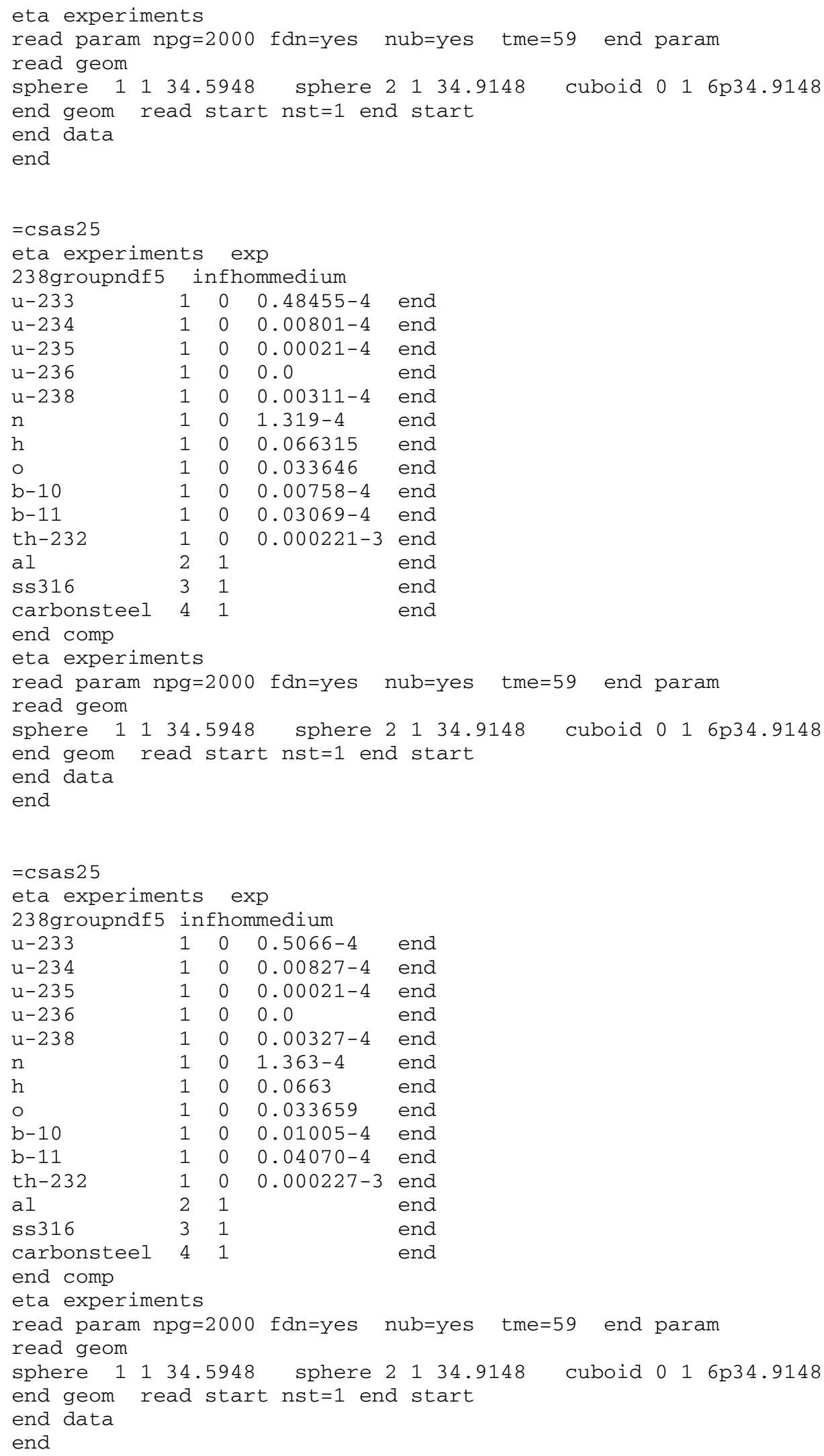




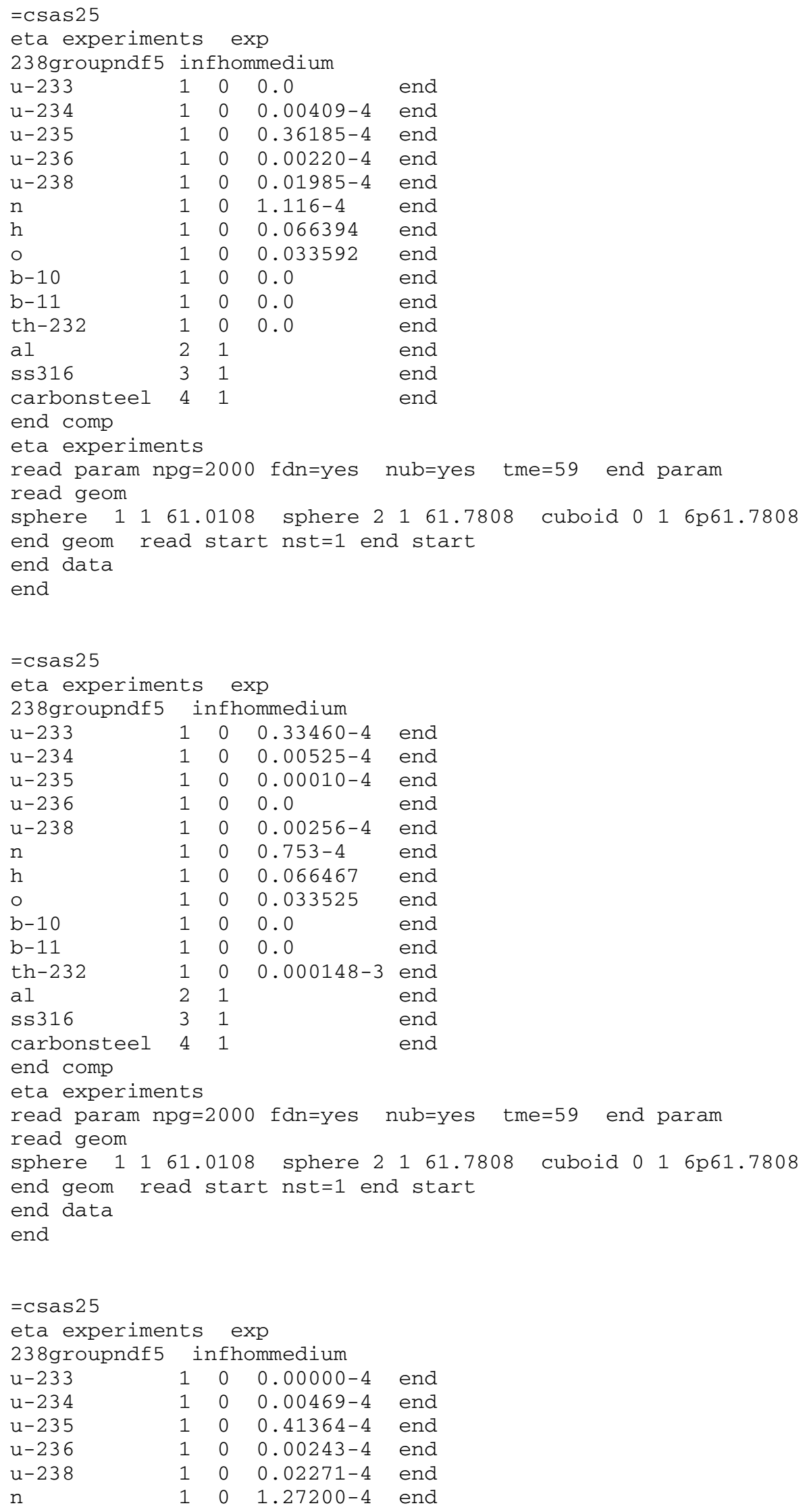




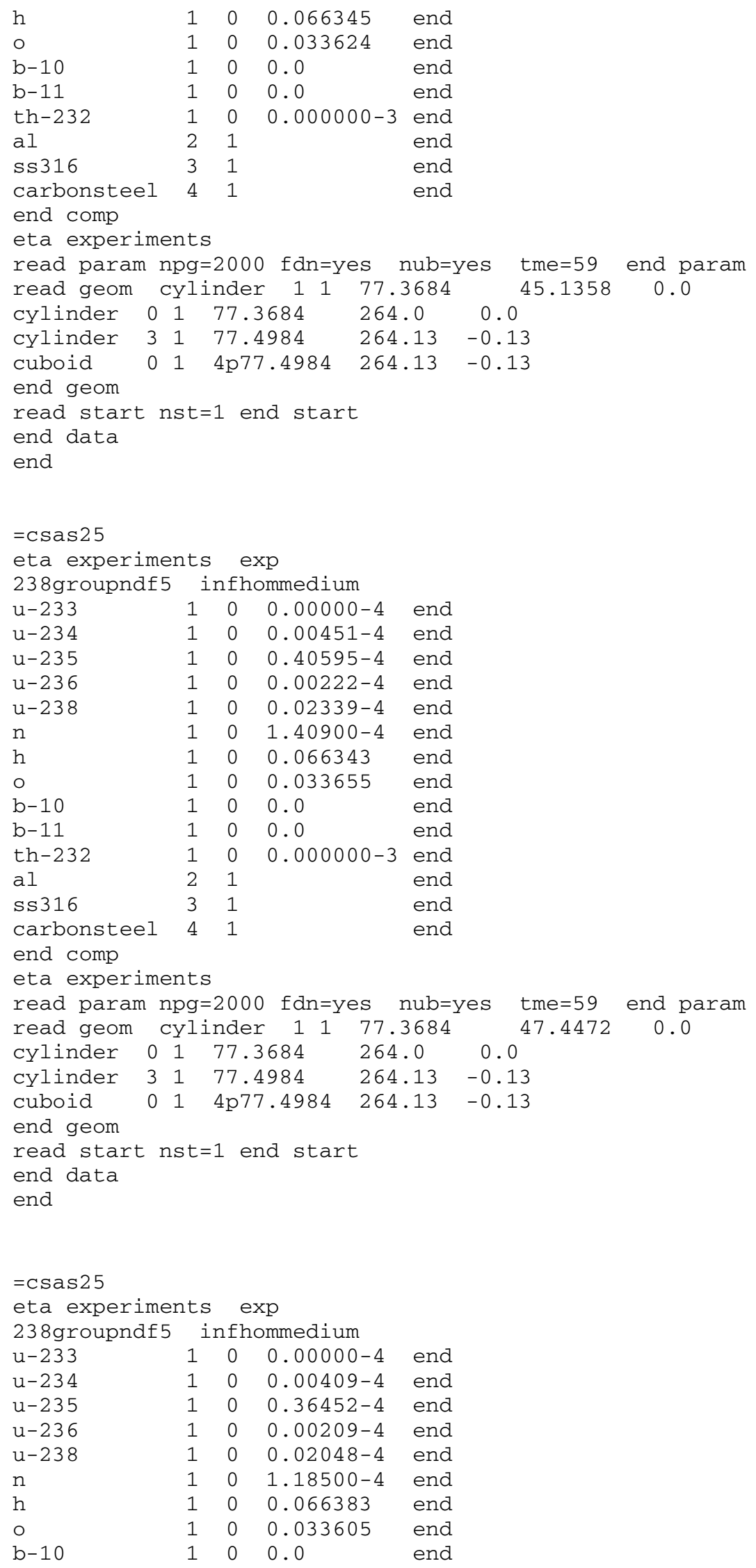




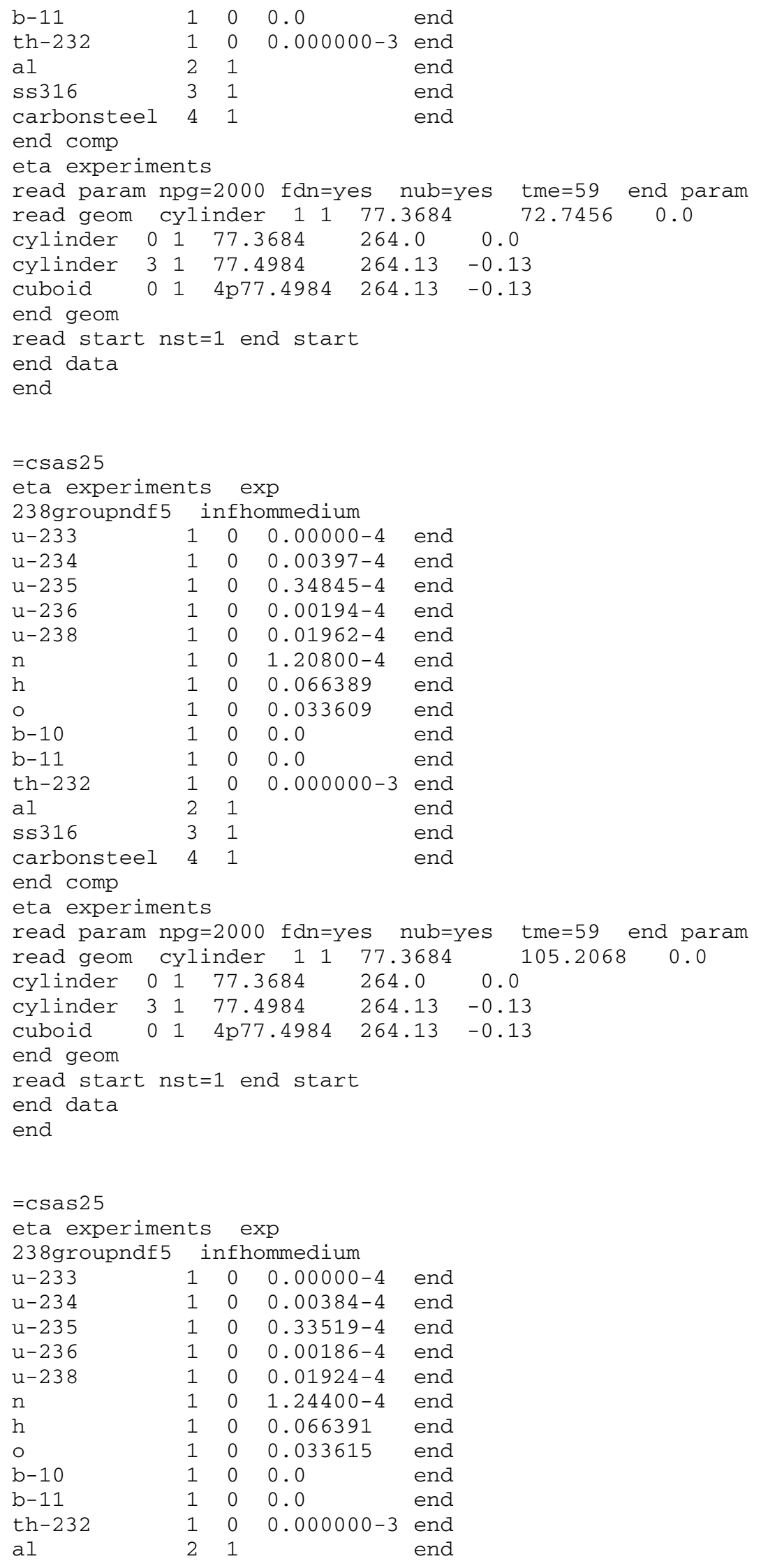




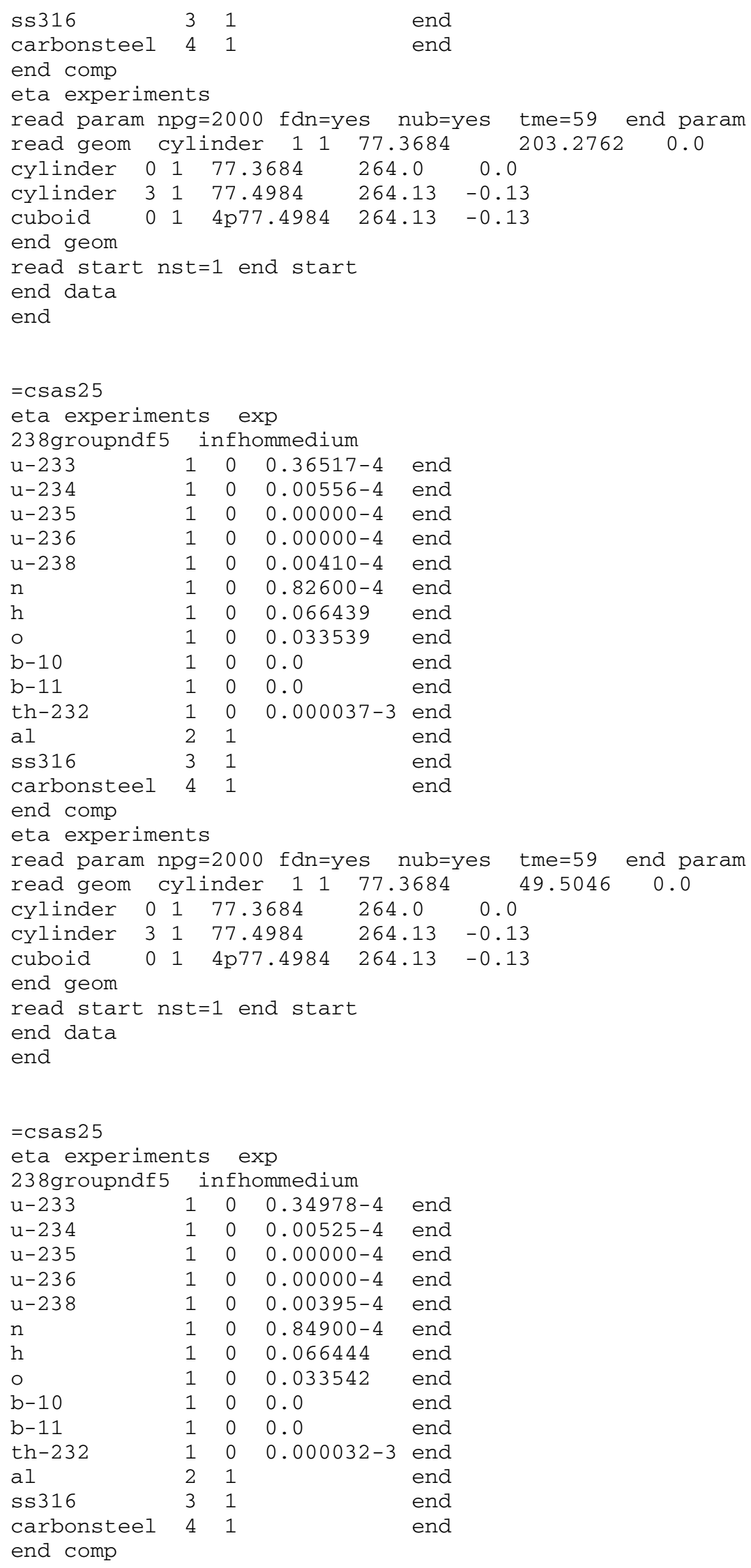




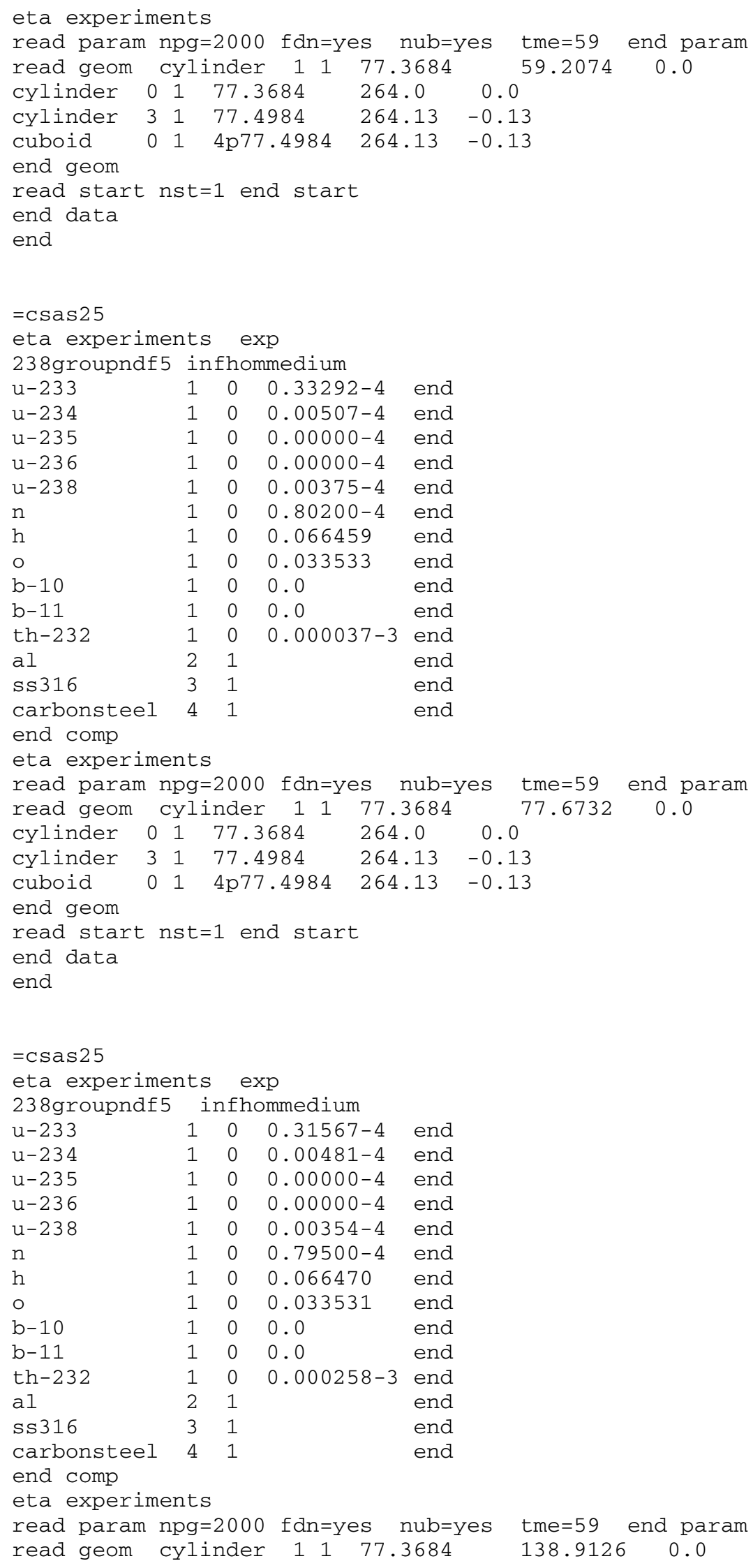




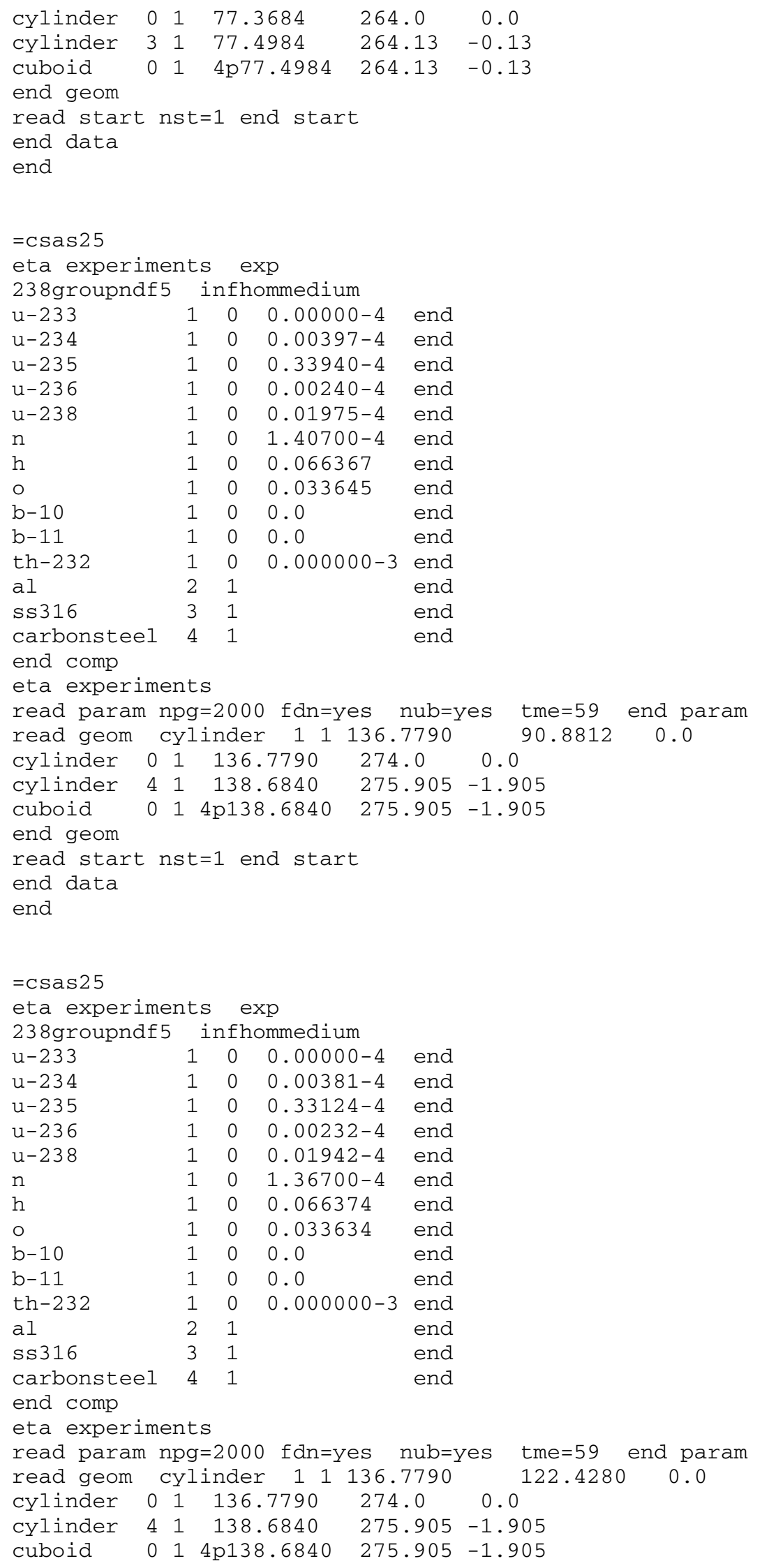




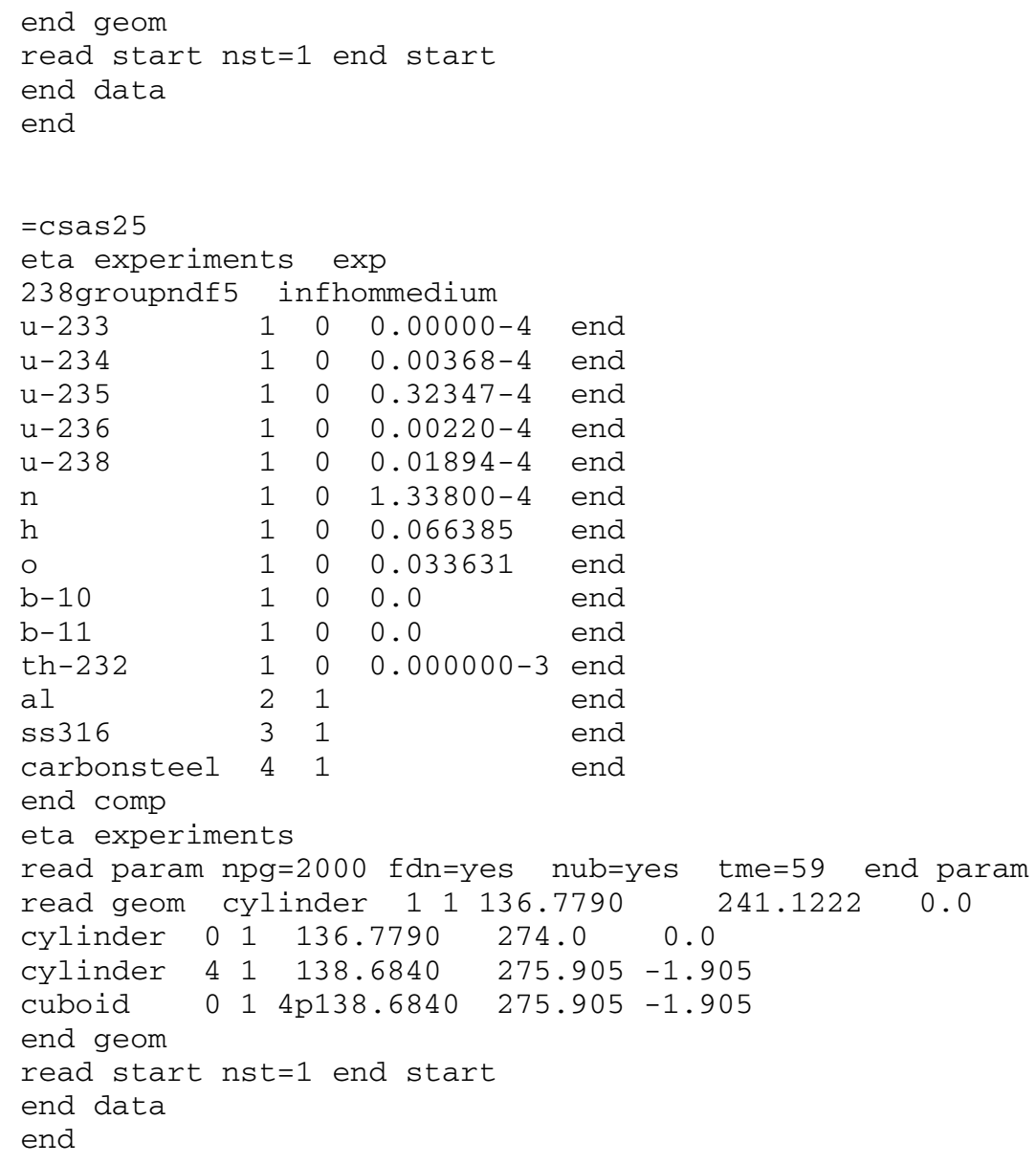




\section{APPENDIX B}

\section{KENO-VI INPUT DATA USED FOR VALIDATION CASES LISTED IN TABLES 1-6 (238 group)}




\title{
APPENDIX B \\ KENO-VI INPUT DATA USED FOR VALIDATION CASES LISTED IN TABLES 1-6 (238 GROUP)
}

\author{
Input data for the validation appears in the same order as listed in Tables 1-6 of Section 1 of this \\ report.

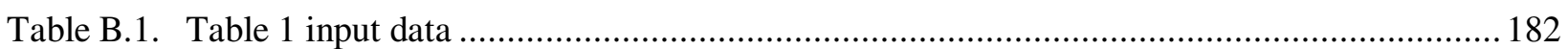

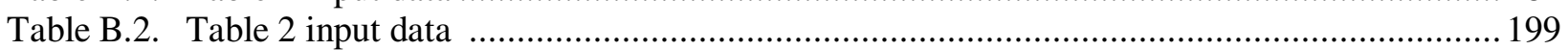

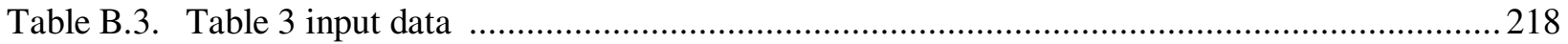

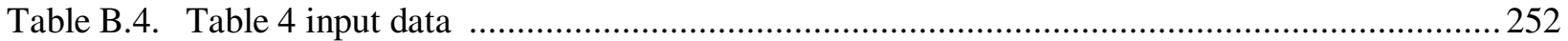

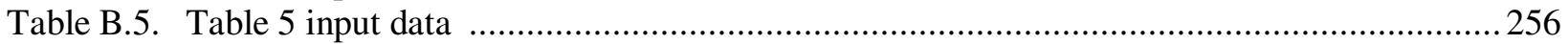

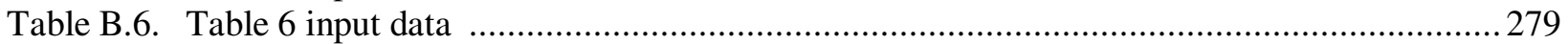


Table B.1. Table 1 input data

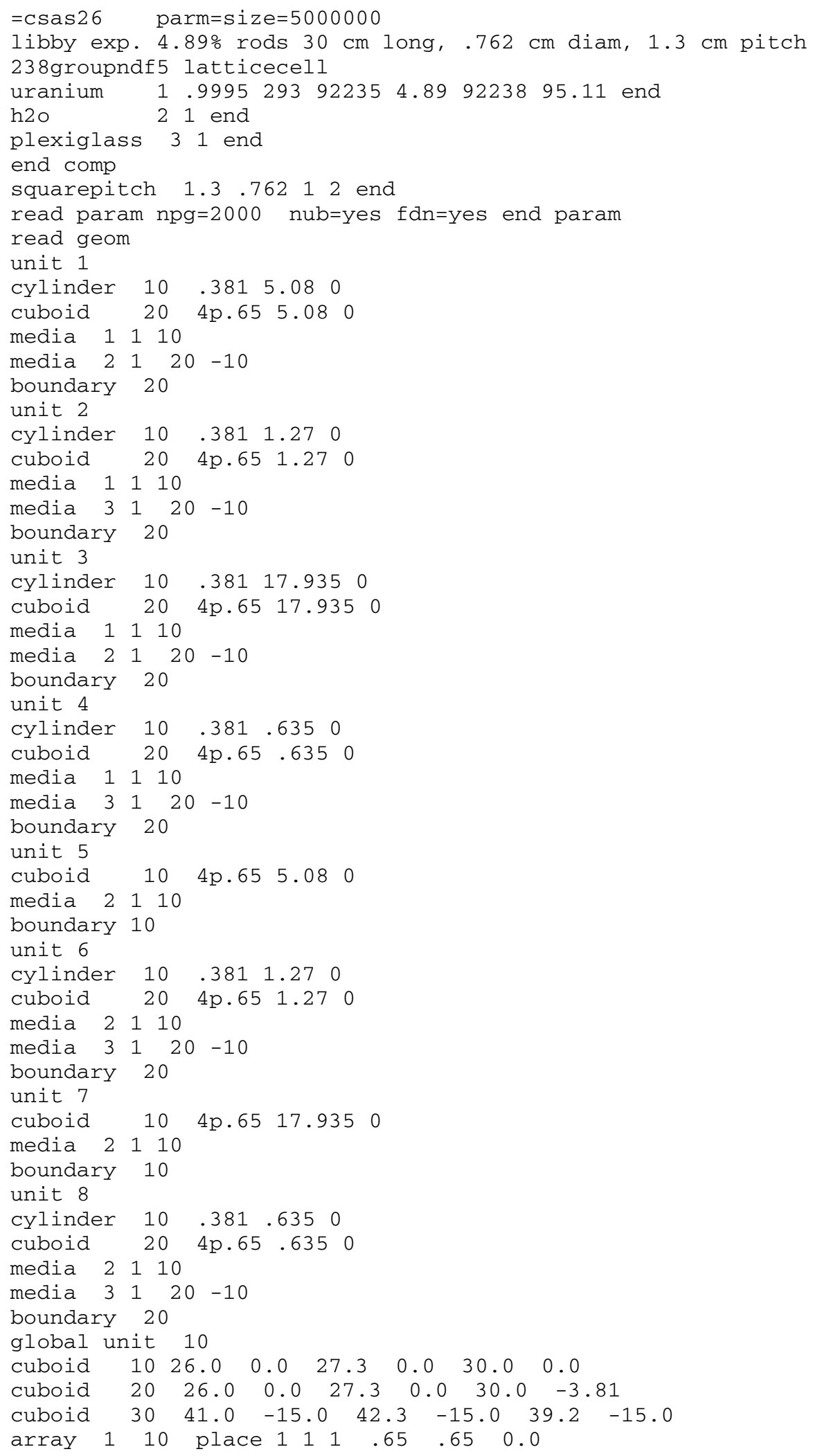




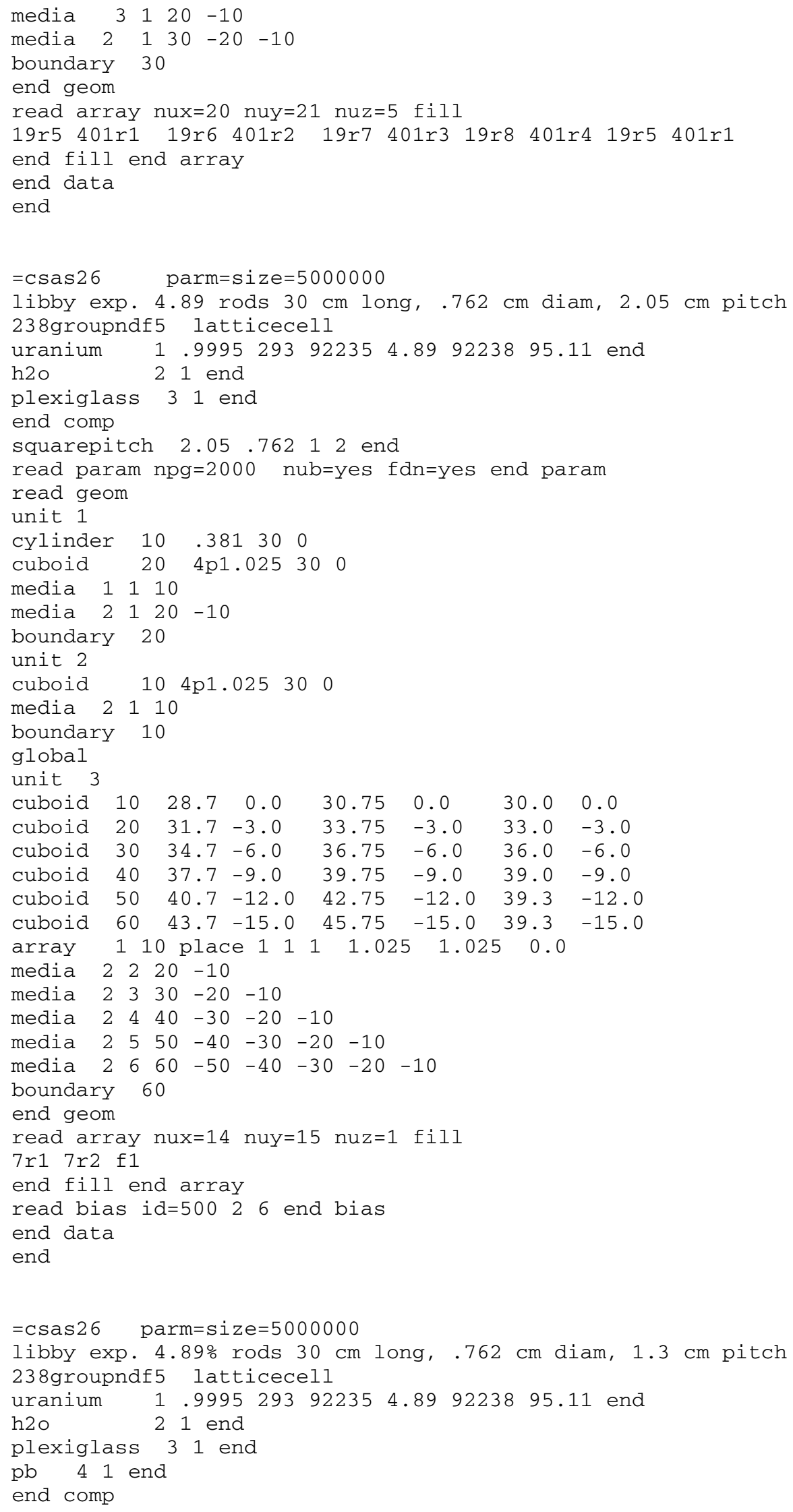




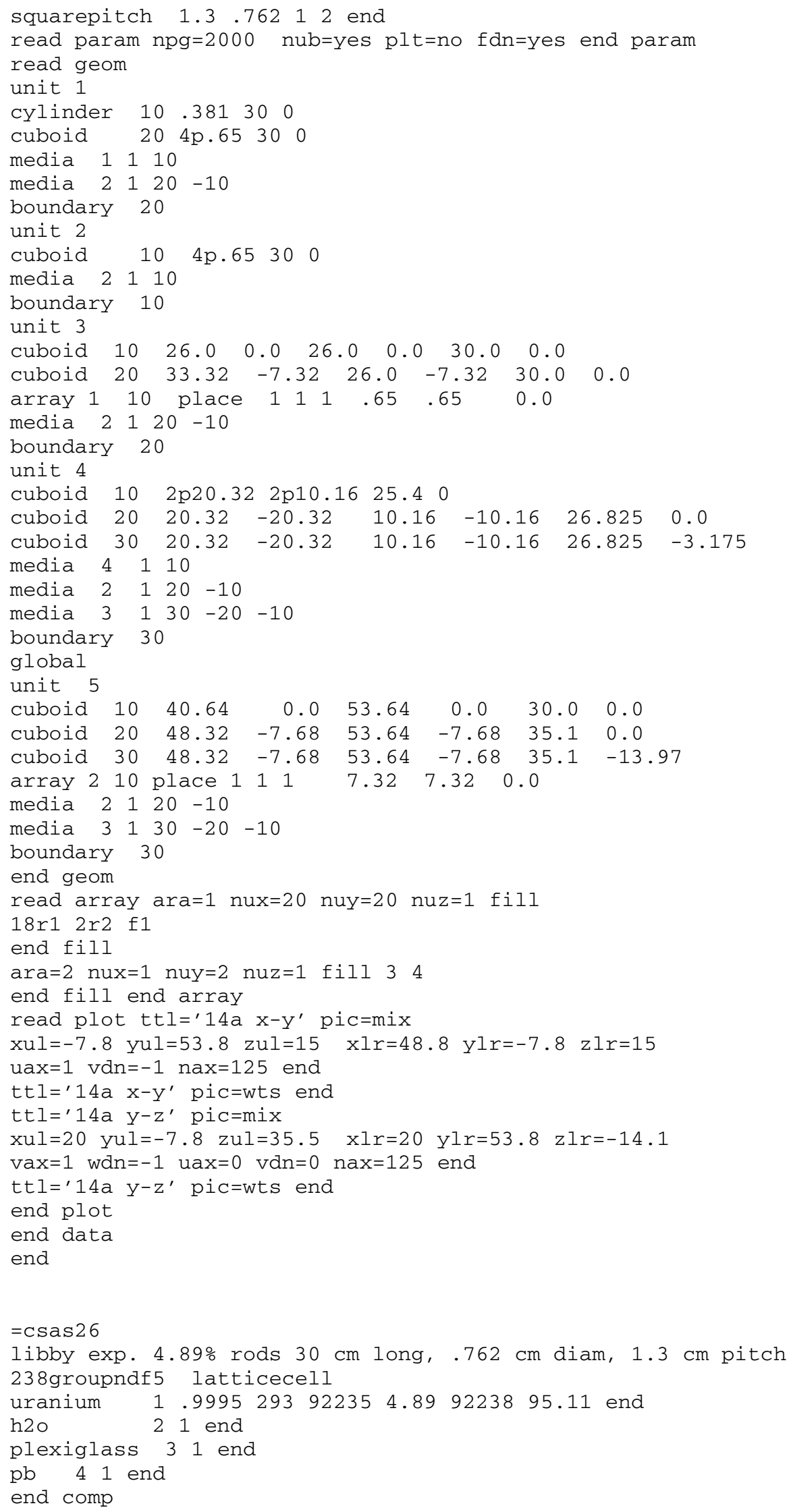




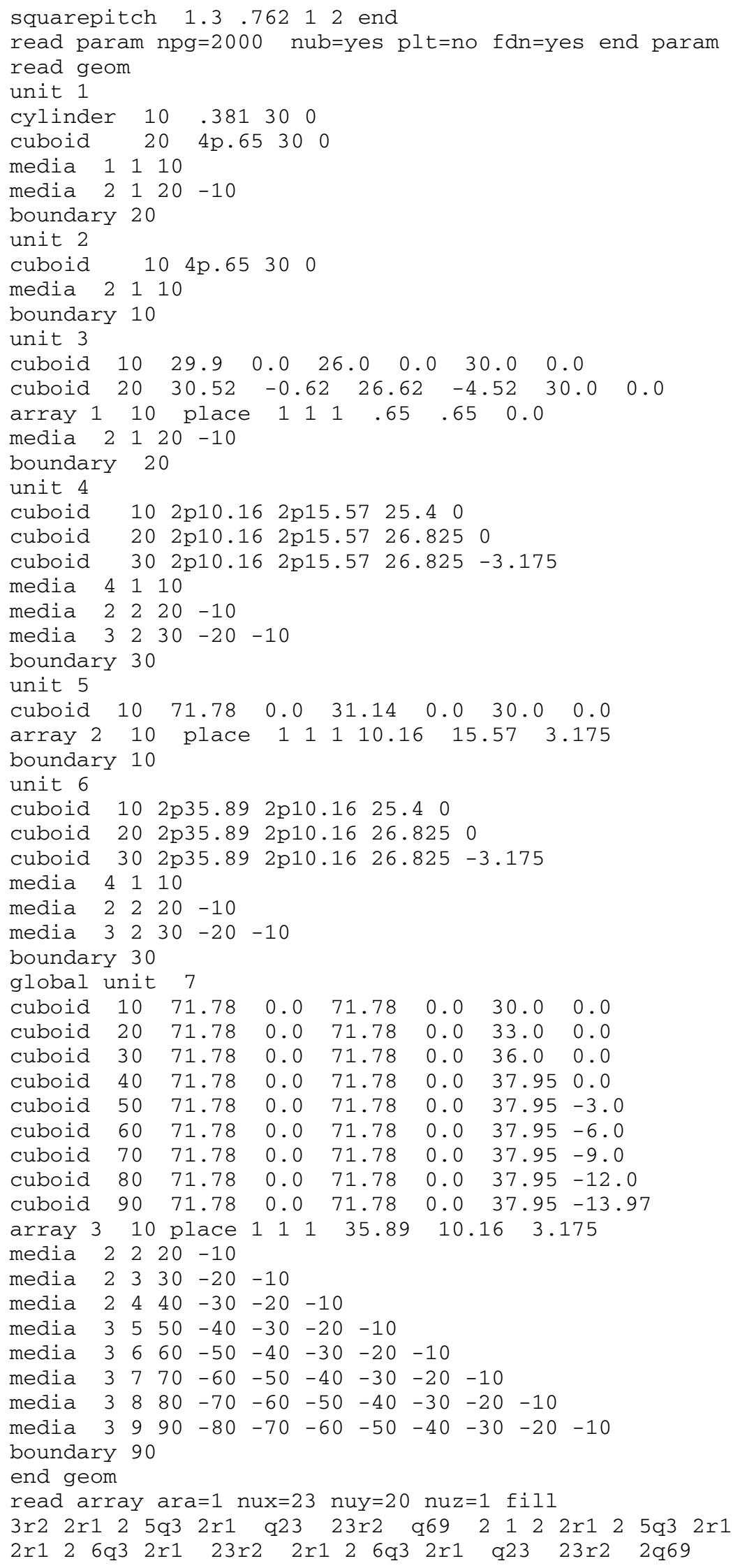




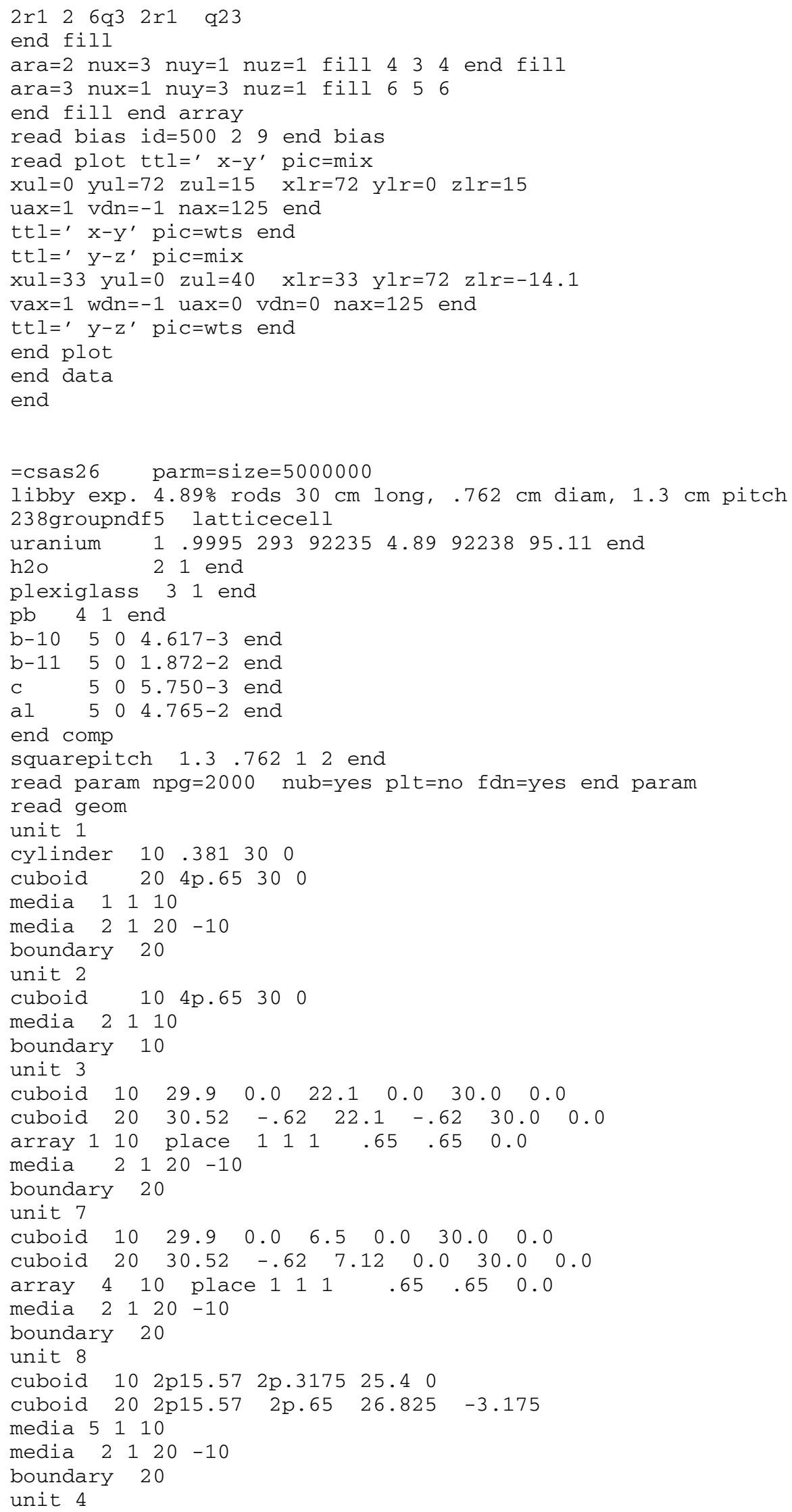




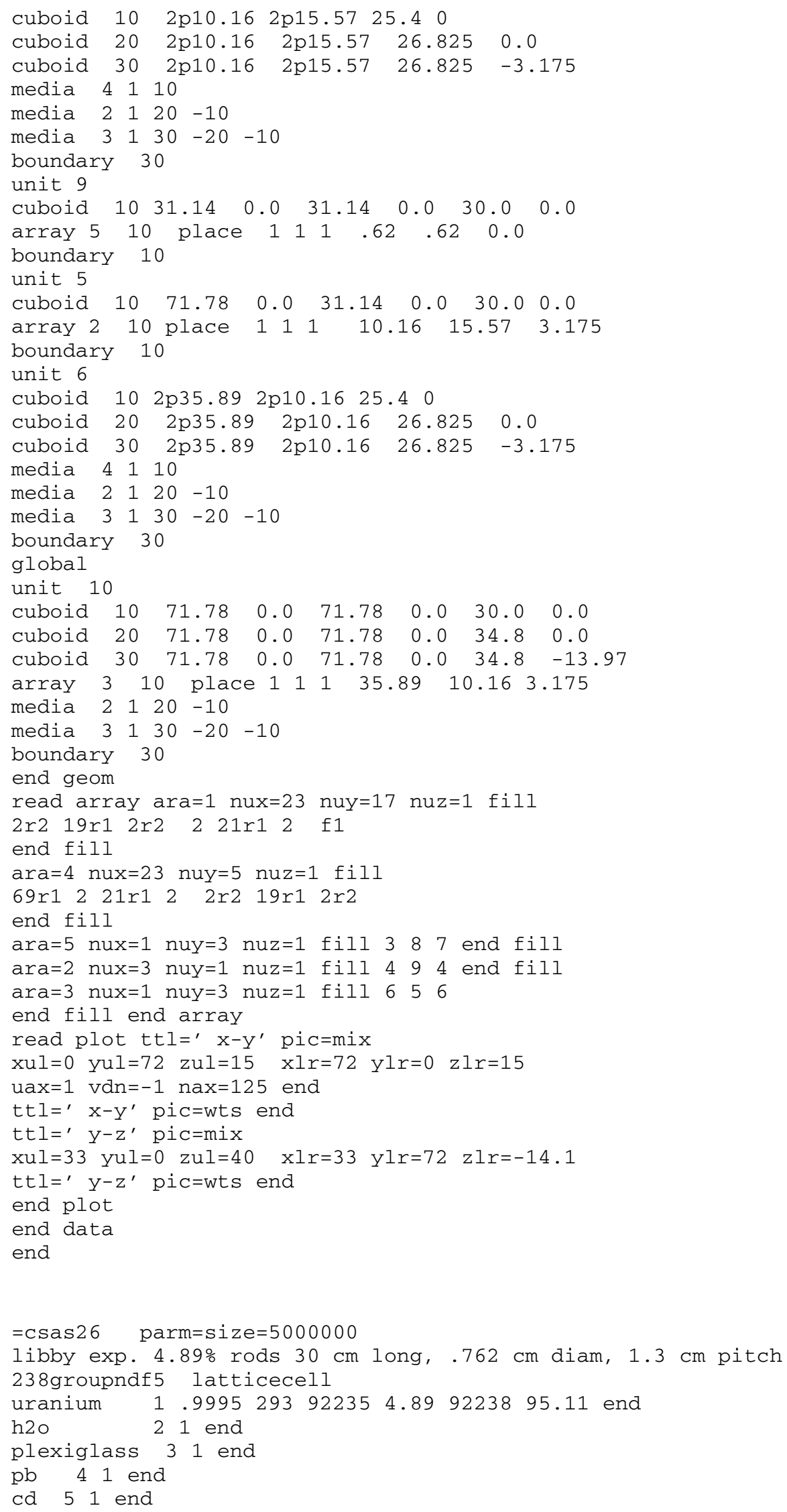




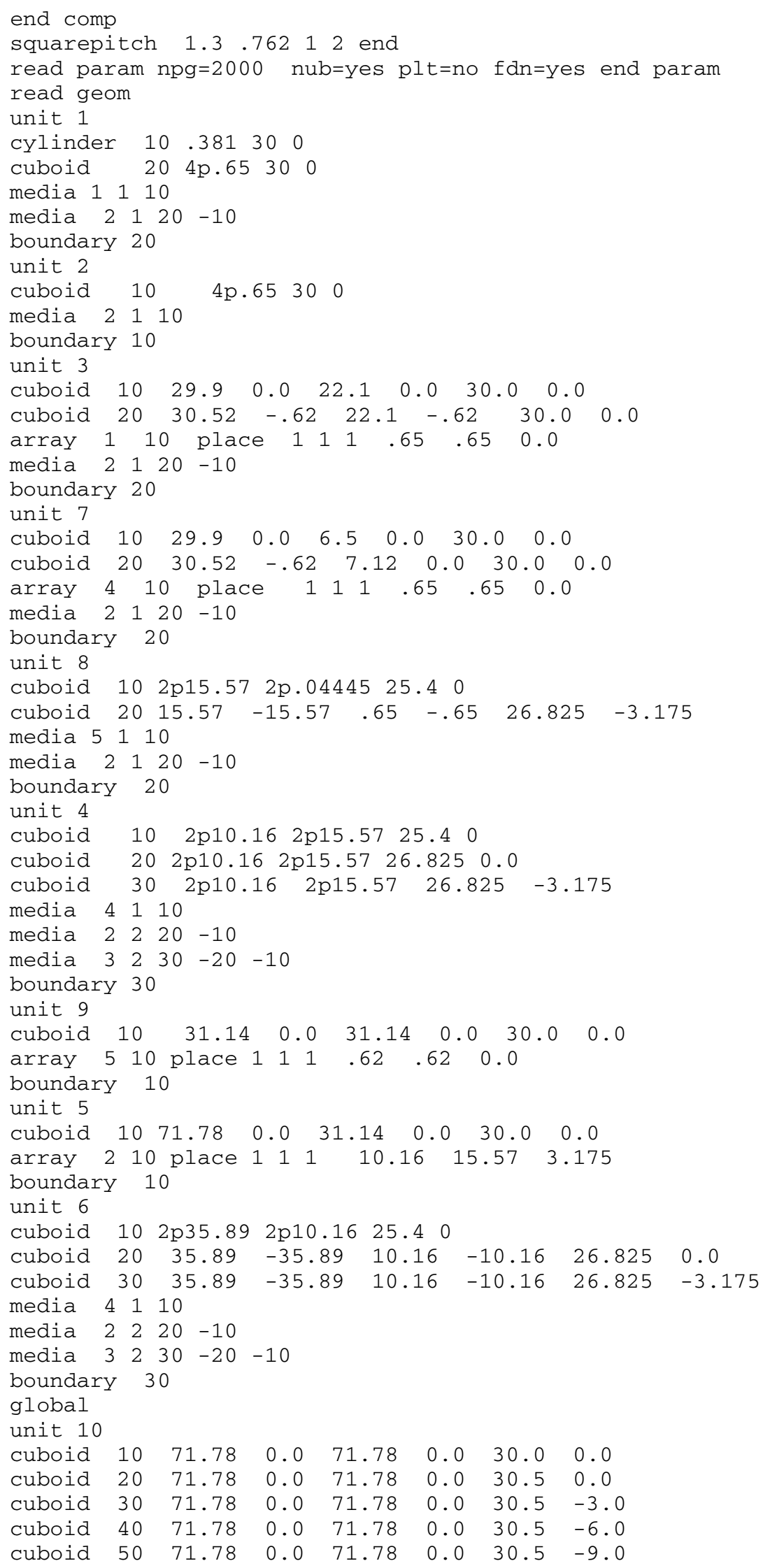




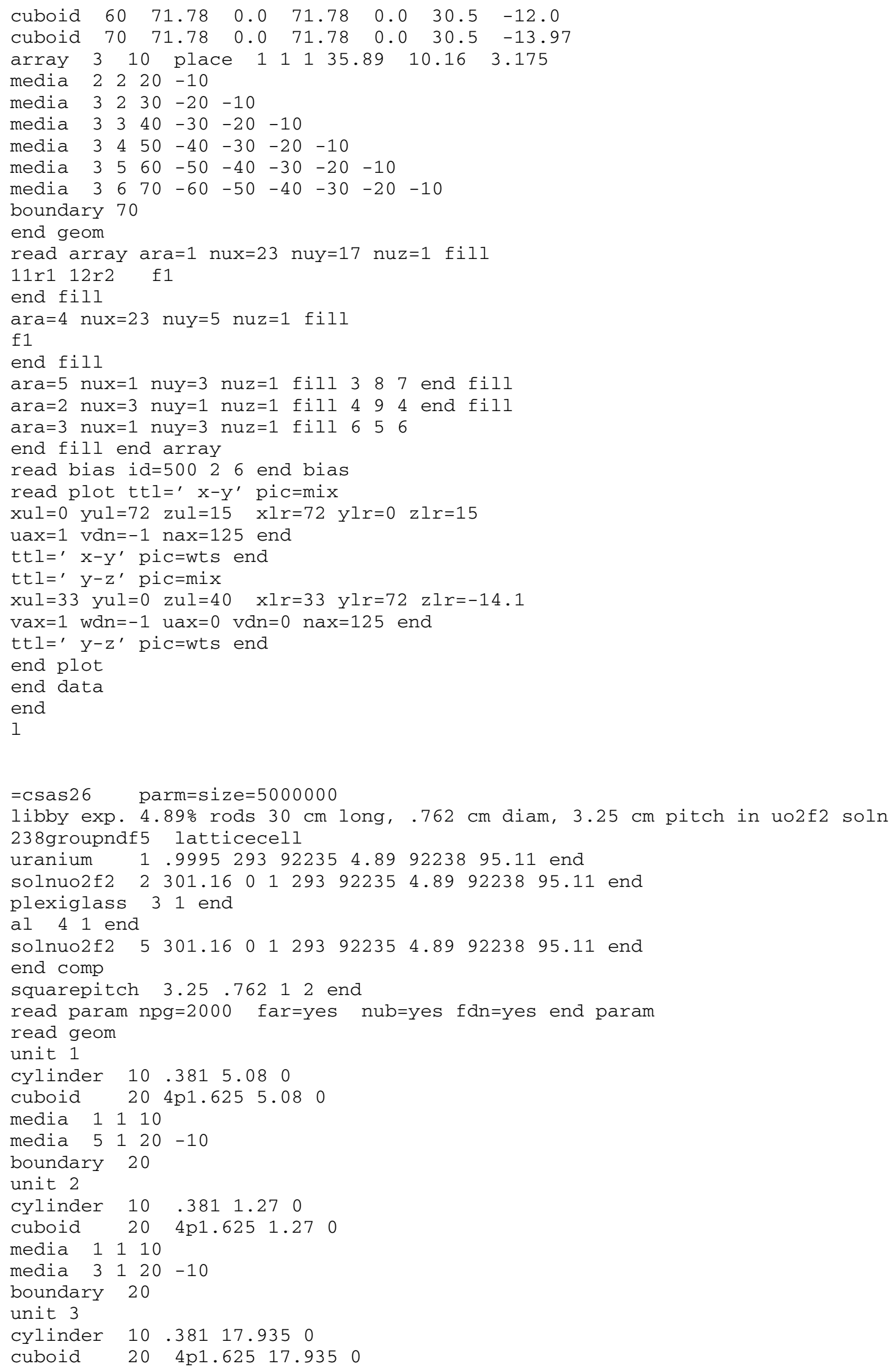




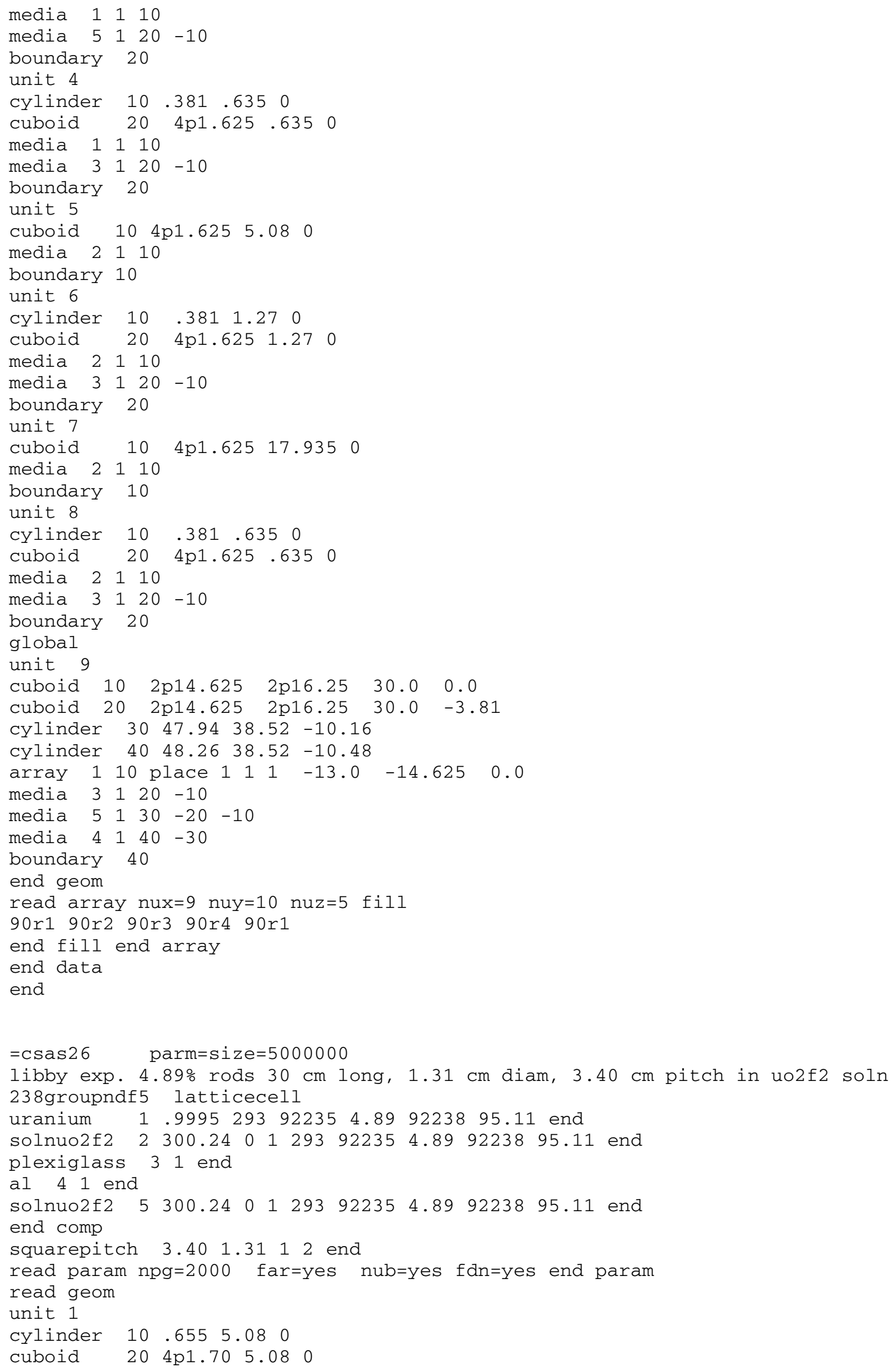




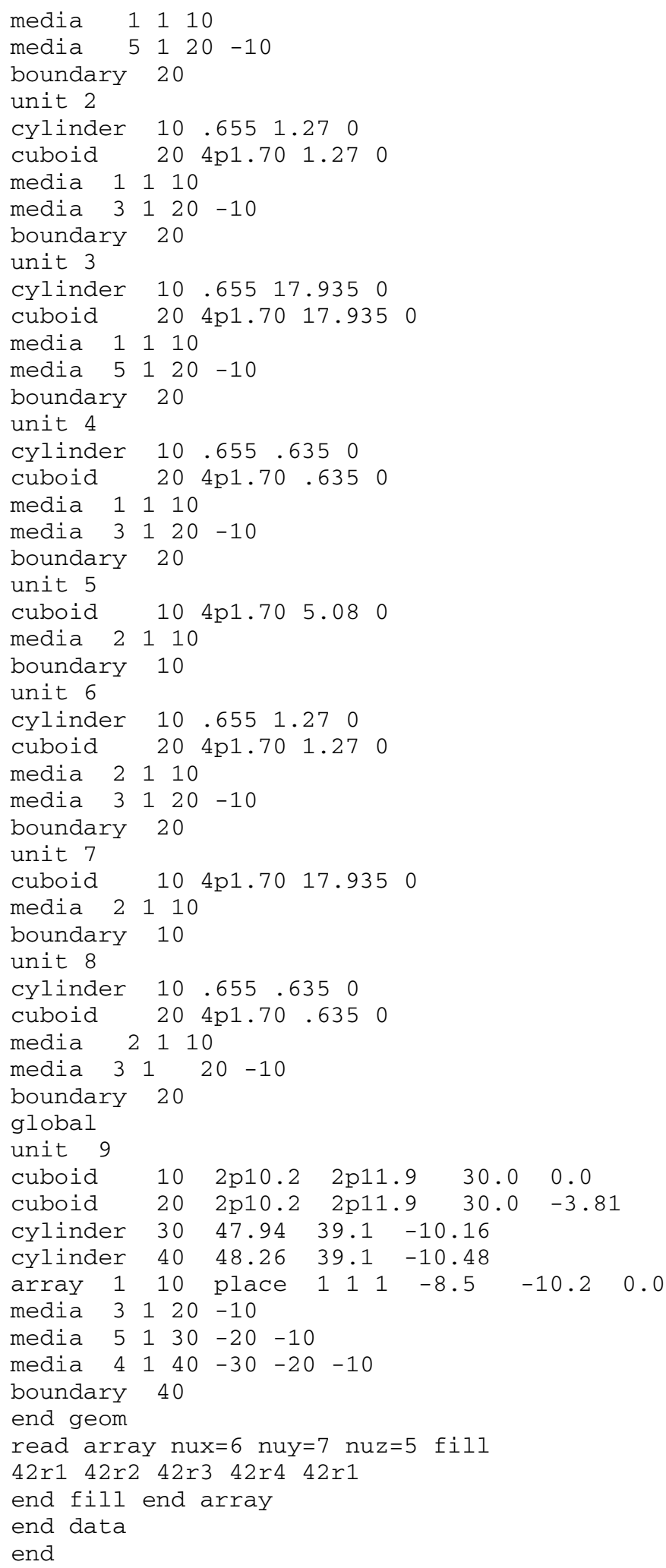




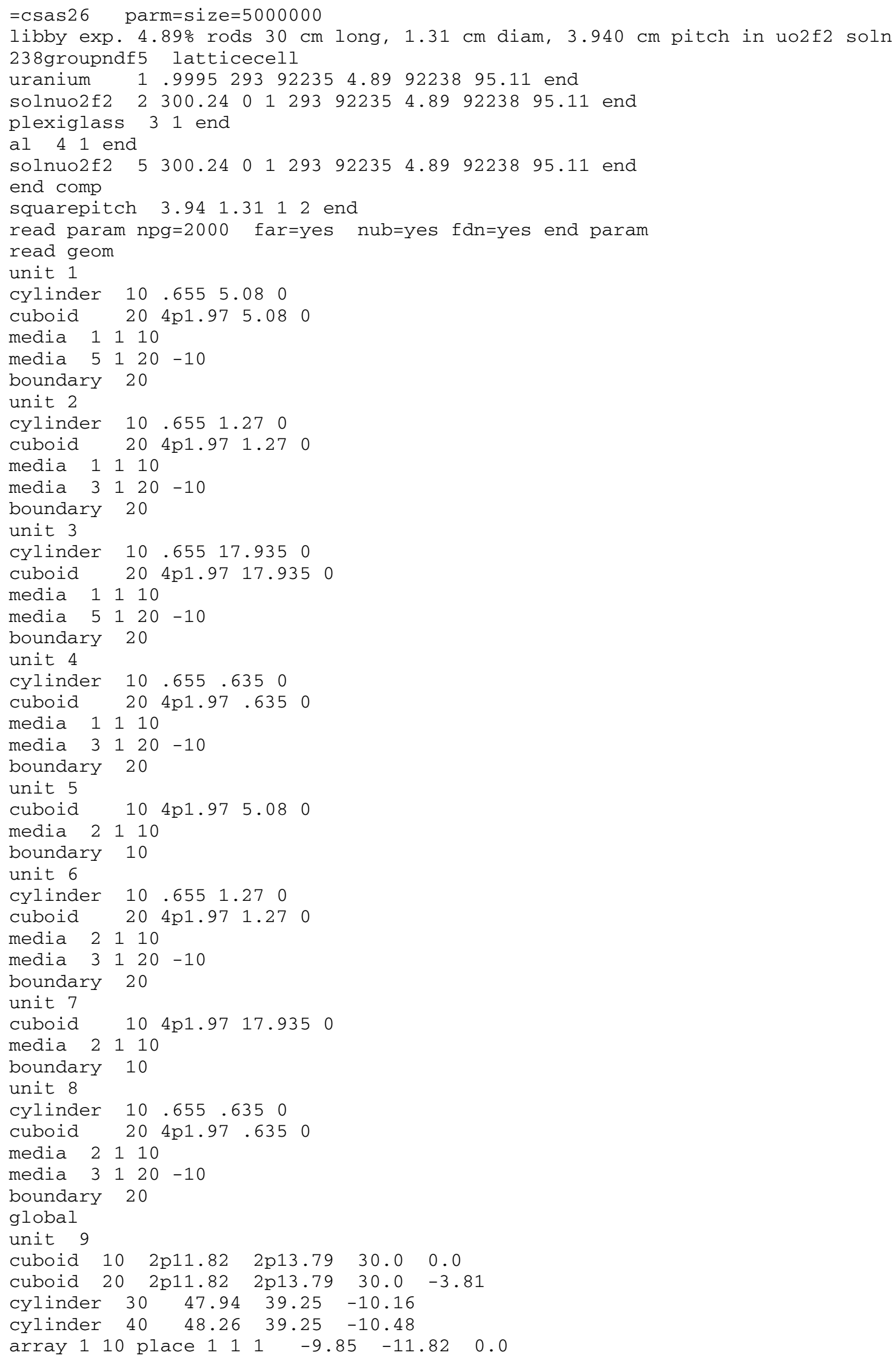




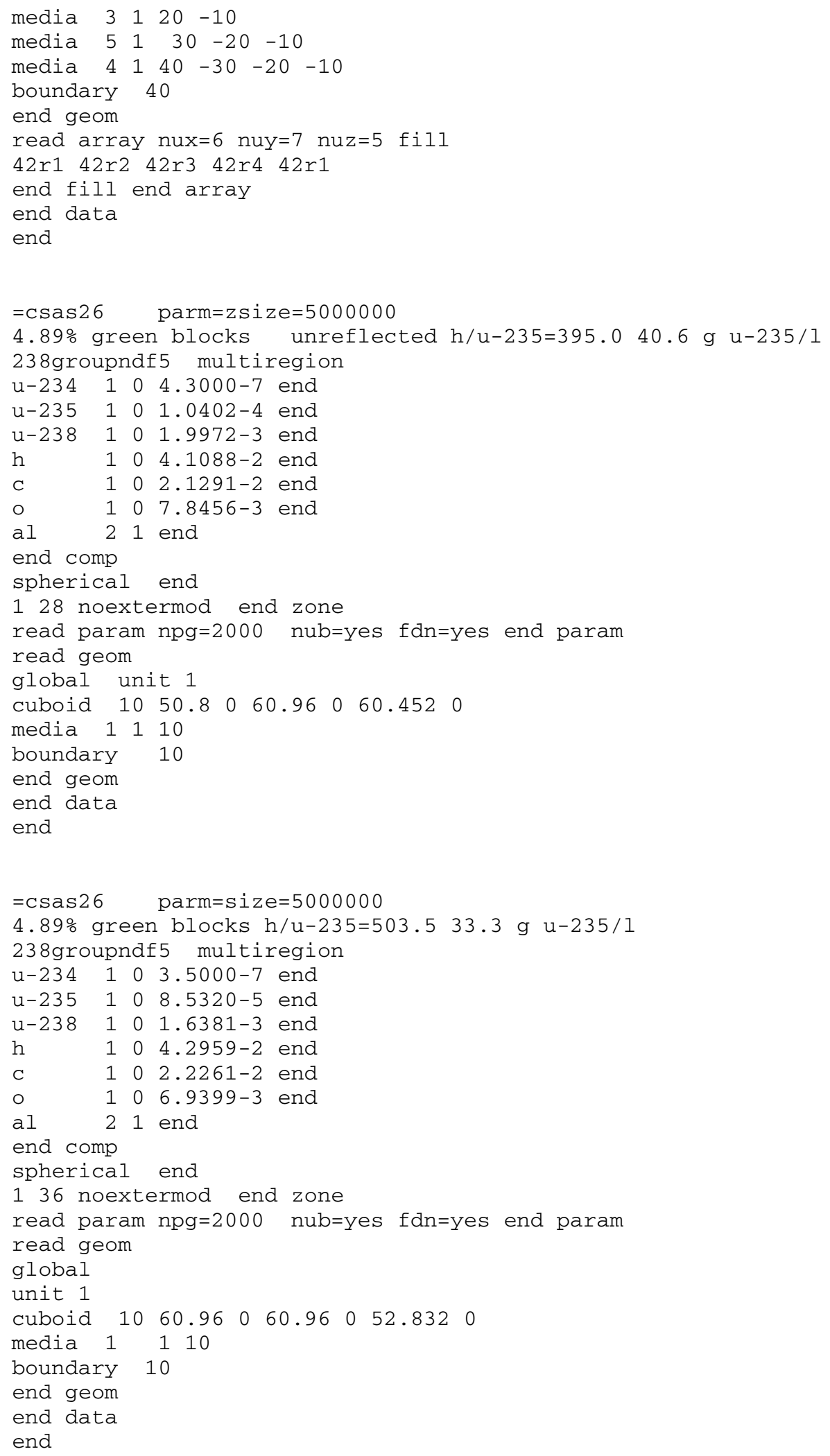




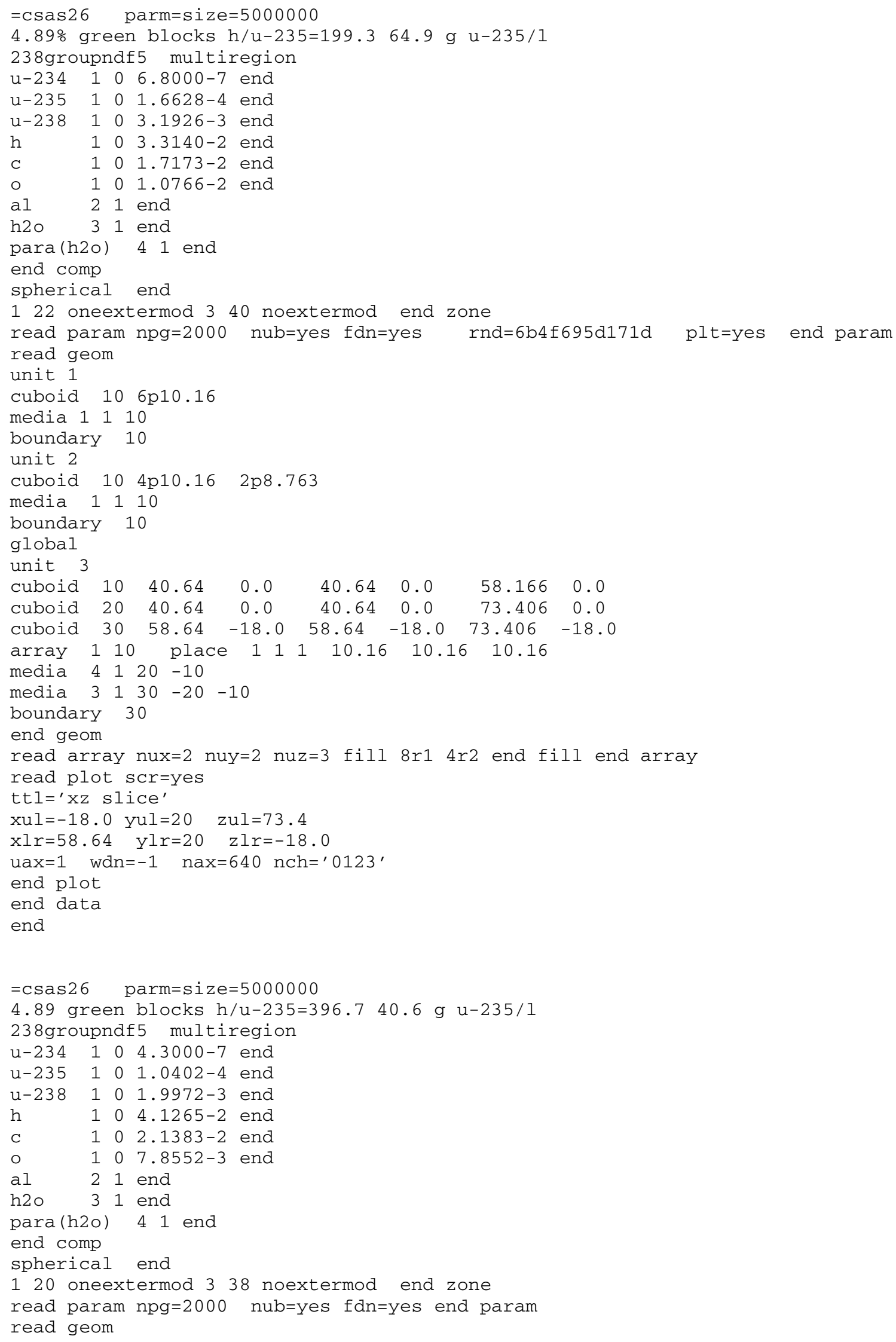




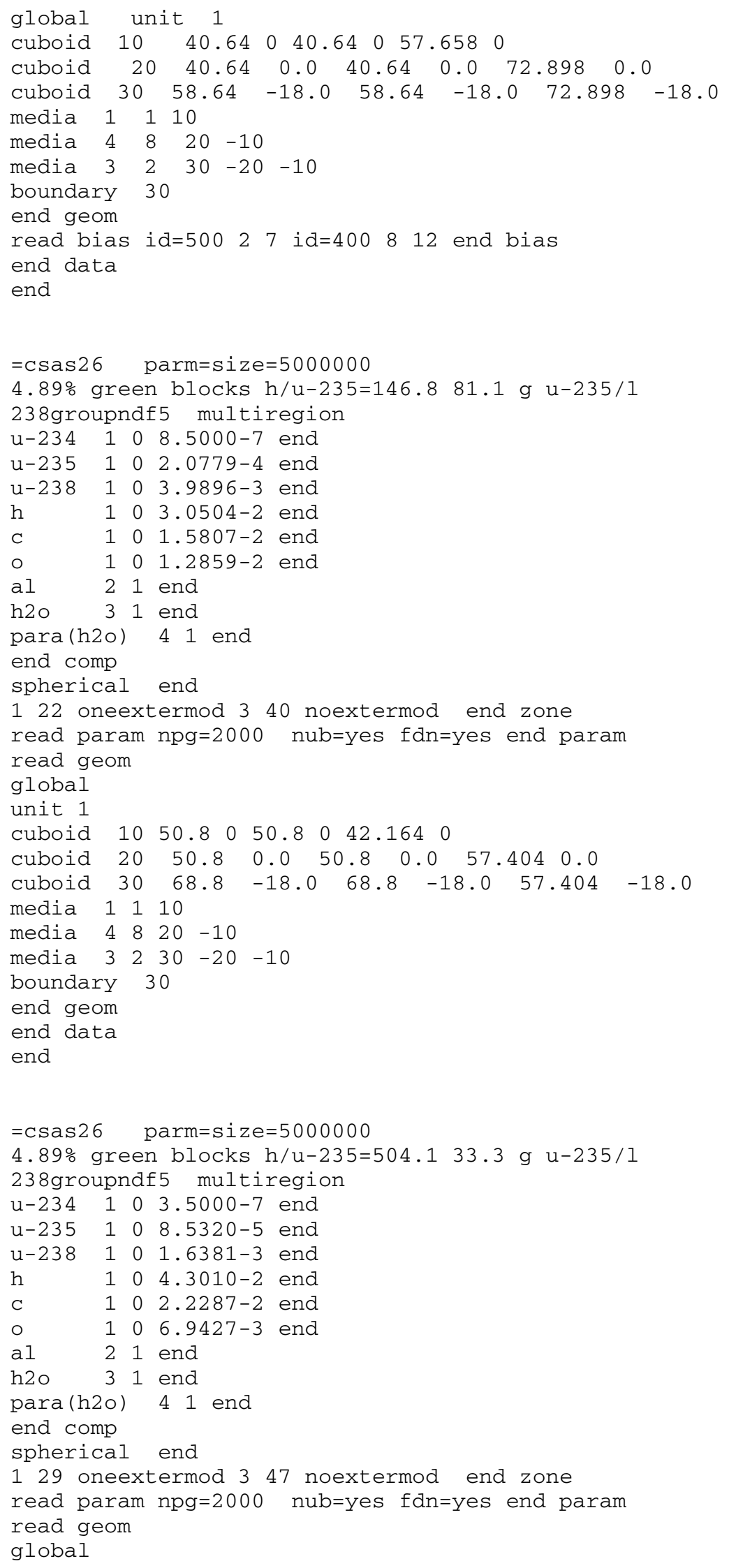




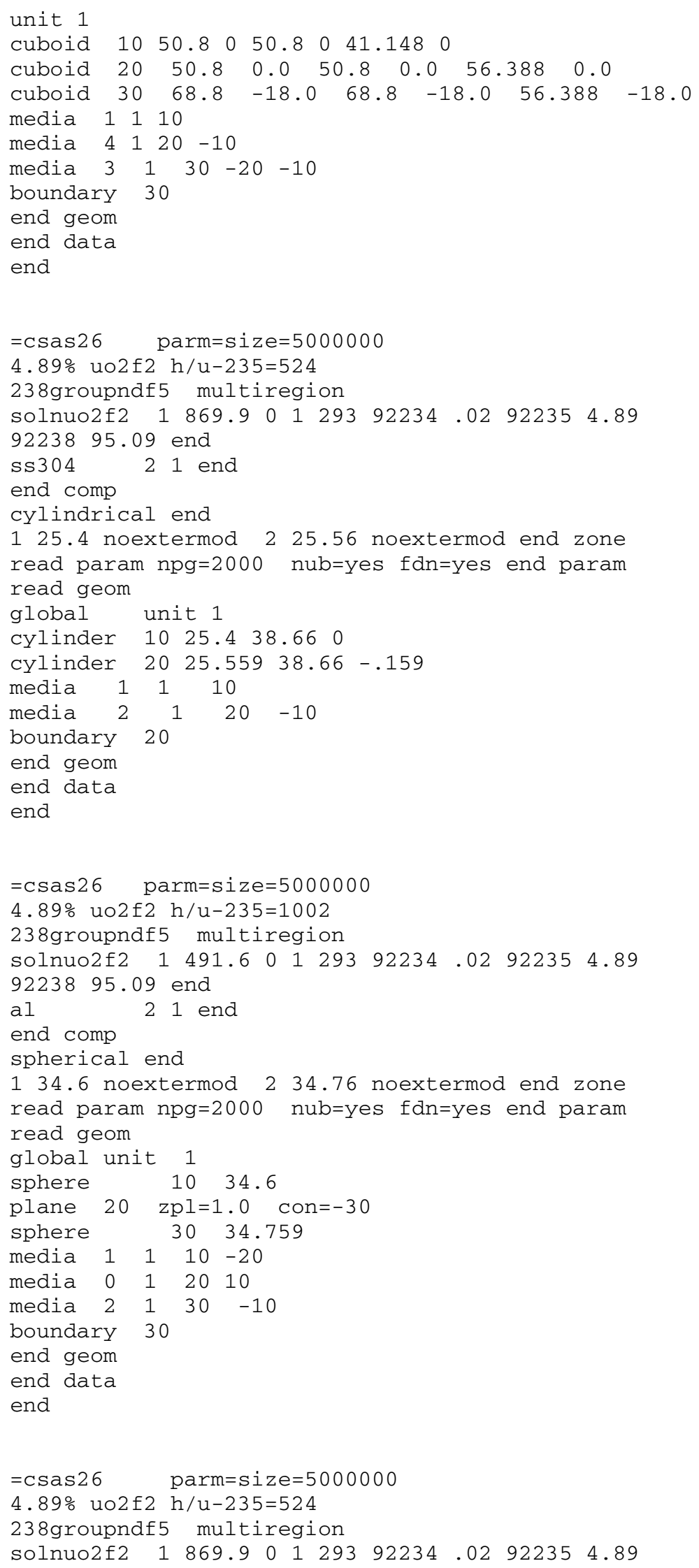




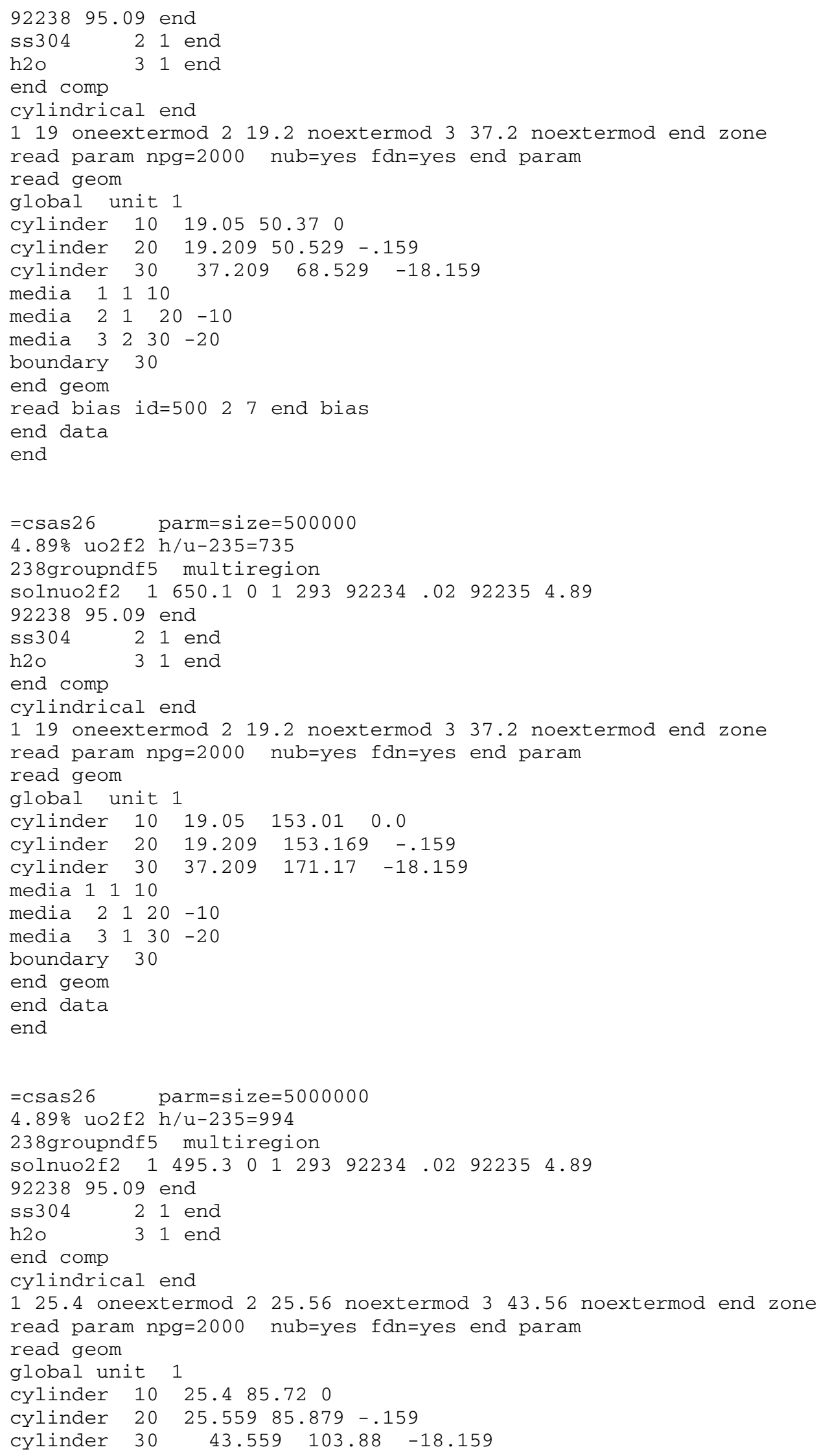


media 1110

media $2 \quad 1 \quad 20-10$

$\begin{array}{lllll}\text { media } & 3 & 1 & 30 & -20\end{array}$

boundary 30

end geom

end data

end 
Table B.2. Table 2 input data

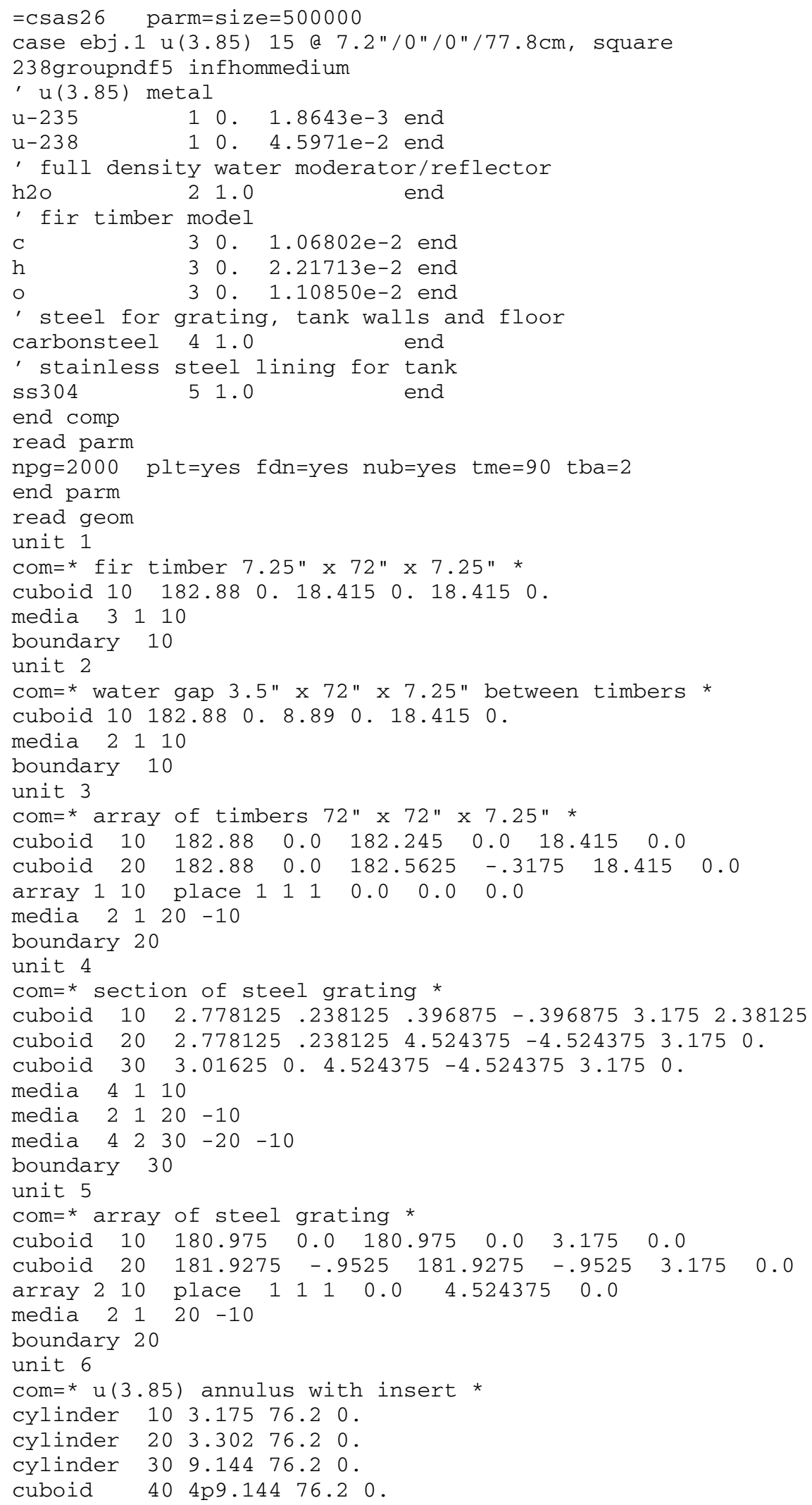




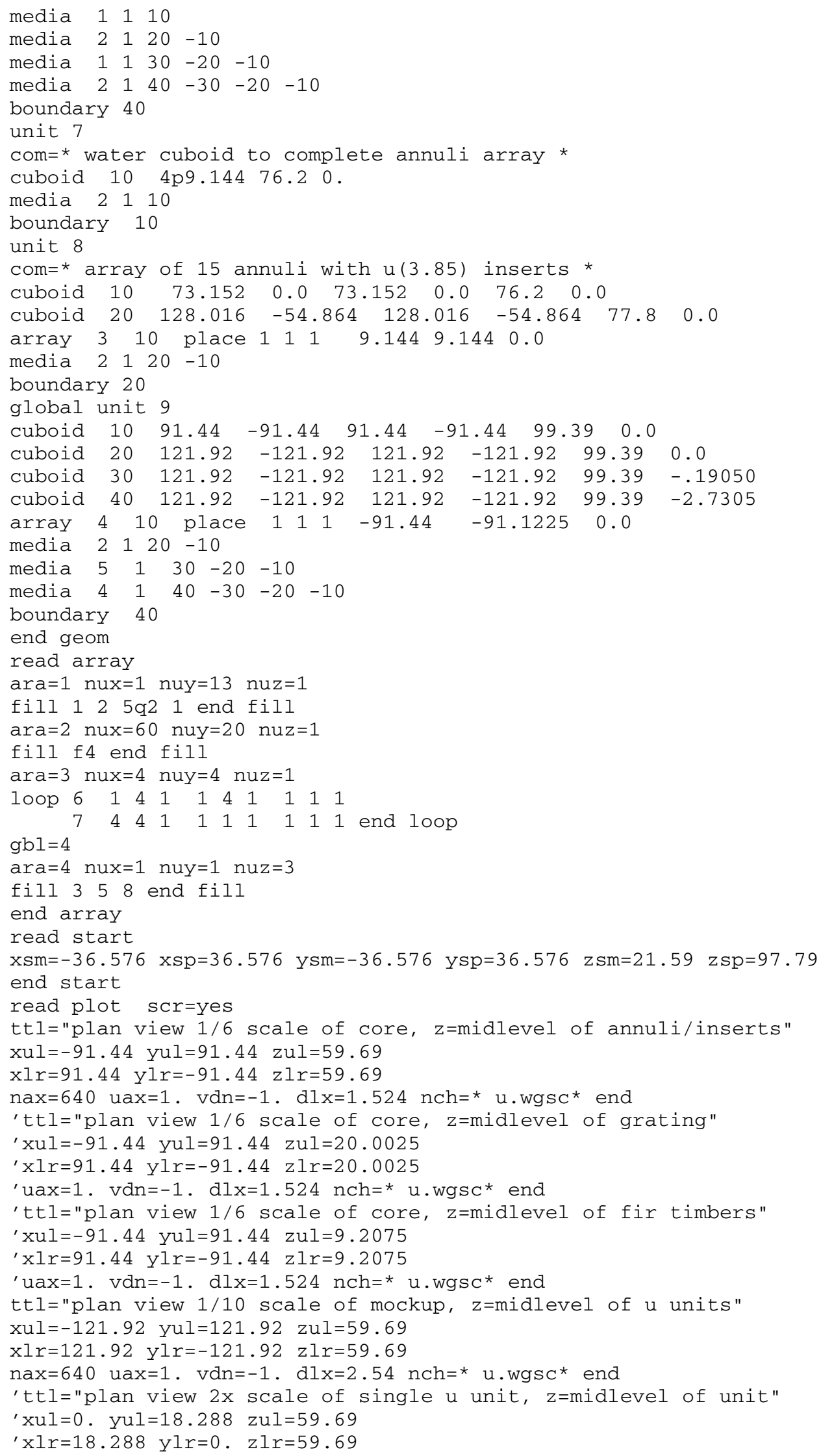




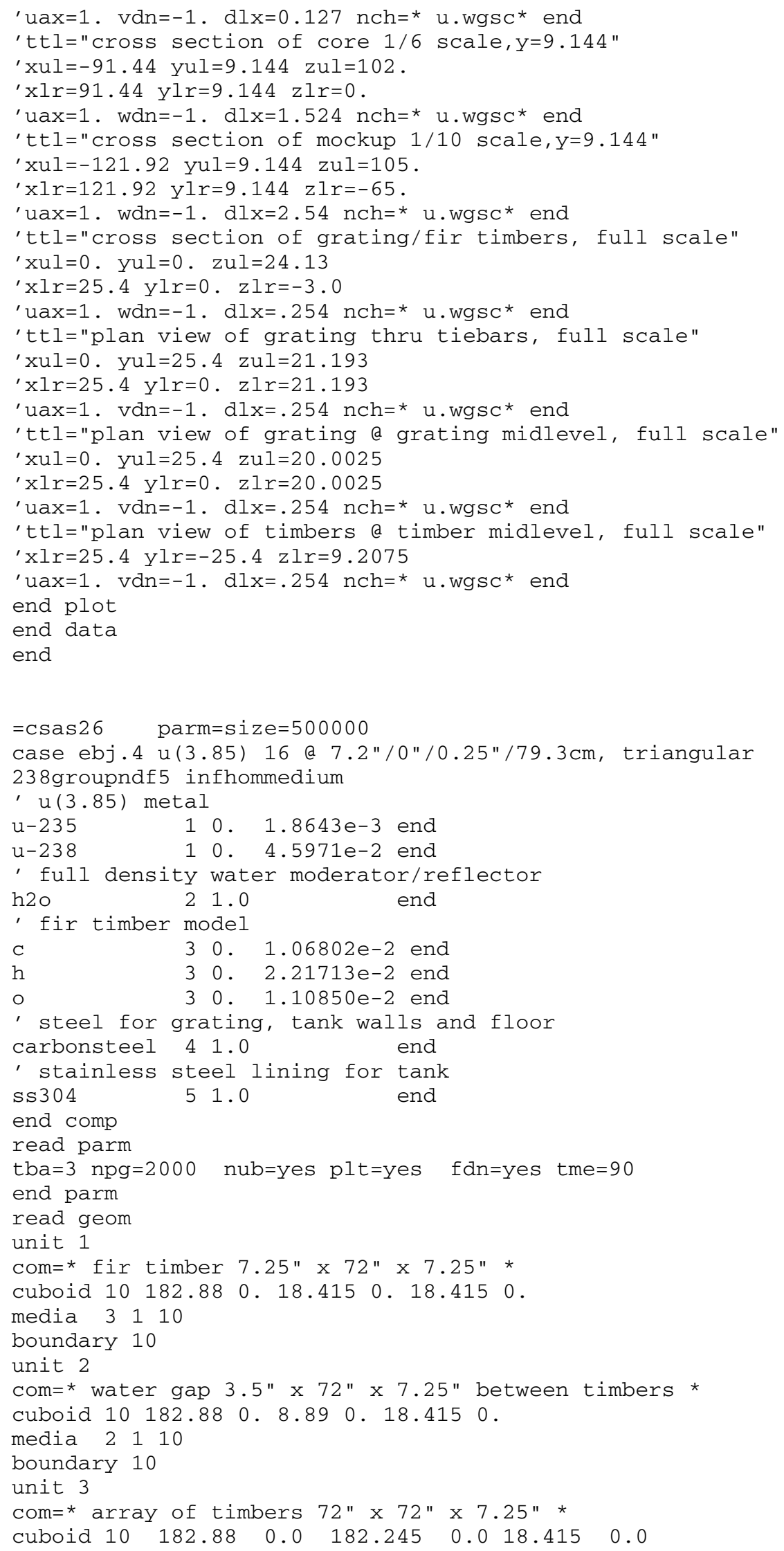




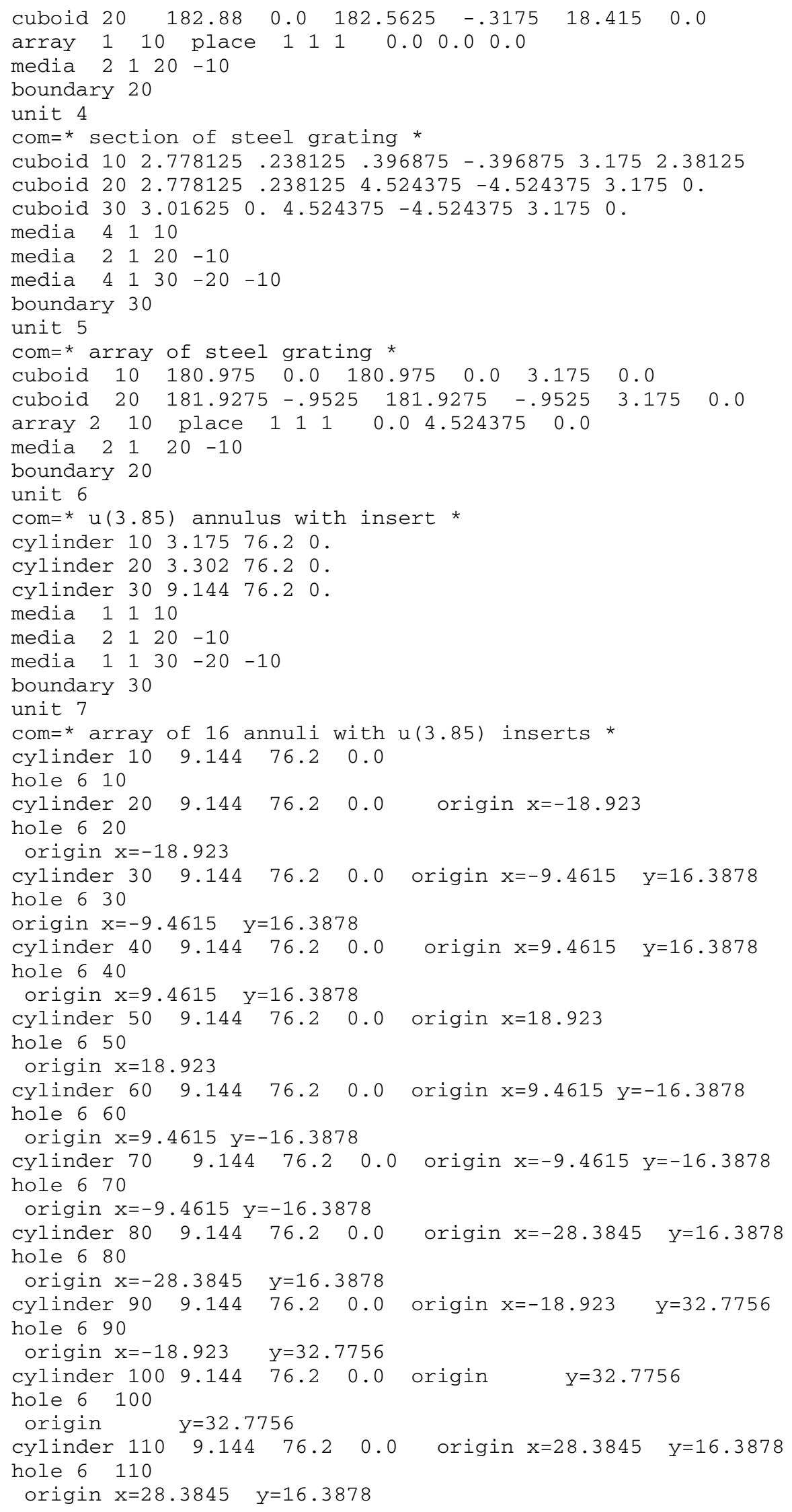




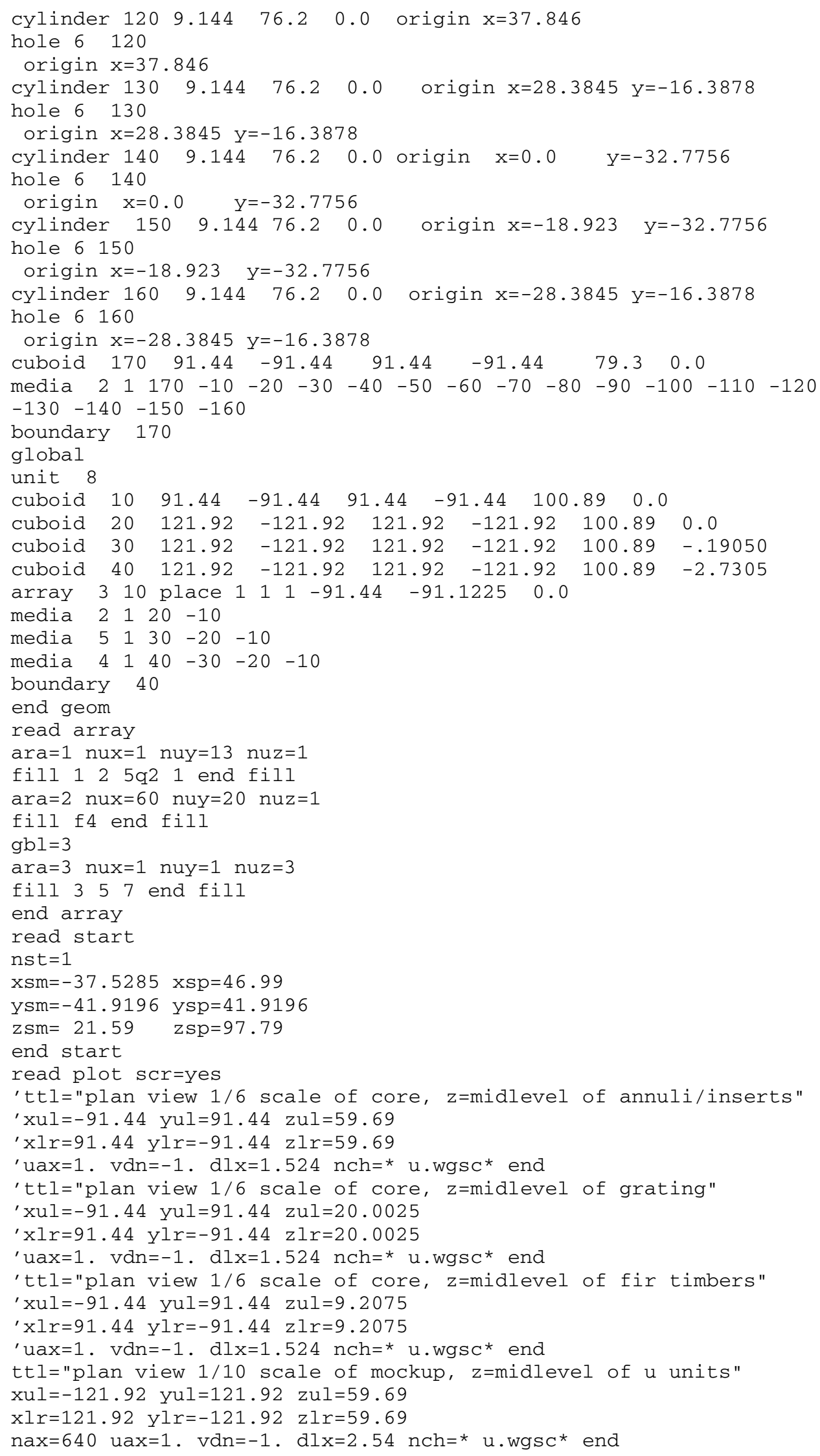




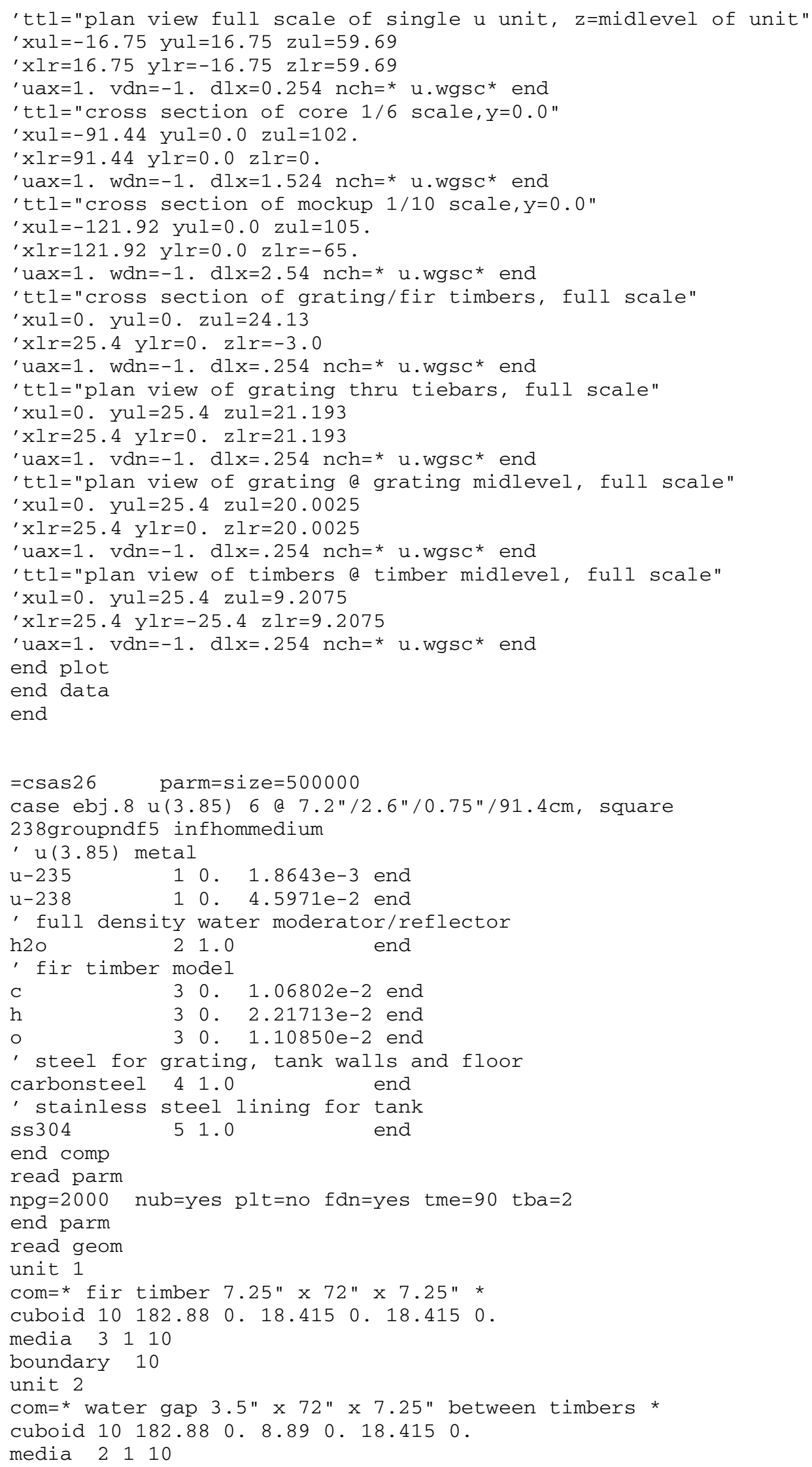




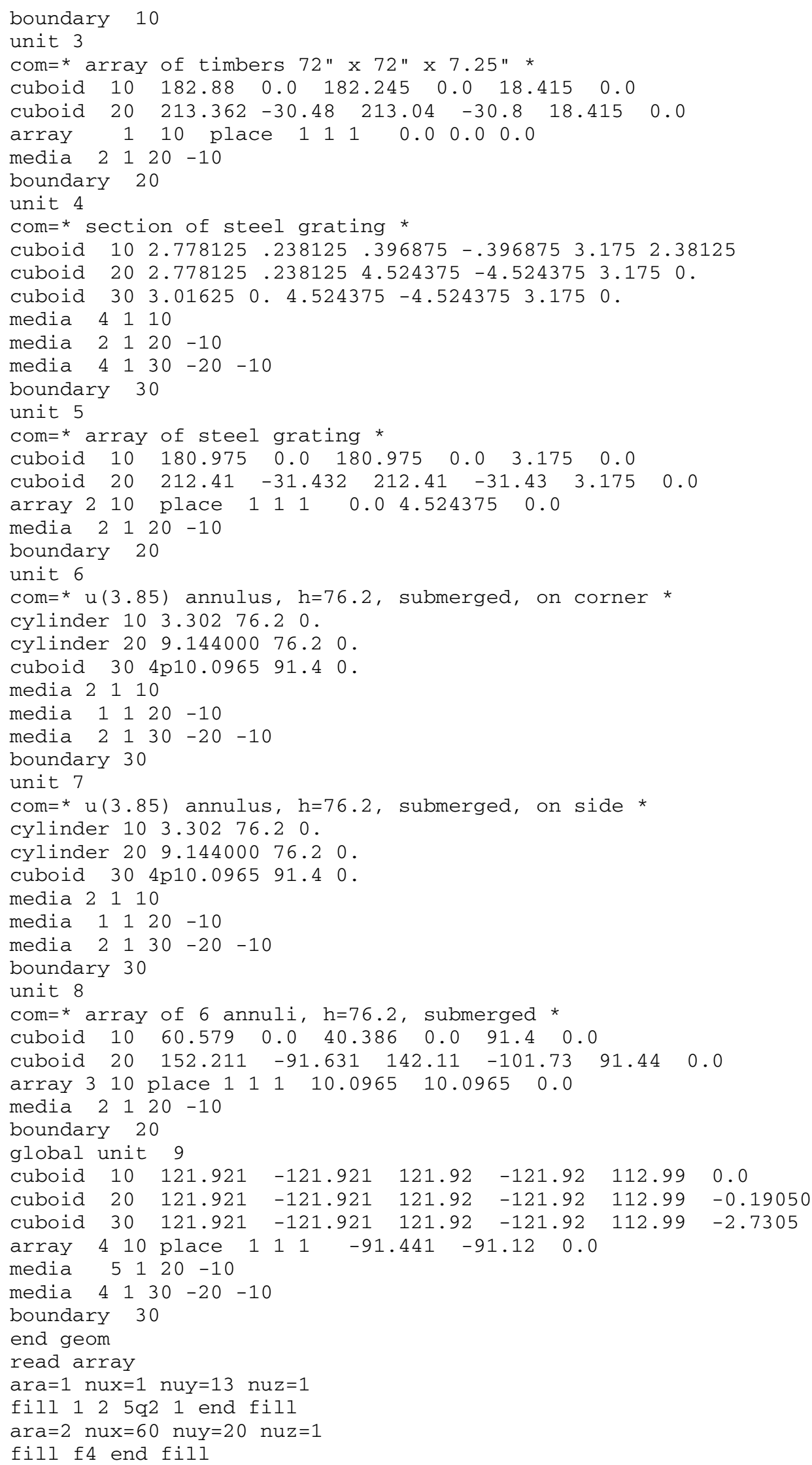




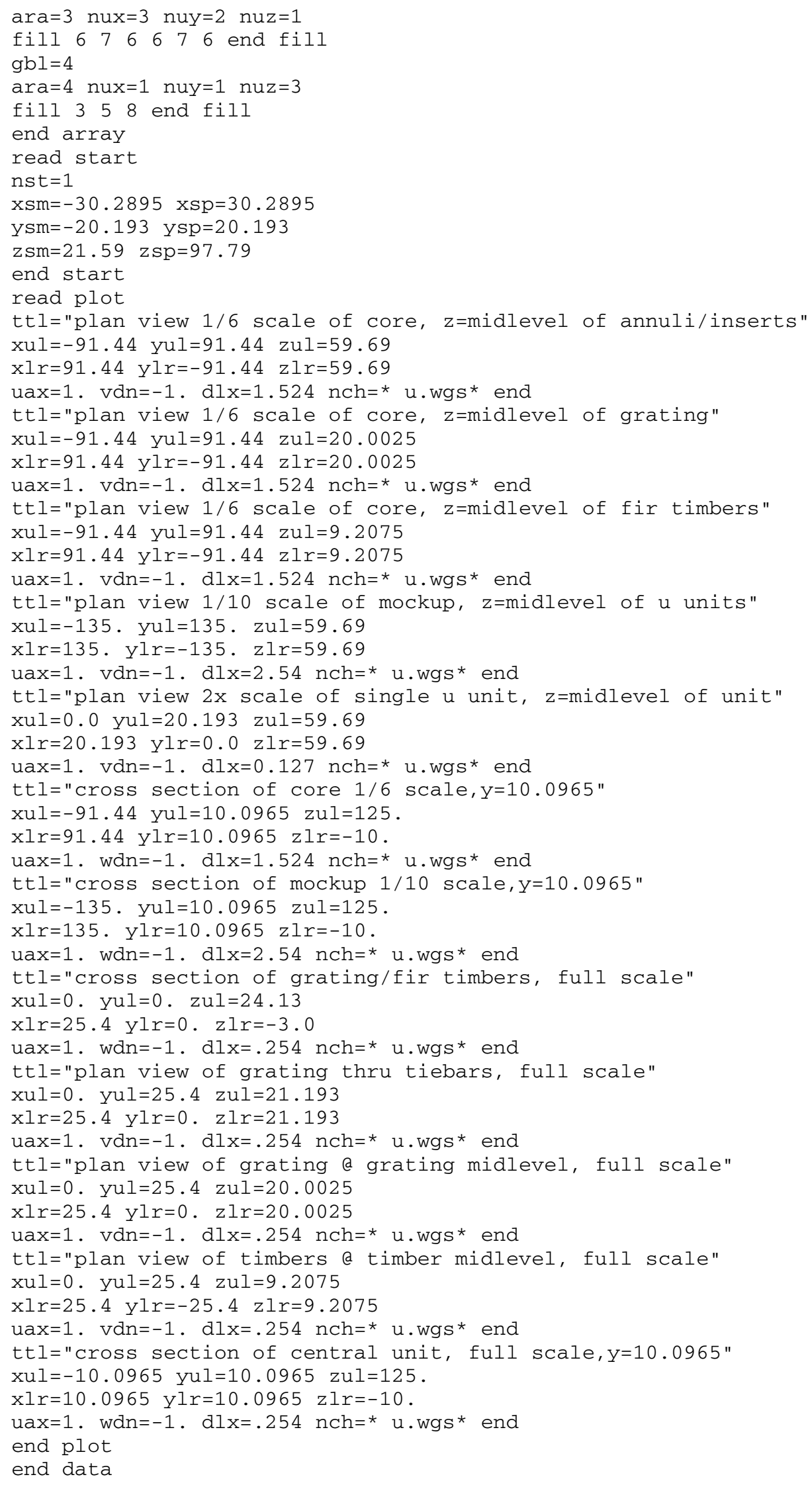


end

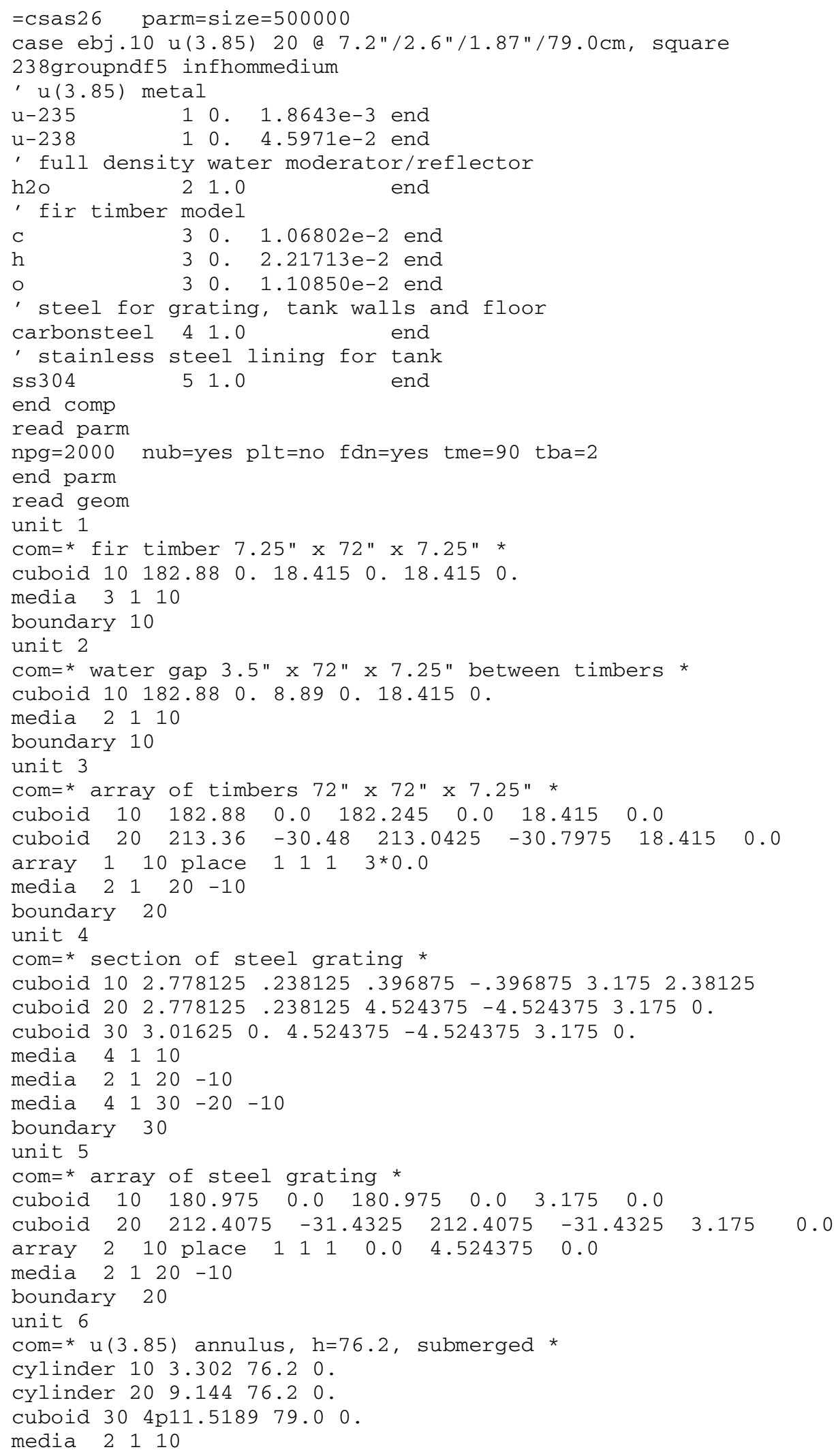




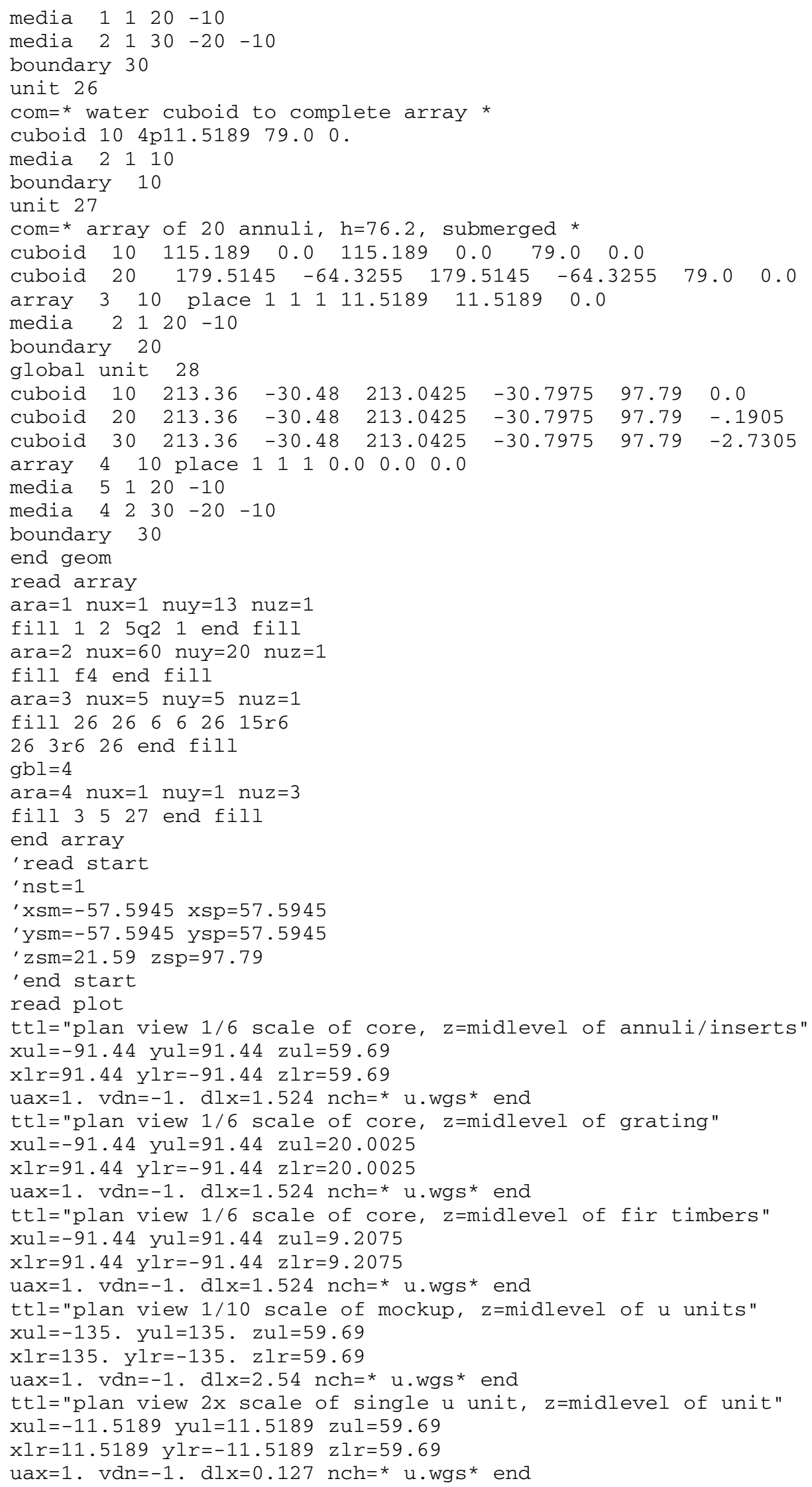




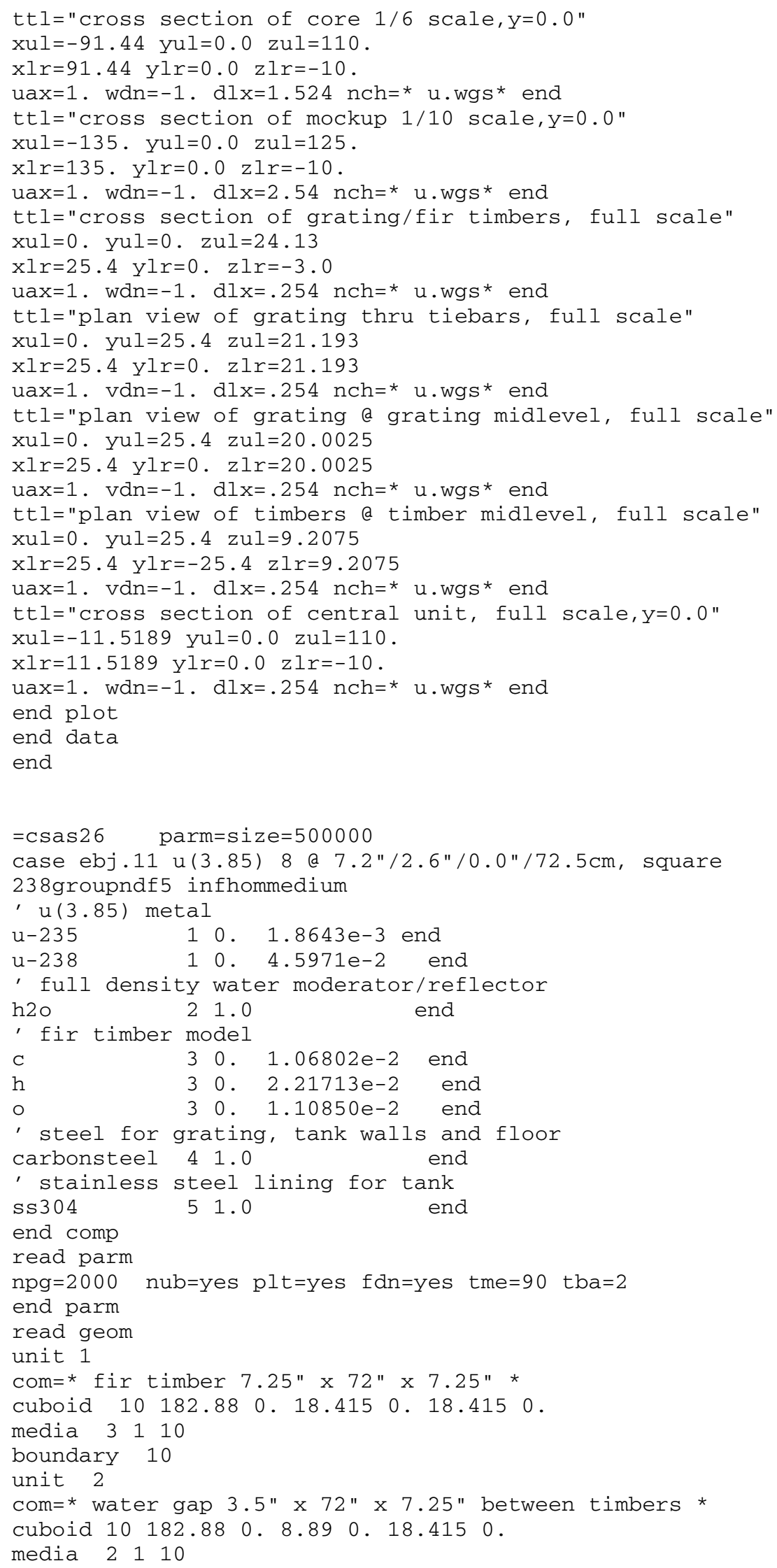




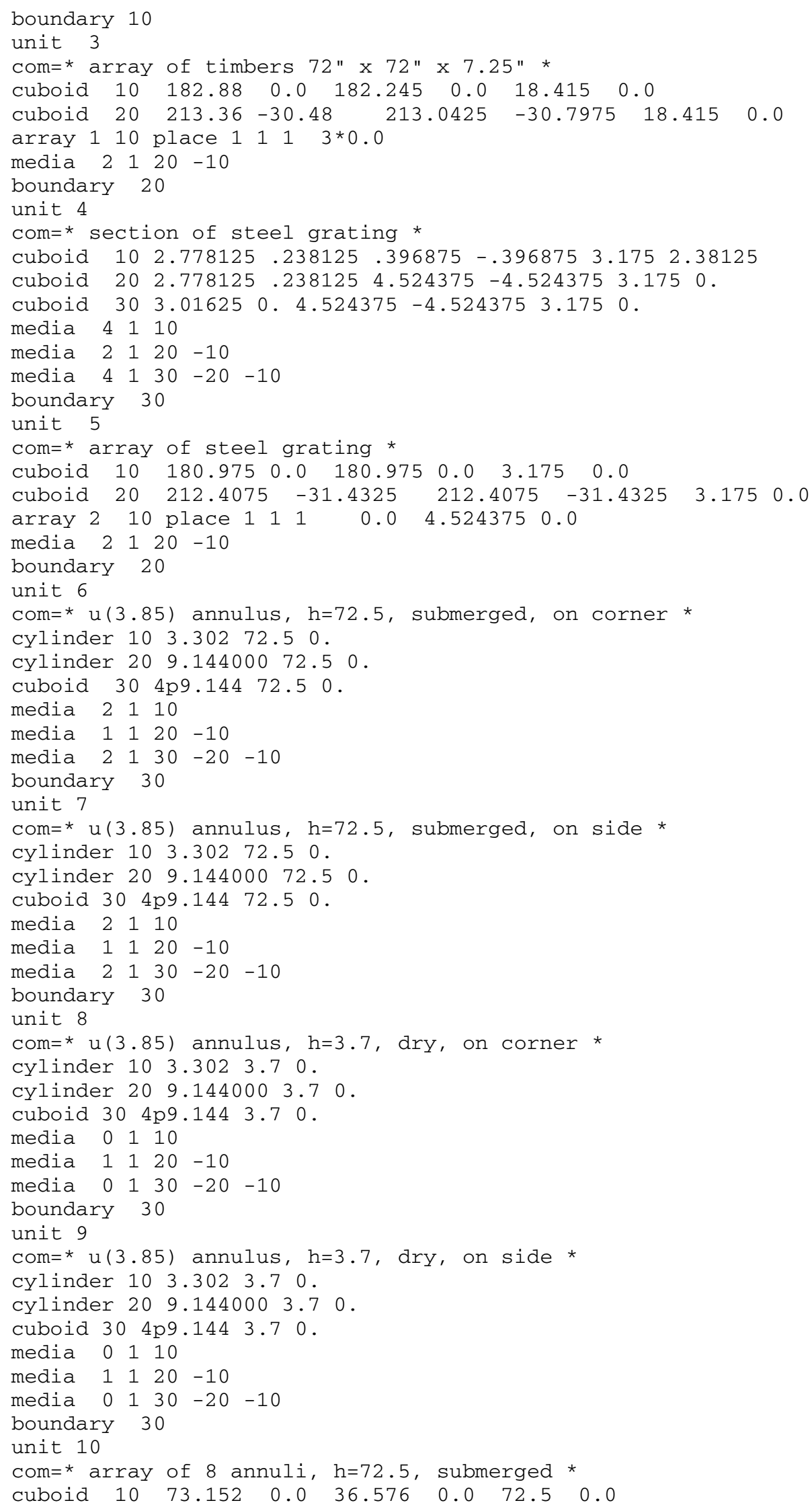




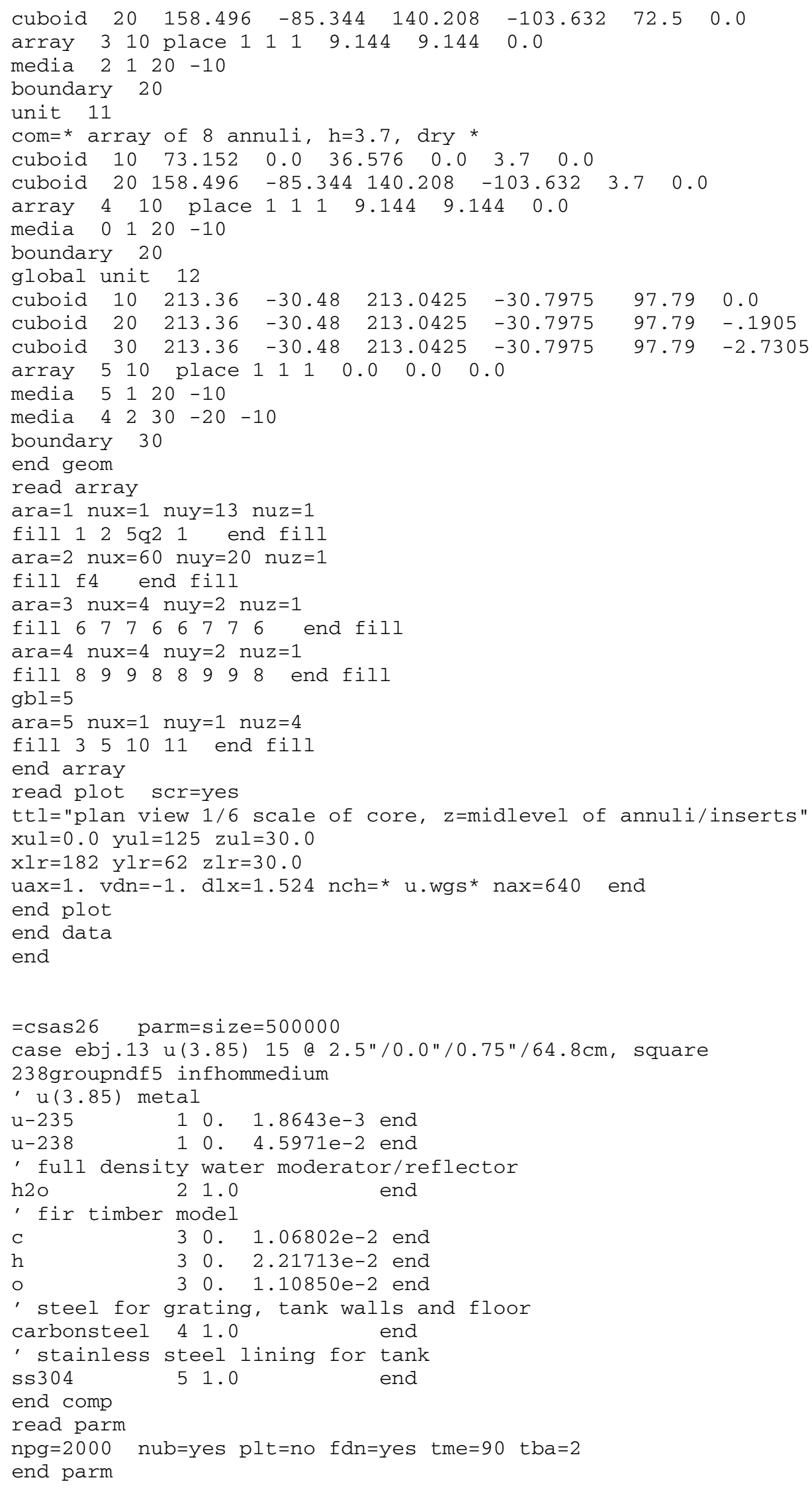




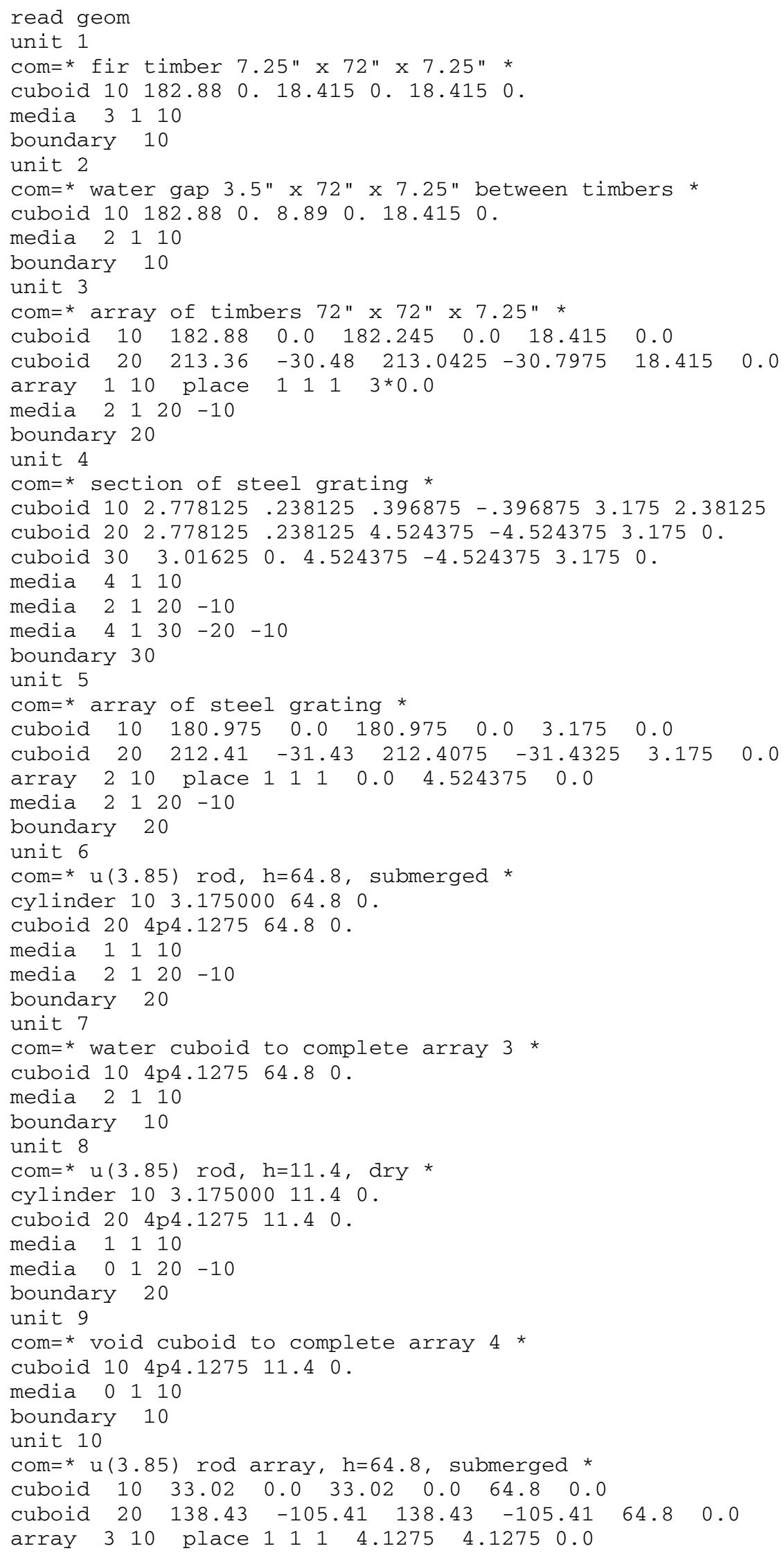




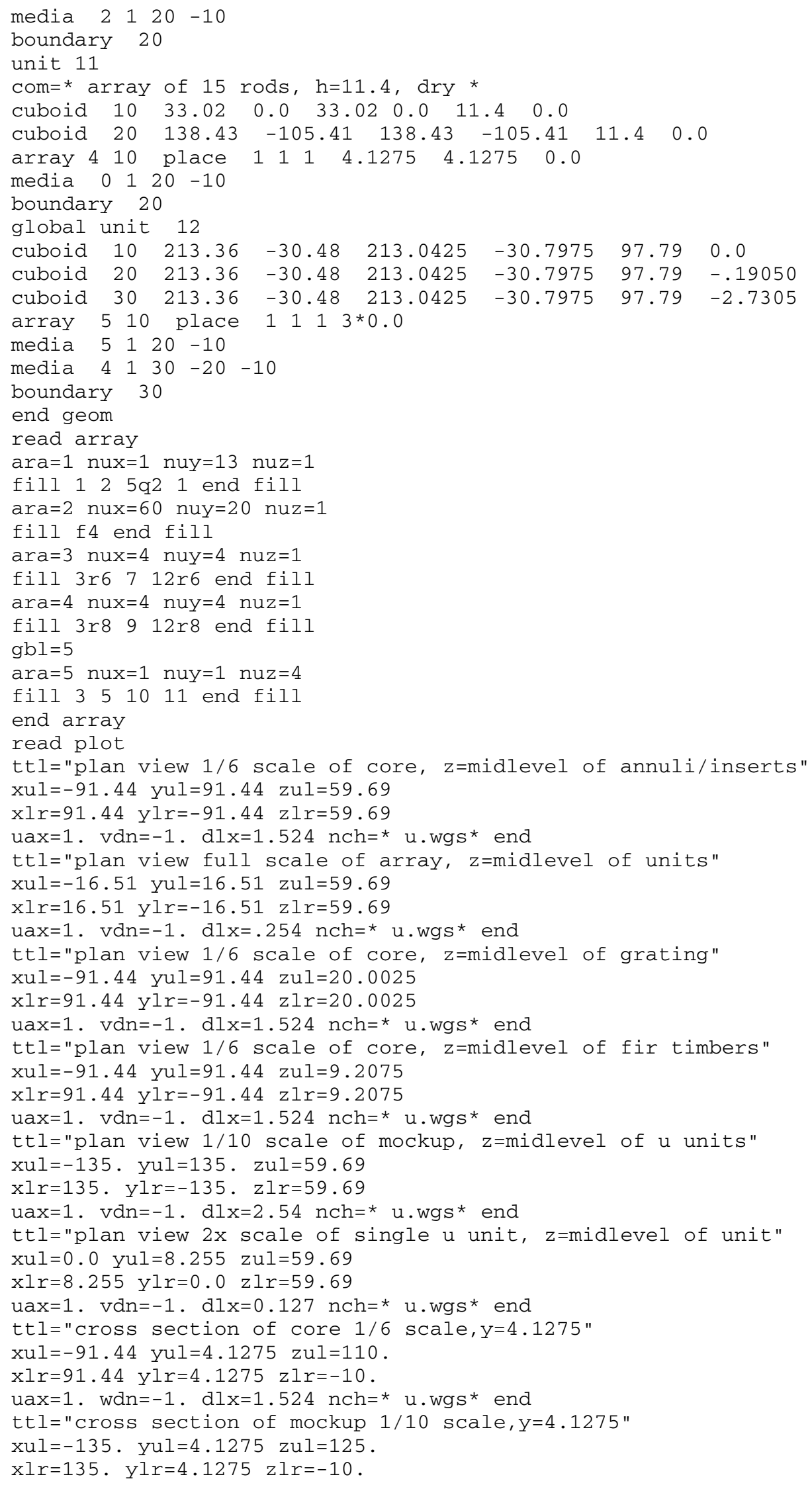




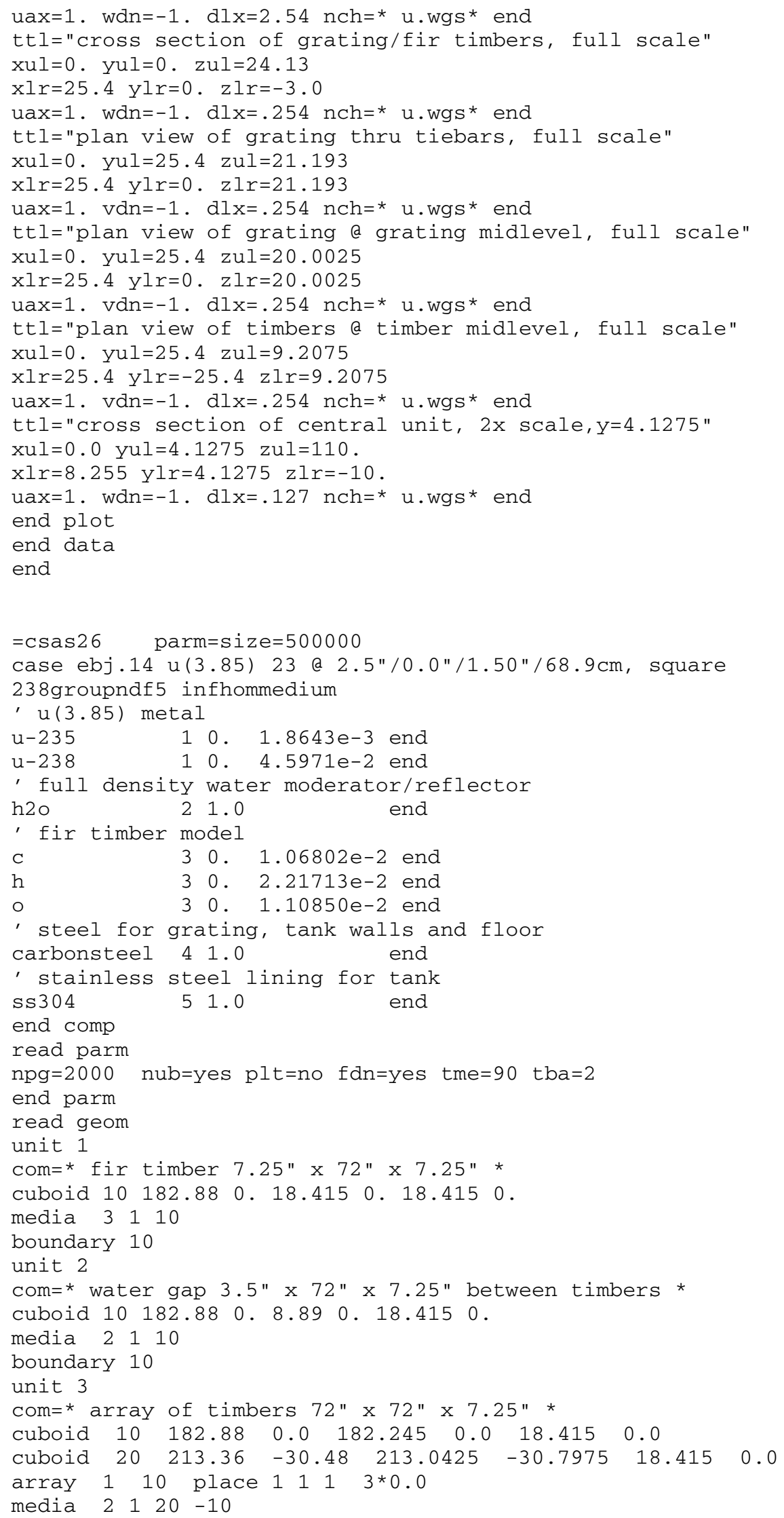




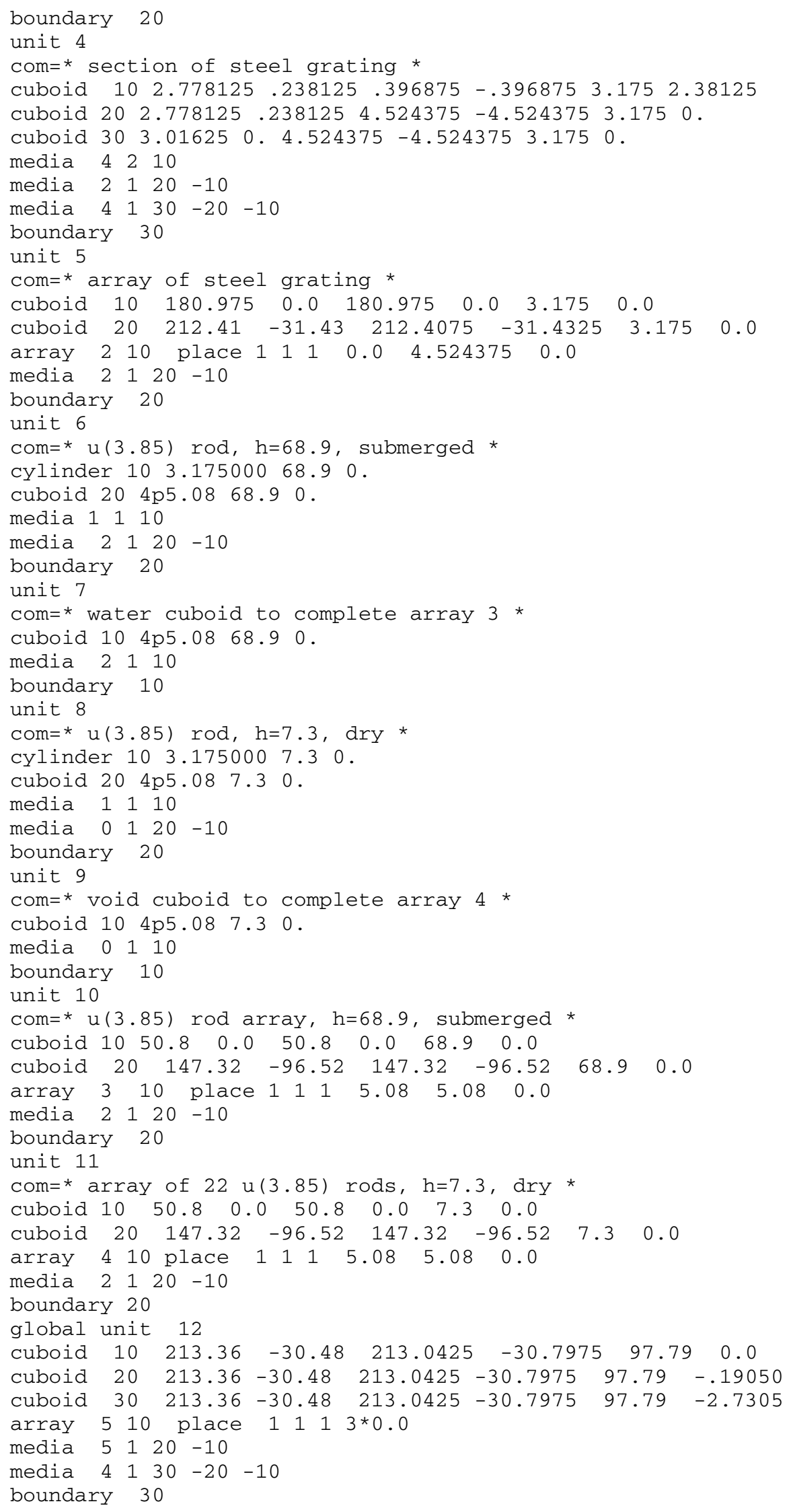




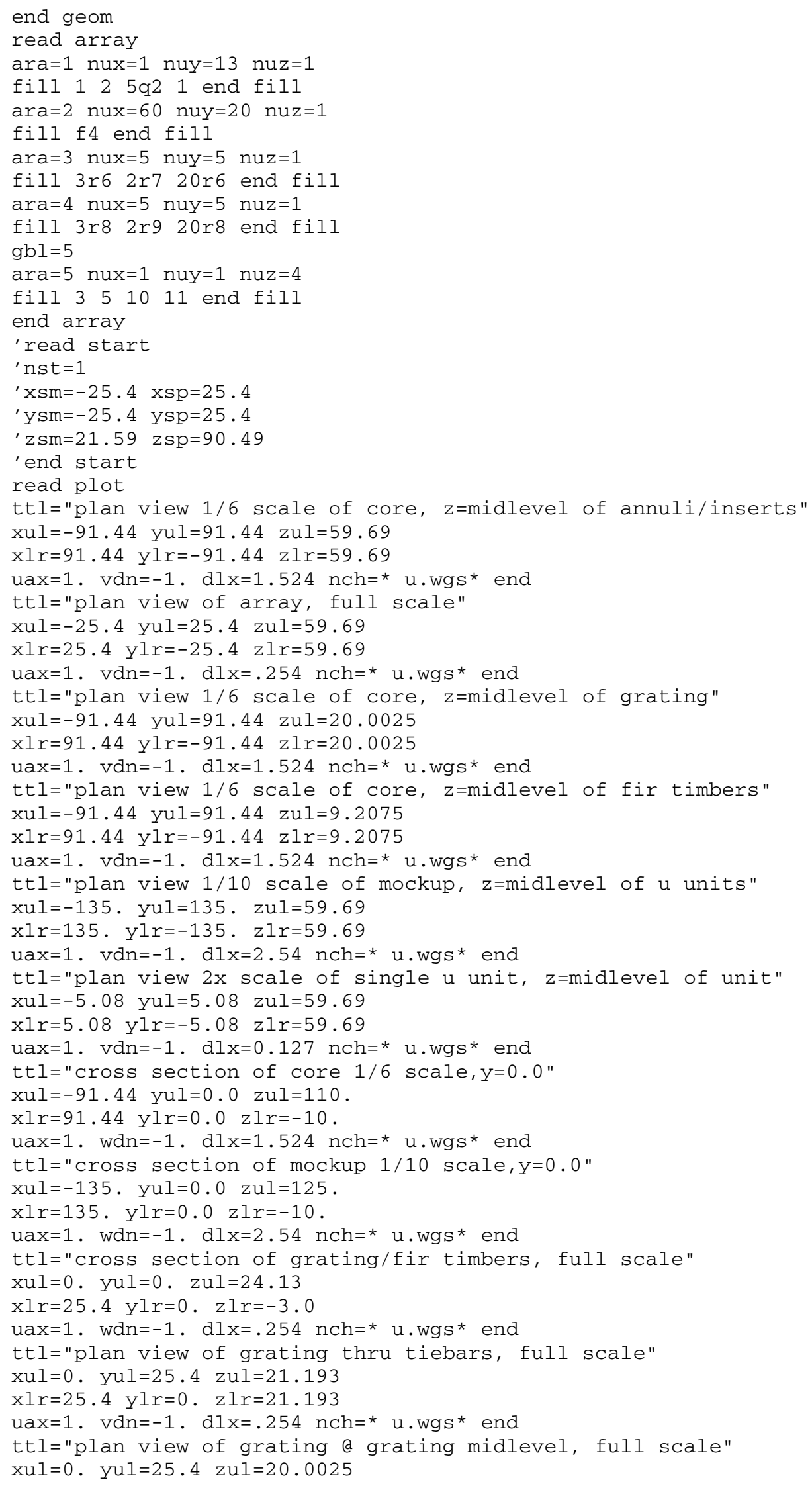


$\mathrm{xlr}=25.4 \mathrm{ylr}=0 . \mathrm{zlr}=20.0025$

uax $=1$. $\operatorname{vdn}=-1 . \mathrm{dlx}=.254 \mathrm{nch}=$ * u.wgs * end

ttl="plan view of timbers a timber midlevel, full scale"

$\mathrm{xul}=0 . \mathrm{yul}=25.4 \mathrm{zul}=9.2075$

$\mathrm{xlr}=25.4$ ylr $=-25.4$ zlr $=9.2075$

uax $=1 . v d n=-1 . d l x=.254$ nch $=$ * u.wgs * end

tt $l="$ cross section of central unit, $2 \mathrm{x}$ scale, $\mathrm{y}=0.0 "$

$\mathrm{xul}=-5.08 \mathrm{yul}=0.0 \mathrm{zul}=110$.

$\mathrm{xlr}=5.08$ ylr=0.0 zlr $=-10$.

uax $=1$. wdn $=-1 . d 1 x=.127 \mathrm{nch}=$ * u.wgs * end

end plot

end data

end 
Table B.3. Table 3 input data

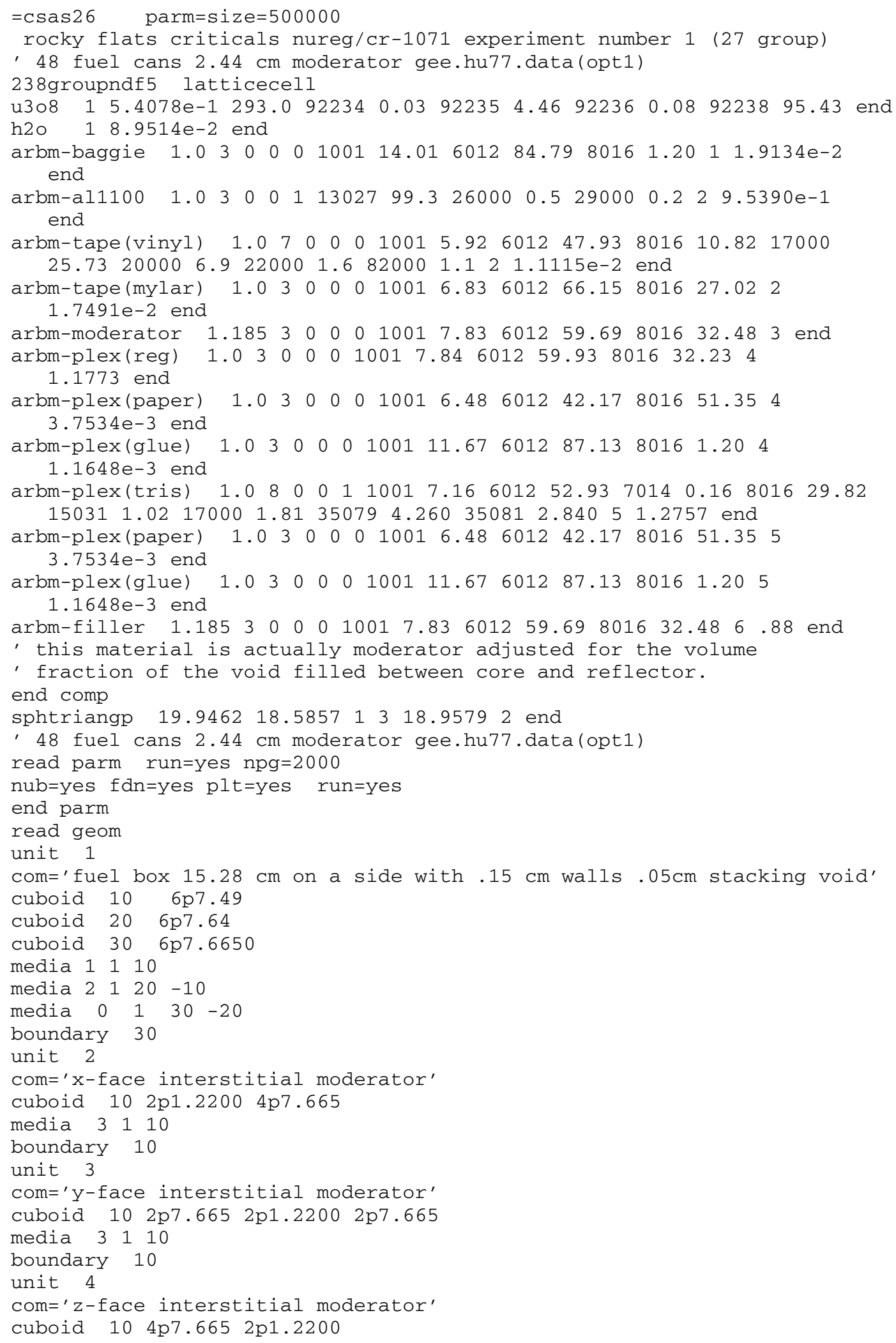




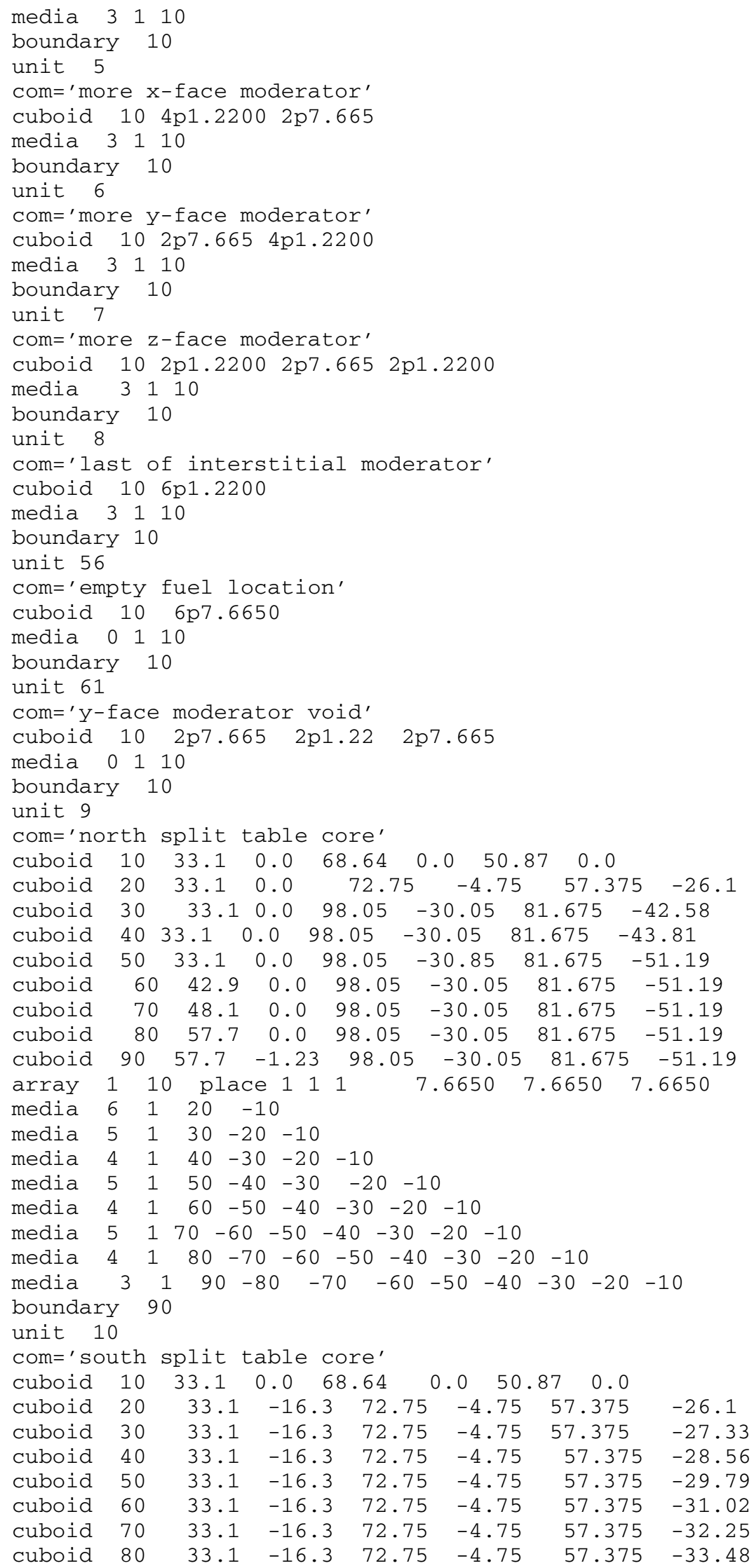




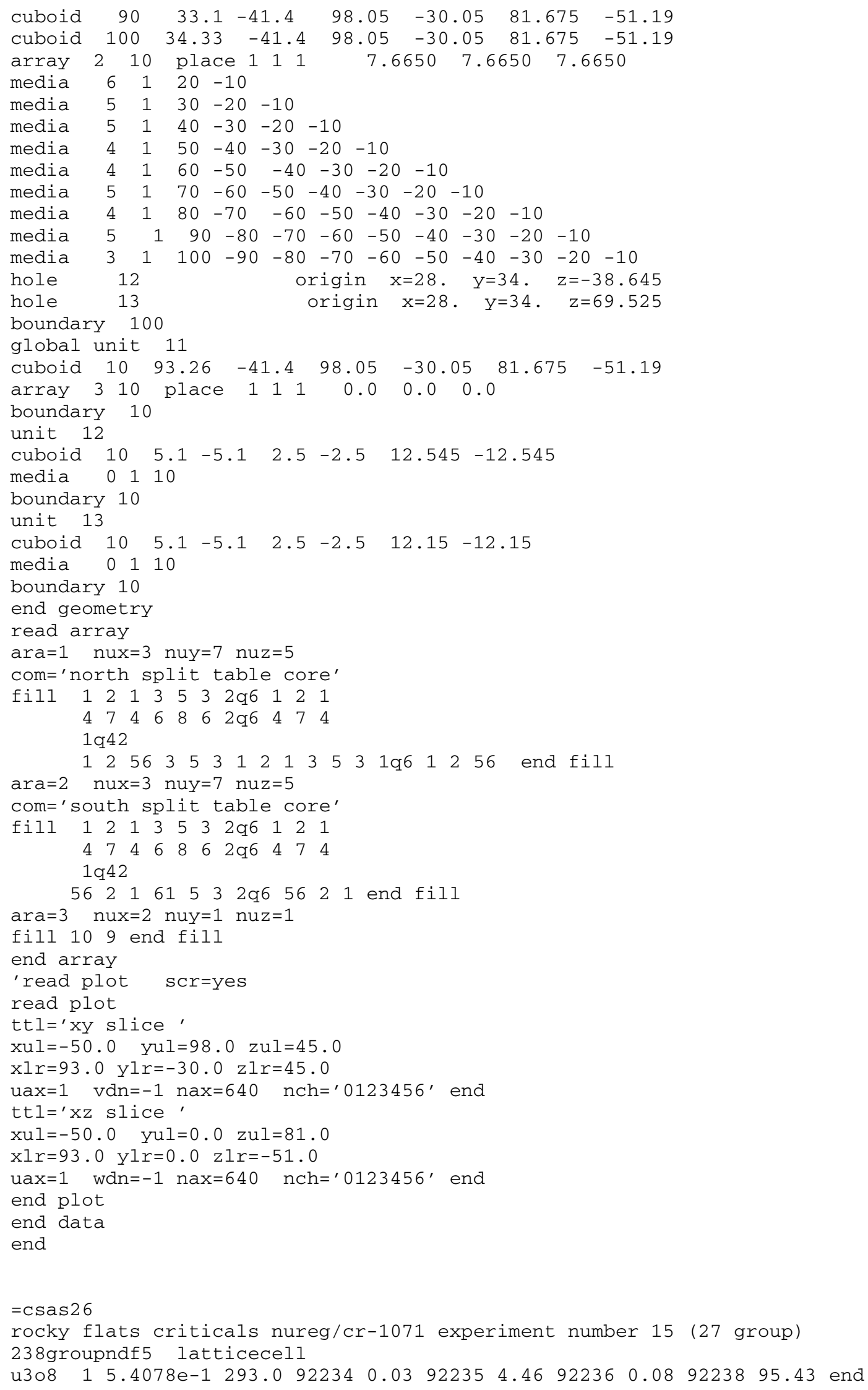




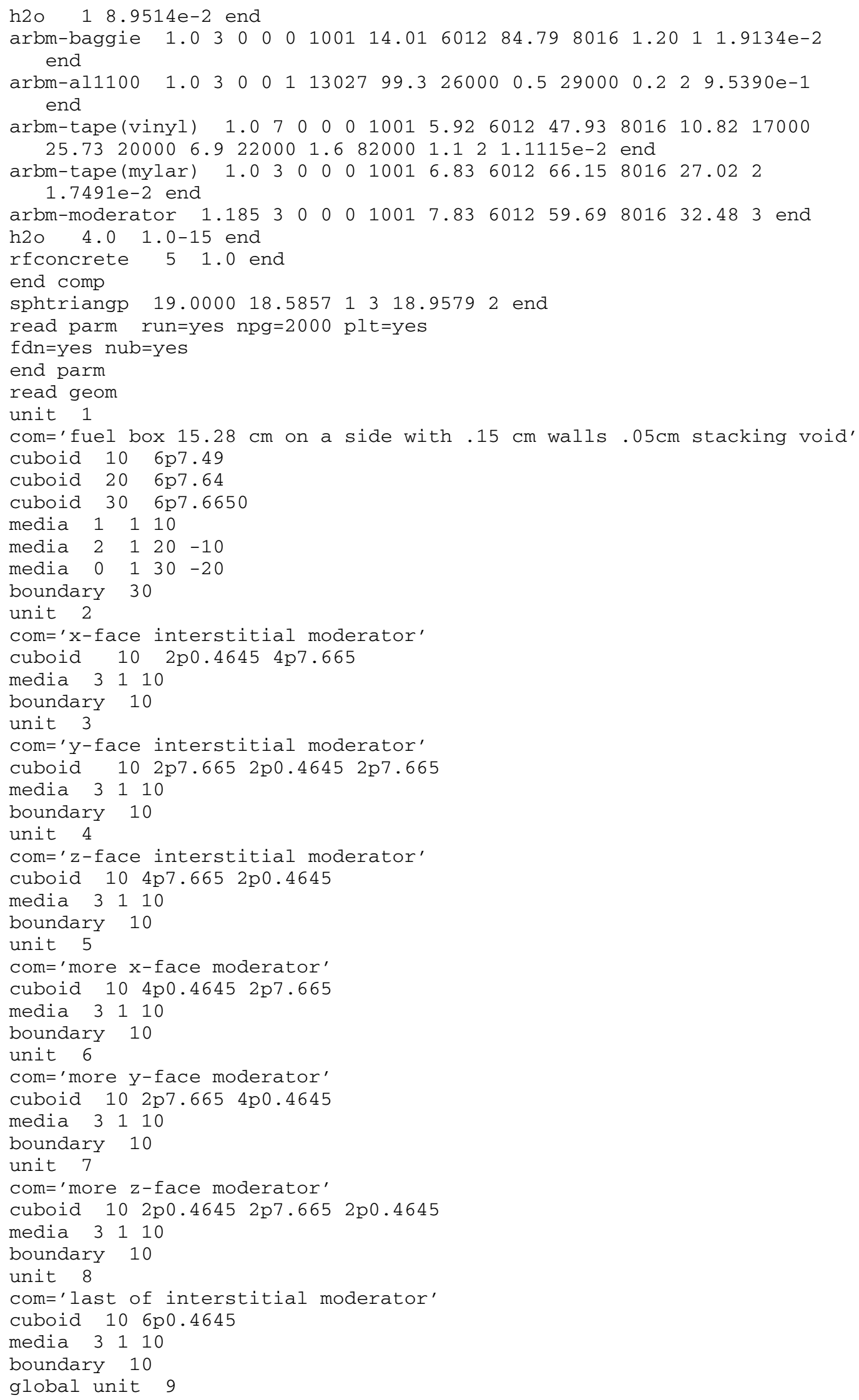




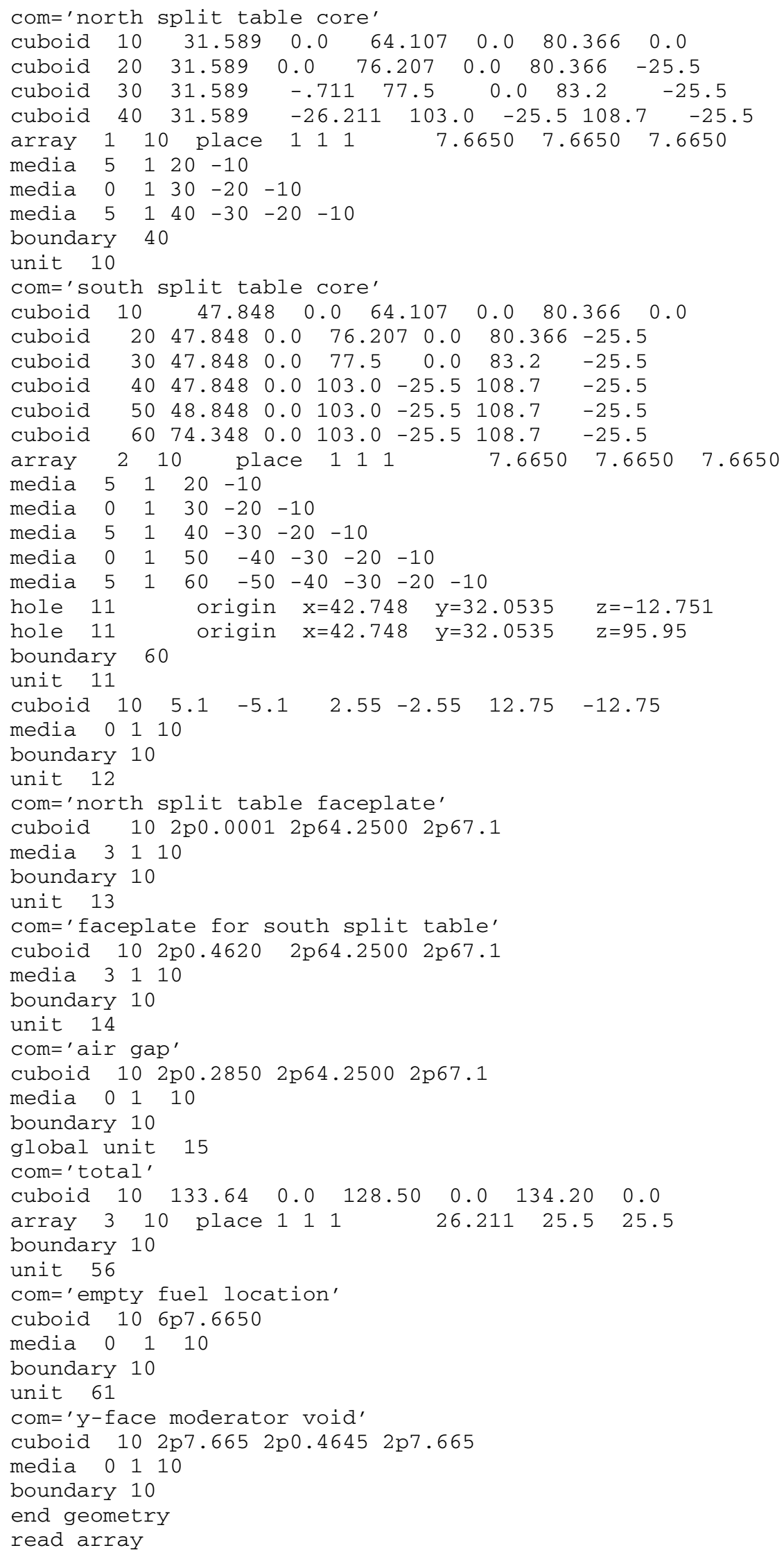




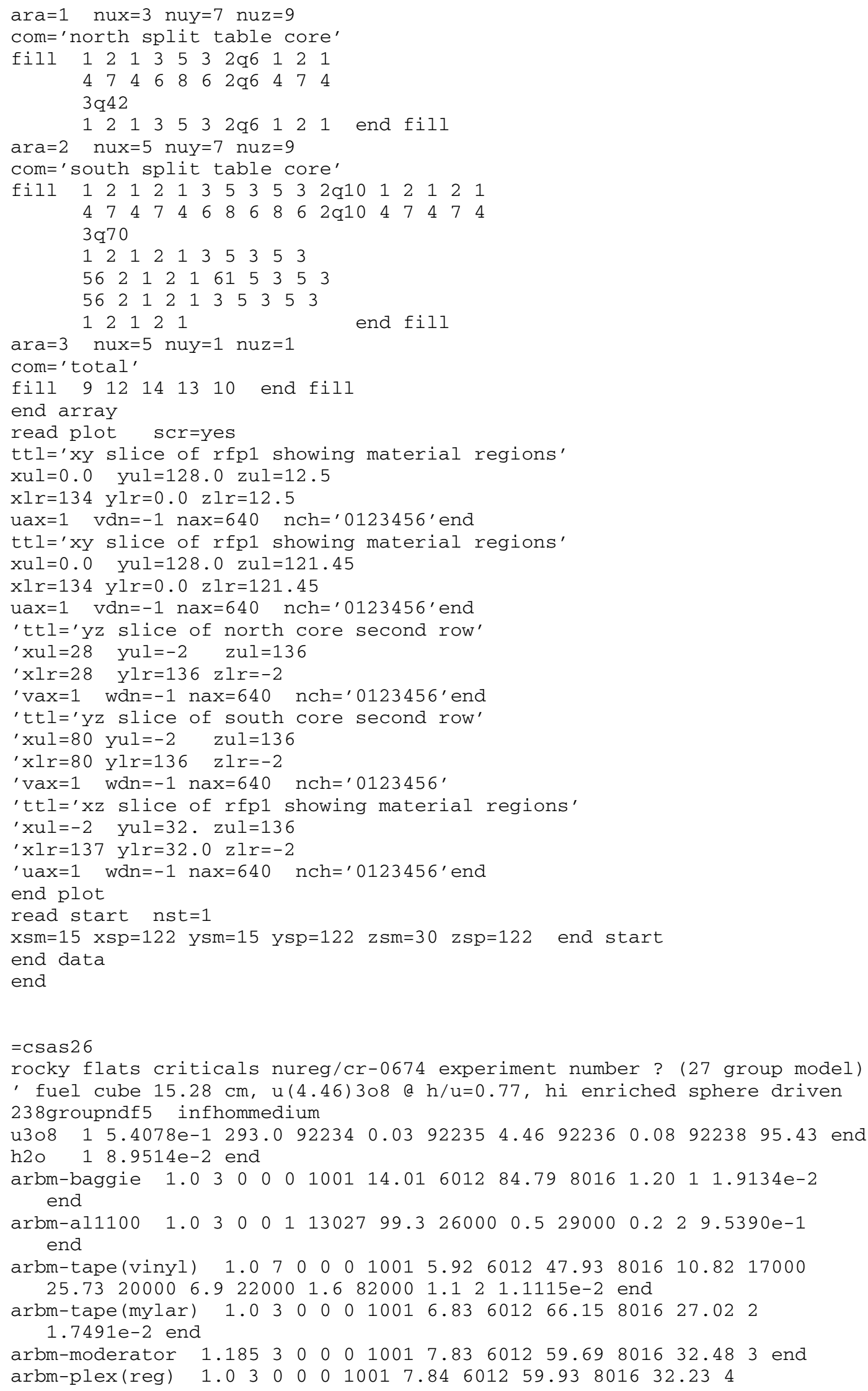




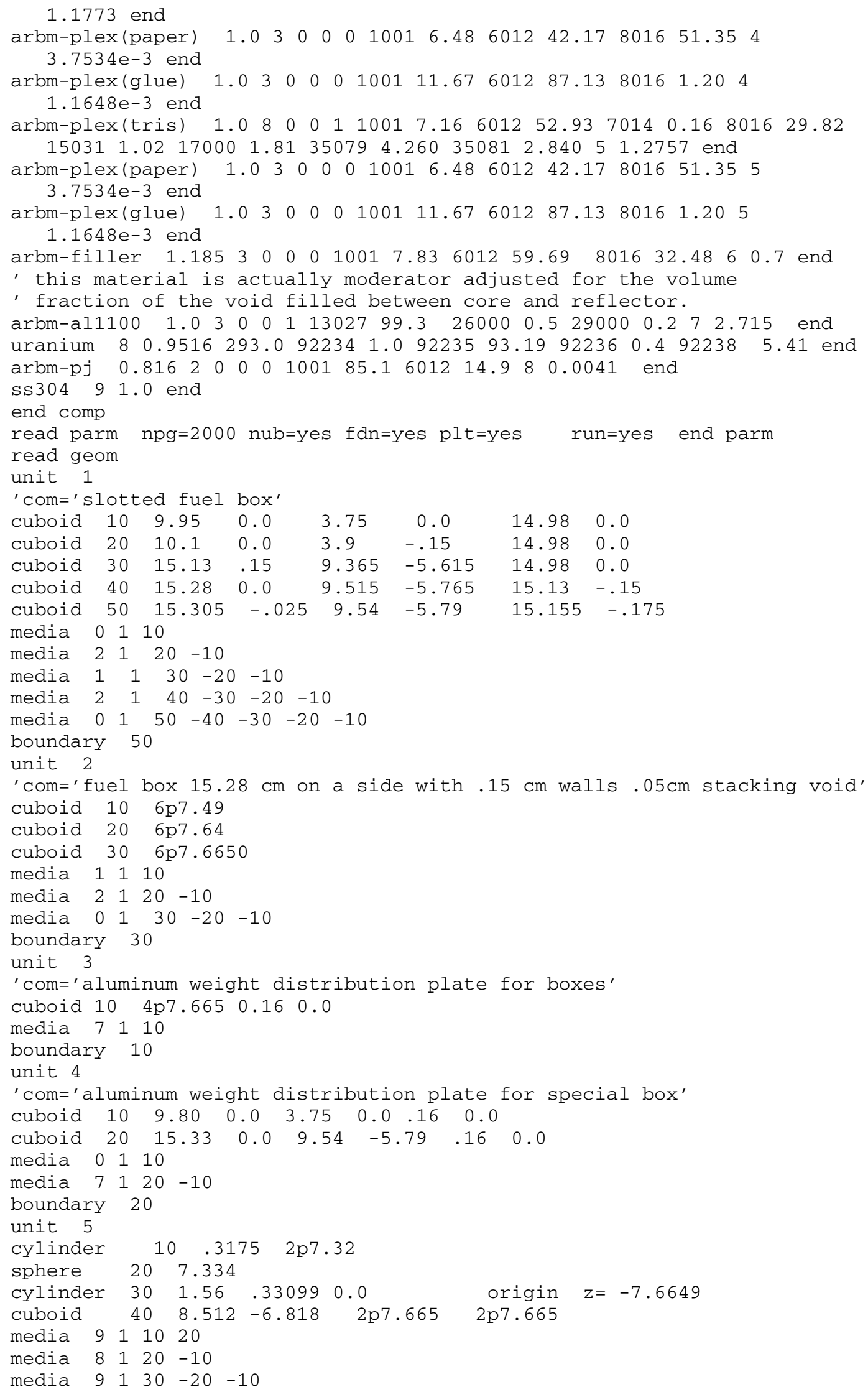




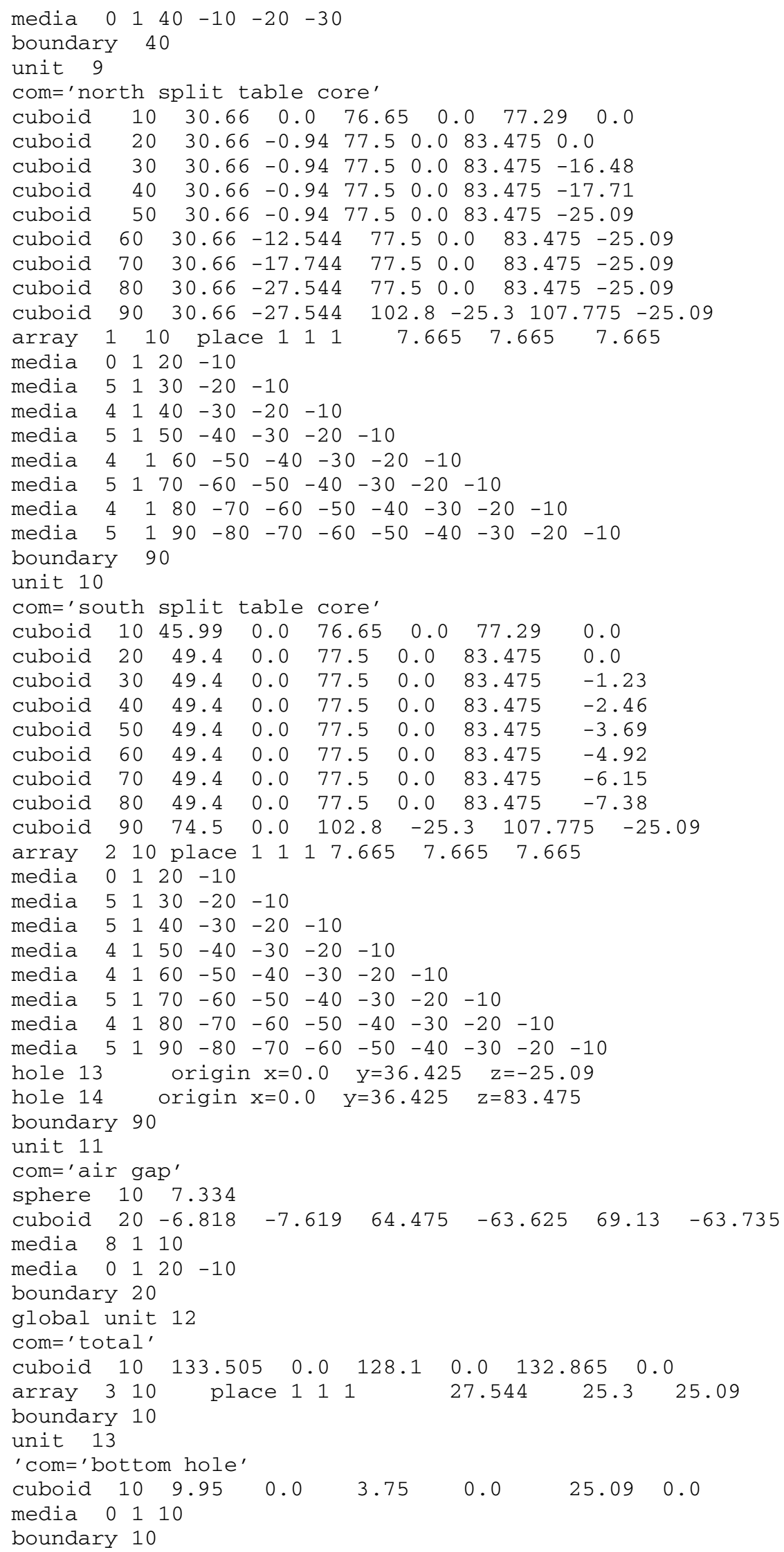




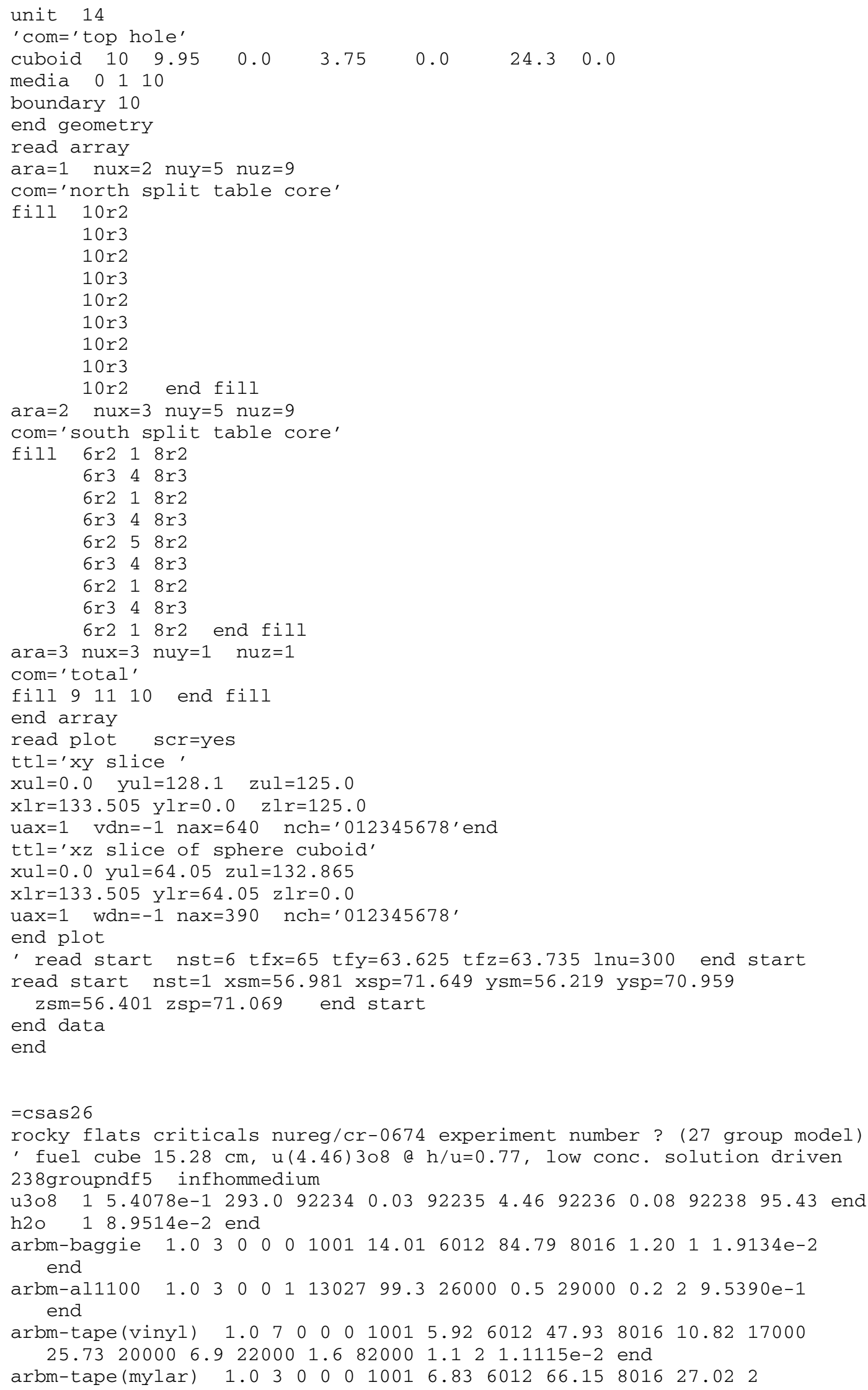




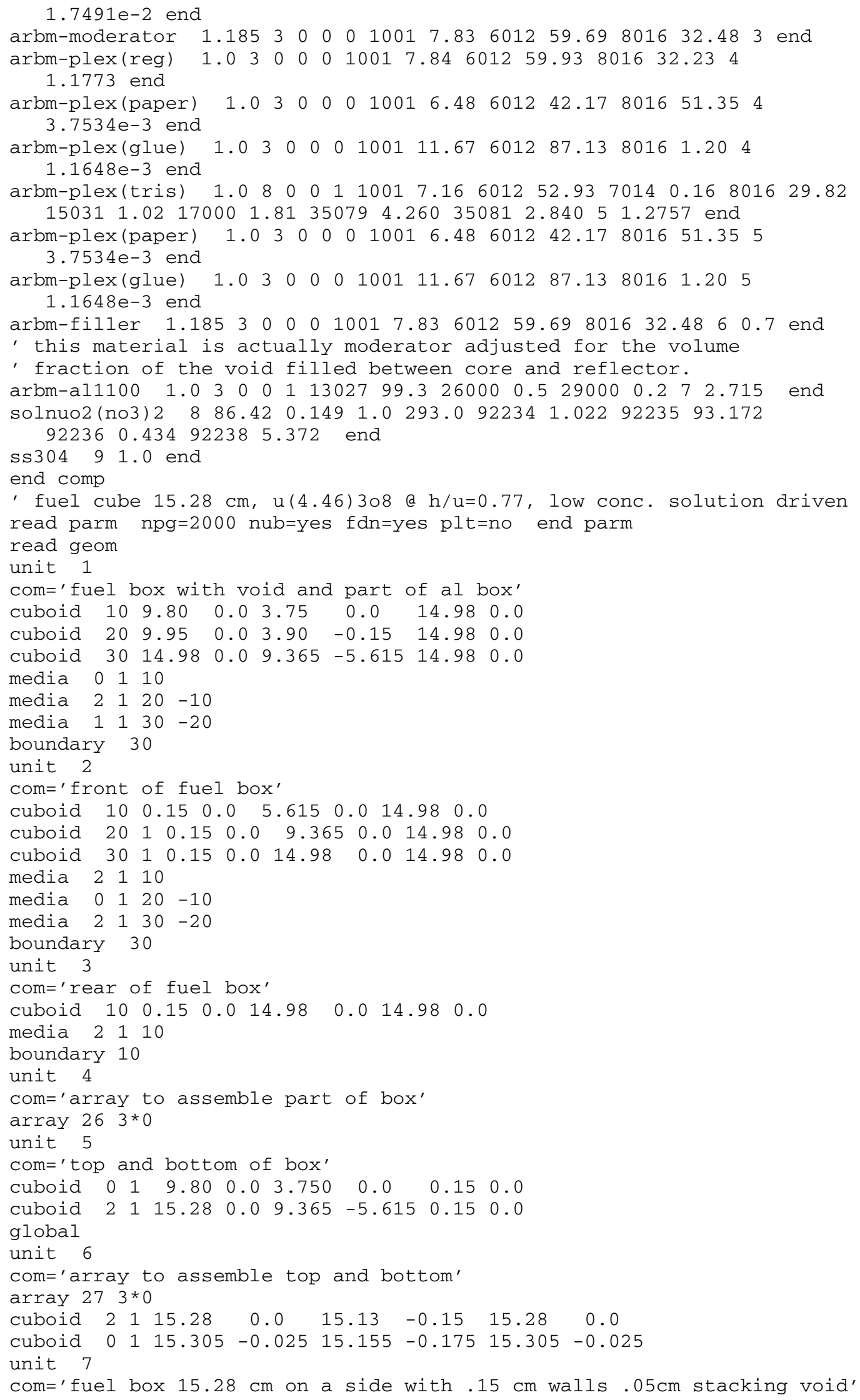


cuboid $1116 \mathrm{p} 7.49$

cuboid $2 \quad 16 \mathrm{p} 7.64$

cuboid $0 \quad 16 \mathrm{p} 7.6650$

unit 34

com='aluminum weight distribution plate for special box'

$\begin{array}{lllllllll}\text { cuboid } & 0 & 1 & 9.80 & 0.0 & 3.750 & 0.0 & 0.16 & 0.0\end{array}$

$\begin{array}{llllllllll}\text { cuboid } 7 & 1 & 15.33 & 0.0 & 9.54 & -5.79 & 0.16 & 0.0\end{array}$

unit 35

com='aluminum weight distribution plate for boxes'

cuboid 714 p 7.6650 .160 .0

unit 9

com $=$ 'north split table core'

array $13 * 0.0$

$\begin{array}{llllllllll}\text { cuboid } 0 & 1 & 30.66 & -0.94 & 77.5 & 0.0 & 83.475 & 0.0\end{array}$

unit 10

com='south split table core'

array $23 * 0.0$

$\begin{array}{llllllllll}\text { cuboid } & 0 & 1 & 49.4 & 0.0 & 77.5 & 0.0 & 83.475 & 0.0\end{array}$

unit 11

com='plexiglass reflector sheet without tris, north bottom reflector'

cuboid $412 \mathrm{p} 15.80002 \mathrm{p} 38.75002 \mathrm{p} 0.6150$

unit 12

com='plexiglass reflector sheet with tris, north bottom reflector'

cuboid $512 \mathrm{p} 15.80002 \mathrm{p} 38.75002 \mathrm{p} 0.6150$

unit 13

com='upper portion north bottom reflector with tris'

cuboid $5 \quad 12 \mathrm{p} 15.8 \quad 2 \mathrm{p} 38.75 \quad 2 \mathrm{p} 8.24$

unit 14

com='lower portion north bottom reflector with tris'

cuboid $5 \quad 12$ p15.8 2 p38.75 2p3.69

unit 15

com='north bottom reflector includes regular and tris'

array $3 \quad 3 * 0.0$

unit 16

com='plexiglas sheet bottom south reflector without tris'

cuboid 0 1 $2 \mathrm{p} 5.12 \mathrm{p} 2.5 \quad 2 \mathrm{p} 0.6150$

cuboid $4 \quad 144.3 \quad-5.1 \quad 2 p 38.75 \quad 2 p 0.615$

unit 17

com='plexiglas sheet bottom south reflector with tris'

cuboid $0 \quad 12 \mathrm{p} 5.12 \mathrm{p} 2.5 \quad 2 \mathrm{p} 0.6150$

cuboid $5144.3 \quad-5.1 \quad 2 \mathrm{p} 38.75 \quad 2 \mathrm{p} 0.6150$

unit 18

com='lower portion south bottom reflector with tris'

cuboid $0 \quad 12 \mathrm{p} 5.12 \mathrm{p} 2.5 \quad 2 \mathrm{p} 8.855$

cuboid 5 1 $44.3 \quad-5.1 \quad 2 \mathrm{p} 38.75 \quad 2 \mathrm{p} 8.855$

unit 19

com='south bottom reflector with regular and tris'

array $4 \quad 3 * 0.0$

unit 20

com='east and west reflectors for north reflector with tris'

cuboid $5 \quad 12 \mathrm{p} 15.8 \quad 2 \mathrm{p} 12.65 \quad 2 \mathrm{p} 54.2825$

unit 21

com='array for east and west reflectors for north reflector'

array $53 * 0.0$

unit 22

com='east and west reflectors for south reflector with tris' cuboid $512 \mathrm{p} 24.700 \quad 2 \mathrm{p} 12.65 \quad 2 \mathrm{p} 54.2825$

unit 23

com='array for east and west reflectors for south reflector'

array $63 * 0.0$

unit 24

com='north top reflector with tris'

cuboid $512 \mathrm{p} 28.35$ 2p64.05 2p12.15 


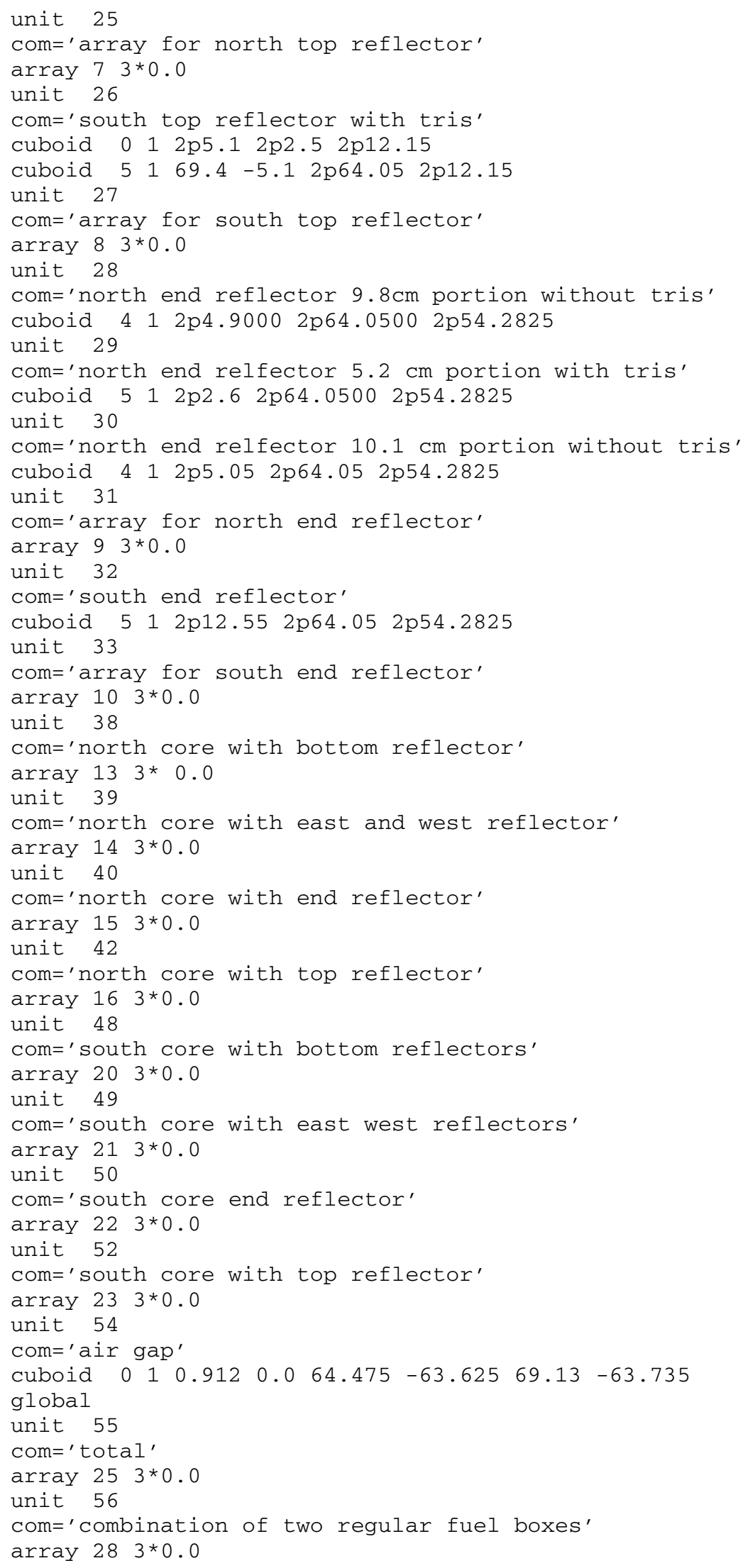




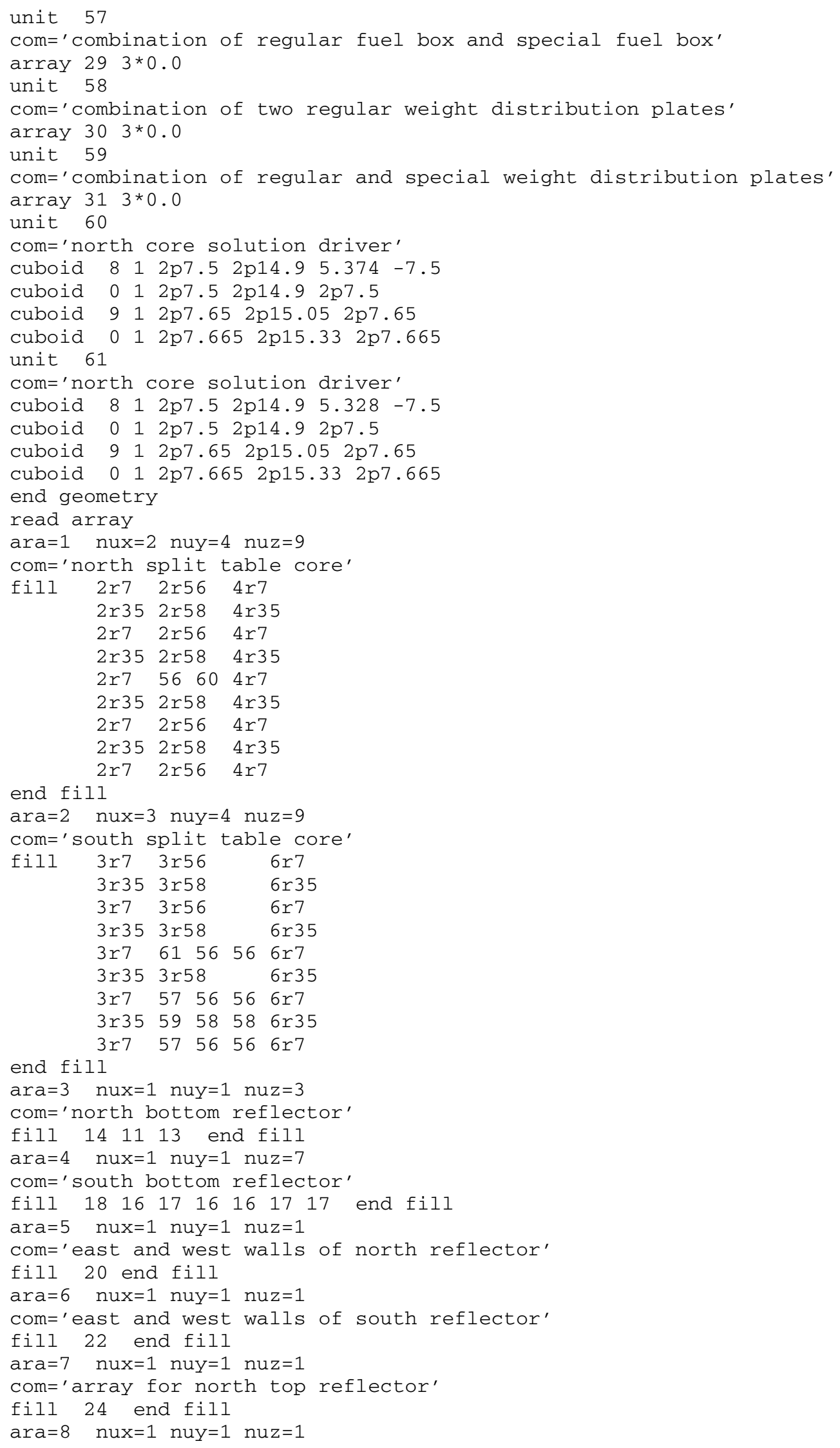




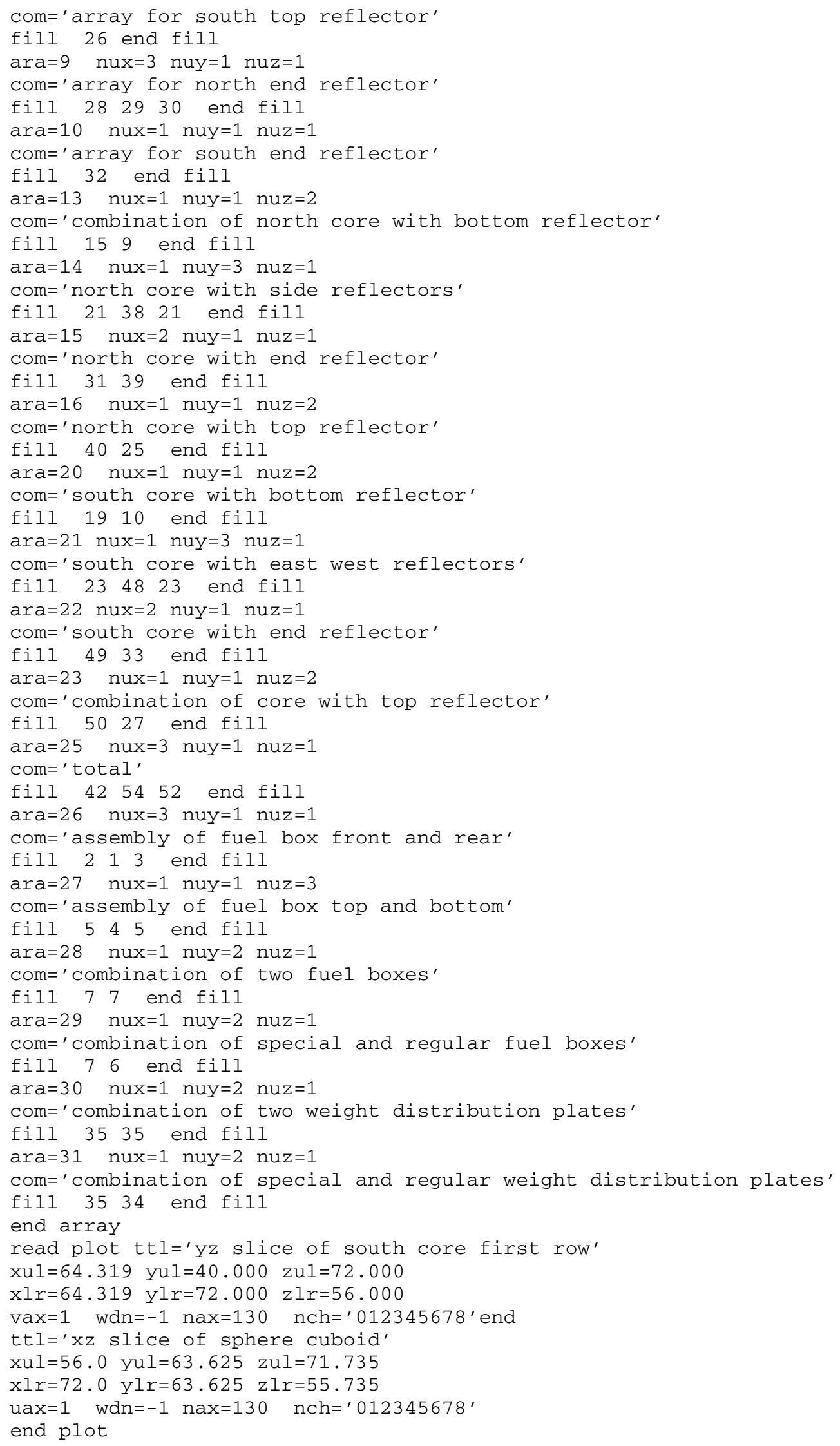




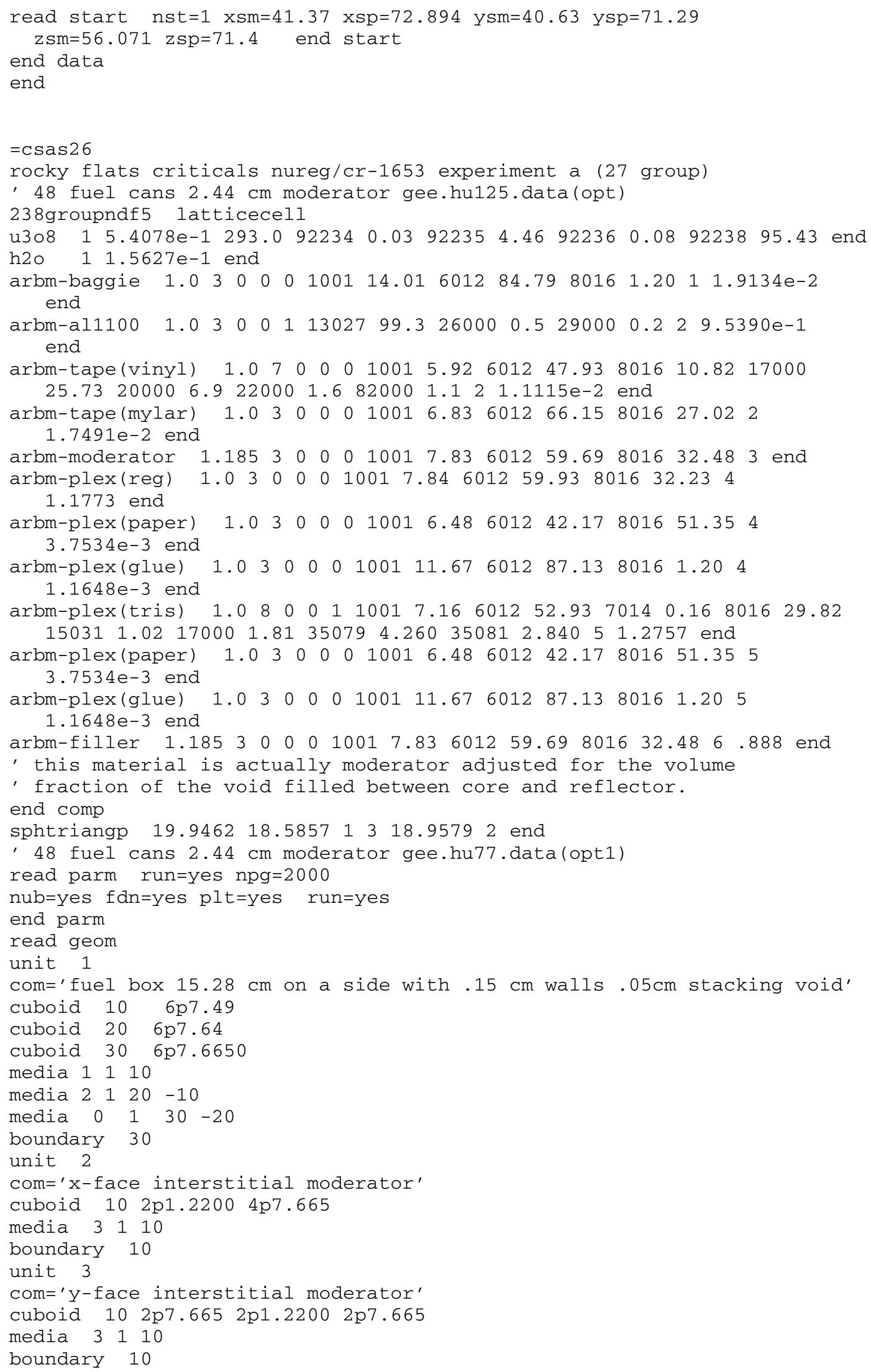




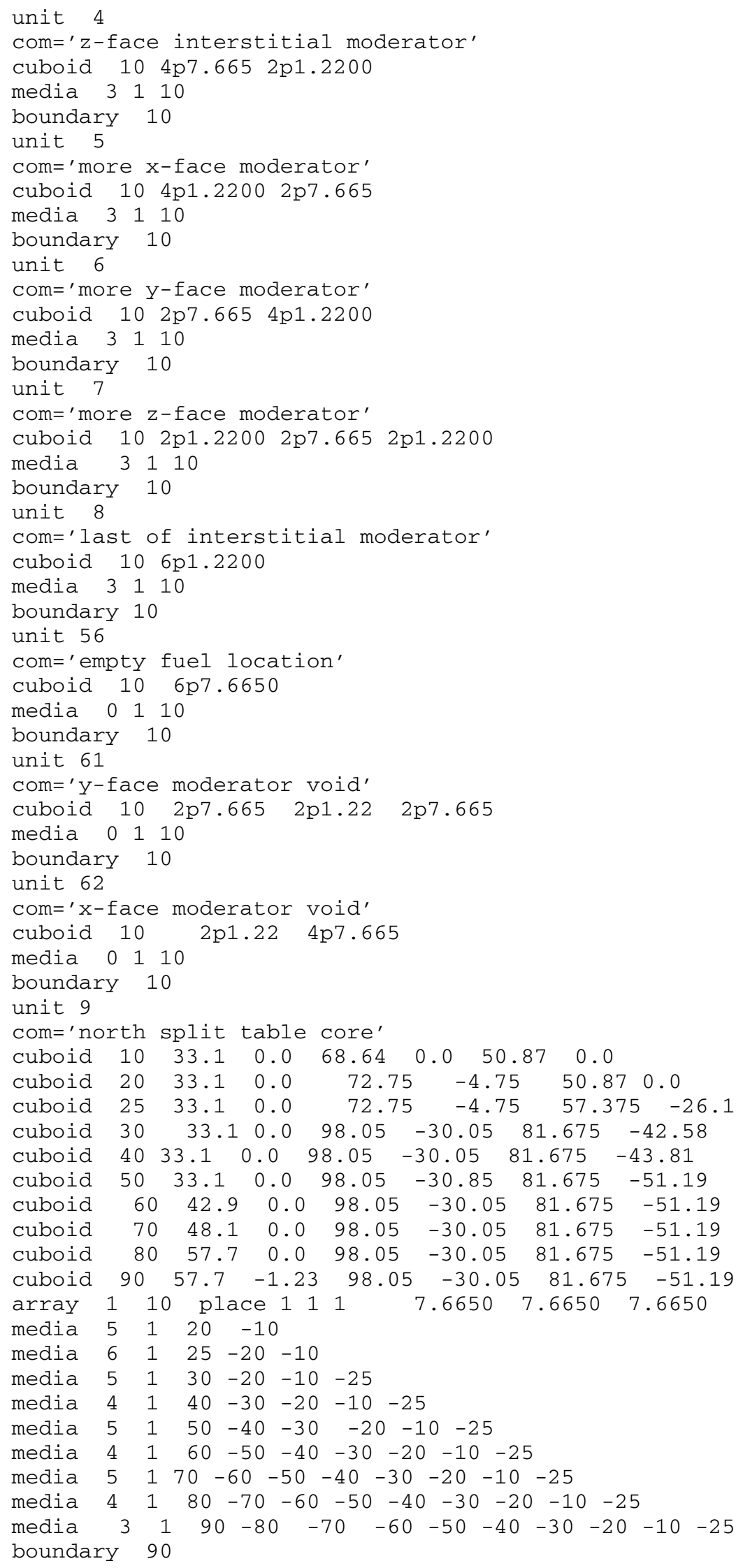




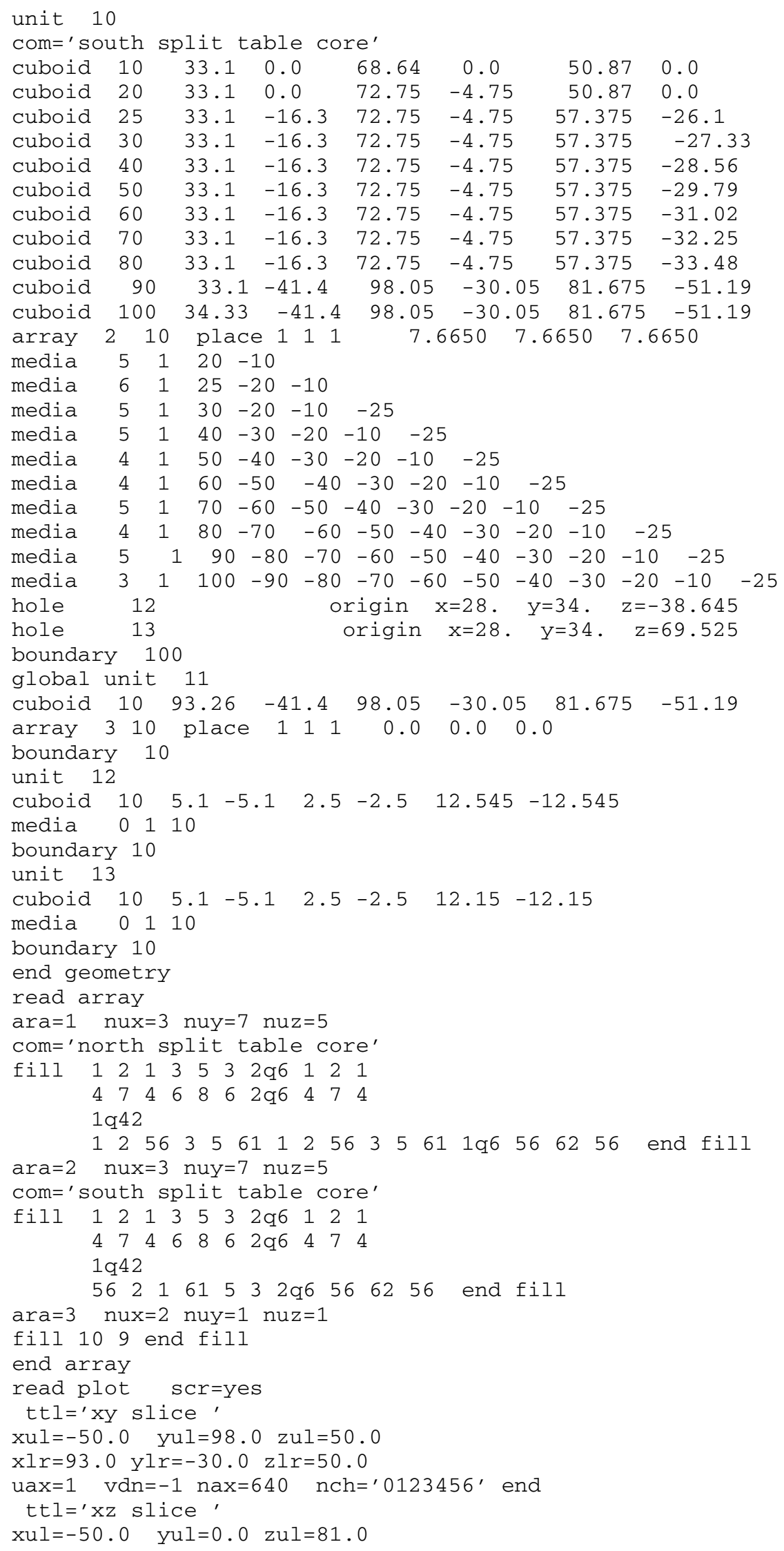




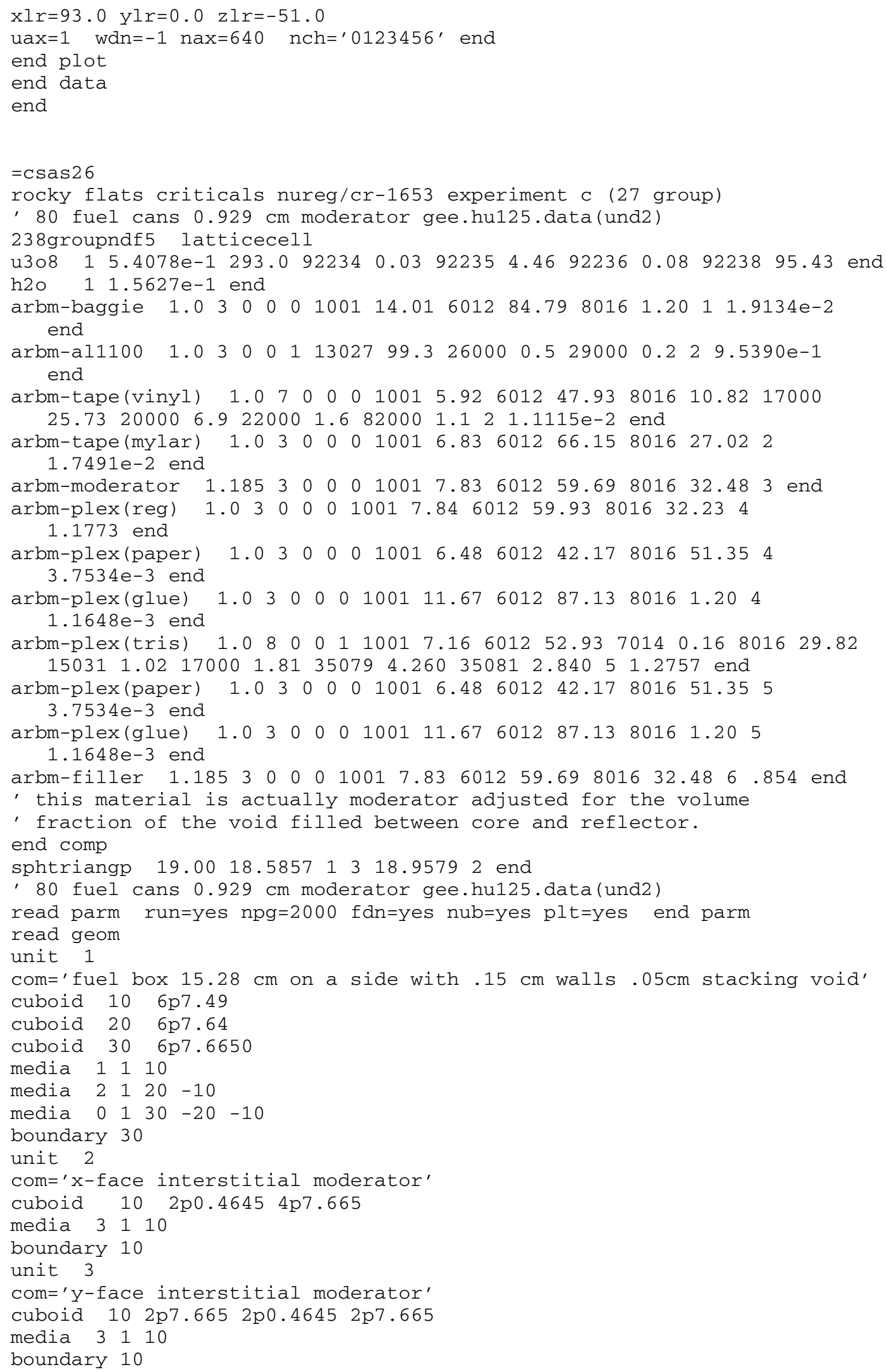




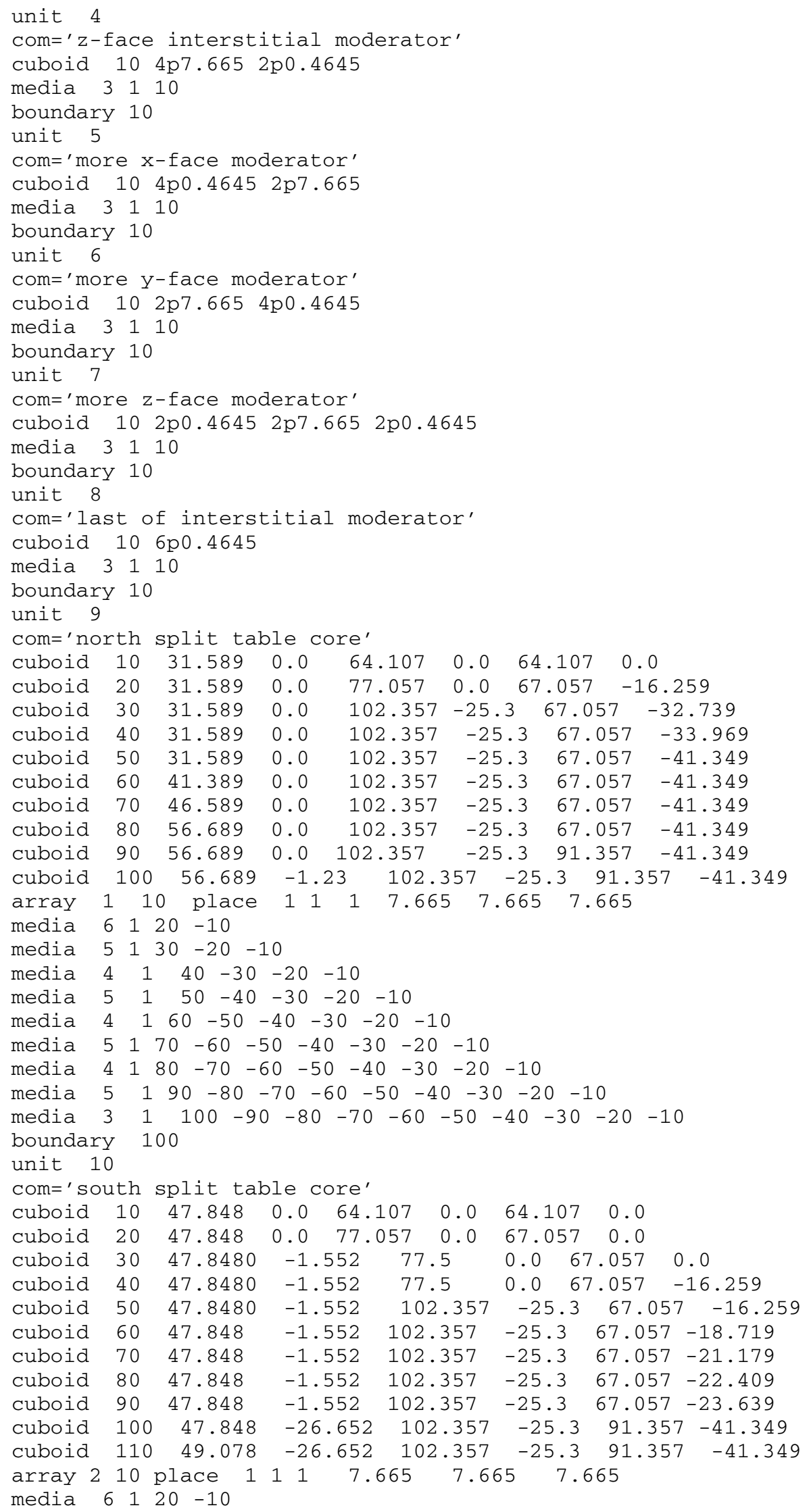




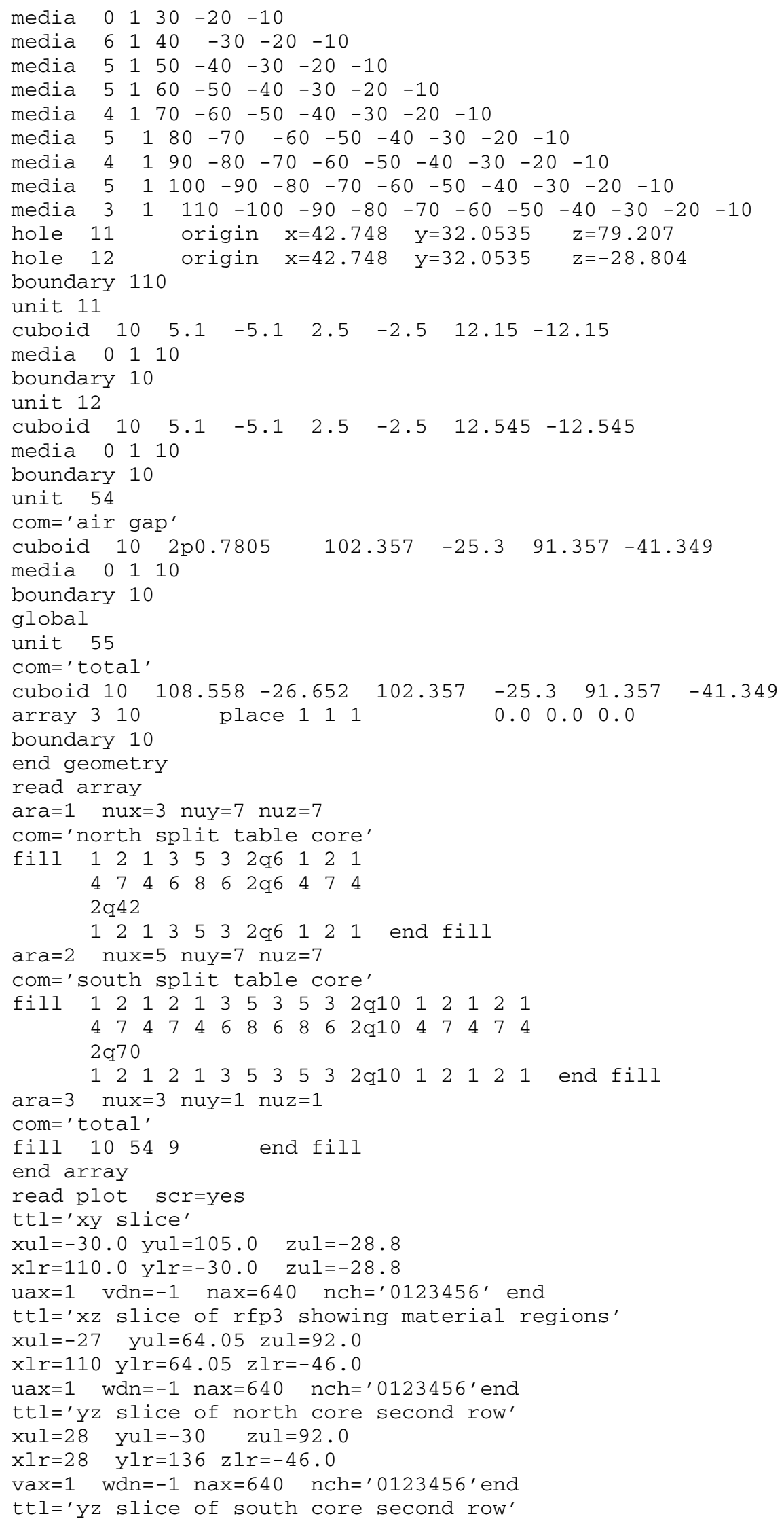


$\mathrm{xul}=80$ yul=-30 $\mathrm{zul}=92.0$

$\mathrm{xlr}=80$ ylr=136 $\mathrm{zlr}=-46.0$

$\operatorname{vax}=1$ wdn $=-1$ nax $=640$ nch='0123456'

end plot

end data

end

$=\operatorname{csas} 26$

rocky flats criticals nureg/cr-1653 experiment number ? (27 group model)

' fuel cube $15.28 \mathrm{~cm}$, u(4.46)308 @ h/u=1.25, hi conc. solution driven 238 groupndf5 infhommedium

u3o8 $15.4078 e-1 \quad 293.0 \quad 92234 \quad 0.0392235 \quad 4.4692236 \quad 0.089223895 .43$ end

h2o $11.5627 e-1$ end

arbm-baggie $1.0 \quad 3 \quad 0 \quad 0 \quad 0 \quad 1001 \quad 14.016012 \quad 84.798016 \quad 1.2011 .9134 \mathrm{e}-2$ end

arbm-al1100 $1.0 \quad 3 \quad 0 \quad 0 \quad 1 \quad 13027 \quad 99.326000 \quad 0.5 \quad 29000 \quad 0.2 \quad 2 \quad 9.5390 e-120$ end

arbm-tape (vinyl) $\quad 1.0 \quad 7 \quad 0 \quad 0 \quad 0 \quad 1001 \quad 5.92 \quad 6012 \quad 47.93 \quad 8016 \quad 10.82 \quad 17000$ $25.73200006 .9220001 .6820001 .121 .1115 \mathrm{e}-2$ end

arbm-tape (mylar) $1.0 \quad 3 \quad 0 \quad 0 \quad 0 \quad 1001 \quad 6.83 \quad 6012 \quad 66.15 \quad 8016 \quad 27.02 \quad 2$ $1.7491 e-2$ end

arbm-moderator $1.185 \quad 3 \quad 0 \quad 0 \quad 0 \quad 1001 \quad 7.83 \quad 6012 \quad 59.698016 \quad 32.48 \quad 3$ end

arbm-plex(reg) $\quad 1.0 \quad 3 \quad 0 \quad 0 \quad 0 \quad 1001 \quad 7.84 \quad 6012 \quad 59.93 \quad 8016 \quad 32.23 \quad 4$ 1.1773 end

arbm-plex(paper) $\quad 1.0 \quad 3 \quad 0 \quad 0 \quad 0 \quad 1001 \quad 6.48 \quad 6012 \quad 42.17 \quad 8016 \quad 51.35 \quad 4$ $3.7534 \mathrm{e}-3$ end

arbm-plex(glue) $\quad \begin{array}{llllllllllllll}1.0 & 3 & 0 & 0 & 0 & 1001 & 11.67 & 6012 & 87.13 & 8016 & 1.20 & 4\end{array}$ $1.1648 e-3$ end

arbm-plex(tris) $\quad 1.0 \quad 8 \quad 0 \quad 0 \quad 1 \quad 1001 \quad 7.16 \quad 6012 \quad 52.93 \quad 7014 \quad 0.16 \quad 8016 \quad 29.82$ $\begin{array}{llllllllll}15031 & 1.02 & 17000 & 1.81 & 35079 & 4.260 & 35081 & 2.840 & 5 & 1.2757\end{array}$ end

arbm-plex(paper) $\quad 1.0 \quad 3 \quad 0 \quad 0 \quad 0 \quad 1001 \quad 6.48 \quad 6012 \quad 42.17 \quad 8016 \quad 51.35 \quad 5$ $3.7534 \mathrm{e}-3$ end

arbm-plex(glue) $\quad 1.0 \quad 3 \quad 0 \quad 0 \quad 0 \quad 1001 \quad 11.67 \quad 6012 \quad 87.13 \quad 8016 \quad 1.205$ $1.1648 \mathrm{e}-3$ end

arbm-filler $1.1853000010017 .83 \quad 6012 \quad 59.698016 \quad 32.48 \quad 6 \quad 0.7$ end

, this material is actually moderator adjusted for the volume

' fraction of the void filled between core and reflector.

arbm-al1100 $1.0 \quad 3 \quad 0 \quad 0 \quad 1 \quad 13027 \quad 99.3 \quad 26000 \quad 0.5 \quad 29000 \quad 0.2 \quad 7 \quad 2.715$ end

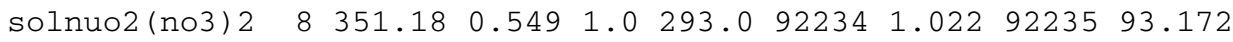

$92236 \quad 0.43492238 \quad 5.372$ end

s.304 91.0 end

end comp

read parm npg=2000 nub=yes fdn=yes plt=yes run=yes end parm

read geom

unit 1

' $\mathrm{com}=$ 'slotted fuel box'

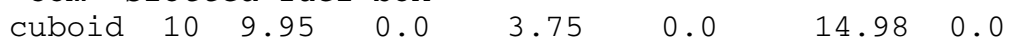

$\begin{array}{llllllll}\text { cuboid } & 20 & 10.1 & 0.0 & 3.9 & -.15 & 14.98 & 0.0\end{array}$

$\begin{array}{llllllll}\text { cuboid } 30 & 15.13 & .15 & 9.365 & -5.615 & 14.98 & 0.0\end{array}$

cuboid $40 \quad 15.28 \quad 0.0 \quad 9.515 \quad-5.765 \quad 15.13 \quad-.15$

cuboid $\begin{array}{lllllll}50 & 15.305 & -.025 & 9.54 & -5.79 & 15.155 & -.175\end{array}$

media 0110

media $2 \begin{array}{llll}1 & 20 & -10\end{array}$

$\begin{array}{llllll}\text { media } & 1 & 1 & 30 & -20 & -10\end{array}$

media $2 \quad 1 \quad 40-30-20-10$

media $\begin{array}{lllllll}0 & 1 & 50 & -40 & -30 & -20 & -10\end{array}$

boundary 50

unit 2

'com='fuel box $15.28 \mathrm{~cm}$ on a side with $.15 \mathrm{~cm}$ walls $.05 \mathrm{~cm}$ stacking void'

cuboid $10 \quad 6 \mathrm{p} 7.49$

cuboid $20 \quad 6 \mathrm{p} 7.64$

cuboid $30 \quad 6 \mathrm{p} 7.6650$ 


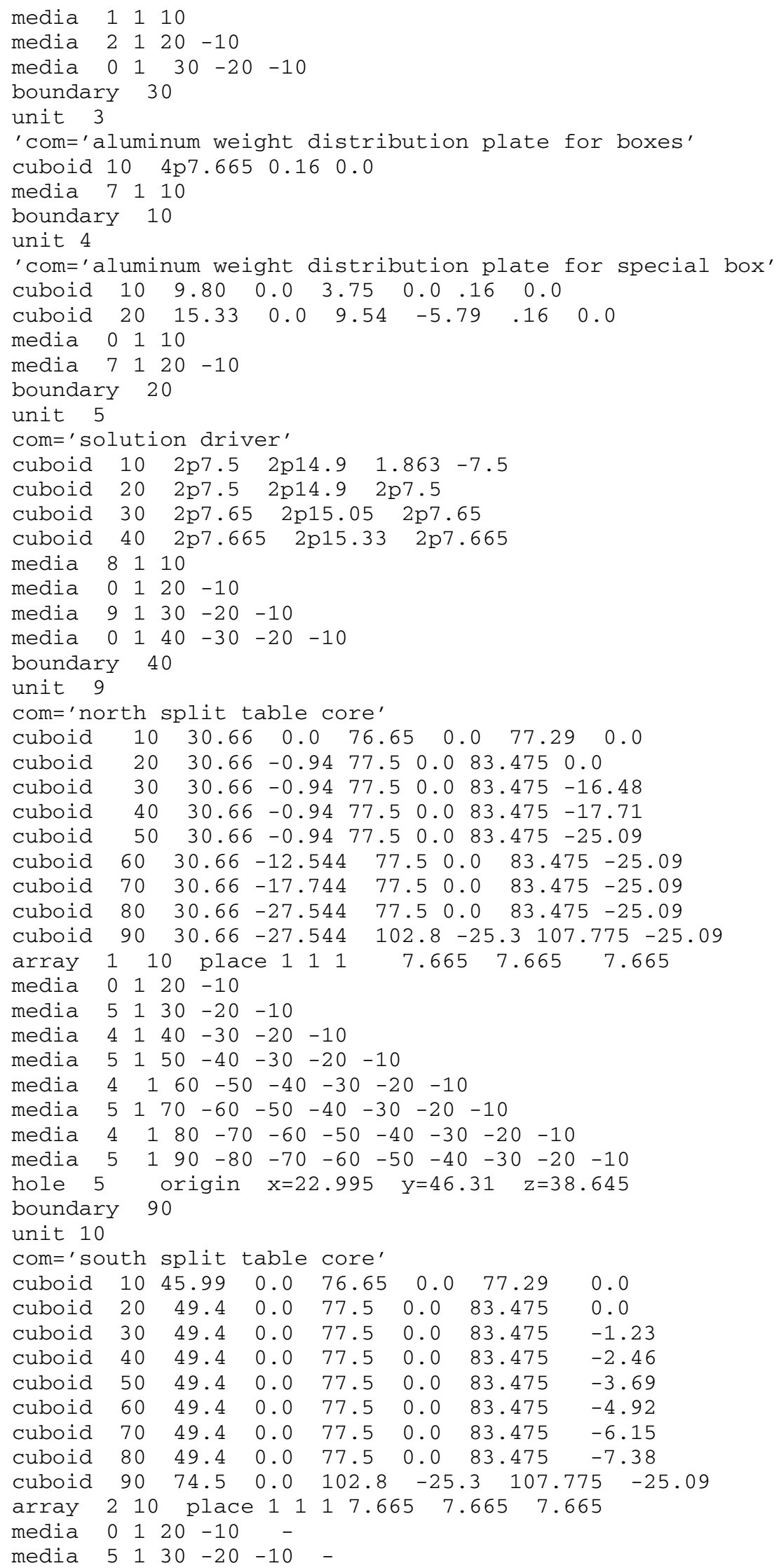




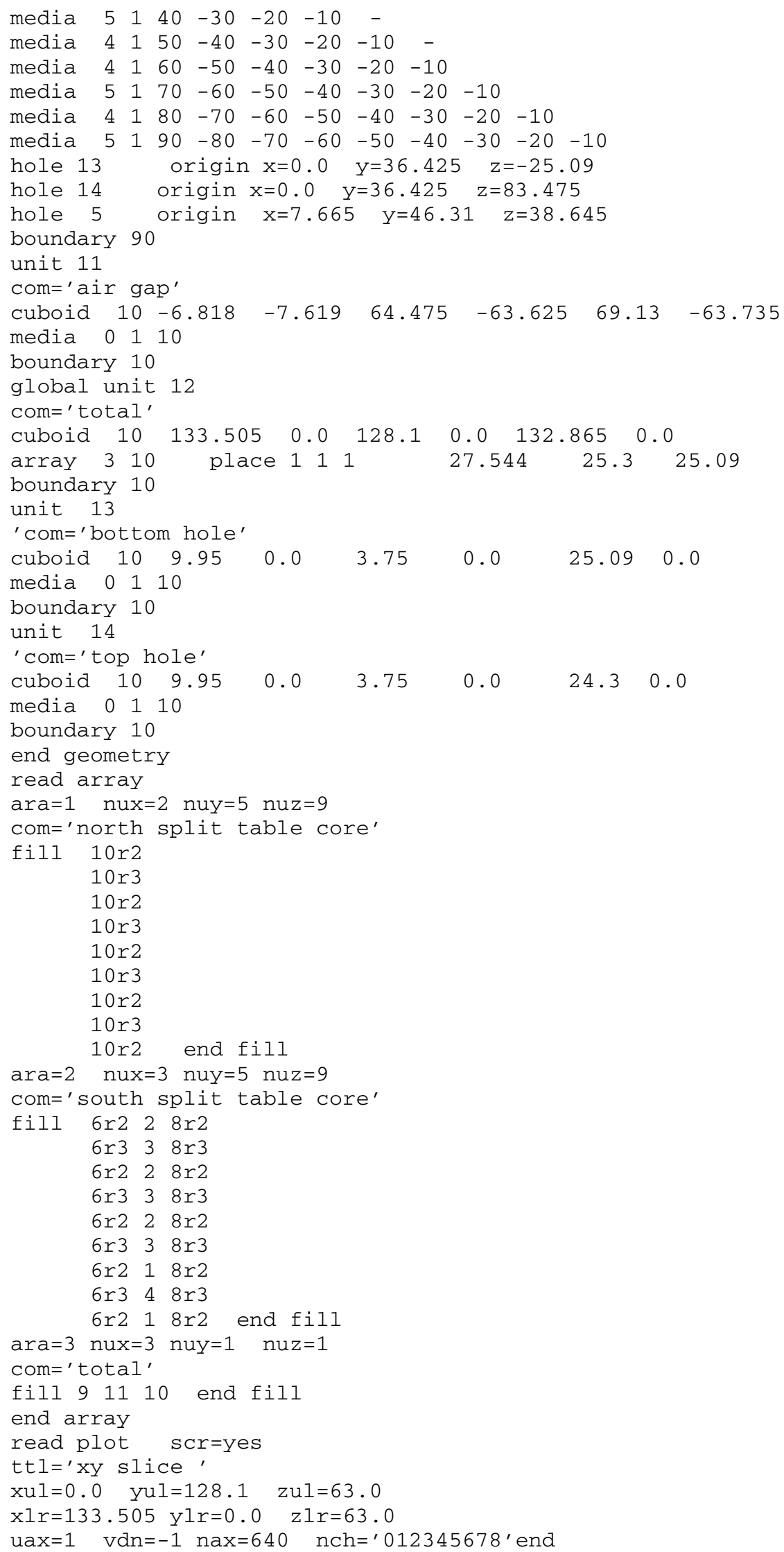




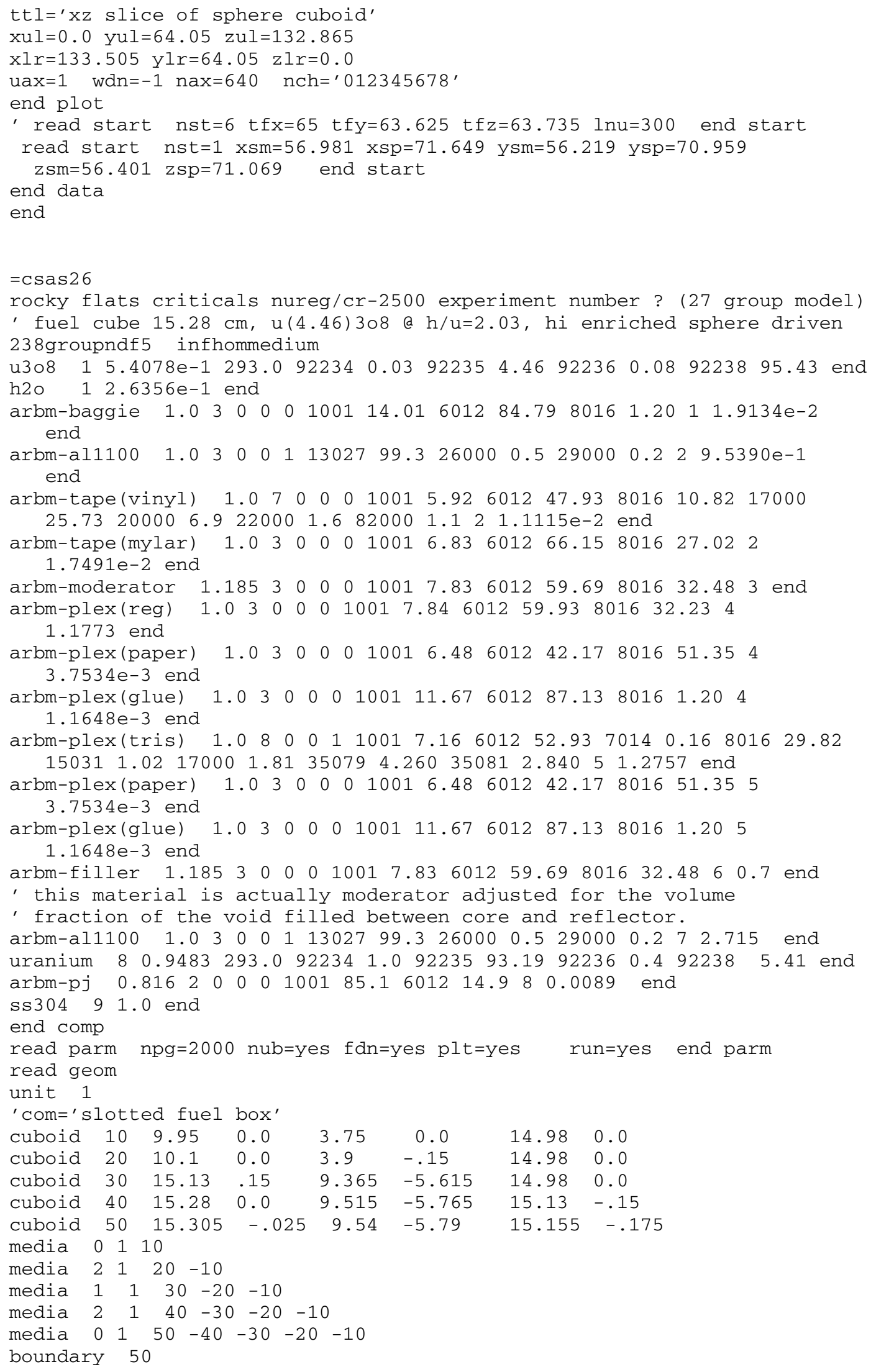




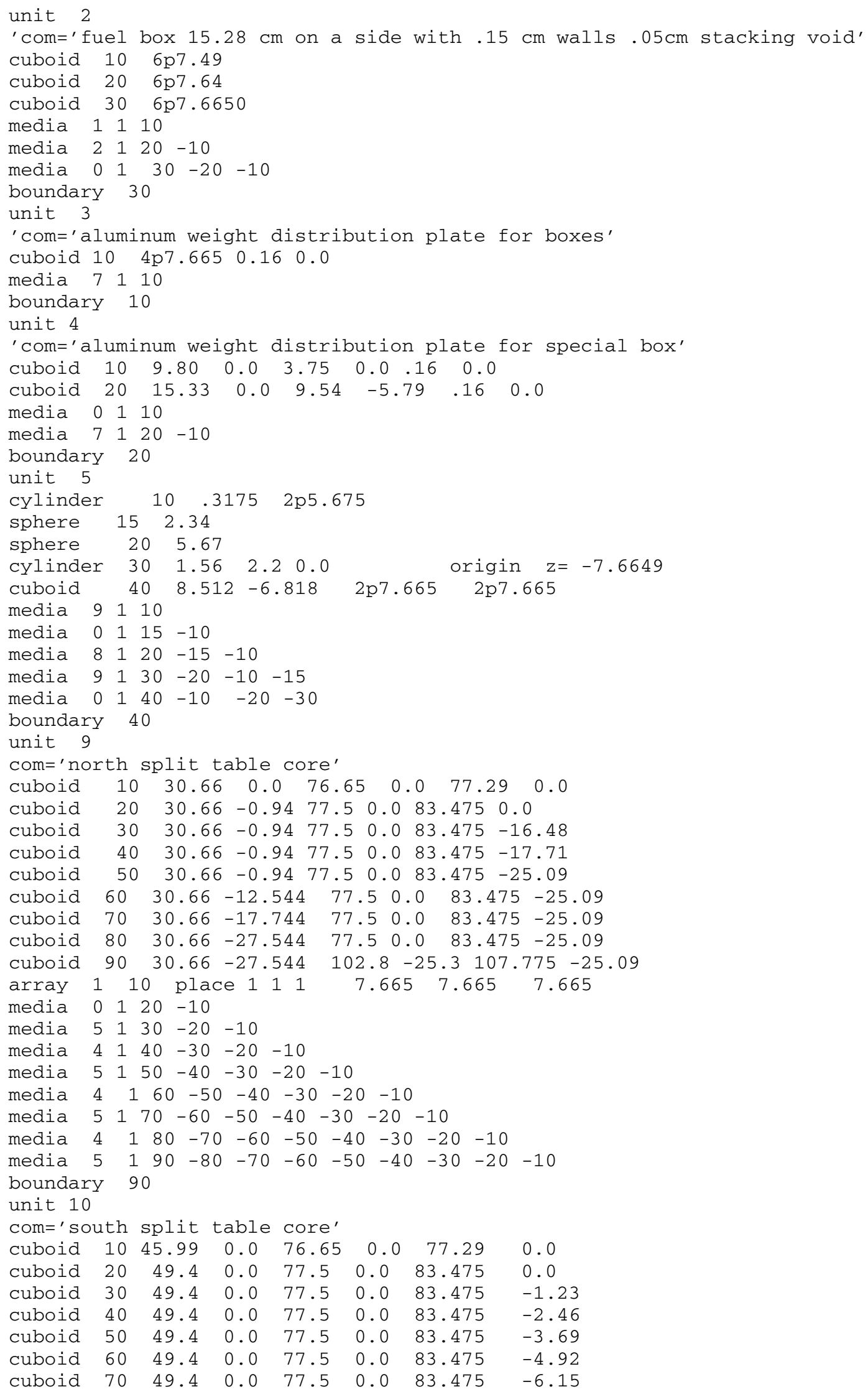




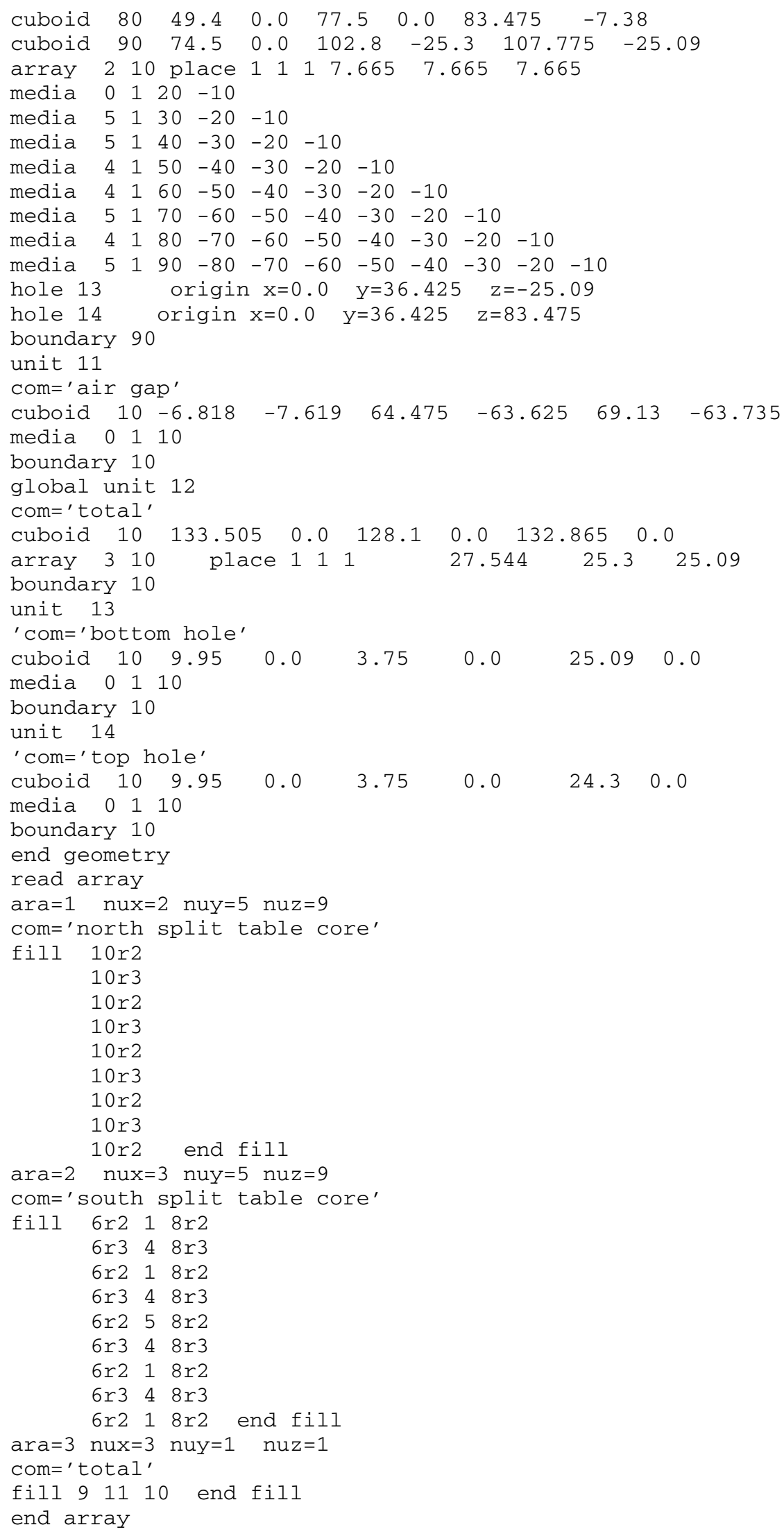




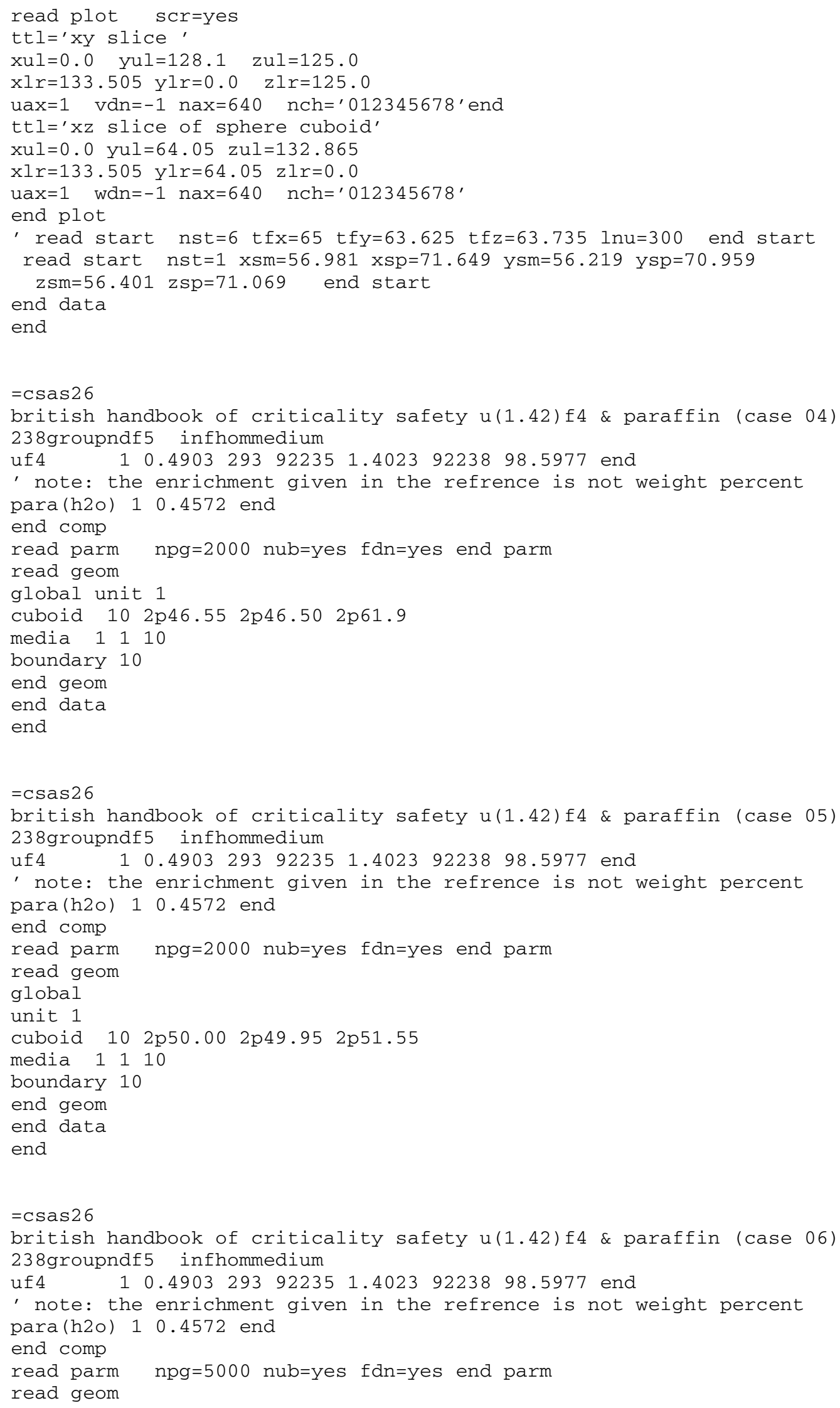




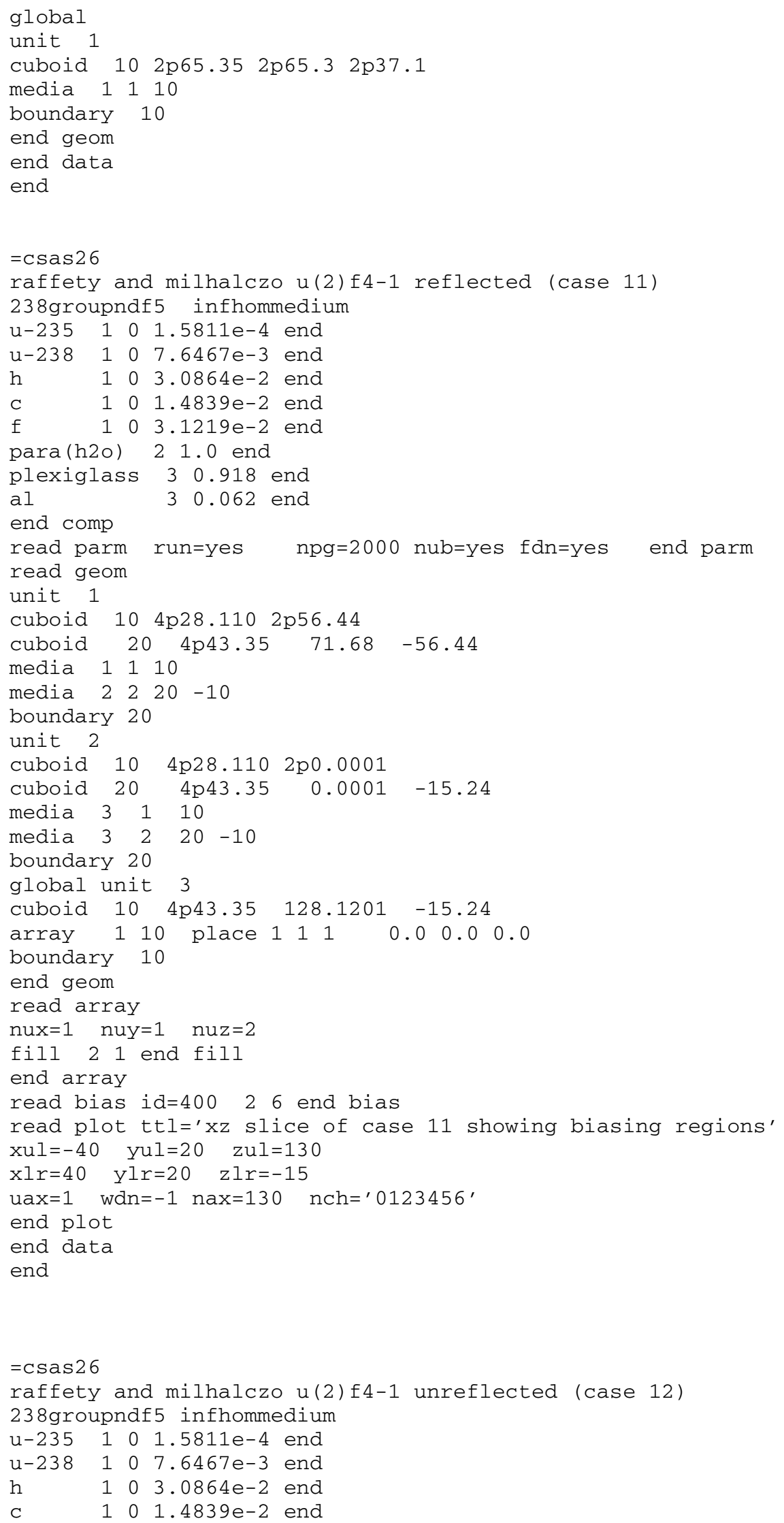




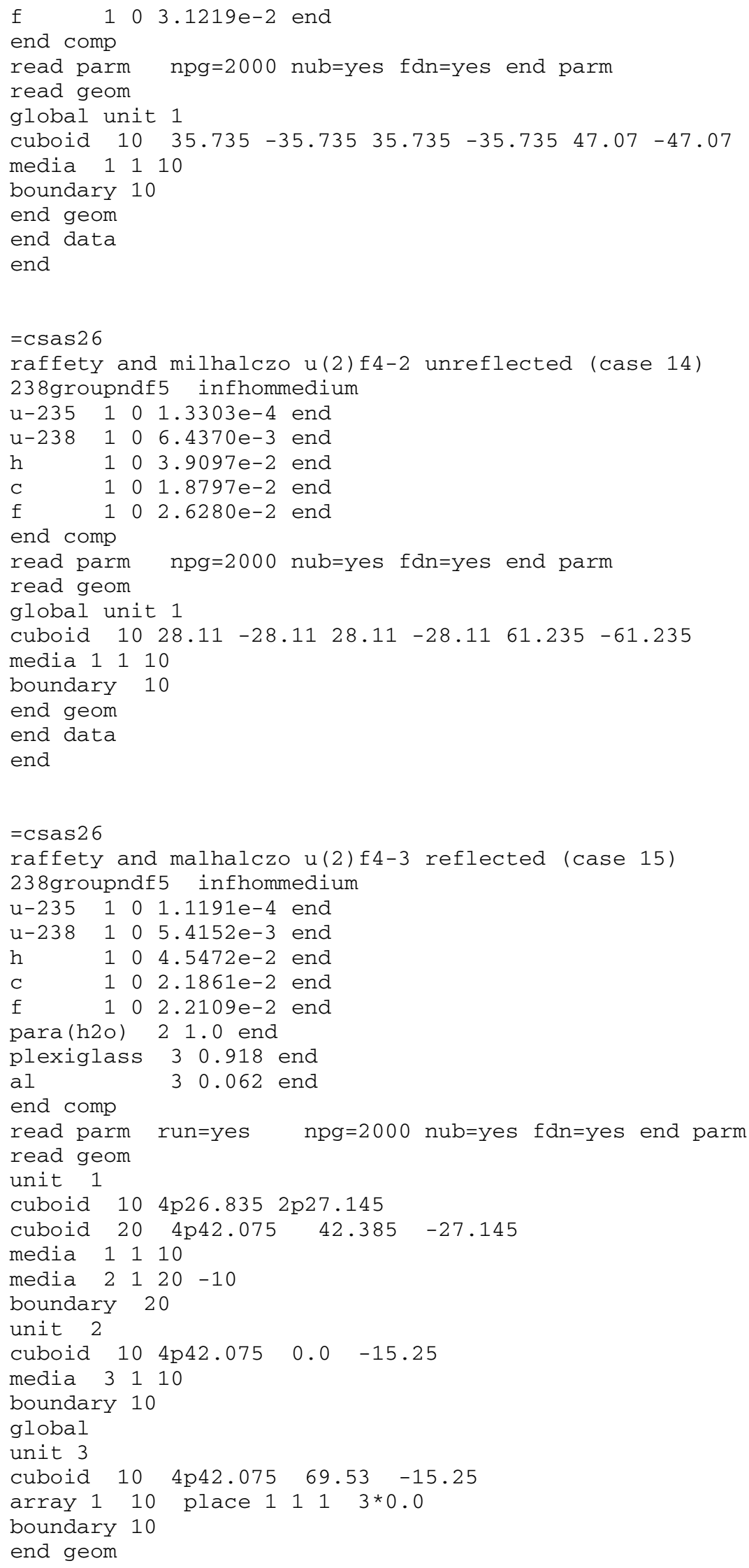




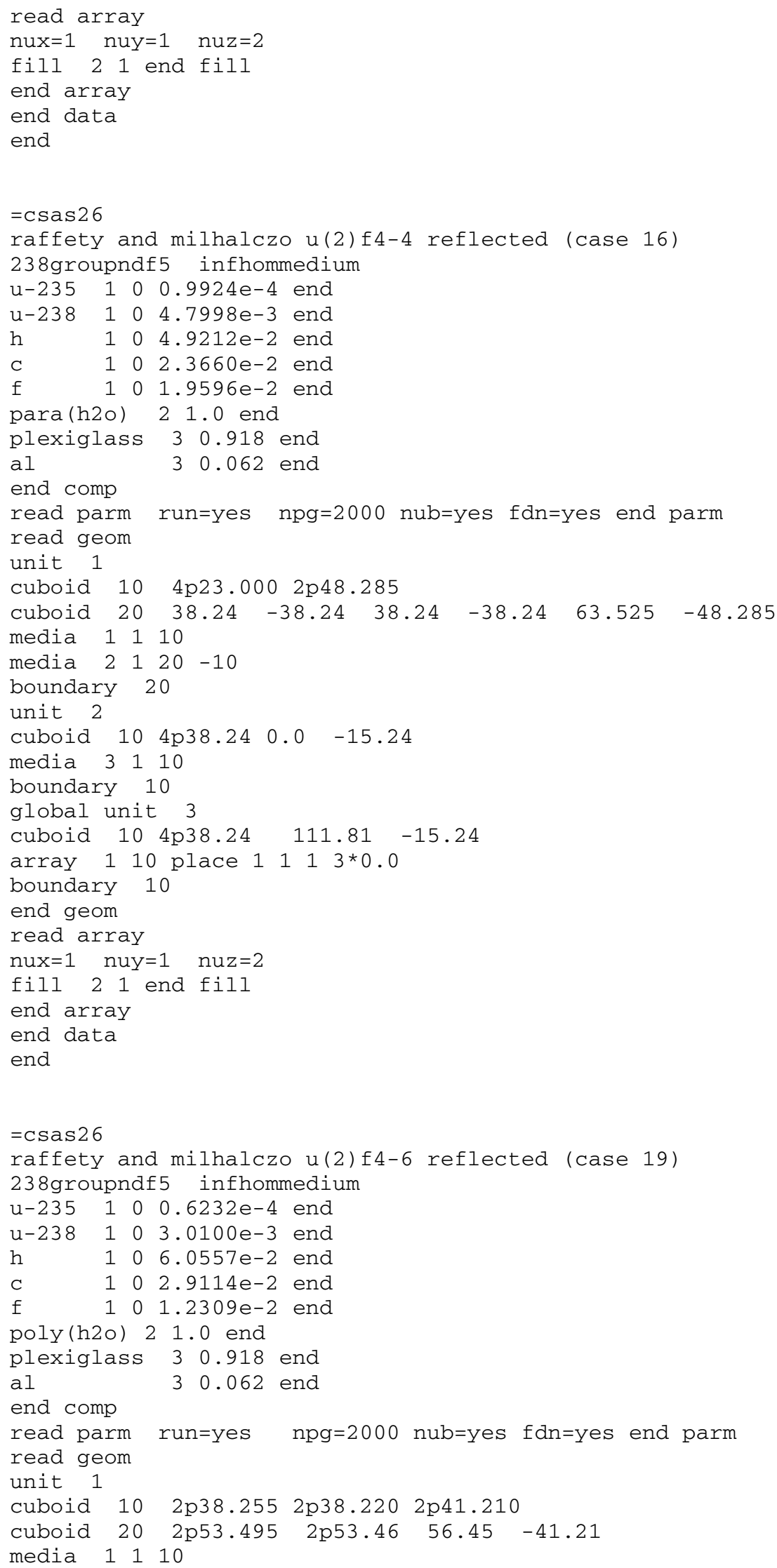




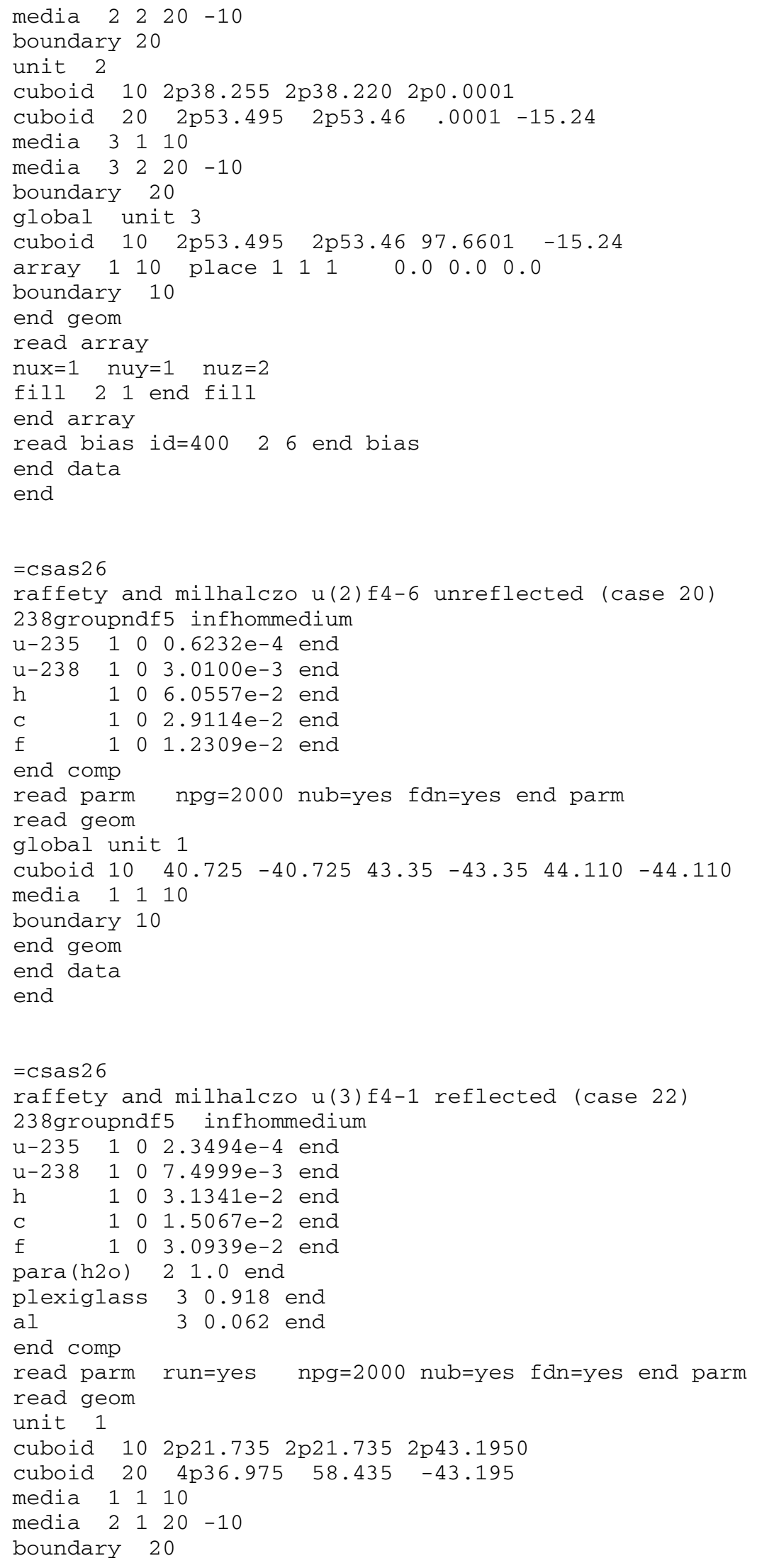




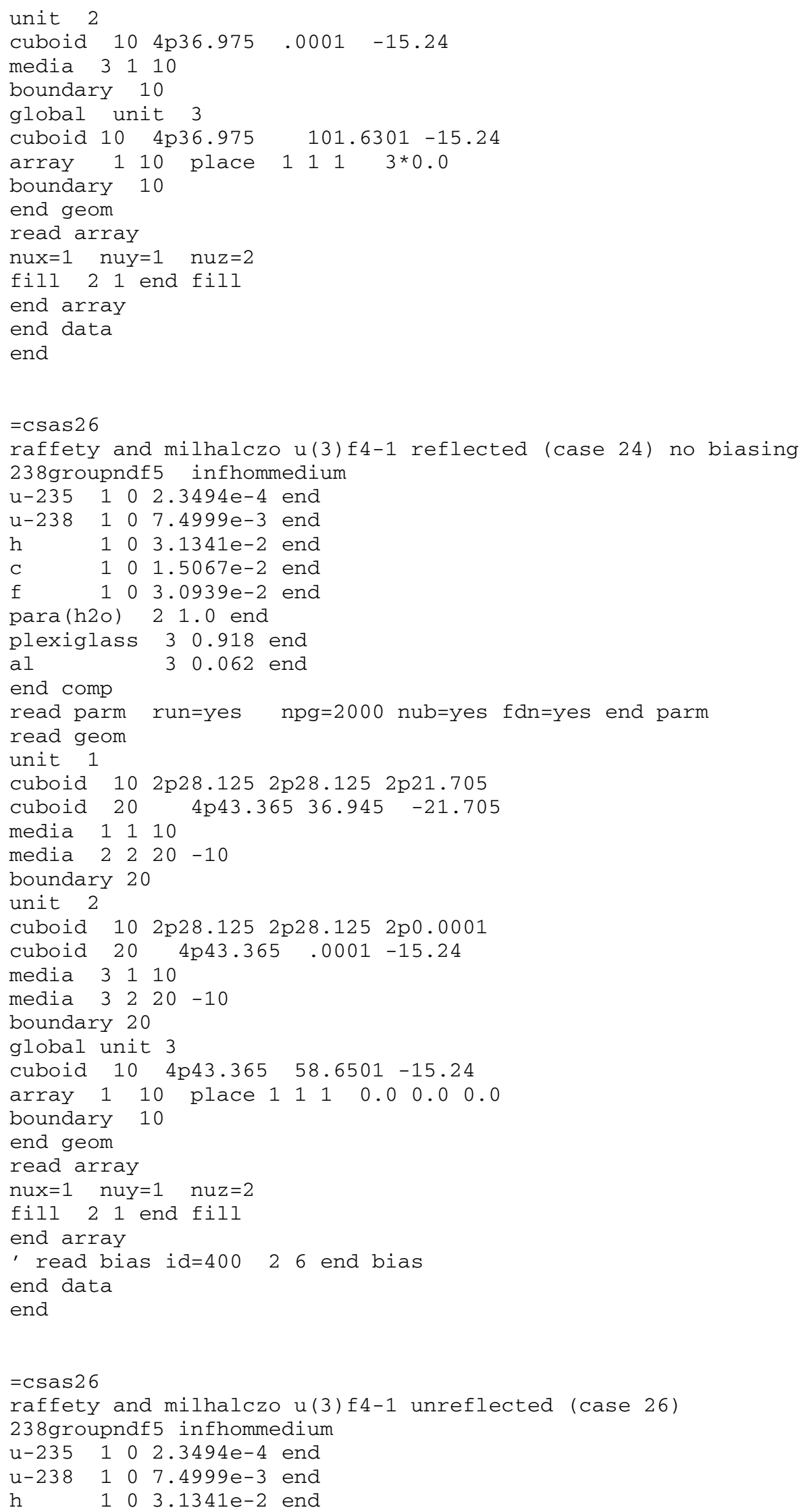




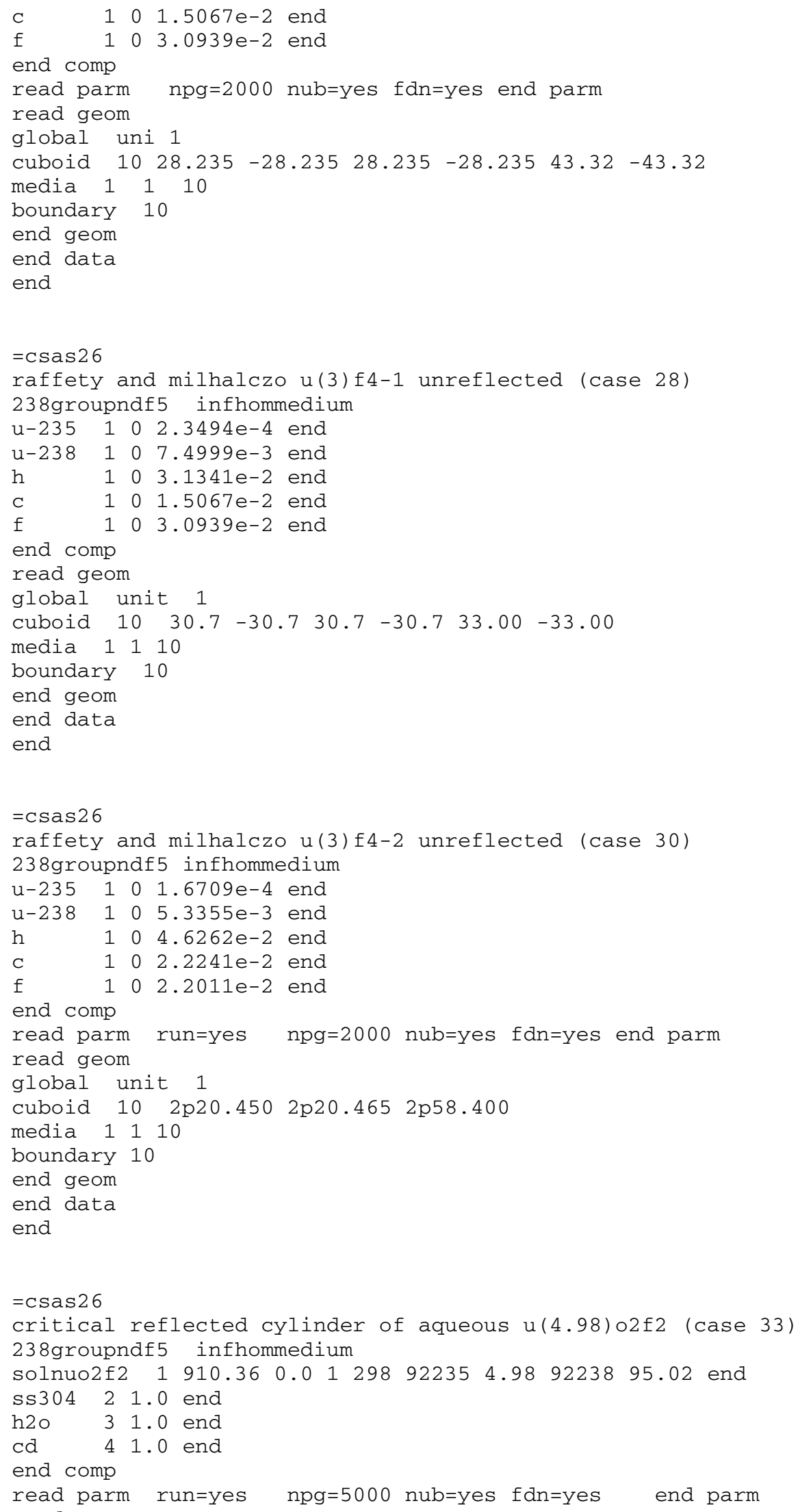




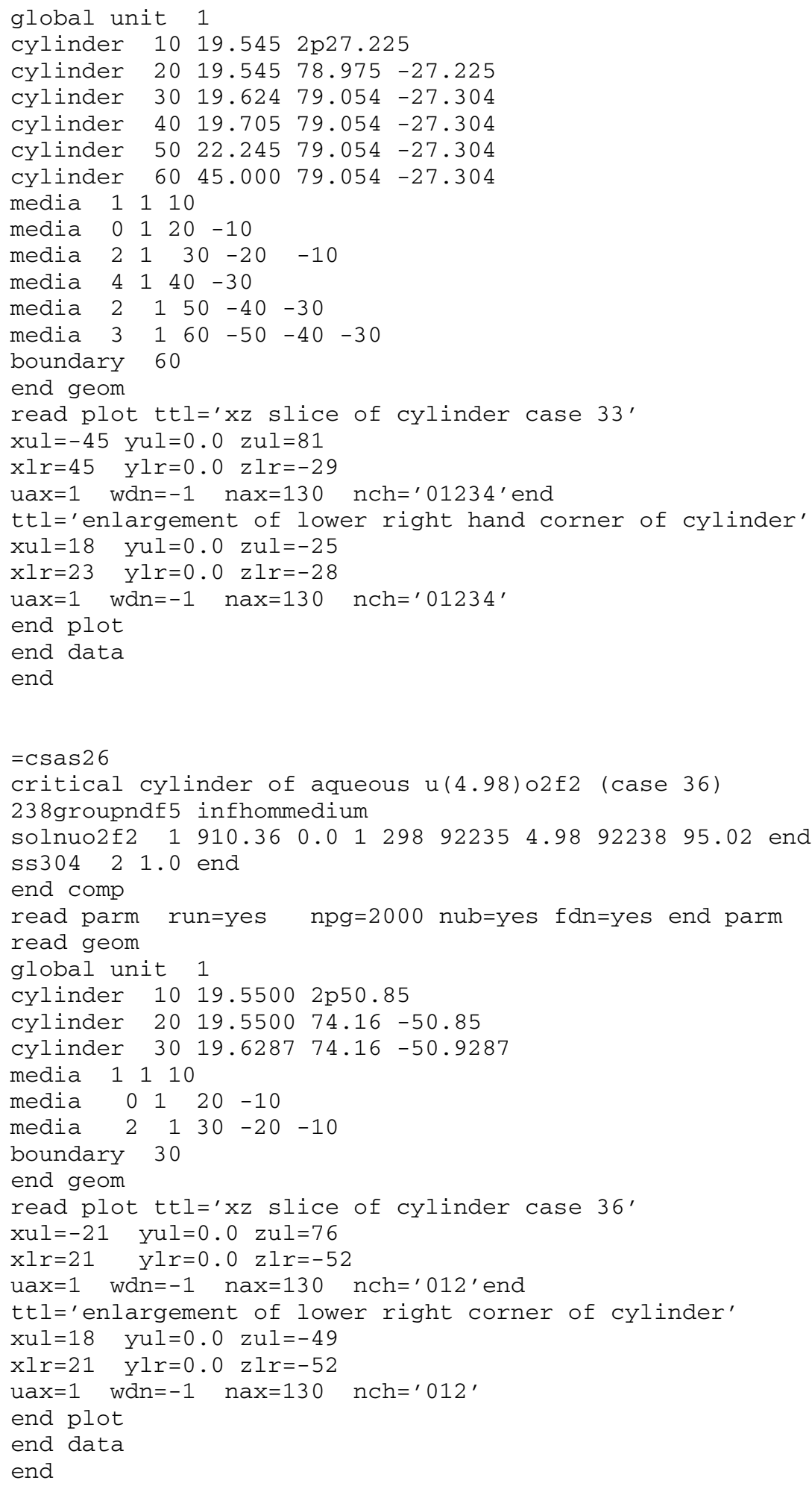


Table B.4. Table 4 input data

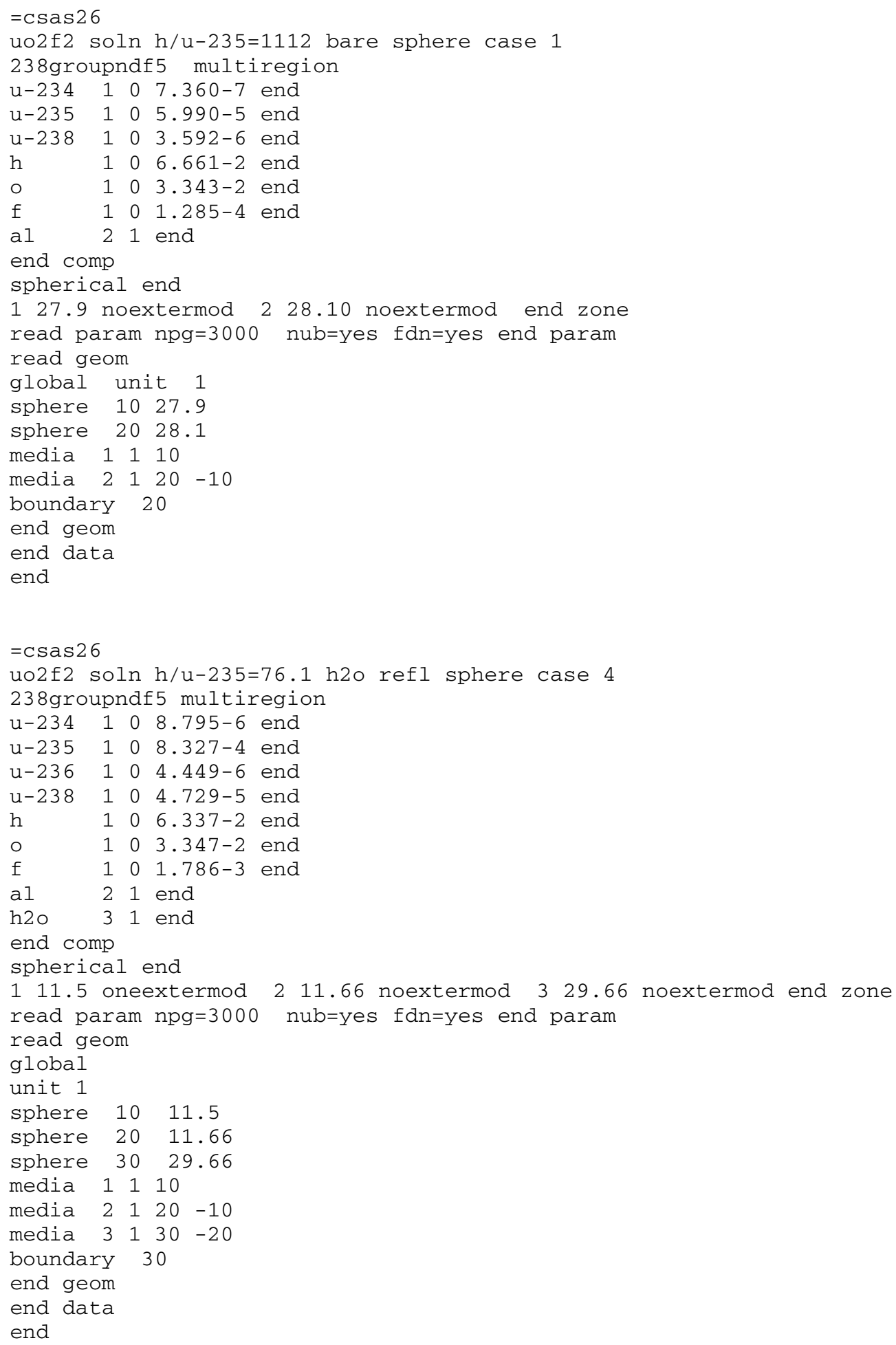




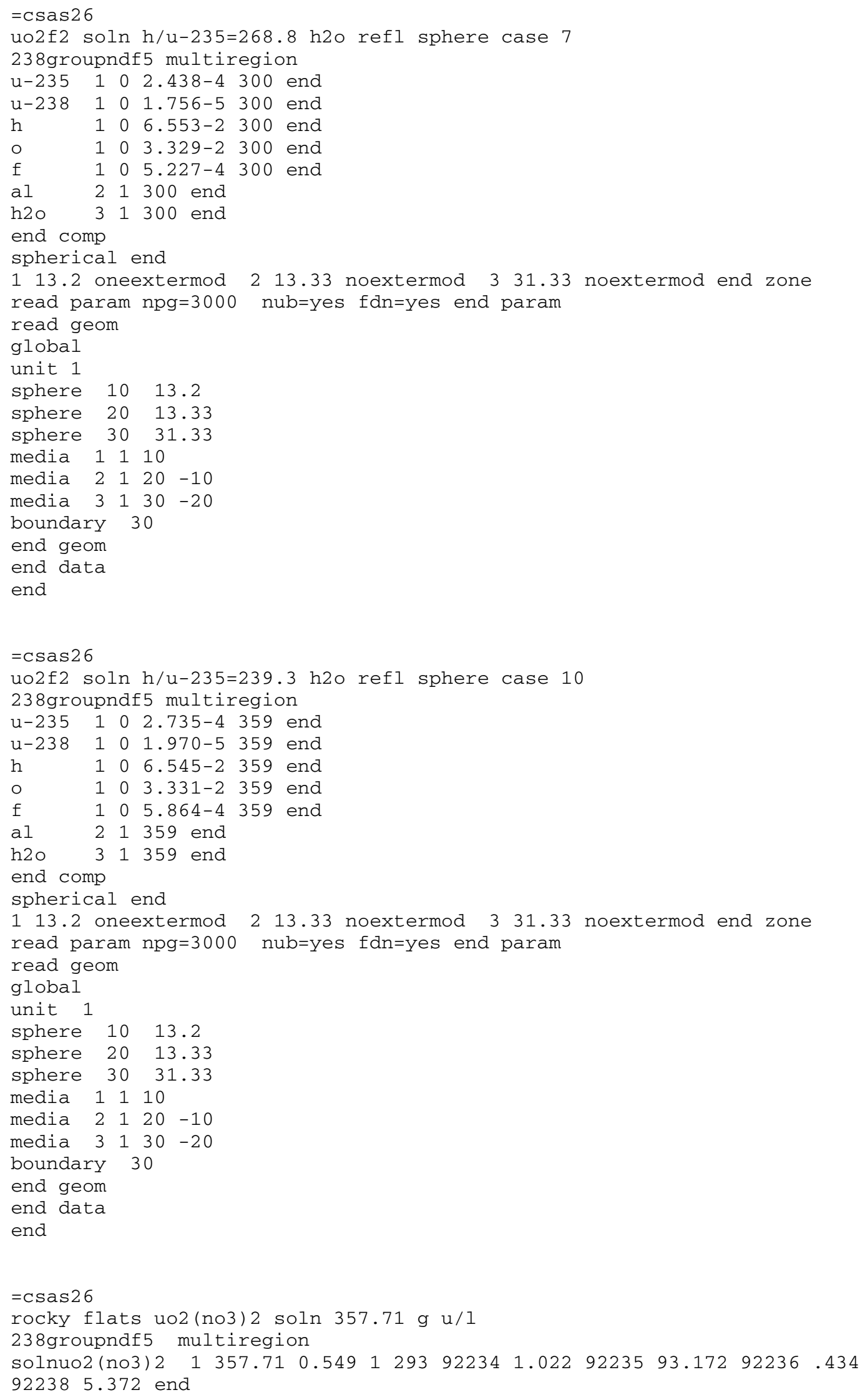




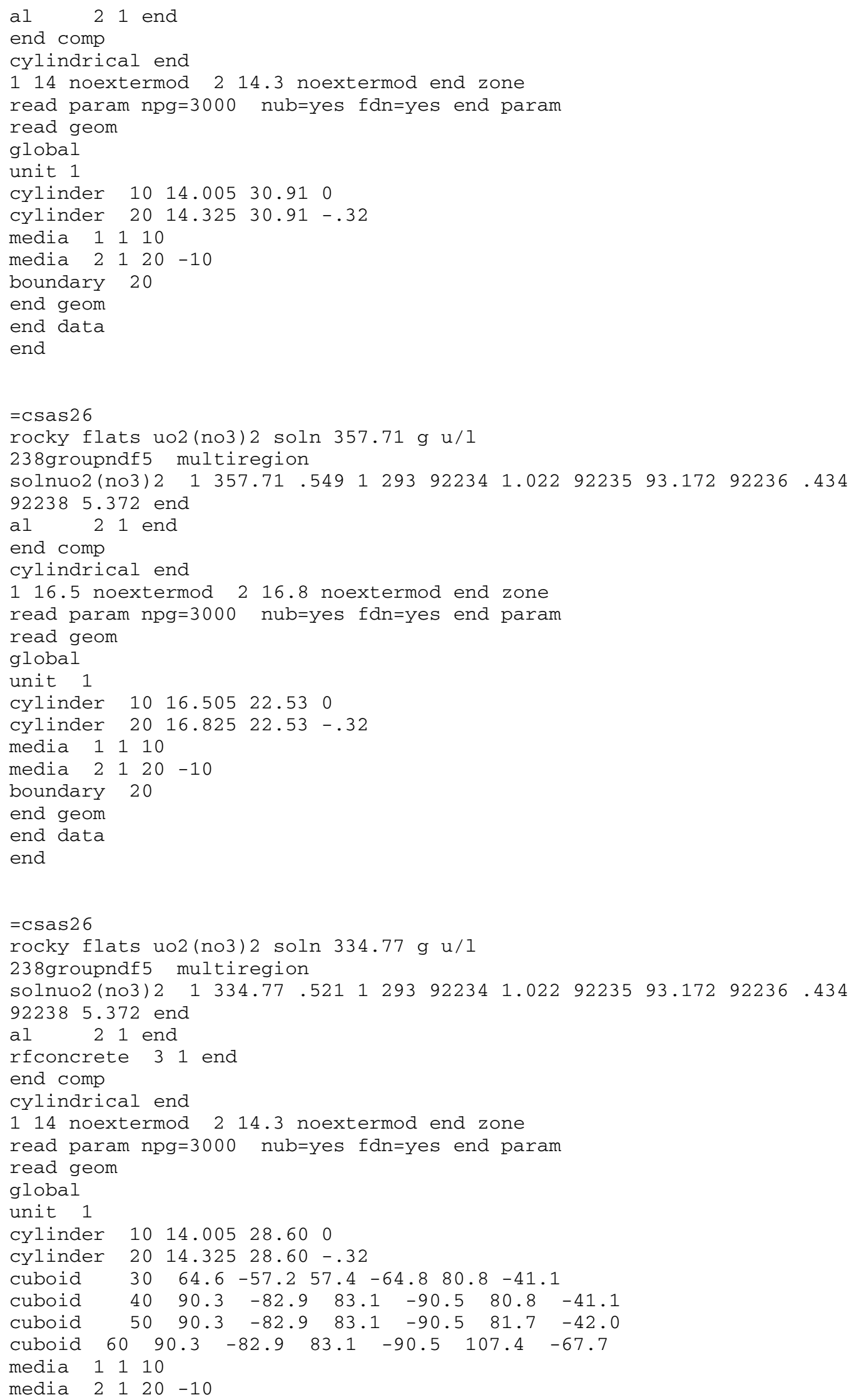




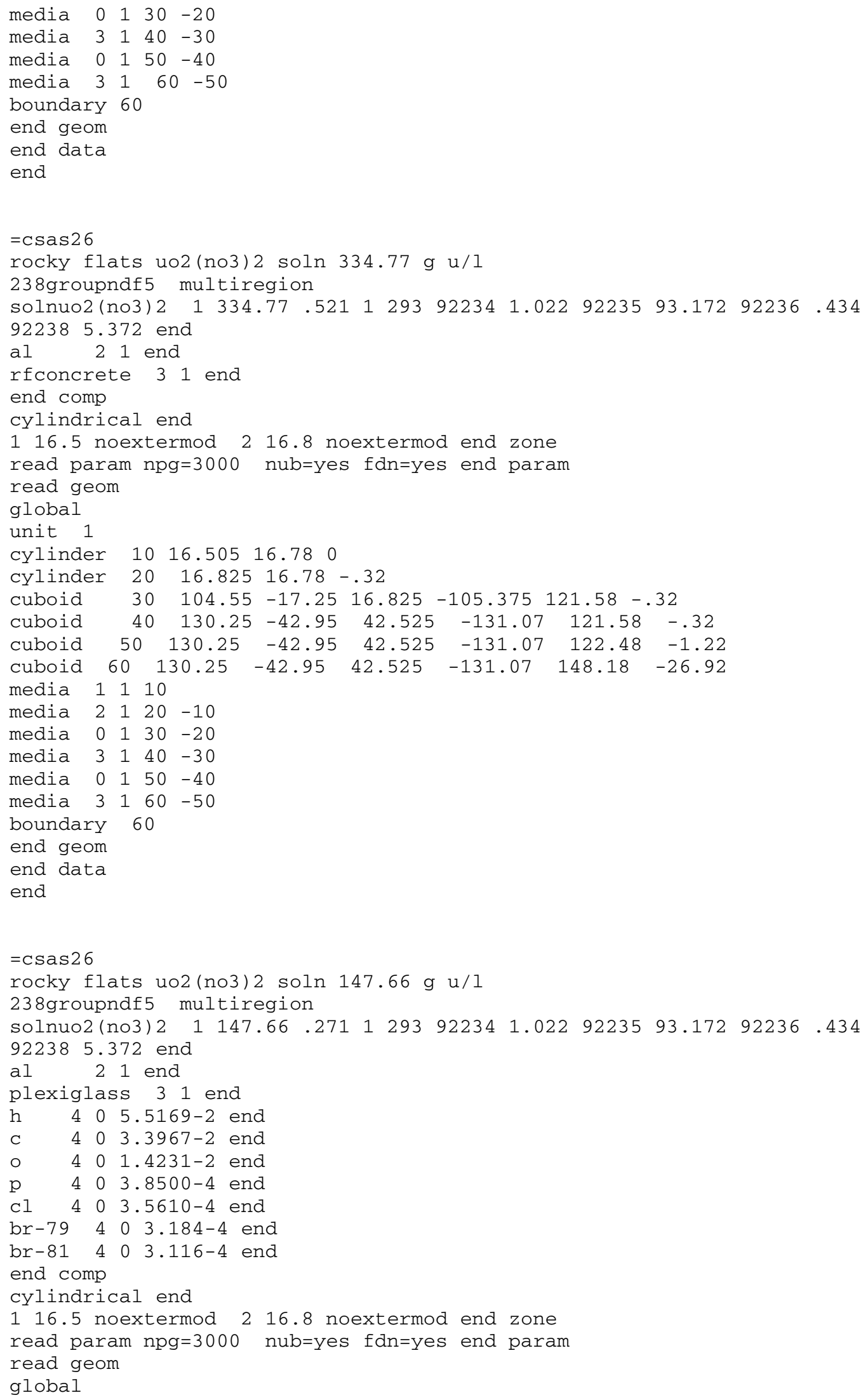




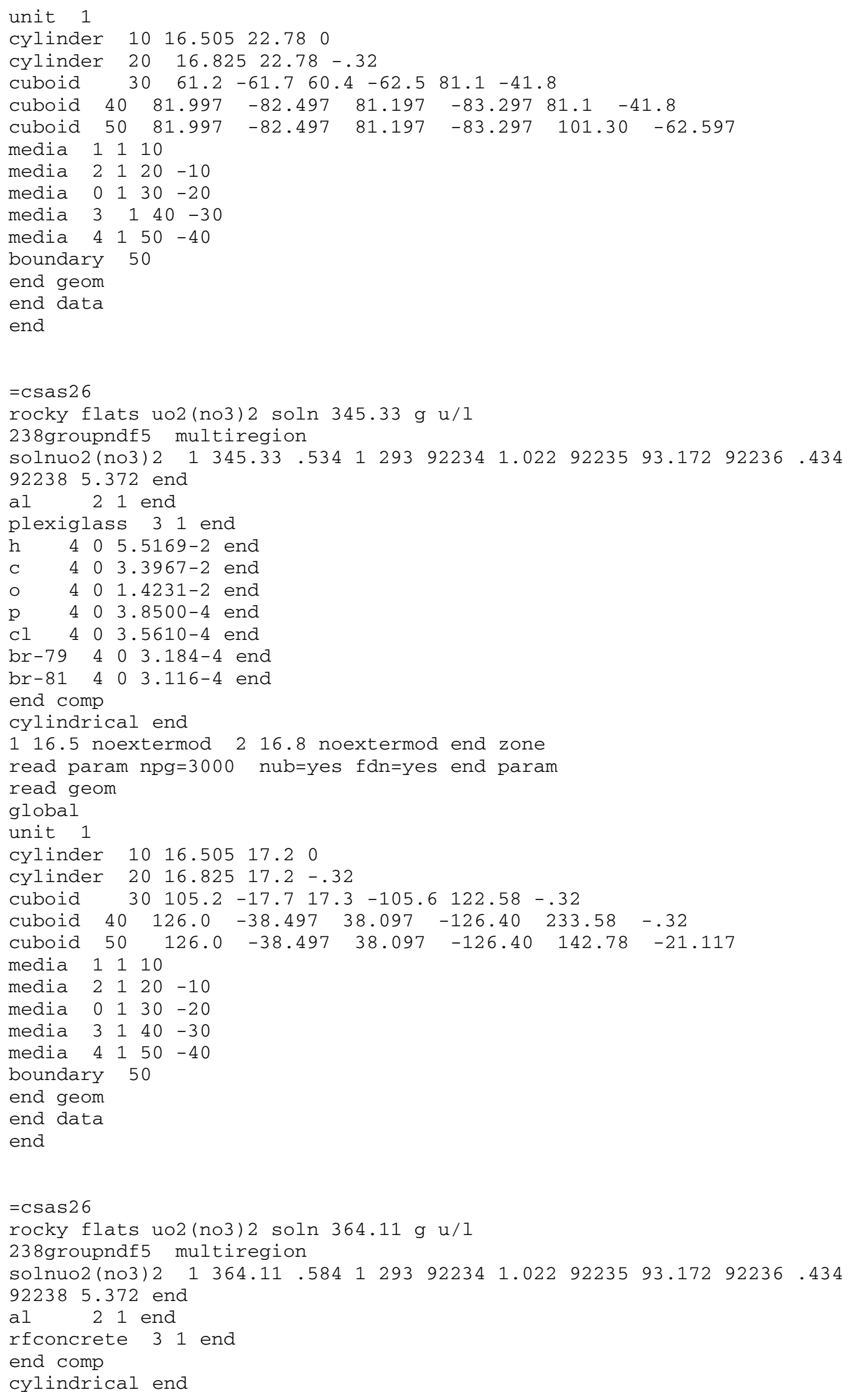




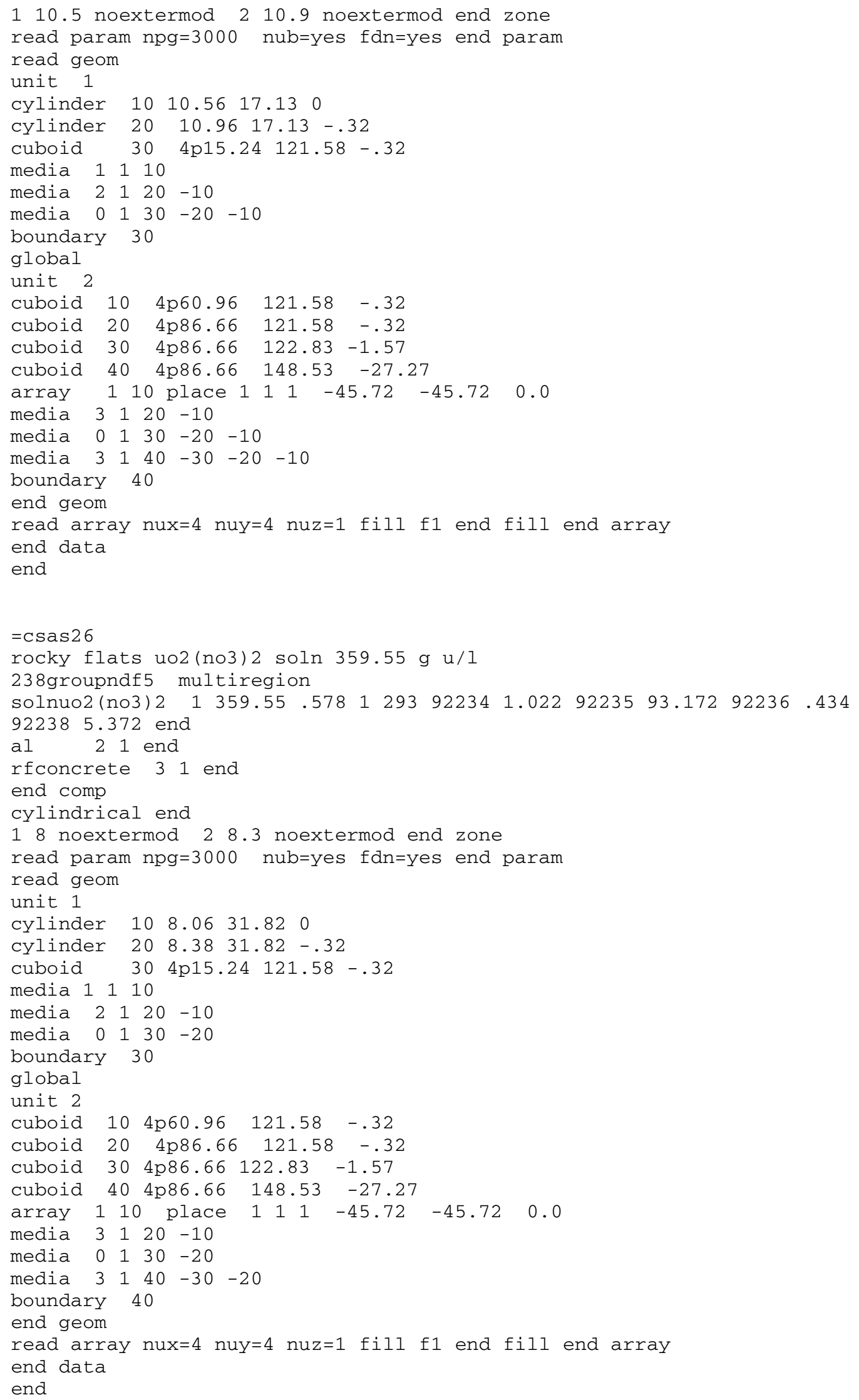




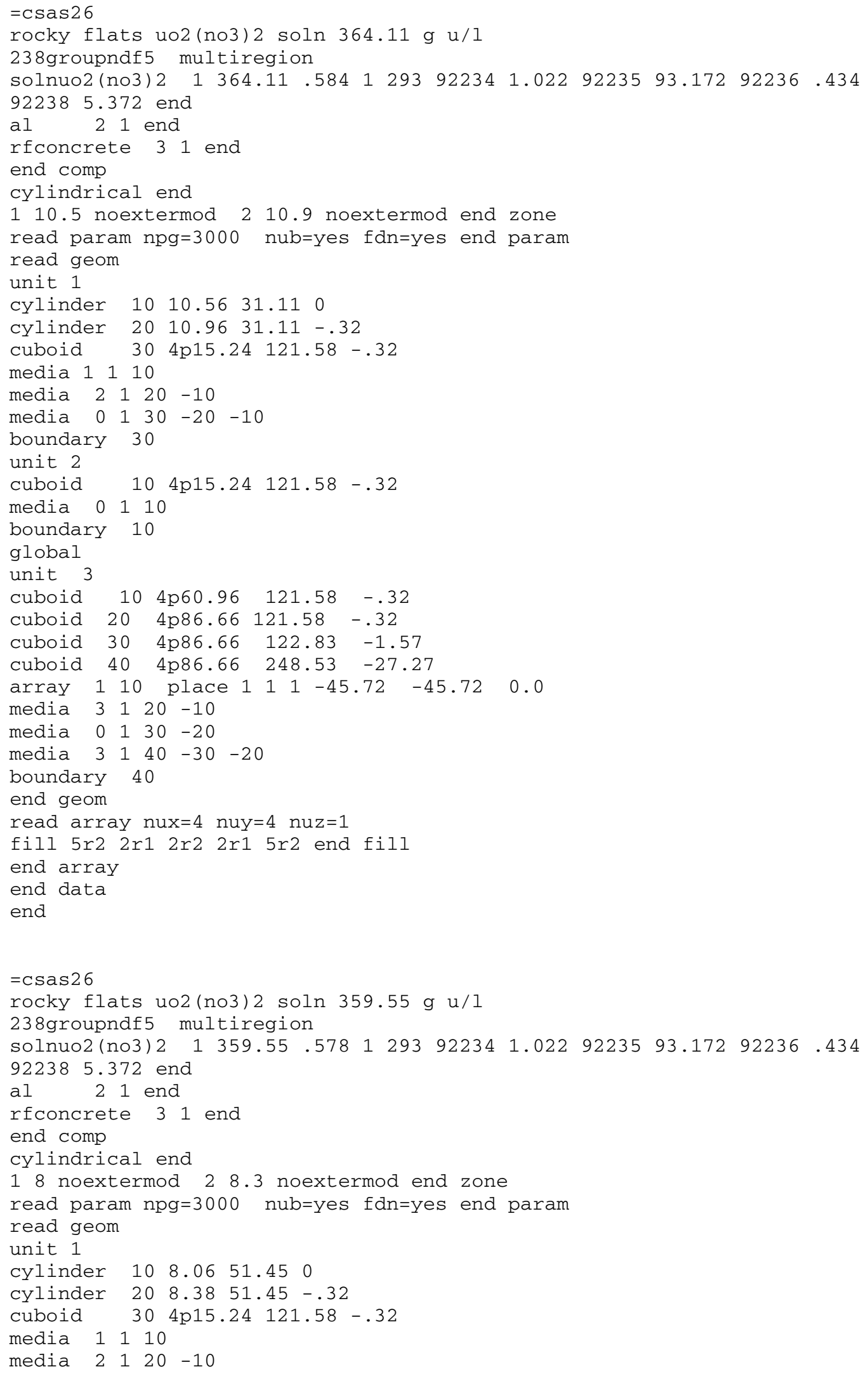




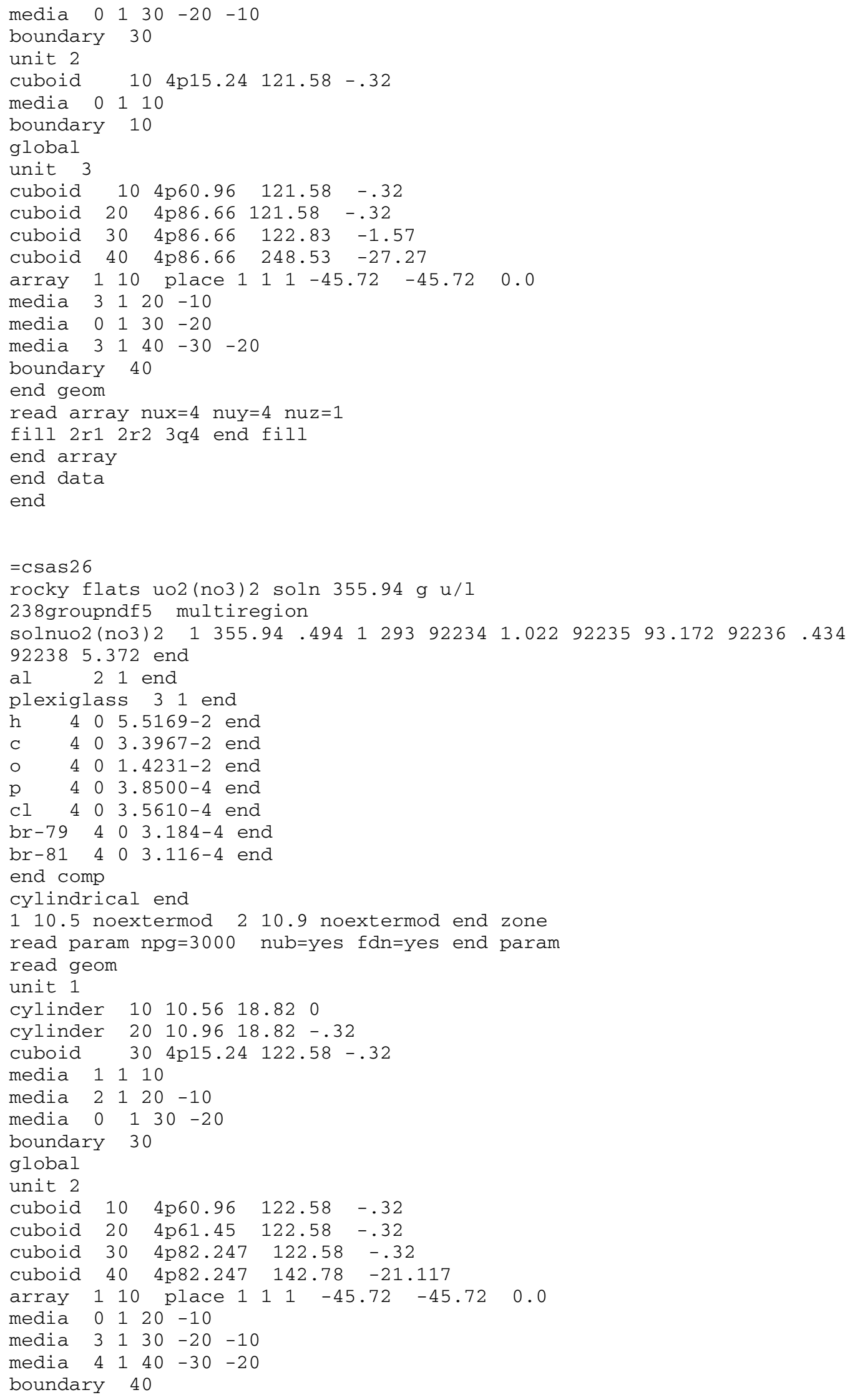




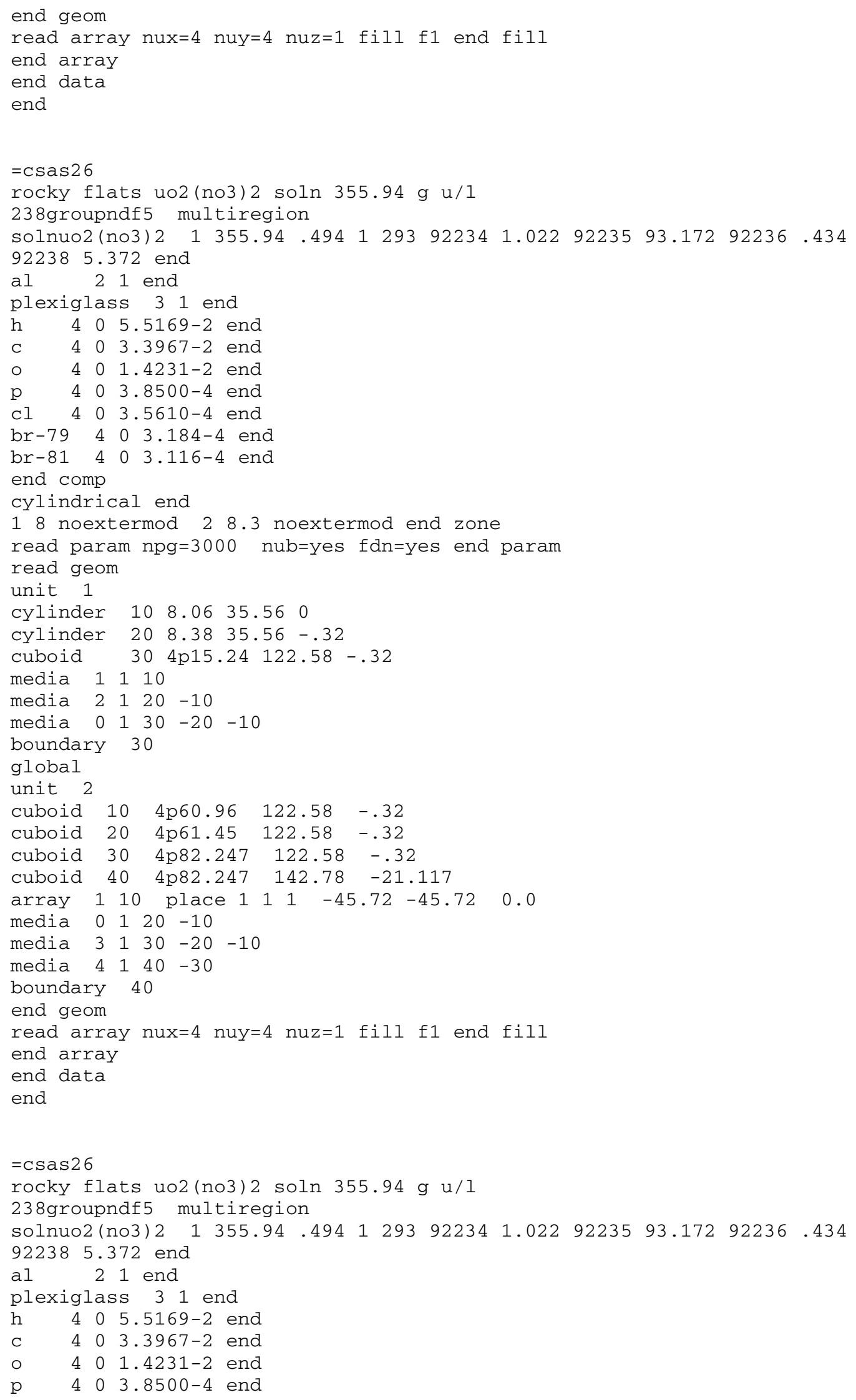




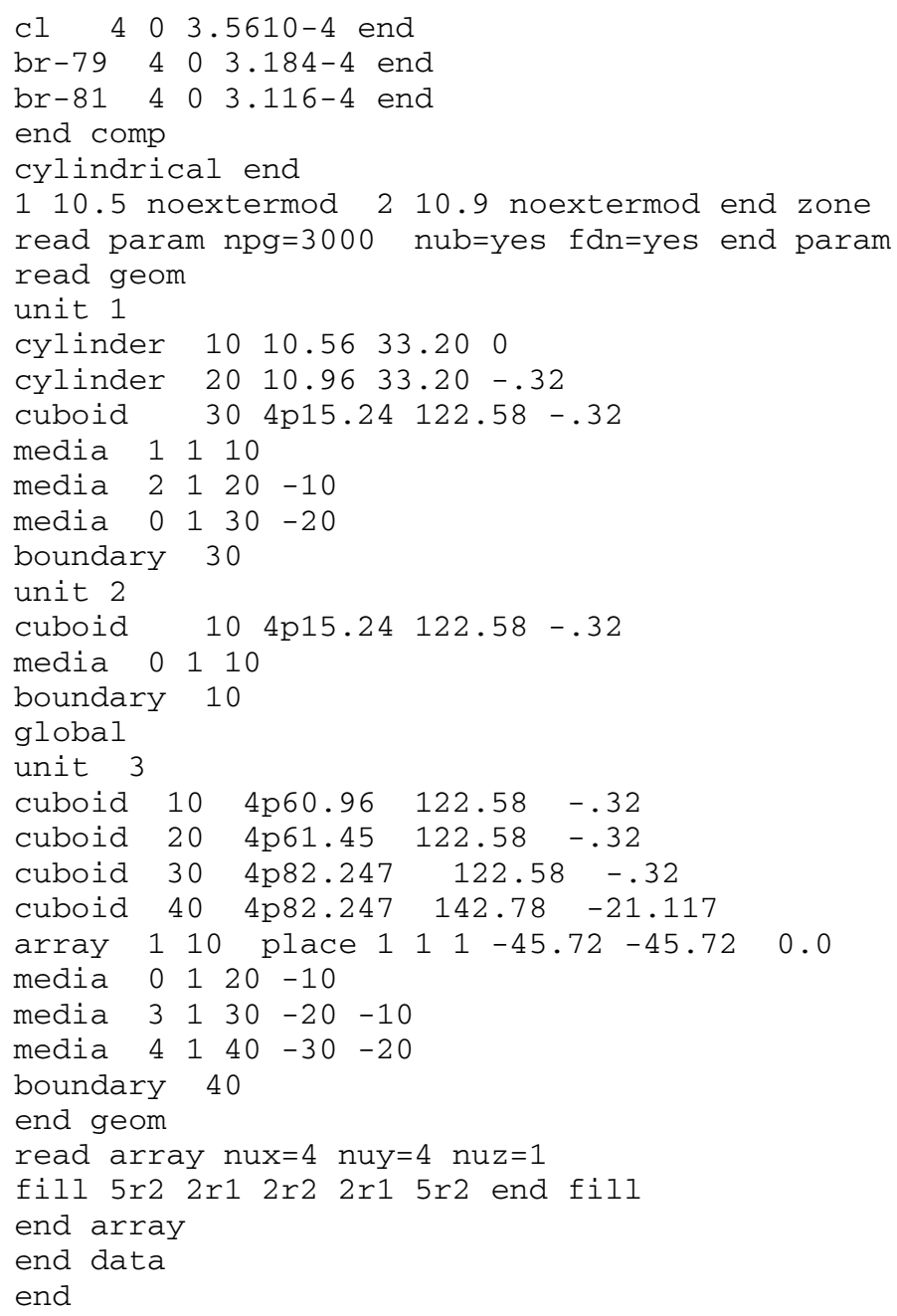


Table B.5. Table 5 input data

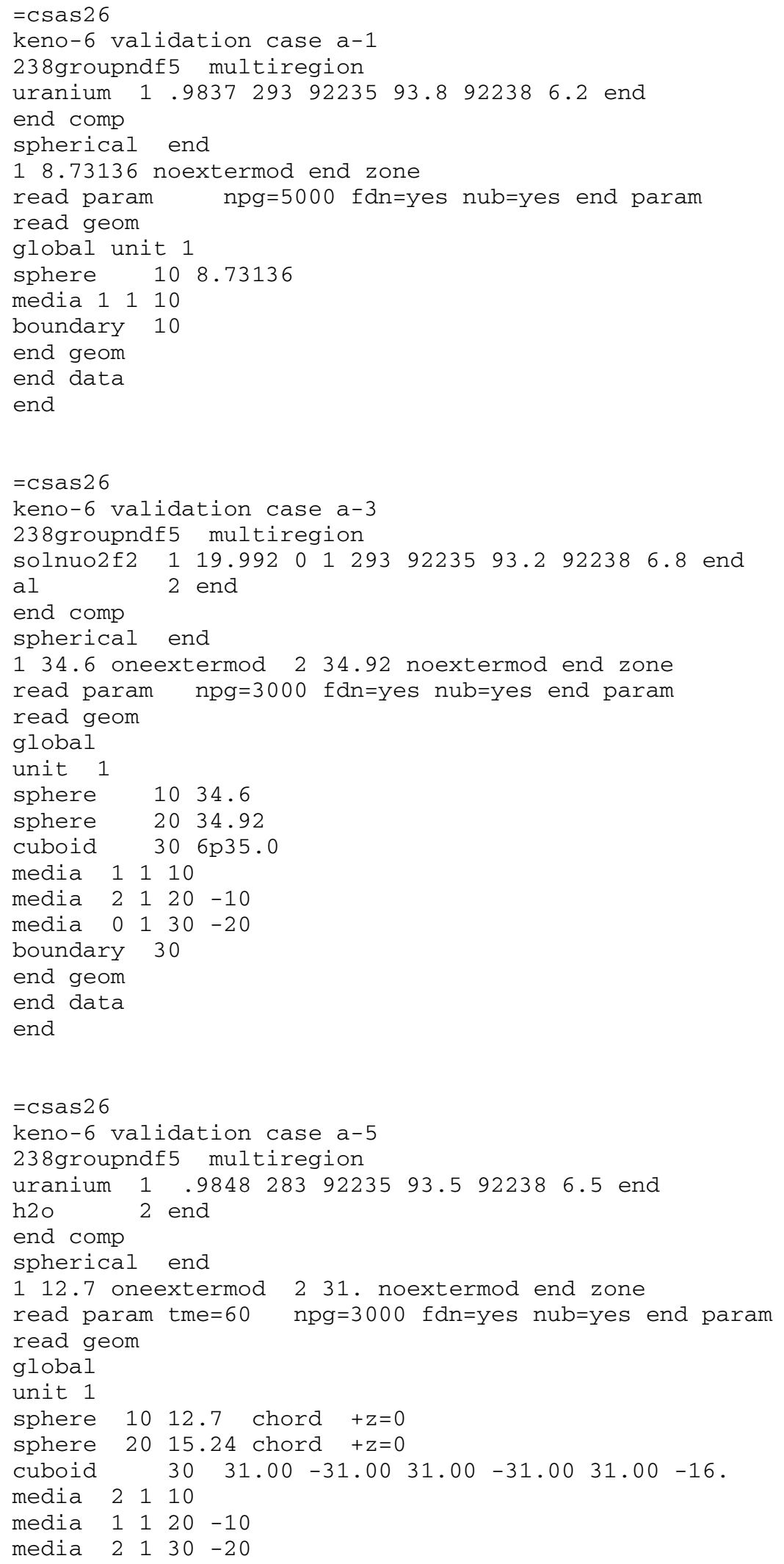




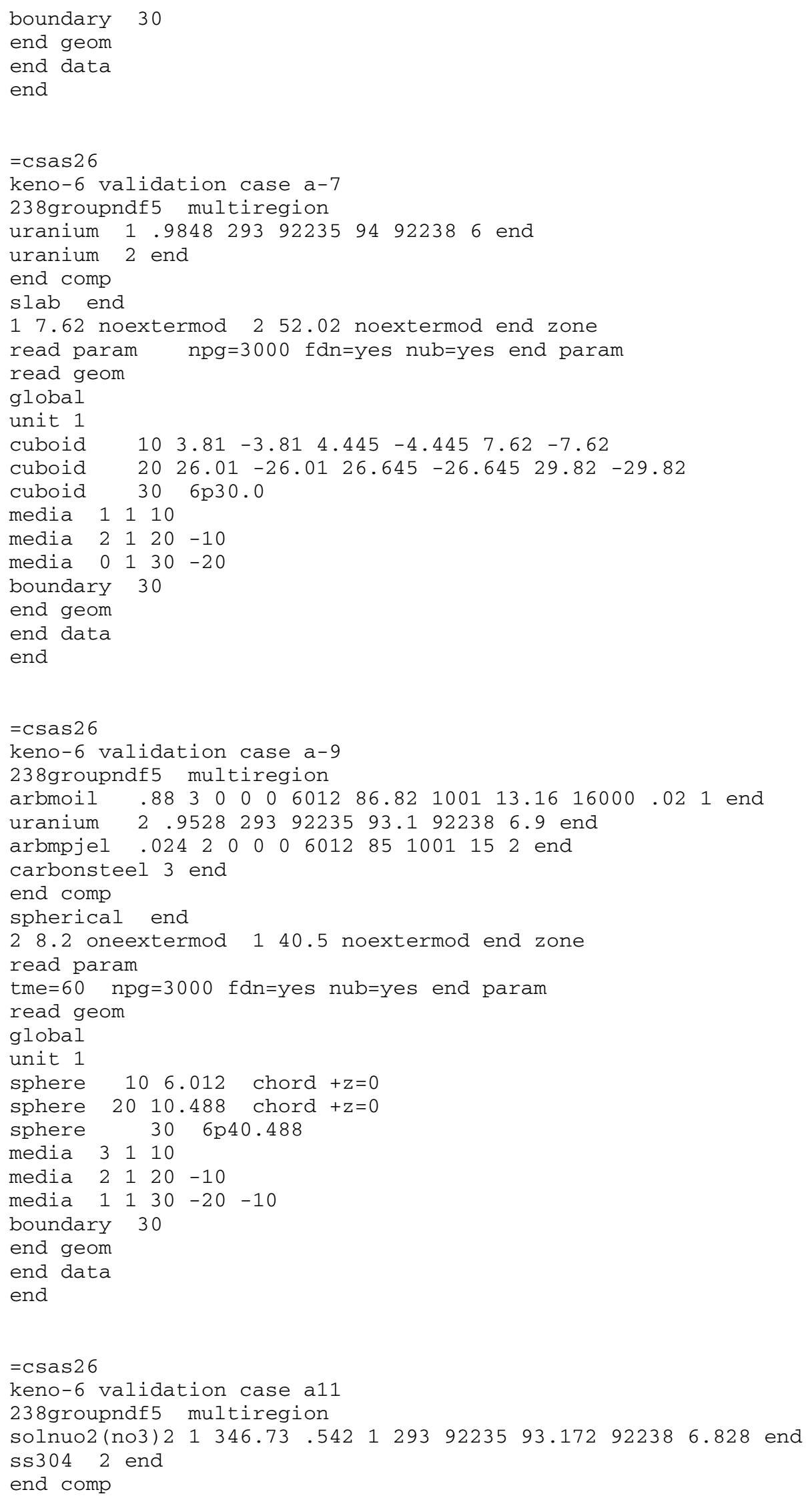


cylindrical end

113.96 oneextermod 214.28 oneextermod end zone read param $\mathrm{npg}=5000$ fdn=yes nub=yes end param

read geom

global unit 1

cylinder $\begin{array}{lllll}10 & 13.96 & 14.465 & -14.465\end{array}$

cylinder $20 \quad 13.96 \quad 27.135-14.465$

cylinder $30 \quad 14.28 \quad 27.135-15.105$

media 1110

media $0 \begin{array}{llll} & 1 & 20 & -10\end{array}$

$\begin{array}{llllll}\text { media } & 2 & 1 & 30 & -20\end{array}$

boundary 30

end geom

end data

end

$=\operatorname{csas} 26$

keno-6 validation case b-5

238 groupndf5 multiregion

uranium $1.98092939223593 .292238 \quad 6.8$ end

end comp

cylindrical end

14.56 noextermod end zone

read param npg=3000 fdn=yes nub=yes end param

read geom

unit 1

cylinder $10 \quad 4.558 \quad 2.16-2.16$

cylinder $20 \quad 5.747 \quad 2.16 \quad-2.16$

cylinder $30 \quad 5.747 \quad 4.851-4.851$

$\begin{array}{lllllllll}\text { cuboid } & 40 & 5.8554 & -5.8554 & 5.8554 & -5.8554 & 4.9771 & -4.9771\end{array}$

media 1110

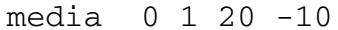

media $1130-20$

media $0 \begin{array}{lllll} & 4 & 4 & -30\end{array}$

boundary 40

global unit 2

cuboid $10 \quad 4 p 11.7108 \quad 2 p 9.9542$

array 110 place $1 \begin{array}{llllll}1 & 1 & 1 & -5.8554 & -5.8554 & -4.9771\end{array}$

boundary 10

end geom

read array ara=1 nux=2 nuy=2 nuz=2 fill fl end fill end array end data

end

$=\operatorname{csas} 26$

keno-6 validation case b-9

238 groupndf5 multiregion

solnuo2(no3)2 $144150012939223592.6 \quad 92238 \quad 7.4$ end

plexiglass 21 end

end comp

cylindrical end

19.52 oneextermod 210.16 noextermod end zone

read param npg $=3000$ fdn=yes nub=yes end param

read geom

unit 1

cylinder $10 \quad 9.52 \quad 8.7804 \quad-8.7804$

cylinder $20 \quad 9.52 \quad 8.9896-8.7804$

cylinder $3010.16 \quad 9.6296-9.4204$

$\begin{array}{llllllll}\text { cuboid } 40 & 13.4 & -13.4 & 13.4 & -13.4 & 12.8696 & -12.6604\end{array}$

media 1110

media $0 \begin{array}{llll} & 1 & 20 & -10\end{array}$ 


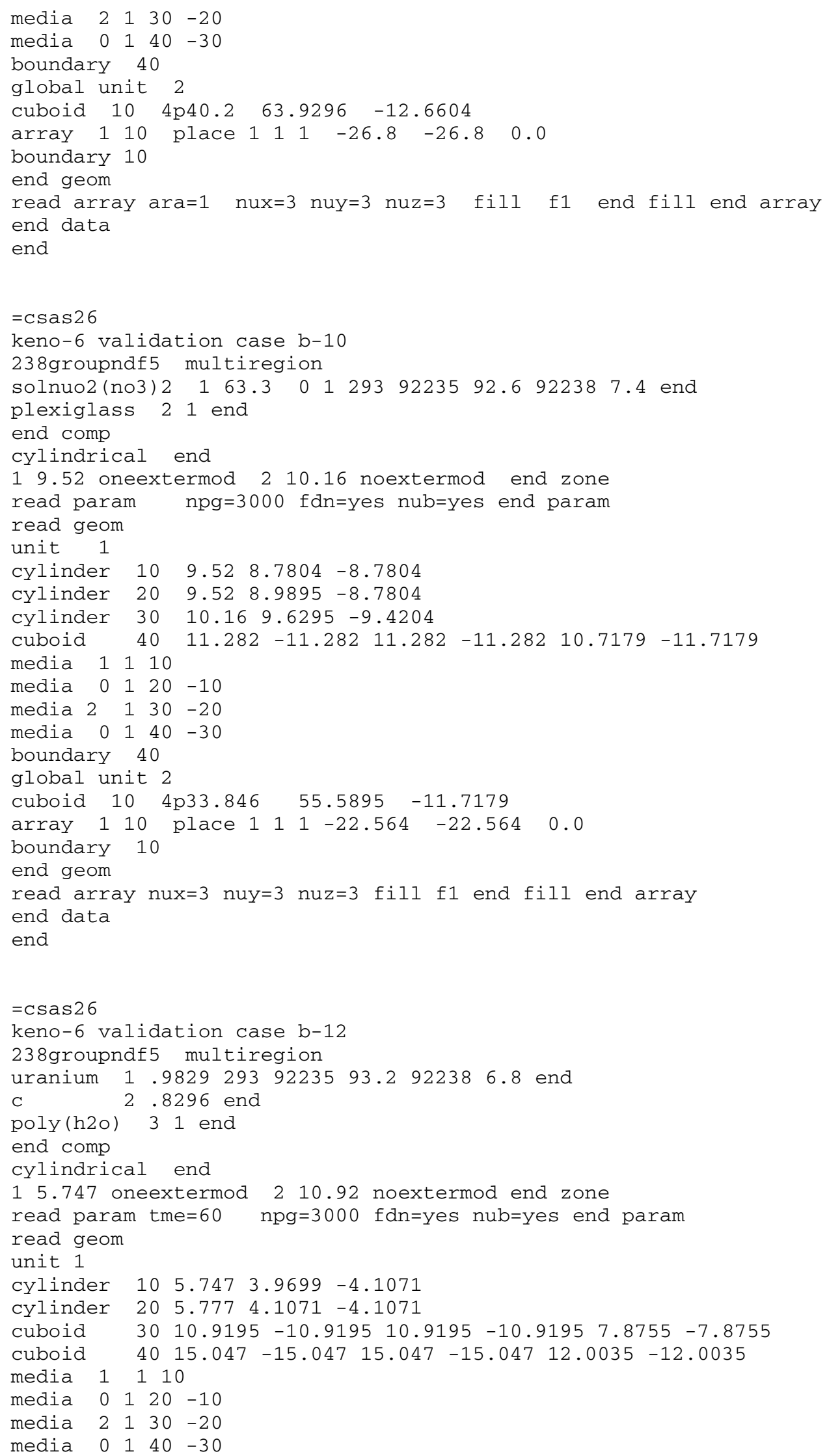




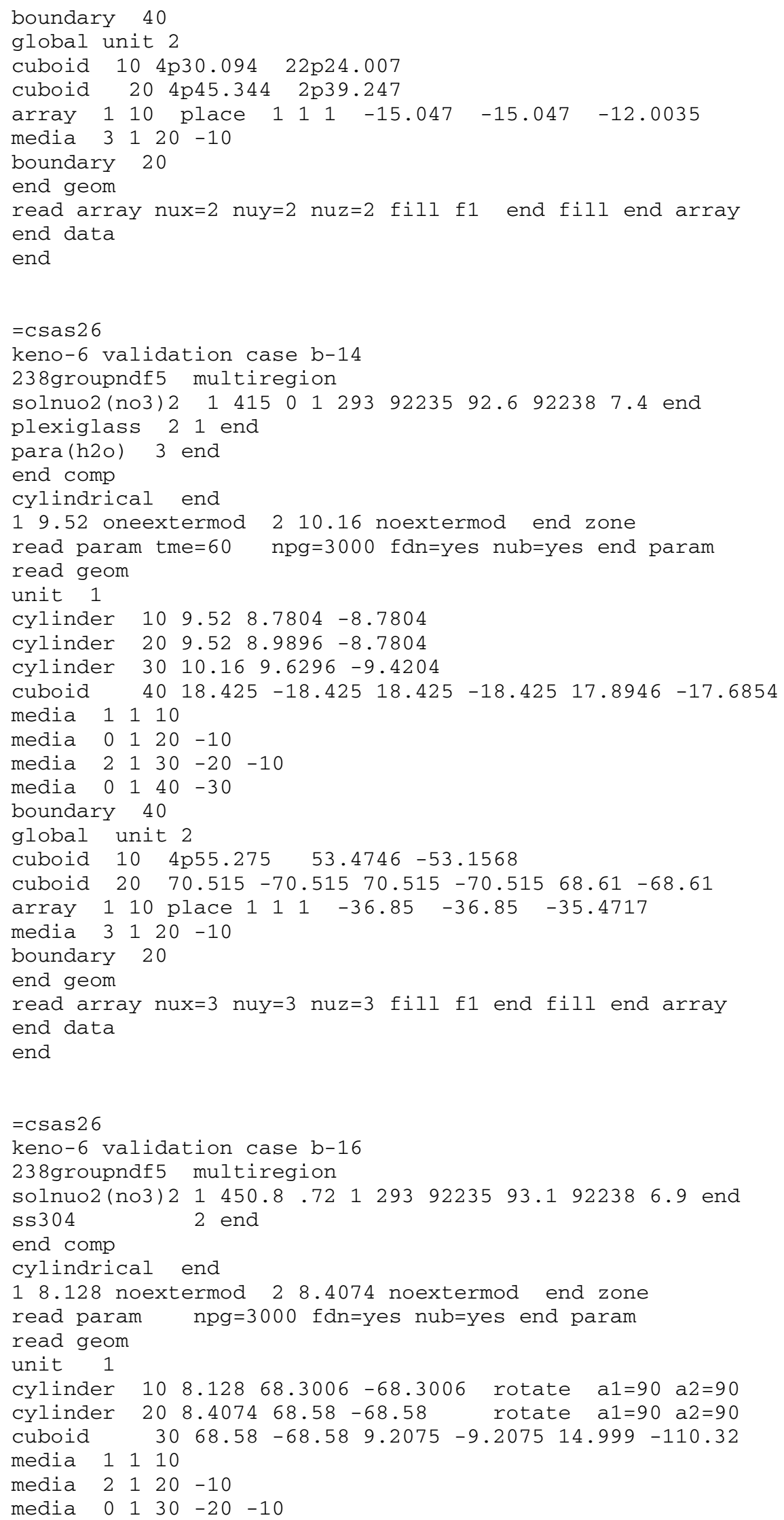




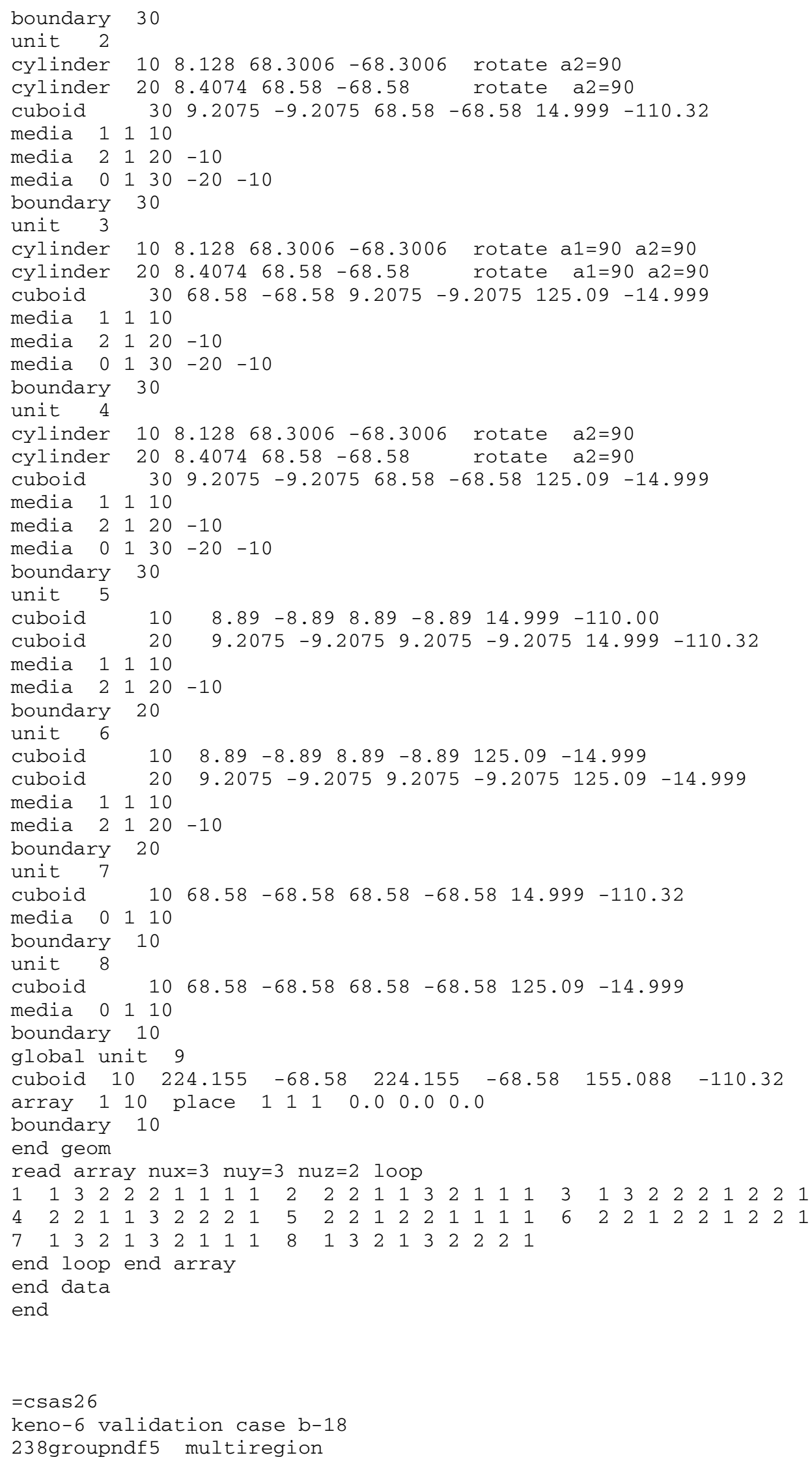




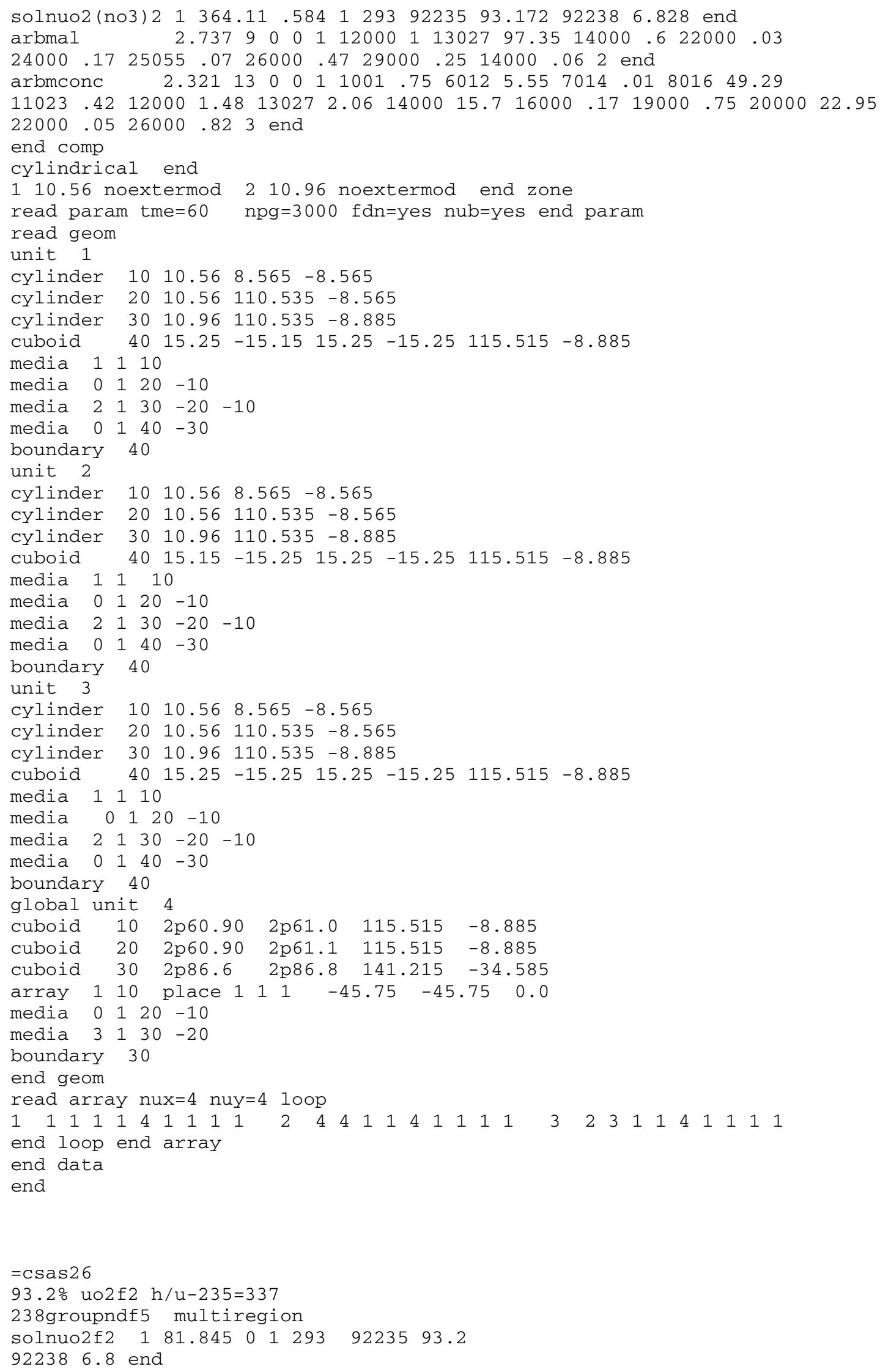




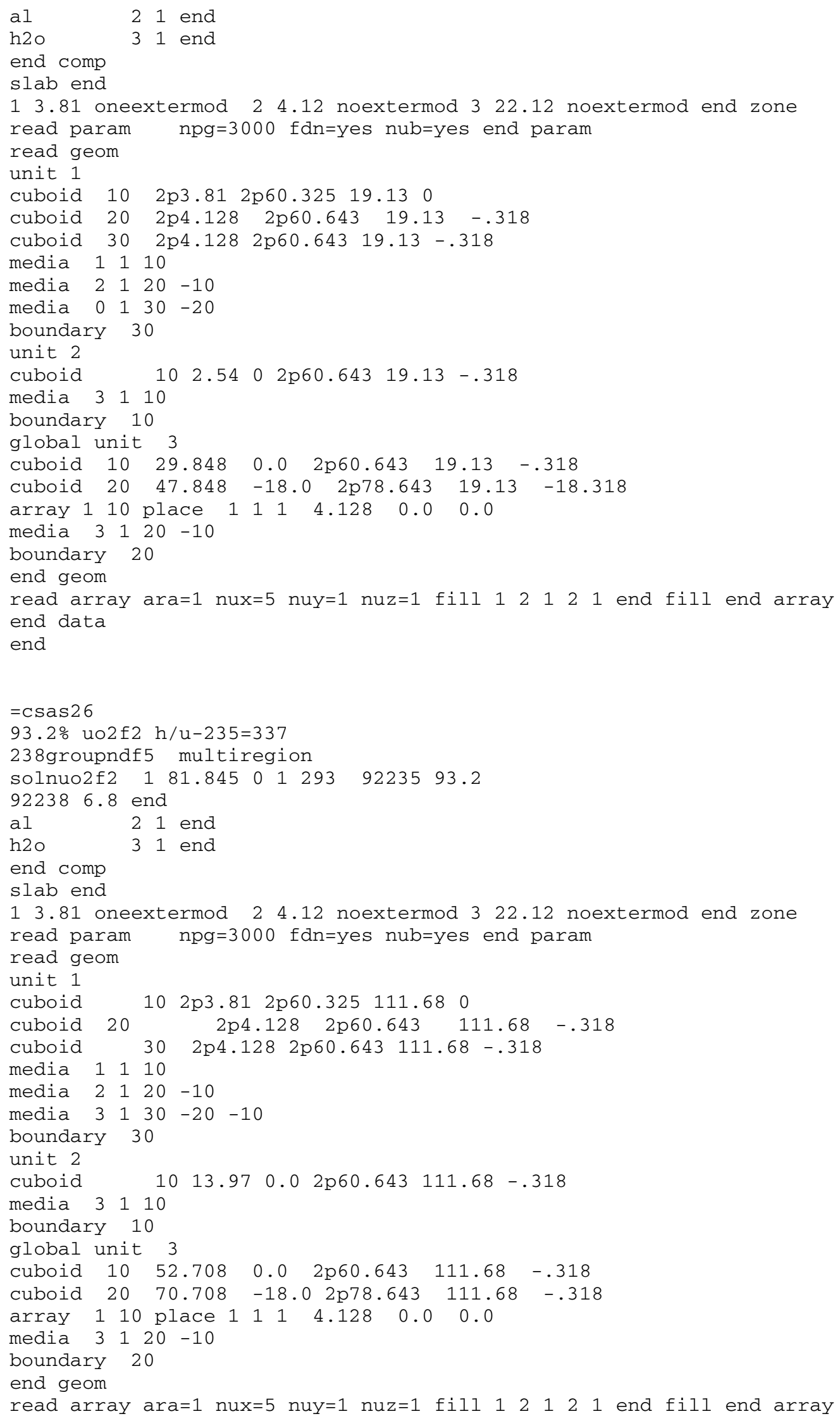




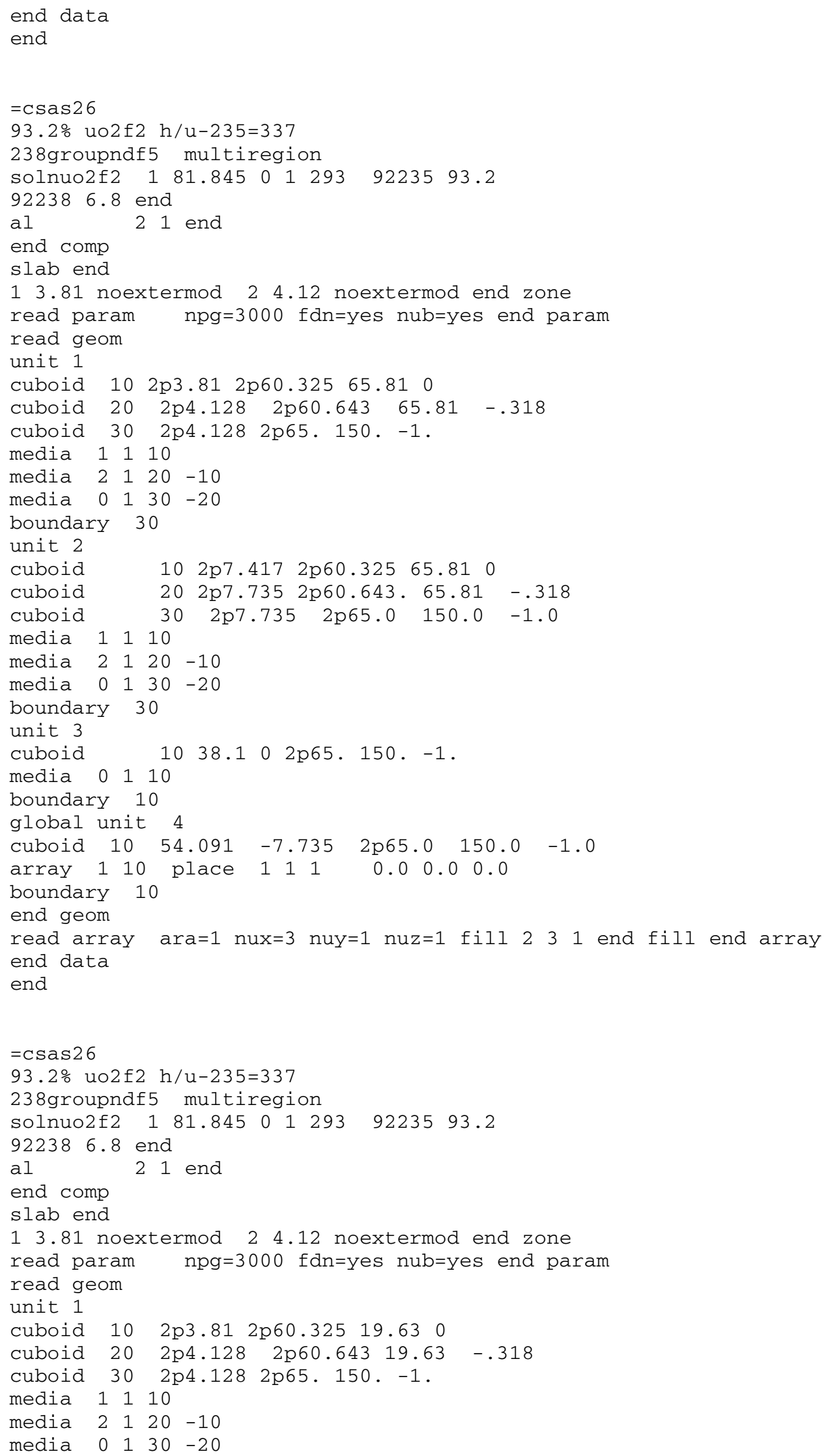




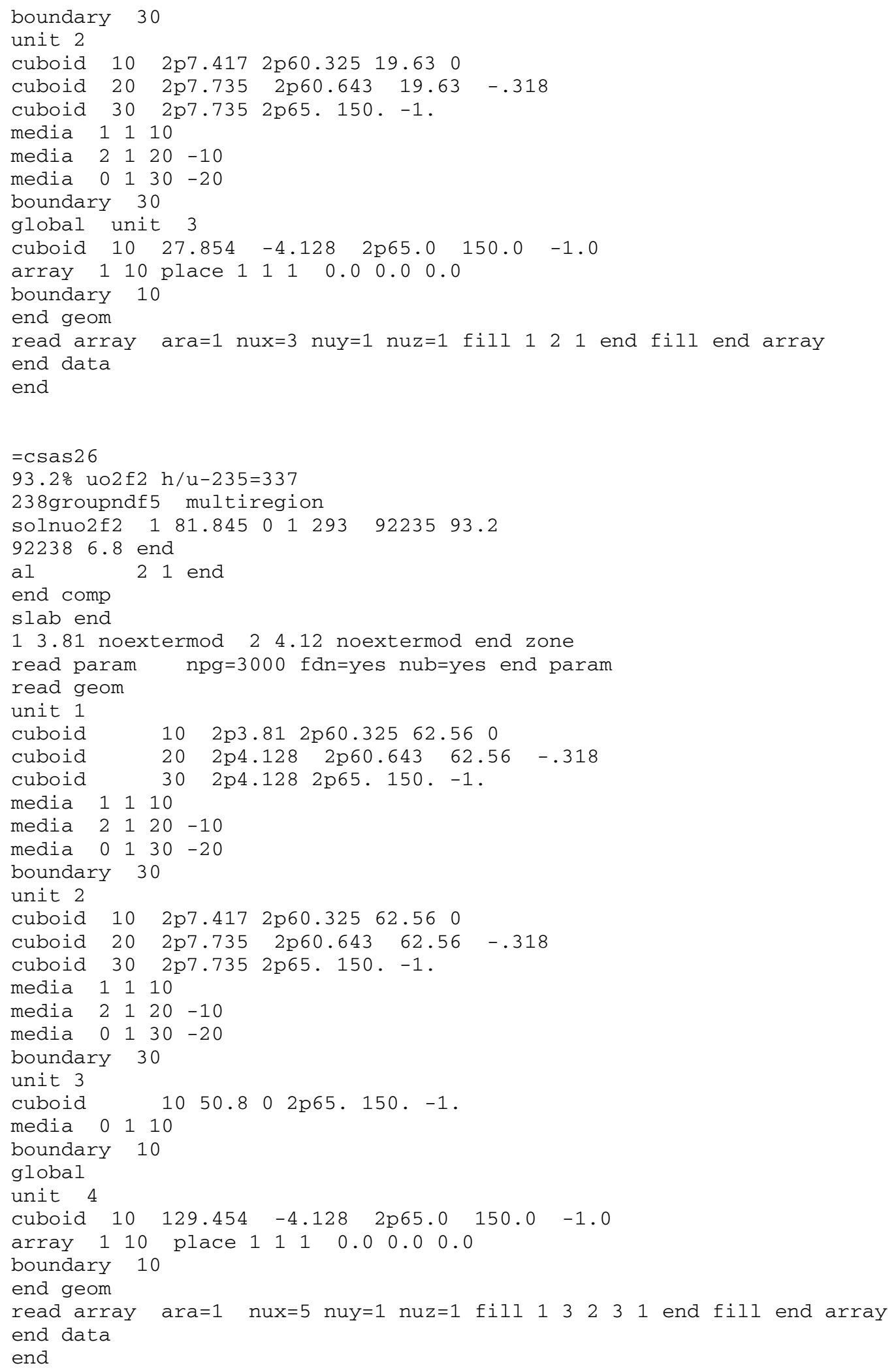




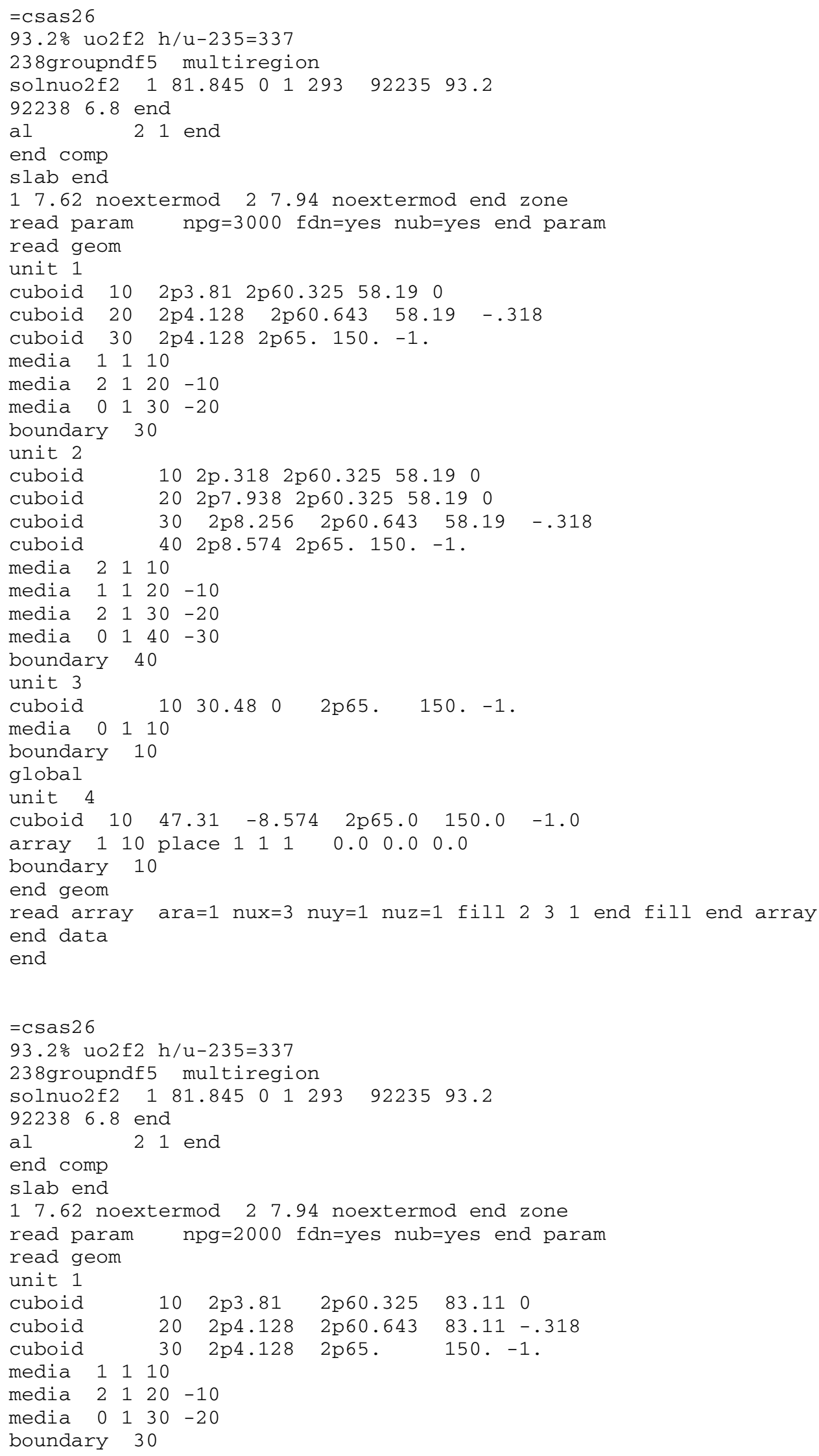




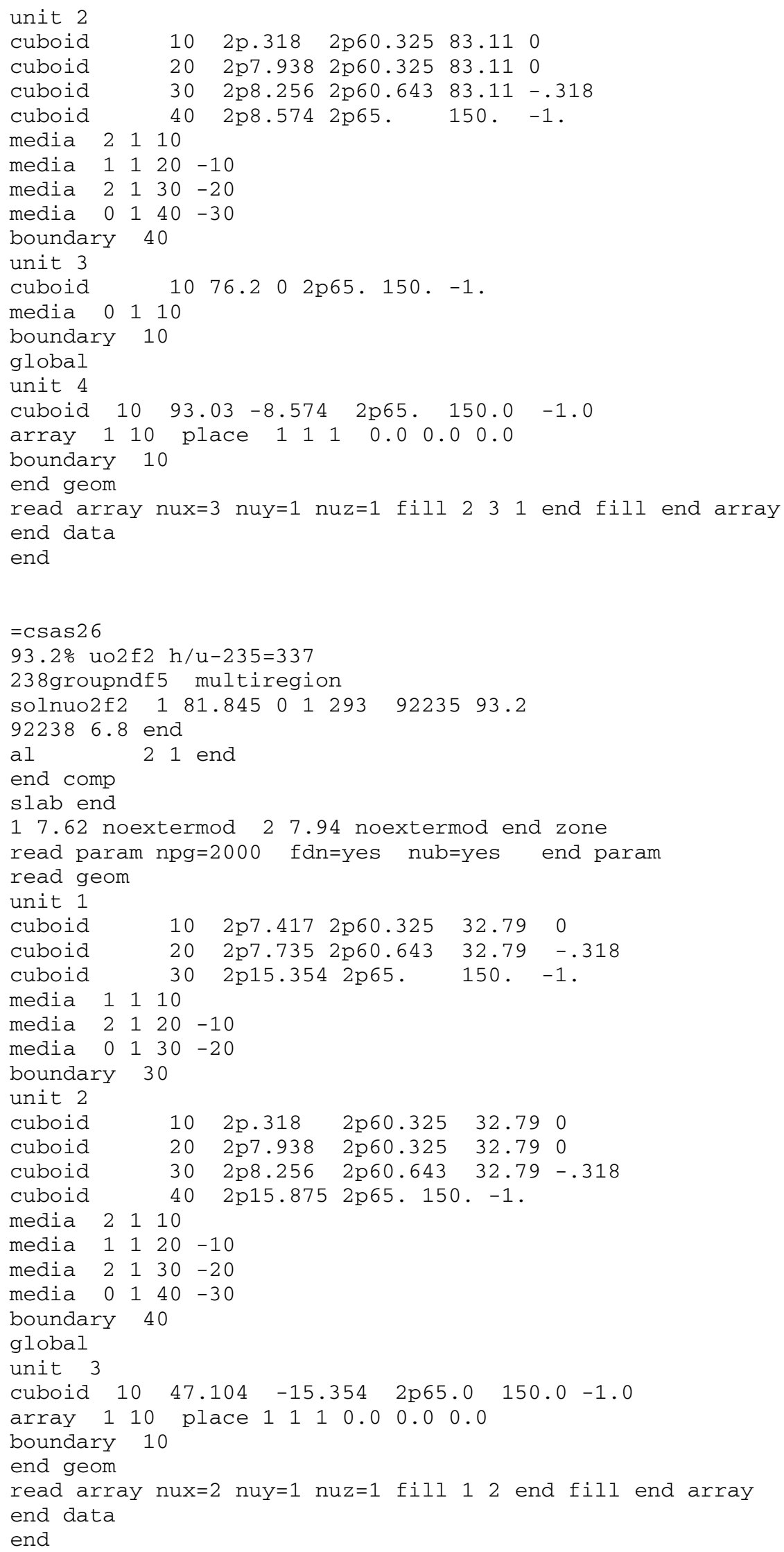




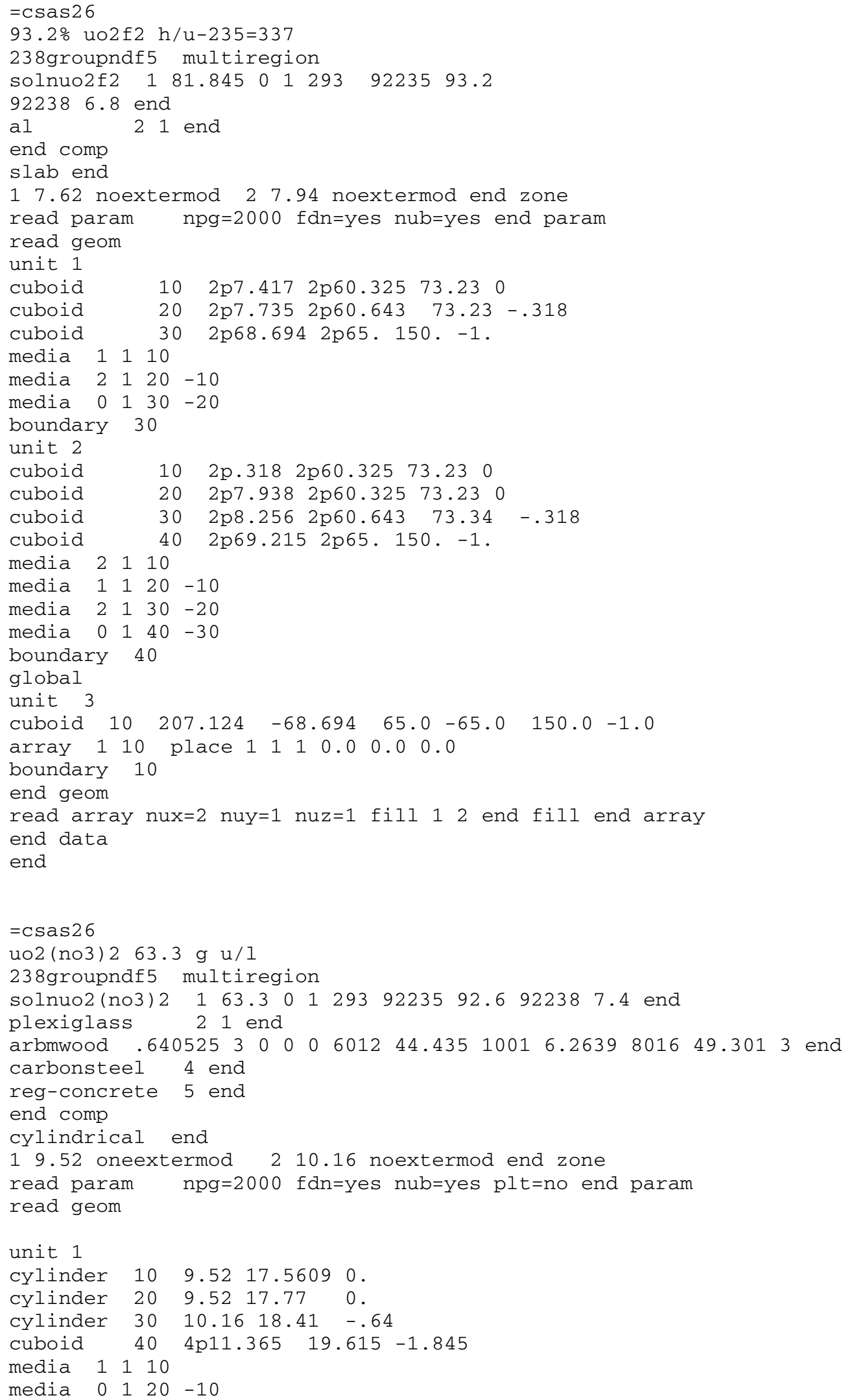




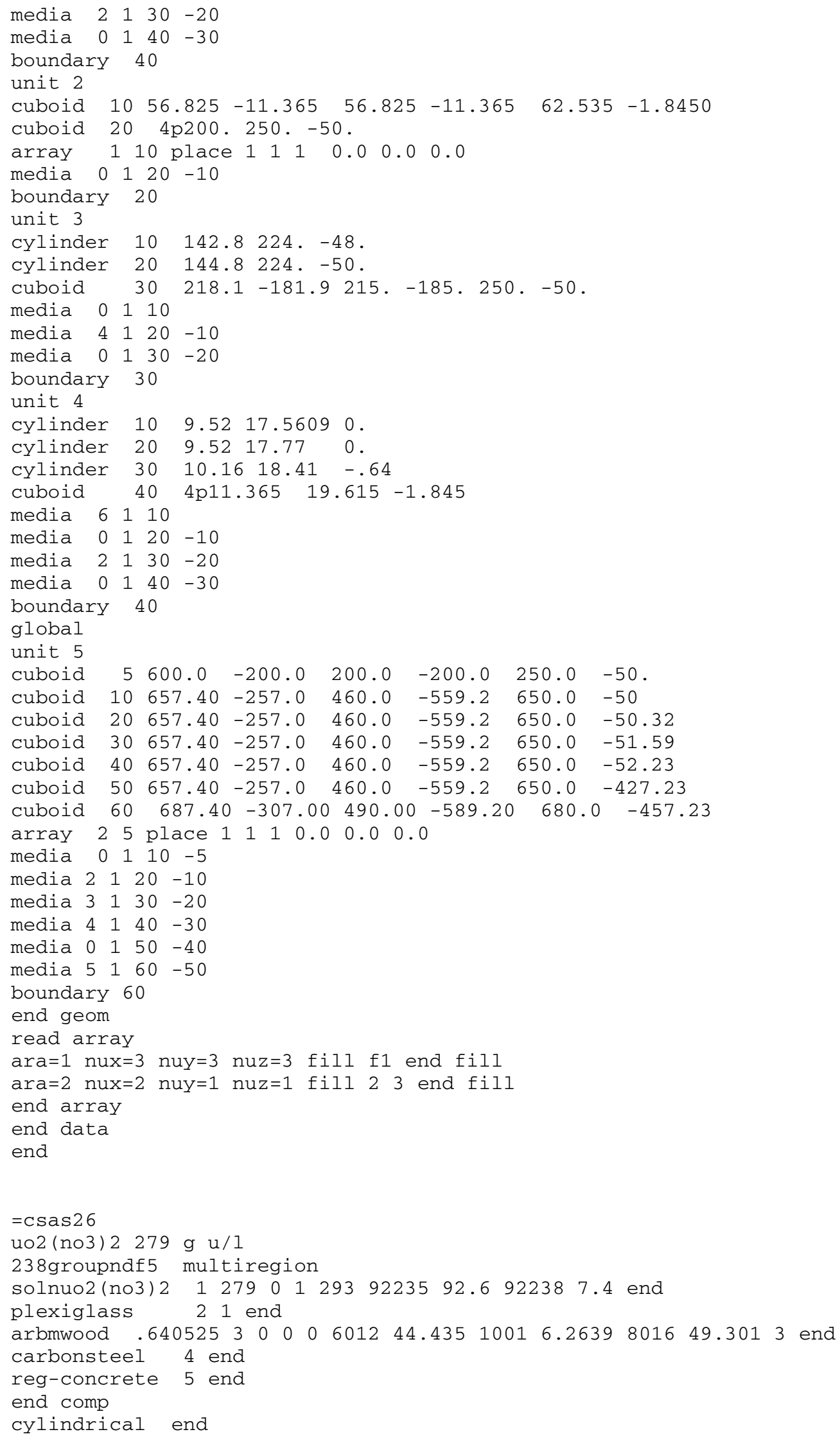




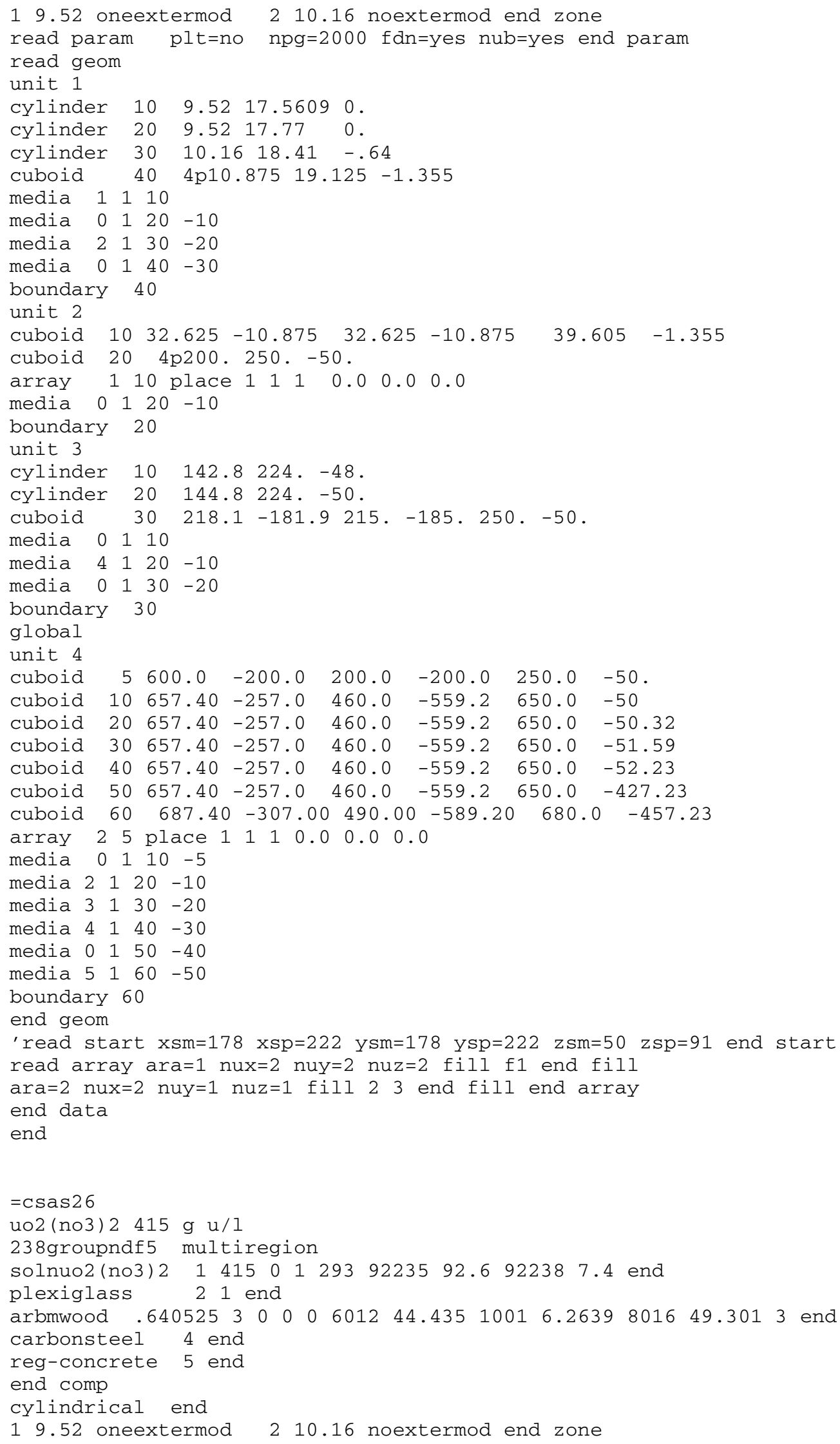




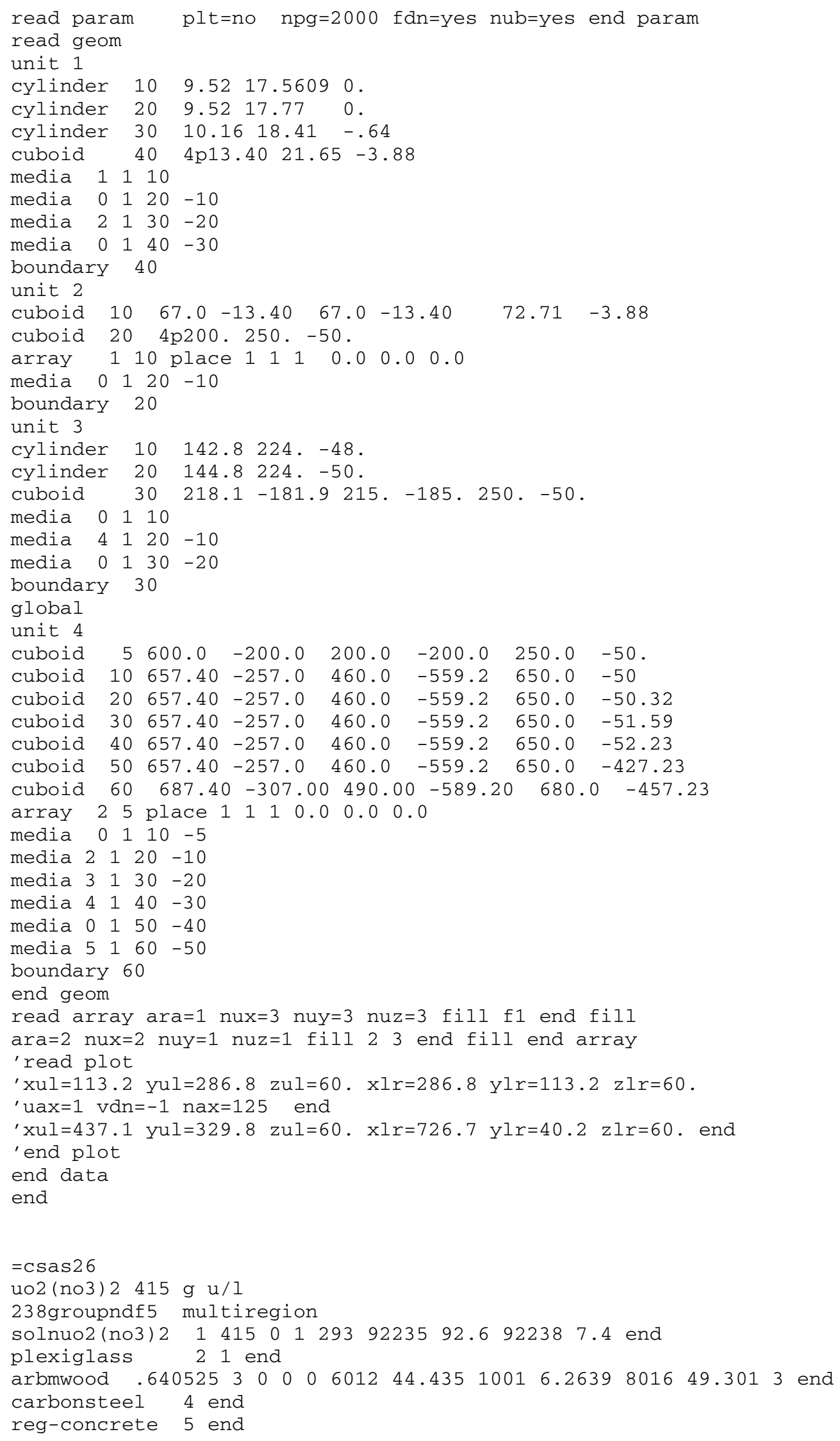




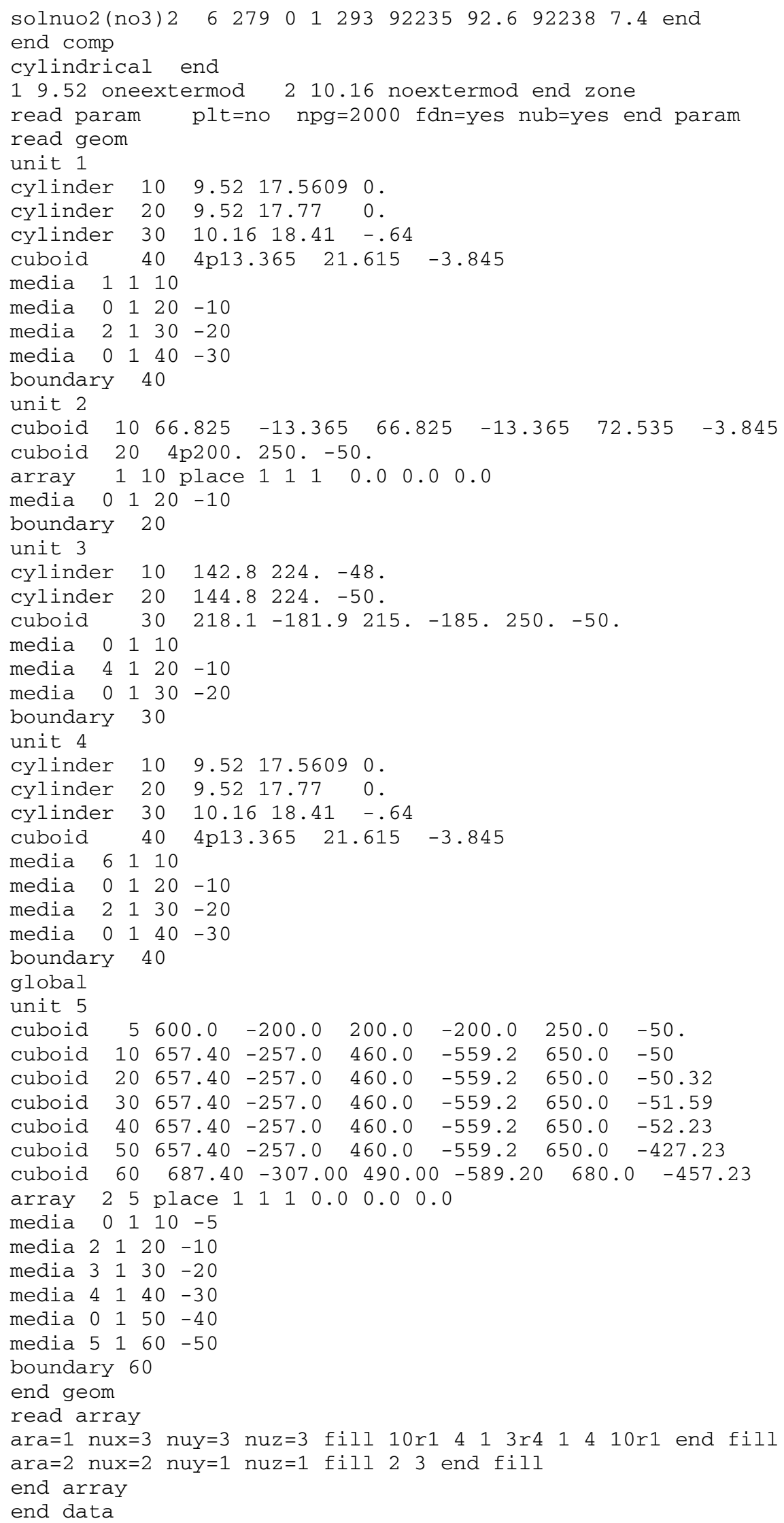




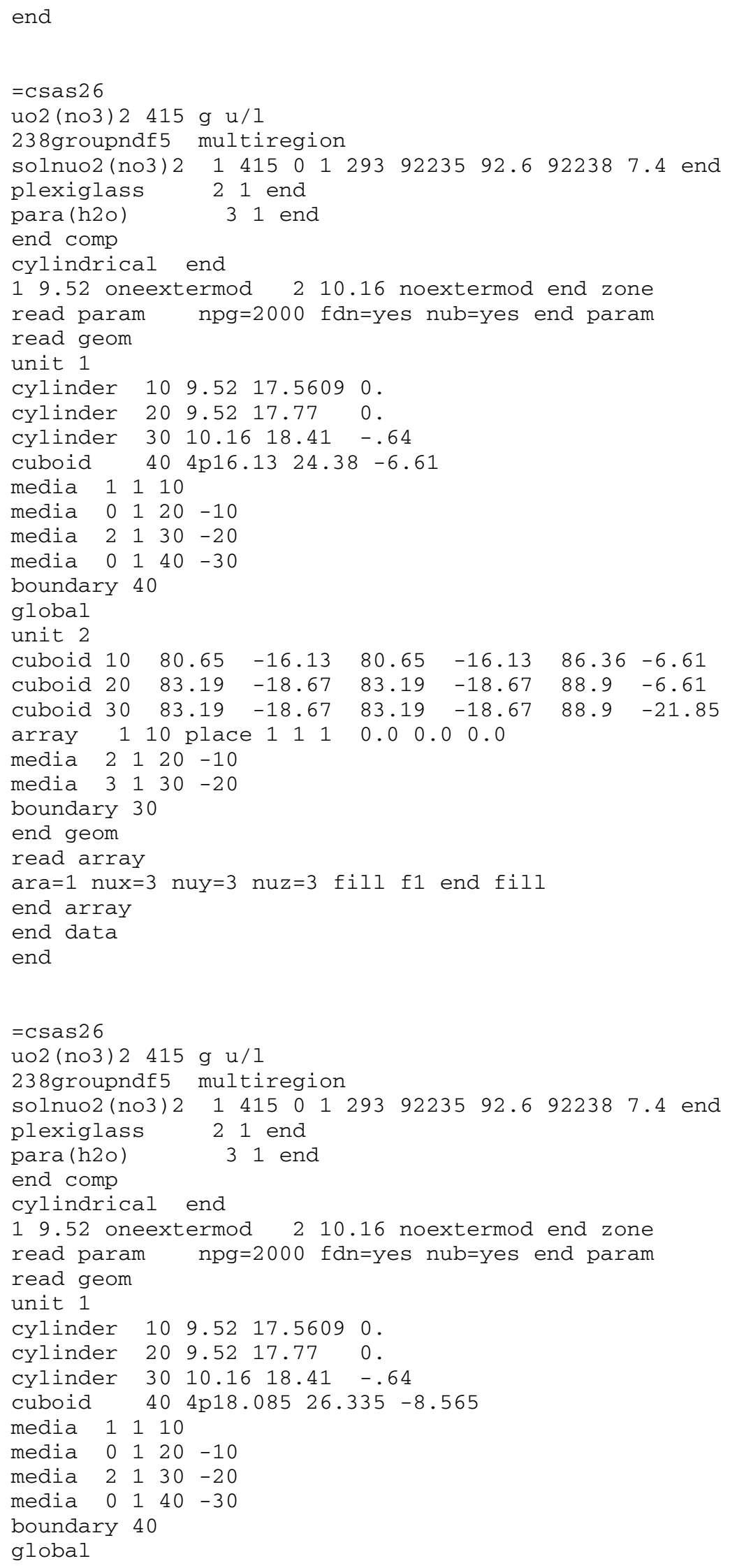




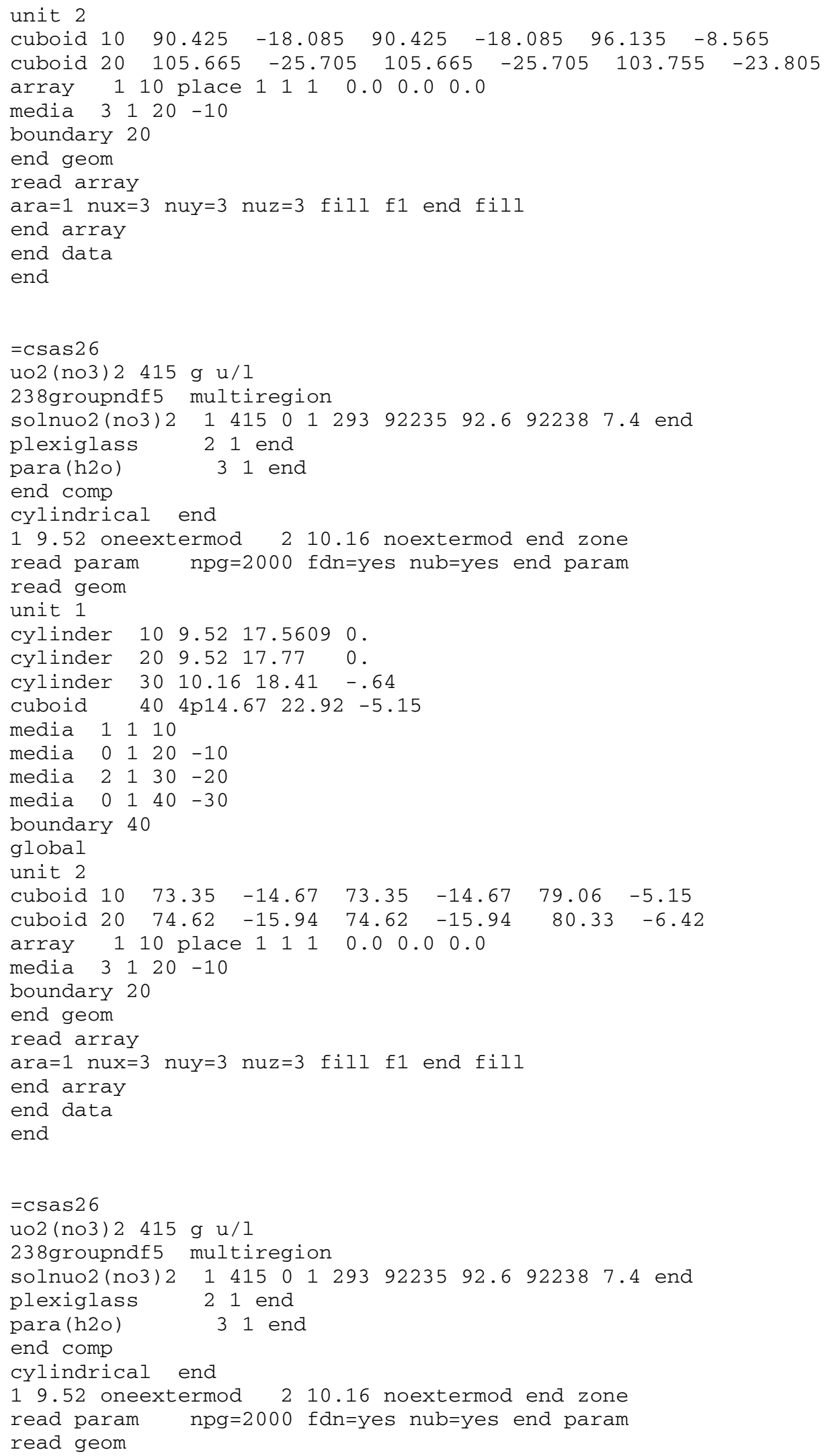




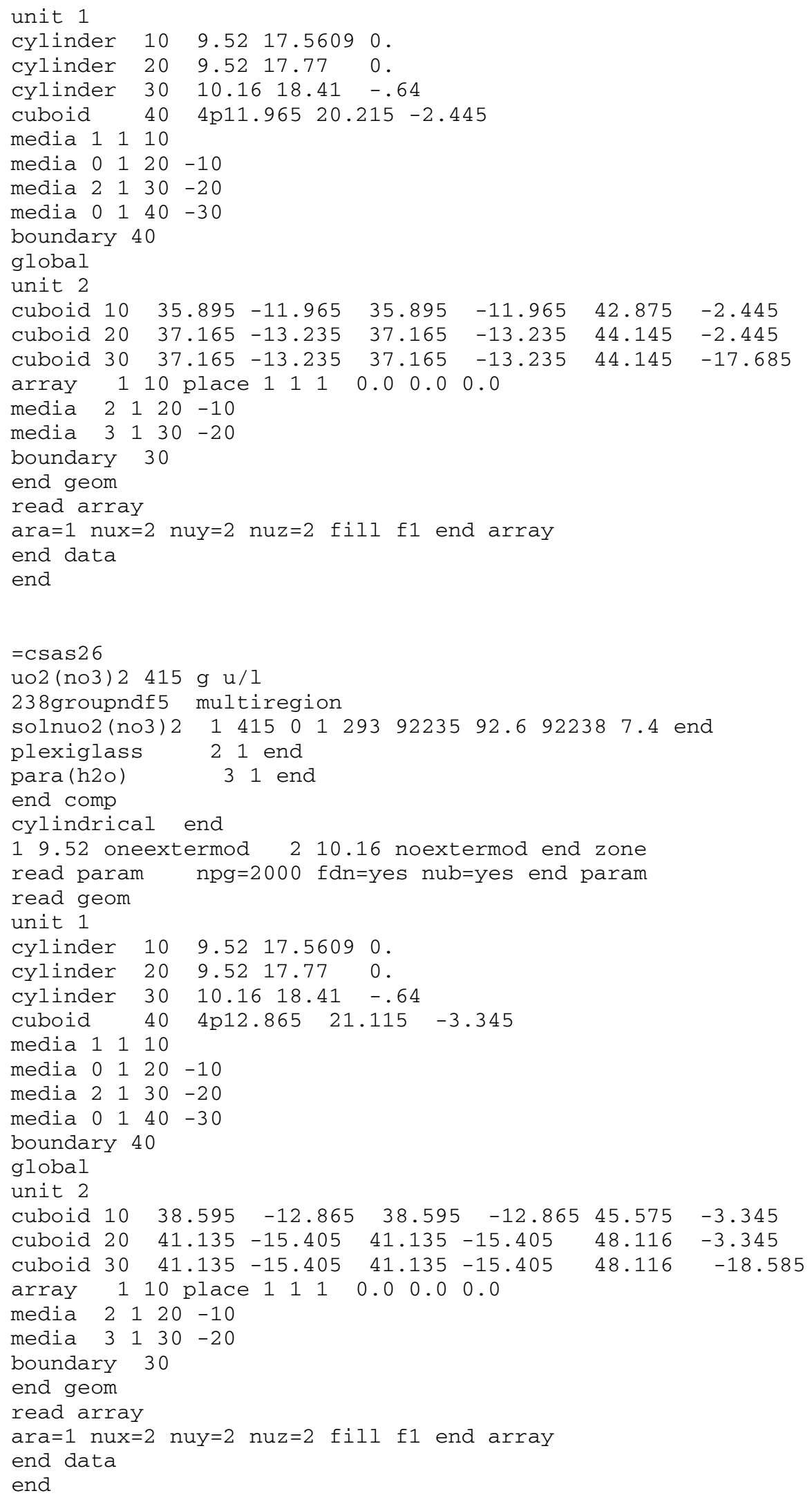




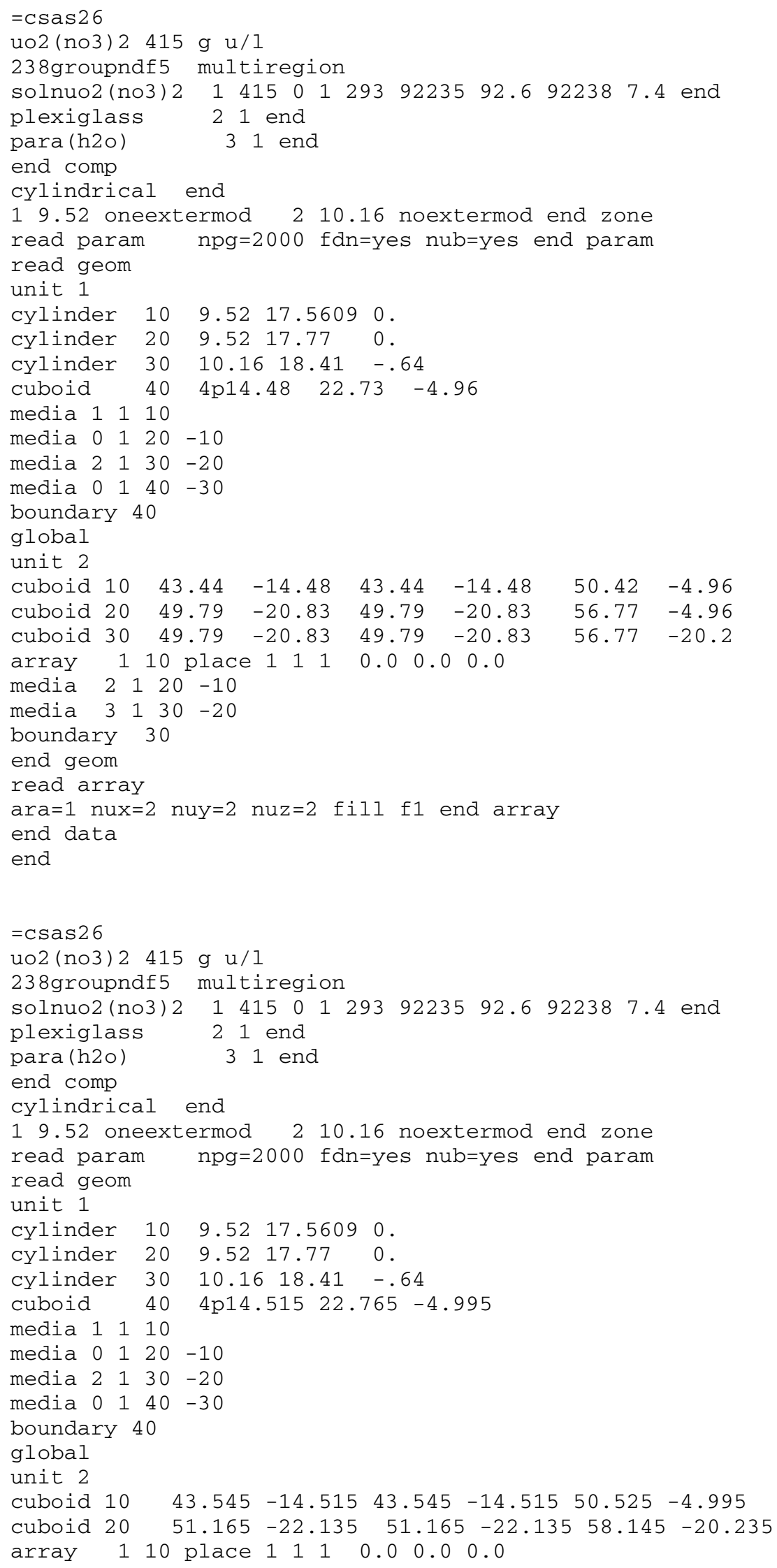




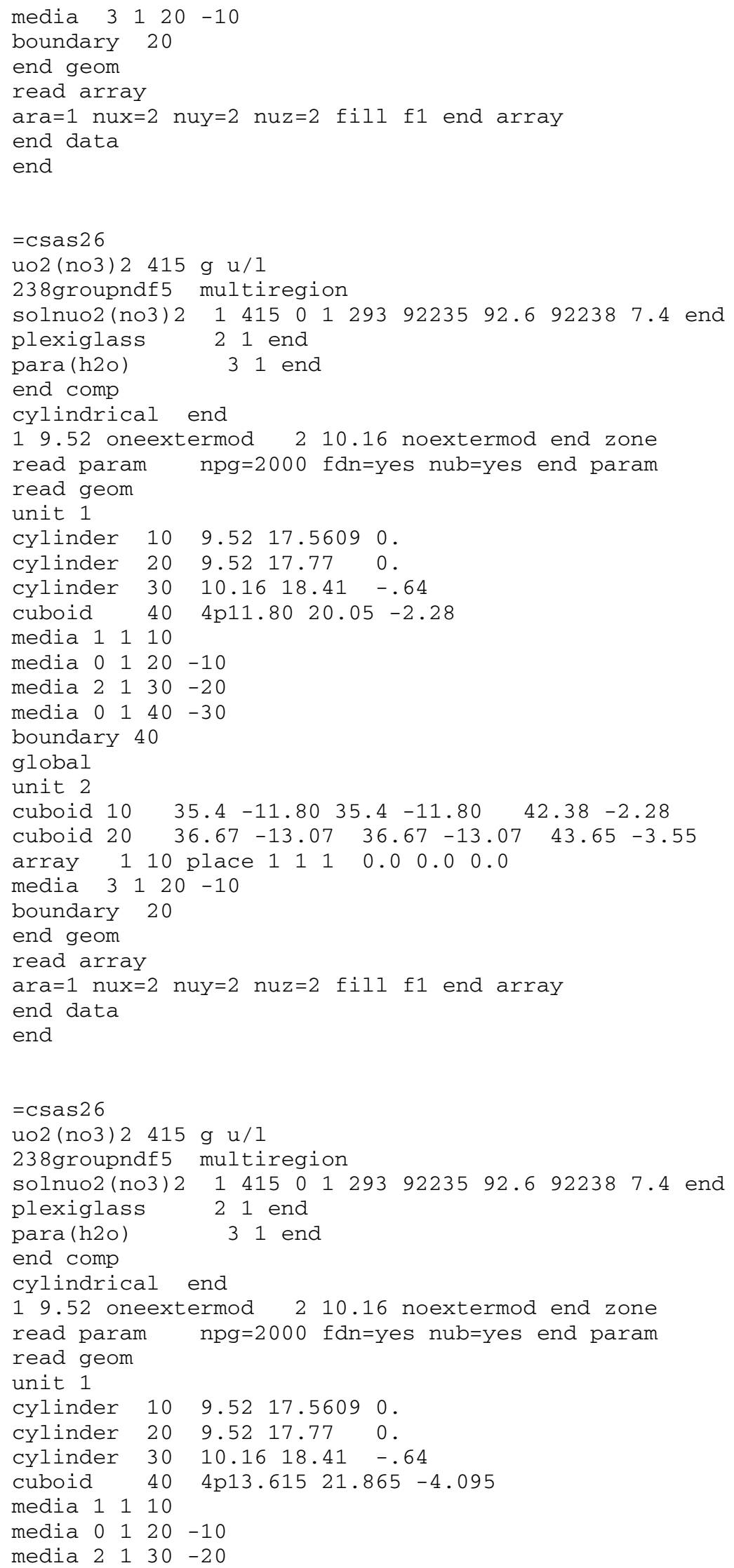


media $0 \begin{array}{llll} & 4 & 4 & -30\end{array}$

boundary 40

global

unit 2

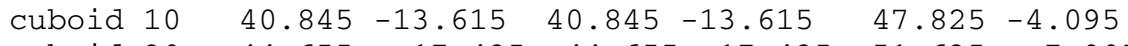

cuboid $20 \quad 44.655 \quad-17.425 \quad 44.655-17.425 \quad 51.635 \quad-7.905$

array 110 place 11110.00 .00 .0

media $3 \quad 120-10$

boundary 20

end geom

read array

ara=1 nux=2 nuy=2 nuz=2 fill fl end array

end data

end 
Table B.6. Table 6 input data

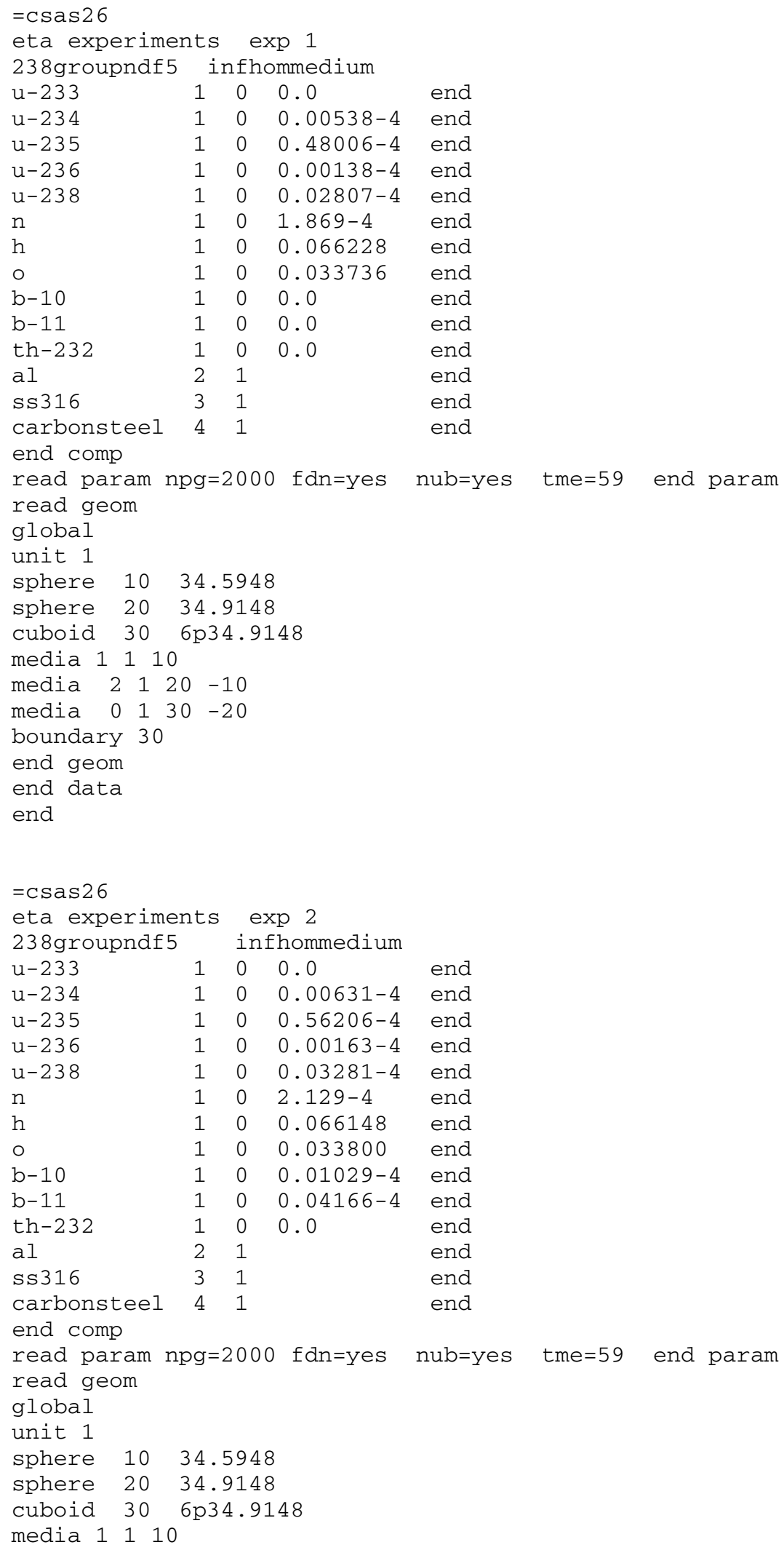




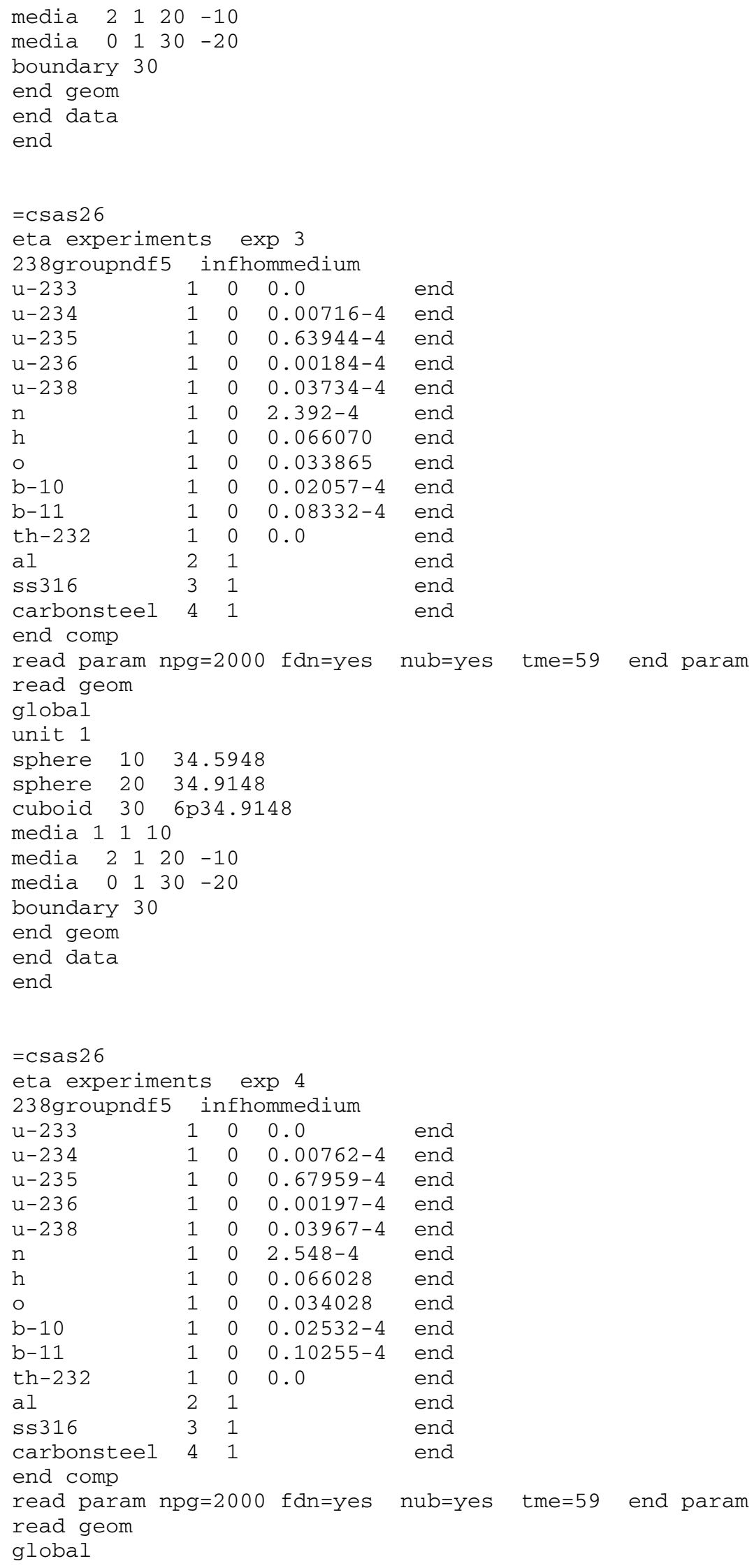




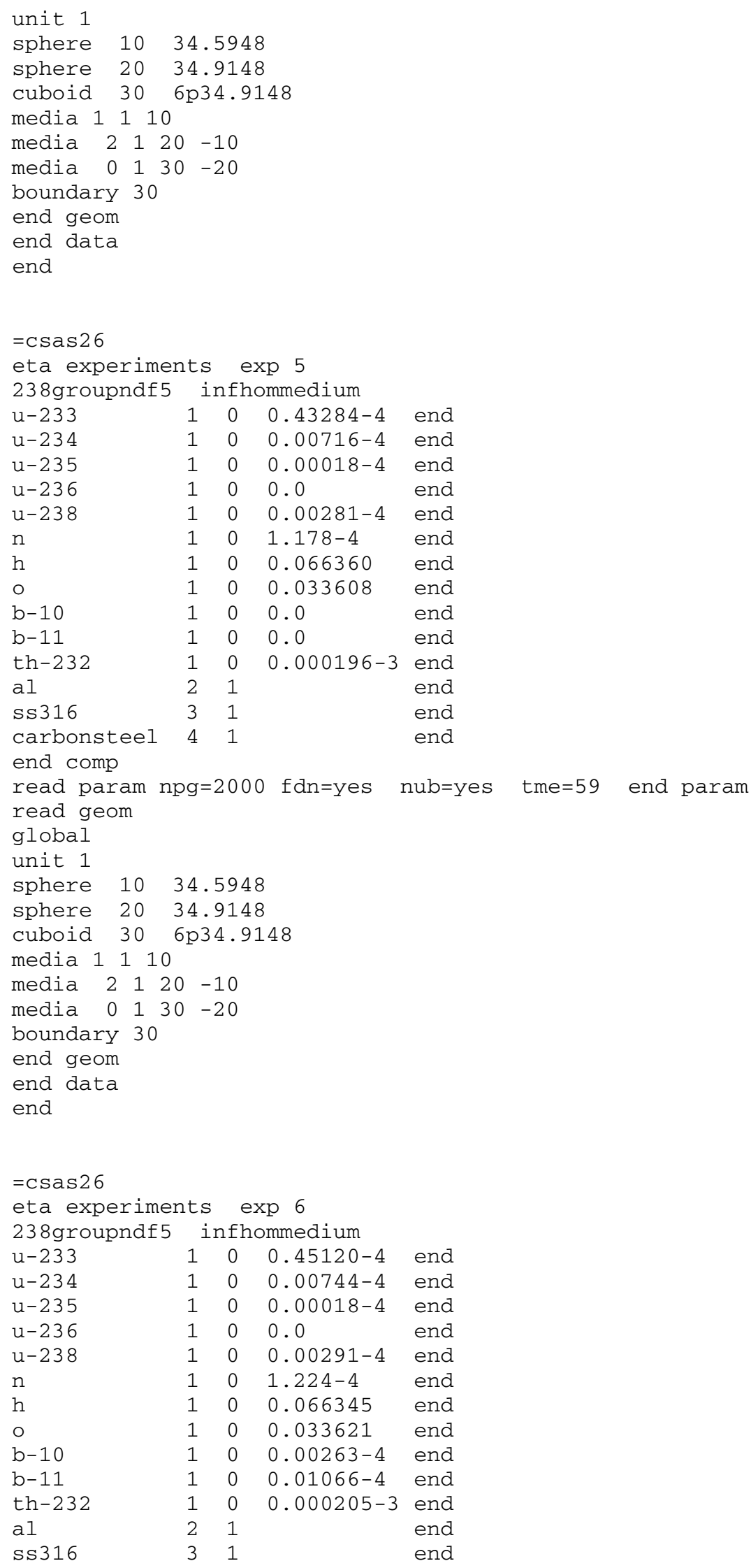




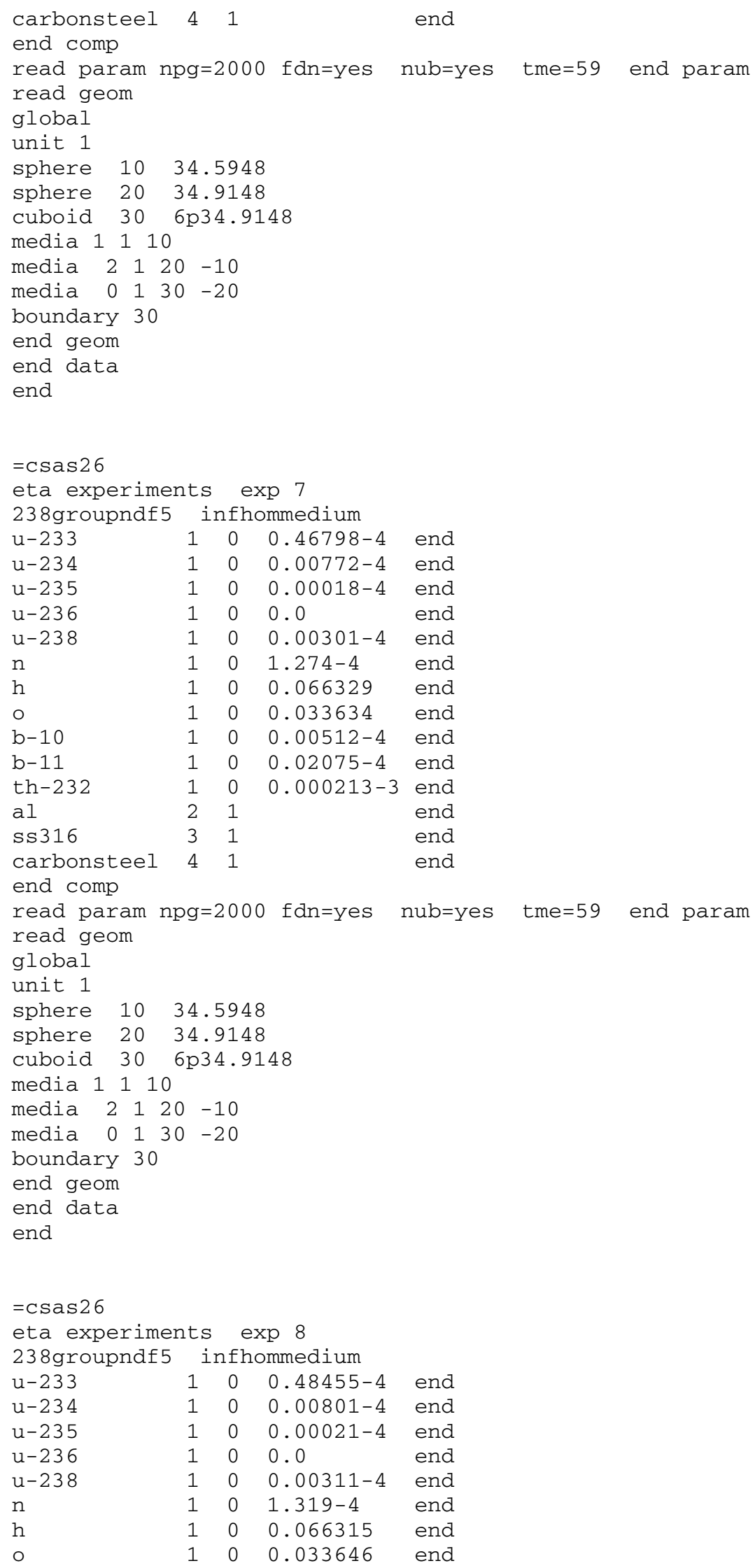




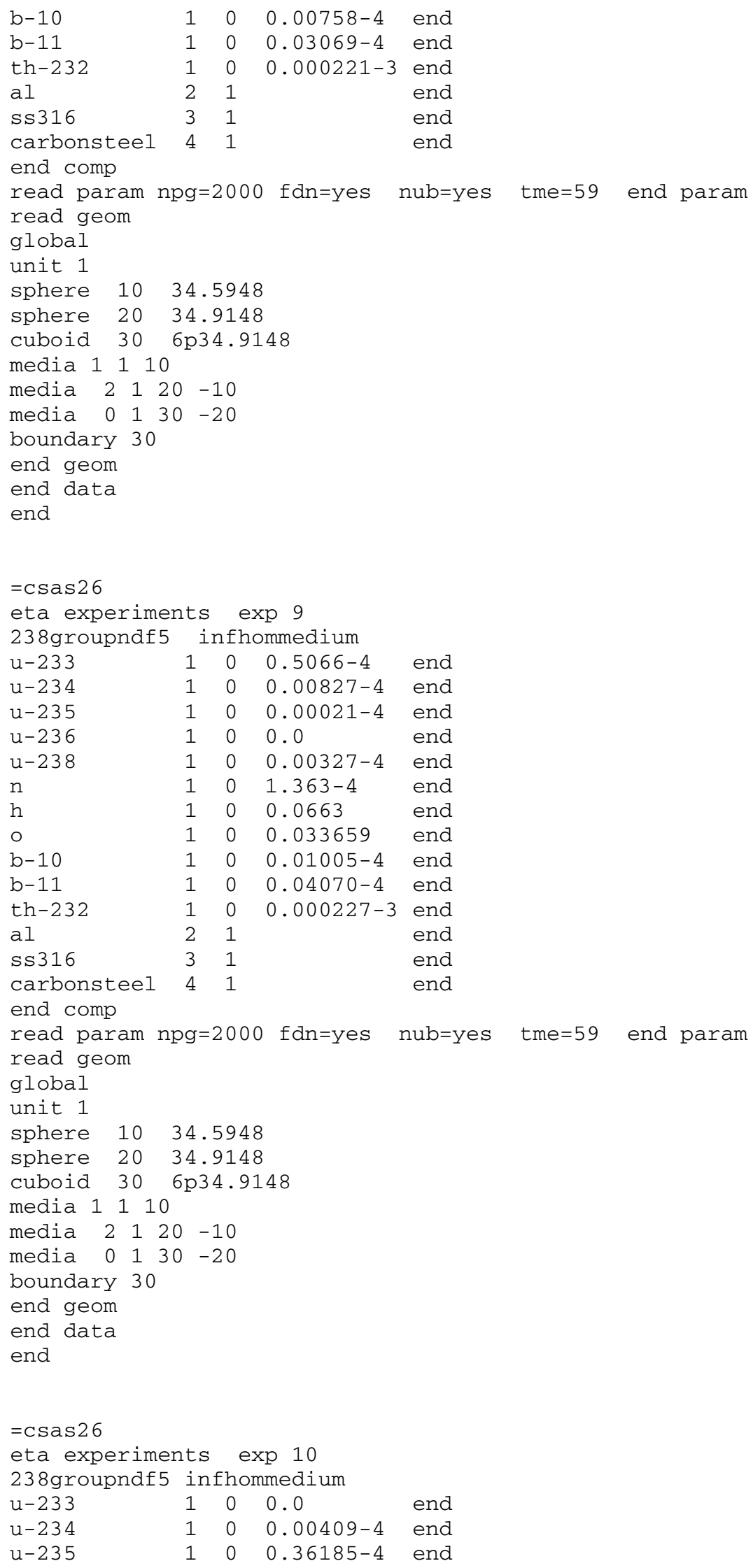




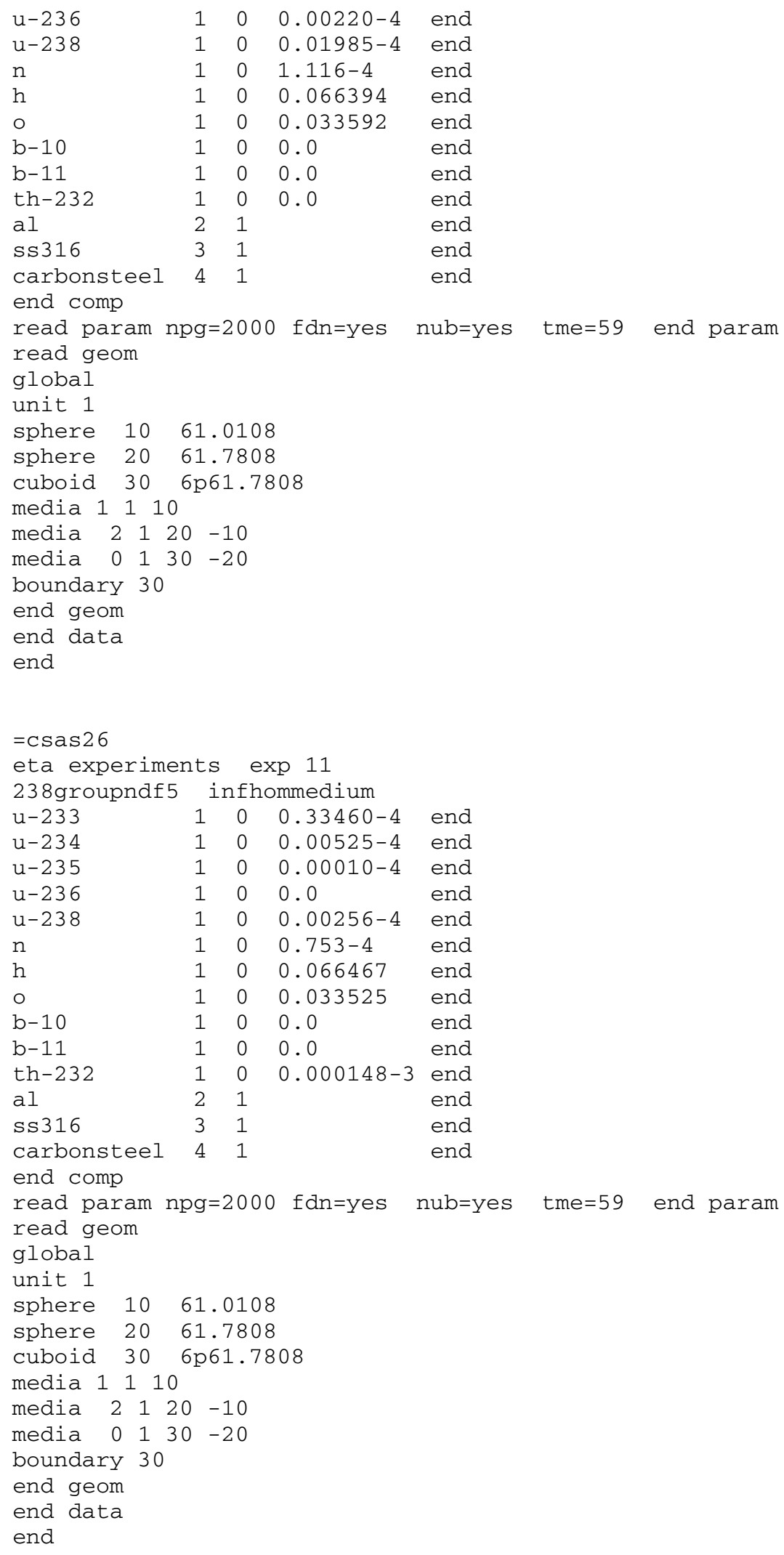




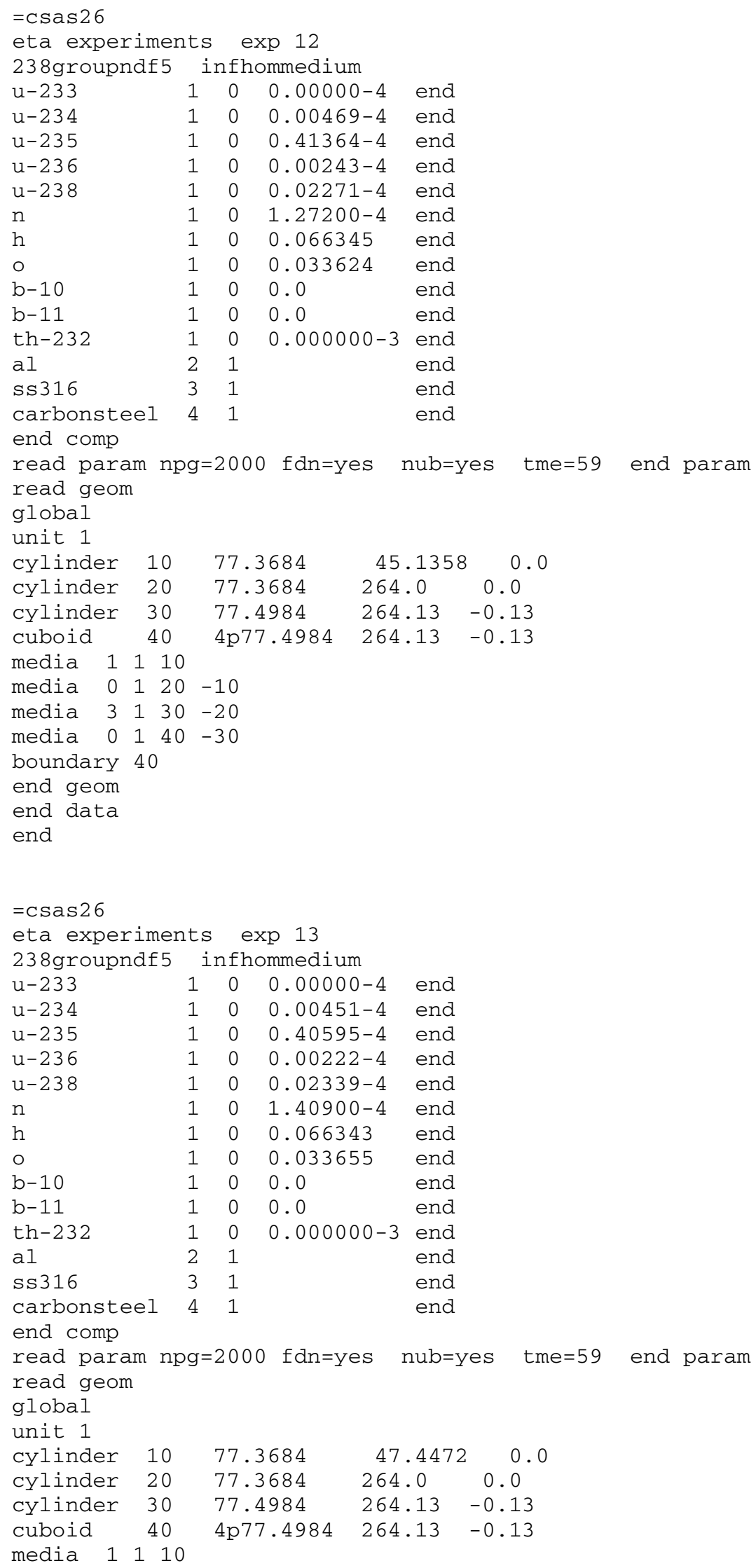




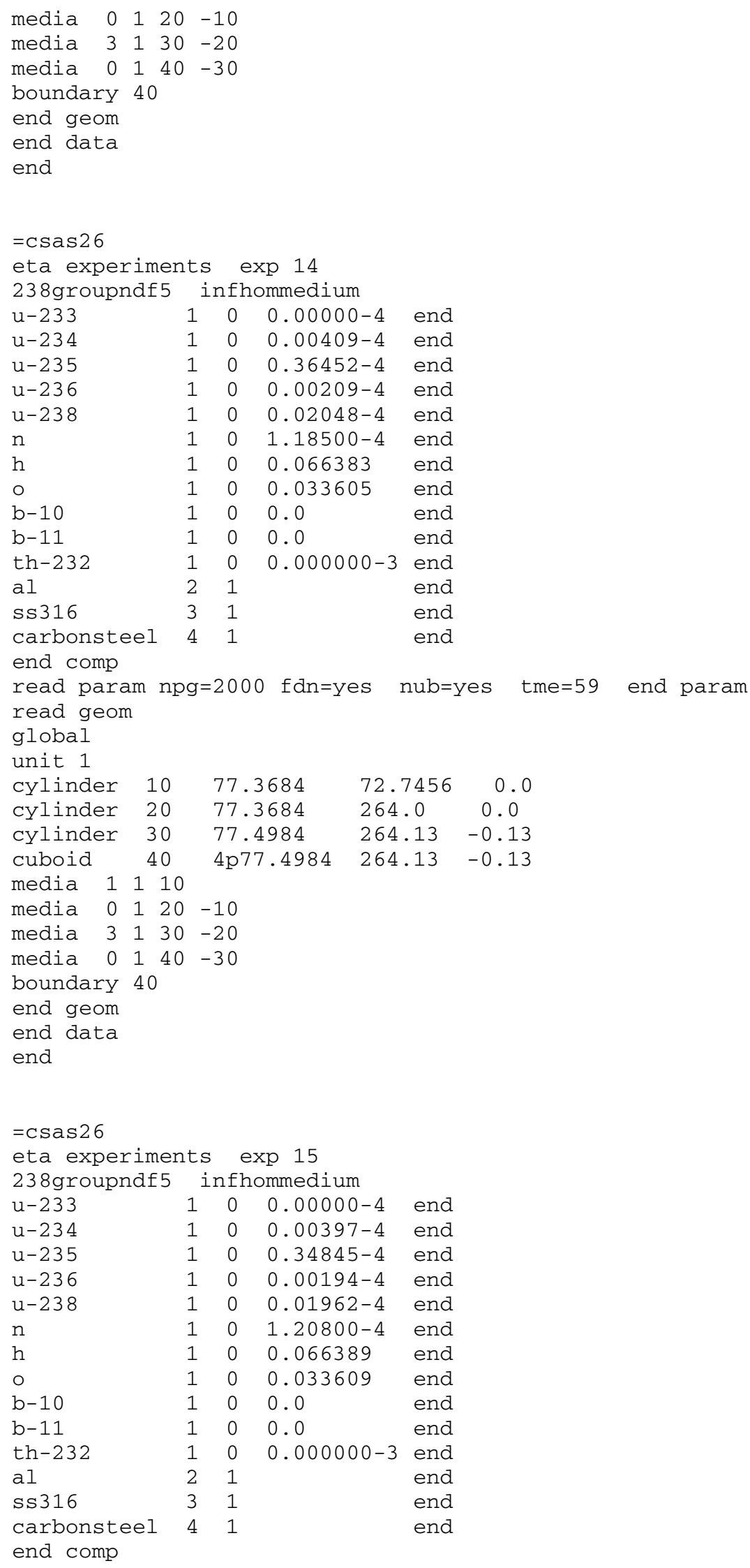




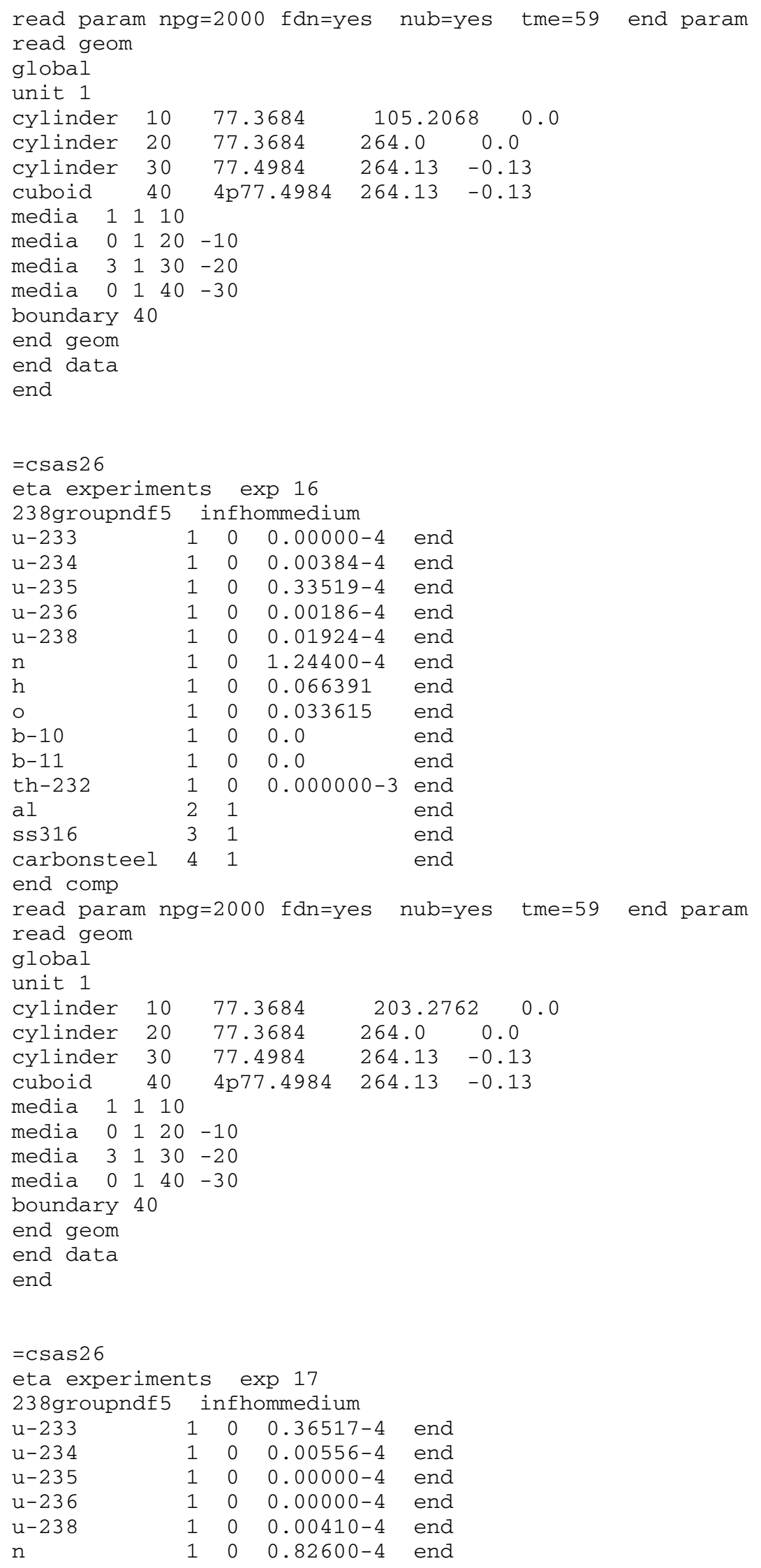




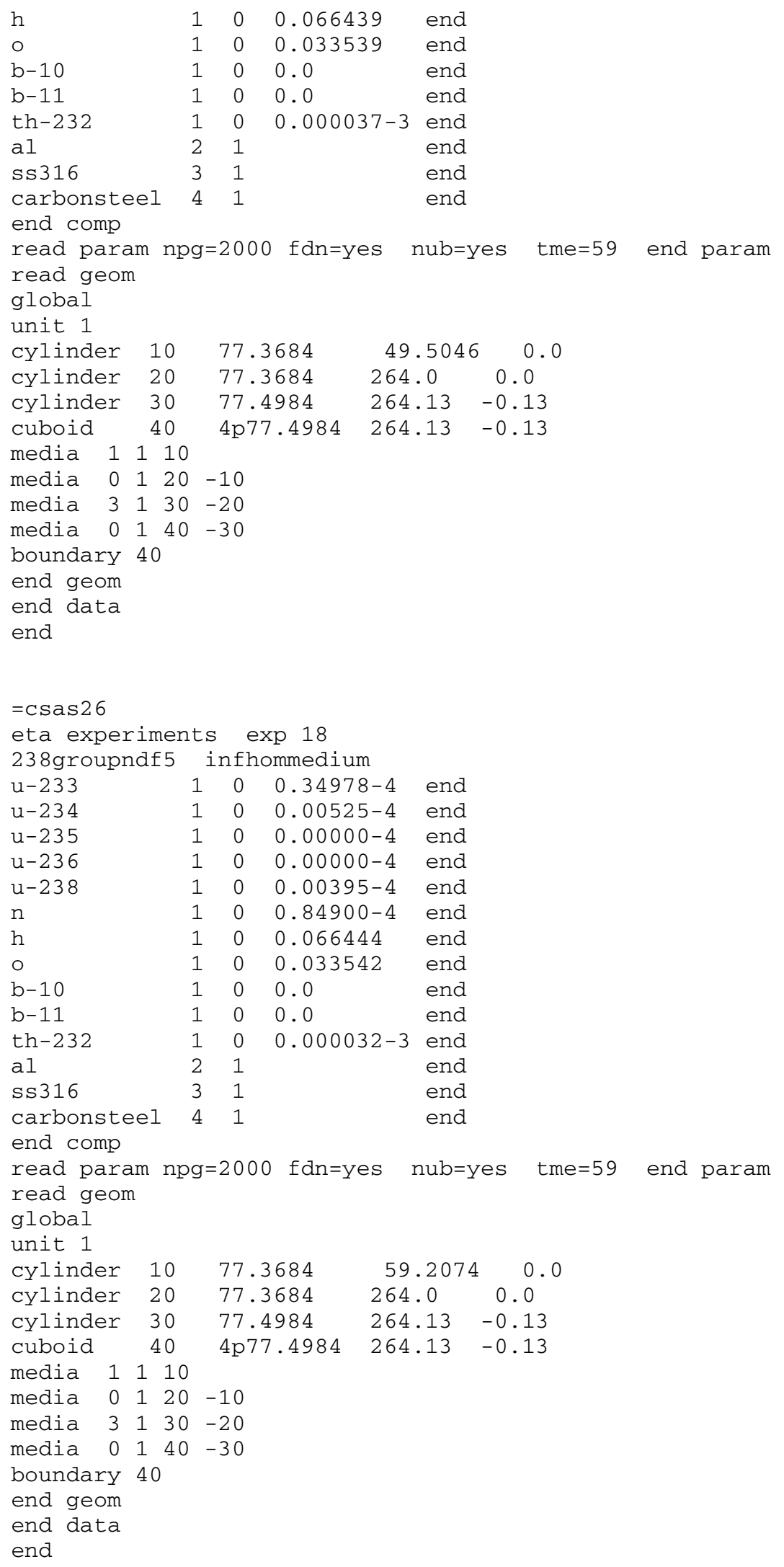




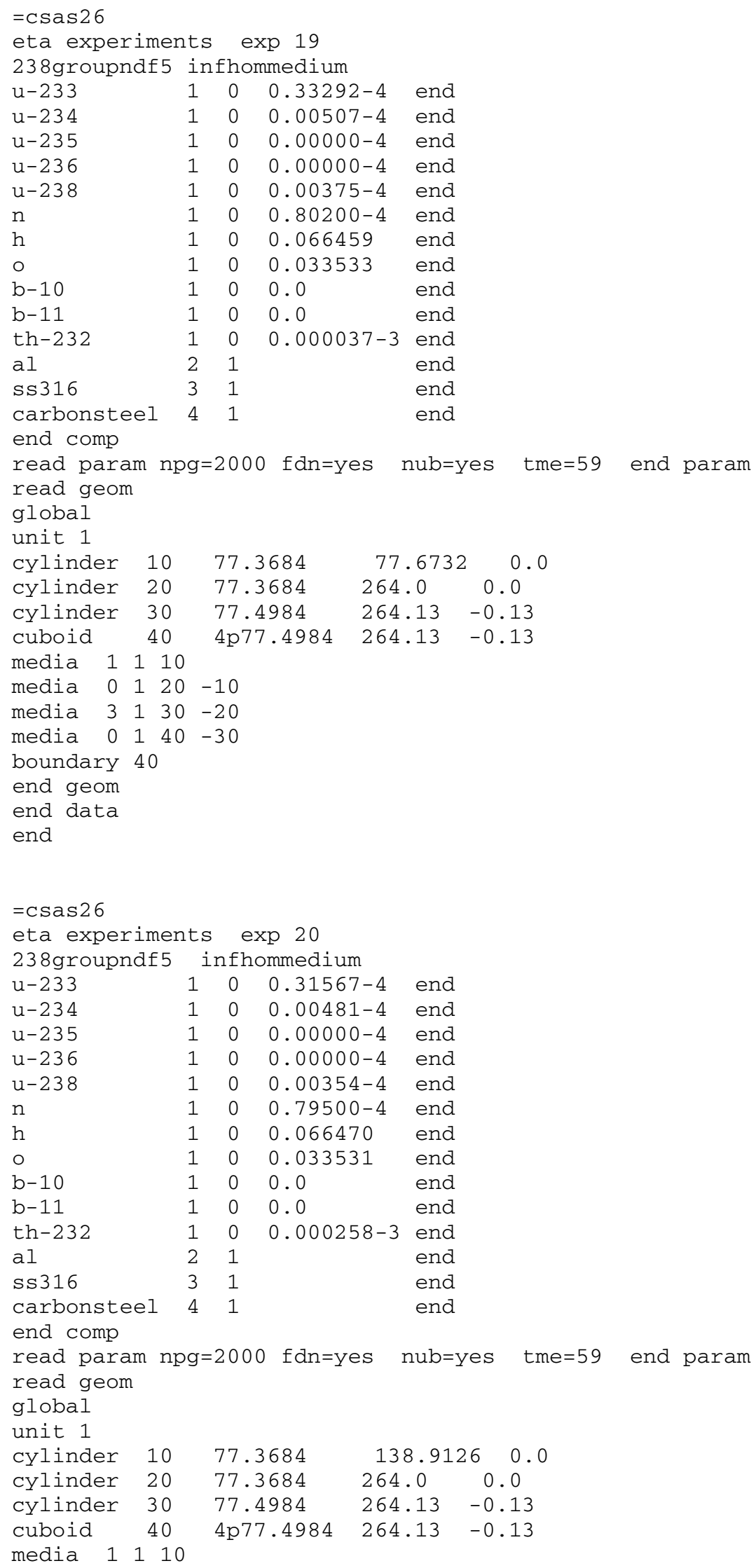




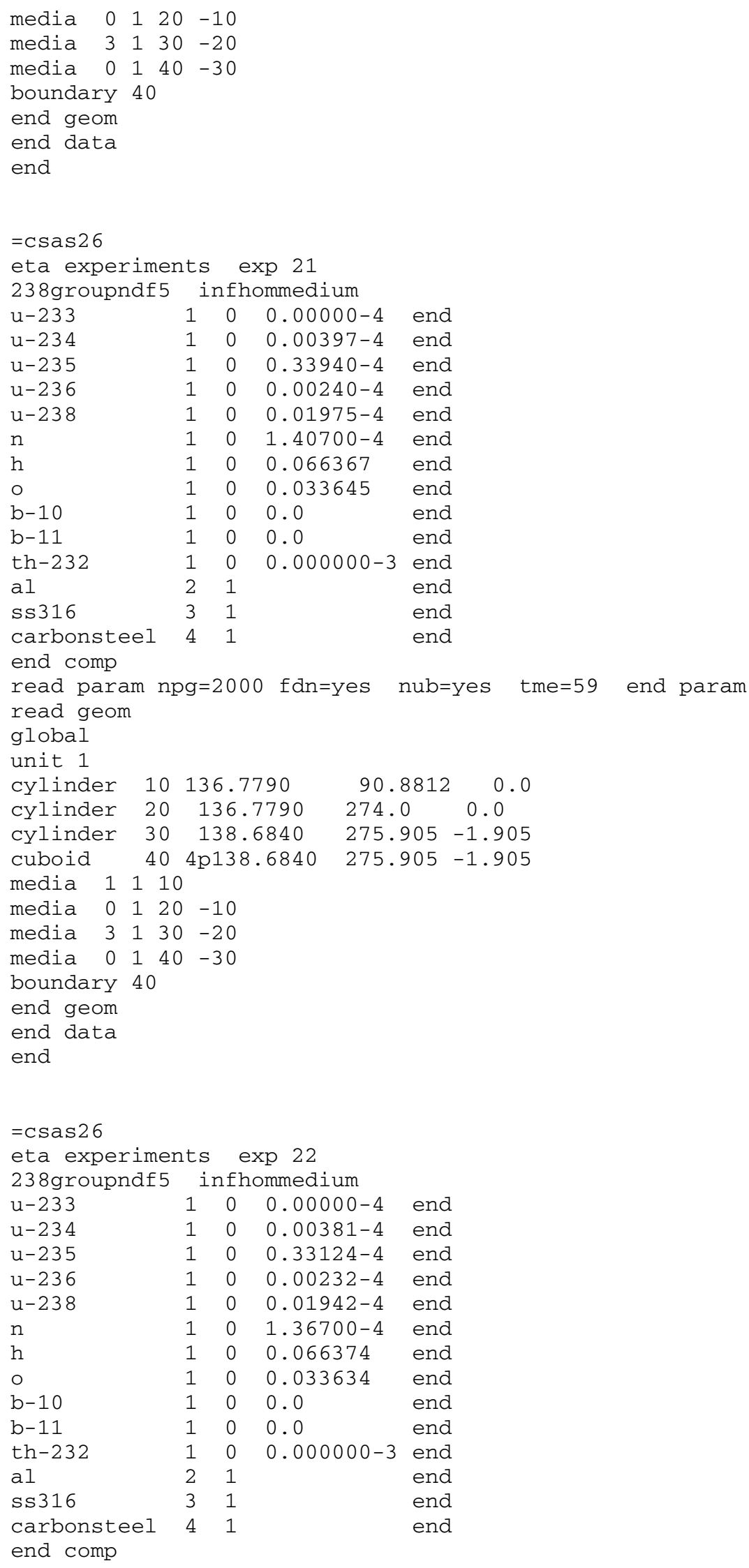




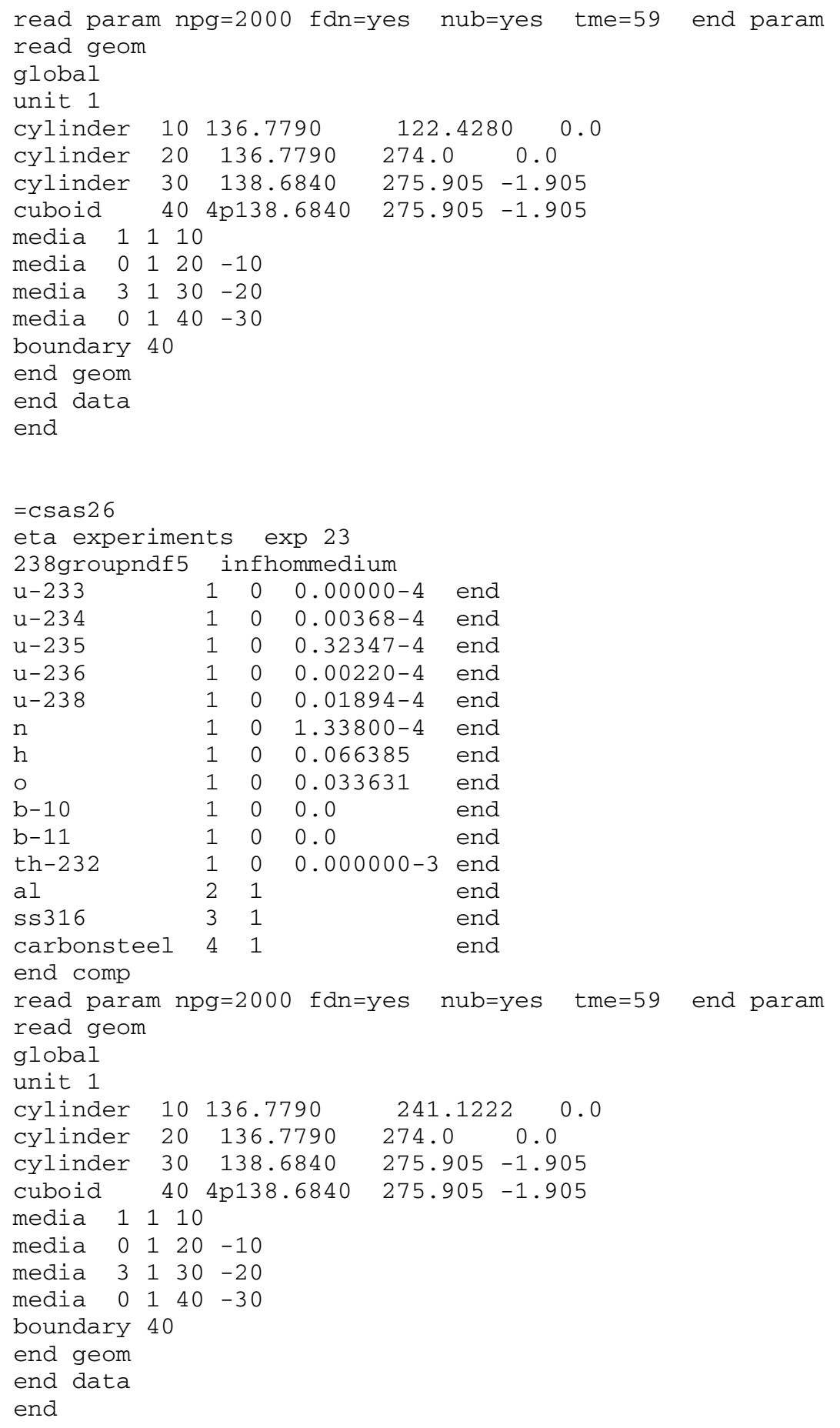


ORNL/TM-2001/110

\section{INTERNAL DISTRIBUTION}

1. S. M. Bowman, 6011, MS-6370

2. B. L. Broadhead, 6011, MS-6370

3. K. J. Carroll, 9110, MS-8238

4. W. C. Carter, 6011, MS-6370

5. M. D. DeHart, 6011, MS-6370

6. M. E. Dunn, 6011, MS-6370

7. K. R. Elam, 6011, MS-6370

8. R. J. Ellis, 6025, MS-6363

9. M. B. Emmett, 6011, MS-6370

10. P. B. Fox, 6011, MS-6370

11. I. C. Gauld, 6011, MS-6370

12. J. C. Gehin, 6025, MS-6363

13. S. Goluoglu, 6011, MS-6370

14. N. M. Greene, 6011, MS-6370

15. J. N. Herndon, 4500N, MS-6228

16. D. F. Hollenbach, 6011, MS-6370

17. C. M. Hopper, 6011, MS-6370

18. D. T. Ingersoll, 6025, MS-6363

19. B. L. Kirk, 6025 , MS-6362

20. L. C. Leal, 6011, MS-6370
21. J. B. Manneschmidt, 6011, MS-6370

22. B. D. Murphy, 6011, MS-6370

23. C. V. Parks, 6011, MS-6370

24. L. M. Petrie, 6011, MS-6370

25. R. T. Primm, III, 6025, MS-6363

26. B. T. Rearden, 6011, MS-6370

27. J. P. Renier, 6025, MS-6363

28. T. E. Valentine, 6025, MS-6362

29. J. C. Wagner, 6011, MS-6370

30. Central Research Library, 4500N, MS-6191

31. ORNL Laboratory Records - RC, 4500N, MS-6254

\section{EXTERNAL DISTRIBUTION}

32. F. M. Alcorn, BWX Technologies, Inc., Naval Nuclear Fuel, 212 Windsor Rd., Lynchburg, VA 24502

33. R. E. Anderson, Los Alamos National Laboratory, PO Box 1663, NIS-6, MS J562, Los Alamos, NM 87545

34. W. L. Andrews, Defense Nuclear Facilities Safety Board, 625 Indiana Ave., Washington, DC 20004

35. C. Apperson, Westinghouse Savannah River Company, Savannah River Site, Bldg. 773-11A, Aiken, SC 29808-0001

36. A. B. Barto, U.S. Nuclear Regulatory Commission, NMSS/SFPO, MS O13 D13, Washington, DC 20555-0001

37. R. N. Blomquist, Argonne National Laboratory, RA/208, 9700 S. Case Ave., Argonne, IL 60439-4842

38. J. B. Briggs, INEEL, PO Box 1625, MS-3855, Idaho Falls, ID 83402 
39. T. Burns, Defense Nuclear Facilities Safety Board, 625 Indiana Ave., Washington, DC 20004

40. R. D. Busch, University of New Mexico, Chemical and Nuclear Engineering Department, FEC 209, Albuquerque, NM 87131-1341

41. D. E. Carlson, U.S. Nuclear Regulatory Commission, NMSS/SFPO, MS O13 D13, Washington, DC 20555-0001

42. P. Cousinou, Institute of Protection and Nuclear Safety, B. P. 6 - 92265 Fontenay-AuxRoses, Cedex, France

43. D. R. Damon, U.S. Nuclear Regulatory Commission, NMSS/FCSS, MS T8 A33, Washington, DC 20555-0001

44. J. R. Davis, U.S. Nuclear Regulatory Commission, NMSS/FCSS, MS T8 A33, Washington, DC 20555-0001

45. H. L. Dodds, University of Tennessee, Nuclear Engineering Dept., 214 Pasqua Engineering Bldg., Knoxville, TN 37922

46. T. W. Doering, 1180 Town Center Drive, Las Vegas, NV 89144

47. H. D. Felsher, U.S. Nuclear Regulatory Commission, NMSS/FCSS, MS T8 A33, Washington, DC 20555-0001

48. J. R. Felty, Science Applications Int'l Corp., 2418 N. Dickerson St., Arlington, VA 22207

49. I. E. Fergus, U.S. Department of Energy, EH-22, 20300 Century Blvd., Germantown, MD 20874

50. E. K. Fujita, Argonne National Laboratory, 9700 South Cass Avenue, RA/208, Argonne, IL 60439-4842

51. A. S. Garcia, U.S. Department of Energy, Idaho Operations Office, 850 Energy Dr., MS 1154, Idaho Falls, ID 83401-1563

52. F. Gee, U.S. Nuclear Regulatory Commission, NMSS/FCSS, MS T8 A33, Washington, DC 20555-0001

53. A. S. Giantelli, U.S. Nuclear Regulatory Commission, NMSS/SFPO/TRD, MS O13 D13, Washington, DC 20555-0001

54. G. R. Handley, BWXT-Y12, PO Box 2009, Bldg. 9110, MS-8238, Oak Ridge, TN 37831-8238

55. S. T. Huang, Lawrence Livermore National Laboratory, MS L-128, PO Box 808, Livermore, CA 94551

56. S. Keeton, Lawrence Livermore National Laboratory, PO Box 808, MS L-634, Livermore, CA 94551-0808

57. E. F. Kendall, U.S. Department of Energy, Oak Ridge Operations Office, YSO, PO Box 2001, Oak Ridge, TN 37831

58. K. D. Kimball, NISYS Corporation, 6055 Atlantic Blvd., Suite G-2, Norcross, GA 30071

59. R. Knief, XE Corporation (XEC), PO Box 90818, Albuquerque, NM 87199-0818

60. M. A. Lee, U.S. Department of Energy, Livermore Operations, 7000 East ST., PO Box 808, L-293, Livermore, CA 94551-0808

61. L. J. Lessler, U.S. Nuclear Regulatory Commission, NMSS/FCSS/FCOB, MS T8 A33, Washington, DC 20555-0001

62. J. R. Liaw, Argonne National Laboratory, Bldg. 208, 9700 S. Cass Avenue, Argonne, IL 60439 
63. R. Libby, Pacific Northwest National Laboratory, PO Box 999, M/S K7-74, Richland, WA 99352

64. J. J. Lichtenwalter, BWXT Y-12, PO Box 2009, Bldg. 9110, MS-8238, Oak Ridge TN 37831-8238

65. R. C. Little, Los Alamos National Laboratory, MS F6663, PO Box 1663, Los Alamos, NM 87545

66. Y. Y. Liu, Argonne National Laboratory, 9700 S. Cass Avenue, 308/D108, Argonne, IL 60439

67. C. D. Manning, Framatome Advanced Nuclear Power, 2101 Horn Rapids Road, Richland, WA 99352-5102

68. R. McBroom, U.S. Department of Energy, Oak Ridge Operations Office, YSO, PO Box 2001, Oak Ridge, TN 37831

69. J. McKamy, U.S. Department of Energy, ED-34, 19901 Germantown Road, Germantown, MD 20874-1290

70. R. D. McKnight, Argonne National Laboratory, 9700 S. Cass Ave., Argonne, IL 604394842

71. T. P. McLaughlin, Los Alamos National Laboratory, PO Box 1663, Los Alamos, NM 87545

72. D. E. I. Mennerdal, E M Systems, Starvãgen 12, Täby, SWEDEN S-18357

73. R. D. Montgomery, Nuclear Fuel Services, Inc., 1205 Banner Hill Rd., Erwin, TN 37650

74. L. Montierth, Idaho National Engineering and Environmental Laboratory, PO Box 1625, MS 3458, Idaho Falls, ID 83415-3458

75. D. C. Morey, U.S. Nuclear Regulatory Commission, NMSS/FCSS/FCOB, MS T8 A33, Washington, DC 20555-0001

76. R. D. Mosteller, Los Alamos National Laboratory, MS J562, Los Alamos, NM 87545

77. J. A. Morman, Argonne National Laboratory, 9700 South Cass Avenue, RA/208, Argonne, IL 60439-4842

78. K. A. Niemer, Duke Engineering \& Services, 400 S. Tryone St., WC26B, PO Box 1004, Charlotte, NC 28201-1004

79. H. Okuno, Japan Atomic Energy Research Institute, 2-4 Shirakara-shirane, Tokai-mura, Ibaraki-ken, JAPAN 319-1195

80. S. A. Parra, Lawrence Livermore National Laboratory, PO Box 808, MS L-128, Livermore, CA 94551

81. L. E. Paulson, Global Nuclear Fuel - America, PO Box 780, Castle Hayne Road, M/C K26, Wilmington, NC 28402-0780

82. S. Payne, U.S. DOE/AL/SASD, PO Box 5400, Albuquerque, NM 87185-5400

83. V. A. Perin, U.S. Nuclear Regulatory Commission, NMSS/DWM/HLWB, MS T10 K8, Washington, DC 20555-0001

84. R. E. Pevey, University of Tennessee, Nuclear Engineering Dept., 214 Pasqua Engineering Bldg., Knoxville, TN 37922

85. J. Philbin, Sandia National Laboratory, PO Box 5800, Albuquerque, NM 87185-1143

86. A. W. Prichard, Pacific Northwest National Laboratory, PO Box 999, MSIN: K8-34, Richland, WA 99352

87. V. L. Putman, Idaho National Engineering and Environmental Laboratory, PO Box 1625, MS 3458, Idaho Falls, ID 83415-3458 
88. M. Rahimi, U.S. Nuclear Regulatory Commission, NMSS/DWM, MS T7 F3, Washington, DC 20555-0001

89. M. Brady Raap, Battelle Pacific Northwest National Lab., PO Box 999 / MS K8-34, Richland, WA 99352

90. C. Reed, BWX Technologies, Inc., Naval Nuclear Fuel, PO Box 785, Lynchburg, VA 24505

91. T. A. Reilly, Westinghouse Safety Management Solutions, 1993 South Centennial Dr., Aiken, SC 29803

92. R. C. Robinson, BWXT-Y12, PO Box 2009, Bldg. 9110, MS-8238, Oak Ridge, TN 37831-8238

93. C. T. Rombough, CTR Technical Services, Inc., 950 Sugarloaf Rd., Manitou Springs, CO 80829

94. B. Rothleder, U.S. Department of Energy, EH-31, 19901 Germantown Rd., Germantown, MD 20874-1290

95. C. A. Snyder, Westinghouse Electric Co., PO Drawer R, Columbia, SC 29250

96. J. Stewart, DETR RMTD 2/33, Great Minster House, 76 Marsham Street, LONDON, UK, SW1P 4DR

97. J. T. Taylor, Idaho National Engineering and Environmental Laboratory, PO Box 1625, MS 3458, Idaho Falls, ID 83415-3458

98. C. Tripp, U.S. Nuclear Regulatory Commission, NMSS/FCSS, MS T8 A33, Washington, DC 20555-0001

99. D. A. Thomas, Framatome Cogema Fuels, 1261 Town Center Drive, Las Vegas, NV 89124

100. J. A. Thornton, Duke Engineering \& Services, 400 S. Tyrone St., WC26B, PO Box 1004, Charlotte, NC 28201-1004

101. W. W. Tippl, Framatome-ANP GmbH, Department NDM3, Postfach 1010 63, Berliner Strasse 295-303, D-63010 Offenbach am Main / Germany

102. H. Toffer, Fluor Daniel Northwest, Inc., PO Box 1050, MSIN B4-44, 1100 Jadwin Ave., Richland, WA 99352-1050

103. E. F. Trumble, Westinghouse Safety Management Solutions, PO Box 5388, 1993 South Centennial Dr., Aiken, SC 29803

104. A. P. Ulses, U.S. Nuclear Regulatory Commission, NRR/DSSA, MS O10 B3, Washington, DC 20555-0001

105. M. Wangler, Office of Site Operations, EM-5/CLV-1059, U.S. Department of Energy, 19901 Germantown Road, Germantown, MD 20874-1290

106. L. Wetzel, BWX Technologies, Inc., Naval Nuclear Fuel, PO Box 785, Lynchburg, VA 24505

107. S. A. Whaley, U.S. Nuclear Regulatory Commission, NMSS/FCSS, MS T8 A33, Washington, DC 20555-0001

108. B. H. White IV, U.S. Nuclear Regulatory Commission, NMSS/SFPO, MS O13 D13, Washington, DC 20555-0001

109. R. E. Wilson, U.S. Department of Energy, 10808 Highway 93,Unit A, Golden, CO 80403

110. C. J. Withee, U.S. Nuclear Regulatory Commission, NMSS/SFPO, MS O13 D13, Washington, DC 20555-0001 